$$
\begin{aligned}
& \equiv 0 \\
& \equiv \infty \\
& \equiv N \\
& 0 \\
& \text { 跤 } \\
& \text { 든 } \\
& \geqslant 0 \\
& \text { D } \\
& \text { 缺 } \\
& 3 \\
& \equiv
\end{aligned}
$$



.

. 

. 



\title{
TRANSFORMATIONS OF SURFACES
}

\author{
BY \\ LUTHER PFAHLER EISENHART \\ PROFESSOR OF MATHEMATICS \\ IN PRINCETON UNIVERSITY
}

PUBLISHED WITH THE COOPERATION

OF THE NATIONAL RESEARCH COUNCIL

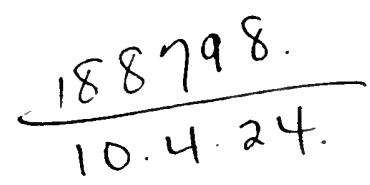

\section{PRINCETON}

PRINCETON UNIVERSITY PRESS

LONDON : HUMPHREY MILFORD

OXFORD UNIVERSITY PRESS 


\section{QA 601 \\ E 37}




\section{Preface}

During the past twenty-five years many of the advances in differential geometry of surfaces in euclidean space have had to do with transformations of surfaces of a given type into surfaces of the same type. Before this period Bianchi and Bäcklund had established their transformations of a pseudospherical surface into pseudospherical surfaces, the essential feature of which is that a given surface and any transform are the focal surfaces of a $W$ congruence. Furthermore, Bianchi (Lezioni, § 383) established the so-called theorem of permutability of such transformations; that is, if $S_{1}$ and $S_{2}$ are two transforms of $S$ there can be found a fourth surface $S^{\prime}$ which is a transform of both $S_{1}$ and $S_{2}$. Later (footnote 41) he showed that there is a similar theorem of permutability for transformations such that a given surface and a transform are the focal surfaces of a $W$ congruence.

In 1899 Guichard (f. n. 100) announced two theorems concerning the detormations of a quadric of revolution which led to the transformations of Darboux of isothermic surfaces. In such a transformation a surface and its transform are the sheets of the envelope of a two-parameter family of spheres with the lines of curvature corresponding on the two sheets. Families of spheres of this type are associated with cyclic systems of circles, which Ribaucour was the first to investigate extensively, and consequently two surfaces which are the sheets of the envelope of a two-parameter family of spheres with lines of curvature in correspondence are said to be in the relation of a transformation of Ribaucour. Bianchi showed that for transtormations of Ribaucour (f. n. 54) and in particular for transformations of Darboux of isothermic surfaces (f. n. 64) there is a theorem of permutability in the sense mentioned above.

When two surfaces are in the relation of a transformation of Ribancour, the lines joining corresponding points on the surfaces form a congruence whose developables meet the surfaces in their 
lines of curvature. The transformations of Darboux are a particular case of transformations of conjugate systems, or nets, with equal point invariants, such that the lines joining corresponding points of such a net and a transform form a congruence whose developables meet the surfaces on which the nets lie in these nets and corresponding points of the two nets divide harmonically the focal segment of the corresponding lines of the congruence; these transformations were first studied by Koenigs (f. n. 17) and are called transformations $K$.

When two nets and the congruence of the joins of corresponding points are so related that the developables of the congruence meet the surfaces on which the nets lie in these nets, we say that either net is obtained from the other by a fundamental transformation, or more briefly a transformation $F$. We have remarked that transformations of Ribaucour and transformations $K$ are of this type. The general transformations $F$ for 3-space have been studied by Jonas and the author (f. n. 15) and a theorem of permutability of these transformations has been established.

Most, if not all, of the transformations which have been developed in recent years are reducible to transformations $F$ or to transformations of the type such that a surface and a transform are focal surfaces of a $W$ congruence. It is the purpose of this book to develop these two types of transformations and thereby to coordinate the results of many investigations.

October, 1922.

Luther Pfahler Eisenhart. 


\section{Contents}

Chapter I.

Section Conjugate nets and congruences. Page

1. Geometric entities of euclidean $n$-space $\ldots \ldots \ldots \ldots \ldots \ldots \ldots \ldots \ldots$

2. Conjugate nets. Normal parameters ................. 3

3. Determination of nets on a surface $\ldots \ldots \ldots \ldots \ldots \ldots \ldots \ldots \ldots \ldots \ldots$

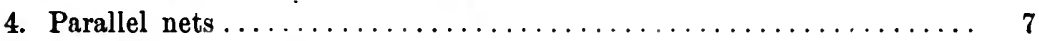

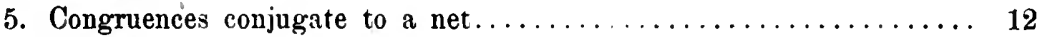

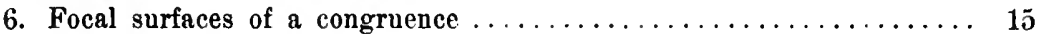

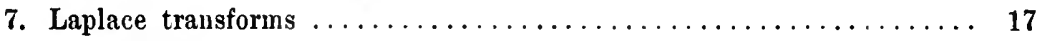

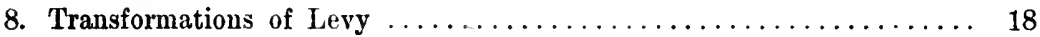

9. Determination of congruences .................... 20

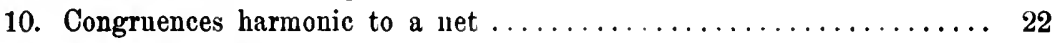

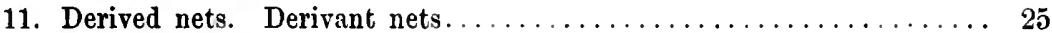

12. Determination of nets harmonic to a given congruence $\ldots \ldots \ldots \ldots .27$

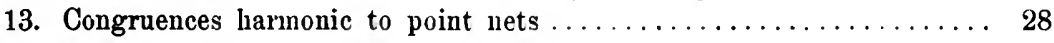

14. Radial transformations $\ldots \ldots \ldots \ldots \ldots \ldots \ldots \ldots \ldots \ldots \ldots \ldots \ldots \ldots$

Cliapter II.

Transformations $F$.

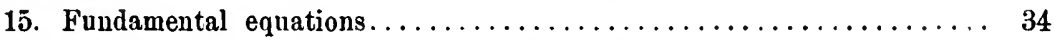

16. Inverse of a transformation $F$. Parallel transformations $F \ldots \ldots \ldots 37$

17. Harmonic congruence of a transformation $F \ldots \ldots \ldots \ldots \ldots \ldots \ldots$

18. Transformations $F$ and radial transformations $\ldots \ldots \ldots \ldots \ldots \ldots \ldots 40$

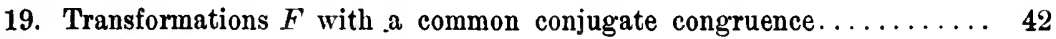

20. Transformations $F$ determined by the same function $\theta \ldots \ldots \ldots \ldots 42$

21. The theorem of permutability of transformations $F \ldots \ldots \ldots \ldots \ldots$.

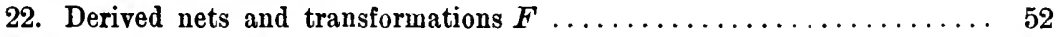

23. Derivant net and derived net of two transformations $F \ldots \ldots \ldots \ldots$ j4

24. The extended theorem of permutability $\ldots \ldots \ldots \ldots \ldots \ldots \ldots \ldots \ldots 5$

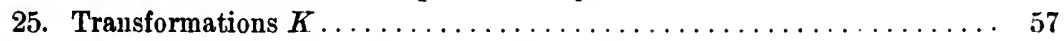

26. Theorem of permutability of transformations $K \ldots \ldots \ldots \ldots \ldots \ldots$

27. Transformations $F$ of applicable nets $\ldots \ldots \ldots \ldots \ldots \ldots \ldots \ldots \ldots \ldots \ldots$

28. Nets corresponding with orthogonality of linear elements ....... 63

Clapter III.

Sequences of Laplace.

29. Homogeneous point coordinates $\ldots \ldots \ldots \ldots \ldots \ldots \ldots \ldots \ldots \ldots \ldots$

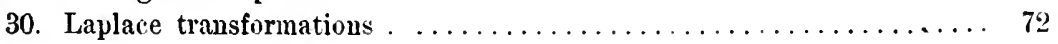


31. Sequences of Laplace $\ldots \ldots \ldots \ldots \ldots \ldots \ldots \ldots \ldots \ldots \ldots \ldots \ldots \ldots$

32. Periodic sequences of Laplace $\ldots \ldots \ldots \ldots \ldots \ldots \ldots \ldots \ldots \ldots \ldots$

33. Harmonic congruences........................ 80

34. Levy sequences of the first order.................. 81

35. Levy sequences of higher order. Derired sequences.......... 83

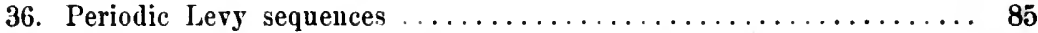

37. Transformations $F$ in lomogreneous coordinates............ 87

38. Transformations $F$ with the same conjugate congruence. Triads of nets 89

39. Theorem of pernutability ...................... 90

Cliapter IV.

\section{Surfaces and congruences in 3-space.}

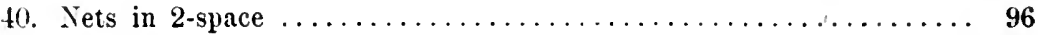

41. Tangential coordinates of a surface in 3 -space........... 97

42. Asymptotic lines $\ldots \ldots \ldots \ldots \ldots \ldots \ldots \ldots \ldots \ldots \ldots \ldots \ldots \ldots \ldots$

43. Nets in 3-space. Point coordinates................. 100

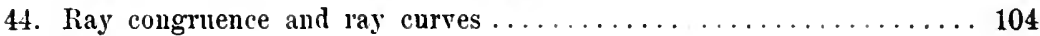

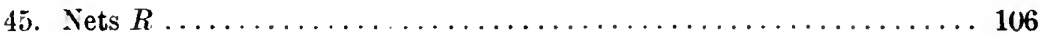

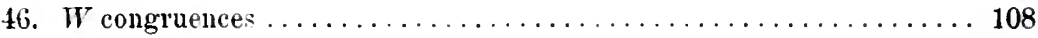

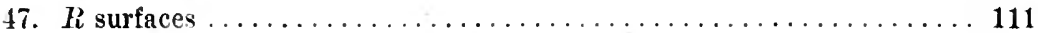

48. $R$ congruences. Transformations $\theta_{1} \ldots \ldots \ldots \ldots \ldots \ldots \ldots \ldots \ldots$

49. Reciprocally derived nets. Transformations $W$ of nets $R \ldots \ldots \ldots \ldots 115$

jo. Theorem of permutability of transformations $W \ldots \ldots \ldots \ldots \ldots \ldots$

\section{( liapter V.}

\section{Transformations $\Omega$. $W$ congruences.}

51. Tangential coordinates of a net. Laplace transforms in tangential

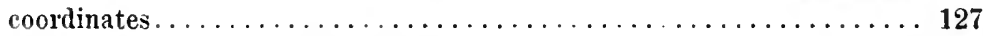

52. Transformations $F$ in taugential coordinates $\ldots \ldots \ldots \ldots \ldots \ldots \ldots \ldots$

33. Transformations $S$ of nets with equal tangential invariants....... 134

כ4. Theorems of perinutability of transformations $\Omega$ and of $W$ congruences 136

5.5. Nets permanent in deformation................... 138

56. Transformations $\Omega$ of permanent nets for which $\tilde{E} \neq 0$ and $\mathbb{E} \neq 0 \ldots 141$

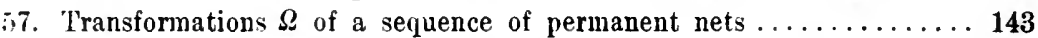

58. Transformations $\Omega$ in point coordinates. Nets in relation $O \ldots \ldots \ldots 146$

59. Transformations $\Omega$ and $K$ of the focal surfaces of a $W$ congruence. . 149

60. Nets with equal point invariants and equal tangential invariants ... 150

Chapter VI.

\section{Orthogonal nets.}

61. Nets $O$ and $p, O$. Congruences $\mathrm{I}$ and $p, \mathrm{I} \ldots \ldots \ldots \ldots \ldots \ldots \ldots$

62. Tets conjugate to congruences $I$ and $p, I \ldots \ldots \ldots \ldots \ldots \ldots$

63. Orthogonal determinants......................... 160

64. Deternination of 0 nets..................... 165 
Section Page

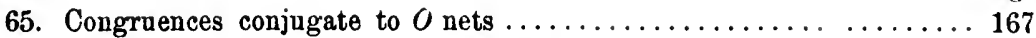

66. Transformations $F$ of an $O$ net for which the conjugate congruence

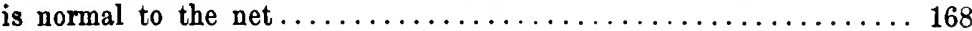

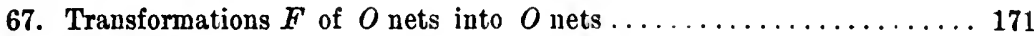

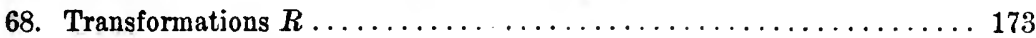

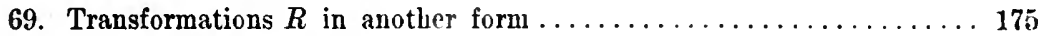

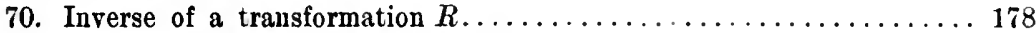

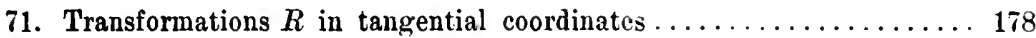

72. Theorem of permutability of transformations $R \ldots \ldots \ldots \ldots \ldots \ldots 179$

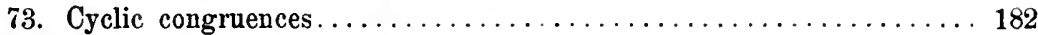

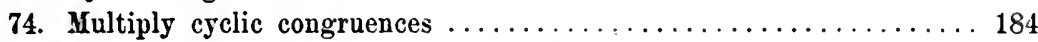

75. Transformations $F$ of $O$ nets into $O$ nets which are not transformations $R \quad 185$

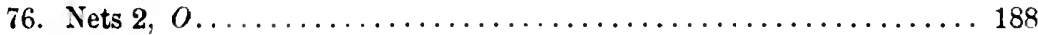

\section{Chapter VII. \\ Transformations of Ribaucour.}

77. Orthogonal determinants and $O$ nets in 3 -space............. 194

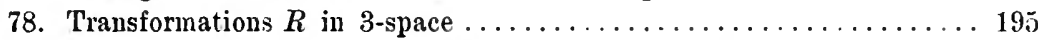

79. The cyclic system associated with a transformation $R \ldots \ldots \ldots \ldots 197$

80. When the circle-planes of a transformation $R$ pass through a point 200

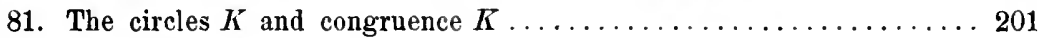

82. Transformations $D_{m}$ of isothermic surfaces ............... 206

83. Theorem of permutability of transformations $D_{n} \ldots \ldots \ldots \ldots \ldots 209$

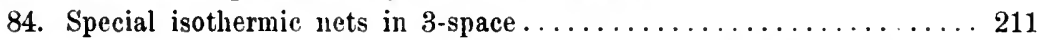

85. Complementary transformations $D_{m}$ of special isothermic nets ..... 212

86. Transformations $D_{m}$ of special isothermic nets ............. 214

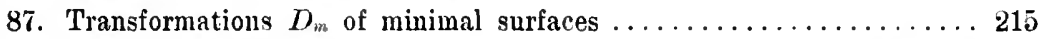

88. Transformations $E_{m}$ of $O$ nets with isothermal spherical representation 215

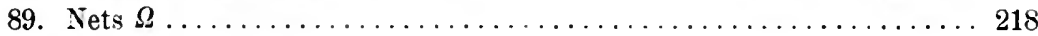

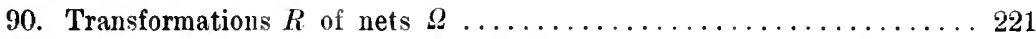

91. Theorem of permutability of the transformations of nets $\Omega \ldots \ldots 223$

92. Surfaces of Guichard of the first kind ................... 224

\section{Chapter VIII. \\ Circles and spheres.}

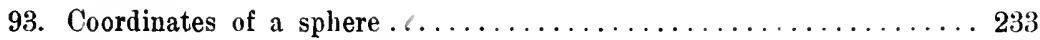

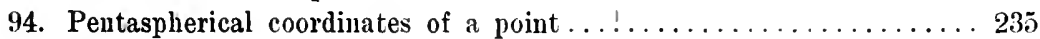

95. Pentaspherical coordinates of an $O$ net $\ldots \ldots \ldots \ldots \ldots \ldots \ldots 237$

96. Congruences of spheres ............................ 237

97. Derired congruences of spheres ...................... 240

98. Congruences of circles . . . . . . . . . . . . . . . . . . 241

99. Congruences of spheres aud circles in cartesian coorlinates ...... 243

100. Congruences $R$ of spheres........................ 245

101. Plücker line coordinates.......................... 247 
Sectiou - Page

102. The Lie line-sphere transtormation $\ldots \ldots \ldots \ldots \ldots \ldots \ldots \ldots \ldots . \ldots 249$

103. The Lie transformations of surfaces . . . . . . . . . . . . 252

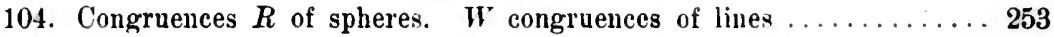

105. Harmonic congruences of spheres and circles $\ldots \ldots \ldots \ldots \ldots \ldots \ldots 255$

106. Representation in 5 -space $\ldots \ldots \ldots \ldots \ldots \ldots \ldots \ldots \ldots \ldots \ldots \ldots \ldots . \ldots \ldots$

107. Conjugate congruences of spheres and circles............. 261

105. Congruences of circles $K$ determined by transformations $k \ldots \ldots 263$

109. Orthogonal congruences of spheres and circles........... 265

110. Transformations $F$ of congruences of circles............. 267

111. Parallel transformations of congruences of circles .......... 271

112. Congruences of spheres with applicable central nets $\ldots \ldots \ldots \ldots 271$

113. Generation of cyclic systems.................... 272

114. Transformations $F$ of cyclic systems................ 274

115. Cyclic systems in 3 -space and nets 0 in 5 -space $\ldots \ldots \ldots \ldots \ldots 277$

116. Cyclic congruences.......................... 280

\section{Chapter IX. \\ Rolling surfaces.}

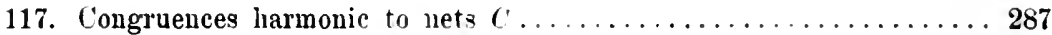

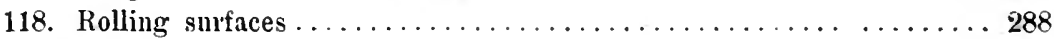

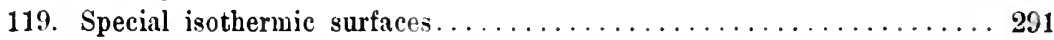

120. Rolling of a surface applicable to a surface of revolution . . . . . 292

121. The fundamental theorems of Guichard . . . . . . . . . . . . 294

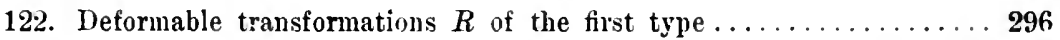

123. Deformable transformations $R$ of the second type .......... 298

124. Deformable transformations of the second type of minimal surfaces 304

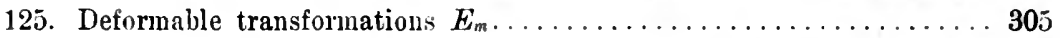

126. Transformations $F$ of deformable transformations $R$ of the second type 306

127. Converses of the theorems of Guichard . . . . . . . . . . . 307

128. Theorems of Ribaucour and Bianchi ................ 310

129. The surface generated by a point in the tangent plane to a surface $\bar{S}$

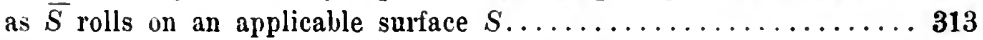

130. Kinematically conjugate directions on rolling surfaces ........ 314

131. Congruences of rolling $\ldots \ldots \ldots \ldots \ldots \ldots \ldots \ldots \ldots \ldots \ldots \ldots$

\section{Chapter $\mathbf{X}$.}

\section{Surfaces applicable to a quadric.}

132. Transformations $F$ of nets on a quadric $\ldots \ldots \ldots \ldots \ldots \ldots \ldots \ldots 2$

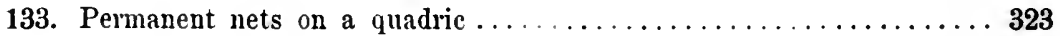

134. The permanent net on a deform of a quadric............. 326

135. Transformations $F_{k}$ of permanent nets on a central quadric . . .... 329

136. Transformations $F_{k}$ of surfaces applicable to a central quadric .... 334

137. Theorem of permutability of transformations $F_{k}$ of surfaces applicable

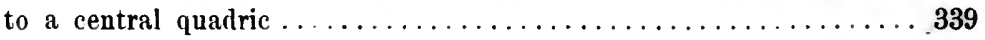


138. Transformations $B_{k}$ of surfaces applicable to a central quadric .... 340

139. Permutability of transformations $F_{k}$ and $B_{k} \ldots \ldots \ldots \ldots \ldots \ldots$

140. Theorem of permutability of transformations $B_{k} \ldots \ldots \ldots \ldots \ldots \ldots$

141. Transformations $F_{k}$ of permanent nets on a paraboloid and of surfaces applicable to a paraboloid ..................... 352

142. Transformations $B_{k}$ of surfaces applicable to a paraboloid ....... 354

143. Determination of the asymptotic lines on a surface.......... 356

144. Deformations of paraboloids and central quadrics of revolution .... 358

145. Surfaces conjugate in deformation ................. 360

146. Transformations $H$ of surfaces applicable to a quadric........ 365

147. Isothermal-conjugate nets on a quadric $\ldots \ldots \ldots \ldots \ldots \ldots \ldots \ldots$

148. Transformations $F$ and $W$ of isothermal-conjugate nets on a central

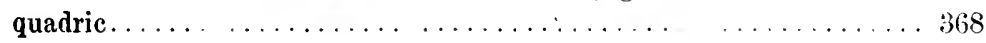





\section{Chapter I. \\ Conjugate nets and congruences.}

1. Geometric entities of euclidean $n$-space. A point in euclidean space of $n$ dimensions is determined by a system of $n$ numbers $x^{1}, \ldots x^{n}$, called the coordinates of the point, which are a generalization of cartesian coordinates in euclidean 3-space. We refer to the point as $P(x)$. Thus $x$ typifies all of the coordinates. In like manner $P(y)$ is the point whose coordinates are $y^{1}, \ldots y^{n}$. The distance $d$ between $P(x)$ and $P(y)$ is defined by the equation

$$
\text { - } d^{2}=\left(y^{1}-x^{1}\right)^{2}+\ldots+\left(y^{n}-x^{n}\right)^{2}=\sum(y-x)^{2} \text {. }
$$

As thus used $\mathbf{\Sigma}$ indicates the sum of all terms of the type $\left(y^{i}-x^{i}\right)^{2}$, but we write it in the above form without subscripts or superscripts, and shall do so in what follows.

If $X^{1}, \ldots X^{n}$ are $n$ numbers, the points whose coordinates are of the form

$$
y=' x+u X,
$$

where $u$ is a parameter, lie on a line through $P(x)$. The quantities $X$ are called direction-parameters of the line. Evidently they are determined only to within a factor. This equation represents each of the $n$ equations $y^{i}=x^{i}+u X^{i}$. It is important that the reader should become familiar with this notation. It is understood that $u$ is the same for all $n$ equations.

Two non-coincident lines whose corresponding direction-parameters $X$ and $Y$ are proportional are said to be parallel. The angle of inclination of two non-parallel lines of direction-parameters $X^{i}$ and $Y^{i}$ is defined to be

$$
\cos \theta=\frac{\sum X Y}{\sqrt{\sum X^{2} \cdot \sum Y^{2}}} .
$$

When $\cos \theta=0$ the lines are said to be perpendicular. 
The locus of the points whose coordinates are of the form

$$
y=x+u X+v Y
$$

where $u$ and $v$ are parameters, is a plane. The locus of the points whose coordinates satisfy a relation of the form

$$
a^{1} x^{1}+a^{2} x^{2}+\ldots+a^{n} x^{n}+a^{n+1}=0
$$

where the $a$ 's are constants, is called a hyperplane. For the sake of brevity we write the above equation in the form $\sum a x+a^{n+1}=0$. In particular, $x_{i} \doteq 0$ is the equation of a coordinate hyperplane.

Two hyperplanes

$$
\sum_{i=1}^{n} a^{i} x+a^{n+1}=0, \sum_{i=1}^{n} b^{i} x+b^{n+1}=0
$$

are said to be parallel when the corresponding quantities $a^{i}$ and $b^{i}$ $(i=1, \ldots n)$ are proportional. The angle of inclination $\theta$ of two non-parallel hyperplanes is defined by

$$
\cos \theta=\frac{\sum_{i=1}^{n} a^{i} b^{i}}{\sqrt{\sum\left(a^{i}\right)^{2} \cdot \sum\left(b^{i}\right)^{2}}} .
$$

When $\cos \theta=0$, the two hyperplanes are said to be perpendicular. In particular, any two coordinate hyperplanes are perpendicular.

A line is a special type of curve, which by definition is the locus of a point whose coordinates $x$ are functions ${ }^{1}$ ) of a parameter $u$. The tangent to a curve at a point is the line through the point whose direction-parameters are $\frac{d x}{d u}$.

A plane is a special case of a surface, the latter being defined to be the locus of a point whose coordinates $x$ are functions of two parameters $u$ and $v$. The points of the surface for which $v$ has the same value is called a parametric curve $v=$ const. There is a one-parameter family of curves $v=$ const. on a surface.

1) In this treatment the parameters may be real or complex, and the only requirements made of the functions is that they and their derivatives (to such order as the latter appear in the development) are uniform and continuous. 
When $u$ and $v$ are replaced by functions of two new parameters $u^{\prime}$ and $v^{\prime}$, we get new parametric curves, and conversely any two one-parameter families of curves can be made parametric.

As in the case of 3-space $[\S 25]^{2}$ ), it can be shown that the tangents to all the curves at an ordinary point $P$ of a surface lie in a plane, called the tangent plane at the point.

2. Conjugate nets. Normal parameters. When the parameters of a surface are such that the coordinates $x$ are solutions of the same equation of the Laplace form,

$$
\frac{\partial^{2} \theta}{\partial u \partial v}=\frac{\partial \log a}{\partial v} \frac{\partial \theta}{\partial u}+\frac{\partial \log b}{\partial u} \frac{\partial \theta}{\partial v}
$$

the parametric curves are said to form a conjugate net, or simply a net. As a consequence of this definition a net in 3-space consists of a conjugate system of curves [§80]. Equation (1) is called the point equation of the net. We speak of the net as $N(x)$.

As in the case of ordinary space, if we put

(2) $E=\sum\left(\frac{\partial x}{\partial u}\right)^{2}, F=\sum \frac{\partial x}{\partial u} \frac{\partial x}{\partial v}, G=\sum\left(\frac{\partial x}{\partial v}\right)^{2}, H^{2}=E G-F^{2}$,

it is readily found that [cf. $\S 63$ ]

$$
\frac{\partial \log a}{\partial v}=\frac{G \frac{\partial E}{\partial v}-F \frac{\partial G}{\partial u}}{2 H^{2}}, \quad \frac{\partial \log b}{\partial u}=\frac{E \frac{\partial G}{\partial u}-F \frac{\partial E}{\partial v}}{2 H^{2}} .
$$

The functions $E, F, G$ are called the fundamental coefficients of the net.

The functions $\frac{\partial x}{\partial u}$ and $\frac{\partial x}{\partial v}$ are direction-parameters of the tangents to the curves $v=$ const. and $u=$ const. respectively, of a net whose point equation is (1). The same is true of the functions $\alpha^{\prime}$ and $\beta^{\prime}$ defined by

$$
\frac{\partial x}{\partial u}=p^{\prime} \alpha^{\prime}, \frac{\partial x}{\partial v}=q^{\prime} \boldsymbol{\beta}^{\prime},
$$

2) A reference in square brackets, thus [\$ 25], is to the author's Differential Geometry, Ginn and Co., 1909 ; in parentheses, thus ( $\$ 25)$, is to the present volume. 
$p^{\prime}$ and $q^{\prime}$ being functions of $u$ and $v$. If these equations be differentiated with respect to $v$ and $u$ respectively, we have in consequence of (1)

$$
\frac{\partial \alpha^{\prime}}{\partial v}=m_{1} \alpha^{\prime}+n_{1} \beta^{\prime}, \quad \frac{\partial \beta^{\prime}}{\partial u}=m_{2} \alpha^{\prime}+n_{2} \beta^{\prime},
$$

where

$$
\begin{array}{ll}
m_{1}=\frac{\partial}{\partial v} \log \frac{a}{p^{\prime}}, & n_{1}=\frac{q^{\prime}}{p^{\prime}} \frac{\partial \log b}{\partial u} \\
m_{2}=\frac{p^{\prime}}{q^{\prime}} \frac{\partial \log a}{\partial v}, & n_{2}=\frac{\partial}{\partial u} \log \frac{b}{q^{\prime}} .
\end{array}
$$

The coordinates of points on the tangents to curves $v=$ const. and $u=$ const. have the respective forms

$$
x+r \alpha^{\prime}, \quad x+t \beta^{\prime} .
$$

By means of (4) we find that the derivatives of these functions with respect to $v$ and $u$ respectively are linear in $\boldsymbol{\alpha}^{\prime}$ and $\boldsymbol{\beta}^{\prime}$.

Conversely, if a surface is referred to any system of parametric curves, a point on the tangent to $v=$ const. has coordinates of the form $x+t \frac{\partial x}{\partial u}$. When $v$ varies the direction-parameters of the tangent are

$$
\frac{\partial x}{\partial v}+\frac{\partial t}{\partial v} \frac{\partial x}{\partial u}+t \frac{\partial^{2} x}{\partial u \partial v} .
$$

Hence if this tangent is to be in the tangent plane to the surface, the preceding expression must be a linear combination of $\frac{\partial x}{\partial u}$ and $\frac{\partial x}{\partial v}$, and we have the theorem:

A necessary and sufficient condition that a system of curves on a surface form a net is that any point on the tangent to a curve $v=$ const. moves in the tangent plane as $v$ varies.

This theorem gives a geometric characterization of a net in the sense that the tangents to $v=$ const. are characteristics of the tangent planes along a curve $u=$ const. It will be convenient at times to refer to the tangent plane to a surface on which a given net lies as the tangent plane of the net. 
Evidently any functions proportional to $\alpha^{\prime}$ and $\beta^{\prime}$ are also direction-parameters. We wish to consider now the particular parameters $\alpha$ and $\beta$, such that

$$
\frac{\partial x}{\partial u}=a \alpha, \quad \frac{\partial x}{\partial v}=b \beta
$$

in which case equations (4) become

$$
\frac{\partial \alpha}{\partial v}=n \beta, \quad \frac{\partial \beta}{\partial u}=m \alpha,
$$

where $m$ and $n$ are functions of $u$ and $v$ given by

$$
\frac{\partial b}{\partial u}=a n, \frac{\partial a}{\partial v}=b m
$$

Following Guichard we say that the $\alpha$ 's and $\beta$ 's are the normal parameters of the net.

Conversely, if we have $n$ pairs of functions $\alpha$ and $\beta$ satisfying equations of the form (6), where $m$ and $n$ are given functions of $u$ and $v$, each pair of functions $a$ and $b$ satisfying (7) leads by quadratures of the form (5) to a net. Thus the complete integration of (7) determines a family of nets, such that at points with the same values of $u$ and $v$ on the nets the tangents to the curves $v=$ const. and $u=$ const. are parallel. A representation of all these nets is given by drawing through the origin lines whose direction-parameters are the $\alpha$ 's and $\beta$ 's. We call this representation a point net.

3. Determination of nets on a surface. Consider the differential equation

$$
A \frac{\partial^{2} \theta}{\partial u^{2}}+2 B \frac{\partial^{2} \theta}{\partial u \partial v}+C \frac{\partial^{2} \theta}{\partial v^{2}}+D \frac{\partial \theta}{\partial u}+E \frac{\partial \theta}{\partial v}=0
$$

where $A, B, C ; D$ and $E$ are functions of $u$ and $v$. If we change the independent variables, putting

$$
u^{\prime}=\varphi_{1}(u, v), \quad v^{\prime}=\varphi_{2}(u, v)
$$


the resulting equation is of the form

(9) $A^{\prime} \frac{\partial^{2} \theta}{\partial u^{\prime 2}}+2 B^{\prime} \frac{\partial^{2} \theta}{\partial u^{\prime} \partial v^{\prime}}+C^{\prime} \frac{\partial^{2} \theta}{\partial v^{\prime 2}}+D^{\prime} \frac{\partial \theta}{\partial u^{\prime}}+E^{\prime} \frac{\partial \theta}{\partial v^{\prime}}=0$, where

$$
\begin{aligned}
& A^{\prime}=A\left(\frac{\partial u^{\prime}}{\partial u}\right)^{2}+2 B \frac{\partial u^{\prime}}{\partial u} \frac{\partial u^{\prime}}{\partial v}+C\left(\frac{\partial u^{\prime}}{\partial v}\right)^{2} \\
& B^{\prime}=A \frac{\partial u^{\prime}}{\partial u} \frac{\partial v^{\prime}}{\partial u}+B\left(\frac{\partial u^{\prime}}{\partial u} \frac{\partial v^{\prime}}{\partial v}+\frac{\partial v^{\prime}}{\partial u} \frac{\partial u^{\prime}}{\partial v}\right)+C \frac{\partial u^{\prime}}{\partial v} \frac{\partial v^{\prime}}{\partial v} \\
& C^{\prime}=A\left(\frac{\partial v^{\prime}}{\partial u}\right)^{2}+2 B \frac{\partial v^{\prime}}{\partial u} \frac{\partial v^{\prime}}{\partial v}+C\left(\frac{\partial v^{\prime}}{\partial v}\right)^{2}
\end{aligned}
$$

From these expressions it follows that if $\varphi_{1}$ and $\varsigma_{2}$ are resolutions of

$$
A\left(\frac{\partial \varphi}{\partial u}\right)^{2}+2 B \frac{\partial \varphi}{\partial u} \frac{\partial \varphi}{\partial v}+C\left(\frac{\partial \varphi}{\partial v}\right)^{2}=0
$$

equation (9) is of the form (1). Since $\varphi_{1}$ and $\varsigma_{2}$ must be functionally independent, they are obtained by solving the two differential equations of the first order which are factors of

$$
A d v^{2}-2 B d u d v+C d u^{2}=0
$$

Darboux ${ }^{3}$ ) has called (10) the differential equation of the characteristics of (8).

There is only one such function $\varphi$ when

$$
A C-B^{2}=0 \text {. }
$$

If we take it for $u^{\prime}$, equation (9) is reducible to the form

$$
\frac{\partial^{2} \theta}{\partial v^{\prime 2}}+a^{\prime} \frac{\partial \theta}{\partial u^{\prime}}+b^{\prime} \frac{\partial \theta}{\partial v^{\prime}}=0 \text {. }
$$

Let $S$ be a surface in 3 -space whose cartesian coordinates $x$ are functions of any two parameters $u$ and $v$. We can find by differentiation an equation of the form (8) satisfied by the three coordinates and by any function of them, say $F\left(x^{1}, x^{2}, x^{3}\right)$. Then

3) Leçons, vol. 1, p. 193. 
the curves defined by (10) form a net, which is determined by the character of $F$; or if (11) is satisfied, a family of asymptotic lines [\$ 77].

If $S$ is a surface in 4-space ${ }^{4}$ ), an equation of the form (8) can be found which is satisfied by the four coordinates, and this equation is unique to within a factor. Consequently there is a unique net on $S$, unless (11) is satisfied, in which case equation (10) defines a self-conjugate family of curves.

When $S$ is a surface in a space of order higher than the fourth, it is not always possible to find an equation of the form (8) satisfied by all of the coordinates of $S$. Consequently in such spaces there are surfaces upon which there are not any nets.

4. Parallel nets. When the points of two surfaces are in a one-to-one correspondence of any sort, and two corresponding systems of curves are taken as parametric, the parameters can be chosen so that $u$ and $v$ have the same values on the two surfaces at corresponding points. It is understood that this plan will be followed hereafter whenever we are dealing with point-to-point correspondence between two surfaces.

We inquire under what conditions the tangents to the curves of the parametric systems at corresponding points on two surfaces are parallel. When these conditions are satisfied we say that the two systems are parallel. The coordinates $x$ and $x^{\prime}$ of the two systems, expressed as functions of the parameters $u$ and $v$, must satisfy the equations

$$
\frac{\partial x^{\prime}}{\partial u}=h \frac{\partial x}{\partial u}, \quad \frac{\partial x^{\prime}}{\partial v}=l \frac{\partial x}{\partial v}
$$

where $h$ and $l$ are fuuctions of $u$ and $v$ such that the conditions

$$
\frac{\partial}{\partial v}\left(\frac{\partial x^{\prime}}{\partial u}\right)=\frac{\partial}{\partial u}\left(\frac{\partial x^{\prime}}{\partial v}\right)
$$

are satisfied. These equations show that the $x$ 's satisfy an equation of the form (1), where now $a$ and $b$ are given by

4) When we speak of a surface in $n$-space, it is meant that the surface is not contained in any space of order less than $n$. 


$$
\left.\frac{\partial h}{\partial v}=(l-h) \frac{\partial \log a}{\partial v}, \quad \frac{\partial l}{\partial u}=(h-l) \frac{\partial \log b}{\partial u}{ }^{3}\right) .
$$

Hence a necessary condition that a system admit a parallel system is that it be a net. Evidently the parallel system also forms a net. In fact, it follows from (12) that the coordinates $x^{\prime}$ are solutions of the equation

$$
\frac{\partial^{2} \theta^{\prime}}{\partial u \partial v}=\frac{l}{h} \frac{\partial \log a}{\partial v} \frac{\partial \theta^{\prime}}{\partial u}+\frac{h}{l} \frac{\partial \log b}{\partial u} \frac{\partial \theta^{\prime}}{\partial v}
$$

which may be written in the form

$$
\frac{\partial^{2} \theta^{\prime}}{\partial u \partial v}=\frac{\partial \log a^{\prime}}{\partial v} \frac{\partial \theta^{\prime}}{\partial u}+\frac{\partial \log b^{\prime}}{\partial u} \frac{\partial \theta^{\prime}}{\partial v}
$$

where

$$
a^{\prime}=a h, \quad b^{\prime}=b l
$$

Assume that we have a net whose equation is (1). Each pair of functions satisfying (13) gives by quadratures (12) a parallel net. If $\theta$ is any solution of (1), the function $\theta^{\prime}$ given by the quadratures

$$
\frac{\partial \boldsymbol{\theta}^{\prime}}{\partial u}=h \frac{\partial \theta}{\partial u}, \quad \frac{\partial \boldsymbol{\theta}^{\prime}}{\partial v}=l \frac{\partial \theta}{\partial v}
$$

is a solution of (14); we call $\theta$ and $\theta^{\prime}$ corresponding solutions of (1) and (14).

The analytical problem of finding parallel nets may be given another form. If we define a function $\varphi$ by

$$
h-l=\varphi,
$$

equations (13) may be replaced by

$$
\frac{\partial l}{\partial u}=\varphi \frac{\partial \log b}{\partial u}, \quad \frac{\partial l}{\partial v}=-\varphi \frac{\partial}{\partial v} \log a \varphi .
$$

5) A particular solution of these equations is $h=l=$ const., in which case the surfaces are homothetic transforms of one another with respect to the origin, to within a translation. We exclude this case hereafter. 
The condition of integrability of these equations leads directly to

(20) $\frac{\partial^{2} \varphi}{\partial u \partial v}+\frac{\partial \log a}{\partial v} \frac{\partial \varphi}{\partial u}+\frac{\partial \log b}{\partial u} \frac{\partial \varphi}{\partial v^{\prime}}+\varphi \frac{\partial^{2}}{\partial u \partial v} \log a b=0$.

Each solution of this equation leads by a quadrature (19) and by (18) to a pair of solutions of equations (13), and consequently to the determination of a parallel net.

Equation (20) is by definition the adjoint of equation (1). Hence we have the theorem:

The determination of nets parallel to a given net $N$ is equivalent to the solution of the adjoint of the point equation of $N$.

The functions $h$ and $l$ are determined by (18) and (19) only to within the same additive constant $k$. Hence if $h$ and $l$ are one set of solutions of (18) and (19), and $x^{\prime}$ the corresponding solution of (12), the other solutions $h+k, l+k$ lead to $x^{\prime}+k x$.

Suppose now that we have two nets $N$ and $N^{\prime}$ such that the tangents to the curves of parameter $u$ at corresponding points are parallel. We shall show that $N$ and $N^{\prime}$ are parallel, unless they are planar nets.

By hypothesis the first of equations (12) holds. Differentiating it whith respect to $v$ and making use of the fact that $x$ satisfies (1) and $x^{\prime}(15)$, we get

$$
\frac{\partial \log b^{\prime}}{\partial u} \frac{\partial x^{\prime}}{\partial v}=h \frac{\partial}{\partial v} \log \frac{h a}{a^{\prime}} \frac{\partial x}{\partial u}+h \frac{\partial \log b}{\partial u} \frac{\partial x}{\partial v} .
$$

If the coefficient of $\frac{\partial x}{\partial u}$ is zero, the theorem is established. If it is not equal to zero, we express the condition of integrability of this equation and the first of (12). The resulting equation is reducible to the form

$$
\frac{\partial^{2} x}{\partial u^{2}}=A \frac{\partial x}{\partial u}+B \frac{\partial x}{\partial v} .
$$

Expressing the condition of integrability of this equation and (1), we get an equation of the form

$$
\frac{\partial^{2} x}{\partial v^{2}}=C \frac{\partial x}{\partial u}+D \frac{\partial x}{\partial v}
$$


In $\S 40$ it will be shown that at most two linearly independent functions can satisfy two such equations and (1). Hence:

If two non-planar nets correspond and the tangents to the parametric curves in one family are parallel, the nets are parallel.

If $N(x)$ and $N^{\prime}\left(x^{\prime}\right)$ are parallel nets, the coordinates of any point on the line $L$ joining corresponding points are of the form

$$
x+t\left(x^{\prime}-x\right) .
$$

In consequence of (12) the derivatives of this expression are reducible to

$$
\begin{aligned}
& \frac{\partial x}{\partial u}(1+t h-t)+\frac{\partial t}{\partial u}\left(x^{\prime}-x\right), \\
& \frac{\partial x}{\partial v}(1+t l-t)+\frac{\partial t}{\partial v}\left(x^{\prime}-x\right) .
\end{aligned}
$$

Hence the points for which $t$ has the respective values

$$
t_{1}=\frac{1}{1-h}, \quad t_{2}=\frac{1}{1-1}
$$

describe surfaces $\Sigma_{1}$ and $\Sigma_{2}$ such that the lines $L$ are tangent to the curves $v=$ const. on $\Sigma_{1}$ and $u=$ const. on $\Sigma_{2}$. The coordinates $y$ and $z$ of $\Sigma_{1}$ and $\Sigma_{2}$ are

$$
y=\frac{x^{\prime}-h x}{1-h}, \quad z=\frac{x^{\prime}-l x}{1-l} .
$$

A one-parameter family of straight lines tangent to a curve or meeting in a point, or having constant direction-parameters is called a developable surface ${ }^{6}$ ): Any other one-parameter family of lines constitutes a skew ruled surface. In a two-parameter family of lines each relation between the parameters determines a surface, developable or skew. Each line of the family belongs to an infinity of these surfaces. In space of three dimensions two of these surfaces are developable [§ 163]. In spaces of higher order there

$\left.{ }^{6}\right)$ cf. [§ 27]. As in 3-space we use the terms cone and cylinder for the second and third types here mentioned. 
are not necessarily two developables of the family through each line. We call a congruence in $n$-space a two-parameter family of lines such that through each line pass two developable surfaces of the family. Hereafter it is understood that the parameters $"$ and $v$ of the congruence are such that these developables are given by $u=$ const. and $v=$ const.

The preceding results may be stated thus:

The lines joining corresponding points on two parallel nets form a congruence whose developables meet the surfaces on which the nets lie in the nets.

The points, $F_{1}$ and $F_{2}$, whose coordinates are given by (21) are called the first and second focal points of the line of the congruence on which they lie; that is, the point at which the line is tangent to the curve of parameter $u$ is called the first focal point. The focal points are also spoken of as being of the first and second rank. The surfaces, $\Sigma_{1}$ and $\Sigma_{2}$, the loci of $F_{1}$ and $F_{2}$ respectively, are called the first and second focal surfaces of the congruence.

We remark that the tangent planes of the second focal surface are the osculating planes of the curves of parameter $u$ of the first focal surface, and the tangent planes of the first focal surface are the osculating planes of the curves of parameter $v$ of the second focal surface [cf. $\S 163$ ].

By differentiating equations (21), we obtain

$$
\left\{\begin{array}{l}
\frac{\partial y}{\partial u}=\frac{x^{\prime}-x}{(1-h)^{2}} \frac{\partial h}{\partial u}, \quad \frac{\partial y}{\partial v}=\frac{l-h}{1-h}\left(\frac{\partial x}{\partial v}+\frac{x^{\prime}-x}{1-h} \frac{\partial \log a}{\partial v}\right) \\
\frac{\partial z}{\partial u}=\frac{h-l}{1-l}\left(\frac{\partial x}{\partial u}+\frac{x^{\prime}-x}{1-l} \frac{\partial \log b}{\partial u}\right), \quad \frac{\partial z}{\partial v}=\frac{x^{\prime}-x}{(1-l)^{2}} \frac{\partial l}{\partial v}
\end{array}\right.
$$

and

$$
\left\{\begin{array}{l}
\frac{\partial^{2} y}{\partial u \partial v}=\frac{\partial}{\partial v} \log \left(\frac{a}{1-h} \frac{\partial h}{\partial u}\right) \frac{\partial y}{\partial u}+\frac{\partial}{\partial u} \log \left(b \frac{l-h}{1-h}\right) \frac{\partial y}{\partial v} \\
\frac{\partial^{2} z}{\partial u \partial v}=\frac{\partial}{\partial v} \log \left(a \frac{h-l}{1-l}\right) \frac{\partial z}{\partial u}+\frac{\partial}{\partial u} \log \left(\frac{b}{1-l} \frac{\partial l}{\partial v}\right) \frac{\partial z}{\partial v}
\end{array}\right.
$$

From these equations it is seen that the parametric curves on $\Sigma_{1}$ and $\Sigma_{2}$ form nets [cf. $\S 163$ ]. In the next section we show that 
any congruence consists of the joins of corresponding points on two parallel nets. Hence:

The developables of a congruence meet each of the focal surfaces in a net.

Following Guichard, we say that a net and a congruence are conjugate when the developables of the congruence meet the surface of the net in the curves of the net, provided that the surface is not a focal surface of the congruence. Accordingly we may state the next to the last theorem in the form:

The lines joining corresponding points of two parallel nets form a congruence conjugate to these nets.

5. Congruences conjugate to a net. We consider a net $N(x)$ and a congruence $G$ of direction-parameters $X$ passing through points of the net. We seek the general conditions to be satisfied by the parameters $X$ in order that $N$ and $G$ shall be conjugate.

A necessary and sufficient condition that $N$ and $G$ be conjugate is that on each line of $G$ there be two points defined by equations of the form

$$
y=x-\lambda X, \quad z=x-\mu X,
$$

such that as $u$ and $v$ vary respectively the corresponding point moves tangentially to the line. This is expressed analytically by

$$
\frac{\partial y}{\partial \imath}=\sigma X, \quad \frac{\partial z}{\partial v}=\tau X
$$

where $\sigma$ and $\tau$ are determinate functions. Substituting the above values, we arrive at equations of the form

$$
\frac{\partial x}{\partial u}=\lambda \frac{\partial X}{\partial u}+v X, \quad \frac{\partial x}{\partial v}=\mu \frac{\partial X}{\partial v}+\varrho X .
$$

Expressing the condition of integrability of these equations, we find that the parameters $X$ must satisfy an equation of the form

$$
\frac{\partial^{2} \theta}{\partial u \partial v}=\frac{\partial \log A}{\partial v} \frac{\partial \theta}{\partial u}+\frac{\partial \log B}{\partial u} \frac{\partial \theta}{\partial v}+C \theta .
$$


Hence we have the theorem:

The direction-parameters of a congruence referred to its developables satisfy an equation of Laplace.

We call (27) the direction equation of the congruence.

When now we require that the point $M$ of coordinates $x$ describe a net whose equation is (1), we find on differentiating equations (26) with respect to $v$ and $u$ respectively that the coefficients in (27) have the respective values

(28)

$A=\frac{a}{\lambda}, \frac{\partial \log B}{\partial u}=\frac{1}{\lambda}\left(\mu \frac{\partial \log b}{\partial u}-\nu\right), C=\frac{\nu}{\lambda}\left(\frac{\partial}{\partial v} \log \frac{a}{\nu}+\frac{\varrho}{\nu} \frac{\partial \log b}{\partial u}\right)$.

and

(29)

$\frac{\partial \log A}{\partial v}=\frac{1}{\mu}\left(\lambda \frac{\partial \log a}{\partial v}-\varrho\right), \quad B=\frac{b}{\mu}, C=\frac{\varrho}{\mu}\left(\frac{\partial}{\partial u} \log \frac{b}{\varrho}+\frac{\nu}{\varrho} \frac{\partial \log a}{\partial v}\right)$.

If these two sets of values of the coefficients be equated, we get the following equations of condition:

(30)

$$
\left\{\begin{array}{c}
\frac{\partial}{\partial v} \frac{1}{\lambda}+\frac{\varrho}{\lambda \mu}+\frac{\partial \log a}{\partial v}\left(\frac{1}{\lambda}-\frac{1}{\mu}\right)=0, \\
\frac{\partial}{\partial u} \frac{1}{\mu}+\frac{\nu}{\lambda \mu}+\frac{\partial \log b}{\partial u}\left(\frac{1}{\mu}-\frac{1}{\lambda}\right)=0, \\
\left.\frac{\partial}{\partial v} \frac{\nu}{\lambda}=\frac{\partial}{\partial u} \frac{\varrho}{\mu}\right)^{\prime} .
\end{array}\right.
$$

If the last of these equations be replaced by

$$
\frac{\nu}{\lambda}=\frac{\partial \log t}{\partial u}, \frac{\varrho}{\mu}=\frac{\partial \log t}{\partial v},
$$

where $t$ is thus defined, the first two are reducible to

$$
\left\{\begin{aligned}
\frac{\partial}{\partial v} \frac{t}{\lambda}+\frac{\partial \log a}{\partial v}\left(\frac{t}{\lambda}-\frac{t}{\mu}\right) & =0 \\
\frac{\partial}{\partial u} \frac{t}{\mu}+\frac{\partial \log b}{\partial u}\left(\frac{t}{\mu}-\frac{t}{\lambda}\right) & =0 .
\end{aligned}\right.
$$

7) We note that if we put $\frac{1}{\gamma}=h, \frac{1}{\mu}=l, \nu=\rho=0$, equations (26) and (30) reduce to (12) and (13). 
Moreover, equations (26) can be written

$$
\frac{\partial x}{\partial u}=\frac{\lambda}{t} \frac{\partial}{\partial u}(X t), \frac{\partial x}{\partial v}=\frac{\mu}{t} \frac{\partial}{\partial v}(X t) .
$$

Comparing these equations with (12), we see that the locus of the point whose coordinates $x^{\prime}$ are given by

$$
x^{\prime}=X t
$$

is a net parallel to $N$. Hence by a quadrature (31) we can determine direction-parameters of the congruence which are the cartesian coordinates of a net $N^{\prime}$ parallel to $N$, and we have the theorem:

If a net $N$ is conjugate to a congruence. $G$, a net $N^{\prime}$ parallel to $N$ can be found by quadratures whose cartesian coordinates are direction-parameters of the congruence.

Conversely, if $N^{\prime}$ is any net parallel to $N$ and through points of the latter we draw lines parallel to lines joining the origin to corresponding points of $N^{\prime}$, that is, lines with direction-parameters $x^{\prime}$, the coordinates of any point $P$ on such a line are of the form

$$
x-r x^{\prime}
$$

'The first derivatives of this expression are of the form

$$
\frac{\partial x}{\partial u}(1-r h)-\frac{\partial r}{\partial u} x^{\prime}, \quad \frac{\partial x}{\partial v}(1-r l)-\frac{\partial r}{\partial v} x^{\prime}
$$

Whell $r$ takes the values $1 / h$ and $1 / l$ respectively, the points are focal points of the line, and hence the lines form a congruence. The coordinates of the focal points are of the form

$$
y=x-\frac{1}{h} x^{\prime}, \quad z=x-\frac{1}{l} x^{\prime}
$$

From these results follows the theorem:

Any congruence conjugate to a given net $N$ can be obtained by drawing through points of $N$ lines parallel to lines joining the origin to corresponding points of a net $N^{\prime}$ parallel to the given net; and every parallel net determines in this way a congruence conjugate to $N$. 
It is evident from the above investigation that lines joining any fixed point to $N^{\prime}$ determine the directions of a congruence conjugate to $N$.

If two congruences with corresponding direction-parameters equal or proportional are said to be parallel, we have, as a corollary of the above results, the theorem:

If two nets are parallel, every congruence conjugate to one is parallel to a congruence conjugate to the other.

From (36) it follows that the point $P$ with coordinates (35) describes a net parallel to $N$ when $r$ is a constant, and only in this case. Hence we have the theorem:

A congruence conjugate to a net $N$ is conjugate to an infinity of nets parallel to $N$.

Combining this result with the third theorem of $\S 4$, we have also:

Any congruence conjugate to a net $N$ consists of the joins of corresponding points of $N$ and of a parallel net.

6. Focal Surfaces of a congruence. From (37) we have by differentiation and reduction by means of (12):

(38)

$$
\left\{\begin{array}{l}
\frac{\partial y}{\partial u}=\frac{1}{h^{2}} \frac{\partial h}{\partial u} x^{\prime}, \quad \frac{\partial y}{\partial v}=\left(1-\frac{l}{h}\right)\left(\frac{\partial x}{\partial v}-\frac{x^{\prime}}{h} \frac{\partial \log a}{\partial v}\right), \\
\frac{\partial z}{\partial u}=\left(1-\frac{h}{l}\right)\left(\frac{\partial x}{\partial u}-\frac{x^{\prime}}{l} \frac{\partial \log b}{\partial u}\right), \quad \frac{\partial z}{\partial v}=\frac{1}{l^{2}} \frac{\partial l}{\partial v} x^{\prime}
\end{array}\right.
$$

and

$$
\left\{\begin{array}{l}
\frac{\partial^{2} y}{\partial u \partial v}=\frac{\partial}{\partial v} \log \left(\frac{a}{h} \frac{\partial h}{\partial u}\right) \frac{\partial y}{\partial u}+\frac{\partial}{\partial u} \log \left(b \frac{h-l}{h}\right) \frac{\partial y}{\partial v} \\
\frac{\partial^{2} z}{\partial u \partial v}=\frac{\partial}{\partial v} \log \left(a \frac{l-h}{l}\right) \frac{\partial z}{\partial u}+\frac{\partial}{\partial u} \log \left(\frac{b}{l} \frac{\partial l}{\partial v}\right) \frac{\partial z}{\partial v}
\end{array}\right.
$$

From these equations we see again that the developables meet the focal surfaces in nets.

In order that the point midway between the focal points shall describe the net $N(x)$ conjugate to the congruence, we must have, as follows from (37),

$$
l=-h .
$$


From (13) and (16) it follows that in all generality we may take

$$
\begin{gathered}
a=b=\frac{1}{\sqrt{h}}, \\
a^{\prime}=-b^{\prime}=\sqrt{h} .
\end{gathered}
$$

We recall that for an equation of the Laplace form

$$
\frac{\partial^{2} \theta}{\partial u \partial v}=a \frac{\partial \theta}{\partial u}+b \frac{\partial \theta}{\partial v}+c \theta
$$

the functions

$$
H=-\frac{\partial a}{\partial u}+a b+c, \quad K=-\frac{\partial b}{\partial v}+a b+c,
$$

are called the invariants of the equation, since these functions are invariant when equation (43) is transformed into an equation of the same form in $\theta_{1}$, where $\theta_{1}=\lambda \theta, \lambda$ being any function of $u$ and $v$.

From (41) and (42) we have for $n$-space the theorem announced by Ribaucour for 3 -space:

A necessary condition that the developables of a congruence meet the middle surface in a net is that the direction equation of the congruence have equal invariants; in this case the point equation of the net on the middle surface also has equal invariants.

We are in position now to establish the theorem:

When two congruences are conjugate to a net, the joins of corresponding focal points of the same rank form a congruence conjugate to the nets described by these focal points.

Let $N(x)$ be the net, and let the direction-parameters of the congruences be the coordinates $x^{\prime}$ and $x^{\prime \prime}$ of nets $N^{\prime}$ and $N^{\prime \prime}$ parallel to $N$, determined by solutions $h, l$ and $h_{1}, l_{1}$ of (13). The coordinates of the focal points of the congruences are of the respective forms (37) and

$$
y_{1}=x-\frac{x^{\prime \prime}}{h_{1}}, \quad z_{1}=x-\frac{x^{\prime \prime}}{l_{1}} .
$$

The coordinates of any point on the line joining the focal points of coordinates $y$ and $y_{1}$ are of the form $y+t\left(y-y_{1}\right)$. If we differentiate this expression with respect to $u$ and $v$ and make use 
of (38) and similar equations for $y_{1}$, we find that its first derivatives are proportional to the corresponding first derivatives of $y$ when $t=h_{1}$. Consequently the points of coordinates $y$ and

$$
y+h_{1}\left(y-y_{1}\right)
$$

generate parallel nets, and hence by the third theorem of $\$ 4$ the congruence is conjugate to these nets. In like manner we can show that it is conjugate to the net of coordinates $y_{1}$.

7. Laplace transforms. In [\$ 165] we have derived for 3 -space the expressions for the cartesian coordinates of the second focal surface of the congruence of tangents to the curves $v=$ const. or $u=$ const. of a net. The method followed is equally valid for spaces of higher order. If (1) is the point equation of the net, the coordinates of these respective second focal nets are of the form

$$
x_{-1}=x-\frac{1}{\frac{\partial \log b}{\partial u}} \frac{\partial x}{\partial u}, \quad r_{1}=x-\frac{1}{\frac{\partial \log a}{\partial v}} \frac{\partial x}{\partial v} .
$$

In fact we have

$$
\frac{\partial x-1}{\partial v}=\frac{b}{\frac{\partial b}{\partial u}} \frac{\partial}{\partial v} \log \left(\frac{\frac{\partial b}{\partial u}}{a b}\right) \frac{\partial x}{\partial u}, \quad \frac{\partial x_{1}}{\partial u}=\frac{a}{\frac{\partial a}{\partial v}} \frac{\partial}{\partial u} \log \left(\frac{\frac{\partial a}{\partial v}}{a b}\right) \frac{\partial x}{\partial v} .
$$

The nets $N_{1}$ and $N_{-1}$ with the respective coordinates $x_{1}$ and $x_{-1}$ are called the first Laplace transform of $N$ and the minus first Laplace transform respectively. If the point equation of $N_{-1}$ is written in the form

$$
\frac{\partial^{2} \theta_{-1}}{\partial u \partial v}=\frac{\partial}{\partial v}\left(\log a_{-1}\right) \frac{\partial \theta_{-1}}{\partial u}+\frac{\partial}{\partial u}\left(\log b_{-1}\right) \frac{\partial \theta_{-1}}{\partial v}
$$

we find that

$$
a_{-1}=\frac{a b}{\frac{\partial b}{\partial u}}, \quad b_{-1}=b \frac{\partial}{\partial v} \log \frac{\frac{\partial b}{\partial u}}{a b}=-\frac{b K}{\frac{\partial \log b}{\partial u}} .
$$

The Laplace transforms of a net parallel to $N$ are defined by equations of the form 


$$
x_{-1}^{\prime}=x^{\prime}-\frac{1}{\frac{\partial \log b^{\prime}}{\partial u}} \frac{\partial x^{\prime}}{\partial u}, \quad x_{1}^{\prime}=x^{\prime}-\frac{1}{\frac{\partial \log a^{\prime}}{\partial v}} \frac{\partial x^{\prime}}{\partial v} .
$$

From these, (14) and (48) we have

$$
\left\{\begin{aligned}
\frac{\partial x_{-1}^{\prime}}{\partial u} & =l \frac{\partial x_{-1}}{\partial u}, & \frac{\partial x_{-1}^{\prime}}{\partial v} & =\frac{l^{2} K^{\prime}}{h K} \frac{\partial x_{-1}}{\partial v}, \\
\frac{\partial x_{1}^{\prime}}{\partial u} & =\frac{h^{2} H^{\prime}}{l H} \frac{\partial x_{1}}{\partial u}, & \frac{\partial x_{1}^{\prime}}{\partial v} & =h \frac{\partial x_{1}}{\partial v},
\end{aligned}\right.
$$

where $H^{\prime}$ and $K^{\prime}$ are the invariants of (15).

Hence we have the theorem:

If $N$ and $N^{\prime}$ are parallel nets, their respective Laplace transforms are parallel.

8. Transformations of Levy. By means of (38) equations(37) can be written in the form

$$
x=y-\frac{\frac{1}{h}}{\frac{\partial}{\partial u} \frac{1}{h}} \frac{\partial y}{\partial u}, \quad x=z-\frac{\frac{1}{l}}{\frac{\partial}{\partial v} \frac{1}{l}} \frac{\partial z}{\partial v} .
$$

It is readily shown that $1 / h$ and $1 / l$ are solutions of the respective equations (39). By a change of notation the second of these equations may be written

$$
y=x-\frac{\theta}{\frac{\partial \theta}{\partial v}} \frac{\partial x}{\partial v}
$$

where now the congruence consists of the tangents to the curves $u=$ const. of $N(x)$ and $\theta$ is a solution of its point equation, and the $y$ 's are the coordinates of a net conjugate to this congruence. In like manner the first of the above equations may be written

$$
z=x-\frac{\theta}{\frac{\partial \theta}{\partial u}} \frac{\partial x}{\partial u}
$$

Since $N(x)$ in (37) may be any net conjugate to the tangents to the curves $u=$ const. of $N(y)$ or $v=$ const. of $N(z)$, we have the first part of the following theorem of Levy: 
The coordinates of any net conjugate to the congruence of tangents to the curves $u=$ const. or $v=$ const. of a net $N(x)$ whose point equation is (1) may be expressed in the respective forms

$$
y=x-\frac{\theta}{\frac{\partial \theta}{\partial v}} \frac{\partial x}{\partial v}, \quad z=x-\frac{\theta}{\frac{\partial \theta}{\partial u}} \frac{\partial x}{\partial u},
$$

where $\theta$ is a solution of (1). Conversely, every solution of (1) gives two nets conjugate to the congruences of tangents.

In order to prove the latter part of this theorem, we note that if $\theta$ is a solution of (1), we have from (53):

$$
\frac{\partial y}{\partial u}=\frac{\partial \log B}{\partial u}(y-z), \quad \frac{\partial z}{\partial v}=\frac{\partial \log A}{\partial v}(z-y)
$$

where

$$
A=\frac{a \theta}{\frac{\partial \theta}{\partial u}}, \quad B=\frac{b \theta}{\frac{\partial \theta}{\partial v}}
$$

From (54) we have by differentiation

$$
\left\{\begin{array}{l}
\frac{\partial^{2} y}{\partial u \partial v}=\frac{\partial}{\partial v} \log \left(A \frac{\partial \log B}{\partial u}\right) \frac{\partial y}{\partial u}+\frac{\partial \log B}{\partial u} \frac{\partial y}{\partial v} \\
\frac{\partial^{2} z}{\partial u \partial v}=\frac{\partial \log A}{\partial v} \frac{\partial z}{\partial u}+\frac{\partial}{\partial u} \log \left(B \frac{\partial \log A}{\partial v}\right) \frac{\partial z}{\partial v} .
\end{array}\right.
$$

Hence the points of coordinates (53) describe nets. We call these nets the Levy transforms of $N$ by means of $\theta$.

As a corollary of the above theorem we have:

There are nets conjugate to any congrience.

It is evident that, if $\theta$ is a solution of (1), the function

$$
\theta_{-1}=\theta-\frac{1}{\frac{\partial \log b}{\partial u}} \frac{\partial \theta}{\partial u}
$$

is a solution of (49), the point equation of the minus first Laplace transform of $N$. We call $\theta_{-1}$ the minus first Laplace transform of $\theta$. From these equations, (47), (48) and (53), we have 


$$
x_{-1}-\frac{\theta_{-1}}{\frac{\partial \theta_{-1}}{\partial v}} \frac{\partial x_{-1}}{\partial v}=x-\frac{\theta}{\frac{\partial \theta}{\partial u}} \frac{\partial x}{\partial u} .
$$

Consequently the net of coordinates $z$ in (53) is the Levy transform of the minus first Laplace transform of $N$ by means of $\theta_{-1}$. Similar results follow when we consider the net of coordinates $y$. Hence:

The Levy transforms of a net determined by a solution $\theta$ of the point equation of $N$ are Levy transforms of the minus first and first Laplace transforms of $N$ by means of the corresponding Laplace transforms of $\theta$.

9. Determination of congruences. We saw in $\S 5$ that the direction-parameters $X$ of a congruence are solutions of an equation of the form (27). If in (27) we put

we obtain

$$
X=x^{\prime} \theta \text {, }
$$

$$
\frac{\partial^{2} x^{\prime}}{\partial u \partial v}=\frac{\partial}{\partial v} \log \left(\frac{A}{\theta}\right) \frac{\partial x^{\prime}}{\partial u}+\frac{\partial}{\partial u} \log \left(\frac{B}{\theta}\right) \frac{\partial x^{\prime}}{\partial v}+C^{\prime} x^{\prime},
$$

where

$$
C^{\prime}=\frac{1}{\theta}\left(\frac{\partial \log A}{\partial v} \frac{\partial \theta}{\partial u}+\frac{\partial \log B}{\partial u} \frac{\partial \theta}{\partial v}+C \theta-\frac{\partial^{2} \theta}{\partial u \partial v}\right)
$$

Hence a necessary and sufficient condition that the quantities $x^{\prime}$ are the cartesian coordinates of a net is that $\theta$ be a solution of (27).

By the above corollary of the theorem of Levy there are nets conjugate to any congruence. From the second theorem of $\S 5$ it follows that any one of these nets is parallel to a net whose coordinates are direction-parameters of the congruence. Hence:

In order to obtain a congruence with a given set of directionparameters $X$, we find a solution $\theta$ of the equation of Laplace satisfied by the $X^{\prime}$ 's: then the quantities

$$
x^{\prime}=\frac{X}{\theta}
$$

are the coordinates of a net $N^{\prime}$; through points of a net $N$ parallel to $N^{\prime}$ draw lines with parameters $X$; these lines form a congruence conjugate to $N$; all congruences with direction-parameters $X$ can be found in this way. 
From (38) we find by means of (12) and (16), that the directionparameters of the tangents to the curves $u=$ const. and $v=$ const. respectively, on the first and second focal surfaces defined by (37) are expressible in the form

$$
\frac{\partial x^{\prime}}{\partial v}-x^{\prime} \frac{\partial \log a^{\prime}}{\partial v}, \quad \frac{\partial x^{\prime}}{\partial u}-x^{\prime} \frac{\partial \log b^{\prime}}{\partial u} .
$$

When $x^{\prime}$ is replaced by the value (59), the resulting expressions are reducible to the same form in terms of $X$ and the coefficients of (27) to within the factor $1 / \theta$. Hence:

If the direction-parameters $X$ of a congruence $G$ are solutions of an equation (27), the functions

$$
\frac{\partial X}{\partial v}-\frac{\partial \log A}{\partial v} X, \quad \frac{\partial X}{\partial u}-\frac{\partial \log B}{\partial u} X
$$

are direction-parameters of the tangents to the curves $u=$ const. and $v=$ const., respectively, on the first and second focal surfaces of $G$.

We say that these congruences of tangents are the first derived and minus first derived congruences of $G$, and we denote them by $G_{1}$ and $G_{-1}$.

As a corollary of this theorem we have:

When two congruences are parallel, their focal nets of the same rank are parallel.

Let $M_{0}\left(x_{0}\right)$ be a generic point on the middle surface of a congruence with direction-parameters $X$, the parameters $u$ and $v$ being those of the developables of the congruence. The coordinates of the focal points are of the form

$$
y=x_{0}+\varrho X, \quad z=x_{0}-\varrho X .
$$

Expressing that these values must satisfy (25), we have equations of the form (26). In order that these equations be consistent, they must reduce, on the assumption that the $X$ 's satisfy (27), to

$$
\left\{\begin{array}{l}
\frac{\partial x_{0}}{\partial u}=-\varrho \frac{\partial X}{\partial u}+2 \varrho X \frac{\partial}{\partial u} \log B \sqrt{\varrho}, \\
\frac{\partial x_{0}}{\partial v}=\varrho \frac{\partial X}{\partial v}-2 \varrho X \frac{\partial}{\partial v} \log A \sqrt{\varrho}
\end{array}\right.
$$


and $\varrho$ must satisfy the equation

(63) $\frac{\partial^{2} \varrho}{\partial u \partial v}+\frac{\partial \log A}{\partial v} \frac{\partial \varrho}{\partial u}+\frac{\partial \log B}{\partial u} \frac{\partial \varrho}{\partial v}+\left(\frac{\partial^{2}}{\partial u \partial v} \log A B-C\right) \varrho=0$,

which is the adjoint of equation (27).

Conversely, each solution of (63) and $n$ linearly independent solutions of (27) determine a congruence for which the surface of coordinates $x_{0}$ is the middle surface.

As a consequence of these results and the preceding corollary we have:

The determination of nets parallel to the focal nets of a congruence is equivalent to the integration of the adjoint of the direction equation of the congruence.

ro. Congruences harmonic to a net. From (54) it follows that the points of coordinates $y$ and $z$ defined by (53) are the focal points of first and second rank respectively of the congruence of lines joining these points. Hence:

The two Levy transforms of a net $N$ by means of the same solution $\theta$ of the point equation of $N$ are the focal points of the congruence of the joins of corresponding points of the transforms; that is, the points defined by (53) are Laplace transforms of one another.

The mutual arrangement of $N$

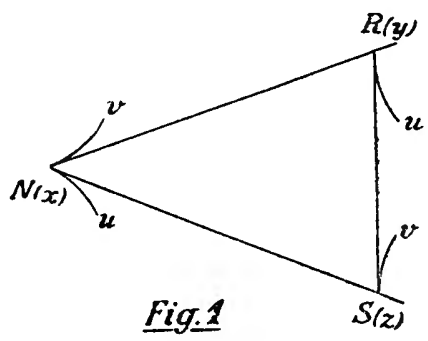
and the congruence is shown in fig. 1 where $u$ and $v$ indicate the parameter varying along the curve; this notation is used in all subsequent figures.

A net and a congruence are said to be harmonic when the foci of the congruence lie on the tangents of the net, and the developables of the congruence correspond to the curves of the net. As a consequence of the above theorem and the first one of $\S 8$ we have:

When a net $N$ is conjugate to a congruence $G$, the congruence of tangents to one family of curves of $N$ is harmonic to one of the focal nets of $G$ and the congruence of tangents of the other family of curves is harmonic to the other focal net of $G$. 
This situation is illustrated by fig. 2. We have also the theorem:

If $N$ is conjugate to a congruence $G$, the osculating planes of the curves of . parameter $u(v)$ of the first (second) focal net of $G$ are determined by the lines of $G$ and the tangents of the curves $u(v)$ of $N$.

Suppose that we have a net $N$ and a congruence whose lines lie in tangent planes of $N$ and the developables of $G$ correspond to the curves of $N$, taken as parametric. As $u$ varies, the corresponding focus of a line of the congruence must lie on the characteristic of the developable of the tangent planes along a curve $v=$ const. $^{8}{ }^{8}$. Since these

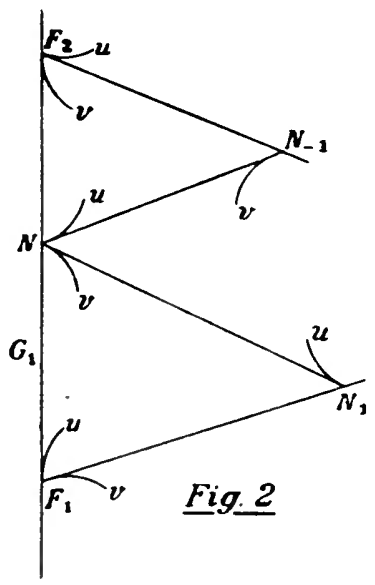
characteristics are tangent to the curves $u=$ const., we have the theorem:

If lines of a congruence lie in tangent planes of a net and developables of the congruence correspond to the curves of the net. the congruence is harmonic to the net.

We shall prove that any congruence harmonic to a net $N(x)$ may be obtained as in the first theorem of this section. In fact, the coordinates $z$ and $y$ of the foci $R$ and $S$ of a congruence harmonic to $N(x)$ are of the form

$$
z=x-r \frac{\partial x}{\partial u}, \quad y=x-t \frac{\partial x}{\partial v} .
$$

Now

$$
\frac{\partial z}{\partial v}=-r \frac{\partial x}{\partial u} \frac{\partial \log r a}{\partial v}+\frac{\partial x}{\partial v}\left(1-r \frac{\partial \log b}{\partial u}\right) .
$$

Expressing the condition that this is proportional to $r \frac{\partial x}{\partial u}-t \frac{\partial x}{\partial v}$, we get

$$
\frac{\partial}{\partial v} \frac{1}{r}=\frac{1}{r} \frac{\partial \log a}{\partial v}+\frac{1}{t} \frac{\partial \log b}{\partial u}-\frac{1}{r t} .
$$

8) This can be shown analytically by making use of the fact that the $x^{\prime} \cdot$ cannot satisfy (1) and two equations of the form referred to in $\S 4$. 
In similar manner the condition that the expression for $\frac{\partial y}{\partial u}$ shall be proportional to $r \frac{\partial x}{\partial u}-t \frac{\partial x}{\partial v}$ is

$$
\frac{\partial}{\partial u} \frac{1}{t}=\frac{1}{r} \frac{\partial \log a}{\partial v}+\frac{1}{t} \frac{\partial \log b}{\partial u}-\frac{1}{t r} .
$$

Hence there must exist a function $\theta$ such that

$$
\frac{1}{r}=\frac{1}{\theta} \frac{\partial \theta}{\partial u}, \frac{1}{t}=\frac{1}{\theta} \frac{\partial \theta}{\partial v}
$$

Substituting these values in (65), we find that $\theta$ is a solution of equation (1), and consequently equations (64) become equivalent to (53). Hence we lave the theorem:

A necessary and sufficient condition that a congruence be harmonic to a net $N(x)$ is that the focal nets of the congruence be Levy transforms of $N$ by means of the same solution $\theta$ of the point equation of $N$.

Since the direction-parameters of the harmonic congruence are of the form

$$
\frac{\partial \theta}{\partial u} \frac{\partial x}{\partial v}-\frac{\partial \theta}{\partial v} \frac{\partial x}{\partial u}
$$

it follows that if a second harmonic congruence, determined by a function $\theta_{1}$, is to be parallel to the given one, $\theta_{1}$ must be a function of $\theta$. Since both must satisfy (1), $\theta_{1}$ is a linear function of $\theta$ with constant coefficients. Hence we have:

A necessary and sufficient condition that two congruences, harmonic to a given net $N$ and determined by solutions $\theta$ and $\theta_{1}$ of the point equation of $N$, be parallel is that $\theta_{1}$ be a linear function of $\theta$ with constant coefficients.

Consider now a congruence $G$ and two nets $N_{1}$ and $N_{2}$ harmonic to $G$. Corresponding tangents to $N_{1}$ and $N_{2}$ meet in a point of a focal net of $G$, and the congruences of these tangents are conjugate to this focal net, by the theorem of Levy. These two congruences and the nets $N_{1}$ and $N_{2}$ are in the relation discussed in the last theorem of $\S 6$. Hence we have the theorem: 
If two nets are harmonic to a congruence, the joins of corresponding points of the nets form a congruence conjugate to the nets.

I I. Derived nets. Derivant nets. Let $G_{1}$ and $G_{2}$ be congruences harmonic to a net $N$, determined by solutions $\theta_{1}$ and $\theta_{2}$ of the point equation (1) of $N$, it being understood that $\theta_{2}$ is $n n t$ a linear function of $\theta_{1}$. The function

$$
\varphi=\theta_{2}-\frac{\theta_{1}}{\frac{\partial \theta_{1}}{\partial u}} \frac{\partial \theta_{2}}{\partial u}
$$

is a solution of the second of equations (56) with $\theta$ replaced by $\theta_{1}$. that is, the point equation of the second. focal net of $G_{1}$. The coordinates of the Levy transform $\bar{N}(\bar{x})$ of this focal net by means of $\varphi$ conjugate to $G_{1}$ are of the form

$$
\bar{x}=z-\frac{\varphi}{\frac{\partial \varphi}{\partial v}} \frac{\partial z}{\partial r},
$$

which in consequence of (53) and (54) is reducible to

$$
\bar{x}=x-\frac{\left(\theta_{1} \frac{\partial \theta_{2}}{\partial v}-\theta_{2} \frac{\partial \theta_{1}}{\partial v}\right) \frac{\partial x}{\partial u}-\left(\theta_{1} \frac{\partial \theta_{2}}{\partial u}-\theta_{2} \frac{\partial \theta_{1}}{\partial u}\right) \frac{\partial x}{\partial v}}{\frac{\partial \theta_{1}}{\partial u} \frac{\partial \theta_{2}}{\partial v}-\frac{\partial \theta_{1}}{\partial v} \frac{\partial \theta_{2}}{\partial u}} .
$$

The coordinates $y_{2}$ and $z_{2}$ of the focal points of $G_{2}$ are givell by (53) when $\theta$ is replaced by $\theta_{2}$. The point equation of the second focal surface of $G_{2}$ admits the solution $\theta_{1}-\theta_{2} \frac{\partial \theta_{1}}{\partial u} / \frac{\partial \theta_{2}}{\partial u}$, which determines a Levy transform of this surface conjugate to $G_{2}$. The expressions for the coordinates of this transform are reducible to (66). Hence:

If two congruences are harmonic to a net $N$, the point of intersection of corresponding lines of the two congruences describes a net conjugate to the two congruences.

This result is illustrated by fig. 3, where $L_{1 u}$ and $L_{1 v}$ are Levy transforms

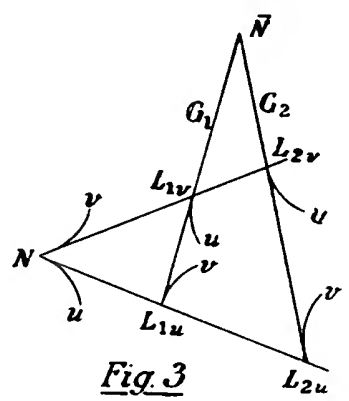


of $N$ by means of $\theta_{1}$ and $L_{2 u}$ and $L_{2 v}$ by means of $\theta_{2} \cdot \bar{N}$ is called the corresponding derived net by Guichard.

We shall prove the following converse of the above theorem:

If two congruences are conjugate to a net $N$, the planes determined by pairs of corresponding lines of the congruences envelop a net harmonic to the congruences.

Let the coordinates of the focal points of the congruences be taken in the forms (37) and (45). We have seen that the point of coordinates (46) describes a net parallel to the net of coordinates $y$, and is conjugate to the congruence $\bar{G}$ of the lines joining the points of coordinates $y$ and $y_{1}$. If the expression (46) is differentiated with respect to $v$, the resulting expression is reducible by means of (12) and (38) to $\bar{l} \frac{\partial y}{\partial v}$, where

$$
\bar{l}=1+\frac{h l_{1}-h_{1} l}{h-l} .
$$

If we apply the formulas (21) to his congruence $\bar{G}$, we find that the coordinates of the second focal net are in the form

which is reducible to

$$
\frac{y+h_{1}\left(y-y_{1}\right)-\bar{l} y}{1-\bar{l}}
$$

$$
x+\frac{x^{\prime}\left(l_{1}-h_{1}\right)-x^{\prime \prime}(l-h)}{h_{1} l-h l_{1}} .
$$

In like manner we find that the coordinates of the first focal point of the congruence conjugate to the nets of coordinates $z$ and $z_{1}$ are of the form (67). Hence the above theorem is proved. We say that the net of coordinates (67) is the derivant net of $N$. From (66) we have by differentiation

$$
\left\{\begin{array}{c}
\frac{\partial \bar{x}}{\partial u}=\frac{\theta_{1} \frac{\partial \theta_{2}}{\partial v}-\theta_{2} \frac{\partial \theta_{1}}{\partial v}}{\frac{\partial \theta_{2}}{\partial u} \frac{\partial \theta_{1}}{\partial v}-\frac{\partial \theta_{2}}{\partial v} \frac{\partial \theta_{1}}{\partial u}} \\
{\left[\frac{\partial^{2} x}{\partial u^{2}}+\frac{\left(\frac{\partial^{2} \theta_{1}}{\partial u^{2}} \frac{\partial \theta_{2}}{\partial v}-\frac{\partial^{2} \theta_{2}}{\partial u^{2}} \frac{\partial \theta_{1}}{\partial v}\right) \frac{\partial x}{\partial u}-\left(\frac{\partial^{2} \theta_{1}}{\partial u^{2}} \frac{\partial \theta_{2}}{\partial u}-\frac{\partial^{2} \theta_{2}}{\partial u^{2}} \frac{\partial \theta_{1}}{\partial u}\right) \frac{\partial x}{\partial v}}{\frac{\partial \theta_{2}}{\partial u} \frac{\partial \theta_{1}}{\partial v}-\frac{\partial \theta_{2}}{\partial v} \frac{\partial \theta_{1}}{\partial u}}\right]}
\end{array}\right.
$$


$(68 \mathrm{~b})$

$$
\left\{\begin{array}{c}
\frac{\partial \bar{x}}{\partial v}=-\frac{\theta_{1} \frac{\partial \theta_{2}}{\partial u}-\theta_{2} \frac{\partial \theta_{1}}{\partial u}}{\frac{\partial \theta_{2}}{\partial u} \frac{\partial \theta_{1}}{\partial v}-\frac{\partial \theta_{2}}{\partial v} \frac{\partial \theta_{1}}{\partial u}} \\
{\left[\frac{\partial^{2} x}{\partial v^{2}}+\frac{\left(\frac{\partial^{2} \theta_{1}}{\partial v^{2}} \frac{\partial \theta_{2}}{\partial v}-\frac{\partial^{2} \theta_{2}}{\partial v^{2}} \frac{\partial \theta_{1}}{\partial v}\right) \frac{\partial x}{\partial u}-\left(\frac{\partial^{2} \theta_{1}}{\partial v^{2}} \frac{\partial \theta_{2}}{\partial u}-\frac{\partial^{2} \theta_{2}}{\partial v^{2}} \frac{\partial \theta_{1}}{\partial u}\right) \frac{\partial x}{\partial v}}{\frac{\partial \theta_{2}}{\partial u} \frac{\partial \theta_{1}}{\partial v}-\frac{\partial \theta_{2}}{\partial v} \frac{\partial \theta_{1}}{\partial u}}\right.}
\end{array}\right]
$$

We remark that if we replace $\theta_{2}$ by $\theta_{2}+$ const. the expressions in the parentheses are unaltered. Consequently:

The $\infty^{1}$ derived nets of $N$ determined by $\theta_{1}$ and $\theta_{\mathbf{z}}+c$, where $c$ is a parameter, are parallel to one another and conjugate to the congruence harmonic to $N$ determined by $\theta_{1}$.

12. Determination of nets harmonic to a given congruence. We establish the following theorem which may be looked upon as a limiting case of the second theorem of the preceding section:

If two congruences are parallel, the point of intersection of lines joining corresponding focal points generates a net harmonic to the congruences.

Let $N(x)$ and $N^{\prime}\left(x^{\prime}\right)$ be the first focal nets of the congruences. The second focal nets are the minus first Laplace transforms of $N$ and $N^{\prime}$, and their coordinates are given by (47) and (51). From these expressions we find that the coordinates of the points of intersection of the lines joining the focal points of the first and second ranks respectively are of the form $\frac{x^{\prime}-l x}{1-l}$. By (21) this is the second focal point of the lines joining corresponding points on $N$ and $N^{\prime}$. In like manner it can be shown that it is the first focal point of the congruence conjugate to $N_{-1}$ and $N_{-1}^{\prime}$.

From these two theorems it follows that the problem of finding nets harmonic to a given congruence $G$ is equivalent to the determination of congruences conjugate to a net conjugate to $G$, or of congruences parallel to $G$, or of nets parallel to either focal net of $G$. In this section we give another means of finding nets harmonic to $G$, arising from the solution of the last problem.

If $G$ and $G^{\prime}$ are parallel congruences, and we use the notation of the above paragraph, we have from (47), (51), (52) and (14), 


$$
\bar{x}=x-\frac{x^{\prime}}{l}=x_{-1}-\frac{x_{-1}^{\prime}}{l},
$$

where $\bar{x}$ is thus defined. Consequently for the congruences conjugate to $N$ and $N_{-1}$ with direction-parameters $x^{\prime}$ and $x_{-1}^{\prime}$ corresponding lines meet in the points of coordinates $\bar{x}$, which is the second and first focal point respectively of these two congruences, in consequence of (52). Hence:

If $G$ and $G^{\prime}$ are parallel congruences, and through the focal points of $G$ lines are drawn parallel to the lines joining a fixed point to the corresponding focal points of $G^{\prime}$, these lines are tangent to the curves of a net harmonic to $G$.

From these results and the last theorem of $\S 9$ we have:

The determination of nets harmonic to a congruence is equivalent to the integration of the adjoint of the direction equation of the congruence.

13. Congruences harmonic to point nets. If $y$ and $z$ are the coordinates of the first and second focal points of a congruence, we have

$$
\frac{\partial y}{\partial u}=\frac{\partial \log q}{\partial u}(z-y), \quad \frac{\partial z}{\partial v}=\frac{\partial \log p}{\partial v}(y-z),
$$

where $p$ and $q$ are determinate functions. These equations are reducible to the normal form (6), if we put

$$
\alpha=z p, \quad \beta=y q
$$

with

$$
\frac{\partial p}{\partial v}=q n, \quad \frac{\partial q}{\partial u}=p m
$$

Hence we have the theorem:

The lines joining the origin to the foci of a congruence constitute a point net.

We say that the congruence is harmonic to the point net.

The direction-parameters of the congruence are given by

$$
Z=\alpha q-\beta p
$$


Conversely, suppose we have any point net of parameters $\alpha$ and $\beta$, and a pair of solutions $p$ and $q$ of the equations (6). The functions $y$ and $z$ given by (71) satisfy the conditions (70). Hence the joins of the points whose coordinates are $y$ and $z$ form a congruence for which these are the focal points, and consequently the congruence is harmonic to the point net. Accordingly we have the theorem:

If the parameters of a point net are in the normal form, each pair of solutions of the corresponding equations (6) gives directly a congruence harmonic to the point net, and all such harmonir congruences are so determined.

It is readily seen that if the parameters are not in the normal form the determination of harmonic congruences reduces to the solution of the equations (4) of the parameters.

Consider a net $N$ with normal parameters of its tangents given by (6). If $p$ and $q$ are a pair of solutions of (6), it follows from (5) that $\theta$, given by the quadrature

$$
\frac{\partial \theta}{\partial \imath}=a p, \quad \frac{\partial \theta}{\partial v}=b q,
$$

is a solution of the point equation (1) of $N$. Making use of this function $\theta$, we get a family of parallel congruences harmonic to $N$ whose direction-parameters are of the form

$$
\frac{\partial x}{\partial u} \frac{\partial \theta}{\partial v}-\frac{\partial x}{\partial v} \frac{\partial \theta}{\partial u}=a b(\alpha q-\beta p) .
$$

From (73) it is seen that these congruences are parallel to those harmonic to the parallel point net determined by $p$ and $q$.

Conversely, when a congruence harmonic to a net $N$ is known, we have by a quadrature at most a solution of the point equation of $N$ in consequence of the theorem of Levy $(\S 8)$. If the parameters of the tangents of $N$ are in the normal form, then $p$ and $q$ given by (74) satisfy the corresponding equations (6). Hence:

When the congruences harmonic to a point net are known, all the congruences harmonic to a parallel net can be found by quadratures; when a congruence harmonic to ary net is known, by a quadrature at most a congruence harmonic to the parallel point net can be found. 
Because of this theorem and the second one of $\S 12$ we have:

If $N$ is a net harmonic to a congruence $G$, and lines be drawn through the focal points of a parallel congruence $G^{\prime}$ parallel to the corresponding tangents to $N$, these lines are tangent to a net harmonic to $G^{\prime}$.

We have also:

Of all the parallel nets harmonic to the family of congruences parallel to a given congruence, one is the point net of the family.

Since the direction-parameters of any congruence harmonic to a net can be given the form (73), we have:

Each pair of solutions $p, q$ of equations (4) satisfied by the direction-parameters of the tangents to a net $N$ determine a congruence harmonic to $N$; its direction-parameters are of the form $\alpha^{\prime} q-\beta^{\prime} p$; all congruences harmonic to $N$ can be obtained in this way. From (73) we have by differentiation, and with the aid of (6) and (72),

$$
\begin{gathered}
\frac{\partial Z}{\partial u}=\frac{\partial \alpha}{\partial u} q-\frac{\partial p}{\partial u} \beta, \quad \frac{\partial Z}{\partial v}=\frac{\partial q}{\partial v} \alpha-\frac{\partial \beta}{\partial v} p \\
\frac{\partial^{2} Z}{\partial u \partial v}=\frac{\partial q}{\partial v} \frac{\partial \alpha}{\partial u}-\frac{\partial p}{\partial u} \frac{\partial \beta}{\partial v}+m n Z
\end{gathered}
$$

Hence the direction equation of the congruence is

$$
\text { (75) } \frac{\partial^{2} Z}{\partial u \partial v}=\frac{\partial \log q}{\partial v} \frac{\partial Z}{\partial u}+\frac{\partial \log p}{\partial u} \frac{\partial Z}{\partial v}+\left(m n-\frac{\partial \log p}{\partial u} \cdot \frac{\partial \log q}{\partial v}\right) Z \text {. }
$$

This is of the form (27), where now

$$
q=A U, \quad p=B V, \quad m n-\frac{\partial \log p}{\partial u} \cdot \frac{\partial \log q}{\partial v}=C,
$$

$U$ and $V$ being functions of $u$ and $v$ alone respectively. Hence:

The direction-parameters of any congruence whose direction equation is (27) are expressible in the form

$$
Z=\alpha A U-\beta B V,
$$


where $\alpha$ and $\beta$ are normal parameters of a net harmonic to the congruence.

14. Radial transformations. Suppose we have a net whose point equation is (1) and let $\theta$ be any solution of (1). From (57), and (58) it follows that the locus of the points of coordinates $\bar{x}$ given by

$$
\bar{x}=\frac{x}{\theta}
$$

is a net $\bar{N}$, whose point equation is

$$
\frac{\partial^{2} \bar{\theta}}{\partial u \partial v}=\frac{\partial}{\partial v} \log \frac{a}{\theta} \frac{\partial \bar{\theta}}{\partial u}+\frac{\partial}{\partial u} \log \frac{b}{\theta} \frac{\partial \bar{\theta}}{\partial v} .
$$

Conversely it follows from (58) that only when $\theta$ is a solution of (1) does the point $\bar{x}$ describe a net. We call $\bar{N}$ the radial transfor $m$ of $N$ by means of $\theta$.

The tangents to the curves $v=$ const. at corresponding points on $N$ and $\bar{N}$ meet in the point whose coordinates are

$$
x-\frac{\theta-1}{\frac{\partial \theta}{\partial u}} \frac{\partial x}{\partial u}=\bar{x}+\frac{1-\frac{1}{\theta}}{\frac{\partial}{\partial u}\left(\frac{1}{\theta}\right)} \frac{\partial \vec{x}}{\partial u},
$$

and the curves $u=$ const. in the point

$$
x-\frac{\theta-1}{\frac{\partial \theta}{\partial v}} \frac{\partial x}{\partial v}=\bar{x}+\frac{1-\frac{1}{\theta}}{\frac{\partial}{\partial v}\left(\frac{1}{\theta}\right)} \frac{\partial \bar{x}}{\partial v} .
$$

These points generate the Levy transforms of $N$ by means of the function $\theta-1$, and of $\bar{N}$ by $1-1 / \theta$. Hence we have the theorem:

The lines of intersection of the tangent planes of two nets $N$ and $\bar{N}$ in the relation of a radial transformation generate a congruence harmonic to both nets.

\section{Exercises.}

1. The coordinates of any point on a line joining two points of coordinates $x_{1}^{i}$ and $x_{2}^{i}$ are expressible in the form $\left(l_{1} x_{1}^{i}+l_{2} x_{2}^{i}\right) /\left(l_{1}+l_{2}\right)$.

2. The coordinates of any point of a plane determined by three points of coordinates $x_{1}^{i}, x_{2}^{i}, x_{3}^{i}$ are expressible in the form $\left(l_{1} x_{1}^{i}+l_{2} x_{2}^{i}+l_{3} x_{3}^{i}\right) /\left(l_{1}+l_{2}+l_{2}\right)$. 
3. The tangents to the curves $v=$ const. of all nets conjugate to. a given congruence at points of the same line of the congruence are coplanar; likewise for the tangents to the curves $u=$ const.

4. A necessary and sufficient condition that a point $P$ on the join of corresponding points of two parallel nets describe a net parallel to them is that $P$ divide the segment between points of the nets in constant ratio.

5. Show that if $h$ in (12) is a function $V$ of $v$ alone, then (1) must be of the form

$$
\frac{\partial^{2} \theta}{\partial u \partial v}=\rho \Gamma^{\prime \prime} \frac{\partial \theta}{\partial u}+\frac{\partial \log \rho}{\partial u} \frac{\partial \theta}{\partial v}
$$

one of whose invariants is zero. In this case $l=V+1 / \rho$.

6. When in equation (1) we have $a=U$ and $b=V$, where $U$ and $V$ are functions of $u$ and $v$ alone respectively, the point equation is $\frac{\partial^{2} \theta}{\partial u \partial v}=0$. In this case we say that $N(x)$ is a net of translation [cf. $\S 81$ ]. Show that all nets parallel to a net of translation are nets of translation.

7. The curves on the surface $S$ of a net $N$ which are defined by $\boldsymbol{E} \boldsymbol{d} \boldsymbol{u}^{2}$ $+2 F d u d v+G d v^{2}=0$ are called the minimal curves of $S$ [cf. $\$ 35$ ]. When the curves of $N$ are the minimal curves of $S, N$ is called a minimal net. Show that every net parallel to a minimal net is a minimal net.

8. If a net $N^{\prime}\left(x^{\prime}\right)$ has equal point iuvariants, that is $b^{\prime}=a^{\prime}$, the equatious

$$
\frac{\partial x}{\partial u}=\frac{1}{a^{\prime 2}} \frac{\partial x^{\prime}}{\partial u}, \quad \frac{\partial x}{\partial v}=-\frac{1}{a^{\prime 2}} \frac{\partial x^{\prime}}{\partial v}
$$

are consistent, and the $x$ s are coordinates of a net $N$. The cougruence conjugate to $N$ and of direction-parameters $x^{\prime}$ has for focal points $x-x^{\prime} / a^{\prime 2}, x+x^{\prime} / a^{\prime 2}$. Consequently $N$ lies on the middle surface of the congruence.

9. If $N$ and $N^{\prime}$ are parallel nets, and $\theta$ and $\theta^{\prime}$ are corresponding solutions of their point equations $(\$ 4)$, the point of coordinates $\left(x \theta^{\prime}-x^{\prime} \theta\right) /\left(\theta^{\prime}-\theta\right)$ describes a net conjugate to the congruence of the lines joining corresponding points on $Y$ and $X^{\prime \prime}$.

10. If $N$ and $N^{\prime}$ are parallel nets, and $\theta$ and $\theta^{\prime}$ are corresponding solutions of their point equations ( $(4)$, the corresponding Levy transforms of $N$ and $N^{\prime}$ by means of these respective functions are parallel nets; also the lines joining corresponding Levy transforms meet in the points of the net of Ex. 9.

Martin, Comptes Rendus, vol. 139 (1904), p. 32.

11. If $\theta_{1}$ is a solution of (1), then $\theta_{1}-\theta \frac{\partial \theta_{1}}{\partial v} / \frac{\partial \theta}{\partial v}$ and $\theta_{1}-\theta \frac{\partial \theta_{1}}{\partial u} / \frac{\partial \theta}{\partial u}$ are solutions of the respective equations (56); and the former admits the latter as its miuns first Laplace transform.

12. To each solution $\varphi$ of the first of equations (56) there correspondends a solution $\theta_{1}$ of (1) such that $\varphi=\theta_{1}-\theta \frac{\partial \theta_{1}}{\partial v} / \frac{\partial \theta}{\partial v}$.

13. If $\bar{N}$ is a derived net of a net $N$, the osculating planes of the curres of parameter $u$ and $v$ of $N$ pass through the corresponding points of the minus first and first Laplace transforms of $\bar{N}$. 
14. If $\bar{N}$ is a derived net of a net $N$, the first and minus first Laplace transforms of $\bar{N}$ are derived nets of the first and minus first Laplace transforms respectively of $N$.

Tzitzeica, Comptes Rendus, vol. 156 (1913), p. 374.

15. If $\bar{N}$ is the derived net of $N$ by means of solutions $\theta_{1}$ and $\theta_{2}$ of (1), the quantities $\bar{x}^{\prime}=\left(\frac{\partial x}{\partial u} \frac{\partial \theta_{1}}{\partial v}-\frac{\partial x}{\partial v} \frac{\partial \theta_{1}}{\partial u}\right) /\left(\frac{\partial \theta_{2}}{\partial u} \frac{\partial \theta_{1}}{\partial v}-\frac{\partial \theta_{2}}{\partial v} \frac{\partial \theta_{1}}{\partial u}\right)$ are the coordinates of a net parallel to $\bar{N}$.

16. A necessary and sufficient condition that a net $N$ and a parallel net $N^{\prime}$ defined by (12) be radial transforms of one another, to within a translation of either net, is that $h=l=$ const., say $c$; then if $N^{\prime}$ is a radial transform, $x^{\prime}=c x$.

17. A necessary and sufficient condition that two nets, $N^{\prime}$ and $N^{\prime \prime}$, parallel to $N$ and determined by pairs of solutions $h_{1}, l_{1}$, and $h_{2}, l_{2}$ of (13) be radial transforms of one another, to within a translation of either, is that $\frac{h_{3}}{h_{1}}=\frac{l_{3}}{l_{1}}=$ const.

18. If more than two ruled surfaces of a congruence are developable, all the ruled surfaces are developable and the lines of the congruence are concurrent.

19. If $\bar{N}$ is a radial transform of a net $N$ by ineans of a solution $\theta$ of the point equation (1) of $N$, the minus first and first Laplace transforms of $\bar{N}$ are radial transforms of the corresponding Laplace transforms of $N$. the respective functions being

$$
\theta-\frac{b}{\frac{\partial b}{\partial u}} \frac{\partial \theta}{\partial u}, \quad \theta-\frac{a}{\frac{\partial a}{\partial v}} \frac{\partial \theta}{\partial v} .
$$

20. If $\bar{N}$ is a radial transform of a net $N$ by means of a solution $\theta$ of the point equation of $N$, and $\theta_{1}$ is any other solution of this equation, then $\theta_{1} / \theta$ is a solution of the point equation of $\vec{N}$. Show that the Levy transforms of $N$ and $\bar{N}$ by means of $\theta_{1}$ and $\theta_{1} / \theta$ are radial transforms of one another.

21. If $G$ and $G_{1}$ are parallel congruences and lines be drawn throngh the focal points of each parallel to the lines joining the corresponding foci of the other to the origin, the two nets determined by the intersections of these pairs of lines are in the relation of a radial transformation. 
Chapter II.

\section{Transformations $F$.}

15. Fundamental equations. In this chapter we are concerned with the determination of all nets $N_{1}$ such that for a net $N_{1}$ and a given net $N$ the lines joining corresponding points form a congruence $G$ conjugate to $N$ and $N_{1}{ }^{9}$ ). These transformations of $N$ into nets $N_{1}$ are fundamental in a general theory of nets, and we call them the fundamental transformations, or for the sake of brevity transformations $F$. We say also that $N$ and $N_{1}$ are in relation $F$. We call $G$ the conjugate congruence of the transformation ${ }^{10}$ ). An example of this relation is afforded by two parallel nets and the lines joining corresponding points (\$ 4). Also the second theorem of $\S 6$ and the last theorem of $\S 11$ may be stated as follows:

When two congruences are conjugate to a net, corresponding focal nets are in relation $F$, or are radial transforms of one another.

When two nets are harmonic to a congruence, they are in relation $F$, $o$ are radial transforms of on another.

We turn now to the general study of this relation. From the second theorem of $\S 5$ it follows that if $N$ and $N_{1}$ are in relation $F$, the direction-parameters of the conjugate congruence of the transformation are proportional to the coordinates $x^{\prime}$ of a net $N^{\prime}$ parallel to $N$, and also to the coordinates $x_{1}^{\prime}$ of a net $N_{1}^{\prime}$ parallel to $N_{1}$. Hence these coordinates must satisfy a relation of the form

$$
x_{1}^{\prime}=\frac{x^{\prime}}{\theta^{\prime}}
$$

9) A statement of the history of these transformations is given in the Preface.

10) Two nets in the relation of a radial transformation (\$14) satisfy this requirement, since all the lines of the congruence meet in a point, and then every ruled surface of the congruence is developable. However, we exclude this exceptional case from the definition of transformations $F$. 
where, as follows from $\S 14, \theta^{\prime}$ is necessarily a solution of the point equation of $N^{\prime}$.

The coordinates $x_{1}$ of $N_{1}$ are necessarily of the form

$$
x_{1}=x-\frac{\theta}{\theta^{\prime}} x^{\prime}
$$

where $\theta$ is to be determined. The coordinates $x^{\prime}$ are given by equations of the form (cf. I, 12)

$$
\frac{\partial x^{\prime}}{\partial u}=h \frac{\partial x}{\partial u}, \frac{\partial x^{\prime}}{\partial v}=l \frac{\partial x}{\partial v} .
$$

Hence the first derivatives of $x_{1}$ are reducible to the forms

(4)

$$
\left\{\begin{array}{l}
\frac{\partial x_{1}}{\partial u}=\left(\frac{\theta^{\prime}}{h}-\theta\right) \frac{\partial x_{1}^{\prime}}{\partial u}+x_{1}^{\prime}\left(\frac{1}{h} \frac{\partial \theta^{\prime}}{\partial u}-\frac{\partial \theta}{\partial u}\right), \\
\frac{\partial x_{1}}{\partial v}=\left(\frac{\theta^{\prime}}{l}-\theta\right) \frac{\partial x_{1}^{\prime}}{\partial v}+x_{1}^{\prime}\left(\frac{1}{l} \frac{\partial \theta^{\prime}}{\partial v}-\frac{\partial \theta}{\partial v}\right) .
\end{array}\right.
$$

From these expressions it follows that $N_{1}$ is parallel to $N_{1}^{\prime}$, if, and only if, $\theta$ and $\theta^{\prime}$ satisfy

$$
\frac{\partial \theta^{\prime}}{\partial u}=l \frac{\partial \theta}{\partial u}, \quad \frac{\partial \theta^{\prime}}{\partial v}=l \frac{\partial \theta}{\partial v}
$$

Expressing the condition of integrability of these equations, we find from $(I, 13)$ that $\theta$ is a solution of the point equation of $N$, namely

$$
\frac{\partial^{2} \theta}{\partial u \partial v}=\frac{\partial \log a}{\partial v} \frac{\partial \theta}{\partial u}+\frac{\partial \log b}{\partial u} \frac{\partial \theta}{\partial v}
$$

Moreover, from (5) it follows that $\theta^{\prime}$ is the corresponding solution of the point equation of $N^{\prime}$ (cf. $\S 4$ ). Conversely, if $\theta$ is any solution of (6) and $\theta^{\prime}$ the corresponding solution of the point equation of $N^{\prime}$, then (2) defines an $F$ transform of $N$. Hence:

Any transformation $F$ of a net $N$ is determined by a net $N^{\prime}$ parallel to $N$, such that the joins of corresponding points of $N$ and $N^{\prime}$ are not concurrent, and by a solution of the point equation of $N$; and any such net $N^{\prime}$ and a solution determine an $F$ transform. 
In consequence of (5) equations (4) may be written

$$
\frac{\partial x_{1}}{\partial u}=-\frac{\tau}{h} \frac{\partial x_{1}^{\prime}}{\partial u}, \quad \frac{\partial x_{1}}{\partial v}=-\frac{\sigma}{l} \frac{\partial x_{1}^{\prime}}{\partial v}
$$

where we have put

$$
\tau=h \theta-\theta^{\prime}, \quad \sigma=l \theta-\theta^{\prime} .
$$

By means of (1), (3), and (5) equations (7) are reducible to

(9) $\frac{\partial x_{1}}{\partial u}=\frac{\tau}{\theta^{\prime 2}}\left(x^{\prime} \frac{\partial \theta}{\partial u}-\theta^{\prime} \frac{\partial x}{\partial u}\right), \quad \frac{\partial x_{1}}{\partial v}=\frac{\sigma}{\theta^{\prime 2}}\left(x^{\prime} \frac{\partial \theta}{\partial v}-\theta^{\prime} \frac{\partial x}{\partial v}\right)$,

which, in consequence of (2), are equivalent to

$$
\left\{\begin{array}{l}
\frac{\partial x_{1}}{\partial u}=-\frac{\tau}{\theta \theta^{\prime}}\left[\left(x_{1}-x\right) \frac{\partial \theta}{\partial u}+\theta \frac{\partial x}{\partial u}\right] \\
\frac{\partial x_{1}}{\partial v}=-\frac{\sigma}{\theta \theta^{\prime}}\left[\left(x_{1}-x\right) \frac{\partial \theta}{\partial v}+\theta \frac{\partial x}{\partial v}\right] .
\end{array}\right.
$$

From (9) it follows that the point equation of $N_{1}$ is reducible to

$$
\frac{\partial^{2} \theta_{1}}{\partial u \partial v}=\frac{\partial \log a_{1}}{\partial v} \frac{\partial \theta_{1}}{\partial u}+\frac{\partial \log b_{1}}{\partial u} \frac{\partial \theta_{1}}{\partial v}
$$

where

$$
a_{1}=a \frac{\tau}{\theta^{\prime}}, \quad b_{1}=b \frac{\sigma}{\theta^{\prime}} .
$$

This equation may also be put in the form

(13) $\frac{\partial^{2} \theta_{1}}{\partial u \partial v}=\frac{\sigma}{\tau}\left(\frac{\partial \log a}{\partial v}-\frac{h}{\theta^{\prime}} \frac{\partial \theta}{\partial v}\right) \frac{\partial \theta_{1}}{\partial u}+\frac{\tau}{\sigma}\left(\frac{\partial \log b}{\partial u}-\frac{l}{\theta^{\prime}} \frac{\partial \theta}{\partial u}\right) \frac{\partial \theta_{1}}{\partial v}$.

In consequence of ( $I, 37)$ equations (2) can also be given the forms

$$
x_{1}=-\frac{\tau}{\theta^{\prime}} x+\frac{\theta h y}{\theta^{\prime}}=-\frac{\sigma}{\theta^{\prime}} x+\frac{\theta l z}{\theta^{\prime}} .
$$


From (8) and (I, 18) follows

$$
\tau-\sigma=\varphi \theta .
$$

Incidentally we observe that $\tau$ and $\sigma$ satisfy

$$
\begin{cases}\frac{\partial \tau}{\partial u}=\theta \varphi \frac{\partial}{\partial u} \log b \varphi, & \frac{\partial \tau}{\partial v}=\theta \varphi \frac{\partial}{\partial v} \log \frac{\theta}{a}, \\ \frac{\partial \sigma}{\partial u}=-\theta \varphi \frac{\partial}{\partial u} \log \frac{\theta}{b}, & \frac{\partial \sigma}{\partial v}=-\theta \varphi \frac{\partial}{\partial v} \log a \varphi .\end{cases}
$$

Suppose we have any congruence $G$. There is a net $N$ conjugate to $G(\S 8)$, and a net $N^{\prime}$ parallel to $N$ whose coordinates $x^{\prime}$ are direction-parameters of $G(\S 5)$. Each radial transform of $N^{\prime}$, say $N_{1}^{\prime}$, determines an $F$ transform $N_{1}$ of $N$, and $N_{1}$ is parallel to $N_{1}^{\prime}$. Since there is an infinity of parallel nets $N_{1}$ satisfying this condition $(\$ 5)$, we have the theorem:

If the coordinates of $a$ net are the direction-parameters of a congruence, there are an infinity of nets parallel to the former. net and conjugate to the congruence.

As a corollary we have:

If two congruences are parallel, every net conjugate to the one is parallel to $\infty^{1}$ nets conjugate to the other.

For, if $N$ is a net conjugate to the first congruence, there is a net $N^{\prime}$, parallel to $N$, whose coordinates are direction-parameters of both congruences, and by the theorem there are $\infty^{1}$ nets conjugate to the second congruence and parallel to $N^{\prime}$.

16. Inverse of a transformation $F$. Parallel transformations $F$. Evidently $N$ can be looked upon as a transform of $N_{1}$ and now we seek the functions $\theta^{-1}$ and $\left(\theta^{\prime}\right)^{-1}$ giving this transformation. Since the roles of $N^{\prime}$ and $N_{1}^{\prime}$ are interchanged it follows from (1) that

$$
\left(\theta^{\prime}\right)^{-1}=\frac{1}{\theta^{\prime}}
$$

Hence if we make (2) conform to

$$
x=x_{1}-\frac{\theta^{-1}}{\left(\theta^{\prime}\right)^{-1}} x_{1}^{\prime}
$$


we find that

$$
\theta^{-1}=-\frac{\theta}{\theta^{\prime}}
$$

It is readily verified that equations (7) are satisfied by these values of $\theta^{-1}$ and $\left(\theta^{\prime}\right)^{-1}$. Hence $\theta^{-1}$ is a solution of (11). Moreover, we have

$$
\left\{\begin{array}{l}
\frac{\partial}{\partial u}\left(\frac{1}{\theta^{-1}}\right)=\tau \frac{\partial}{\partial u}\left(\frac{1}{\theta}\right), \\
\frac{\partial}{\partial v}\left(\frac{1}{\theta^{-1}}\right)=\sigma \frac{\partial}{\partial v}\left(\frac{1}{\theta}\right) .
\end{array}\right.
$$

Hence equations (9) can be written

$$
\left\{\begin{array}{l}
\frac{\partial}{\partial u}\left(\frac{x_{1}}{\theta^{-1}}\right)=\boldsymbol{\tau} \frac{\partial}{\partial u}\left(\frac{x}{\theta}\right), \\
\frac{\partial}{\partial v}\left(\frac{x_{1}}{\theta^{-1}}\right)=\sigma \frac{\partial}{\partial v}\left(\frac{x}{\theta}\right) .
\end{array}\right.
$$

From these equations follows:

If $N$ and $N_{1}$ are in the relation $F$ determined by a solution $\theta$ of the point equation of $N$, and $\theta^{-1}$ is the solution of the point equation of $N_{1}$ likewise determining the transformation, the radial transforms of $N$ and $N_{1}$ by means of $\theta$ and $\theta^{-1}$ respectively are parallel.

We have obsèrved in $\S 15$ that two parallel nets are in relation $F$, since they are conjugate to the congruence of lines joining corresponding points. We wish to find the form of equation (2) in this case.

From (9) we see that:

$A$ necessary and sufficient condition that $N_{1}$ be parallel to $N$ $i s$ that $\theta$ be a constant.

Now $\theta^{\prime}$ also is a constant, which must be different from zero. Hence the equations of the parallel transforms are of the form

$$
x_{1}=x-c x^{\prime}
$$

where $c$ is an arbitrary constant.

In a general transformation $F$ the function $\theta$ corresponding to a given $\theta^{\prime}$ is determined by (5) only to within an additive 
constant. Suppose we consider the transforms $N_{1}$ and $N_{2}$, corresponding to the values $\theta$ and $\theta+c$, where $c$ is a constant and to the same $\theta^{\prime}$. Now the coordinates of $N_{1}$ are given by (2) and those of $N_{2}$ by

$$
x_{2}=x-\frac{\theta+c}{\theta^{\prime}} x^{\prime} \text {. }
$$

From these follows

$$
x_{2}=x_{1}-c x_{1}^{\prime} \text {. }
$$

In consequence of the above results we have:

When the conjugate congruence of two transformations is the same, and the two functions $\theta$ differ by an additive constant while $\theta^{\prime}$ is the same, the two transforms are parallel to one another.

In the definition of transformations $F$ we have required that $\Lambda^{\prime \prime}$ be not a radial transform of $N$. It is readily shown that in order that $N^{\prime}$, parallel to $N$, be a radial transform of $N$ it is necessary and sufficient that $x^{\prime}=c x$, where $c$ is a constant. In this case corresponding solutions $\theta$ and $\theta^{\prime}$ of the point equations of $N$ and $N^{\prime}$ are in the relation $\theta^{\prime}=c \theta+d$. Substituting in (2), we have

$$
x_{1}=\frac{x d}{c \theta+d}
$$

that is $N_{1}$ is a radial transform of $N$. Conversely, in order that (2) define a radial transform of $N$ it is necessary that $N^{\prime}$ be a radial transform of $N$. Consequently, if the restriction is removed from the definition of transformations $F$, radial transformations form a sub-group of transformations $F$. But we shall retain the restriction and thus distinguish between the two types of transformations.

17. Harmonic congruence of a transformation $F$. From (20) and (21) we have

$$
\left\{\begin{array}{l}
x_{1}-\frac{\theta^{-1}}{\frac{\partial \theta^{-1}}{\partial u}} \frac{\partial x_{1}}{\partial u}=x-\frac{\theta}{\frac{\partial \theta}{\partial u}} \frac{\partial x}{\partial u} \\
x_{1}-\frac{\theta^{-1}}{\frac{\partial \theta^{-1}}{\partial v}} \frac{\partial x_{1}}{\partial v}=x-\frac{\theta}{\frac{\partial \theta}{\partial v}} \frac{\partial x}{\partial v}
\end{array}\right.
$$

"Hence the corresponding tangents to the curves $u=$ const. of the nets $N$ and $N_{1}$ meet in points of a net which is a Levy transform 
of $N$ by $\theta$ and $N_{1}$ by $\theta^{-1}$, and likewise the tangents to the curves $v=$ const. Furthermore as follows from the theorem of Levy $(\S 8)$ the line joining these points of intersection generates a congruence harmonic to the nets $N$ and $N_{1}$. Hence we have the following converse of the second theorem of $\S 15$ :

When two nets $N, N_{1}$ are in the relation of a transformation $F$, their corresponding tangent planes meet in a line generating the congruence harmonic to $N$ determined by the function $\theta$ and harmonic to $N_{1}$ determined by $\theta^{-1}$.

We call this the harmonic congruence of the transformation.

From the above theorem and the second of $\S 15$ follows:

If $N$ is a net and $G$ a congruence harmonic to it, the nets harmonic to $G$ are obtainable from $N$ by transformations $F$ involving the same function $\theta$, or by radial transformations of $N$.

Since $\theta^{\prime}$ is determined by (5) only to within an additive constant, we have as a corollary to this theorem:

All the nets $N_{1}$ obtained from $N$ by transformations $F$ determined bysthe same function $\theta$, and differing only in the additive constant of $\boldsymbol{\theta}^{\prime}$, are harmonic to the same congruence, and consequently their tangent planes form linear pencils.

The coordinates of the point of intersection of the conjugate congruence of a transformation $F$ and the hyperplane $x^{i}=0$ (cf. $\S 1$ ) are of the form

$$
x-\frac{x^{i}}{x^{i^{\prime}}} x^{\prime}
$$

Since $x^{i}$ and $x^{i \prime}$ are corresponding solutions of the point equations of $N$ and $N^{\prime}$, we have the result:

The developables of any congruence meet a hyperplane in a net.

In the above case the $i$ th coordinate of $N_{1}^{\prime}$ is 1 , as follows from (1). Consequently $N_{1}^{\prime}$ also is a net in a hyperplane.

18. Tansformations $F$ and radial transformations. Let $\bar{N}(x)$ be a radial transform of $N(x)$ by means of a solution $\omega$ of the point equation (6) of $N$, so that $\bar{x}=x / \omega$. From $\S 14$ we have that the point equation of $\bar{N}$ is

$$
\frac{\partial^{2} \bar{\theta}}{\partial u \partial v}=\frac{\partial}{\partial v} \log \frac{a}{\omega} \frac{\partial \bar{\theta}}{\partial u}+\frac{\partial}{\partial u} \log \frac{b}{\omega} \frac{\partial \bar{\theta}}{\partial v} .
$$


If $\theta$ is any other solution of (6), then $\bar{\theta}=\theta / \omega$ is a solution of (23). Also it can be shown that if $\varphi$ is a solution of the adjoint of (6), then $\bar{\varphi}=\varphi \omega$ is a solution of the adjoint of (23).

From (16) it follows that if $\boldsymbol{r}$ and $\sigma$ are functions of the transformation $F$ of $N$ by means of $\theta$ and $\varphi$, these functions, $\tau$ and $\sigma$, serve also for the transformation $F$ of $\bar{N}$ into a net $\overline{N_{1}}\left(\bar{x}_{1}\right)$, determined by $\bar{\theta}$ and $\bar{\varphi}$. Hence similarly to (20) and (21) we have

(24)

$$
\frac{\partial}{\partial u}\left(\frac{1}{\bar{\theta}^{-1}}\right)=\boldsymbol{x} \frac{\partial}{\partial u}\left(\frac{1}{\bar{\theta}}\right)=\boldsymbol{\tau} \frac{\partial}{\partial u}\left(\frac{\omega}{\theta}\right), \frac{\partial}{\partial v}\left(\frac{1}{\bar{\theta}^{-1}}\right)=\sigma \frac{\partial}{\partial v}\left(\frac{\omega}{\theta}\right)
$$

(25) $\frac{\partial}{\partial u}\left(\frac{\bar{x}_{1}}{\bar{\theta}^{-1}}\right)=\boldsymbol{\tau} \frac{\partial}{\partial u}\left(\frac{\bar{x}}{\bar{\theta}}\right)=\boldsymbol{\tau} \frac{\partial}{\partial u}\left(\frac{x}{\theta}\right), \frac{\partial}{\partial v}\left(\frac{\bar{x}_{1}}{\bar{\theta}^{-1}}\right)=\boldsymbol{\sigma} \frac{\partial}{\partial v}\left(\frac{x}{\theta}\right)$.

From these equations and (21) we have

$$
\frac{\bar{x}_{1}}{\overline{\theta^{-1}}}=\frac{x_{1}}{\theta^{-1}},
$$

to within an additive constant of integration. If we define a function $\omega_{1}$ by

equations (24) become

$$
\bar{\theta}^{-1} \omega_{1}=\theta^{-1},
$$

$$
\frac{\partial}{\partial u}\left(\frac{\omega_{1}}{\theta^{-1}}\right)=\boldsymbol{\tau} \frac{\partial}{\partial u}\left(\frac{\omega}{\theta}\right), \frac{\partial}{\partial v}\left(\frac{\omega_{1}}{\theta^{-1}}\right)=\sigma \frac{\partial}{\partial v}\left(\frac{\omega}{\theta}\right) .
$$

Comparing these equations with (21), we note that $\omega_{1}$ is a solution of the point equation of $N_{1}$. Hence from (26) it follows that $\bar{N}_{1}$ is the radial transform of $N_{1}$ by means of $\omega_{1}$. Thus by the quadrature (27) we obtain a net $\bar{N}_{1}$ which is an $F$ transform of $\bar{N}$. Moreover, there are an infinity of such nets $\bar{N}_{1}$, since $\omega_{c}=\omega_{1}+c \theta^{-1}$, where $c$ is an arbitrary constant, satisfies (27). Hence:

If $N$ and $\bar{N}$ are nets in the relation of a radial transformation, and $N_{1}$ is an $F$ transform of $N$, there can be found by a quadrature $\infty^{1}$ nets $\bar{N}_{1}$, which are $F$ transforms of $\bar{N}$. and radial transforms of $N_{1}$.

When in particular $\theta=\omega$, then $\bar{\theta}=1$ and consequently $\bar{N}$ and the nets $\bar{N}_{1}$ are parallel, the functions $\omega_{1}$ being $c \theta^{-1}$. Hence: 
A transformation $F$ is equivalent to the combination of a radial, a parallel and a radial transformation ${ }^{11}$ ).

19. Transformations $F$ with a common conjugate congruence. Suppose we have two solutions $\theta_{1}$ and $\theta_{2}$ of equation (6). We seek the two nets obtained from $N$ by transformations $F$ determined by these respective functions and by a net $N^{\prime}$ parallel to $N$. We denote these nets by $N_{1,1}$ and $N_{2,1}{ }^{12}$ ).

The point coordinates of $N_{1,1}$ and $N_{2,1}$ are expressible in the forms

$$
x_{1,1}=x-\frac{\theta_{1}}{\theta_{1}^{\prime}} x^{\prime}, \quad x_{2,1}=x-\frac{\theta_{2}}{\theta_{2}^{\prime}} x^{\prime}
$$

where $\theta_{1}, \theta_{1}^{\prime}$ and $\theta_{2}, \theta_{2}^{\prime}$ are pairs of corresponding solutions of the point equations of $N$ and $N^{\prime}$, that is $\theta_{1}^{\prime}$ and $\theta_{2}^{\prime}$ are obtained from $\theta_{1}$ and $\theta_{2}$ respectively by quadratures of the form (5).

We consider the functions

$$
\theta_{2}-\frac{\theta_{1}}{\theta_{1}^{\prime}} \theta_{2}^{\prime}, \quad \frac{\theta_{2}^{\prime}}{\theta_{1}^{\prime}}
$$

From (2) and (1) it follows that these functions are solutions of the point equations of $N_{1,1}$ and $N_{1,1}^{\prime}$ respectively, and their derivatives are in relations analogous to (7). Hence a transformation of $N_{1,1}$ is given by

$$
x_{1,1}-\frac{\theta_{2} \theta_{1}^{\prime}-\theta_{1} \theta_{2}^{\prime}}{\theta_{2}^{\prime}} x_{1,1}^{\prime}
$$

By substitution we find that this expression is reducible to that of $x_{2,1}$, given by (28). Hence $N_{2,1}$ is the transform of $N_{1,1}$ by means of $N_{1,1}^{\prime}$, and the functions (29). It follows then from $\S 16$ that $N_{1,1}$ is obtained from $N_{2,1}$ by the functions $\theta_{1}-\theta_{2} \theta_{1}^{\prime} / \theta_{2}^{\prime}$ and $\theta_{1}^{\prime} / \theta_{2}^{\prime}$.

20. Transformations $F$ determined by the same function $\theta$. Let $N^{\prime}$ and $N^{\prime \prime}$ be two nets parallel to a given net $N$

ii) In fact Jonas dereloped the transformations from this point of view (see Preface); this theorem follows also from the first of $\S \mathbf{1 6}$.

12) In this notation the first subscripts refer to the subscripts of $\theta_{1}$ and $\theta_{3}$ and the second to the subscript of common function, $\varphi_{1}$, determining the conjugate congruence. 
which are not radial transforms of one another. The coordinates $x^{\prime}$ and $x^{\prime \prime}$ of $N^{\prime}$ and $N^{\prime \prime}$ respectively are given by quadratures of the form

$$
\begin{cases}\frac{\partial x^{\prime}}{\partial u}=h_{1} \frac{\partial x}{\partial u}, & \frac{\partial x^{\prime}}{\partial v}=l_{1} \frac{\partial x}{\partial v}, \\ \frac{\partial x^{\prime \prime}}{\partial u}=h_{2} \frac{\partial x}{\partial u}, & \frac{\partial x^{\prime \prime}}{\partial v}=l_{2} \frac{\partial x}{\partial v},\end{cases}
$$

where $h_{1}, l_{1}$ and $h_{2}, l_{2}$ are pairs of solutions of equations $(\mathrm{I}, 13)$. The coordinates $x^{\prime}$ and $x^{\prime \prime}$ are the direction-parameters of two congruences, $G^{\prime}$ and $G^{\prime \prime}$ respectively, conjugate to $N$. By means of them and a solution $\theta_{1}$ of the point equation of $N$ we obtain two transforms $N_{1,1}$ and $N_{1,2}$ of $N$, whose point coordinates are of the respective forms

$$
x_{1,1}=x-\frac{\theta_{1}}{\theta_{1}^{\prime}} x^{\prime}, \quad x_{1,2}=x-\frac{\theta_{1}}{\theta_{1}^{\prime \prime}} x_{1}^{\prime \prime} .
$$

It is our purpose to show that $N_{1,1}$ and $N_{1,2}$ are in relation $F$.

There is a net which is the $F$ transform of $N^{\prime \prime}$ by means of $\theta_{1}^{\prime \prime}$ and $N^{\prime}$. Its coordinates are of the form

$$
x_{1,1}^{\prime \prime \prime}=x^{\prime \prime}-\frac{\theta_{1}^{\prime \prime}}{\theta_{1}^{\prime}} x^{\prime}
$$

Differentiating and making use of (19), (31) and similar equations for $\theta_{1}^{\prime}$ and $\theta_{1}^{\prime \prime}$, we get

$$
\left\{\begin{array}{l}
\frac{\partial x_{1,1}^{\prime \prime \prime}}{\partial u}=\left(h_{1} \theta_{1}^{\prime \prime}-h_{2} \theta_{1}^{\prime}\right) \frac{1}{\tau_{1}} \cdot \frac{\partial x_{1,1}}{\partial u} \\
\frac{\partial x_{1,1}^{\prime \prime \prime}}{\partial v}=\left(l_{1} \theta_{1}^{\prime \prime}-l_{2} \theta_{1}^{\prime}\right) \frac{1}{\sigma_{1}} \cdot \frac{\partial x_{1,1}}{\partial v} .
\end{array}\right.
$$

Hence $N_{1,1}^{\prime \prime \prime}$ as defined by (33) is parallel to $N_{1,1}{ }^{13}$ ).

We have seen that the solution of the point equation of $N_{1,1}$ giving $N$ by the inverse transformation is $-\theta_{1} / \theta_{1}^{\prime}$. When $x_{1,1}$ in (34) is replaced by this value, we have by a quadrature a solution of the point equation of $N_{1,1}^{\prime \prime \prime}$. By means of (20) we find that the

${ }^{13}$ ) We have used the notation $x_{1,1}^{\prime \prime \prime}$ to mean that the net is parallel to $N_{1,1}$ and determines a congruence $G^{\prime \prime \prime}$ conjugate to $N_{1,1}$. 
corresponding solution of (34) is $-\theta_{1}^{\prime \prime} / \theta_{1}^{\prime}$. Hence a transformation $F$ of $N_{1,1}$ is given by equations of the form

$$
x_{1,1}-\frac{\theta_{1}}{\theta_{1}^{\prime \prime}} x_{1,1}^{\prime \prime \prime}
$$

By substituting the above values we find that this expression is reducible to the second of (32). Thus $N_{1,2}$ is a transform of $N_{1,1}$ by means of the same function, $-\theta_{1} / \theta_{1}^{\prime}$, which gives the transformation of $N_{1,1}$ into $N$. Hence we have the theorem:

If a net $N$ is transformed into two nets $N_{1,1}$ and $N_{1,2}$ by means of the same function $\theta_{1}$, the latter two nets are in the relation of a transformation $F$; moreover, in the triad of nets $N, N_{1,1}$ and $N_{1,2}$, any two are the transforms of the third by means of the same solution of its point equation.

Hereafter we say that three nets so related form a triad under transformations $F$. Now equation (33) may be interpreted as follows:

If the nets $N, N_{1}$, and $N_{2}$ form a triad, and if $N^{\prime}$ and $N^{\prime \prime}$ are the nets parallel to $N$ determining the transformations from $N$ to $N_{1}$ and $N_{2}$ respectively, the net $N_{1,1}^{\prime \prime \prime}$ determining the congruence of the transformation from $N_{1}$ to $N_{2}$ can be so placed in space that it is an $F$ transform of $N^{\prime \prime}$, the conjugate congruence of the latter transformation being determined by $N^{\prime}$.

As a particular case of this result, suppose we use for $\theta_{1}$ the coordinate $x^{(n)}$. Then the two transforms are the nets in which the hyperplane $x^{(n)}=0$ is met by the lines of the two congruences, and in accordance with the above theorem these two nets in the hyperplane $x^{(n)}=0$ are in the relation of a transformation $F$. In general, we have:

If $a$ net $N$ is conjugate to two congruences $G^{\prime}$ and $G^{\prime \prime}$, the developables of these congruences meet any hyperplane in two nets in the relation $F$.

We shall prove the converse of the above theorem:

If two nets $N_{1}$ and $N_{2}$, transforms of a net $N$ by means of congruences $G^{\prime}$ and $G^{\prime}$, are $F$ transforms of one another, the three nets form a triad, unless $N_{1}$ and $N_{2}$ are parallel transforms of $N$.

Let the coordinates of $x_{1}$ and $x_{2}$ of $N_{1}$ and $N_{2}$ be given by

$$
x_{1}=x-\frac{\theta_{1}}{\theta_{1}^{\prime}} x^{\prime}, \quad x_{2}=x-\frac{\theta_{2}}{\theta_{2}^{\prime \prime}} x^{\prime \prime}
$$


If these nets are to be in relation $F$, it is necessary and sufficient that on the lines joining corresponding points there be focal points, that is that there exist functions $\lambda_{1}, \lambda_{2}, \mu_{1}, \mu_{2}$ such that

$$
\begin{aligned}
& \frac{\partial}{\partial u}\left[x_{1}+\lambda_{1}\left(x_{1}-x_{2}\right)\right]=\mu_{1}\left(x_{1}-x_{2}\right), \\
& \frac{\partial}{\partial v}\left[x_{1}+\lambda_{2}\left(x_{1}-x_{2}\right)\right]=\mu_{2}\left(x_{1}-x_{2}\right) .
\end{aligned}
$$

When the above values are substituted in these equations, we get equations of the form

$$
A_{1} x^{\prime}+B_{1} x^{\prime \prime}+C_{1} \frac{\partial x}{\partial u}=0, \quad A_{2} x^{\prime}+B_{2} x^{\prime \prime}+C_{2} \frac{\partial x}{\partial v}=0 .
$$

Evidently we must have

$$
A_{1}=B_{1}=C_{1}=A_{2}=B_{2}=C_{2}=0 .
$$

These conditions are equivalent to

$$
\begin{aligned}
\left(1+\lambda_{1}\right) \tau_{1} \theta_{2}^{\prime \prime}-\lambda_{1} \tau_{2} \theta_{1}^{\prime} & =0, \\
\lambda_{1} \tau_{2} \frac{\partial \theta_{1}}{\partial u}+\theta_{1} \theta_{2}^{\prime \prime}\left(\mu_{1}-\frac{\partial \lambda_{1}}{\partial u}\right) & =0, \quad \lambda_{1} \tau_{2} \frac{\partial \theta_{2}}{\partial u}+\theta_{2} \theta_{2}^{\prime \prime}\left(\mu_{1}-\frac{\partial \lambda_{1}}{\partial u}\right)=0 \\
\lambda_{2} \sigma_{2} \frac{\partial \theta_{1}}{\partial v}+\theta_{1} \theta_{2}^{\prime \prime}\left(\mu_{2}-\frac{\partial \lambda_{2}}{\partial v}\right) & =0, \quad \lambda_{2} \sigma_{2} \frac{\partial \theta_{2}}{\partial v}+\theta_{2} \theta_{2}^{\prime \prime}\left(\mu_{2}-\frac{\partial \lambda_{2}}{\partial v}\right)=0 .
\end{aligned}
$$

If $\tau_{1}=\sigma_{1}=\tau_{2}=\sigma_{2}=0$, then $h_{1}=l_{1}=$ const. and $h_{2}=l_{2}=$ const. $(\S 4)$, and consequently $G^{\prime}$ and $G^{\prime \prime}$ are not distinct. If $\theta_{1}$ and $\theta_{2}$ are constants, then $N_{1}$ and $N_{2}$ are parallel $(\S 16)$. Excluding these cases, we find that the above equations necessitate $\theta_{2} / \theta_{1}=$ const., that is $N, N_{1}, N_{2}$ form a triad.

21. The theorem of permutability of transformations $F$. The equations (1) and (2) apply to any pairs of solutions of the point equations of $N$ and $N_{1,1}$. Making use of (1), (2) and (18) we can show that any solution of the point equation of $N_{1,1}$ is expressible in the form

$$
\theta_{12}=\theta_{2}-\frac{\theta_{1}}{\theta_{1}^{\prime}} \theta_{2}^{\prime},
$$


where $\theta_{2}$ is a solution of (6). Incidentally we remark that from (21) it follows that

$$
\left\{\begin{array}{l}
\frac{\partial}{\partial u}\left(\theta_{12} \frac{\theta_{1}^{\prime}}{\theta_{1}}\right)=-\tau_{1} \frac{\partial}{\partial u}\left(\frac{\theta_{2}}{\theta_{1}}\right), \\
\frac{\partial}{\partial v}\left(\theta_{12} \frac{\theta_{1}^{\prime}}{\theta_{1}}\right)=-\sigma_{1} \frac{\partial}{\partial v}\left(\frac{\theta_{2}}{\theta_{1}}\right) .
\end{array}\right.
$$

This function $\theta_{12}$ determines a transform of $N_{1,1}$ such that its points lie on the lines joining corresponding points on $N_{1,1}$ and $N_{1,2}$, that is, $G^{\prime \prime \prime}$ is the conjugate congruence. From (33) it is seen that the corresponding function $\theta_{12}^{\prime \prime}$ is given by

$$
\theta_{12}^{\prime \prime \prime}=\theta_{2}^{\prime \prime}-\frac{\theta_{1}^{\prime \prime}}{\theta_{1}^{\prime}} \theta_{2}^{\prime}
$$

Hence the coordinates of the transform $N_{12}$ are of the form

$$
x_{12}=x_{1,1}-\frac{\theta_{2} \theta_{1}^{\prime}-\theta_{1} \theta_{2}^{\prime}}{\theta_{2}^{\prime \prime} \theta_{1}^{\prime}-\theta_{1}^{\prime \prime} \theta_{2}^{\prime}} x_{1,1}^{\prime \prime} \text {. }
$$

The function $\theta_{2}$ and the congruences $G^{\prime}$ and $G^{\prime \prime}$ define two transforms of $N$, namely $N_{2,1}$ and $N_{2,2}$, whose coordinates are respectively of the forms

$$
x_{2,1}=x-\frac{\theta_{\mathbf{2}}}{\theta_{2}^{\prime}} x^{\prime}, \quad x_{2,2}=x-\frac{\theta_{\mathbf{2}}}{\theta_{2}^{\prime \prime}} x^{\prime \prime} .
$$

Corresponding points of the nets $N, N_{1,2}$ and $N_{2,2}$ lie on a line, and from (29), (32) and (40) we have that $N_{2,2}$ is a transform of $N_{1,2}$ by means of the function

$$
\theta_{2}-\frac{\theta_{1}}{\theta_{1}^{\prime \prime}} \theta_{2}^{\prime \prime}
$$

In like manner it follows from (29) and (35) that $N_{12}$ is obtainable from $N_{1,2}$ by means of the function $\theta_{12}-\theta_{1} \theta_{12}^{\prime \prime \prime} / \theta_{1}^{\prime \prime}$. But by means of (36) and (38) we show that this expression is reducible to (41). Hence $N_{2,2}$ and $N_{12}$, being transforms of $N_{1,2}$ by means of the same solution of the latter's point equation are themselves. in relation $F$. We wish to show further that $N_{12}$ bears to $N_{2,2}$ and $N_{2,1}$ a relation analogons to that born to $N_{1,1}$ and $N_{1,2}$ used to determine $N_{12}$. 
Since $N_{2,2}$ and $N_{2,1}$ are obtained from $N$ by $\theta_{2}$, they are in relation $F$. The corresponding net parallel to $N_{2,2}$ is defined by equations of the form (cf.(33))

$$
\left.x_{2,2}^{\prime \prime \prime}=x^{\prime}-\frac{\theta_{2}^{\prime}}{\theta_{2}^{\prime \prime}} x^{\prime \prime 14}\right)
$$

A solution of the point equation of $N_{2,2}$ is

$$
\theta_{21}=\theta_{1}-\frac{\theta_{2}}{\theta_{2}^{\prime \prime}} \theta_{1}^{\prime \prime}
$$

and the corresponding function $\theta_{21}^{\prime \prime \prime}$ is given by

$$
\theta_{21}^{\prime \prime \prime}=\theta_{1}^{\prime}-\frac{\theta_{2}^{\prime}}{\theta_{2}^{\prime \prime}} \theta_{1}^{\prime \prime} .
$$

The net $N_{21}$ obtained by this transformation is defined by equations of the form

$$
x_{21}=x_{2,2}-\frac{\theta_{1} \theta_{2}^{\prime \prime}-\theta_{2} \theta_{1}^{\prime \prime}}{\theta_{1}^{\prime} \theta_{2}^{\prime \prime}-\theta_{2}^{\prime} \theta_{1}^{\prime \prime}} x_{2,2}^{\prime \prime \prime}
$$

Making use of the above values, we find

$$
x_{12}-x=x_{21}-x=\frac{\left(\theta_{1}^{\prime \prime} \theta_{2}-\theta_{2}^{\prime \prime} \theta_{1}\right) x^{\prime}+\left(\theta_{2}^{\prime} \theta_{1}-\theta_{1}^{\prime} \theta_{2}\right) x^{\prime \prime}}{\theta_{1}^{\prime} \theta_{2}^{\prime \prime}-\theta_{1}^{\prime \prime} \theta_{2}^{\prime}} .
$$

Hence the nets $N_{12}$ and $N_{21}$ coincide and the congruences $G^{r}$ and $G^{\prime \prime \prime \prime}$ are conjugate to $N_{2,1}{ }^{15}$ ).

In view of the above results we have that when two nets $N^{\prime}$ and $N^{\prime \prime}$ parallel to $N$ are known, and two solutions $\theta_{1}$ and $\theta_{2}$ of equation (6) are given, the four functions $\theta_{1}^{\prime}, \theta_{1}^{\prime \prime}, \theta_{2}^{\prime}$ and $\theta_{2}^{\prime \prime}$ (each involving an additive constant of integration) can be found by as many quadratures. When these are known, we have a group of

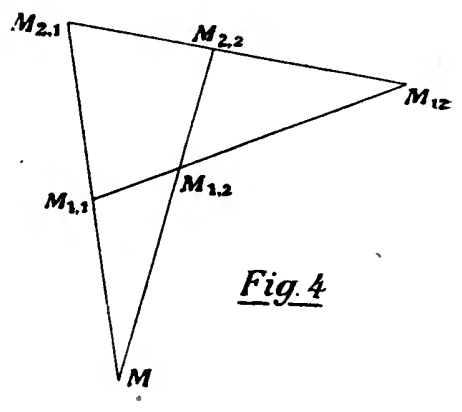

${ }^{14}$ ) The mark "'" indicates that the net is the parallel to $N_{2,2}$ determining the conjugate congruence $G^{\prime \prime \prime \prime}$.

${ }^{15)}$ Cf. Jonas, Sitzungsberichte Berl. Math. Gesell., vol. 14 (1915), p. 103 ; also 'Transactions, vol. 18 (1917), p. 111. 
six nets pictured schematically in the fig. 4 by corresponding points of these nets. We say that any four nets as $N, N_{1,1}, N_{2,2}, N_{12}$, such that the first and fourth are in relations $F$ with each of the second and third form a quatern.

We note that $N_{1,1}$ and $N_{2,2}$ determine the additive constants in $\theta_{1}^{\prime}$ and $\theta_{2}^{\prime \prime}$, but that the additive constants of $\theta_{2}^{\prime}$ and $\theta_{1}^{\prime \prime}$ are arbitrary and consequently there are $\infty^{2}$ transforms $N_{12}$ of a given $N_{1,1}$ and $N_{2,2}$. However, it follows from (33) and (42) that when one of these constants is fixed, the $\infty^{1}$ transforms $N_{12}$ are conjugate to the same congruence.

We may gather together the foregoing results into the following fundamental theorem of permutability:

If $N_{1,1}$ and $N_{2,2}$ are two transforms of $N$ by means of functions $\theta_{1}$ and $\theta_{2}$ and congruences $G^{\prime}$ and $G^{\prime \prime}$, there exist $\infty^{2}$ nets $N_{12}$, each of which is an $F$ transform of $N_{1,1}$ and $N_{2,2}$, their determination involving two quadratures; there are obtained incidentally two other nets $N_{2,1}$ and $N_{1 ; 2}$ such that $N_{2,1}, N_{1,1}, N_{2,2}, N_{12}$ is a quatern, and also $N, N_{2,1}, N_{1,2}, N_{12}$, Moreover, the six nets can be associated into the triads $N, N_{1,1}, N_{1,2} ; N, N_{2,1}, N_{2,2} ; N_{1,2}, N_{2,2}, N_{12} ; N_{2,1}, N_{1,1}, N_{12}$.

Any set of corresponding points in the above configuration are the vertices of the complete quadrilateral formed by corresponding lines of the four conjugate congruences of the transformations. Each point generates a net which may be taken in place of $N$ as the given net from which the configuration is obtained.

With each net there are associated two parallel nets whose coordinates are direction-parameters of the two congruences conjugate to the former net. These twelve auxiliary nets may be so chosen that they may be constituted into four groups such that corresponding points of the three nets of a group lie on a line through the origin (cf. (1)). These four groups are $N^{\prime}, N_{1,1}^{\prime}, N_{2,1}^{\prime}$; $\left.N^{\prime \prime}, N_{1,2}^{\prime \prime}, N_{2,2}^{\prime \prime} ; N_{1,1}^{\prime \prime \prime}, N_{1,2}^{\prime \prime \prime}, N_{12}^{\prime \prime \prime} ; N_{2,1}^{\prime \prime \prime}, N_{2,2}^{\prime \prime \prime}, N_{12}^{\prime \prime \prime \prime 16}\right)$.

In consequence of (32), (36), (38), (40) and (43) equation (46) can be written

(47) $\theta_{12}^{\prime \prime \prime} x_{12}=\theta_{2}^{\prime \prime}\left(\frac{\theta_{21}}{\theta_{1}} x_{1,1}+\frac{\theta_{12}}{\theta_{2}} x_{2,2}\right)+\left(\theta_{12}^{\prime \prime \prime}-\frac{\theta_{2}^{\prime \prime}}{\theta_{1}} \theta_{21}-\frac{\theta_{2}^{\prime \prime}}{\theta_{2}} \theta_{12}\right) x$.

$\left.{ }^{16}\right)$ In this notation $N_{i, j}^{\prime \prime \prime}$ means that the radius vector is parallel to a line of $G^{\prime \prime \prime}$ and the net is parallel to $N_{i, j}$. 
From the expressions (36), (38), (43) and (44) we find

$$
\theta_{1}^{\prime} \theta_{12}^{\prime \prime \prime}=\theta_{2}^{\prime \prime} \theta_{21}^{\prime \prime \prime}=\frac{\theta_{1}^{\prime} \theta_{2}^{\prime \prime}}{\theta_{1} \theta_{2}}\left(\theta_{2} \theta_{21}+\theta_{1} \theta_{12}-\theta_{12} \theta_{21}\right) \text {. }
$$

Hence the above equation may be written

$$
\theta_{1} \theta_{12}^{\prime \prime \prime} x_{12}=\frac{\theta_{2}^{\prime \prime}}{\theta_{2}}\left(\theta_{2} \theta_{21} x_{1,1}+\theta_{1} \theta_{12} x_{2,2}-\theta_{12} \theta_{21} x\right) .
$$

From (34) it follows that the functions $l_{12}$ and $l_{12}$ of the parallel transformation determining $G^{\prime \prime \prime}$, the congruence conjugate to $N_{1,1}$ and $N_{12}$, have the values

$$
h_{12}=\frac{h_{1} \theta_{1}^{\prime \prime}-l_{2} \theta_{1}^{\prime}}{\tau_{1}}, \quad l_{12}=\frac{l_{1} \theta_{1}^{\prime \prime}-l_{2} \theta_{1}^{\prime}}{\sigma_{1}} .
$$

The functions $\tau_{12}, \sigma_{12}, \rho_{12}$ of the transformations from $N_{1,1}$ into $N_{12}$ are given by equations similar to (S) and (15), namely

$$
\tau_{12}=h_{12} \theta_{12}-\theta_{12}^{\prime \prime \prime}, \quad \sigma_{12}=l_{12} \theta_{12}-\theta_{12}^{\prime \prime}, \quad \varphi_{12} \theta_{12}=\tau_{12}-\sigma_{12} .
$$

In consequence of the above values these functions have the expressions

$$
\left\{\begin{array}{l}
\tau_{12}=-\frac{1}{\tau_{1}} \frac{\theta_{1}^{\prime}}{\theta_{1}} \frac{\theta_{2}^{\prime \prime}}{\theta_{2}}\left(\frac{\theta_{2}}{\theta_{1}^{\prime}} \theta_{21} \tau_{1}+\frac{\theta_{1}}{\theta_{2}^{\prime \prime}} \theta_{12} \tau_{2}+\theta_{12} \theta_{21}\right), \\
\sigma_{12}=-\frac{1}{\sigma_{1}} \frac{\theta_{1}^{\prime}}{\theta_{1}} \frac{\theta_{2}^{\prime \prime}}{\theta_{2}}\left(\frac{\theta_{2}}{\theta_{1}^{\prime}} \theta_{21} \sigma_{1}+\frac{\theta_{1}}{\theta_{2}^{\prime \prime}} \theta_{12} \sigma_{2}+\theta_{12} \theta_{21}\right), \\
\varphi_{12}=\frac{1}{\sigma_{1} \tau_{1}} \frac{\theta_{1}^{\prime}}{\theta_{2}}\left(\tau_{1} \sigma_{2}-\tau_{2} \sigma_{1}+\varphi_{1} \theta_{2}^{\prime \prime} \theta_{21}\right) .
\end{array}\right.
$$

In an analogous manner the functions $h_{21}$ and $l_{21}$ of the transformation from $N_{2,2}$ to $N_{12}$ are of the form

$$
h_{21}=\frac{h_{2} \theta_{2}^{\prime}-h_{1} \theta_{2}^{\prime \prime}}{\tau_{2}}, \quad l_{21}=\frac{l_{2} \theta_{2}^{\prime}-l_{1} \theta_{2}^{\prime \prime}}{\sigma_{9}}
$$

and the values of the corresponding functions $\tau_{21}, \sigma_{21}, \varphi_{21}$, defined by

$$
\tau_{21}=h_{21} \theta_{21}-\theta_{21}^{\prime \prime \prime}, \quad \sigma_{21}=l_{21} \theta_{21}-\theta_{21}^{\prime \prime \prime}, \quad \varphi_{21} \theta_{21}=\tau_{21}-\sigma_{21},
$$


are reducible to

$$
\left\{\begin{array}{c}
\tau_{2} \tau_{21}=\tau_{1} \tau_{12}, \quad \sigma_{2} \sigma_{21}=\sigma_{1} \sigma_{12} \\
\varsigma_{21}=\frac{1}{\sigma_{2} \tau_{2}} \frac{\theta_{2}^{\prime \prime}}{\theta_{1}}\left(\tau_{2} \sigma_{1}-\tau_{1} \sigma_{2}+\varsigma_{2} \theta_{1}^{\prime} \theta_{12}\right)
\end{array}\right.
$$

Consider in particular the case when $N_{2,2}$ is a parallel transform of $N$. If we take

then

$$
\theta_{2}=\theta_{2}^{\prime \prime}=1, \quad \theta_{2}^{\prime}=0
$$

$$
\theta_{12}=\theta_{12}^{\prime \prime \prime}=1
$$

and consequently $N_{12}$ is a parallel transform of $N_{1,1}$. From (43) we have

$$
\theta_{21}=\theta_{1}-\theta_{1}^{\prime \prime},
$$

and consequently from (49)

$$
\theta_{1}\left(x_{12}-x_{2,2}\right)=\left(\theta_{1}-\theta_{1}^{\prime \prime}\right)\left(x_{1,1}-x\right) .
$$

Since $\theta_{1}^{\prime \prime}$ involves an additive arbitrary constant, we have the theorem:

If $N_{1}$ is any transform of a net $N$ and $N_{2}$ is parallel to $N$, there are $\infty^{1}$ transforms $N_{12}$ of $N_{1}$ and $N_{2}$ which are parallel to $N_{1}$; corresponding points of these nets lie on the line through $M_{2}$ parallel to $M M_{1}$.

Consider also the case where $\theta_{2}=\theta_{1}+c, c$ being a constant. We have accordingly

(57) $\left\{\begin{array}{c}\theta_{2}^{\prime}=\theta_{1}^{\prime}+c^{\prime}, \quad \theta_{2}^{\prime \prime}=\theta_{1}^{\prime \prime}+c^{\prime \prime}, \\ \theta_{12}=c-\frac{\theta_{1}}{\theta_{1}^{\prime}} c^{\prime}, \quad \theta_{21}=-c+\frac{\theta_{2}}{\theta_{2}^{\prime \prime}} c^{\prime \prime}, \quad \theta_{12}^{\prime \prime \prime}=c^{\prime \prime}-\frac{\theta_{1}^{\prime \prime}}{\theta_{1}^{\prime}} c^{\prime},\end{array}\right.$

where $c^{\prime}$ and $c^{\prime \prime}$ are constants. If $c^{\prime}=0, \theta_{12}$ is constant; it follows from $\S 16$ that $N_{12}$ is parallel to $N_{1,1}$. Also $N_{2,2}$ is parallel to $N_{12}$ if $c^{\prime \prime}=0$.

In order to determine the effect of the additive arbitrary constants of $\theta_{2}^{\prime}$ and $\theta_{1}^{\prime \prime}$, we replace them by $\theta_{2}^{\prime}+(c-1)$ and $\theta_{1}^{\prime \prime}+(e-1)$ in (33) and (36). If we denote the new functions by $x_{e, 1}^{\prime \prime \prime}$ and $\theta_{c 2}$, we find in consequence of (1) and (19)

$$
x_{e, 1}^{\prime \prime}=x_{1,1}^{\prime \prime \prime}-(e-1) x_{1}^{\prime}, \quad \theta_{c 2}=\theta_{12}+(c-1) \theta_{1}^{-1} \text {. }
$$


Hence the $\infty^{1}$ nets $N_{12}$ obtained by varying $c$ and holding $e$ fixed are conjugate to the same congruence conjugate to $N_{1,1}$ also; similarly as $e$ varies and $c$ remains fixed the $\infty^{1}$ nets $N_{12}$ and $N_{2,2}$ are conjugate to the same congruence.

The foregoing formulas are interesting also in another connection. Thus if we look upon $N$ and a net $N_{12}$ as $F$ transforms of $N_{2,2}$, there are $\infty^{2}$ nets, including $N_{1,1}$, each of which forms a quatern with $N_{2,2}, N$ and $N_{12}$. The above results lead to a means of finding these nets $N_{c, e}$. In fact we replace $N_{1,1}$ by the transform of $N$ by means of the functions

$$
\theta_{c}=\theta_{1}+(c-1) \theta_{2}, \quad x^{(e)}=x^{\prime}+(e-1) x^{\prime \prime},
$$

the quantities $x^{(e)}$ being taken as direction-parameters of the congruence of the transformation. Now we have

where

$$
\frac{\partial x^{(e)}}{\partial u}=h_{e} \frac{\partial x}{\partial u}, \quad \frac{\partial x^{(e)}}{\partial v}=l_{e} \frac{\partial x}{\partial v},
$$

also

$$
h_{e}=h_{1}+(e-1) h_{\mathbf{2}}, \quad l_{e}=l_{1}+(e-1) l_{\mathbf{2}} ;
$$

$$
\begin{gathered}
\theta_{2}^{(e)}=\theta_{2}^{\prime}+(e-1) \theta_{2}^{\prime \prime}, \quad \theta_{c}^{\prime \prime}=\theta_{1}^{\prime \prime}+(c-1) \theta_{2}^{\prime \prime}, \\
\theta_{c}^{(e)}=\theta_{1}^{\prime}+(c-1) \theta_{2}^{\prime}+(e-1) \theta_{1}^{\prime \prime}+(c-1)(e-1) \theta_{2}^{\prime \prime} .
\end{gathered}
$$

When these values are substituted in the following expression which is the analogue of the right-hand member of (46), it is formd that the result is reducible to the latter:

$$
\frac{\left(\theta_{c}^{\prime \prime} \theta_{2}-\theta_{2}^{\prime \prime} \theta_{c}\right) x^{(e)}+\left(\theta_{2}^{(e)} \theta_{c}-\theta_{c}^{(e)} \theta_{2}\right) x^{\prime \prime}}{\theta_{c}^{(e)} \theta_{2}^{\prime \prime}-\theta_{c}^{\prime \prime} \theta_{2}^{(e)}} .
$$

Hence:

Let $N_{1,1}$ and $N_{2,2}$ be $F$ transforms of $N$ by means of functions $\theta_{1}$ and $\theta_{\mathbf{2}}, x^{\prime}$ and $x^{\prime \prime}$ being the direction-parameters of the congruences of the transformations; if $N_{c, e}$ is the transform of $N$ by means of $\theta_{1}+(c-1) \theta_{2}$ and a congruence of direction-parameters $x^{\prime}+(e-1) x^{\prime \prime}$, $c$ and $e$ being constants, the $\infty^{2}$ nets $N_{12}$ forming quaterns with $N$, $N_{1,1}$ and $N_{2,2}$ form quaterns also with $N, N_{c, e}$ and $N_{2,2}$ whatever be $c$ and $e$. 
22. Derived nets and transformations $F$. If $N(x)$ is a net with the point equation (6), and $\theta_{1}$ and $\theta_{2}$ are solutions of (6), they determine a derived net $\bar{N}(\bar{x})$ of $N$ (cf. $\S 11$ ), whose equations may be written

$$
\bar{x}=x+p \frac{\partial x}{\partial u}+q \frac{\partial x}{\partial v},
$$

where

$$
\left\{\begin{array}{c}
p=\frac{1}{A}\left(\theta_{1} \frac{\partial \theta_{2}}{\partial v}-\theta_{2} \frac{\partial \theta_{1}}{\partial v}\right) \\
q=\frac{1}{\Delta}\left(\theta_{2} \frac{\partial \theta_{1}}{\partial u}-\theta_{1} \frac{\partial \theta_{2}}{\partial u}\right), \quad \Delta=\frac{\partial \theta_{2}}{\partial u} \frac{\partial \theta_{1}}{\partial v}-\frac{\partial \theta_{2}}{\partial v} \frac{\partial \theta_{1}}{\partial u}
\end{array}\right.
$$

Let $\theta_{3}$ be another solution of (6), and $N^{\prime}$ a net parallel to $N$. An $F$ transform $N_{3}$ of $N$ is given by

$$
x_{3}=x-\frac{\theta_{3}}{\theta_{3}^{\prime}} x^{\prime} .
$$

The functions $\theta_{31}$ and $\theta_{32}$ defined by

$$
\theta_{3 i}=\theta_{i}-\frac{\theta_{\mathbf{3}}}{\theta_{3}^{\prime}} \theta_{i}^{\prime} \quad(i=1,2),
$$

where

$$
\frac{\partial \theta_{i}^{\prime}}{\partial u}=h \frac{\partial \theta_{i}}{\partial u}, \quad \frac{\partial \theta_{i}^{\prime}}{\partial v}=l \frac{\partial \theta_{i}}{\partial v}(i=1,2,3),
$$

are solutions of the point equation of $N_{3}$. They determine a derived net $\overline{N_{3}}$ of $N_{3}$, whose coordinates are of the form

$$
\bar{x}_{3}=x_{3}+p_{3} \frac{\partial x_{3}}{\partial u}+q_{3} \frac{\partial x_{3}}{\partial v}
$$

where $p_{3}$ and $q_{3}$ are given by (65) when $\theta_{1}$ and $\theta_{2}$ are replaced by $\theta_{31}$ and $\theta_{32}$ respectively.

From (66) and (67) we have

$$
\begin{aligned}
& \frac{\partial x_{3}}{\partial u}=\frac{\theta_{3} h-\theta_{3}^{\prime}}{\theta_{3}^{\prime 2}}\left(x^{\prime} \frac{\partial \theta_{3}}{\theta u}-\theta_{3}^{\prime} \frac{\partial x}{\partial u}\right), \\
& \frac{\partial x_{3}}{\partial v}=\frac{\theta_{3} l-\theta_{3}^{\prime}}{\theta_{3}^{\prime 2}}\left(x^{\prime} \frac{\partial \theta_{3}}{\partial v}-\theta_{3}^{\prime} \frac{\partial x}{\partial v}\right),
\end{aligned}
$$




$$
\begin{aligned}
& \frac{\partial \theta_{3 i}}{\partial u}=\frac{\theta_{3} h-\theta_{3}^{\prime}}{\theta_{3}^{\prime 2}}\left(\theta_{i}^{\prime} \frac{\partial \theta_{3}}{\partial u}-\theta_{3}^{\prime} \frac{\partial \theta_{i}}{\partial u}\right), \\
& \frac{\partial \theta_{3 i}}{\partial v}=\frac{\theta_{3} l-\theta_{3}^{\prime}}{\theta_{3}^{\prime 2}}\left(\theta_{i}^{\prime} \frac{\partial \theta_{3}}{\partial v}-\theta_{3}^{\prime} \frac{\partial \theta_{i}}{\partial v}\right),(i=1,2) .
\end{aligned}
$$

On substituting these expressions in (69), the resulting expression in reducible to

$$
\bar{x}_{\mathbf{3}}=x+\frac{1}{A}\left\{x^{\prime}\left|\begin{array}{ccc}
\theta_{1} & \theta_{2} & \theta_{3} \\
\frac{\partial \theta_{1}}{\partial u} & \frac{\partial \theta_{2}}{\partial u} & \frac{\partial \theta_{3}}{\partial u} \\
\frac{\partial \theta_{1}}{\partial v} & \frac{\partial \theta_{2}}{\partial v} & \frac{\partial \theta_{3}}{\partial v}
\end{array}\right|+\frac{\partial x}{\partial u}\left|\begin{array}{ccc}
\theta_{1}^{\prime} & \theta_{2}^{\prime} & \theta_{3}^{\prime} \\
\theta_{1} & \theta_{2} & \theta_{3} \\
\frac{\partial \theta_{1}}{\partial v} & \frac{\partial \theta_{2}}{\partial v} & \frac{\partial \theta_{3}}{\partial v}
\end{array}\right|-\frac{\partial x}{\partial v}\left|\begin{array}{ccc}
\theta_{1}^{\prime} & \theta_{2}^{\prime} & \theta_{3}^{\prime} \\
\theta_{1} & \theta_{2} & \theta_{3} \\
\frac{\partial \theta_{1}}{\partial u} & \frac{\partial \theta_{2}}{\partial u} & \frac{\partial \theta_{3}}{\partial u}
\end{array}\right|,,\right.
$$

where

$$
\begin{gathered}
A=\theta_{1}^{\prime}\left(\frac{\partial \theta_{\mathbf{2}}}{\partial v} \frac{\partial \theta_{3}}{\partial u}-\frac{\partial \theta_{\mathbf{2}}}{\partial u} \frac{\partial \theta_{3}}{\partial v}\right)+\theta_{2}^{\prime}\left(\frac{\partial \theta_{3}}{\partial v} \frac{\partial \theta_{1}}{\partial u}-\frac{\partial \theta_{3}}{\partial u} \frac{\partial \theta_{1}}{\partial v}\right) \\
+\theta_{3}^{\prime}\left(\frac{\partial \theta_{1}}{\partial v} \frac{\partial \theta_{2}}{\partial u}-\frac{\partial \theta_{1}}{\partial u} \frac{\partial \theta_{2}}{\partial v}\right) .
\end{gathered}
$$

Since $\theta_{1}^{\prime}$ and $\theta_{2}^{\prime}$ are solutions of the point equation of $N^{\prime}$, a derived net $\bar{N}^{\prime}$ of $N^{\prime}$ is given by equations of the form

$$
\bar{x}^{\prime}=x^{\prime}+p^{\prime} \frac{\partial x^{\prime}}{\partial u}+q^{\prime} \frac{\partial x^{\prime}}{\partial v}
$$

where $p^{\prime}$ and $q^{\prime}$ are obtained from (65) by replacing $\theta_{1}$ and $\theta_{2}$ by $\theta_{1}^{\prime}$ and $\theta_{2}^{\prime}$. From equations analogous to $(I, 68)$, we find

$$
\frac{\partial \bar{x}^{\prime}}{\partial u}=\bar{h} \frac{\partial \bar{x}}{\partial u}, \quad \frac{\partial \bar{x}^{\prime}}{\partial v}=\bar{l} \frac{\partial \bar{x}}{\partial v}
$$

where

$$
\bar{h}=\frac{\theta_{1}^{\prime} \frac{\partial \theta_{2}}{\partial v}-\theta_{2}^{\prime} \frac{\partial \theta_{1}}{\partial v}}{\theta_{1} \frac{\partial \theta_{2}}{\partial v}-\theta_{2} \frac{\partial \theta_{1}}{\partial v}}, \quad \bar{l}=\frac{\theta_{1}^{\prime} \frac{\partial \theta_{2}}{\partial u}-\theta_{2}^{\prime} \frac{\partial \theta_{1}}{\partial u}}{\theta_{1} \frac{\partial \theta_{2}}{\partial u}-\theta_{2} \frac{\partial \theta_{1}}{\partial u}}
$$

Consequently $\overline{N^{\prime}}$ is parallel to $\bar{N}$. 
The functions $\overline{\theta_{3}}$ and $\overline{\theta_{3}^{\prime}}$ defined by

$$
\bar{\theta}_{3}=\theta_{3}+p \frac{\partial \theta_{3}}{\partial u}+q \frac{\partial \theta_{3}}{\partial v^{\prime}}, \quad \overline{\theta_{3}^{\prime}}=\theta_{3}^{\prime}+p^{\prime} \frac{\partial \theta_{3}^{\prime}}{\partial u}+q^{\prime} \frac{\partial \theta_{3}^{\prime}}{\partial v}
$$

are corresponding solutions of the point equations of $\bar{N}$ and $\overline{N^{\prime}}$. Hence quantities of the form

$$
\bar{x}-\frac{\bar{\theta}_{3}}{\bar{\theta}_{3}^{\prime}} \bar{x}^{\prime}
$$

are the coordinates of an $F$ transform of $\bar{N}$. When the above expressions are substituted in this quantity, it is reducible to (70). Since $\theta_{1}^{\prime}$ and $\theta_{2}^{\prime}$, as given by (68) involve additive arbitrary constants, there are $\infty^{2}$ nets $\bar{N}_{3}$. Hence we have the theorem:

If $\bar{N}$ is a derived net of $N$ and $N_{3}$ is any $F$ transform of $N$, there can be found by two quadratures $\infty^{2}$ nets $\overline{N_{3}}$, each of which is a derived net of $N_{3}$ and an $F$ transform of $\bar{N}$.

23. Derivant net and derived net of two transformations $F$. We note that the corresponding points of six nets in the relation of the theorem of permutability are coplanar. From the second theorem of $\S 11$ it follows that these planes envelope a derivant net $\hat{N}$ of $N$. Since the four congruences of the configuration are harmonic to $\hat{N}$, it is a derivant net of each of the six nets. By means of (32), (40) and equations similar to (8) and (15) the expressions $(\mathrm{I}, 67)$ for the coordinates of $\hat{N}$ are reducible to

$$
\left\{\begin{array}{c}
\left(\frac{\tau_{1} \sigma_{2}-\tau_{2} \sigma_{1}}{\theta_{1} \theta_{2}}+\frac{\theta_{2}^{\prime \prime}}{\theta_{2}} \varphi_{1}-\frac{\theta_{1}^{\prime}}{\theta_{1}} \varphi_{2}\right) \hat{x} \\
=\frac{\tau_{1} \sigma_{2}-\tau_{2} \sigma_{1}}{\theta_{1} \theta_{2}} x+\frac{\theta_{2}^{\prime \prime}}{\theta_{2}} \varphi_{1} x_{2,2}-\frac{\theta_{1}^{\prime}}{\theta_{1}} \varphi_{2} x_{1,1} .
\end{array}\right.
$$

From $\S 17$ it follows that corresponding tangent planes of $N$, $N_{1,1}$ and $N_{2,2}$ meet in the point which generates the derived net $\bar{N}$ of $N$ determined by the solutions $\theta_{1}$ and $\theta_{2}$ of the point equation of $N$. We shall show that the corresponding tangent planes of the $\infty^{2}$ nets $N_{12}$ pass through this point. In fact from (I, 66) it follows that the coordinates $\bar{x}$ of this point may be given the form

$$
\bar{x}=x+\frac{\frac{\partial}{\partial v}\left(\frac{\theta_{2}}{\theta_{1}}\right) \frac{\partial x}{\partial u}-\frac{\partial}{\partial u}\left(\frac{\theta_{2}}{\theta_{1}}\right) \frac{\partial x}{\partial v}}{\theta_{1}\left[\frac{\partial}{\partial u}\left(\frac{1}{\theta_{1}}\right) \frac{\partial}{\partial v}\left(\frac{\theta_{2}}{\theta_{1}}\right)-\frac{\partial}{\partial v}\left(\frac{1}{\theta_{1}}\right) \frac{\partial}{\partial u}\left(\frac{\theta_{2}}{\theta_{1}}\right)\right]} .
$$


Consider now the net $N_{1,1}$ and look upon $N$ and a net $N_{12}$ as $F$ transforms of it by means of $\theta_{1}^{-1}=-\theta_{1} / \theta_{1}^{\prime}$ and $\theta_{12}$. The corresponding formula for this case is

$$
x_{1}-\frac{\theta_{1}^{\prime}}{\theta_{1}} \frac{\frac{\partial}{\partial v}\left(\theta_{12} \frac{\theta_{1}^{\prime}}{\theta_{1}}\right) \frac{\partial x_{1}}{\partial u}-\frac{\partial}{\partial u}\left(\theta_{12} \frac{\theta_{1}^{\prime}}{\theta_{1}^{\prime}}\right) \frac{\partial x_{1}}{\partial v}}{\frac{\partial}{\partial u}\left(\frac{1}{\theta_{1}^{-1}}\right) \frac{\partial}{\partial v}\left(\theta_{12} \frac{\theta_{1}^{\prime}}{\theta_{1}}\right)-\frac{\partial}{\partial v}\left(\frac{1}{\theta_{1}^{-1}}\right) \frac{\partial}{\partial u}\left(\theta_{12} \frac{\theta_{1}^{\prime}}{\theta_{1}}\right)} .
$$

By means of (9), (20) and (37) this is reducible to the preceding form. Hence:

The corresponding tangent planes of $N, N_{1,1}, N_{2,2}$ and the $\infty^{2}$ nets $N_{12}$ meet in the point which generates the derived net of $N$ by means of the functions $\theta_{1}$ and $\theta_{2}$ of the transformations of $N$ into $N_{1,1}^{r}$ and $N_{2,2}$.

24. The extended theorem of permutability. In this section we extend the theorem of permutability so as to involve three transforms of $N$. Let $N_{1}, N_{2}$ and $N_{3}$ be these transforms of $N$ by means of the functions $\theta_{i}$ and $w_{i}(i=1,2,3)$, where

$$
w_{1}=\frac{\theta_{1}^{\prime}}{\theta_{1}}, \quad w_{2}=\frac{\theta_{2}^{\prime \prime}}{\theta_{2}}, \quad w_{12}=\frac{\theta_{12}^{\prime \prime \prime}}{\theta_{12}}, \quad w_{21}=\frac{\theta_{21}^{\prime \prime \prime}}{\theta_{21}^{\prime \prime}}, \ldots
$$

Applying the theorem of permutability to the three pairs of these nets, we get three families of nets $N_{12}, N_{23}$ and $N_{31}$, since $N_{i j}=N_{j i}$. From (48) and (49) we have

$$
\left\{\begin{aligned}
\theta_{i} \theta_{i j} w_{i j} & =w_{j}\left(\theta_{j} \theta_{j i}+\theta_{i} \theta_{i j}-\theta_{i j} \theta_{j i}\right) \\
\theta_{i} \theta_{i j} w_{i j} x_{i j} & =w_{j}\left(\theta_{j} \theta_{j i} x_{i}+\theta_{i} \theta_{i j} x_{j}-\theta_{i j} \theta_{j i} x\right) \quad(i \neq j)
\end{aligned}\right.
$$

Since a net $N_{12}$ and a net $N_{13}$ are transforms of $N_{1}$, there exist $\infty^{2}$ nets $\bar{N}$ for each of which $N_{1}, N_{12}, N_{13}$ and $\bar{N}$ form a quatern. It is our purpose to show that one of these nets $\bar{N}$ is such that $N_{2}, N_{12}, N_{23}, \bar{N}$ form a quatern; and likewise $N_{3}, N_{13}, N_{23}$ and $\bar{N}$. We denote by $\bar{\theta}_{i j}$ and $\bar{w}_{i j}$ the functions by means of which $N_{i j}$ is transformed into $\bar{N}$; from their definition it follows that $\bar{\theta}_{i j}=\bar{\theta}_{j i}$ and $\bar{w}_{i j}=\bar{w}_{j i}$. According as we look upon $\bar{N}$ as belonging to the one or the other of the quaterns, $N_{1}, N_{12}, N_{13}, \bar{N} ; N_{2}, N_{12}$, $N_{23}, \bar{N}$, the coordinates $\bar{x}$ of $\bar{N}$ are given by the respective equations 


$$
\left\{\begin{array}{l}
\theta_{12} \bar{\theta}_{12} \bar{w}_{12} \bar{x}=w_{13}\left(\theta_{13} \bar{\theta}_{13} x_{12}+\theta_{12} \bar{\theta}_{12} x_{13}-\bar{\theta}_{12} \bar{\theta}_{13} x_{1}\right) \\
\theta_{21} \bar{\theta}_{21} \bar{w}_{21} \bar{x}=w_{23}\left(\theta_{23} \bar{\theta}_{23} x_{12}+\theta_{21} \bar{\theta}_{21} x_{23}-\bar{\theta}_{21} \bar{\theta}_{23} x_{2}\right)
\end{array}\right.
$$

which are analogous to (72). When we equate these two values for $\bar{x}$, the resulting equation is reducible by means of (72) to an equation of the form $A x_{1}+B x_{2}+C x=0$. Since $A, B$ and $C$ are necessarily equal to zero, we have the following equations of condition:

$\theta_{1} w_{12} \bar{\theta}_{12}\left(\theta_{1} w_{13} \bar{\theta}_{13}-\theta_{3} w_{3} \frac{\theta_{12} \theta_{31}}{\theta_{13}}\right)-\theta_{1} w_{13} \bar{\theta}_{13} \theta_{2} w_{2} \frac{\theta_{21} \theta_{13}}{\theta_{12}}+\theta_{2} w_{23} \bar{\theta}_{23} \theta_{1} w_{2} \theta_{23}=0$ $\theta_{1} w_{12} \bar{\theta}_{12}\left(\theta_{2} w_{23} \bar{\theta}_{23}-\theta_{3} w_{3} \frac{\theta_{21} \theta_{32}}{\theta_{23}}\right)-\theta_{2} w_{23} \bar{\theta}_{23} \theta_{1} w_{2} \theta_{23}+\theta_{1} w_{13} \bar{\theta}_{13} \theta_{2} w_{2} \frac{\theta_{21} \theta_{18}}{\theta_{12}}=0$ $\theta_{1} w_{12} \bar{\theta}_{12}\left(\theta_{1} w_{3} \theta_{32}-\theta_{2} w_{3} \theta_{31}\right)-\theta_{1} w_{13} \bar{\theta}_{13} \theta_{2} w_{2} \frac{\theta_{13} \theta_{21}}{\theta_{12}}+\theta_{2} w_{23} \bar{\theta}_{23} \theta_{1} w_{2} \theta_{23}=0$.

By subtracting the last of these equations from the first and adding it to the second we find that the above system is equivalent to

$$
\left\{\begin{array}{l}
\theta_{1} \theta_{12} w_{12} \bar{\theta}_{12}=w_{2}\left(\theta_{2} \theta_{21} \theta_{13}+\theta_{1} \theta_{12} \theta_{23}-\theta_{3} \theta_{12} \theta_{21}\right) \\
\theta_{1} \theta_{13} w_{13} \bar{\theta}_{13}=w_{3}\left(\theta_{3} \theta_{31} \theta_{12}+\theta_{1} \theta_{13} \theta_{32}-\theta_{2} \theta_{13} \theta_{31}\right) \\
\theta_{2} \theta_{23} w_{23} \bar{\theta}_{23}=w_{3}\left(\theta_{3} \theta_{32} \theta_{21}+\theta_{2} \theta_{23} \theta_{31}-\theta_{1} \theta_{23} \theta_{32}\right)
\end{array}\right.
$$

From (38) and (44) it follows that

$$
w_{1} u_{12} \theta_{1} \theta_{12}=w_{2} w_{21} \theta_{2} \theta_{21}
$$

so that the above equations are consistent with the requirement that $\overline{\boldsymbol{\theta}}_{i j}=\overline{\boldsymbol{\theta}}_{j i}$. When equations (74) are compared with the second of (72), it is found that $\bar{\theta}_{i j}$ is a solution of the point equation of $N_{i j}$; which is a necessary condition. The analogue of the first of (72) is

$$
\theta_{12} \bar{\theta}_{12} \bar{w}_{12}=u_{13}\left(\theta_{13} \bar{\theta}_{13}+\theta_{12} \bar{\theta}_{12}-\bar{\theta}_{12} \bar{\theta}_{13}\right)
$$

Substituting in the right-hand member the expressions for $\bar{\theta}_{12}$ and $\overline{\boldsymbol{\theta}}_{13}$ from (7t), we have 


$$
\begin{aligned}
\Phi=\frac{\theta_{1} \theta_{12} w_{12} \bar{\theta}_{12} \bar{w}_{12}}{w_{2} w_{3}}= & \theta_{1}\left(\theta_{12} \theta_{23}+\theta_{13} \theta_{32}-\theta_{32} \theta_{23}\right) \\
& +\theta_{2}\left(\theta_{23} \theta_{31}+\theta_{21} \theta_{13}-\theta_{13} \theta_{31}\right) \\
& +\theta_{3}\left(\theta_{31} \theta_{12}+\theta_{32} \theta_{21}-\theta_{21} \theta_{12}\right) \\
& -\theta_{12} \theta_{23} \theta_{31}-\theta_{21} \theta_{13} \theta_{32},
\end{aligned}
$$

which in consequence of (75) is consistent with the requirement that $\bar{w}_{i j}=\bar{w}_{j i}$. By means of these results equations (73) reduce to

$$
\left\{\begin{aligned}
\Phi \bar{x}= & x_{1}\left(\theta_{2} \theta_{23} \theta_{31}+\theta_{3} \theta_{32} \theta_{21}-\theta_{1} \theta_{23} \theta_{32}\right) \\
& +x_{3}\left(\theta_{3} \theta_{31} \theta_{12}+\theta_{1} \theta_{13} \theta_{32}-\theta_{2} \theta_{31} \theta_{13}\right) \\
& +x_{3}\left(\theta_{1} \theta_{12} \theta_{23}+\theta_{2} \theta_{21} \theta_{13}-\theta_{3} \theta_{12} \theta_{21}\right) \\
& -x\left(\theta_{12} \theta_{23} \theta_{31}+\theta_{21} \theta_{13} \theta_{32}\right) .
\end{aligned}\right.
$$

Since this expression is symmetrical in the functions involved, it follows that $\bar{N}$ defined by (76) forms a quatern with $N_{\mathfrak{g}}, N_{12}$ and $N_{23}$ and also with $N_{3}, N_{13}$ and $N_{23}$.

25. Transformations $K$. We inquire under what conditions a net $N$ and a transform $N_{1}$ meet the lines of the congruence in points harmonic to the focal points. From (I, 37) and (2) we have that the necessary and sufficient condition is that

$$
\theta^{\prime}=\frac{1}{2}(h+l) \theta
$$

When we require that this function satisfy (5), the resulting equations are reducible, in consequence of $(I, 13,18)$, to

$$
\frac{\partial}{\partial u} \log \frac{\theta}{\varphi}=2 \frac{\partial \log b}{\partial u}, \quad \frac{\partial}{\partial v} \log \frac{\theta}{\varphi}=2 \frac{\partial \log a}{\partial v} .
$$

From this it follows that the point equation of $N$ is necessarily of the form

$$
\frac{\partial^{2} \theta}{\partial u \partial v}=\frac{\partial \log \sqrt{\varrho}}{\partial v} \frac{\partial \theta}{\partial u}+\frac{\partial \log \sqrt{\varrho}}{\partial u} \frac{\partial \theta}{\partial v},
$$

where $\varrho$ is defined by

$$
\varrho \varphi=2 \theta \text {. }
$$


Then from (I, 18) and (77) we have

$$
h=\frac{\theta}{\varrho}+\frac{\theta^{\prime}}{\theta}, \quad l=-\frac{\theta}{\varrho}+\frac{\theta^{\prime}}{\theta} .
$$

Now the equations (5) may by written

$$
\frac{\partial}{\partial u}\left(\frac{\theta^{\prime}}{\theta}\right)=\frac{1}{\varrho} \frac{\partial \theta}{\partial u}, \quad \frac{\partial}{\partial v}\left(\frac{\theta^{\prime}}{\theta}\right)=-\frac{1}{\varrho} \frac{\partial \theta}{\partial v} .
$$

From (8) it follows that

$$
\tau=-\sigma=\frac{\theta^{2}}{\varrho} \text {. }
$$

Hence equations (10) become

$$
\left\{\begin{array}{l}
\frac{\partial x_{1}}{\partial u}=-\frac{1}{\varrho} \frac{\theta}{\theta^{\prime}}\left(\left(x_{1}-x\right) \frac{\partial \theta}{\partial u}+\theta \frac{\partial x}{\partial u}\right), \\
\frac{\partial x_{1}}{\partial v}=\frac{1}{\varrho} \frac{\theta}{\theta^{\prime}}\left(\left(x_{1}-x\right) \frac{\partial \theta}{\partial v}+\theta \frac{\partial x}{\partial v}\right),
\end{array}\right.
$$

and the point equation of $N_{1}$ is

$$
\frac{\partial^{2} \theta_{1}}{\partial u \partial v}=\frac{\partial \log \sqrt{\varrho_{1}}}{\partial v} \frac{\partial \theta_{1}}{\partial u}+\frac{\partial \log \sqrt{\varrho_{1}}}{\partial u} \frac{\partial \theta_{1}}{\partial v}
$$

where

$$
\sqrt{\varrho_{1}}=\frac{\theta^{2}}{\sqrt{\varrho} \theta^{\prime}} .
$$

We note that the invariants $H$ and $K$ of equation (79) are equal, or, in other words, $N$ has equal point invariants. Since the same in true of $N_{1}$, as shown by (85), we have the theorem:

In order that a net $N$ and a $F$ transform $N_{1}$ meet the lines of the conjugate congruence in points harmonic to the focal points, it is necessary that both $N$ and $N_{1}$ have equal point invariants.

Koenigs solved this problem for 3-space ${ }^{17}$ ). Accordingly we call a transformation of this sort for space of any order a transformation $K$.

17) Comptes Rendus, vol. 113 (1891), p. 1022. 
When two nets in the relation of the above transformation are parallel we say they are associate ${ }^{18}$ ). In all generality equations (84) are in this case

$$
\frac{\partial x_{1}}{\partial u}=-\frac{1}{\varrho} \frac{\partial x}{\partial u}, \quad \frac{\partial x_{1}}{\partial v}=\frac{1}{\varrho} \frac{\partial x}{\partial v} .
$$

Suppose now that $N$ has equal point invariants. The knowledge of a parallel net $N^{\prime}$ gives $h$ and $l$, and consequently a solution $\varphi$ of the adjoint $(I, 20)$ of the point equation which in this case is reducible to

$$
\frac{\partial^{2}}{\partial u \partial v}(\varphi \sqrt{\varrho})=\sqrt{\varrho} \frac{\partial^{2}}{\partial u \partial v}\left(\frac{1}{\sqrt{\varrho}}\right) \cdot(\varphi \sqrt{\varrho}) .
$$

Since equation (79) can be written

$$
\frac{\partial^{2}}{\partial u \partial v}\left(\frac{\theta}{\sqrt{\varrho}}\right)=\sqrt{\varrho} \frac{\partial^{2}}{\partial u \partial v}\left(\frac{1}{\sqrt{\varrho}}\right) \cdot \frac{\theta}{\sqrt{\varrho}},
$$

a solution of this equation is given by (80). It is readily shown that $\theta^{\prime}$ given by (77) satisfies (5), and thus we have:

When $N$ has equal point invariants, each parallel net $N^{\prime}$ determines without quadrature a transformation $K$ into a net $N_{1}$, the function $\theta$ of the transformation having the value $\varphi \cdot \varrho / 2, \theta^{\prime}$ being given by (77).

In particular, $\varphi=\frac{2}{\varrho}$ is a solution of (88). In this case $\theta=1$ from (80) and consequently $N_{1}$ is associate to $N$.

Suppose conversely that we have a solution $\theta$ of the point equation. From (88) and (89) it follows that $\varphi=2 \theta / \varrho$ is a solution of the adjoint equation. From $\S 4$ we know that by means of $\varphi$ we find $\infty^{1}$ nets $N^{\prime}$ parallel to $N$, of the form $x^{\prime}+k x$, where $x^{\prime}$ are the coordinates of one of these nets and $k$ is a constant. Accordingly we have the theorem:

When $N$ has equal point invariants, each solution of the point equation determines by a quadrature, an infinity of transformations $K$,

18) This is a generalization of the idea of associate surfaces in 3-space [§ 155 and Ex. 22, p. 425]. 
such that corresponding lines of the conjugate congruences of the transformations which pass through a point of $N$ are coplanar.

26. Theorem of permutability of transformations $K$. If $N_{1}$ and $N_{2}$ are two nets, each in the relation of a transformation $K$ with a given net $N$, we apply the theorem of permutability of general transformations $F$, and seek the nets $N_{12}$, which are $K$ transforms of $N_{1}$ and $N_{2}$. For this case we must have

Since

$$
\tau_{12}=-\sigma_{12}, \quad \tau_{21}=-\sigma_{21} .
$$

$$
\tau_{1}=-\sigma_{1}=\frac{\theta_{1}^{2}}{\varrho}, \quad \tau_{2}=-\sigma_{2}=\frac{\theta_{2}^{2}}{\varrho},
$$

these conditions are equivalent, in consequence of (52), to

$$
\theta_{21} \frac{\theta_{2}^{\prime \prime}}{\theta_{2}}+\theta_{12} \frac{\theta_{1}^{\prime}}{\theta_{1}}=0
$$

In consequence of (90) equations (37) for the case of transformations $K$ are reducible to

$$
\left\{\begin{array}{l}
\frac{\partial}{\partial u}\left(\theta_{12} \frac{\theta_{1}^{\prime}}{\theta_{1}}\right)=\frac{1}{\varrho}\left(\theta_{2} \frac{\partial \theta_{1}}{\partial u}-\theta_{1} \frac{\partial \theta_{2}}{\partial u}\right), \\
\frac{\partial}{\partial v}\left(\theta_{12} \frac{\theta_{1}^{\prime}}{\theta_{1}}\right)=-\frac{1}{\varrho}\left(\theta_{2} \frac{\partial \theta_{1}}{\partial v}-\theta_{1} \frac{\partial \theta_{2}}{\partial v}\right) .
\end{array}\right.
$$

From these equations and similar ones in $\theta_{21}$ we find that the lefthand member of (91) is necessarily constant. Since each term of this expression is determined to within an additive constant, there are $\infty^{1}$ sets of solutions satisfying (91). In fact, in consequence of (36) and (43) we can put (91) in the form

$$
\frac{\theta_{2}^{\prime \prime} \theta_{1}}{\theta_{\mathbf{2}}}-\theta_{1}^{\prime \prime}+\frac{\theta_{1}^{\prime} \theta_{\mathbf{2}}}{\theta_{1}}-\theta_{2}^{\prime}=0 .
$$

From (52), (86) and (93) it follows that

$$
\varsigma_{12}=2 \frac{\theta_{12}}{\varrho_{1}}, \quad \varphi_{21}=2 \frac{\theta_{21}}{\varrho_{2}} .
$$

Hence each $N_{12}$ for which (93) is satisfied is a $K$ transform of $N_{1}$ and $N_{2}$. 
Now equations (48) and (49) become

(94)

$$
\left\{\begin{aligned}
\theta_{1} \theta_{12}^{\prime \prime \prime} & =\theta_{12}\left(\theta_{1} \frac{\theta_{2}^{\prime \prime}}{\theta_{2}}-\theta_{2} \frac{\theta_{1}^{\prime}}{\theta_{1}}+\theta_{12} \frac{\theta_{1}^{\prime}}{\theta_{1}}\right), \\
\theta_{1} \theta_{12}^{\prime \prime \prime} x_{12} & =\theta_{12}\left(\theta_{1} \frac{\theta_{2}^{\prime \prime}}{\theta_{2}} x_{2,2}-\theta_{2} \frac{\theta_{1}^{\prime}}{\theta_{1}} x_{1,1}+\theta_{12} \frac{\theta_{1}^{\prime}}{\theta_{1}} x\right) .
\end{aligned}\right.
$$

By means of these results the coordinates (71) of the point of contact $\hat{M}$ of the plane of the quatern with its envelope are reducible to

$$
\hat{x}=\frac{\theta_{2}^{\prime \prime} \theta_{1}^{2} x_{2,2}-\theta_{1}^{\prime} \theta_{2}^{2} x_{1,1}}{\theta_{2}^{\prime \prime} \theta_{1}^{2}-\theta_{1}^{\prime} \theta_{2}^{2}}=\frac{\theta_{12}^{\prime \prime \prime} \theta_{1}^{2} x_{12}-\theta_{1}^{\prime} \theta_{12}^{2} x}{\theta_{12}^{\prime \prime \prime} \theta_{1}^{2}-\theta_{1}^{\prime} \theta_{12}^{2}} .
$$

Hence $\hat{M}$ is the intersection of the lines $M M_{12}$ and $M_{1} M_{2}$; consequently the points $M_{12}$ of the $\infty^{1}$ nets lie on a line, $M M_{12}$. Therefore in consequence of the theorem of permutability of general transformations $F$ ( $\S \S 21,23)$, we have the following theorem of permutability of transformations $K$ :

If $N_{1}$ and $N_{2}$ are $K$ transforms of a net $N$ with equal point invariants, there can be found by a quadrature $\infty^{1}$ nets $N_{12}$ which are $K$ transforms of $N_{1}$ and $N_{2}$; corresponding points $M_{12}$ of these nets $N_{12}$ lie on a line $l$ through the corresponding point $M$ of $N$ and in the plane $\pi$ determined by $M$ and the corresponding points $M_{1}$ and $M_{2}$ of $N_{1}$ and $N_{2}$; the plane $\pi$ touches its envelope at the intersection of $l$ and the line $M_{1} M_{2}$; the parametric lines on the envelope form a net to which are harmonic the congruences generated by the lines $M M_{1}, M M_{2}, M_{1} M_{12}, M_{2} M_{12}$, and the tangents to this net are harmonic to $l$ and $M_{1} M_{2}$.

27. Transformations $F$ of applicable nets. We say that a net $N$ in $n$-space and a net $\bar{N}$ in $p$-space are applicable, when their coordinates $x$ and $\bar{x}$ satisfy the condition

which is equivalent to

$$
\sum_{1}^{n} d x^{2}=\sum_{1}^{p} d \bar{x}^{2}
$$

$$
\begin{gathered}
E=\sum_{1}^{n}\left(\frac{\partial x}{\partial u}\right)^{2}=\sum_{1}^{p}\left(\frac{\partial \bar{x}}{\partial u}\right)^{2}, F=\sum_{1}^{n} \frac{\partial x}{\partial u} \frac{\partial x}{\partial v}=\sum_{1}^{p} \frac{\partial \bar{x}}{\partial u} \frac{\partial \bar{x}}{\partial v} \\
G=\sum_{1}^{n}\left(\frac{\partial x}{\partial v}\right)^{2}=\sum_{1}^{p}\left(\frac{\partial \bar{x}}{\partial v}\right)^{2}
\end{gathered}
$$


From (I, 3) it follows that the point equations of the two nets are the same. Hence a pair of solutions $h$ and $l$ of equations (I, 13) determine a net $\overline{N^{\prime}}$ parallel to $\bar{N}$, as well as a net $N^{\prime}$ parallel to $N$. Moreover, it is evident that $N^{\prime}$ and $\overline{N^{\prime}}$ are applicable. Hence:

If $N$ and $\bar{N}$ are applicable nets, the knowledge of a net parallel to either enables one to find by quadratures a net parallel to the other, to which it is applicable.

Suppose now that we subject $N$ to a transformation $F$ determined by a parallel net $N^{\prime}$ and a solution $\theta$ of the point equation of $N$. From (9) it follows that the fundamental coefficients of the new net $N_{1}$ are given by

$$
\begin{aligned}
& E_{1}=\frac{\tau^{2}}{\theta^{\prime 4}}\left(\sum x^{\prime 2} \cdot\left(\frac{\partial \theta}{\partial u}\right)^{2}-2 \sum x^{\prime} \frac{\partial x}{\partial u} \theta^{\prime} \frac{\partial \theta}{\partial u}+\theta^{\prime 2} E\right), \\
& F_{1}=\frac{\tau \sigma}{\theta^{\prime 4}}\left(\sum x^{\prime 2} \cdot \frac{\partial \theta}{\partial u} \frac{\partial \theta}{\partial v}-\sum x^{\prime} \frac{\partial x}{\partial u} \theta^{\prime} \frac{\partial \theta}{\partial v}-\sum x^{\prime} \frac{\partial x}{\partial v} \theta^{\prime} \frac{\partial \theta}{\partial u}+\theta^{\prime 2} F\right), \\
& G_{1}=\frac{\sigma^{2}}{\theta^{\prime 4}}\left(\sum x^{\prime 2} \cdot\left(\frac{\partial \theta}{\partial v}\right)^{2}-2 \sum x^{\prime} \frac{\partial x}{\partial v} \theta^{\prime} \frac{\partial \theta}{\partial v}+\theta^{\prime 2} G\right) .
\end{aligned}
$$

We transform $\bar{N}$ by means of the net $\bar{N}^{\prime}$, applicable to $N^{\prime}$, and the same function $\theta$ used above. In order that $\bar{N}_{1}$ and $N_{1}$ shall be applicable, the expressions for the fundamental coefficients for $\bar{N}_{1}$ must be equal to the above. Equating the corresponding expressions, we get three equations, which in fact are equivalent to the two

$$
\begin{aligned}
& \left(\sum x^{\prime 2}-\sum \bar{x}^{\prime 2}\right) \frac{\partial \theta}{\partial u}=2\left(\sum x^{\prime} \frac{\partial x}{\partial u}-\sum \bar{x}^{\prime} \frac{\partial \bar{x}}{\partial u}\right) \theta^{\prime} \\
& \left(\sum x^{\prime 2}-\sum \bar{x}^{\prime 2}\right) \frac{\partial \theta}{\partial v}=2\left(\sum x^{\prime} \frac{\partial x}{\partial v}-\sum \bar{x}^{\prime} \frac{\partial \bar{x}}{\partial v}\right) \theta^{\prime} .
\end{aligned}
$$

By integration we find, to within a negligible constant factor,

$$
\theta^{\prime}=\sum x^{\prime 2}-\sum \bar{x}^{\prime 2}
$$

It is readily shown that this function is a solution of the common point equation of the applicable nets $N^{\prime}$ and $\overline{N^{\prime}}$. Consequently the $F$ transforms of $N$ and $\bar{N}$ by means of $\theta^{\prime}$ and $\theta$ given by the quadrature 
$\frac{\partial \theta}{\partial u}=2\left(\sum x^{\prime} \frac{\partial x}{\partial u}-\sum \bar{x}^{\prime} \frac{\partial \bar{x}}{\partial u}\right), \quad \frac{\partial \theta}{\partial v}=2\left(\sum x^{\prime} \frac{\partial x}{\partial v}-\sum \bar{x}^{\prime} \frac{\partial \bar{x}}{\partial v}\right)$,

are applicable. Hence $\left.{ }^{19}\right)$ :

If $N$ and $\bar{N}$ are applicable nets, each net parallel to $N$ determines a pair of applicable nets $N_{1}$ and $\bar{N}_{1}$ which are respectively $F$ transforms of $N$ and $\bar{N}$.

We shall establish a theorem of permutability of these transformations. Let two applicable nets $N$ and $\bar{N}$ be transformed into pairs of applicable nets $N_{1}, \bar{N}_{1}$ and $N_{2}, \bar{N}_{2}$ be means of parallel nets $N^{\prime}, \bar{N}^{\prime}$ and $N^{\prime \prime}, \bar{N}^{\prime \prime}$ and functions $\theta_{1}^{\prime}$ and $\theta_{2}^{\prime \prime}$ given by

$$
\theta_{1}^{\prime}=\sum x^{\prime 2}-\sum \bar{x}^{\prime 2}, \quad \theta_{2}^{\prime \prime}=\sum x^{\prime \prime 2}-\sum \bar{x}^{\prime \prime 2} .
$$

We apply the results of $\S 21$ to this case and seek whether $N_{12}$ and $\bar{N}_{12}$ are applicable. To this end we take

$$
\theta_{12}^{\prime \prime \prime}=\sum x_{1}^{\prime \prime \prime 2}-\sum \bar{x}_{1}^{\prime \prime \prime 2}, \quad \theta_{21}^{\prime \prime \prime}=\sum x_{2}^{\prime \prime \prime 2}-\sum \bar{x}_{2}^{\prime \prime \prime \prime 2} .
$$

Substituting the values of these functions from $\S \S 20,21$, we get

$$
\theta_{1}^{\prime \prime}+\theta_{2}^{\prime}-2\left(\sum x^{\prime} x^{\prime \prime}-\sum \bar{x}^{\prime} \bar{x}^{\prime \prime}\right)=0 \text {. }
$$

In consequence of (96) we find by differentiation that the left-hand member of (97) is constant. We have observed that in the general case $\theta_{1}^{\prime \prime}$ and $\theta_{2}^{\prime}$ involve additive arbitrary constants. Hence they can be chosen in an infinity of ways to satisfy (97), and we have:

of the $\infty^{2}$ transforms $N_{12}$ and $\bar{N}_{12}, \infty^{1}$ pairs are applicable, these cases arising when the constants in $\theta_{1}^{\prime \prime}$ and $\theta_{2}^{\prime}$ are chosen so that (97) is satisfied.

28. Nets corresponding with orthogonality of linear elements. Two nets in $n$-space are said to correspond with orthogonality of linear elements, if corresponding directions on the surfaces of these nets are orthogonal to one another. We say that two such nets are in relation $O$. A necessary and sufficient condition that $N(x)$ and $\bar{N}(\bar{x})$ are in relation $O$ is [cf. $\S 153$ ]

(98) $\sum \frac{\partial x}{\partial u} \frac{\partial \bar{x}}{\partial u}=0, \quad \sum \frac{\partial x}{\partial u} \frac{\partial \bar{x}}{\partial v}+\sum \frac{\partial x}{\partial v} \frac{\partial \bar{x}}{\partial u}=0, \quad \sum \frac{\partial x}{\partial v} \frac{\partial \bar{x}}{\partial v}=0$.

19) Transactions, vol. 19 (1918) p. 170. 
Let (6) be the point equation of $N(x)$ and let

$$
\frac{\partial^{2} \theta}{\partial u \partial v}=\frac{\partial \log \bar{a}}{\partial v} \frac{\partial \theta}{\partial u}+\frac{\partial \log b}{\partial u} \frac{\partial \theta}{\partial v}
$$

be the point equation of $\bar{N}(\bar{x})$. If the first and third of (98) be differentiated with respect to $v$ and $u$ respectively, we have in consequence of the second of (98) that $N$ and $\bar{N}$ have the same point equation. Hence:

When $N$ and $\bar{N}$ are two nets in relation $O$, they have the same point equation.

From this result and $\$ 4$ it follows that if $h$ and $l$ are a pair of solutions of

$$
\frac{\partial h}{\partial r}=(l-h) \frac{\partial \log a}{\partial v}, \frac{\partial l}{\partial u}=(h-l) \frac{\partial \log b}{\partial u},
$$

the nets $N^{\prime}\left(x^{\prime}\right)$ and $\overline{N^{\prime}}\left(\bar{x}^{\prime}\right)$, whose coordinates are given by

$$
\text { (100) } \frac{\partial x^{\prime}}{\partial u}=h \frac{\partial x}{\partial u}, \frac{\partial x^{\prime}}{\partial \imath}=l \frac{\partial x}{\partial v} ; \quad \frac{\partial \bar{x}^{\prime}}{\partial u}=h \frac{\partial \bar{x}}{\partial u}, \frac{\partial \bar{x}^{\prime}}{\partial v}=l \frac{\partial \bar{x}}{\partial v}
$$

are parallel to $N$ and $\bar{N}$ respectively. Moreover, from (98) it follows that $N^{\prime}$ and $\overline{N^{\prime}}$ are in relation $O$. If we say that $N^{\prime}$ and $\overline{N^{\prime}}$ are corresponding parallel nets' of $N$ and $\bar{N}$, we have:

If $N$ and $\vec{N}$ are two nets in relation $O$, corresponding parallel. nets are in relation $O$.

We seek transformations $F$ of nets $N$ and $\bar{N}$ in relation $O$ into nets $N_{1}$ and $\overline{N_{1}}$ in relation $O$. Let $N^{\prime}$ and $\overline{N^{\prime}}$ be two corresponding parallel nets of $N$ and $\bar{N}$, and let $\theta$ and $\theta^{\prime}$ be corresponding solutions of the point equations of $N$ and $N^{\prime}$ respectively, that is

$$
\frac{\partial \boldsymbol{\theta}^{\prime}}{\partial \imath}=h \frac{\partial \theta}{\partial \imath}, \quad \frac{\partial \boldsymbol{\theta}^{\prime}}{\partial \imath}=l \frac{\partial \boldsymbol{\theta}}{\partial \imath}
$$

The equations of the transformations are

$$
x_{1}=x-\frac{\theta}{\theta^{\prime}} x^{\prime}, \quad \bar{x}_{1}=\bar{x}-\frac{\theta}{\theta^{\prime}} \bar{x}^{\prime} .
$$


From the first we have by differentiation equations (9) and similar expressions for $\frac{\partial \bar{x}_{1}}{\partial \imath}$ and $\frac{\partial \bar{x}_{1}}{\partial v}$.

When we express the condition that these quantities satisfy equations of the form (98), the resulting equations give, to within a negligible constant factor,

$$
\theta^{\prime}=\sum x^{\prime} \bar{x}^{\prime} .
$$

Since $N^{\prime}$ and $\overline{N^{\prime}}$ are in the relation $O$, this function $\theta^{\prime}$ satisfies their common point equation.

\section{'Hence:}

If $N(x)$ and $\bar{N}(\bar{x})$ are nets in relation $O$, and $N^{\prime}\left(x^{\prime}\right)$ and $\overline{N^{\prime}}\left(\bar{x}^{\prime}\right)$ are two corresponding parallel nets, the nets $N_{1}$ and $\vec{N}_{1}$ which are $F$ transforms of $N$ and $\vec{N}$ respectively by means of the equations

$$
x_{1}=x-\frac{\theta}{\sum x^{\prime} \bar{x}^{\prime}} x^{\prime}, \quad \bar{x}_{1}=\bar{x}-\frac{\theta}{\sum x^{\prime} \bar{x}^{\prime}}-\bar{x}^{\prime},
$$

where $\theta$ is given by

$$
\frac{\partial \theta}{\partial u}=\frac{1}{h} \frac{\partial}{\partial u} \sum x^{\prime} \bar{x}^{\prime}, \quad \frac{\partial \theta}{\partial v}=\frac{1}{l} \frac{\partial}{\partial v} \sum x^{\prime} \bar{x}^{\prime},
$$

are in relation $O$.

We shall establish a theorem of permutability of these transformations. Let two nets $N$ and $\bar{N}$ in relation $O$ be transformed into pairs of nets $N_{1}, \bar{N}_{1}$ and $N_{2}, \bar{N}_{2}$ by means of parallel nets $N^{\prime}, \overline{N^{\prime}}$ and $N^{\prime \prime}, \bar{N}^{\prime \prime}$ and the functions $\theta_{1}^{\prime}$ and $\theta_{2}^{\prime \prime}$ given by

$$
\theta_{1}^{\prime}=\sum x^{\prime} \bar{x}^{\prime}, \quad \theta_{2}^{\prime \prime}=\sum x^{\prime \prime} \bar{x}^{\prime \prime}
$$

We apply the results of $\S 21$ to this case and seek under what condition $N_{12}$ and $\bar{N}_{12}$ are in relation $O$. For this to be the case we must have

$$
\theta_{12}^{\prime \prime \prime}=\sum x_{1}^{\prime \prime \prime} \bar{x}_{1}^{\prime \prime \prime}, \quad \theta_{21}^{\prime \prime \prime}=\sum x_{2}^{\prime \prime \prime \prime} \bar{x}_{2}^{\prime \prime \prime},
$$

where $x_{1}^{\prime \prime \prime}$ and $x_{2}^{\prime \prime \prime \prime}$ are given by (33) and (42) and $\bar{x}_{1}^{\prime \prime \prime}$ and $\bar{x}_{2}^{\prime \prime \prime \prime}$ have analogous expressions. Substituting the values of $\theta_{12}^{\prime \prime \prime}$ and $\theta_{21}^{\prime \prime \prime}$ from $\$ 21$, we have

$$
\theta_{1}^{\prime \prime}+\theta_{2}^{\prime}-\sum\left(x^{\prime \prime} \bar{x}^{\prime}+x^{\prime} \bar{x}^{\prime \prime}\right)=0 .
$$


In consequence of (106) we find by differentiation that the left-hand member of (107) is constant. We have observed ( $\$ 21)$ that in the general case of the theorem of permutability the functions $\theta_{1}^{\prime \prime}$ and $\theta_{2}$ are determined to within additive arbitrary constants. Since these can be chosen in an infinity of ways so that (107) holds, we have:

Of the $\infty^{2} F$ transforms $N_{12}$ and $\bar{N}_{12}$ arising from the theorem of per'mutalility of any nets $N$ and $\vec{N}$ in relation $O$, there are $\infty^{1}$ pairs in relation $O$; they arise when the constants in $\theta_{2}^{\prime}$ and $\theta_{1}^{\prime \prime}$ are rhosen so that (107) is satisfied; all these $\infty^{1}$ pairs of transforms can be found by two quadratures, when $N_{1}, \bar{N}_{1}$ and $N_{2}, \overline{N_{2}^{*}}$ are known.

\section{Exercises.}

1. If $N$ is a net of translation (cf. I, Ex. 6), the ouly $F$ transforms of $N$ which are nets of translation are parallel to $N$.

2. If $N$ is a minimal net (cf. I, Ex. 7), the only $F$ transforms of $N$ which are minimal nets are parallel to $N$.

3. If $N$ is any net and $N_{1}$ is the $F$ transform defined by (2), and $(N)_{1}$, $\left(N_{1}\right)_{1}$ and $\left(N^{\prime}\right)_{1}$ are the first Laplace transforms of $N, N_{1}$ and $N^{\prime}$ respectively, then

where

$$
\left(x_{1}\right)_{1}=(x)_{1}-\frac{(\theta)_{1}}{\left(\theta^{\prime}\right)_{1}}\left(x^{\prime}\right)_{1},
$$

$$
(\theta)_{1}=\theta-\frac{1}{\frac{\partial \log a}{\partial v}} \frac{\partial \theta}{\partial v}, \quad\left(\theta^{\prime}\right)_{1}=\theta^{\prime}-\frac{1}{\frac{\partial \log a h}{\partial v}} \frac{\partial \theta^{\prime}}{\partial v} .
$$

4. If $N_{1}$ is an $F$ transform of a net $N$, any Laplace transform of $N$ admits as $H^{\prime}$ transform the corresponding Laplace transform of $N_{1}$, the equations of the transformation being

$$
\left(x_{1}\right)_{r}=(x)_{r}-\frac{(\theta)_{r} \cdot}{\left(\theta^{\prime}\right)_{r}}\left(x^{\prime}\right)_{r}
$$

where $(\theta)_{r},\left(\theta^{\prime}\right)_{r}$ and $\left(x^{\prime}\right)_{r}$ are the $r^{\text {th }}$ Laplace transforms of $\theta, \theta^{\prime}$ and $x^{\prime}$.

5. If $N$ and $N_{1}$ are in relation $F$, and $\varphi$ and $\varphi_{1}$ are corresponding solutions of their point equations, the Levy transforms of $N$ and $N_{1}$ on the tangents to the curves $v=$ const. of $N$ and $N_{1}$ are in relation $F$, and also the Levy transform on the tangents to the curves $u=$ const.; moreover, the lines of the conjugate congruences of these transformations are tangent to the net which is the $F$ transform' of $N$ by $\varphi$ conjugate to the congruence of the transformation from $N$ into $N_{1}$.

6. By means of Ex. 5 and $\S 11$ show that if $N$ and $N_{1}$ are nets in relation $F$, and $\varphi, \varphi_{1}$ and $\psi, \zeta^{\prime \prime}$ are corresponding pairs of solutions of the point equations 
of $N$ and $N_{1}$, the derived net of $N^{r}$ by $\varphi$ and $\psi$ is in relation $F$ with the derived net of $N_{1}$ by $\varphi_{1}$ and $\psi_{1}$.

7. If $N$ and $N_{1}$ are nets in relation $F$, and $N_{2}$ and $N_{12}$ are parallel to $N$ and $N_{1}$ respectively in accordance with the second theorem of $\$ 21$, corresponding lines of the congruences of parameters $x_{2}$ and $x_{12}$ conjugate to $N$ and $N_{1}$ meet in a point describing a net forming a triad with $N$ and $N_{1}$.

8. Show that if in $\S 24$ we take $\theta_{3}=w_{3}=1, \theta_{3}^{\prime}=\theta_{3}^{\prime \prime}=0$, the nets $N_{3}^{\prime}$, $N_{13}, N_{28}, \bar{N}$ are parallel to $N, N_{1}, N_{4}, N_{12}$ respectively.

9. If $N, N_{1}, N_{2}, N_{12}$ are nets of a quatern, the respective functions $\theta_{2}, \theta_{12}$, $-\theta_{2} / \theta_{2}^{\prime \prime},-\theta_{12} / \theta_{12}^{\prime \prime \prime}$ determine radial transforms $\bar{N}, \bar{N}_{1}, \bar{N}_{2}, \bar{N}_{12}$, forming a quatern under trausformations $F$.

Transactions, vol. 18 (1917), p. 123.

10. If $N$ is a net and $\theta$ any solution of its point equation, the equations $x_{1}=x-c \theta$, where the $c$ 's are constants define a net $N_{1}$ which is a transform of $N$, the congruence of the transformation consisting of parallel lines. The tangent planes to $N$ and $N_{1}$ meet in the harmonic congruence of $N$ determined by $\theta$.

11. If $N$ and $\bar{N}$ are applicable nets, the equations $x_{1}=x-c \theta, \bar{x}_{1}=\bar{x}-\bar{c} \theta$ determine applicable nets $N_{1}$ and $\bar{N}_{1}$, if $\theta=2\left(\Sigma_{c} \bar{x}-\Sigma_{c x}\right) /\left(\Sigma_{c}^{2}-\Sigma_{c^{2}}\right)$.

12. When the functions $a$ and $b$ in equation (6) satisfy the condition

$$
a \frac{\partial^{2} a}{\partial u \partial v}-b \frac{\hat{o}^{2} b}{\partial u \partial v}=\left(\frac{a}{b}-\frac{b}{a}\right) \frac{\partial a}{\partial v} \frac{\partial b}{\partial u},
$$

the equations

$$
\frac{\partial \log \sqrt{\rho}}{\partial u}=\frac{1}{a} \frac{\partial b}{\partial u}, \quad \frac{\partial \log \sqrt{\prime \prime}}{\partial v}=\frac{1}{b} \frac{\partial a}{\partial r}
$$

are consistent, and the functions

$$
h_{1}=\frac{\sqrt{\rho}}{a}, l_{1}=\frac{\sqrt{\rho}}{b} ; h_{9}=\frac{1}{\sqrt{\rho} a}, l_{2}=-\frac{1}{\sqrt{p^{b}}}
$$

satisfy equations $(I, 13)$. The two nets $N$ and $N_{0}$ parallel to a net $N^{\prime}$ with equation (6) detemmined by the solutions (ii) have equal point invariants, and are associate $(\S 25)$ to one allother.

Conversely, if a net $N^{\prime}$ with point equation (6) admits a parallel net with equal point invariants equation (i) nust be satisfiel.

13. When the condition (i) of Ex. 12 is satisfied, equation (6) admits the solution $\theta^{\prime}=\frac{1}{4}\left(a^{2}-b^{2}\right)$. If we put $\theta=\frac{1}{2} \sqrt{\rho}(a-b)$, we have $\frac{\partial \theta^{\prime}}{\partial u}=\frac{a}{\sqrt{\rho}}-\frac{\partial \theta}{\partial u}$ $\frac{\partial \theta^{\prime}}{\partial v}=\frac{b}{\sqrt{\rho}} \frac{\partial \theta}{\partial v}$. These values satisfy (81), and consequently $\theta$ and $\theta^{\prime}$ and the congruence conjugate to $N$ whose direction-parameters are the coordinates $x^{\prime}$ of $N^{\prime}$ determine a $K$ transform of $N$.

14. Let $M, M M_{1}, M_{2}, M_{12}$ be corresponding points of four nets $\mathrm{N}, N_{1}, N_{2}, N_{12}$ of a quatern under transformations $F$. Show that a necessary and sufficient condition that another net $N_{12}$ obtained by varying the additive constants of $\theta_{1}^{\prime \prime}$ and $\theta_{2}$ be such that its points lie on the corresponding lines $\boldsymbol{M} \boldsymbol{M}_{12}$ is that the transformations be $K$. 
15. If $S^{*}$ is a net with equal point invariants, $N_{1}, N_{2}, N_{s}$ are $K$ transforms of $N_{\text {, and }} \Lambda_{12}, N_{13}, N_{33}$ are the nets with equal point invariants which together with the respective groups $N, N_{1}, N_{2} ; N_{1}, N_{1}, N_{3} ; N, N_{2}, N_{3}$ form quaterns under transformations $K$, then $\bar{N}$ defined by (76) is a $K$ transform of $N_{12}, N_{13}, N_{23}$.

Transactions, vol. 16 (1915), p. 296.

16. Determine whether transfornations $K$ are the only transformations $\boldsymbol{F}$ of a net with equal point inrariants into nets with equal point invariants.

17. In order that the Levy transforms conjugate to the tangents to the curves of parameter $u$ of a net $N$ with the point equation (6) and determined by solutions $\theta$ and $\theta_{1}$ of (6) be $K$ transforms of one another, it is necessary that $\theta \theta_{1}=b^{2} V$, where $V$ is a function of $v$ alone.

18. If $N$ and $N_{1}$ are two nets in relation $K$; their respective associates can be so placed in space that they shall be in relation $K$.

19. If in (46) $\theta_{1}^{\prime \prime}$ and $\theta_{2}^{\prime}$ are replaced by $\theta_{1}^{\prime \prime}+c$ and $\theta_{2}^{\prime}-c$, where $c$ is a parameter, the corresponding points of the $\mathfrak{o}^{1}$ nets $N_{12}$ lie on a conic which passes through the corresponding points of $N, N_{1}$ and $N_{2}$; this conic is degenerate when the transformations are $K$, and only in this case (cf. Ex. 14). 
Chapter III.

\section{Sequences of Laplace.}

29. Homogeneous point coordinates. If the cartesian coordinates of a point $P$ are $x^{1}, \ldots x^{n}$, the $n+1$ quantities $y$, of which $y^{n+1} \neq 0$, satisfying the conditions

$$
r^{1}=\frac{y^{1}}{y^{n+1}} \ldots x^{n}=\frac{y^{n}}{y^{n+1}}
$$

are called lomogeneons coorlinates of $P$. If the coordinates $y$ are given, $P$ is determined, but if the cartesian coordinates $x$ are given, the homogeneous coordinates $y$ are defined only to within a factor.

In homogeneous coordinates the equation of a hyperplane is of the form $(\S 1)$

$$
a^{1} y^{1}+\ldots+a^{n \cdot 1} y^{n \cdot 1}=0 .
$$

Now $y^{i}=0(i=1, \ldots, n)$ is the equation of a coordinate hyperplane. Also $y^{n+1}=0$ is taken as the equation of a hyperplane, namely the hyperplane at infinity. This hyperplane likewise is a coordinate hyperplane in homogeneous coordinates. Thus we have $n+1$ hyperplanes forming a coordinate $(n+1)$-hedron. Noreover, a point all of whose homogeneous coordinates save one, say $y^{i}$, are zero lies in all of these hyperplanes except the hyperplane $y^{i}=0$; it is a rertex of the coordinate $(n+1)$-hedron.

Suppose now that we have any $n+1$ hyperplanes, say

$$
a_{i}^{1} y^{1}+a_{i}^{2} y^{2}+\ldots+a_{i}^{n} \quad 1 y^{n}: 1=0 \quad(i=1, \ldots, n+1),
$$

subject to the single condition that they do not lave a point in common, that is, the determinant of the $a$ 's is not equal to zero; thus $\left|c_{i}^{j}\right| \neq 0$. If we put

$$
\underline{Q} z^{i}=a_{i}^{1} y^{1}+\ldots+a_{i}^{n+1} y^{n-1} \quad(i=1, \ldots, n+1),
$$


where $\varrho$ is a factor independent of $i$, the quantities $z^{i}$ serve also as point coordinates. For when the y's are given for a point the $z$ 's are uniquely determined except for a factor. Conversely, when the $z$ 's are given, the determination of the corresponding $y$ 's requires the solution of a linear system of equations, whose determinant is different from zero. We call the z's general homogeneous point coordinates, and as such they are distinguished from the particular system of $y$ 's, corresponding to the case where $n$ of the coordinate planes are mutually perpendicular, and the other is at infinity. As in the case of tetrahedral coordinates in 3-space, the transformation is not completely determined by the $(n+1)$-hedron, but is defined when it is required that a particular point, not on the faces of the new $(n+1)$-hedron is to have the coordinates $(1,1, \ldots, 1)$ in the new system.

Since the steps used in the preceding are reversible, we have that there exist linear transformations $P_{0}$ by means of which from a general system of homogeneous coordinates $z$ we pass to a system $y$ so that $y^{1} / y^{n+1} \ldots y^{n /} / y^{n+1}$ are cartesian coordinates. Hereafter we understand that when homogeneous coordinates are used they are of the general type.

We are prepared to prove the theorem:

The homogeneous coordinates of any point on the line joining two points $P_{1}\left(z_{1}\right)$ and $P_{2}\left(z_{2}\right)$ are of the form

and conversely.

$$
\lambda z_{1}+\mu z_{2}
$$

Consider the point with these coordinates and apply a transformation $P_{0}$ (referred to above). The resulting expressions will be of the form $\lambda y_{1}+\mu y_{2}$. Hence the cartesian coordinates of the point are

$$
\frac{\lambda y_{1}^{i}+\mu y_{2}^{i}}{\lambda y_{1}^{n-1}+\mu y_{2}^{n+1}}=\frac{\lambda y_{1}^{n+1} x_{1}^{i}+\mu y_{2}^{n+1} \cdot u_{2}^{i}}{\lambda y_{1}^{n+1}+\mu y_{2}^{n+1}} \quad(i=1,2, \ldots n)
$$

which shows that the point lies on $P_{1} P_{2}$ (cf. I, Ex. 1), Evidently the converse also is true.

In like manner it can be shown (cf. I, Ex. 2) that:

The homogeneous coordinates of any point on the plane through three points $P_{1}\left(z_{1}\right)$. $P_{2}\left(z_{2}\right) P_{3}\left(z_{3}\right)$ are of the form 


$$
\lambda z_{1}+\mu z_{2}+\nu_{z_{3}}
$$

and conversely.

From $\S 1$ the locus of a point whose homogeneous coordinates $z$ are functions of a parameter $u$ is a curve. We wish to prove:

The homogeneous coordinates of any point on the tangent to a curce $z$ (u) are of the form

$$
\lambda z+\mu \frac{d z}{d u}
$$

and conversely.

If the coordinates of a point are in this form, and we apply a transformation $P_{0}$ we get for the new coordinates of the point $\lambda y+\mu \frac{d y}{d l u}$. Hence the cartesian coordinates of the point are

$$
\frac{\lambda y^{i}+\mu \frac{d y^{i}}{d u}}{\lambda y^{n-1}+\mu \frac{d y^{n+1}}{d u}}=x^{i}+\frac{\frac{d x^{i}}{d u}}{\frac{\lambda}{\mu}+\frac{d}{d u} \log y^{n+1}} .
$$

As these are of the form $x+i \frac{d x}{d u}$, the point lies on the tangent (§1). Conversely, the cartesian coordinates of any point on the tangent being of this form are readily transformable into the form of the theorem.

Consider a point on the tangent to a curve with its coordinates in the form $\lambda z+\mu \frac{d z}{d u}$. Two functions $\varrho$ and $t$ are defined by

$$
\varrho \frac{d t}{d u}=\lambda, \quad \varrho t=\mu .
$$

In terms of $\varrho$ and $t$ the coordinates of the point are of the form $o \frac{d}{d u}(t z)$. Hence we have:

The homogeneous point coordinates $z$ of a curve can be chosen so that the coordinates of a given point on the tangent other than the point of contact are of the form $\frac{d z}{d u}$.

The osculating plane of a curve $z(u)$ at a representative point is by definition the locus of points whose coordinates are of the form 
$\lambda z+u \frac{d z}{d u}+v \frac{d^{2} z}{d u^{2}}$. If we apply a transformation $P_{0}$ to the coordinates and proceed as above, we can show that the cartesian coordinates of any point of the osculating plane are of the form

$$
x+\lambda \frac{d x}{d u}+\mu \frac{d^{2} x}{d u^{2}}
$$

We obselve that this is the result previously found for 3-space [\$ 7].

30. Laplace transformations. We have seen in $\$ 2$ that a necessary and sufficient condition that a system of paranetric curves be a net is that any point on the tangent to a curve $r=$ const. of a system moves in the tangent plane to the surface as $v$ varies. Given a net $N$ with $n+1$ homogeneous coordinates $x$. The coordinates of any point $P$ on the tangent to a curve $v=$ const. are of the form $\lambda x+\mu \frac{\partial x}{\partial u}$. As $v$ varies, the point moves in the tangent plane, provided $\frac{\partial}{\partial v}\left(2 x+\mu \frac{\partial x}{\partial u}\right)$ is expressible linearly in terms of $x, \frac{\partial x}{\partial u}$, and $\frac{\partial x}{\partial v}$. Hence a net is characterised analytically by the condition that its homogeneous coordinates are solutions of the same equation of the form

$$
\frac{\partial^{2} \theta}{\partial u \partial v}=\frac{\partial \log a}{\partial v} \frac{\partial \theta}{\partial u}+\frac{\partial \log b}{\partial u} \frac{\partial \theta}{\partial v}+r \theta .
$$

We call this the point equation of the net.

If, in particular, the point $P$ moves tangentially to the tangent to $v=$ const., that is if $P$ describes the minus first Laplace transform of $N$, we must liave

$$
\lambda+\mu \frac{\partial \log l}{\partial u}=0
$$

Since similar results follow for the first Laplace transform, we have:

The coordinates of the minus first and first Laplace transforms of a net with the point equation (1) can be taken in the form

$$
x_{-1}=\frac{\partial x}{\partial u}-\frac{\partial \log b}{\partial u} x, \quad x_{1}=\frac{\partial x}{\partial \iota}-\frac{\partial \log a}{\partial v} x .
$$


In consequence of the fourth theorem of $\S 29$ the equations of a Laplace transformation can be put in the simple form indicated in the following theorem:

The homogeneous coorlinates $z^{i}$ and $y^{i}$ of the fockl points of a congruence ran be chosen so that

$$
\frac{\partial z}{\partial u}=m y, \quad \frac{\partial y}{\partial t}=n z
$$

From (2) we have by differentiation

$$
\frac{\partial x_{-1}}{\partial v}=\frac{\partial \log a}{\partial v} x_{-1}+K x, \quad \frac{\partial x_{1}}{\partial u}=\frac{\partial \log b}{\partial u} x_{1}+H x
$$

where $H$ and $K$ are the invariants of (1) namely

$$
\left\{\begin{array}{l}
H=-\frac{\partial^{2} \log a}{\partial u \partial v}+\frac{\partial \log a}{\partial v} \frac{\partial \log b}{\partial u}+c \\
K=-\frac{\partial^{2} \log b}{\partial u \partial v}+\frac{\partial \log a}{\partial v} \frac{\partial \log b}{\partial u}+c
\end{array}\right.
$$

If $K=0$, we get on integration $r_{-1}=U a$, where $U$ is an arbitrary function of $u$ alone. As the arbitrary function $U$ varies with the integral but a remains the same, the point $M_{-1}$ describes a curve, and not a net; we say that $\nu_{-1}$ is degenerate. Substituting in the first of (2) and integrating the resulting equation, we find

$$
x=b\left(v+\int \frac{U a}{b} d u\right)
$$

$V$ being an arbitrary function of $v$ alone.

In like manner, if $H=0$, the integral of (1) can be given the form

$$
x=a\left(U+\int \frac{v b}{a} d v\right)
$$

Hence we have the theorem:

When either invariant of an equation of Laplace is equal to zero, the equation can be integrated by quadratures.

3r. Sequences of Laplace. When the invariants $H$ and $K$ of (1) are different from zero, by the iteration of the first Laplace 
transformation upon a net $N$ with (1) for its point equation, and also of the minus first transformation, we get a sequence of nets

$$
\ldots N_{-j}, N_{-(j-1)}, \ldots N_{-1}, N, N_{1}, \ldots, N_{i}, \ldots
$$

such that any net of the sequence is a first Laplace transform of its predecessor (sense being from left to right), and a minus first transform of its successor. We say that these nets form a sequence of Laplace.

We are interested in finding the Laplace equation of each net of the sequence. In order to write these equations and others associated with (1) in abbreviated form, we denote by

$$
\left[\theta_{i} ; a_{i}, b_{i}, e_{i}\right]
$$

the equation

$$
\left\{\begin{array}{c}
\frac{\partial^{2} \theta_{i}}{\partial u \partial v}=\frac{\partial \log a_{i}}{\partial v} \frac{\partial \theta_{i}}{\partial u}+\frac{\partial \log b_{i}}{\partial u} \frac{\partial \theta_{i}}{\partial v} \\
+\left(\frac{\partial^{2}}{\partial u \partial v} \log e_{i}-\frac{\partial \log a_{i}}{\partial v} \frac{\partial \log b_{i}}{\partial u}+\frac{\partial \log a}{\partial v} \frac{\partial \log b}{\partial u}+c\right) \theta_{i}
\end{array}\right.
$$

Then from (5) it follows that

$$
\left\{\begin{array}{l}
H_{i}=\frac{\partial^{2}}{\partial u \partial v} \log \frac{e_{i}}{a_{i}}+\frac{\partial \log a}{\partial v} \frac{\partial \log b}{\partial u}+c \\
K_{i}=\frac{\partial^{2}}{\partial u \partial v} \log \frac{e_{i}}{b_{i}}+\frac{\partial \log a}{\partial v} \frac{\partial \log b}{\partial u}+c .
\end{array}\right.
$$

If in equation (1) we put

$$
x=\frac{x^{\prime}}{\hat{\gamma}},
$$

where $z$ is a function of $\|$ and $v$, the Laplace equation satisfied by $x^{\prime}$ is denoted by

$$
\left[\theta^{\prime} ; a \lambda, b \lambda, \lambda\right]
$$

Then from (10) we have $H^{\prime}=H, K^{\prime}=K$, showing that $H$ and $K$ are invariants of (1) for transformations of the form (11). 
In order to find the Laplace equation of $N_{1}$, we differentiate the second of (4) with respect to $v$. Making use of (2), we find that the Laplace equation of $N_{1}$ is denoted by

$$
\left[\theta_{1} ; a H, b, \frac{b}{a}\right]
$$

and that

$$
H_{1}=H-\frac{\partial^{2}}{\partial u \partial v} \log \frac{a H}{b}, \quad K_{1}=H .
$$

Proceeding in a like manner with the first of (4). we find that the Laplace equation of $N_{-1}$ is denoted by

$$
\left[\theta-1 ; a, b K, \frac{a}{b}\right]
$$

and that

$$
H_{-1}=K, K_{-1}=K-\frac{\partial^{2}}{\partial u \partial v} \log \frac{b K}{a}
$$

The coordinates $x_{2}$ of $N_{2}$ are given by the equations

$$
x_{\mathbf{2}}=\frac{\partial x_{1}}{\partial v}-x_{1} \frac{\partial}{\partial r} \log a H
$$

analogous to (2), and the point of equation of $\Lambda_{2}$ is denoted by

$$
\left[\theta_{2} ; a H H_{1}, b, \frac{b^{2}}{a^{2} H}\right] \text {. }
$$

In general the coordinates of $\lambda_{r}$ are given by

$$
x_{r}=\frac{\partial x_{r-1}}{\partial v}-\frac{\partial}{\partial v} \log \left(a H H_{1} \ldots H_{r-2}\right) x_{r-1}
$$

and the point equation is

$$
\left[\theta_{r} ; a H H_{1} \ldots H_{r-1}, b, \frac{b^{r}}{a^{r} H^{r-1} H_{1}^{r-2} \ldots H_{r-2}}\right] \text {. }
$$


The equations analogous to (4) and (17) are

$$
\left\{\begin{array}{l}
\frac{\partial x_{r}}{\partial u}=\frac{\partial \log b}{\partial u} x_{r}+H_{r-1} r_{r-1}, \\
\frac{\partial x_{r}}{\partial r}=\frac{\partial}{\partial r} \log \left(a H \ldots H_{r-1}\right) x_{r}+r_{r} \cdot 1
\end{array}\right.
$$

The coordinates of $N_{-r}$ are given by

$$
x_{-r}=\frac{\partial x_{-r} 1}{\partial u}-\frac{\partial}{\partial u} \log \left(b K K_{-1} \ldots K_{-r+2}\right) x-r+1,
$$

and the point equation is

$$
\left[\theta_{-r} ; a, b K K_{-1} \ldots K_{-r+1}, \frac{a^{r}}{b^{r} K^{r-1} K_{-1}^{r-2} \ldots K_{-r+2}}\right]
$$

Also we have

$$
\left\{\begin{array}{l}
\frac{\partial x_{-r}}{\partial u}=\frac{\partial}{\partial u} \log \left(b K \ldots K_{-r+1}\right) x_{-r+r_{-r-1}} \\
\frac{\partial x_{-r}}{\partial v}=\frac{\partial \log a}{\partial v} x_{-r}+K_{-r+1} \cdot x_{-r+1} .
\end{array}\right.
$$

32. Periodic sequences of Laplace. Ordinarily a sequence of Laplace is unlimited in both directions. If $H_{r}$ or $K_{-r}$ is zero, the sequence terminates in the positive or negative sense (cf. III, Ex. 5). In the present section we are concerned with the case when the sequence is periodic, that is when a certain net $N_{p}$ coincides with $N$. In this case we must have

$$
x_{p}=m x
$$

where $m$ is at most a function of $u$ and $v$ which is the same for all $n$ coordinates $x^{i}$. Since $n$ is at least equal to 3 , the coefficients of (12) and (20) for $r=p$ must be equal. Hence we must have

$$
\begin{gathered}
\frac{\partial}{\partial v} \log \frac{H \ldots H_{p-1}}{m}=0, \quad \frac{\partial}{\partial u} \log m=0, \\
\frac{\partial^{2}}{\partial u \partial v} \log \frac{b^{p}}{a^{p} H^{p-1} H_{1}^{p-2} \ldots . H_{p-\vartheta}}=0 .
\end{gathered}
$$


Differentiating the first of (26) with respect to $u$ and making use of the second, we find that

$$
H H_{1} \ldots H_{p-1}=U r
$$

where $U$ and $V$ are functions of $u$ and $v$ respectively. If we change the independent variables in accordance with the equations

$$
u=\varphi\left(u^{\prime}\right), \quad v=\psi\left(r^{\prime}\right),
$$

we find that the invariants $H^{\prime}, H_{1}^{\prime}, \ldots . H_{p}^{\prime}$ of the transformed equations of the nets $N, N_{1}, \ldots N_{p}$ are given by

$$
H^{\prime}=\varphi^{\prime}\left(u^{\prime}\right) \psi^{\prime}\left(v^{\prime}\right) H, \quad H_{i}^{\prime}=\varphi^{\prime}\left(u^{\prime}\right) \psi^{\prime}\left(v^{\prime}\right) H_{i} \quad(i=1, \ldots p),
$$

where $\varphi^{\prime}$ and $\psi^{\prime}$ denote the derivates of $\varphi$ and $\psi$. Hence $\varphi$ and $\psi$ can be chosen so that (28) becomes

$$
H H_{1} \ldots H_{p-1}=1
$$

Then from (26) it follows that $m$ in (25) is constant ${ }^{20}$ ).

Suppose now that (27) and (29) are satisfied for equation (1). Applying (10) to (20) for $r=p-1$, we get, in consequence of (27),

(30)

$$
\left\{\begin{array}{c}
H_{p-1}=H-\frac{\partial^{2}}{\partial u \partial \iota} \log \frac{a^{p-1} H^{p-1} H_{1}^{p-2} \ldots . H_{p-2}}{b^{p-1}} \\
=H-\frac{\partial^{2}}{\partial u \partial v} \log \frac{b}{u}=K
\end{array}\right.
$$

In like manner, making use of (27), (29) and (30), we obtain

$$
\begin{gathered}
H_{p-2}=H-\frac{\partial^{2}}{\partial u \partial v} \log \frac{a^{p-2} H^{p-2} \ldots H_{p-3}}{l^{p-2}} \\
=H-\frac{\partial^{2}}{\partial u \partial v} \log \frac{b^{2} H_{p-1}}{a^{2}}=K-\frac{\partial^{2}}{\partial u \partial v} \log \frac{b K}{a}=K-1 .
\end{gathered}
$$

20) Cf. Tzitzeica, Comptes Rendus, vol. 157 (1913), p. 908; also Hammond, Annals of Mathematics, ser. 2, vol. 22 (1921), p. 245. 
Hence in general we have

$$
H_{p-i}=K_{-i+1} .
$$

If we differentiate (25) with respect to $"$, and make nse of (2) and (21), we can reduce the resulting equation to

$$
H_{p-1} x_{p-1}=m x_{-1}
$$

which gives the analytical form of the condition that the nets $N_{p-1}$ and $N_{-1}$ coincide. Again differentiating (32) with respect to $u$, we get, in consequence of (21), (24), (30) and (32),

$$
H_{p-1} H_{p-2} x_{p-2}=m x_{-2} .
$$

And in general because of (31) we have

$$
H_{p-1} H_{p-2} \ldots . H_{p-i} x_{p-i}=m x_{-i}
$$

which in consequence of (29) is equivalent to

$$
r_{p-i}=m H H_{1} \ldots H_{p-i-1} x_{-i}
$$

Finally we have

$$
x=m x_{-p},
$$

showing that $N$ and $N_{-p}$ coincide.

Conversely if we differentiate (35) with respect to $v$ we get (34) with $i=p-1$. In general, if we differentiate (34) with respect to $v$, we get (34) with $i$ replaced by $i-1$. Hence according as we differentiate (33) or (34) with respect to $u$ or $v$ we increase or diminish $i$ by 1 . In order to point out the significance of this observation, we note that the expressions (19) and (22) for $x_{r}$ and $x_{-r}$ are expressible in the forms

$$
\begin{aligned}
x_{r} & =\frac{\partial^{r} x}{\partial v^{r}}+A_{r, r-1} \frac{\partial^{r-1} x}{\partial v^{r-1}}+\ldots+A_{r, o} x, \\
x_{-r} & =\frac{\partial^{r} x}{\partial u^{r}}+B_{r, r-1} \frac{\partial^{r-1} x}{\partial u^{r-1}}+\ldots+B_{r, o} x,
\end{aligned}
$$


where $A$ 's and $B$ 's are determinate functions of the derivates of $a$, $b$ and $c$ obtained by repeated use of (19) and (22). Hence equation (33) involves the derivatives of $x$ with respect to $v$ of orders $1, \ldots, p-i$, and of $x$ with respect to $u$ of orders $1, \ldots i$. If this equation is differentiated with respect to $u$ and all derivatives with respect to $u$ and $v$ are eliminated by means of (1) and the equations obtained by differentiating (1), we obtain an equation involving derivatives of $x$ with respect to $v$ of orders $1, \ldots p-i-1$ and with respect to $u$ of orders $1, \ldots i+1$, which necessarily is (33) with $i$ replaced by $i-1$.

Suppose now that we consider a periodic net of odd order, and write $p=2 n+1$. If in (34) we put $i=n$ and in (33) $i=n+1$, we get

$$
\left\{\begin{aligned}
x_{n+1} & =m H H_{1} \ldots H_{n} x_{-n}, \\
x_{-n-1} & =\frac{1}{m} H_{2 n} H_{2 n-1} \ldots H_{n} x_{n} .
\end{aligned}\right.
$$

These two equations express $\frac{\partial^{n+1} x}{\partial v^{n+1}}$ and $\frac{\partial^{n+1} x}{\partial u^{n+1}}$ linearly in terms of the $2 n+1$ quantities

$$
\frac{\partial^{n} x}{\partial u^{n}}, \ldots, \frac{\partial x}{\partial u}, \frac{\partial^{n} x}{\partial \iota^{n}}, \ldots, \frac{\partial x}{\partial v}, x .
$$

We have seen that the consistency of (36) and (1) leads to other equations of the series (33) and (34), by means of which and (1) we can express all the derivatives of order higher than $n$ in terms of (37). Since $m$ does not appear in (27) and (29), for each value of $m$ there exist $p$ independent solutions of (1), (33) and (34) including the condition (25); and not more than $p$ independent solutions.

When $p$ is evell, and we put $p=2 n$, we have in place of (36), $x_{n}=m H H_{1} \ldots H_{n-1} x_{-n}, \quad x_{-n-1}=\frac{1}{m} H_{2 n-1} \ldots H_{n-1} x_{n-1}$.

Then all the derivatives of order $n$ and higher are expressible linearly in terms of the $p$ quantities

$$
\frac{\partial^{n} x}{\partial u^{n}}, \ldots, \frac{\partial x}{\partial u}, \frac{\partial^{n-1} x}{\partial v^{n-1}}, \ldots \frac{\partial x}{\partial v}, x .
$$


Hence we have the theorem:

When an equation of Laplace (1) satisfies the conditions (27) and (29), an infinity of sequences of Laplace of order $p$ exist in space of order $p-1$.

33. Harmonic congruences. We wish to establish the theorem:

The homogeneous coordinates $x$ of a net $N$ can be so chosen that homogeneous coordinates of the focal points of any harmonic congruence are $\frac{\partial x}{\partial u}$ and $\frac{\partial x}{\partial v}$ respectively.

Let $N(x)$ be a net with homogeneous coordinates $x$ satisfying (1). Let $F_{1}$ and $F_{2}$ be the foci of any harmonic congruence, these points being on the tangents to the curves $v=$ const., $u=$ const., respectively. Evidently $x$ can be chosen so that the coordinates of $F_{1}$ are $\frac{\partial x}{\partial u}(\S 29)$. Then the coordinates of $F_{2}$ according as it is looked upon as on the tangent to the curves $u=$ const. at $M$ or on the tangent to the curves $\iota=$ const. at $F_{1}$ are of the respective forms $\lambda x+\mu \frac{\partial x}{\partial v}, \quad \sigma \frac{\partial x}{\partial u}+\tau \frac{\partial^{2} x}{\partial u \partial v}$. These forms must be proportional to one another in consequence of $x$ being a solution of the corresponding equation (1). Expressing this requirement, we find that both of these must be proportional to $\frac{\partial \log b}{\partial u} \frac{\partial x}{\partial v}+c x$. Evidently $\frac{\partial \log b}{\partial \imath} \neq 0$, otherwise $F_{2}$ and $M$ coincide. Looking upon $F_{1}$ as the minus first Laplace transform of $F_{2}$ we must have

$$
\frac{\partial x}{\partial u}=l\left(\frac{\partial \log b}{\partial u} \frac{\partial x}{\partial v}+c x\right)+m \frac{\partial}{\partial u}\left(\frac{\partial \log b}{\partial u} \frac{\partial x}{\partial v}+c x\right) .
$$

As this equation must be satisfied identically, we must have either $c=0$, or $V^{\prime} \frac{\partial \log b}{\partial u}=V c$, where $V$ is a function of $v$ alone and $V^{\prime}$ its derivative with respect to $r$. In the latter case the coordinates of $F_{z}$ can be chosen of the form $\frac{\partial}{\partial v}(V x)$. Replacing $V x$ by $x$, which does not change the form $\frac{\partial x}{\partial \imath}$ of the coordinates of $F_{1}$, we have the result stated in the theorem. 
Furthermore, when the coordinates of $F_{1}$ and $F_{2}$ are of the form $\frac{\partial x}{\partial u}$ and $\frac{\partial x}{\partial v}$, in the point equation of $N$ we have $c=0$. If we put $\theta=\theta_{1} \theta_{0}$ where $\theta_{0}$ is any function of $u$ and $v$, in equation (1), the function $\theta_{1}$ satisfies an equation of the type (1), and for this new equation $c=0$ in case $\theta_{0}$ is a solution of (1) and only in this case. As a result we have the corollary:

The homogeneous coordinates of the focal points of any congruence harmonic to a net $N(x)$ are expressible in the form

$$
\frac{\partial}{\partial u}\left(\frac{x}{\theta}\right), \frac{\partial}{\partial v}\left(\frac{x}{\theta}\right)
$$

in which case $\theta$ is a solution of the point equation of $N$.

We have also the converse theorem:

If $\theta$ is any solution of the point equation (1) of a net $N$, the points whose coordinates are of the form (38) are the focal points of a congruence harmonic to $N$.

For, as $v$ varies the direction-parameters of the path of the first of these points are of the form

$$
\frac{\partial}{\partial v}\left(\frac{\partial}{\partial u}\left(\frac{x}{\theta}\right)\right)=\frac{\partial}{\partial v} \log \frac{a}{\theta} \cdot \frac{\partial}{\partial u}\left(\frac{x}{\theta}\right)+\frac{\partial}{\partial u} \log \frac{b}{\theta} \cdot \frac{\partial}{\partial v}\left(\frac{x}{\theta}\right)
$$

which evidently are the parameters of the line joining the two points; similarly when $u$ varies.

We have also the theorem:

When $\theta=\sum a_{i} x^{i}$, where the a's are constants, the points of coordinates (38) lie in the hyperplane $\sum a_{i} x^{i}=0$.

34. Levy sequences of the first order. If $\theta$ is any solution of the point equation (1) of a net $N$, from (2) it follows that the functions

$$
\theta_{-1}=\frac{\partial \theta}{\partial u}-\frac{\partial \log b}{\partial u} \theta, \quad \theta_{1}=\frac{\partial \theta}{\partial v}-\frac{\partial \log a}{\partial v} \theta
$$

are solutions of the point equations of $N_{-1}$ and $N_{1}$ respectively. We call them the minus first and first Laplace transforms of $\theta$. 
The points of coordinates (38) are the Levy transforms of $N$ by means of $\theta$ (cf. I, 53). In consequence of (2) and (39), we can take as homogeneous coordinates of these respective points

$$
x_{-1,1}=x-\frac{\theta}{\theta_{-1}} x_{-1}, \quad x_{0,1}=x_{1}-\frac{\theta_{1}}{\theta} x .
$$

Moreover, the net of coordinates $x_{0,1}$ is the first Laplace transform of the net of coordinates $x_{-1,1}$.

By differentiation and reduction by means of (2), (4) and (39), we find that the point equations of these nets are denoted by (cf. $\S 31$ )

$$
\begin{gathered}
{\left[\theta_{-1,1} ; \frac{a \theta}{\theta_{-1}}, b, \frac{a}{\theta_{-1}}\right],} \\
{\left[\theta_{0,1} ; \frac{a \theta_{1}}{\theta}, b, \frac{b}{\theta}\right] .}
\end{gathered}
$$

From the form of (40) it follows that $N_{-1,1}$ and $N_{o, 1}$ are Levy transforms of $N_{-1}$ and $N_{1}$ by means of $\theta_{-1}$ and $\theta_{1}$. Moreover, from $\S 10$ we have that the tangents to the curves of parameter $v$ of $N_{o, 1}$ are harmonic to $N_{1}$, and consequently this harmonic congruence $G_{1}$ is determined by the solution $\theta_{1}$ of the point equation of $N_{1}$. Its focal point of the first order generates a Levy transform $N_{1,1}$ of $N_{1}$ whose coordinates are given (analogously to (40)) by

$$
x_{1,1}=x_{2}-\frac{\theta_{2}}{\theta_{1}} x_{1},
$$

where $\theta_{2}$ is the second Laplace transform of $\theta$. In like manner the function $\theta_{-1}$ determines a congruence $G_{-1}$ harmonic to $N_{-1}$ whose focal nets are $N_{-1,1}$ and $N_{-2,1}$, where

$$
x_{-2,1}=x_{-1}-\frac{\theta_{-1}}{\theta_{-2}} x_{-2},
$$

$\theta_{-2}$ being the minus second Laplace transform of $\theta$.

Continuing this process we obtain a sequence of Laplace whose focal nets are Levy transforms of the nets of the Laplace sequence arising from $N$. We call it the first Levy sequence of $N$ determined by $\theta$. The coordinates of the nets $N_{r, 1}$ for positive and negative values of $r$ are of the form 


$$
x_{r, 1}=x_{r+1}-\frac{\theta_{r+1}}{\theta_{r}} x_{r},
$$

where $\theta_{r}$ is the $r^{\text {th }}$ Laplace transform of $\theta$.

35. Levy sequences of higher order. Derived sequences. Let $\theta$ and $\theta^{\prime}$ be two solutions of the point equation (1) of $N$ linearly independent of the coordinates of $N$. By means of $\theta$ and $\theta^{\prime}$ we determine two first Levy sequences $N_{r, 1}$ and $N_{r, 1}^{\prime}$. These nets are the focal nets of two sequences of congruences $G_{r}$ and $G_{r}^{\prime}$ harmonic to the nets $N_{r}$ respectively of the Laplace sequence obtained from $N$. From $\S 11$ it follows that the point of intersection of corresponding lines of $G_{r}$ and $G_{r}^{\prime}$ describe a derived net of $N_{r}$. Moreover, from the results of $\S 11$ it follows that these derived nets form first Levy sequences of the sequences of $N_{r, 1}$ and $N_{r, 1}^{\prime}$ and consequently we call them Levy sequences of the second order. We shall obtain the analytical expressions for their coordinates.

We consider first the derived net of $N$ by means of $\theta$ and $\theta^{\prime}$. The functions $\theta_{-1,1}$ and $\theta_{0,1}$ defined by

$$
\theta_{-1,1}=\theta^{\prime}-\frac{\theta}{\theta_{-1}} \theta_{-1}^{\prime}, \quad \theta_{0,1}=\theta_{1}^{\prime}-\frac{\theta_{1}}{\theta} \theta^{\prime}
$$

where $\theta_{-1}^{\prime}$ and $\theta_{1}^{\prime}$ are the minus first and first Laplace transforms of $\theta^{\prime}$, are evidently corresponding solutions of $N_{-1,1}$ and $N_{0,1}$, which as we have seen are Laplace transforms of one another. Hence the coordinates of the Levy transform of $N_{-1,1}$ by means of $\theta_{-1,1}$ are the form

$$
x_{-1,2}=x_{0,1}-\frac{\theta_{0,1}}{\theta_{-1,1}} x_{-1,1}
$$

In consequence of $(40)$ and (46) this is equivalent to

$$
x_{-1,2}=\frac{1}{\theta_{-1} \theta_{-1,1}}\left|\begin{array}{ccc}
\theta_{-1} & \theta_{-1}^{\prime} & x_{-1} \\
\theta & \theta^{\prime} & x \\
\theta_{1} & \theta_{1}^{\prime} & x_{1}
\end{array}\right| \equiv \frac{1}{\theta_{-1} \theta_{-1,1}}\left|\theta_{-1} \theta^{\prime} x_{1}\right|,
$$

if we make use of the following lemma concerning determinants: 
If $D=\left\|a_{l m}\right\|(l, m=1, \ldots n)$ is any determinant of the $n^{\text {th }}$ order, and we write

then

$$
a_{r+1, s}-\frac{a_{r+1,1} a_{r, s}}{a_{r, 1}}=A_{r, s}
$$

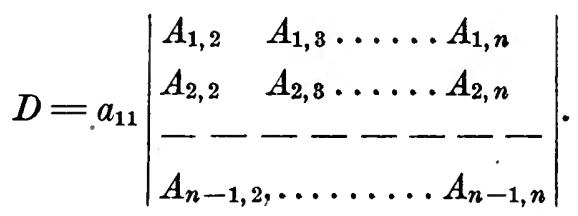

Hence the coordinates of $N_{-1,2}$ can be taken in the form

$$
x_{-1,2}=\left|\begin{array}{lll}
\theta_{-1} & \theta^{\prime} & x_{1}
\end{array}\right| \text {. }
$$

From the symmetry of this expression it follows that $N_{-1,2}$ is also a Levy transform of $N_{-1,1}^{\prime}$, which shows that it is the derived net of $N$ for the functions $\theta$ and $\theta^{\prime}$. Evidently the derived net of $N_{1}$ for $\theta_{1}$ and $\theta_{1}^{\prime}$ and of $N_{-1}$ for $\theta_{-1}$ and $\theta_{-1}^{\prime}$ are given by

$$
x_{0,2}=\left|\begin{array}{lll}
\theta & \theta_{1}^{\prime} & x_{2}
\end{array}\right|, \quad x_{-2,2}=\left|\theta_{-2} \theta_{-1}^{\prime} x\right| \text {. }
$$

Since $N_{-1,2}$ and $N_{0,2}$ are the Levy transforms of $N_{0,1}$ determined by $\theta_{0,1}$ they are Laplace transforms of one another; similarly $N_{-2,2}$ and $N_{-1,2}$ are the Levy transforms of $N_{-1,1}$ determined by $\theta_{-1,1}$. Hence the solutions $\theta$ and $\theta^{\prime}$ determine a Laplace sequence of nets $N_{r, 2}$ which are derived nets of the nets $N_{r+1}$, and a first Levy sequence of the nets $N_{r, 1}$. The coordinates of $N_{r, 2}$ are of the form

$$
x_{r, 2}=\left|\theta_{r} \theta_{r+1}^{\prime} x_{r+2}\right|,
$$

for $r$ positive and negative, and where $N_{0}=N$.

If $\theta^{\prime \prime}$ is another solution of (1) independent $\theta, \theta^{\prime}$ and the coordinates of $N$, the functions

$$
\theta_{-1,1}^{\prime}=\theta^{\prime}-\frac{\theta}{\theta_{-1}} \theta_{-1}^{\prime}, \quad \theta_{-1,1}^{\prime \prime}=\theta^{\prime \prime}-\frac{\theta}{\theta_{-1}} \theta_{-1}^{\prime \prime},
$$

solutions of the point equation of $N_{-1,1}$, determine a derived net of $N_{-1,1}$. Analogously to (49) we have that the coordinates of this net are of the form

$$
\left|\theta_{-2,1}^{\prime} \theta_{-1,1}^{\prime \prime} x_{0,1}\right| \text {. }
$$


Applying the lemma to this case, we have that the coordinates of the net may be taken in the form

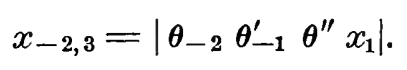

Hence the functions $\theta, \theta^{\prime}$ and $\theta^{\prime \prime}$ determine a sequence of Laplace whose coordinates are of the form

$$
x_{r, 3}=\left|\theta_{r} \theta_{r+1}^{\prime} \theta_{r+2}^{\prime \prime} x_{r+3}\right| .
$$

We remark that from the symmetry of (52) it follows that the net $N_{-2,3}$ is a derived net also of the Levy transforms $N_{-1,1}^{\prime}$ and $N_{-1,1}^{\prime \prime}$ determined by $\theta^{\prime}$ and $\theta^{\prime \prime}$ respectively.

In general $m$ independent solutions $\theta, \theta^{\prime}, \ldots \theta^{(m-1)}$ of (1) determine a Levy sequence of order $m$ whose coordinates are of the form

$$
x_{r, m}=\left|\theta_{r} \theta_{r+1}^{\prime} \theta_{r+2}^{\prime \prime} \ldots \theta_{r+m-1}^{(m-1)}, \quad x_{r+m}\right|,
$$

for positive and negative values of $r^{21}$ ).

36. Periodic Levy sequences. Suppose that we have a Laplace sequence of period $p$. We seek under what condition the Levy sequence of the first order determined by a solution $\theta$ of (1) is also of period $p$. It is necessary that $x_{p, 1}=\lambda x_{0,1}$, where $\lambda$ is at most a function of $u$ and $v$. In consequence of (40) and (45) this condition is equivalent to

$$
x_{p+1}-\frac{\theta_{p+1}}{\theta_{p}} x_{p}=\lambda\left(x_{1}-\frac{\theta_{1}}{\theta} x\right) .
$$

From (19), (25) and (29) we have

$$
x_{p+1}=m x_{1}, \quad \theta_{p+1}=\frac{\partial \theta_{p}}{\partial v}-\frac{\partial}{\partial v} \log a \cdot \theta_{p} .
$$

Consequently from (55) it follows that

$$
\lambda=m, \quad \frac{\partial}{\partial v} \log \frac{\theta_{p}}{\theta}=0 .
$$

${ }^{21)}$ Cf. Hammond, l.c., p. 252. 
In like manner the condition $x_{p-1,1}=\mu x_{-1,1}$ is equivalent in consequence of (21) and (32) to

$$
\mu=m, \quad \frac{\partial}{\partial u} \log \frac{\theta_{p}}{\theta}=0 .
$$

Hence we must have

$$
\theta_{p}=m_{1} \theta,
$$

where $m_{1}$ is a constant. We have seen that equation (1) admits solutions of this type. If we have such a solution, the Laplace transforms of $\theta$ satisfy equations (33) and (34) with $m$ replaced by $m_{1}$. Hence we have

$$
x_{p-i, 1}=x_{p-i+1}-\frac{\theta_{p-i+1}}{\theta_{p-i}} x_{p-i}=m H \ldots H_{p-i} x_{-i, 1} .
$$

Therefore:

If $N$ is a net of period $p$ in ( $p-1)$-space such that $x_{p}=m x$ and $\theta$ is a solution of the point equation of $N$ such that $\theta_{p}=m_{1} \theta$, where $m_{1}$ is a constant, the Levy sequence determined by $\theta$ is of period $p$; when $m_{1}=m, \theta$ is necessarily a linear function of the $x$ 's and the nets of the Levy sequence lie in ( $p-2)$-space.

The latter part of the theorem is a consequence of the last theorem of $\S 33$.

If we take two solutions $\theta$ and $\theta^{\prime}$ of (1) satisfying the conditions $\theta_{p}=m_{1} \theta, \theta_{p}^{\prime}=m_{2} \theta^{\prime}$, the first Levy sequences determined by $\theta$ and $\theta^{\prime}$ are periodic. Also in consequence of (56) and (33) we have from (51)

$$
\begin{aligned}
x_{p, 2} & =m m_{1} m_{2} x_{o, 2}, \\
x_{p-i, 2} & =m m_{1} m_{2}\left(H H_{1} \ldots H_{p-i-1}\right)^{3} H_{p-i}^{2} H_{p-i+1} x_{-i, 2} .
\end{aligned}
$$

Consequently the second Levy sequence determined by $\theta$ and $\theta^{\prime}$ is of period $p$.

Similar results hold for the Levy sequences of higher order. Consider in particular, the case of the Levy sequence of order $p$ determined by the $p$ independent solutions $\theta, \theta^{\prime}, \ldots \theta^{(p-1)}$ of (1) for which $\theta_{p}^{(i)}=m_{1} \theta^{(i)}(i=0, \ldots p-1)$. From (54) we have

$$
x_{o, p}=\left|\begin{array}{llll}
\theta & \theta_{1}^{\prime} & \theta_{2}^{\prime \prime} \ldots & \theta_{p-1}^{(p-1)} x_{p}
\end{array}\right|=\left(m-m_{1}\right)\left|\theta \theta_{1}^{\prime} \ldots \theta_{p-1}^{(p-1)}\right| x .
$$

Similar results hold for $x_{r, p}$. Hence: 
If $N$ is a net of period $p$ in $(p-1)$-space, such that $x_{p}=m x$ and $\theta, \theta^{\prime}, \theta^{\prime \prime}, \ldots \theta^{(p-1)}$ are $p$ independent solutions of the point equation of $N$ such that $\theta_{p}^{(i)}=m_{1} \theta^{(i)}(i=o, \ldots p-1)$, the $p^{\text {th }}$ Levy sequence coincides with the given sequence $\left.{ }^{2 \mathrm{z}}\right)$.

37. Transformations $F$ in homogeneous coordinates. When two nets $N$ and $N_{1}$ are in the relation $F$, the tangents to the curves $v=$ const. at corresponding points $M$ and $M_{1}$ meet in the focal points of a congruence harmonic to both $N$ and $N_{1}(\S 17)$. In accordance with the first theorem of $\S 33$ the coordinates of $N$ and $N_{1}$ can be chosen so that we have

$$
\frac{\partial x_{1}}{\partial u}=\tau_{0} \frac{\partial x}{\partial u}, \quad \frac{\partial x_{1}}{\partial v}=\sigma_{0} \frac{\partial x}{\partial v},
$$

where $\tau_{0}$ and $\sigma_{0}$ are functions of $u$ and $v$. Hence the equations of any transformation $F$ can be given this form. As previously remarked, in this case $c=0$ in (1) and likewise $c_{1}=0$ in the point equation of $N_{1}$.

When $c \neq 0$ in (1), the equation can be reduced to this special form by replacing $x$ by $x \theta$, where $\theta$ is any solution of (1). Hence when the point equation of the net $N$ has the general form (1), the equations of a transformation $F$ are

$$
\frac{\partial x_{1}}{\partial u}=\boldsymbol{\tau} \frac{\partial}{\partial u}\left(\frac{x}{\theta}\right), \frac{\partial x_{1}}{\partial v}=\sigma \frac{\partial}{\partial v}\left(\frac{x}{\theta}\right) .
$$

In order that the conditions of integrability of (60) be satisfied for any solutions $x$ and $\theta$ of (1), it is necessary and sufficient that $\tau$ and $\sigma$ be solutions of

$$
\frac{\partial \boldsymbol{x}}{\partial v}=(\boldsymbol{x}-\sigma) \frac{\partial}{\partial v} \log \frac{\theta}{a}, \frac{\partial \sigma}{\partial u}=(\sigma-\tau) \frac{\partial}{\partial u} \log \frac{\theta}{b},
$$

or in other form

(62) $\frac{\partial}{\partial v} \log \frac{\tau a}{\theta}=\frac{\sigma}{\tau} \frac{\partial}{\partial v} \log \frac{a}{\theta}, \frac{\partial}{\partial u} \log \frac{\sigma b}{\theta}=\frac{\tau}{\sigma} \frac{\partial}{\partial u} \log \frac{b}{\theta}$.

22) Cf. Hammond, l. c., p. 256. 
If we put

$$
x-\sigma=\varphi \theta,
$$

we get equations ( $\Pi, 16$ ), and find that $\varphi$ is a solution of the adjoint of (1), which is denoted by (cf. $\S 31$ )

$$
\left[\varphi ; \frac{1}{a}, \frac{1}{b}, \frac{1}{a b}\right] .
$$

Suppose we have any pair of solutions of (61), and the net $N_{1}$ whose coordinates $x_{1}$ are given by the corresponding equations (60). The points $F_{1}$ and $F_{2}$ whose coordinates $y$ and $z$ are of the forms

$$
y=x_{1}-\tau \frac{x}{\theta}, \quad z=x_{1}-\sigma \frac{x}{\theta},
$$

lie on the line joining corresponding points of the nets $N$ and $N_{1}$. Moreover, as $u$, or $v$, varies the point $F_{1}$, or $F_{2}$, moves tangentially to this line. Hence $F_{1}$ and $F_{2}$ are the focal points of the congruences of these lines, and $N$ and $N_{1}$ are in relation $F$.

With the aid of (61) we show that the point equation of $N_{1}$ is

$$
\frac{\partial^{2} \theta_{1}}{\partial u \partial v}=\frac{\partial}{\partial v} \log \frac{\tau a}{\theta} \frac{\partial \theta_{1}}{\partial u}+\frac{\partial}{\partial u} \log \frac{\sigma b}{\theta} \frac{\partial \theta_{1}}{\partial v} .
$$

When we put $\theta=1$ in (61), we get the conditions of integrability of (59), namely

$$
\frac{\partial \tau_{0}}{\partial v}=\left(\sigma_{0}-\tau_{0}\right) \frac{\partial}{\partial v} \log a, \quad \frac{\partial \sigma_{0}}{\partial u}=\left(\tau_{0}-\sigma_{0}\right) \frac{\partial}{\partial u} \log b .
$$

As a consequence of these results we have:

Whenever the homogeneous coordinates of two nets are in either of the relations (59) or (60), the nets are in relation $F$.

From the manner in which equations (59) were obtained it is evident that the transformations $F$ obtained by taking all possible solutions of (67) possess the property that all the corresponding tangent planes of the nets pass through the line of the congruence whose focal points have the coordinates $\frac{\partial x}{\partial u}$ and $\frac{\partial x}{\partial v}$. Hence in 
order to obtain general transformations $F$, especially in dealing with two such transformations, it is desirable to take the equations in the form (60). However, when a solution of (1) is known, we can write (60) in the form (59), and then the analysis of the transformations $F$ in homogeneous coordinates is the same as that of parallel nets in cartesian coordinates.

38. Transformations $F$ with the same conjugate congruence. Triads of nets. Suppose we have a transformation in the form (59), so that the new net $N^{\prime}\left(x^{\prime}\right)$ is given by

$$
\frac{\partial x^{\prime}}{\partial u}=\tau_{0} \frac{\partial x}{\partial u}, \quad \frac{\partial x^{\prime}}{\partial v}=\sigma_{0} \frac{\partial x}{\partial v}
$$

Now the point equation of $N$ is necessarily of the form (1) with $c=0$. If $\theta$ is any solution of this equation and $\theta^{\prime}$ is given by the quadratures

$$
\frac{\partial \theta^{\prime}}{\partial u}=\tau_{0} \frac{\partial \theta}{\partial u}, \quad \frac{\partial \theta^{\prime}}{\partial v}=\sigma_{0} \frac{\partial \theta}{\partial v},
$$

then the functions $x_{1}$, defined by

$$
x_{1}=x-\frac{\theta}{\theta^{\prime}} x^{\prime}
$$

are the homogeneous coordinates of a net $N_{1}$, since

$$
\left\{\begin{array}{l}
\frac{\partial}{\partial u}\left(\frac{x_{1} \theta^{\prime}}{\theta}\right)=\left(\theta^{\prime}-\tau_{0} \theta\right) \frac{\partial}{\partial u}\left(\frac{x}{\theta}\right), \\
\frac{\partial}{\partial v}\left(\frac{x_{1} \theta^{\prime}}{\theta}\right)=\left(\theta^{\prime}-\sigma_{0} \theta\right) \frac{\partial}{\partial v}\left(\frac{x}{\theta}\right) .
\end{array}\right.
$$

From the form of (70) it follows that corresponding points $M$, $M^{\prime}, M_{1}$ on the three nets $N, N^{\prime}, N_{1}$ are collinear. Hence not only is $N_{1}$ an $F$ transform of $N$, but also of $N^{\prime}$. In the latter respect it differs from the case of $\S 15$ (cf.III, Ex. 24).

As an application of the foregoing results we prove the theorem ${ }^{\mathbf{2 3}}$ ):

${ }^{23}$ ) This theorem for 3-space is due to Ribaucour, Comptes Rendus, vol. 74 (1872), p. 1491. 
If a net $N$ lies on a hyperquadric

$$
\sum_{i, k} a_{i k} x^{(i)} x^{(k)}=0,
$$

each congruence conjugate to $N$ meets the hyperquadric again in a net, which consequently is an $F$ transform of $N$.

We assume that the given conjugate congruence is conjugate to a net $N^{\prime}$ whose coordinates are given by (68). From the above equation we have by differentiation

$$
\sum a_{i k}\left(\frac{\partial x^{(i)}}{\partial u} \frac{\partial x^{(k)}}{\partial v}+\frac{\partial x^{(i)}}{\partial v} \frac{\partial x^{(k)}}{\partial u}\right)=0
$$

since the coordinates $x$ satisfy (1) with $c=0$. In consequence of this result it follows readily that $\theta$ given by

$$
\theta=\sum a_{i k}\left(x^{(i)} x^{\prime(k)}+x^{(k)} x^{\prime(i)}\right)
$$

is a solution of (1), and that $\theta^{\prime}$ where

$$
\theta^{\prime}=\sum a_{i k} x^{\prime(i)} x^{\prime(k)}
$$

satisfies (69). If $N_{1}$ denotes the corresponding transform of $N$ with the equations (70), it is found that $N_{1}$ lies on the quadric.

If $\tau_{1}, \sigma_{1}$ and $x_{2}, \sigma_{2}$ are two sets of solutions of (61) for the same $\theta$, by means of equations of the form (60), we get by quadratures two nets, $N_{1}$ and $N_{2}$, transforms of $N$. From their equations we find

$$
\frac{\partial x_{2}}{\partial u}=\frac{\tau_{2}}{\tau_{1}} \frac{\partial x_{1}}{\partial u}, \quad \frac{\partial x_{2}}{\partial v}=\frac{\sigma_{2}}{\sigma_{1}} \frac{\partial x_{1}}{\partial v} .
$$

Consequently $N_{1}$ and $N_{2}$ are in relation $F$, and form with $N$ a triad $(\S 20)$.

39. Theorem of permutability. In view of the remarks of the preceding section it follows that the results of $\S \S 20,21$ can be translated at once into analogous forms for transformations $F$ in homogeneous coordinates.

Suppose then that we have two solutions $\theta_{1}, \theta_{2}$ of (1) and two solutions $\varphi_{1}, \varphi_{2}$ of the adjoint of (1), so that by quadratures 
of the form (II, 16) two pairs of functions $\tau_{1}, \sigma_{1} ; \tau_{2}, \sigma_{2}$ are to be found. Transforms $N_{1}\left(x_{1}\right)$ and $N_{2}\left(x_{2}\right)$ are given by

$$
\frac{\partial x_{i}}{\partial u}=\boldsymbol{r}_{i} \frac{\partial}{\partial u}\left(\frac{x}{\theta_{i}}\right), \frac{\partial x_{i}}{\partial v}=\sigma_{i} \frac{\partial}{\partial v}\left(\frac{x}{\theta_{i}}\right), \quad(i=1,2) .
$$

Solutions of the point equations of $N_{1}$ and $N_{2}$ are given by

$$
\frac{\partial \theta_{i j}}{\partial u}=\tau_{i} \frac{\partial}{\partial u}\left(\frac{\theta_{j}}{\theta_{i}}\right), \quad \frac{\partial \theta_{i j}}{\partial v}=\sigma_{i} \frac{\partial}{\partial v}\left(\frac{\theta_{j}}{\theta_{i}}\right) \quad\left(\begin{array}{l}
i=1,2, i \neq j) \\
j=1,2,
\end{array}\right)
$$

The coordinates of the transform $N_{12}\left(x_{12}\right)$ are given by (it being understood that $N_{12}$ and $N_{21}$ are the same net)

$$
\frac{\partial x_{i j}}{\partial u}=\tau_{i j} \frac{\partial}{\partial u}\left(\frac{x_{i}}{\theta_{i j}}\right), \frac{\partial x_{i j}}{\partial v}=\sigma_{i j} \frac{\partial}{\partial v}\left(\frac{x_{i}}{\theta_{i j}}\right)\left(\begin{array}{l}
i=1,2, \\
j=1,2,
\end{array} ; \neq j\right)
$$

Comparing these results with (60), (71) and with (II, 21, 37) we have from (II, 52, 49)

and

$$
\left\{\begin{aligned}
\tau_{1} \tau_{12}=\tau_{2} \tau_{21} & =\frac{\theta_{2} \theta_{21} \tau_{1}}{\theta_{1}}+\frac{\theta_{1} \theta_{12} \tau_{2}}{\theta_{2}}-\theta_{12} \theta_{21} \\
\sigma_{1} \sigma_{12}=\sigma_{2} \sigma_{21} & =\frac{\theta_{2} \theta_{21} \sigma_{1}}{\theta_{1}}+\frac{\theta_{1} \theta_{12} \sigma_{2}}{\theta_{2}}-\theta_{12} \theta_{21} \\
\varphi_{12} & =\frac{\theta_{1}}{\theta_{2} \sigma_{1} \tau_{1}}\left(\theta_{21} \theta_{2} \varphi_{1}+\sigma_{1} \tau_{2}-\sigma_{2} \tau_{1}\right)
\end{aligned}\right.
$$

$$
\left.\theta_{1} \theta_{12} x_{12}=\theta_{2} \theta_{21} x_{1}+\theta_{1} \theta_{12} x_{2}-\theta_{12} \theta_{21} x^{24}\right) \text {. }
$$

\section{Exercises.}

1. The homogeneous point coordinates of any point of the tangent plane to a surface are expressible in the form

$$
\lambda x+\mu \frac{\partial x}{\partial u}+\nu \frac{\partial x}{\partial v} .
$$

2. Show that equations (3) hold for the special homogeneous coordinates such that $y^{4} / y^{n+1}$ and $z^{4} / z^{n+1}$ are cartesian coordinates of the foci; also that if the direction-parameters of the congruence are taken in the form

24) In making this comparison it must be noted that the quantities $x_{i} \theta_{i}^{\prime} / \theta_{i}$, $\theta_{i j} \theta_{i}^{\prime} / \theta_{i}, x_{12} \theta_{12}^{\prime \prime \prime} / \theta_{12}$ of Chapter II must be replaced by $-x_{i},-\theta_{i j},-x_{12}$ respectively in order to conform to the usages of the present chapter. 
we have

$$
X^{i}=\frac{y^{4}}{y^{n+1}}-\frac{z^{i}}{z^{n+1}}
$$

$$
\frac{\partial^{2} X}{\partial u \partial v}+\frac{\partial \log y^{n+1}}{\partial v} \frac{\partial X}{\partial u}+\frac{\partial \log z^{n+1}}{\partial u} \frac{\partial X}{\partial v}+C X=0 .
$$

3. The invariants of the Laplace transforms $N_{1}$ and $N_{-i}$ of a net $N$ are given by

$$
\begin{aligned}
& H_{1}=2 H-K-\frac{\partial^{2}}{\partial u \partial v} \log H, \quad K_{1}=H ; \\
& H_{-1}=K, \quad K_{-1}=2 K-H-\frac{\partial^{2}}{\partial u \partial v} \log K .
\end{aligned}
$$

Darboux, Leçons, vol. 2, p. 28.

4. The invariants of the Laplace transforms $N_{r}$ and $N_{-r}$ of a net $N$ are given by

$$
\begin{gathered}
H_{r}=H_{r-1}+H-K-\frac{\partial^{2}}{\partial u \partial v} \log H \ldots H_{r-1}, \quad K_{r}=H_{r-1} ; \\
H_{-r}=K_{-r+1}, \quad K_{-r}=K_{-r+1}+K-H-\frac{\partial^{2}}{\partial u \partial v} \log K \ldots K_{-r+1 .} \\
\text { Darboux, Leçons, vol. } 2, \text { p. } 30 .
\end{gathered}
$$

5. If $H_{i}=0$ for a Laplace sequence, then

$$
x=A\left(U+\int V \beta d v\right)+A_{1}\left(U^{\prime}+\int V \frac{\partial \beta}{\partial u} d v\right)+\ldots+A_{i}\left(U^{(i)}+\int V \frac{\partial^{i} \beta}{\partial u^{j}} d v\right),
$$

where $\beta, A, \ldots A_{i}$ are determinate functions; $U$ and $V$ are functions of $u$ and $v$ respectively, and $U^{(i)}$ is the $i^{\text {th }}$ derivative of $U$ with respect to $u$.

Darboux, Leçons, vol. 2, p. 33.

6. When an equation of Laplace admits a solution of the form

$$
x=A U+A_{1} U^{\prime}+\ldots+A_{i} U^{(i)},
$$

where the $A^{\prime}$ 's are functions of $u$ and $v$ and $U^{(i)}$ is the $i^{\text {th }}$ derivative with respect to $u$ of a function $U$ of $u$, then $H_{i}=0$.

Darboux, Leçons, vol. 2, p. 35.

7. If $H_{i}=0$ and $K_{-j}=0$ for a sequence of Laplace, the point equation for $N$ admits solutions of the form

$$
x=A U+\ldots+A_{\imath} U^{(i)}+B V+\ldots+B_{j} V^{(j)},
$$

where the $A$ 's and $B$ 's are determinate functions; $U$ and $\nabla$ are arbitrary functions of $u$ and $v$ respectively, and $V^{(i)}$ and $V^{(i)}$ denote $i^{\text {th }}$ derivatives with respect to $u$ and $v$.

Darboux, Leçons, vol. 2, p. 38.

8. A necessary and sufficient condition that the point coordinates of a net can be chosen so that the coordinates of the minus first and first Laplace transforms are $\frac{\partial x}{\partial u}$ and $\frac{\partial x}{\partial v}$ respectively is that the point equation of the net have equal invariants.

9. If the parametric curves on a surface $S$ form a net $N$, the tangents to the curves defined by $A d u^{2}+B d v^{2}=0$ meet the lines joining the points $M_{-1}$ and $M_{1}$ of the Laplace transforms of $N$ in points harmonic to $M_{-1}$ and $M_{1}$. 
10. Show that if a point equation (1) satisfies the conditions (27) and (29), it satisfies also the conditions

$$
K K_{-1} \ldots K_{-p+1}=1, \frac{\partial^{2}}{\partial u \partial v} \log \frac{a^{p}}{b^{p} K^{p-1} K_{-1}^{p-2} \ldots K_{-p+2}}=0 .
$$

11. Show that if equation (1) admits solutions of period $p$, so also does its adjoint equation [cf. $\$ 37$ ].

Hammond, Annals, vol. 22 (1921), p. 260.

12. The point equation of $N_{1,1}$ defined by (43) is denoted by

$$
\left[\theta_{1,1} ; a H \frac{\theta_{2}}{\theta_{1}}, b, \frac{b^{2}}{a \theta_{1}}\right]
$$

This may be obtained from (13) by multiplying the second term by $\theta_{2} / \theta_{1}$, retaining the third term, and multiplying together the third and fourth terms of (13) and dividing by $\theta_{1}$. Show that the same method applied to (1), denoted by $[\theta ; a, b, 1]$ gives (42).

13. Apply the method of the preceding exercise to (20) and obtain for the point equation of $N_{r, 1}$ the following

$$
\left[\theta_{r, 1} ; a H H_{1} \ldots H_{r-1} \frac{\theta_{r+1}}{\theta_{r}}, b, \frac{b^{r+1}}{a^{r} H^{r-1} H_{1}^{r-2} \ldots H_{r-1} \theta_{r}}\right] .
$$

Verify this result directly for $r=2$.

14. The point equation of $N_{-2,1}$ is denoted by

Hammond, l. c. p. 249.

$$
\left[\theta_{-2,1} ; a \frac{\theta_{-1}}{\theta_{-2}}, b K, \frac{a^{2}}{b \theta_{-2}}\right] \text {. }
$$

This may be obtained from (15), by multiplying the second term of the latter by $\theta_{-1} / \theta_{-2}$, retaining the third term and taking for the fourth term the product of the second and fourth of (15) divided by $\theta_{-2}$. Show that (41) is obtained from (1) by the same method.

15. Apply the method of the preceding exercise to (23) and obtain for the point equation of $N_{-(r+1), 1}$

$$
\left[\theta_{-(r+1), 1} ; \frac{d \theta_{-r}}{\theta_{-r-1}}, b K K_{-1} \ldots K_{-r+1}, \frac{a^{r+1}}{b^{r} K^{r-1} \ldots K_{-r+2} \theta_{-r-1}}\right] \text {. }
$$

Hammond, l. c.

16. Show that for the derived net $N_{-s, m}$ for $m>s$ the coordinates are expressible in the form

$$
x_{-s, m}=\left[\frac{\partial^{s} \theta}{\partial u^{s}}, \frac{\partial^{s-1} \theta^{\prime}}{\partial u^{s-1}}, \ldots, \theta^{(s)}, \ldots, \frac{\partial^{m-s-1} \theta^{(m-1)}}{\partial v^{m-s-1}}, \frac{\partial_{x}^{m-s}}{\partial v^{m-s}}\right] .
$$

Tzitzeica, Comptes Rendus, vol. 156 (1913), p. 375.

17. If two nets $N$ and $N_{1}$ are in relation $F$ so also are the nets resulting from a projective transformation of $N$ and $N_{1}$. 
18. Show that equation (66) is denoted by

$$
\left[\theta_{1} ; \frac{a \tau}{\theta}, \frac{b \sigma}{\theta}, \frac{1}{\theta}\right]
$$

19. From (65) we have

$$
\frac{\partial y}{\partial u}=-x \varphi_{-1}, \frac{\partial y}{\partial v}=-(x)_{1} \varphi ; \frac{\partial z}{\partial u}=(x)_{-1} \varphi, \frac{\partial z}{\partial v}=x \varphi_{1},
$$

where $(x)_{1}$ and $(x)_{-1}$ denote coordinates of the first and minus first Laplace transforms of $N$, and $\varphi_{1}$ and $\varphi_{-1}$ are first and minus first Laplace transforms of $\varphi$ with respect to (64). Show that the coordinates of the first Laplace transform of $F_{1}$ and the minus first of $F_{2}$ are of the forms

$$
(x)_{1} \varphi+y \frac{\partial}{\partial v} \log a \varphi_{-1}, \quad(x)_{-1} \varphi-z \frac{\partial}{\partial u} \log b \varphi_{1} .
$$

20. If $N$ and $N_{1}$ are nets in relation $F$, the lines joining corresponding points of their first Laplace transforms (minus first Laplace transforms) meet the corresponding lines of the conjugate congruence of the transformation in the focal points of the first (second) order (cf. Ex. 19).

21. When two nets $N$ and $N_{1}$ are in relation $F$, so also are their Laplace transforms of the same orders; the equations of these transformations are

$$
\frac{\partial}{\partial u}\left(x_{1}\right)_{r}=\tau_{r} \frac{\partial}{\partial u}\left(\frac{(x)_{r}}{\theta_{r}}\right), \quad \frac{\partial}{\partial v}\left(x_{1}\right)_{r}=\sigma_{r} \frac{\partial}{\partial v}\left(\frac{(x)_{r}}{\theta_{r}}\right)
$$

where

$$
\tau_{r}=\tau_{r-1}+\frac{\theta_{r} \varphi_{-r}}{H H_{1} \ldots H_{r-1}}, \sigma_{r}=\tau_{r-1}
$$

for $r$ positive.

Hammond, 1. c., p. 260.

22. If the coordinates of a net $N$ of period $p$ satisfy (25), and $\theta$ is a solution of its point equation (1) such that $\theta_{p}=m_{1} \theta$ and $\varphi$ is a solution of the adjoint (64) of (1) such that $\varphi=n \varphi_{-p}$, where $m_{1}$ and $n$ are constants, the $F$ transform of $N$ by means of $\theta$ and $\varphi$ is a periodic net of the same period as $N$.

Hammond, l. c., p. 261.

23. If $N(x)$ and $N_{1}\left(x_{1}\right)$ are nets in relation $F$ on the hyperquadric $\sum_{i, k} a_{i k} x^{(i)} x^{(k)}=0$, then $\sum_{i, k} a_{i k}\left(x^{(i)} x_{1}^{(k)}+x^{(k)} x_{1}^{(i)}\right)=k$, where $k$ is a constant. When $k=0$, the congruence of the transformation consists of generdtors of the hyperquadric [cf. § 38].

24. Show that if corresponding points of three nets in relation $\boldsymbol{F}$ are collinear the relation between their coordinates may be put in the form (70).

25. Show that for a net with the point equation (1) with $a=b=\sqrt{\rho}$ the equations of a transformation $K(\S 25)$ are

$$
\frac{\partial x_{1}}{\partial u}=\frac{\theta^{2}}{\rho} \frac{\partial}{\partial u}\left(\frac{x}{\theta}\right), \quad \frac{\partial x_{1}}{\partial v}=-\frac{\theta^{2}}{\rho} \frac{\partial}{\partial v}\left(\frac{x}{\theta}\right) .
$$

26. Let $N$ be a net in 3-space and $N_{1}$ an $F$ transform of $N$ given by (60). Let corresponding points $M$ and $M_{1}$ of these nets and the two focal points $F_{1}$ 
and $F_{2}$ of the harmonic congruence of the transformation be taken as the vertices of a tetrahedron of reference of homogeneous coordinates $x, y, z, w$ such

$$
M(0,1,0,0), \quad M_{1}(0,0,0,1), \quad F_{1}(1,0.0,0), \quad F_{2}(0,0,1,0) .
$$

Show that the pencil of conics tangent to the lines $M F_{1}$ and $M F_{2}$ at the points $(M)_{-1}$ and $(M)_{1}$ of the Laplace transforms $(N)_{-1}$ and $(N)_{1}$ of $N$ are given by

$$
2 \lambda x z=\left(\frac{\partial}{\partial u} \log \frac{\theta}{b} \cdot x-\frac{y}{\theta}+\frac{\partial}{\partial v} \log \frac{\theta}{a} \cdot z\right)^{2}
$$

where $\lambda$ is a parameter; also that according as $\lambda$ is $K$, or $H$, the conic osculates the curve $u=$ const. of $(N)_{-1}$ at $(M)_{-1}$ or $v=$ const. of $(N)_{1}$ at $(M)_{1}$.

Annals, vol. 18 (1916), p. 11.

27. A necessary and sufficient condition that a conic of the pencil of Ex. 26 osculate the curve $u=$ const. at $(M)_{-1}$ and $v=$ const. at $(M)_{1}$ is that $N$ have equal point invariants.

Darboux, Lecons, vol. 4, p. 38.

28. Show that the pencil of conics tangent to the lines $M_{1} F_{1}$ and $M_{1} F_{2}$ at the points of the Laplace transforms of $N_{1}$ are defined, in the coordinates of Ex. 26, by

$$
2 x z=\lambda_{1}\left(\frac{x}{\sigma} \frac{\partial}{\partial u} \log \frac{b}{\theta}+\frac{z}{\tau} \frac{\partial}{\partial v} \cdot \log \frac{a}{\theta}+\frac{w}{\theta^{2}}\right)_{;}^{2}
$$

that this pencil and the pencil of Ex. 26 determine involutions on the line $F_{1} F_{2}$; and that a necessary and sufficient condition that the two involutions be identical is that the transformation $F$ be $K$.

Annals, l. c., p. 12.

29. If $N$ and $N_{1}$ are two nets in relation $K$, any two conics of the two pencils of Exs. 26 and 28, meeting on the line $F_{1} F_{2}$ determine a pencil of quadrics which cut the line $M M_{1}$ in an involution whose double points are the focal points of this line for the congruence of these lines, and the two cones of the pencil are the quadrics tangent to $M M_{1}$ at these focal points.

Annals, l. c. p. 15 .

30. If $N$ and $N_{1}$ are nets in relation $K$, the doubly osculating conics of the pencils of Exs. 26 and 28 meet on the line $F_{1} F_{2}$, and the vertices of the two cones in the pencil of quadrics determined by these conics are the corresponding focal points of the conjugate congruence of the transformation.

Tzitzeica, Comptes Rendus, vol. 147 (1908), p. 1036; also Annals, l. c., p. 16. 
Chapter IV.

\section{Surfaces and congruences in 3-space.}

40. Nets in 2-space. It is evident that any three functions of two parameters, $u$ and $v$, satisfy an equation of the form

$$
\frac{\partial^{2} \theta}{\partial u \partial v}=\frac{\partial \log a}{\partial v} \frac{\partial \theta}{\partial u}+\frac{\partial \log b}{\partial u} \frac{\partial \theta}{\partial v}+c \theta .
$$

Consequently any two families of curves in 2-space form a net. It is likewise true that we can find two equations of the form

$$
\left\{\begin{array}{l}
\frac{\partial^{2} \theta}{\partial u^{2}}=a_{1} \frac{\partial \theta}{\partial u}+b_{1} \frac{\partial \theta}{\partial v}+c_{1} \theta, \\
\frac{\partial^{2} \theta}{\partial v^{2}}=a_{2} \frac{\partial \theta}{\partial u}+b_{2} \frac{\partial \theta}{\partial v}+c_{2} \theta .
\end{array} .\right.
$$

which the three given functions satisfy.

Conversely, we seek the conditions which must be satisfied by the coefficients in (1) and (2) in order that they admit three linearly independent solutions. To this end the following conditions of integrability must be satisfied:

$$
\frac{\partial}{\partial v}\left(\frac{\partial^{2} \theta}{\partial u^{2}}\right)=\frac{\partial}{\partial u}\left(\frac{\partial^{2} \theta}{\partial u \partial v}\right), \quad \frac{\partial}{\partial v}\left(\frac{\partial^{2} \theta}{\partial u \partial v}\right)=\frac{\partial}{\partial u}\left(\frac{\partial^{2} \theta}{\partial v^{2}}\right) .
$$

Reducing the resulting expressions by means of (1) and (2), we get two equations of the form

(3) $A_{1} \frac{\partial \theta}{\partial u}+B_{1} \frac{\partial \theta}{\partial v}+C_{1} \theta=0, \quad A_{2} \frac{\partial \theta}{\partial u}+B_{2} \frac{\partial \theta}{\partial v}+C_{2} \theta=0$,

where $A_{1}, B_{1} \ldots C_{2}$ are determinate functions, which must vanish, if equations (2) are to hold for three linearly independent solutions. Putting them equal to zero, we get the following conditions: 


$$
\left\{\begin{array}{c}
a_{2} b_{1}+\frac{\partial a_{1}}{\partial v}=\frac{\partial \log a}{\partial v} \frac{\partial \log b}{\partial u}+c+\frac{\partial^{2} \log a}{\partial u \partial v} \\
a_{1} \frac{\partial \log b}{\partial u}+b_{1} b_{2}+c_{1}+\frac{\partial b_{1}}{\partial v}=\frac{\partial \log a}{\partial v} b_{1}+\left(\frac{\partial \log b}{\partial u}\right)^{2}+\frac{\partial^{2} \log b}{\partial u^{2}} \\
a_{1} c+b_{1} c_{2}+\frac{\partial c_{1}}{\partial v}=\frac{\partial \log a}{\partial v} c_{1}+\frac{\partial \log b}{\partial u} c+\frac{\partial c}{\partial u} \\
a_{2} b_{1}+\frac{\partial b_{2}}{\partial u}=\frac{\partial \log a}{\partial v} \frac{\partial \log b}{\partial u}+c+\frac{\partial^{2} \log b}{\partial u \partial v} \\
b_{2} \frac{\partial \log a}{\partial v}+a_{1} a_{2}+c_{2}+\frac{\partial a_{2}}{\partial u}=\frac{\partial \log b}{\partial u} a_{2}+\left(\frac{\partial \log a}{\partial v}\right)^{2}+\frac{\partial^{2} \log a}{\partial v^{2}} \\
b_{2} c+a_{2} c_{1}+\frac{\partial c_{2}}{\partial u}=\frac{\partial \log b}{\partial u} c_{2}+\frac{\partial \log a}{\partial v} c+\frac{\partial c}{\partial v}
\end{array}\right.
$$

When these conditions are satisfied, the system (1) and (2) is completely integrable and there are at most three linearly independent solutions. For, the derivatives of the second and higher orders of $\theta$ are linearly expressible in terms of $\frac{\partial \theta}{\partial u}, \frac{\partial \theta}{\partial v}$, and $\theta$. Hence all the integrals are expressible as linear functions, with constant coefficients, of three solutions. Therefore we have the theorem:

When the homogeneous coordinates of a net satisfy equations of the form (2), the net is in 2-space; and all nets whose homogeneous coordinates satisfy the same equations (1) and (2) are projective transforms of one another.

4I. Tangential coordinates of a surface in 3-space. Let $x, y, z, w$ be the homogeneous point coordinates of a surface $S$ in 3-space referred to any system of parametric curves $u=$ const., $v=$ const. Since the tangents to the parameteric curves at a point of the surface lie in the tangent plane to the surface at that point, the equation of the tangent plane must be satisfied not only by $x, y, z$, and $w$, but also by the coordinates of any point on these tangents, that is by expressions of the form

$$
\lambda_{1} x+\mu_{1} \frac{\partial x}{\partial \imath}, \lambda_{2} x+\mu_{2} \frac{\partial x}{\partial v}
$$


for any values of $\lambda_{1}, \mu_{1}, \lambda_{2}$ and $\mu_{2}$. Hence there exist four functions, $X, Y, Z, W$, of $u$ and $v$, the tangential coordinates of the surface, [cf. §67] satisfying identically the three conditions

$$
\left\{\begin{array}{c}
X x+Y y+Z z+W w=0 \\
\sum X \frac{\partial x}{\partial u}=0, \quad \sum X \frac{\partial x}{\partial v}=0
\end{array}\right.
$$

where $\sum$ indicates the sum of four terms obtained by replacing $x$ and $X$ by $y$ and $Y ; z$ and $Z ; w$ and $W$ respectively.

In consequence of the last two we have, on differentiating the first, the two equations

$$
\sum x \frac{\partial X}{\partial u}=0, \quad \sum x \frac{\partial X}{\partial v}=0 .
$$

42. Asymptotic lines. An asymptotic line on a surface is characterized by the property that its osculating plane at a point is tangent to the surface at that point [§55]. Hence along an asymptotic line $\frac{d v}{d u}$ must equal such a function of $u$ and $v$ that the equation of the tangent plane is satisfied by $\lambda x+\mu d x+\nu d^{2} x$, . for all values of $\lambda, \mu$, and $\nu$ [cf. $\S 30$ ]. Hence we must have

$$
\sum X d^{2} x=0
$$

Eliminating $X, Y, Z$, and $W$ from this equation and (5), we have

where

$L=\left|\begin{array}{l}x \frac{\partial x}{\partial u} \frac{\partial x}{\partial v} \frac{\partial^{2} x}{\partial u^{2}} \\ y \frac{\partial y}{\partial u} \frac{\partial y}{\partial v} \frac{\partial^{2} y}{\partial u^{2}} \\ z \frac{\partial z}{\partial u} \frac{\partial z}{\partial v} \frac{\partial^{2} z}{\partial u^{2}} \\ w \frac{\partial w}{\partial u} \frac{\partial w}{\partial v} \frac{\partial^{2} w}{\partial u^{2}}\end{array}\right|,\left|\begin{array}{l}x \frac{\partial x}{\partial u} \frac{\partial x}{\partial v} \frac{\partial^{2} x}{\partial u \partial v} \\ y----\end{array}\right|, N=\left|\begin{array}{l}x \frac{\partial x}{\partial u} \frac{\partial x}{\partial v} \frac{\partial^{2} x}{\partial v^{2}} \\ y---- \\ z-\ldots-\ldots--\end{array}\right|$. 
This is the equation of the asymptotic lines on $S$. We have immediately the theorem [cf. $\S 77$ ]:

$A$ necessary and sufficient condition that four functions $x, y, z, w$ be the homogeneous point coordinates of a surface referred to its asymptotic lines is that $x, y, z, w$ be four linearly independent solutions of two partial differential equations of the form

$$
\left\{\begin{array}{l}
\frac{\partial^{2} \theta}{\partial u^{2}}=a_{1} \frac{\partial \theta}{\partial u}+b_{1} \frac{\partial \theta}{\partial v}+c_{1} \theta \\
\frac{\partial^{2} \theta}{\partial v^{2}}=a_{2} \frac{\partial \theta}{\partial u}+b_{2} \frac{\partial \theta}{\partial v}+c_{2} \theta
\end{array}\right.
$$

We seek now the conditions upon the coefficients of equations (9) so that two equations (9) shall have four linearly independent solutions. It is necessary that the following condition be satisfied:

$$
\frac{\partial^{2}}{\partial v^{2}}\left(\frac{\partial^{2} \theta}{\partial u^{2}}\right)=\frac{\partial^{2}}{\partial u^{2}}\left(\frac{\partial^{2} \theta}{\partial v^{2}}\right)
$$

When the above expressions are substituted, the resulting equation is reducible by means of (9) to an equation of the form

$$
A \frac{\partial^{2} \theta}{\partial u \partial v}+B \frac{\partial \theta}{\partial u}+C \frac{\partial \theta}{\partial v}+D \theta=0,
$$

where $A, B, C$ and $D$ are determinate functions of the coefficients of (9) and their derivatives. These functions must be equal to zero, otherwise we can have at most three linearly independent solutions of (9) [cf. $\S 40$ ].

Putting them equal to zero, we obtain the four equations of condition

0)

$$
\frac{\partial a_{1}}{\partial v}-\frac{\partial b_{2}}{\partial u}=0
$$

$\frac{\partial^{2} a_{2}}{\partial u^{2}}-\frac{\partial^{2} a_{1}}{\partial v^{2}}+\frac{\partial}{\partial u}\left(a_{1} a_{2}\right)+b_{2} \frac{\partial a_{1}}{\partial v}-a_{2} \frac{\partial b_{1}}{\partial v}-\frac{\partial}{\partial v}\left(a_{2} b_{1}\right)+2 \frac{\partial c_{2}}{\partial u}=0$, $\left\{\begin{array}{l}\frac{\partial^{2} b_{1}}{\partial v^{2}}-\frac{\partial^{2} b_{2}}{\partial u^{2}}+\frac{\partial}{\partial v}\left(b_{1} b_{2}\right)+a_{1} \frac{\partial b_{2}}{\partial u}-b_{1} \frac{\partial a_{2}}{\partial u}-\frac{\partial}{\partial u}\left(a_{2} b_{1}\right)+2 \frac{\partial c_{1}}{\partial v}=0, \\ \frac{\partial^{2} c_{2}}{\partial u^{2}}-\frac{\partial^{2} c_{1}}{\partial v^{2}}+2 c_{1} \frac{\partial a_{2}}{\partial u}+a_{2} \frac{\partial c_{1}}{\partial u}-a_{1} \frac{\partial c_{2}}{\partial u}-2 c_{2} \frac{\partial b_{1}}{\partial v}-b_{1} \frac{\partial c_{2}}{\partial v}+b_{2} \frac{\partial c_{1}}{\partial v}=0 .\end{array}\right.$ 
When these conditions are satisfied, the system is completely integrable, and as all the higher derivatives are expressible linearly in terms of $x, \frac{\partial x}{\partial u}, \frac{\partial x}{\partial v}$ and $\frac{\partial^{2} x}{\partial u \partial v}$, there are four linearly independent solutions, and only four. Hence we have the theorem: $A$ necessary and sufficient condition that a system (9) admit four linearly independent solutions is that the coefficients satisfy (10). All surfaces whose four point coordinates satisfy the same system (9) are projective transforms of one another.

When the surface $S$ is subjected to a polar transformation with respect to the quadric

$$
x^{2}+y^{2}+z^{2}+w^{2}=0,
$$

the point and tangential coordinates of $S$ are tangential and point coordinates respectively of the transform $S^{\prime}$. Since asymptotic lines are transformed into asymptotic lines on $S^{\prime}$ [cf. $\S 84$ ], we have the theorem:

Any four linearly independent solutions of the system (9) are tangential coordinates of a surface referred to its asymptotic lines; all surfaces whose tangential coordinates satisfy the same system of equations (9) are projective transforms of one another.

43. Nets in 3-space. Point coordinates. Consider a surface $S$ referred to any system of curves, $u=$ const., $v=$ const., and upon it a net, or conjugate system. Any point $P$ on a tangent at $M$ to a curve of a family of the net has homogeneous point coordinates of the form

$$
\bar{x}=\lambda x+\mu\left(\frac{\partial x}{\partial u} d u+\frac{\partial x}{\partial v} d v\right) .
$$

A necessary and sufficient condition ( $(2)$ that two families of curves determined by $\frac{d v}{d u}$ and $\frac{\delta v}{\delta u}$ form a net is that, as $M$ moves along the curve of the second family through it, $P$ moves in the tangent plane to the surface at $M$. Hence the point whose coordinates are of the form $\bar{x}+\delta \bar{x}=\bar{x}+\frac{\partial \bar{x}}{\partial u} \delta u+\frac{\partial \bar{x}}{\partial v} \delta v$ must lie in the tangent plane. This gives the equation of condition

$$
X \delta \bar{x}+Y \delta \bar{y}+Z \delta \bar{z}+W \delta \bar{w}=0 .
$$


Combining this equation with the identities (5), we get

$$
L d u \delta u+M(d u \delta u+\delta u d v)+N d v \delta v=0,
$$

where $L, M$ and $N$ are given by (8). This is in keeping with (7) which defines the asymptotic or self-conjugate directions, and could have been inferred directly from (7), since these differential equations in the parameters are independent of the point coordinates and consequently should be equivalent to the similar equations found when cartesian rectangular coordinates are used [cf. $\$ \S 54,55$ ]. As an immediate consequence of these observations and the results of $[\S 56]$ we have:

$A$ necessary and sufficient condition that the curves defined by $R d u^{2}+2 S d u d v+T d v^{2}=0$ form a conjugate system is

$$
R N+T L-2 S M=0 .
$$

From this result, and from (12) also, it follows that a necessary and sufficient condition that the parametric curves form a net is that $\boldsymbol{M}=0$. But from (8) this means merely that $x, y, z$ and $w$ are linearly independent solutions of an equation of the form (1). In this case the equation of the asymptotic lines is of the form

$$
d u^{2}+r d u^{2}=0
$$

where $r=N / L$ is a function of $u$ and $v$. Comparing this equation with (7), we have in consequence of (8),

$$
\left|\begin{array}{cccc}
x & \frac{\partial x}{\partial u} & \frac{\partial x}{\partial v} & r \frac{\partial^{2} x}{\partial u^{2}}-\frac{\partial^{2} x}{\partial v^{2}} \\
y & \\
z &
\end{array}\right|=0 .
$$

Hence we have the theorem:

The homogeneous point coordinates of a net in 3-space are simultaneous solutions of two equations of the form (1) and

$$
\frac{\partial^{2} \theta}{\partial v^{2}}=r \frac{\partial^{2} \theta}{\partial u^{2}}+a^{\prime} \frac{\partial \theta}{\partial u}+b^{\prime} \frac{\partial \theta}{\partial v}+c^{\prime} \theta
$$


Conversely, we shall show that two equations of the form (1) and (15) admit at most four linearly independent solutions. In the first place in order that they admit a common solution it is necessary that they satisfy the condition of integrability

$$
\frac{\partial}{\partial v}\left(\frac{\partial^{2} \theta}{\partial u \partial v}\right)=\frac{\partial}{\partial u}\left(\frac{\partial^{2} \theta}{\partial v^{2}}\right) .
$$

When the expression from (1) and (15) are substituted, the resulting equation is reducible to

$$
\frac{\partial^{3} \theta}{\partial u^{3}}=A_{1} \frac{\partial^{2} \theta}{\partial u^{2}}+B_{1} \frac{\partial \theta}{\partial u}+C_{1} \frac{\partial \theta}{\partial v}+D_{1} \theta,
$$

where

$$
\text { (17) }\left\{\begin{array}{l}
A_{1}=\frac{\partial}{\partial u} \log \frac{b}{r}-\frac{a^{\prime}}{r}, B_{1}=\frac{1}{r}\left(\frac{1}{a} \frac{\partial^{2} a}{\partial v^{2}}+a^{\prime} \frac{\partial}{\partial u} \log \frac{b}{a^{\prime}}-b^{\prime} \frac{\partial \log a}{\partial v}-c^{\prime}\right), \\
C_{1}=\frac{1}{r}\left(K+2 \frac{\partial^{2} \log b}{\partial u \partial v}-\frac{\partial b^{\prime}}{\partial u}\right), \\
D_{1}=\frac{1}{r}\left(c \frac{\partial \log a}{\partial v}+c^{\prime} \frac{\partial \log b}{\partial u}+\frac{\partial c}{\partial v}-c b^{\prime}-\frac{\partial c^{\prime}}{\partial u}\right),
\end{array}\right.
$$

where $K$ is one of the invariants of (1) [cf. I, 44].

From (1) we have also by differentiation

$$
\frac{\partial^{3} \theta}{\partial u^{2} \partial v}=A_{2} \frac{\partial^{2} \theta}{\partial u^{2}}+B_{2} \frac{\partial \theta}{\partial u}+C_{2} \frac{\partial \theta}{\partial v}+D_{2} \theta
$$

where

$\stackrel{(19)}{A_{2}}=\frac{\partial \log a}{\partial v}, B_{2}=H+2 \frac{\partial^{2} \log a}{\partial u \partial v}, C_{2}=\frac{1}{b} \frac{\partial^{2} b}{\partial u^{2}} . D_{2}=c \frac{\partial}{\partial u} \log b c$.

Also from (15) we obtain $\frac{\partial^{3} \theta}{\partial u \partial v^{2}}$ and $\frac{\partial^{3} \theta}{\partial v^{3}}$ expressed linearly in terms of $\theta, \frac{\partial \theta}{\partial u}, \frac{\partial \theta}{\partial v}$ and $\frac{\partial^{2} \theta}{\partial u^{2}}$. There remains the condition

$$
\frac{\partial}{\partial u}\left(\frac{\partial^{3} \theta}{\partial u^{2} \partial v}\right)=\frac{\partial}{\partial v}\left(\frac{\partial^{3} \theta}{\partial u^{3}}\right) .
$$


By means of (16) and (18) this condition is reducible to the form

(20) 、

$$
P \frac{\partial^{2} \theta}{\partial u^{2}}+Q \frac{\partial \theta}{\partial u}+R \frac{\partial \theta}{\partial v}+S \theta=0
$$

where $P, Q, R$ and $S$ are determinate functions. If the coeffieients in (1) and (15) are not such that

$$
P=Q=R=S=0
$$

we have a system to be satisfied similar to (1) and (2), which, as we saw in $\S 40$, admits at most three linearly independent solutions. Hence we must have (21) satisfied, in which case the third and higher derivatives in $\theta$ are linearly expressible in terms of $\theta, \frac{\partial \theta}{\partial u}$, $\frac{\partial \theta}{\partial v}, \frac{\partial^{2} \theta}{\partial u^{2}}$. Since all further conditions of integrability are satisfied, we see that there are at most four linearly independent solutions of a completely integrable system of equations of the type (1) and (15). Accordingly we have the theorem:

The homogeneous point coordinates of a net in 3-space satisfy a system of equations of the form (1) and (15); conversely, a net whose coordinates satisfy such a system lies in 3-space. Any four linearly independent solutions of the same system of equations (1) and (15) are the homogeneous point coordinates of a net projective with the given net.

When the expressions $P, Q, R$ and $S$ in (20) are calculated, it is found that equations (21) reduce to

(22)

$$
\begin{gathered}
\frac{\partial A_{1}}{\partial v}+C_{1} r=\frac{\partial A_{2}}{\partial u}+B_{2} \\
\frac{\partial B_{1}}{\partial v}+A_{1} B_{2}+a^{\prime} C_{1}=\frac{\partial B_{2}}{\partial u}+C_{2} \frac{\partial \log a}{\partial v}+D_{2} \\
\frac{\partial C_{1}}{\partial v}+A_{1} C_{2}+B_{1} \frac{\partial \log b}{\partial u}+b^{\prime} C_{1}+D_{1}=\frac{\partial C_{2}}{\partial u}+A_{2} C_{1}+C_{2} \frac{\partial \log b}{\partial u} \\
\frac{\partial D_{1}}{\partial v}+A_{1} D_{2}+B_{1} c+C_{1} c^{\prime}=\frac{\partial D_{2}}{\partial u}+A_{2} D_{1}+C_{2} c
\end{gathered}
$$


When the point coordinates of a net $N$ are cartesian, we have from (14) and [(40) §55] that $r=D^{\prime \prime} / D$. Consequently by the elimination of $X$ from the first and third of the Gauss equations [(7) § 64] we find that the cartesian coordinates of $N$ satisfy an equation of the form (15) with

$$
a^{\prime}=\left\{\begin{array}{c}
22 \\
1
\end{array}\right\}-r\left\{\begin{array}{c}
11 \\
1
\end{array}\right\} \quad b^{\prime}=\left\{\begin{array}{c}
22 \\
2
\end{array}\right\}-r\left\{\begin{array}{c}
11 \\
2
\end{array}\right\}, \quad c^{\prime}=0 .
$$

From (14) and [§ 82] it follows that a necessary and sufficient condition that $N$ be isothermal-conjugate is that $r=U / V$, where $U$ and $V$ are functions of $u$ and $v$ alone respectively. As a consequence of the preceding theorem, we have:

An isothermal-conjugate net is transformed into an isothermalconjugate net by a projective transformation.

44. Ray congruence and ray curves. Consider a net $N$ in $n$-space, and the system of lines joining corresponding points of the first and minus first Laplace transforms of $N$. If this system of lines is to form a congruence, there must be two points of a line generating curves to which the line is tangent. The coordinates of any point are of the form $\lambda x_{-1}+\mu x_{1}$. Hence it must be possible for $u$ and $v$ to vary in such a way that $d\left(\lambda x_{-1}+\mu x_{1}\right)$ is a linear function of $x_{-1}$ and $x_{1}$. From (III, 2,4) we have

$$
\left\{\begin{array}{l}
\frac{\partial x_{-1}}{\partial u}=\frac{\partial^{2} x}{\partial u^{2}}-\frac{1}{b} \frac{\partial^{2} b}{\partial u^{2}} x-\frac{\partial \log b}{\partial u} x_{-1}, \quad \frac{\partial x_{-1}}{\partial v}=\frac{\partial \log a}{\partial v} x_{-1}+K x, \\
\frac{\partial x_{1}}{\partial u}=\frac{\partial \log b}{\partial u} x_{1}+H x, \quad \frac{\partial x_{1}}{\partial v}=\frac{\partial^{2} x}{\partial v^{2}}-\frac{1}{a} \frac{\partial^{2} a}{\partial v^{2}} x-\frac{\partial \log a}{\partial v} x_{1},
\end{array}\right.
$$

where $H$ and $K$ are the invariants of (1). Expressing the above condition, we get equations of the following form to be satisfied by the coordinates $x$ :

$$
\left\{\begin{array}{c}
\lambda\left[\left(\frac{\partial^{2} x}{\partial u^{2}}-\frac{1}{b} \frac{\partial^{2} b}{\partial u^{2}} x\right) d u+x K d v\right]+\mu\left[x H d u+\left(\frac{\partial^{2} x}{\partial v^{2}}-\frac{1}{a} \frac{\partial^{2} a}{\partial v^{2}} x\right) d v\right] \\
+v\left(\frac{\partial x}{\partial u}-\frac{\partial \log b}{\partial u} x\right)+\sigma\left(\frac{\partial x}{\partial v}-\frac{\partial \log a}{\partial v} x\right)=0
\end{array}\right.
$$

where $\nu$ and $\sigma$ are to be determined. 
Equation (24) is linear in $\frac{\partial^{2} x}{\partial u^{2}}, \frac{\partial^{2} x}{\partial v^{2}}, \frac{\partial x}{\partial u}, \frac{\partial x}{\partial v}$ and $x$. If $n>3$, this equation must be satisfied by five or more linearly independent functions $x$, which is possible only when the coefficients of the quantities $\frac{\partial^{2} x}{\partial u^{2}}, \ldots, x$ are zero. This gives $\lambda d u=\mu d v=0$, which from (24) is seen to be impossible.

When $n=3$, by means of (15), (24) is reducible to an expression linear in $\frac{\partial^{2} x}{\partial u^{2}}, \frac{\partial x}{\partial u}, \frac{\partial x}{\partial v}, x$. The coefficients of the latter expression must then be zero, which gives the four equations of condition

$$
\left\{\begin{array}{c}
\lambda d u+\mu r d v=0, \mu a^{\prime} d v+v=0, \mu b^{\prime} d v+\sigma=0 \\
\lambda\left(\frac{1}{b} \frac{\partial^{2} b}{\partial u^{2}} d u-K d v\right)+\mu\left[-H d u+\left(\frac{1}{a} \frac{\partial^{2} a}{\partial v^{2}}-c^{\prime}\right) d v\right] \\
+v \frac{\partial \log b}{\partial u}+\sigma \frac{\partial \log a}{\partial v}=0
\end{array}\right.
$$

Eliminating $\lambda, \mu, \nu$ and $\sigma$ from these equations, we obtain

$$
H d u^{2}+R d u d v-r K d v^{2}=0
$$

where

$$
R=\frac{r}{b} \frac{\partial^{2} b}{\partial u^{2}}-\frac{1}{a} \frac{\partial^{2} a}{\partial v^{2}}+c^{\prime}+a^{\prime} \frac{\partial \log b}{\partial u}+b^{\prime} \frac{\partial \log a}{\partial v} .
$$

Hence the system of lines forms a congruence. Following Wilczynski ${ }^{25}$ ) we call it the ray congruence of the net $N$, and the curves on the surface of the net defined by (26) the ray curves. Since any one parameter family of lines in a plane has an envelope, the developables of such a congruence for nets in 2-space have no significance. Hence we have the theorem:

The lines joining corresponding points of the first and minus first Laplace transforms of a net form a congruence only when the net lies in 3-space.

From (13), (14) and (26) we have the theorem of Wilczynski $\left.{ }^{26}\right)$ :

25) Transactions of the American Mathematical Society, vol. 16 (1915), p. 318.

26) I. c. p. 319 . 
A necessary and sufficient condition that the ray, curves for a net $N$ form a net is that $N$ have equal point invariants.

In consequence of the fourth theorem of $\S 10$ we have:

The ray congruence of a net with equal point invariants is harmonic to the corresponding net of ray curves.

45. Nets $R$. We determine the condition that the tangents to the curves $v=$ const. of a net $N$ form a $W$ congruence, that is the asymptotic lines on the surface of $N$ and its minus first Laplace transform correspond [§ 172].

From (23) we have by differentiation, making use of (16),

$$
\left\{\begin{aligned}
\frac{\partial^{2} x_{-1}}{\partial u^{2}}= & \left(A_{1}-\frac{\partial \log b}{\partial u}\right) \frac{\partial x_{-1}}{\partial u}+\left(B_{1}-C_{\mathbf{2}}\right) \frac{\partial x}{\partial u}+C_{1} \frac{\partial x}{\partial v} \\
& +\left(A_{1} C_{2}-\frac{\partial C_{2}}{\partial u}+D_{1}\right) x+\left(A_{1} \frac{\partial \log b}{\partial u}-\frac{\partial^{2} \log b}{\partial u^{2}}\right) x_{-1} \\
\frac{\partial^{2} x_{-1}}{\partial v^{2}}= & \frac{\partial \log a}{\partial v} \frac{\partial x_{-1}}{\partial v}+K \frac{\partial x}{\partial v}+\frac{\partial K}{\partial v} x+\frac{\partial^{2} \log a}{\partial v^{2}} x_{-1}
\end{aligned}\right.
$$

If we replace $x$ and $\frac{\partial x}{\partial u}$ by the linear expressions for them obtained from (III, 2,4) and then eliminate $\frac{\partial x}{\partial v}$ from these two equations, we obtain for the net $N_{-1}$ an equation of the form (15), where $r_{-1}$ has the value $K / C_{1}$.

From this result and (14) it follows that a necessary and sufficient condition that the asymptotic lines correspond on the two surfaces is that $C_{1} r=K$, which by (17) is equivalent to

$$
2 \frac{\partial^{2} \log b}{\partial u \partial v}-\frac{\partial b^{\prime}}{\partial u}=0
$$

In like manner a necessary and sufficient condition that the tangents to the curves $u=$ const. of $N$ form a $W$ congruence is

$$
2 \frac{\partial^{2} \log a}{\partial u \partial v}+\frac{\partial}{\partial v}\left(\frac{a^{\prime}}{r}\right)=0
$$


Tzitzeica ${ }^{27}$ ) has defined an $R$ net to be one for which the tangents to the curves of both families form $W$ congruences. Equations (29) and (30) are the analytical condition that $N$ be an $R$ net. When these conditions are satisfied, the first of (22) reduces to $\frac{\partial^{2}}{\partial u \partial v} \log r=0$. Hence we have the theorem of Tzitzeica:

An $R$ net is isothermal-conjugate.

By means of the first of equations (22) we establish the converse theorem due to Demoulin ${ }^{28}$ ):

If the tangents to the curves in either family of an isothermalconjugate net form a $W$ congruence, it is an $R$ net.

Since an $R$ net is isothermal-conjugate, the parameters can be chosen so that $r=-1$. Since $a$ and $b$ in (1) are determined only to within factors, which are functions of $u$ and $v$ respectively, these can be chosen so that (29) and (30) may be replaced by

$$
b^{\prime}=2 \frac{\partial \log b}{\partial v}, \quad a^{\prime}=2 \frac{\partial \log a}{\partial u} .
$$

Hence:

The two differential equations satisfied by the homogeneous point coordinates of an $R$ net are reducible to the form

$$
\left\{\begin{array}{c}
\frac{\partial^{2} \theta}{\partial u \partial v}=\frac{\partial \log a}{\partial v} \frac{\partial \theta}{\partial u}+\frac{\partial \log b}{\partial u} \frac{\partial \theta}{\partial v}+c \theta \\
\frac{\partial^{2} \theta}{\partial v^{2}}+\frac{\partial^{2} \theta}{\partial u^{2}}=2 \frac{\partial \log a}{\partial u} \frac{\partial \theta}{\partial u}+2 \frac{\partial \log b}{\partial v} \frac{\partial \theta}{\partial v}+c^{\prime} \theta
\end{array}\right.
$$

We return to the consideration of the net $N_{-1}$. From (23) we have by differentiation

$$
\left\{\begin{array}{c}
\frac{\partial^{2} x_{-1}}{\partial u \partial v}=\frac{\partial \log a}{\partial v} \frac{\partial x_{-1}}{\partial u}+\frac{\partial}{\partial u} \log K b \frac{\partial x_{-1}}{\partial v} \\
+\left(\frac{\partial^{2} \log \frac{a}{b}}{\partial u \partial v}-\frac{\partial \log K}{\partial u} \frac{\partial \log a}{\partial v}+c\right) x_{-1} .
\end{array}\right.
$$

27) Comptes Rendus, vol. 152 (1911), p. 1077.

${ }^{28}$ ) Comptes Rendus, vol. 153 (1911), p. 592. 
Since $r=-1$, it follows from (17) that $C_{1}=-K$. Making use of (III, 2,4$)$ and the third of $(22)$, we find from (28)

$$
\left\{\begin{array}{c}
\frac{\partial^{2} x_{-1}}{\partial v^{2}}+\frac{\partial^{2} x_{-1}}{\partial u^{2}} \\
=2 \frac{\partial \log a}{\partial u} \frac{\partial x_{-1}}{\partial u}+2 \frac{\partial \log K b}{\partial v} \frac{\partial x_{-1}}{\partial v}+c_{-1}^{\prime} x_{-1}
\end{array}\right.
$$

where $c_{-1}^{\prime}$ is a determinate function. Comparing equations (31) and (33), we see that $N_{-1}$ is an $R$ net. Similar results hold for the first Laplace transform of $N$. Consequently:

The Laplace transforms of an $R$ net are $R$ nets.

Making use of the terminology of [\$ 165], we have:

If either of the first derived congruences of a $W$ congruence is $a W$ congruence, then all of the derived congruences are $W^{\mathbf{2 9}}$ ).

46. $W$ congruences. Let $S$ be a surface referred to its asymptotic lines $\alpha=$ const., $\beta=$ const. The cartesian coordinates, $x^{(1)}, x^{(2)}, x^{(3)}$, of $S$ are given by the Lelieuvre formulas of the form $[\S 79]$

$$
\frac{\partial x^{(1)}}{\partial \alpha}=\left|\begin{array}{cc}
\nu_{2} & \nu_{3} \\
\frac{\partial \nu_{2}}{\partial \alpha} & \frac{\partial \nu_{3}}{\partial \alpha}
\end{array}\right|, \quad \frac{\partial x^{(1)}}{\partial \beta}=\left|\begin{array}{cc}
\nu_{2} & \nu_{3} \\
\frac{\partial \nu_{2}}{\partial \beta} & \frac{\partial \nu_{3}}{\partial \beta}
\end{array}\right|
$$

where $\nu_{1}, \nu_{2}, \nu_{3}$ are proportional to the direction-cosines of the normal to $S$, such that the total curvature of $S$ is given by

$$
K=-\frac{1}{\left(\nu_{1}^{2}+\nu_{2}^{2}+\nu_{s}^{2}\right)^{2}}=-\frac{1}{\varrho^{2}} .
$$

The functions $\nu_{i}$ are solutions of an equation of the form

$$
\frac{\partial^{2} \theta}{\partial \alpha \partial \beta}=M \theta
$$

where $M$ is a function of $\alpha$ and $\beta$.

29) Cf. Demoulin, l. c., p. 591. 
If $\theta_{1}$ is any solution of (36), the equations [cf. $§ 172$ ]

(37)

$$
\frac{\partial\left(\theta_{1} \bar{\nu}_{i}\right)}{\partial \alpha}=-\left|\begin{array}{cc}
\theta_{1} & \nu_{i} \\
\frac{\partial \theta_{1}}{\partial \alpha} & \frac{\partial \nu_{i}}{\partial \alpha}
\end{array}\right|, \quad \frac{\partial\left(\theta_{1} \bar{\nu}_{i}\right)}{\partial \beta}=\left|\begin{array}{cc}
\theta_{1} & \nu_{i} \\
\frac{\partial \theta_{1}}{\partial \beta} & \frac{\partial \nu_{i}}{\partial \beta}
\end{array}\right|
$$

are consistent, and the functions $\overline{\nu_{i}}$ are solutions of

$$
\frac{\partial^{2} \bar{\theta}}{\partial \alpha \partial \beta}=\theta_{1} \frac{\partial^{2}}{\partial \alpha \partial \beta}\left(\frac{1}{\theta_{1}}\right) \bar{\theta}
$$

The equations of the form

$$
\bar{x}^{(1)}=x^{(1)}+\nu_{2} \bar{\nu}_{3}-\nu_{3} \bar{\nu}_{2}
$$

define the coordinates, $\bar{x}^{(i)}$, of a surface $\bar{S}$, such that $S$ and $\bar{S}$ are the focal surfaces of the congruence of lines joining corresponding points on $S$ and $\bar{S}$; this is a W congruence, since the asymptotic lines are parametric on $\bar{S}$. Moreover, any $W$ congruence with $S$ as a focal surface may be obtained in this way. We shall give this result another form.

From (9) and the first of equations (10) it follows that the coordinates $x^{(i)}$ satisfy equations of the form

$$
\frac{\partial^{2} \theta}{\partial \alpha^{2}}=\frac{\partial \varphi}{\partial \alpha} \frac{\partial \theta}{\partial \alpha}+b \frac{\partial \theta}{\partial \beta}, \quad \frac{\partial^{2} \theta}{\partial \beta^{2}}=a_{1} \frac{\partial \theta}{\partial \alpha}+\frac{\partial \varphi}{\partial \beta} \frac{\partial \theta}{\partial \beta} .
$$

Differentiating (34), we obtain

$$
\frac{\partial^{2} x^{(1)}}{\partial \alpha^{2}}=\left|\begin{array}{cc}
\nu_{2} & \nu_{3} \\
\frac{\partial^{2} \nu_{2}}{\partial \alpha^{2}} & \frac{\partial^{2} \nu_{3}}{\partial \alpha^{2}}
\end{array}\right|, \quad \frac{\partial^{2} x^{(1)}}{\partial \beta^{2}}=-\left|\begin{array}{cc}
\nu_{2} & \nu_{3} \\
\frac{\partial^{2} \nu_{2}}{\partial \beta^{2}} & \frac{\partial^{2} \nu_{3}}{\partial \beta^{2}}
\end{array}\right| .
$$

The functions $\nu_{i}$ satisfy three equations of the form (1) and (2). From (36), (40), (41) and the first, second and fifth of equations (4), it follows that these equations are 


$$
\left\{\begin{array}{l}
\frac{\partial^{2} \nu_{i}}{\partial \alpha^{2}}=\frac{\partial \varphi}{\partial \alpha} \frac{\partial \nu_{i}}{\partial \alpha}-b \frac{\partial \nu_{i}}{\partial \beta}+\left(b \frac{\partial \varphi}{\partial \beta}+\frac{\partial b}{\partial \beta}\right) \nu_{i}, \\
\frac{\partial^{2} \nu_{i}}{\partial \alpha \partial \beta}=\left(\frac{\partial^{2} \varphi}{\partial \alpha \partial \beta}+a_{1} b\right) \nu_{i}, \\
\frac{\partial^{2} \nu_{i}}{\partial \beta^{2}}=-a_{1} \frac{\partial \nu_{i}}{\partial \alpha}+\frac{\partial \varphi}{\partial \beta} \frac{\partial \nu_{i}}{\partial \beta}+\left(a_{1} \frac{\partial \varphi}{\partial \alpha}+\frac{\partial a_{1}}{\partial \alpha}\right) \nu_{i} .
\end{array}\right.
$$

We write

$$
\theta_{1} \overline{\nu_{i}}=k \nu_{i}+l \frac{\partial \nu_{i}}{\partial \alpha}+m \frac{\partial \nu_{i}}{\partial \beta}
$$

and seek the conditions which $k, l$ and $m$ must satisfy in order that this expression may satisfy equations (37) for $i=1,2,3$. By means of (42) we find

$$
\begin{gathered}
\frac{\partial l}{\partial \beta}-a_{1} m=0, \quad \frac{\partial m}{\partial \alpha}-b l=0 \\
k=-\frac{1}{2}\left(\frac{\partial l}{\partial \alpha}+\frac{\partial m}{\partial \beta}+l \frac{\partial \varphi}{\partial \alpha}+m \frac{\partial \varphi}{\partial \beta}\right) \\
\theta_{1}=-\frac{1}{2}\left(\frac{\partial l}{\partial \alpha}-\frac{\partial m}{\partial \beta}+l \frac{\partial \varphi}{\partial \alpha}-m \frac{\partial \varphi}{\partial \beta}\right)
\end{gathered}
$$

and$$
\left\{\frac{\partial k}{\partial \alpha}-\frac{\partial \theta_{1}}{\partial \alpha}+l\left(b \frac{\partial \varphi}{\partial \beta}+\frac{\partial b}{\partial \beta}\right)+m\left(\frac{\partial^{2} \varphi}{\partial \alpha \partial \beta}+a_{1} b\right)=0\right.
$$$$
\frac{\partial k}{\partial \beta}+\frac{\partial \theta_{1}}{\partial \beta}+l\left(\frac{\partial^{2} \varphi}{\partial \alpha \partial \beta}+a_{1} b\right)+m\left(a_{1} \frac{\partial \varphi}{\partial \alpha}+\frac{\partial a_{1}}{\partial \alpha}\right)=0 .
$$

Conversely, if $l$ and $m$ are any pair of solutions of (44), the functions $k$ and $\theta_{1}$, defined by (45) satisfy (46). Furthermore, if equations (46) are differentiated with respect to $\beta$ and $\alpha$ respectively, and the resulting equations are substracted, we find that $\theta_{1}$ is a solution of the second of equations (42), in consequence of (46) and the equations for (42) analogous to the third and last of (4). Hence $^{30}$ ):

The determination of the $W$ congruences with a given focal surface $S$ is equivalent to the solution of equations (44), in which $a_{1}$

30) Cf. Jonas, Jahresbericht der deutschen Mathematiker-Vereinigung, vol. 29 (1920), p. 52 . 
and $b$ are the functions appearing in the equations (40) of the surface; when a pair of solutions is known, the coordinates $\bar{x}^{(i)}$ of the other focal surface of the congruence are given by

$$
\bar{x}^{(i)}=x^{(i)}+2 \frac{l \frac{\partial x^{(i)}}{\partial a}-m \frac{\partial x^{(i)}}{\partial \beta}}{\frac{\partial m}{\partial \beta}-\frac{\partial l}{\partial \alpha}-l \frac{\partial \varphi}{\partial \alpha}+m \frac{\partial \varphi}{\partial \beta}} .
$$

This expression follows from (39), (43), (45) and (34).

47. $R$ surfaces. In $\S 45$ we established the conditions to be satisfied by a net $N$ in order that it be an $R$ net. A surface is said to be an $R$ surface when it contains an $R$ net. In this section we establish an analytical characterization of $R$ surfaces.

Let $S$ be a surface referred to its asymptotic lines $\alpha=$ const., $\beta=$ const. Its cartesian coordinates satisfy equations of the form (40). Any isothermal-conjugate system of curves on $S$ is defined by [cf. $§ 82$ ]

$$
u=A(\alpha)+B(\beta), \quad v=A(\alpha)-B(\beta),
$$

when $A$ and $B$ are arbitrary functions of $\alpha$ and $\beta$ respectively. If we effect the transformation of variables (48), equations (40) become

where

$$
\begin{aligned}
\frac{\partial^{2} \theta}{\partial u^{2}}+\frac{\partial^{2} \theta}{\partial v^{2}} & =(K+L) \frac{\partial \theta}{\partial u}+(M+N) \frac{\partial \theta}{\partial v} \\
\frac{\partial^{2} \theta}{\partial u \partial v} & =\frac{1}{2}(K-L) \frac{\partial \theta}{\partial u}+\frac{1}{2}(M-N) \frac{\partial \theta}{\partial v}
\end{aligned}
$$

$$
\begin{aligned}
& K=\frac{1}{2} \frac{1}{A^{\prime 2}}\left(\frac{\partial \varphi}{\partial \alpha} A^{\prime}+b B^{\prime}-A^{\prime \prime}\right), L=\frac{1}{2 B^{\prime 2}}\left(a_{1} A^{\prime}+\frac{\partial \varphi}{\partial \beta} B^{\prime}-B^{\prime \prime}\right) \\
& M=\frac{1}{2} \frac{1}{A^{\prime 2}}\left(\frac{\partial \varphi}{\partial \alpha} A^{\prime}-b B^{\prime}-A^{\prime \prime}\right), N=\frac{1}{2 B^{\prime 2}}\left(a_{1} A^{\prime}-\frac{\partial \varphi}{\partial \beta} B^{\prime}+B^{\prime \prime}\right)
\end{aligned}
$$

the primes indicating differentiation.

From (31) it follows that a necessary and sufficient condition that the net of parameters $u$ and $v$ be an $R$ net is that

$$
\frac{\partial}{\partial v}(K+L)=\frac{\partial}{\partial u}(K-L), \quad \frac{\partial}{\partial v}(M-N)=\frac{\partial}{\partial u}(M+N),
$$


or in terms $\alpha$ and $\beta$

$$
\frac{1}{B^{\prime}} \frac{\partial K}{\partial \beta}-\frac{1}{A^{\prime}} \frac{\partial L}{\partial \alpha}=0, \quad \frac{1}{B^{\prime}} \frac{\partial M}{\partial \beta}+\frac{1}{A^{\prime}} \frac{\partial N}{\partial \alpha}=0 .
$$

Substituting the above expressions for $K, L, M, N$, we are brought to the single condition

$$
\left.B^{\prime 2} \frac{\partial b}{\partial \beta}+B^{\prime} B^{\prime \prime} b=A^{\prime 2} \frac{\partial a_{1}}{\partial \alpha}+A^{\prime} A^{\prime \prime} a_{1}^{31}\right)
$$

Since the quadrics are characterized by the condition that $a_{1}=b=0$, we have the theorem ${ }^{32}$ ):

Every isothermal-conjugate net on a quadric is an $R$ net.

If the condition (49) is satisfied for two sets of functions $A, B$ and $A_{1}, B_{1}$, it is satisfied also by $\bar{A}, \bar{B}$, where

$$
{\overline{A^{\prime}}}^{2}=A^{\prime 2}+k A_{1}^{2}, \bar{B}^{\prime 2}=B^{\prime 2}+k B_{1}^{2},
$$

$k$ being a constant. Hence we have the theorem of Demoulin ${ }^{33}$ ):

If a surface is an $R$ surface in two ways, it is an $R$ surface in an infinity of ways; that is an infinity of nets $R$ lie upon it.

If the condition (49) is satisfied by a surface, the parameters of the asymptotic lines can be chosen so that $A=\alpha, B=\beta$. Consequently:

$A$ necessary and sufficient condition that a surface be an $R$ surface is that its cartesian coordinates satisfy equations of the form

$$
\left\{\begin{array}{l}
\frac{\partial^{2} \theta}{\partial \alpha^{2}}=\frac{\partial \varphi}{\partial \alpha} \frac{\partial \theta}{\partial \alpha}+\frac{\partial \psi}{\partial \alpha} \frac{\partial \theta}{\partial \beta}, \\
\frac{\partial^{2} \theta}{\partial \beta^{2}}=\frac{\partial \psi}{\partial \beta} \frac{\partial \theta}{\partial \alpha}+\frac{\partial \varphi}{\partial \beta} \frac{\partial \theta}{\partial \beta} .
\end{array}\right.
$$

When the conditions (10) are applied to (50) we obtain the equations to be satisfied by $\varphi$ and $\psi$, whose solution gives the complete determination of $R$ surfaces.

31) Cf. Demoulin, Comptes Rendus, vol. 153 (1911), p. 799.

${ }^{32}$ ) Cf. Bianchi, Rendiconti dei Lincei, ser. 5, vol. 22², (1913), p. 5.

33) L. c. 
For this case equations (44) become

$$
\frac{\partial l}{\partial \beta}-\frac{\partial \psi^{\prime}}{\partial \beta} m=0, \quad \frac{\partial m}{\partial \alpha}-\frac{\partial \psi^{\prime}}{\partial \alpha} l=0
$$

of which a solution is $l=m=e^{\psi}$. Making use of (45), the equation (43) becomes in this case

$(51)^{4}$

$$
\left\{\begin{array}{c}
{\left[\frac{\partial}{\partial \alpha}\left(\varphi+\psi^{\prime}\right)-\frac{\partial}{\partial \beta}\left(\varphi+\psi^{\prime}\right)\right] \bar{\nu}_{i}=} \\
{\left[\frac{\partial}{\partial \alpha}(\varphi+\psi)+\frac{\partial}{\partial \beta}\left(\varphi+\psi^{\prime}\right)\right] \nu_{i}-2 \frac{\partial \nu_{i}}{\partial \alpha}-2 \frac{\partial \nu_{i}}{\partial \beta} .}
\end{array}\right.
$$

48. $R$ Congruences. Transformations $\theta_{1}$. By definition the $W$ congruence of tangents to the curves of either family of an $R$ net is an $R$ congruence. In this section we show that when an $R$ surface is known, a congruence $R$ can be found by quadratures, whose lines are parallel to the normals to the given surface $R^{34}$ ).

Equations (50) can be written

(52) $\frac{\partial}{\partial \boldsymbol{\alpha}}\left(e^{-\varphi} \frac{\partial \theta}{\partial \boldsymbol{\alpha}}\right)=e^{-\varphi} \frac{\partial \psi}{\partial \boldsymbol{\alpha}} \frac{\partial \theta}{\partial \boldsymbol{\beta}}, \quad \frac{\partial}{\partial \boldsymbol{\beta}}\left(e^{-\varphi \frac{\partial \theta}{\partial \beta}}\right)=e^{-\varphi} \frac{\partial \psi}{\partial \beta} \frac{\partial \theta}{\partial \boldsymbol{\alpha}}$.

Hence if we put

$$
\bar{\eta}_{i}+\eta_{i}=2 e^{-\varphi} \frac{\partial x^{(i)}}{\partial \alpha}, \quad \bar{\eta}_{i}-\eta_{i}=2 e^{-\varphi \frac{\partial x^{(i)}}{\partial \beta}},
$$

we have

(54) $\frac{\partial}{\partial \boldsymbol{\alpha}}\left(\bar{\eta}_{i}+\eta_{i}\right)=\frac{\partial \psi}{\partial \boldsymbol{\alpha}}\left(\bar{\eta}_{i}-\eta_{i}\right), \quad \frac{\partial}{\partial \boldsymbol{\beta}}\left(\bar{\eta}_{i}-\eta_{i}\right)=\frac{\partial \psi}{\partial \boldsymbol{\beta}}\left(\bar{\eta}_{i}+\eta_{i}\right)$.

Differentiating these equations with respect to $\beta$ and $\boldsymbol{\alpha}$ respectively, we find that

$$
\frac{\partial^{2} \eta_{i}}{\partial \boldsymbol{\alpha} \partial \boldsymbol{\beta}}=e^{\psi} \frac{\partial^{2} e^{-\psi}}{\partial \boldsymbol{\alpha} \partial \boldsymbol{\beta}} \eta_{i}, \quad \frac{\partial^{2} \bar{\eta}_{i}}{\partial \boldsymbol{\alpha} \partial \boldsymbol{\beta}}=e^{-\psi} \frac{\partial^{2} e^{\psi}}{\partial \boldsymbol{\alpha} \partial \boldsymbol{\beta}} \bar{\eta}_{i}
$$

34) Demoulin, Comptes Rendus, vol. 153 (1911), p. 798; also, Jonas, 1. c., pp.67 et seq. 
Since equations (54) may be written in the form (37) with $\theta_{1}$ replaced by $e^{-\psi}$ and $\nu_{i}, \bar{\nu}_{i}$ by $\eta_{i}, \bar{\eta}_{i}$, it follows that the surfaces $\Sigma$ and $\bar{\Sigma}$ of coordinates $\xi^{(i)}$ and $\bar{\xi}^{(i)}$ given by equations of the form (34) in $\eta_{i}$ and analogously to (39) by equations of the form

$$
\bar{\xi}^{(1)}=\xi^{(1)}+\eta_{2} \bar{\eta}_{3}-\eta_{3} \vec{\eta}_{2}
$$

are the focal surfaces of a $W$ congruence $\Gamma$. In consequence of (53) we have

$$
\sum\left(\bar{\xi}^{(i)}-\xi^{(i)}\right) \frac{\partial x^{(i)}}{\partial \boldsymbol{\mu}}=\sum\left(\bar{\xi}^{(i)}-\xi^{(i)}\right) \frac{\partial x^{(i)}}{\partial \beta}=0 .
$$

Consequently the lines of the $W$ congruence are parallel to the normals to the given $R$ surface.

From (53) we have

$$
\eta_{i}=e^{-\varphi}\left(\frac{\partial x^{(i)}}{\partial \alpha}-\frac{\partial x^{(i)}}{\partial \beta}\right)
$$

If we differentiate this equation with respect to $\alpha$ and $\beta$ and add the resulting equations, we find in consequence of $(50)$ and (53)

$$
\begin{gathered}
\frac{\partial \eta_{i}}{\partial \boldsymbol{\mu}}+\frac{\partial \eta_{i}}{\partial \beta}+\frac{1}{2} \eta_{i}\left[\frac{\partial}{\partial \alpha}(\varphi+\psi)+\frac{\partial}{\partial \beta}(\varphi+\psi)\right] \\
=\frac{1}{2} \bar{\eta}_{i}\left[\frac{\partial}{\partial \alpha}(\varphi+\psi)-\frac{\partial}{\partial \beta}(\varphi+\psi)\right] .
\end{gathered}
$$

Comparing this result with an equation analogous to (43) written

$$
\vartheta_{1} \bar{\eta}_{i}=l_{i} \eta_{i}+\lambda \frac{\partial \eta_{i}}{\partial \alpha}+\mu \frac{\partial \eta_{i}}{\partial \beta}
$$

we see that $\lambda=\mu$, and hence from equations analogous to (44) and (49) it follows that $\Sigma$ is an $R$ surface, and consequently the congruence $\Gamma$ is $R$. Moreover, from (53) it is seen that the normals to $\Sigma$ and $\bar{\Sigma}$ are parallel to the tangents to the curves of the $R$ net $\alpha-\beta=$ const., $\alpha+\beta=$ const. on $S$.

The equations for $\Sigma$ analogous to (50) for $S$ are obtained from (50) by replacing $\varphi$ and $\psi$ by functions $\varsigma_{1}$ and $\psi_{1}$, where $\lambda=\mu=e^{\psi_{1}}$, 
as follows from equations analogous to (44). From (58) and equations similar to (51), we find that $\varphi_{1}$ can be chosen so that

$$
\varphi_{1}+\psi_{1}+\varphi+\psi^{\prime}=0 \text {. }
$$

Also from ( 54$)$ we have that $\vartheta_{1}=\frac{1}{2} e^{-\psi}$. Hence equations (53) can be written

$$
\vartheta_{1}\left(\bar{\eta}_{i}+\eta_{i}\right)=e^{\varphi_{1}+\zeta_{1}^{\prime}} \frac{\partial x^{(i)}}{\partial \boldsymbol{\omega}}, \quad \vartheta_{1}\left(\bar{\eta}_{i}-\eta_{i}\right)=e^{\varphi_{1}+\psi_{1}} \frac{\partial x^{(i)}}{\partial \beta} .
$$

Suppose conversely that we have an $R$ congruence for which one of the focal surfaces is $S$ whose point coordinates $x^{(i)}$ satisfy (50), and for which the functions $\bar{\nu}_{i}$ are given by (51). In consequence of (37) and (51), the equations

$$
\frac{\partial \bar{\xi}^{(i)}}{\partial \boldsymbol{\alpha}}=\theta_{1}\left(\overline{\nu_{i}}+\nu_{i}\right) e^{-(\boldsymbol{\varphi}+\psi)}, \quad \frac{\partial \xi^{(i)}}{\partial \boldsymbol{\beta}}=\theta_{1}\left(\overline{\nu_{i}}-\nu_{i}\right) e^{-(\boldsymbol{\psi}+\varphi)},
$$

are consistent, and $\xi^{(i)}$ are solutions of

$$
\begin{aligned}
& \frac{\partial^{2} \theta}{\partial \boldsymbol{\alpha}^{2}}=\frac{\partial}{\partial \boldsymbol{\alpha}}\left(\log \theta_{1}-\varphi-\psi\right) \frac{\partial \theta}{\partial \alpha}-\frac{\partial \log \theta_{1}}{\partial \boldsymbol{\alpha}} \frac{\partial \theta}{\partial \beta}, \\
& \frac{\partial^{2} \theta}{\partial \beta^{2}}=-\frac{\partial \log \theta_{1}}{\partial \beta} \frac{\partial \theta}{\partial \alpha}+\frac{\partial}{\partial \beta}\left(\log \theta_{1}-\varphi-\psi\right) \frac{\partial \theta}{\partial \beta} .
\end{aligned}
$$

Hence $\boldsymbol{\xi}^{(i)}$ are the coordinates of a surface $R$.

Accordingly we have the theorem of Demoulin:

When an $R$ surface is lnown, an associated $R$ congruence can be found by quadratures, and conversely; the lines of the congruence are parallel to the normals of the associated $R$ surface, and the normals to the focul surfaces of the congruence are parallel to the tangents to the $R$ net on the $R$ surface.

Demoulin has called this reciprocal relation a tranformation $\theta_{1}$.

49. Reciprocally derived nets. Transformations $W$ of nets $R$. In $\S 11$ we remarked that if $\theta_{1}$ and $\theta_{2}$ are solutions of the point equation

$$
\frac{\partial^{2} \theta}{\partial u \partial v}=\frac{\partial \log a}{\partial v} \frac{\partial \theta}{\partial u}+\frac{\partial \log b}{\partial u} \frac{\partial \theta}{\partial v}
$$


of the cartesian coordinates of a net $N(x)$, they determine a derived net $\bar{N}(x)$ of $N$. whose coordinates are given by equations of the form

$$
\bar{x}=x+p \frac{\partial x}{\partial u}+q \frac{\partial x}{\partial v},
$$

where

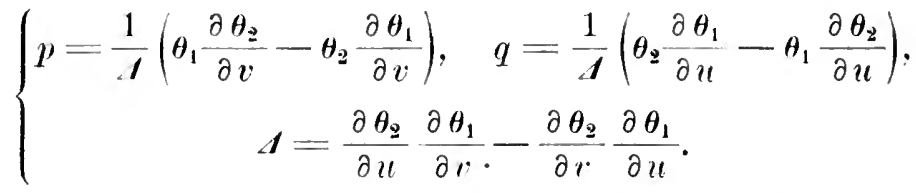

From these equations we have

$$
\theta_{1}+p \frac{\partial \theta_{1}}{\partial u}+q \frac{\partial \theta_{1}}{\partial r}=0, \quad \theta_{2}+p \frac{\partial \theta_{2}}{\partial u}+q \frac{\partial \theta_{2}}{\partial r}=0
$$

Ordinarily $N$ is not a derived net of $\bar{N}$. When it is, we say that $N$ and $\bar{N}$ are reciprocally derived nets ${ }^{35}$ ). If $N$ and $\bar{N}$ are reciprocally derived nets. the tangents planes at corresponding points, $M$ and $\bar{M}$, must pass through $\bar{M}$ and $M$ respectively. Hence the surfaces, $S$ and $\bar{S}$, on which $N$ and $\bar{N}$ lie are the focal surfaces of the congruence $\bar{G}$ of lines $M \bar{M}$. Since the lines of $\bar{G}$ are not tangents to the curves of $N$ or $\bar{N}$, there exist upon $S$ and $\bar{S}$ corresponding nets to which these lines are tangent, namely the focal nets. Consequently $S$ and $\bar{S}$ are surfaces in 3 -space $(\S 3)$, and since two nets on $S$ correspond to two nets on $\bar{S}$, the asymptotic lines correspond on $S$ and $\bar{S}$. Hence $\bar{G}$ is a $W$ congruence.

Since $\theta_{1}$ and $\theta_{2}$ are solutions of (60), we have from (62)

$$
\begin{aligned}
& \left(\frac{\partial p}{\partial u}=-1-q \frac{\partial \log a}{\partial r}+\frac{p}{d}\left(\frac{\partial^{2} \theta_{1}}{\partial u^{2}} \frac{\partial \theta_{2}}{\partial v}-\frac{\partial^{2} \theta_{2}}{\partial u^{2}} \frac{\partial \theta_{1}}{\partial v}\right),\right. \\
& \frac{\partial p}{\partial v}=-p \frac{\partial \log a}{\partial v}+\frac{1}{\lambda}\left(\theta_{1} \frac{\partial^{2} \theta_{2}}{\partial v^{2}}-\theta_{2} \frac{\partial^{2} \theta_{1}}{\partial v^{2}}\right)-\frac{p}{\lambda}\left(\frac{\partial \theta_{2}}{\partial u} \frac{\partial^{2} \theta_{1}}{\partial v^{2}}-\frac{\partial \theta_{1}}{\partial u} \frac{\partial^{2} \theta_{2}}{\partial v^{2}}\right), \\
& \frac{\partial q}{\partial u}=-q \frac{\partial \log b}{\partial u}+\frac{1}{d}\left(\theta_{2} \frac{\partial^{2} \theta_{1}}{\partial u^{2}}-\theta_{1} \frac{\partial^{2} \theta_{2}}{\partial u^{2}}\right)-\frac{q}{d}\left(\frac{\partial \theta_{1}}{\partial \imath} \frac{\partial^{2} \theta_{2}}{\partial u^{2}}-\frac{\partial \theta_{2}}{\partial v} \frac{\partial^{2} \theta_{1}}{\partial u^{2}}\right), \\
& \frac{\partial q}{\partial \imath}=-1-p \frac{\partial \log b}{\partial u}+\frac{q}{d}\left(\frac{\partial^{2} \theta_{2}}{\partial \iota^{2}} \frac{\partial \theta_{1}}{\partial u}-\frac{\partial^{2} \theta_{1}}{\partial \imath^{2}} \frac{\partial \theta_{\underline{\Xi}}}{\partial u}\right) \text {. }
\end{aligned}
$$

3.5) Cf. Tzitzeica, Comptes Rendus, vol. 156 (1913), p. 666. 
With the aid of these expressions we show that the point equation of $\bar{N}$ is

$$
\frac{\partial^{2} \theta}{\partial u \partial v}=\frac{\partial}{\partial v} \log a p \frac{\partial \theta}{\partial u}+\frac{\partial}{\partial u} \log b q \frac{\partial \theta}{\partial v} .
$$

If $N$ is to be a derived net of $\bar{N}$, we must have

$$
r=\bar{x}+\bar{p} \frac{\partial \bar{x}}{\partial u}+\bar{q} \frac{\partial \bar{x}}{\partial r},
$$

and the point equation of $N$ is, analogously to (65),

$$
\frac{\partial^{2} \theta}{\partial u \partial v}=\frac{\partial}{\partial v} \log a p \bar{p} \frac{\partial \theta}{\partial u}+\frac{\partial}{\partial u} \log h q \bar{q} \frac{\partial \theta}{\partial \imath} \text {. }
$$

Comparing this equation with (60), we have

$$
p \bar{p}=U, \quad q \bar{q}=V,
$$

where $U$ and $V$ are functions of $u$ and $v$ alone. From (61) and (66) it is seen that the parameters can be chosen so that

$$
p \bar{p}=q \bar{q}=-\frac{1}{c},
$$

where $c$ is a constant.

When the expression (61) for $r$ is substituted in (66), the result is reducible to

$$
\frac{\partial^{2} x}{\partial u^{2}}+\frac{\partial^{2} x}{\partial v^{2}}=a^{\prime} \frac{\partial x}{\partial u}+b^{\prime} \frac{\partial x}{\partial v},
$$

where

$$
\left\{\begin{array}{l}
-a^{\prime}=-c p+\frac{1}{p}+\frac{\partial \log p}{\partial u}+\left(\frac{q}{p}+\frac{p}{q}\right) \frac{\partial \log a}{\partial v}+\frac{1}{q} \frac{\partial p}{\partial v} \\
-b^{\prime}=-c q+\frac{1}{q}+\frac{\partial \log q}{\partial v}+\left(\frac{p}{q}+\frac{q}{p}\right) \frac{\partial \log b}{\partial u}+\frac{1}{p} \frac{\partial q}{\partial u}
\end{array}\right.
$$

By means of (64) equations (71) are reducible to

$$
\begin{aligned}
& \frac{\partial \theta_{2}}{\partial v}\left(\frac{\partial^{2} \theta_{1}}{\partial u^{2}}+\frac{\partial^{2} \theta_{1}}{\partial v^{2}}-a^{\prime} \frac{\partial \theta_{1}}{\partial u}-c \theta_{1}\right)=\frac{\partial \theta_{1}}{\partial v}\left(\frac{\partial^{2} \theta_{2}}{\partial u^{2}}+\frac{\partial^{2} \theta_{2}}{\partial v^{2}}-a^{\prime} \frac{\partial \theta_{2}}{\partial u}-c \theta_{2}\right), \\
& \frac{\partial \theta_{2}}{\partial u}\left(\frac{\partial^{2} \theta_{1}}{\partial u^{2}}+\frac{\partial^{2} \theta_{1}}{\partial v^{2}}-b^{\prime} \frac{\partial \theta_{1}}{\partial v}-c \theta_{1}\right)=\frac{\partial \theta_{1}}{\partial u}\left(\frac{\partial^{2} \theta_{2}}{\partial u^{2}}+\frac{\partial^{2} \theta_{2}}{\partial v^{2}}-b^{\prime} \frac{\partial \theta_{2}}{\partial v}-c \theta_{2}\right),
\end{aligned}
$$


from which it follows that $\theta_{1}$ and $\theta_{2}$ are solutions of

$$
\frac{\partial^{2} \theta}{\partial u^{2}}+\frac{\partial^{2} \theta}{\partial v^{2}}=a^{\prime} \frac{\partial \theta}{\partial u}+b^{\prime} \frac{\partial \theta}{\partial v}+c \theta .
$$

In order that equations (60) and (70) shall admit three independent solutions, we must have equations (22) satisfied with $c=r^{\prime}=0$. When we make use of these results in obtaining equation (20) for the system (60) and (72), we find that $P=Q=R=0$ and consequently $S=0$. But this is the last of (22) and it reduces to

$$
\frac{\partial b^{\prime}}{\partial u}-2 \frac{\partial^{2} \log b}{\partial u \partial v}=0
$$

Then from the first of (22) we have

$$
\frac{\partial a^{\prime}}{\partial v}-2 \frac{\partial^{2} \log a}{\partial u \partial v}=0 .
$$

From the results of $\S 45$ it follows that $N$ is an $R$ net, whose cartesian coordinates satisfy equations which can be written in the forms

$$
\left\{\begin{array}{c}
\frac{\partial^{2} \theta}{\partial u^{2}}+\frac{\partial^{2} \theta}{\partial \iota^{2}}=2 \frac{\partial \log a}{\partial u} \frac{\partial \theta}{\partial u}+2 \frac{\partial \log b}{\partial v} \frac{\partial \theta}{\partial v} \\
\frac{\partial^{2} \theta}{\partial u \partial v}=\frac{\partial \log a}{\partial v} \frac{\partial \theta}{\partial u}+\frac{\partial \log b}{\partial u} \frac{\partial \theta}{\partial v} .
\end{array}\right.
$$

Now equation (72), to be satisfied by $\theta_{1}$ and $\theta_{2}$, is

$$
\frac{\partial^{2} \theta}{\partial u^{2}}+\frac{\partial^{2} \theta}{\partial v^{2}}=2 \frac{\partial \log a}{\partial u} \frac{\partial \theta}{\partial u}+2 \frac{\partial \log b}{\partial v} \frac{\partial \theta}{\partial v}+c \theta
$$

Since (60) and (74) satisfy the corresponding equations (22), they admit four independent solutions.

If in (61) the expression (66) for $x$ is substituted, we find in consequence of (69) and (71) that $\bar{x}$ is a solution of

$$
\frac{\partial^{2} \theta}{\partial u^{2}}+\frac{\partial^{2} \theta}{\partial v^{2}}=2 \frac{\partial}{\partial u} \log a p \frac{\partial \theta}{\partial u}+2 \frac{\partial}{\partial v} \log b q \frac{\partial \theta}{\partial v} .
$$

Hence $\bar{N}$ is an $R$ net whose equations are (65) and (75). 
In $\S 50$ it is shown that, if $\theta_{3}$ and $\theta_{4}$ are two solutions of (60) and (74) independent of $\theta_{1}$ and $\theta_{2}$, the functions $\bar{\theta}_{3}$ and $\bar{\theta}_{4}$, defined by

$$
\bar{\theta}_{i}=\theta_{i}+p \frac{\partial \theta_{i}}{\partial \imath}+q \frac{\partial \theta_{i}}{\partial \imath}(i=3,4)
$$

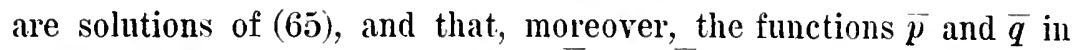
(66) are expressible in terms of $\overline{\theta_{3}}$ and $\overline{\theta_{4}}$ in the form analogous to (62). Hence $N$ is a derived net of $\bar{N}$, and consequently is reciprocally derived. We say that $\bar{N}$ is obtained from $N$ by a transformation $W_{c}$. Since any solution of $(60)$ and $(74)$ is expressible linearly in terms of four independent solutions, it follows from the form of $(62)$ that there are $\infty^{5}$ transformations $W_{c}$ for each value of ' , different from zero, in (74). Hence:

Any $R$ net admits of $\infty^{5}$ transformations $W_{c}$ into $R$ nets, for each value of the constant c different from zero ${ }^{36}$ ).

50. Theorem of permutability of transformations $W$. Let $\theta_{3}$ and $\theta_{4}$ be two solutions of the equation (60) and

$$
\frac{\partial^{2} \theta}{\partial u}+\frac{\partial^{2} \theta}{\partial \vartheta^{2}}=2 \frac{\partial \log a}{\partial u} \frac{\partial \theta}{\partial u}+2 \frac{\partial \log b}{\partial v} \frac{\partial \theta}{\partial u^{\circ}}+c^{\prime} \theta
$$

and consider the functions (76). Analogously to (I, 68) we liave

$$
(77)\left\{\begin{aligned}
& \frac{\partial \bar{\theta}_{i}}{\partial u}=p\left\{\frac{\partial^{2} \theta_{i}}{\partial u^{2}}+\frac{1}{t}\left[\frac{\partial \theta_{i}}{\partial u}\left(\frac{\partial \theta_{2}}{\partial v} \frac{\partial^{2} \theta_{1}}{\partial u^{2}}-\frac{\partial \theta_{1}}{\partial v} \frac{\partial^{2} \theta_{2}}{\partial u^{2}}\right)\right.\right.\left.\left.-\frac{\partial \theta_{i}}{\partial v}\left(\frac{\partial \theta_{2}}{\partial u} \frac{\partial^{2} \theta_{1}}{\partial u^{2}}-\frac{\partial \theta_{1}}{\partial u} \frac{\partial^{2} \theta_{\mathbf{z}}}{\partial u^{2}}\right)\right]\right\}, \\
&=q\left\{\frac{\partial^{2} \theta_{i}}{\partial v^{2}}+\frac{1}{\lambda}\left[\frac{\partial \theta_{i}}{\partial u}\left(\frac{\partial \theta_{2}}{\partial v} \frac{\partial^{2} \theta_{1}}{\partial v^{2}}-\frac{\partial \theta_{1}}{\partial v} \frac{\partial^{2} \theta_{2}}{\partial v^{2}}\right)\right.\right. \\
&\left.\left.-\frac{\partial \theta_{i}}{\partial v}\left(\frac{\partial \theta_{2}}{\partial u} \frac{\partial^{2} \theta_{1}}{\partial v^{2}}-\frac{\partial \theta_{1}}{\partial u} \frac{\partial^{2} \theta_{2}}{\partial \iota^{2}}\right)\right]\right\}, \\
&=q\left\{-\frac{1}{p} \frac{\partial \overline{\theta_{i}}}{\partial u}+\left(c^{\prime}-c\right) \theta_{i}+c \overline{\theta_{i}}\right\} .
\end{aligned}\right.
$$

36) Amuals of Mathematics, vol. 22 (1921), p. 170; these transformations are given in a different form by Demoulin, Bulletin de la Classe des Sciences, Académie Royale de Belgique, 1920, p. 226, and by Jonas, Jahresbericht, vol. 29 (1920), p. 58 . 
Differentiating the first of these equations with respect to $"$, we get

$$
\begin{gathered}
\frac{\partial^{2} \vec{\theta}_{i}}{\partial \|^{2}}=\frac{\partial \vec{\theta}_{i}}{\partial u}\left[\frac{\partial}{\partial u} \log p a^{2} b+\frac{1}{\prime}\left(\frac{\partial \theta_{2}}{\partial r} \frac{\partial^{2} \theta_{1}}{\partial u^{2}}-\frac{\partial \theta_{1}}{\partial v} \frac{\partial^{2} \theta_{\mathbf{2}}}{\partial u^{2}}\right)\right] \\
\left.+c^{\prime}-c\right) \frac{\partial \theta_{i}}{\partial u}-p \frac{\partial \log b}{\partial u}\left[c^{\prime} \theta_{i}+c\left(p \frac{\partial \boldsymbol{\theta}_{i}}{\partial u}+\eta \frac{\partial \boldsymbol{\theta}_{i}}{\partial v}\right)\right] .
\end{gathered}
$$

Making use of the expression (64) for $\frac{\partial p}{\partial u}$ and (76), we reduce this to

$$
\begin{gathered}
\frac{\partial^{2} \bar{\theta}_{i}}{\partial u^{2}}=\frac{\partial \bar{\theta}_{i}}{\partial u}\left[\frac{\partial}{\partial u} \log p^{2} a^{2} b+\frac{1}{p}+\frac{q}{p} \frac{\partial \log a}{\partial v}\right]+p\left(c^{\prime}-c\right) \frac{\partial \theta_{i}}{\partial u} \\
-p \frac{\partial \log b}{\partial u}\left[\left(c^{\prime}-c\right) \theta_{i}+c \overline{\theta_{i}}\right] .
\end{gathered}
$$

Also we find that

$$
\frac{\partial^{2} \overline{\theta_{i}}}{\partial u \partial v}=\frac{\partial}{\partial v} \log a p \frac{\partial \overline{\theta_{i}}}{\partial u}+\frac{\partial}{\partial u} \log b q \frac{\partial \overline{\theta_{i}}}{\partial v} .
$$

From the second of $(7 \tau)$ we get

$\frac{\partial^{2} \overline{\theta_{i}}}{\partial r^{2}}=-\frac{q}{p} \frac{\partial \log a}{\partial v} \frac{\partial \overline{\theta_{i}}}{\partial u}+\left(\frac{\partial \log q}{\partial r}-\frac{q}{p} \frac{\partial \log b q}{\partial u}+c q\right) \frac{\partial \bar{\theta}_{i}}{\partial v}+q\left(c^{\prime}-c\right) \frac{\partial \theta_{i}}{\partial v}$.

With the aid of (71) and (77) we obtain

$$
\frac{\partial^{2} \overline{\boldsymbol{\theta}}_{i}}{\partial \boldsymbol{u}^{2}}+\frac{\partial^{2} \overline{\boldsymbol{\theta}}_{\boldsymbol{i}}}{\partial v^{2}}=2 \frac{\partial \log a p}{\partial u} \frac{\partial \overline{\boldsymbol{\theta}}_{i}}{\partial u}+2 \frac{\partial \log b q}{\partial v} \frac{\partial \overline{\boldsymbol{\theta}}_{i}}{\partial v}+c^{\prime} \overline{\boldsymbol{\theta}}_{i}
$$

Hence the functions $\overline{\theta_{i}}$ determine a $W_{c^{\prime}}$ transformation of $\bar{N}$. The coordinates of the transform $\hat{N}$ are of the form

$$
\hat{x}=\bar{x}+\bar{p} \frac{\partial \bar{x}}{\partial u}+\bar{q} \frac{\partial \bar{x}}{\partial v},
$$


where (cf. I, 68)

$$
\begin{aligned}
\frac{\partial \bar{x}}{\partial u}=p\left\{\frac{\partial^{2} x}{\partial u^{2}}+\frac{1}{1}\left[\frac { \partial x } { \partial u } \left(\frac{\partial \theta_{2}}{\partial v} \frac{\partial^{2} \theta_{1}}{\partial u^{2}}\right.\right.\right. & \left.-\frac{\partial \theta_{1}}{\partial \imath} \frac{\partial^{2} \theta_{2}}{\partial u^{2}}\right) \\
& \left.\left.-\frac{\partial x}{\partial v}\left(\frac{\partial \theta_{2}}{\partial u} \frac{\partial^{2} \theta_{1}}{\partial u^{2}}-\frac{\partial \theta_{1}}{\partial u} \frac{\partial^{2} \theta_{2}}{\partial u^{2}}\right)\right]\right\},
\end{aligned}
$$

$\frac{\partial \bar{x}}{\partial v}=q\left[-\frac{1}{p} \frac{\partial \bar{x}}{\partial u}+c\left(p \frac{\partial x}{\partial u}+q \frac{\partial x}{\partial v}\right)\right]$,

and

$$
\begin{gathered}
\overline{\boldsymbol{p}}=\frac{1}{\overline{\boldsymbol{A}}}\left(\overline{\boldsymbol{\theta}}_{3} \frac{\partial \overline{\boldsymbol{\theta}}_{4}}{\partial v}-\overline{\boldsymbol{\theta}}_{4} \frac{\partial \overline{\boldsymbol{\theta}}_{3}}{\partial v}\right), \quad \bar{q}=\frac{1}{\overline{\boldsymbol{A}}}\left(\overline{\boldsymbol{\theta}}_{4} \frac{\partial \overline{\boldsymbol{\theta}}_{3}}{\partial u}-\overline{\boldsymbol{\theta}}_{3} \frac{\partial \overline{\boldsymbol{\theta}}_{4}}{\partial u}\right), \\
\overline{\boldsymbol{A}}=\left(\frac{\partial \overline{\boldsymbol{\theta}_{4}}}{\partial u} \frac{\partial \overline{\boldsymbol{\theta}}_{3}}{\partial v}-\frac{\partial \overline{\boldsymbol{\theta}}_{4}}{\partial v} \frac{\partial \overline{\boldsymbol{\theta}}_{3}}{\partial u}\right) .
\end{gathered}
$$

From (77) and (63) we have

$$
\begin{aligned}
& \bar{A}=q\left[\left(c^{\prime}-c\right)\left(\boldsymbol{\theta}_{3} \frac{\partial \overline{\boldsymbol{\theta}}_{4}}{\partial u}-\boldsymbol{\theta}_{4} \frac{\partial \overline{\boldsymbol{\theta}}_{3}}{\partial u}\right)+c\left(\overline{\boldsymbol{\theta}}_{3} \frac{\partial \overline{\boldsymbol{\theta}}_{4}}{\partial u}-\overline{\boldsymbol{\theta}}_{\mathbf{4}} \frac{\partial \overline{\boldsymbol{\theta}}_{3}}{\partial u}\right)\right] \\
& =\frac{p q}{\Delta}\left\{c ^ { \prime } \left[\left(\frac{\partial \theta_{2}}{\partial u} \frac{\partial \theta_{1}}{\partial v}-\frac{\partial \theta_{2}}{\partial v} \frac{\partial \theta_{1}}{\partial u}\right)\left(\theta_{3} \frac{\partial^{2} \theta_{4}}{\partial u^{2}}-\theta_{4} \frac{\partial^{2} \theta_{3}}{\partial u^{2}}\right)\right.\right. \\
& +\left(\theta_{3} \frac{\partial \theta_{4}}{\partial \imath}-\theta_{4} \frac{\partial \theta_{3}}{\partial u}\right)\left(\frac{\partial \theta_{2}}{\partial v} \frac{\partial^{2} \theta_{1}}{\partial \iota^{2}}-\frac{\partial \theta_{1}}{\partial v} \frac{\partial^{2} \theta_{2}}{\partial \iota^{2}}\right) \\
& \left.-\left(\theta_{3} \frac{\partial \theta_{4}}{\partial v}-\theta_{4} \frac{\partial \theta_{3}}{\partial v}\right)\left(\frac{\partial \theta_{2}}{\partial u} \frac{\partial^{2} \theta_{1}}{\partial u^{2}}-\frac{\partial \theta_{1}}{\partial u} \frac{\partial^{2} \theta_{2}}{\partial u^{2}}\right)\right] \\
& +c\left[\left(\frac{\partial \theta_{4}}{\partial u} \frac{\partial \theta_{3}}{\partial v}-\frac{\partial \theta_{4}}{\partial v} \frac{\partial \theta_{3}}{\partial u}\right)\left(\theta_{1} \frac{\partial^{2} \theta_{2}}{\partial \iota^{2}}-\theta_{2} \frac{\partial^{2} \theta_{1}}{\partial u^{2}}\right)\right. \\
& +\left(\theta_{1} \frac{\partial \theta_{2}}{\partial u}-\theta_{2} \frac{\partial \theta_{1}}{\partial u}\right)\left(\frac{\partial \theta_{4}}{\partial v} \frac{\partial^{2} \theta_{3}}{\partial u^{2}}-\frac{\partial \theta_{3}}{\partial v} \frac{\partial^{2} \theta_{4}}{\partial u^{2}}\right) \\
& \left.\left.-\left(\theta_{1} \frac{\partial \theta_{2}}{\partial v}-\theta_{2} \frac{\partial \theta_{1}}{\partial v}\right)\left(\frac{\partial \theta_{4}}{\partial u} \frac{\partial^{2} \theta_{3}}{\partial u^{2}}-\frac{\partial \theta_{3}}{\partial u} \frac{\partial^{2} \theta_{4}}{\partial u^{2}}\right)\right]\right\} .
\end{aligned}
$$


By means of the above formulas equation (78) is reducible to

$$
\begin{aligned}
\hat{\imath}=x+q & \frac{c^{\prime}-c}{\bar{A}}\left\{\left(p \frac{\partial x}{\partial u}+q \frac{\partial x}{\partial v}\right)\left(\theta_{3} \frac{\partial \overline{\theta_{4}}}{\partial u}-\theta_{4} \frac{\partial \overline{\theta_{3}}}{\partial u}\right)+\left(\overline{\theta_{3}} \theta_{4}-\overline{\theta_{4}} \theta_{3}\right) \frac{\partial \bar{x}}{\partial u}\right\} \\
=x+ & \frac{c^{\prime}-c}{d \bar{I}} p q\left\{\frac { \partial x } { \partial u } \left[\left(\theta_{1} \frac{\partial \theta_{2}}{\partial v}-\theta_{2} \frac{\partial \theta_{1}}{\partial v}\right)\left(\theta_{3} \frac{\partial^{2} \theta_{1}}{\partial u^{2}}-\theta_{4} \frac{\partial^{2} \theta_{3}}{\partial u^{2}}\right)\right.\right. \\
& \left.-\left(\theta_{3} \frac{\partial \theta_{4}}{\partial v}-\theta_{4} \frac{\partial \theta_{3}}{\partial v}\right)\left(\theta_{1} \frac{\partial^{2} \theta_{2}}{\partial u^{2}}-\theta_{2} \frac{\partial^{2} \theta_{1}}{\partial u^{2}}\right)\right] \\
& -\frac{\partial x}{\partial v}\left[\left(\theta_{1} \frac{\partial \theta_{2}}{\partial u}-\theta_{2} \frac{\partial \theta_{1}}{\partial u}\right)\left(\theta_{3} \frac{\partial^{2} \theta_{4}}{\partial u^{2}}-\theta_{4} \frac{\partial^{2} \theta_{3}}{\partial u^{2}}\right)\right. \\
& \left.-\left(\theta_{3} \frac{\partial \theta_{4}}{\partial u}-\theta_{4} \frac{\partial \theta_{3}}{\partial u}\right)\left(\theta_{1} \frac{\partial^{2} \theta_{2}}{\partial u^{2}}-\theta_{2} \frac{\partial^{2} \theta_{1}}{\partial u^{2}}\right)\right] \\
& +\frac{\partial^{2} x}{\partial u^{2}}\left[\left(\theta_{1} \frac{\partial \theta_{2}}{\partial v}-\theta_{2} \frac{\partial \theta_{1}}{\partial v}\right)\left(\theta_{4} \frac{\partial \theta_{3}}{\partial u}-\theta_{3} \frac{\partial \theta_{4}}{\partial u}\right)\right. \\
& \left.\left.-\left(\theta_{1} \frac{\partial \theta_{2}}{\partial u}-\theta_{2} \frac{\partial \theta_{1}}{\partial u}\right)\left(\theta_{4} \frac{\partial \theta_{3}}{\partial v}-\theta_{3} \frac{\partial \theta_{4}}{\partial v}\right)\right]\right\} .
\end{aligned}
$$

In consequence of (80) this expression is symmetrical in $c$ and $c^{\prime}$, and the pairs of functions $\theta_{1}, \theta_{2}$ and $\theta_{3}, \theta_{4}$. Hence the net $\hat{N}$ can be obtained also by applying to $N$ the $W_{c^{\prime}}$ transformation determined by $\theta_{3}$ and $\theta_{4}$, and then to the resulting net $\overline{\bar{N}}$ the transformation determined by the functions

$$
\overline{\theta_{i}}=\theta_{i}+\frac{\left(\theta_{t} \frac{\partial \theta_{3}}{\partial v}-\theta_{3} \frac{\partial \theta_{4}}{\partial v}\right) \frac{\partial \theta_{i}}{\partial u}-\left(\theta_{4} \frac{\partial \theta_{3}}{\partial u}-\theta_{3} \frac{\partial \theta_{4}}{\partial u}\right) \frac{\partial \theta_{i}}{\partial v}}{\frac{\partial \theta_{3}}{\partial u} \frac{\partial \theta_{4}}{\partial v}-\frac{\partial \theta_{3}}{\partial v} \frac{\partial \theta_{4}}{\partial u}}(i=1,2),
$$

which are analogous to (76).

If $c^{\prime}=c$, the net $\hat{N}$ coincides with $N$. Hence if a net $\bar{N}$ is a $W_{c}$ transform of $N$ by means of functions $\theta_{1}$ and $\theta_{2}$, solutions of (60) and (72), and $\theta_{3}$ and $\theta_{4}$ are two other solutions linearly independent of $\theta_{1}$ and $\theta_{2}$, the functions (76) determine $N$ as a $W_{c}$ transform of $\bar{N}$.

When $c^{\prime} \neq c$, we have that $\hat{N}$ is a $W_{c^{\prime}}$ transform of $\bar{N}$ and a $W_{c}$ transform of $\overline{\bar{N}}$. Hence: 
If $N$ is an $R$ net, and $N_{1}^{r}$ and $N_{2}$ are obtained from $N$ by transformations $W_{c_{1}}$ and $W_{c_{2}}$, there can be found directly an $R$ net $N_{12}$ which is a $W_{c_{2}}$ transform of $N_{1}$ and a $W_{c_{1}}$ transform of $N_{9}{ }^{37}$ ).

\section{Exercises.}

1. If $x_{i}(i=1,2,3,4)$ satisfy equations of the form (9), from the first of (10) and the first and fourth of (4) it follows that $x_{1}, x_{2}, x_{3}$ satisfy an equation of the form (1) with equal invariants. Hence the projection from a point upon any plane of the asymptotic lines of a surface is a plane net with equal point invariants.

Koenigs, Comptes Rendus, vol. 114 (1892), p. 5̃.

2. If $x_{1}, x_{z}, x_{z}$ are the coordinates of a planar net satisfying equations (2) and

the equations

$$
\frac{\partial^{2} \theta}{\partial u \partial v}=\frac{\partial \log \sigma}{\partial v} \frac{\partial \theta}{\partial u}+\frac{\partial \log \sigma}{\partial u} \frac{\partial \theta}{\partial v},
$$

$$
\frac{\partial y_{1}}{\partial u}=\frac{1}{\sigma^{2}}\left(x_{2} \frac{\partial x_{3}}{\partial u}-x_{s} \frac{\partial x_{2}}{\partial u}\right), \quad \frac{\partial y_{1}}{\partial v}=-\frac{1}{\sigma^{2}}\left(x_{2} \frac{\partial x_{3}}{\partial v}-x_{3} \frac{\partial x_{2}}{\partial v}\right)
$$

are consistent. In like manner we obtain by quadratures two functions $y_{2}$ and $y_{3}$ by permuting the subscripts of the $x$ 's cyclicly. Show that the function

$$
x_{4}=x_{1} y_{1}+x_{4} y_{2}+x_{3} y_{3}
$$

satisfies equations (2). Hence $x_{i}(i=1,2,3,4)$ are the coordinates of a surface $S$ referred to its asymptotic lines.

Annals, vol. 18 (1917), p. 224.

3. The functions $y_{1}, y_{2}, y_{s},-1$ in Ex. 2 are the homogeneous point coordinates of the asymptotic lines of a surface which is the polar reciprocal of $S$ with respect to the quadric $x_{1}^{2}+x_{2}^{2}+x_{3}^{2}+x_{4}^{2}=0$.

Annals, l. c., p. 225.

4. A necessary and sufficient condition that the surface $S$ whose coordinates satisfy (9) be a ruled surface is that $a_{z}=0$ or $b_{1}=0$; in this case the curves $u=$ const. or $v=$ const. are straight lines.

5. If in accordance with the first of (10) we put $a_{1}=2 \frac{\partial \log \lambda}{\partial u}, \quad b_{3}=2 \frac{\partial \log \lambda}{\partial v}$; then the coordinates $\bar{x}^{i}=x^{i} / \lambda$ satisfy equations of the form

$$
\frac{\partial^{2} \theta}{\partial u^{2}}=b_{1} \frac{\partial \theta}{\partial v}+c_{1} \theta, \quad \frac{\partial^{2} \theta}{\partial v^{2}}=u_{2} \frac{\partial \theta}{\partial u}+c_{2} \theta
$$

called by Wilczynski the canonical form of (9). Trans., vol. 8 (1907), p. 247.

6. Let $S$ be a ruled surface for which $u=$ const. are the straight lines and the equations are in the canonical form (Ex. 5). Now $a_{2}=0$, and from (10) we have $\frac{\partial c_{2}}{\partial u}=0$. The tangents to the curved asymptotic lines at points of a generator $u=$ const. form a ruled surface $R_{u}$ whose coordinates are given by

$$
y=v x+\frac{\partial x}{\partial u}
$$

$\left.{ }^{37}\right)$ Annals, l. c., p. 172. 
It is readily found that the $y$ s satisfy the equations

$$
\frac{\partial^{2} y}{\partial v^{2}}=c_{2} y, \quad \frac{\partial^{2} y}{\partial w^{2}}=0 .
$$

Hence $k_{u}$ is a quadric, which osculates $S$ along the given generator.

7. If two surfaces $S$ and $\bar{S}$ in 3-space are so related that each net on $\bar{S}$ is a radial transform of a net on $S$, then

where $a_{j}, d$ are constauts.

$$
\bar{x}^{(i)}=\frac{x^{(i)}}{\sum_{j} a_{j} x^{(j)}+d},
$$

8. If two parallel nets in 3-space have areas preserved, the total curvature is the same at corresponding points.

Guichard, Comptes Rendus, vol.136, p.151.

9. The focal points of a ray congruence are given by $\lambda x_{-1}+\mu x_{1}$, where $K \lambda^{2}+R \lambda \mu-r H \mu^{2}=0$; they are the intersections of the lines of the congruence and the tangents to the curres

$$
H^{*} d u^{2}+R d u d v-v H d v^{2}=0 .
$$

10. A necessary and sufficient condition that the tangents to the ray curves of a net $X$ pass through the corresponding focal points of the ray congruence is that $Y$ have equal point invariants.

Green, Amer. Journ., vol. 38 (1916), p. 313.

11. Let $N$ be a net with equal points invariants on a surface $S$ and $N_{0}$ its associate net on a surface $S_{0}(\$ 25)$; also let $\Sigma$ and $\Sigma_{0}$ be the surfaces corresponding with orthogonality of linear elements to $S$ and $S_{0}$ as determined by $N_{0}$ and $N$ respectively [ $\S 157$ ]. If $N_{1}$ is a $K$ transform of $N$, it is possible to place the associate, $N_{10}$, of $N_{1}$ so that $N_{0}$ and $N_{10}$ are in relation $K$ (II. Ex. 18). These nets determine surfaces $\Sigma_{1}$ and $\Sigma_{10}$ corresponding to $S_{1}$ and $S_{10}$ by orthogonality of linear elements. Show that the pairs of surfaces $\Sigma, \Sigma_{1} ; \Sigma, \Sigma_{0} ; \Sigma_{10}, \Sigma_{0}$ and $\Sigma_{10} \Sigma_{1}$ are the focal surfaces of $W$ congruences; that is the $K$ transformation from $N$ into $N_{1}$ determines a quatern of $W$ congruences.

Trans., vol. 15 (1914), p. 415.

12. Four nets $N, N_{1}, N_{2}, N_{12}$ forming a quateru under transformations $K$ determine twelve $W$ congruences, forming six quatems.

Trans. 1. c., p. 416.

13. The focal nets of the congruence of normals to a surface of constant currature are $R$ nets.

Tzitzeica, Comptes Rendus, vol. 152 (1911), p.1078.

14. The lines of curvature of a surface of constant curvature and the normals to the surface are in relation $\theta_{1}$. Demoulin, Comptes Rendus, vol.153(1911), p. 798.

15. An isothermal orthogonal system on a sphere and the normals to the mininal surface, whose lines of curvature admit this system for spherical representation, are in relation $\theta_{1}$.

Demoulin, 1. c.

16. An $R$ net $N$ admits $\infty^{4}$ parallel nets determining congruences $G$ of transformations $F$ of $N$ into $R$ nets $N_{1}$; when such a parallel net is known, each solution $\theta$ of equations (60) and (74) defines a transform $N_{1}$.

Anuals, vol. 22 (1921), p. 176. 
17. If $N$ is an $R$ net, and $N_{1}$ and $N_{2}$ are two $F$ transforms of $N$ by means of functions $\theta_{1}$ and $\theta_{2}$ which are solutions of equations (60) and (74) for the same constant $c$, all of the $\infty^{2}$ nets $N_{12}$ which are $F$ transforms of $N_{1}$ and $N_{2}(\S 21)$ are $\boldsymbol{R}$ nets and their determination requires two quadratures; when the constant $c$ in (74) is different for $\theta_{1}$ and $\theta_{2}$, there is a unique net $N_{12}$ which is an $R$ ret and it can be found without quadratures.

Anuals, vol. 22 (1921), p. 178.

18. If $\bar{N}$ is a $W$ transform of an $R$ net $N$ by means of solutions $\theta_{1}$ and $\theta_{2}$ of equations (60) and (74), and $N_{3}$ is an $R$ net, which is an $F$ transform of $N$ by means of a solution $\theta_{3}$ of (60) and (74) with $c$ replaced by $c^{\prime}$, there can be found directly a unique net $\bar{N}_{3}$ which is a $W$ transform of $N_{3}$ and an $F$ transform of $\bar{N}$; when $c=c^{\prime}$, there are $\infty^{2}$ such nets $\bar{N}_{3}$ obtained by two quadratures.

Annals, vol. 22 (1921), p. 181.

19. From the equation (15) of a net $N(x)$ on a surface $S$ it follows that the point of coordinates $z=\frac{\partial^{2} x}{\partial u^{2}}+\frac{a^{\prime}}{r} \frac{\partial x}{\partial u}$ lies on the intersection $l$ of the osculating planes of the curves of $N$ at the corresponding point. This line generates a congruence whose focal points are determined by functions $\lambda$ and $\mu$ such that $d(\lambda x+\mu z)$ is a linear functions of $x$ and $z$. Proceeding as in $\S 44$, we show that the developables of the congruence are determined by the equation

$$
r C_{1} d u^{2}+R d u d v-r\left(B_{2}+\frac{\partial}{\partial v}\left(\frac{a^{\prime}}{r}\right)\right) d v^{2}=0
$$

where $R$ is given by (2 7 ). The curves on $S$ defined by (i) are called the axis curves and the congruence the axis congruence of $\mathrm{X}$. By means of (22) equation (i) may be written

$$
v C_{1} d u^{2}+h d u d v-v\left(H-K+v C_{1}-\frac{\partial^{2}}{\partial u \partial v} \log v\right) d v^{2}=0
$$

Wilczynski, Trans., vol. 16 (1915), p. 316;

Green, Amer. Journ., vol. 38 (1916), p. 308.

20. A necessary and sufficient condition that the axis curres form a conjugate system is

$$
H-K^{*}-\frac{\partial^{2}}{\partial u \partial v} \log r=0
$$

Green, 1. c., p. 311.

21. When a net has equal point invariants and is isothermal-conjugate, the ray curves and the axis curves form nets; and conversely.

Green, l. c., p. 321.

22. When a net is subjected to a polar reciprocation the axis and ray congruences are interchanged.

Wilczynski, 1. c.. p.317.

23. Show that at any point on a surface $S$ the tangents to the two systems of curves defined by the equations

$$
\begin{aligned}
& a_{1} d u^{2}+2 b_{1} d u d v+c_{1} d v^{2}=0, \\
& a_{2} d u^{2}+2 b_{2} d u d v+c_{2} d v^{2}=0,
\end{aligned}
$$


are separated harmonically by the tangents to the curves of the system defined by

$$
\left|\begin{array}{ll}
a_{1} d u+b_{1} d v, & b_{1} d u+c_{1} d v \\
a_{2} d u+b_{2} d v, & b_{2} d u+c_{2} d v
\end{array}\right|=0 .
$$

24. If the parametric curves on a surface $S$ form a net $N$ with equations (1) and (15), the curves defined by

$$
d u^{2}-v d r^{2}=0
$$

form a net, whose tangents at a point separate harnonically the tangents of $N$ and the asymptotic tangents.

25. A necessary and sufficient condition that a net $N(x)$ on a surface be isothermal-conjugate, that is $\frac{\partial^{2}}{\partial u \partial v} \log r=0$, is that the tangents to the axis curves, the curves defined by Ex. 24 and the curves defined by $H d u^{2}-R d u d v$ $-r K d v^{2}=0$ be pairs of the same involution, provided that the double lines of the involution are not the tangents of the net.

Green, Amer. Journ., vol. 38 (1916), p. 323.

Wilczynski, Amer. Journ., vol. 42 (1920), p. 216. 


\section{Chapter V.}

\section{Transformations $\Omega$. $W$ Congruences.}

5I. Tangential coordinates of a net. Laplace transforms in tangential coordinates. In [\$ 84] we found that a necessary and sufficient condition that the parametric curves on a surface in 3-space form a net is that the tangential coordinates $X, Y, Z, W$ satisfy a Laplace equation

$$
\frac{\partial^{2} \lambda}{\partial u \partial v}=\frac{\partial \log \alpha}{\partial v} \frac{\partial \lambda}{\partial u}+\frac{\partial \log \beta}{\partial u} \frac{\partial \lambda}{\partial v}+\gamma \lambda
$$

From $[22, \S 66]$ and $[34, \S 67]$ it follows that the tangential coordinates satisfy also the equation

$$
\frac{\partial^{2} \lambda}{\partial v^{2}}=r \frac{\partial^{2} \lambda}{\partial u^{2}}+\alpha^{\prime} \frac{\partial \lambda}{\partial u}+\beta^{\prime} \frac{\partial \lambda}{\partial v}+\gamma^{\prime} \lambda
$$

where in terms of the spherical representation of the net

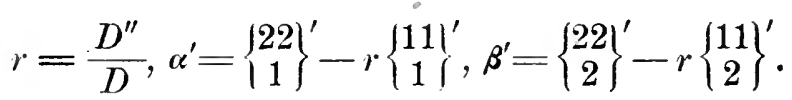

Conversely, if two equations of the form (1) and (2) admit four linearly independent solutions, the latter may be taken as the tangential coordinates of a net $N$, whose homogeneous point coordinates $x, y, z, w$ are given directly by the equations

$$
\sum X x=0, \sum \frac{\partial X}{\partial u} x=0, \sum \frac{\partial X}{\partial v} x=0 .
$$

We have also the following theorem analogous to the third theorem of $\S 43$ : 
Any four linearly independent solutions of equations (1) and (2) are the tangential coordinates of a net which is a mojective transform of the given net $N$.

We call (1) the tangential equation of the net $N$. If this equation has equal invariants, we say that the net has equal tangential invariants.

The homogeneous point coordinates $x, y, z, w$ and the tangential coordinates $X, Y, Z, W$ of a net in 3-space, satisfy respectively equations (IV, 1) and (1), and also the relations (IV, 5). If the second and third of the latter be differentiated with respect to " and $"$, we have accordingly

$$
\left\{\begin{array}{c}
\sum X \frac{\partial^{2} x}{\partial u^{2}}+\sum \frac{\partial X}{\partial u} \frac{\partial x}{\partial u}=0, \\
\sum \frac{\partial X}{\partial u} \frac{\partial x}{\partial v}=0, \quad \sum \frac{\partial X}{\partial v} \frac{\partial x}{\partial u}=0 \\
\sum X \frac{\partial^{2} x}{\partial v^{2}}+\sum \frac{\partial X}{\partial v} \frac{\partial x}{\partial v}=0 .
\end{array}\right.
$$

From the first and last of (5) we have in consequence of $(\mathrm{IV}, 5)$

$$
\sum \frac{\partial X}{\partial v} \frac{\partial x}{\partial r}-r \sum \frac{\partial X}{\partial u} \frac{\partial x}{\partial u}=0
$$

When the second and third of (5) are differentiated with respect to $v$ and $u$ respectively, the resulting equations are reducible by means of (1) and (5) to

$$
\left\{\begin{array}{l}
\sum\left(\frac{\partial X}{\partial u}-\frac{\partial \log \beta}{\partial u} X\right) \frac{\partial^{2} x}{\partial v^{2}}=0 \\
\Sigma\left(\frac{\partial X}{\partial v}-\frac{\partial \log \alpha}{\partial v} X\right) \frac{\partial^{2} x}{\partial u^{2}}=0 .
\end{array}\right.
$$

The minus first and first Laplace transforms of equation (1) are given by

$$
X_{-1}=\frac{\partial X}{\partial u}-\frac{\partial \log \beta}{\partial u} X, \quad X_{1}=\frac{\partial X}{\partial v}-\frac{\partial \log \alpha}{\partial v} X .
$$


From $\S 30,(5)$ and (7) we have

$$
\begin{aligned}
\sum X_{-1} x_{1}=0, & \sum X_{1} x_{-1}=0 \\
\sum X_{-1} \frac{\partial x_{1}}{\partial u}=0, & \sum X_{1} \frac{\partial x_{-1}}{\partial u}=0 \\
\sum X_{-1} \frac{\partial x_{1}}{\partial v}=0, & \sum X_{1} \frac{\partial x_{-1}}{\partial v}=0 .
\end{aligned}
$$

Hence we have the theorem:

When the Laplace transformations are applied to the tangential coordinates of a net $N$, the resulting functions are the tangential coordinates of the Laplace transforms of $N$, but in opposite sense.

52. Transformations $F$ in tangential coordinates. Since the analytical processes of $\S 37$ are independent of their geometrical interpretation, it follows that if $\lambda$ is a solution of (1) and $\mu$ a solution of its adjoint, namely

(9) $\frac{\partial^{2} \mu}{\partial u \partial v}=-\frac{\partial \log \alpha}{\partial v} \frac{\partial \mu}{\partial u}-\frac{\partial \log \beta}{\partial u} \frac{\partial \mu}{\partial v}+\left(\gamma-\frac{\partial^{2}}{\partial u \partial v} \log \alpha \beta\right) \mu$, there exist two functions $\bar{\tau}$ and $\bar{\sigma}$, defined to within additive constants by

$$
\begin{cases}\frac{\partial \bar{\tau}}{\partial u}=\lambda \mu \frac{\partial}{\partial u} \log \mu \beta, & \frac{\partial \bar{\tau}}{\partial v}=\lambda \mu \frac{\partial}{\partial v} \log \frac{\lambda}{\alpha} \\ \frac{\partial \bar{\sigma}}{\partial u}=-\lambda \mu \frac{\partial}{\partial u} \log \frac{\lambda}{\beta}, & \frac{\partial \bar{\sigma}}{\partial v}=-\lambda \mu \frac{\partial}{\partial v} \log \mu \alpha .\end{cases}
$$

Consider now a net $N$ with tangential coordinates $X, Y, Z, W$ satisfying (1). Then the functions $X_{1}, Y_{1}, Z_{1}, W_{1}$, given by quadratures of the form

$$
\frac{\partial X_{1}}{\partial u}=\bar{\tau} \frac{\partial}{\partial u}\left(\frac{X}{\lambda}\right), \quad \frac{\partial X_{1}}{\partial v}=\bar{\sigma} \frac{\partial}{\partial v}\left(\frac{X}{\lambda}\right),
$$

(which are consistent in consequence of (10)) satisfy the equation

$$
\frac{\partial^{2} \lambda_{1}}{\partial u \partial v}+\frac{\bar{\sigma}}{\bar{\tau}} \frac{\partial}{\partial v} \log \frac{\lambda}{\alpha} \frac{\partial \lambda_{1}}{\partial u}+\frac{\bar{\tau}}{\bar{\sigma}} \frac{\partial}{\partial u} \log \frac{\lambda}{\beta} \frac{\partial \lambda_{1}}{\partial v}=0,
$$


and consequently are tangential coordinates of a net $N_{1}$. It is our purpose to slow that $N$ and $N_{1}$ are in the relation $F$, and furthermore to find $\lambda$ in terms of the functions defining the transformation $F$, when $N$ and $N_{1}$ are given in terms of homogeneous point coordinates, $x$ and $x_{1}$.

On the supposition that $N$ and $N_{1}$ are in the relation $F$, their homogeneous point coordinates are related as in (III, 65). Necessarily we must have

$$
\sum x_{1} \frac{\partial X_{1}}{\partial u}=0, \quad \sum x_{1} \frac{\partial X_{1}}{\partial v}=0
$$

which in consequence of (III, 65) and (11) lead to

$$
\frac{\partial \log \lambda}{\partial u}=\frac{\sum y \frac{\partial X}{\partial u}}{\sum y X}, \quad \frac{\partial \log \lambda}{\partial v}=\frac{\sum y \frac{\partial X}{\partial v}}{\sum y X} .
$$

From (III, 60, 65) it follows that

$$
\sum X \frac{\partial y}{\partial u}=0, \quad \sum X \frac{\partial y}{\partial v}=0 .
$$

Hence, to within a constant factor, the integral of the above equations is

$$
\lambda=\sum y X
$$

In consequence of (5) we have by differentiation

$$
\frac{\partial \lambda}{\partial u}=\sum y \frac{\partial X}{\partial u}, \frac{\partial \lambda}{\partial v}=\sum y \frac{\partial X}{\partial v}, \frac{\partial^{2} \lambda}{\partial u \partial v}=\sum y \frac{\partial^{2} X}{\partial u \partial v}
$$

so that $\lambda$ is a solution of (1). From (II, 65) it follows that .

$$
\lambda=\sum y X=\sum z X .
$$

The analogues of equations (III, 65) are

$$
\bar{X}_{1}=X_{1}=\bar{\tau} \frac{X}{\lambda}, \quad \bar{X}_{2}=X_{1}-\bar{\sigma} \frac{X}{\lambda} .
$$


These functions $\bar{X}_{1}$ and $\bar{X}_{2}$ satisfy the equations

$$
\begin{cases}\frac{\partial \bar{X}_{1}}{\partial u}=-X \mu \frac{\partial}{\partial u} \log \mu \beta, & \frac{\partial \bar{X}_{1}}{\partial v}=-X \mu \frac{\partial}{\partial v} \log \frac{X}{\alpha} \\ \frac{\partial \bar{X}_{\mathbf{2}}}{\partial u}=X \mu \frac{\partial}{\partial u} \log \frac{X}{\beta}, & \frac{\partial \bar{X}_{2}}{\partial v}=X \mu \frac{\partial}{\partial v} \log \mu \alpha .\end{cases}
$$

We must have also

$$
\sum X_{1} \frac{\partial x_{1}}{\partial u}=0, \quad \sum X_{1} \frac{\partial x_{1}}{\partial v}=0,
$$

which by means of (III, 60) and (15) are reducible to

$$
\frac{\partial \log \theta}{\partial u}=\frac{\sum \bar{X}_{1} \frac{\partial x}{\partial u}}{\sum \bar{X}_{1} x}, \quad \frac{\partial \log \theta}{\partial v}=\frac{\sum \bar{X}_{1} \frac{\partial x}{\partial v}}{\sum \bar{X}_{1} x} .
$$

From these we find that we may take

$$
\theta=\sum \bar{X}_{1} x=\sum \bar{X}_{2} x .
$$

Moreover, this expression for $\theta$ satisfies equation (III, 1).

As a consequence of (13) and (17) we have that $\sum x_{1} x_{1}$ is a constant. However, we wish it to be equal to zero. Substituting from (III, 65) and (15), we find with the aid of (14) and (18) that this is accomplished, if the additive constants of integration of $\overline{\boldsymbol{\tau}}$ and $\overline{\boldsymbol{\sigma}}$ are chosen so that

$$
\left\{\begin{array}{l}
\tau+\bar{\tau}-\sum y \bar{X}_{1}=0 \\
\sigma+\bar{\sigma}-\sum z \bar{X}_{2}=0
\end{array}\right.
$$

Thus we have established that the net $N_{1}$ whose tangential coordinates are given by (11) is in the relation $F$ with $N$. Moreover, when the transformation is given in point coordinates, the functions $\lambda$ and $\mu$ follow from (14), (11) and (10); and conversely, when the transformation is defined in terms of tangential coordinates.

In consequence of $(\mathrm{III}, 65)$ and (15) the expressions for $\lambda$ and $\theta$ can be given also the forms

$$
\lambda=\sum X x_{1}, \quad \theta=\sum X_{1} x .
$$


By making use of the results of $\S 39$, we can obtain the equations of the theorem of permutability of transformations $F$ from the standpoint of tangential coordinates. The functions $\lambda_{12}$ and $\lambda_{21}$ must satisfy

$$
\frac{\partial \lambda_{i j}}{\partial u}=\overline{\boldsymbol{x}}_{i} \frac{\partial}{\partial u}\left(\frac{\lambda_{j}}{\lambda_{i}}\right), \quad \frac{\partial \lambda_{i j}}{\partial v}=\overline{\boldsymbol{\sigma}}_{i} \frac{\partial}{\partial v}\left(\frac{\lambda_{j}}{\lambda_{i}}\right)(i, j=1,2, i \neq j) .
$$

The functions $\bar{\sigma}_{12}, \bar{\sigma}_{21}, \bar{\tau}_{12}, \bar{\tau}_{21}$ are given by

$$
\left\{\begin{array}{l}
\bar{\tau}_{1} \bar{\tau}_{12}=\bar{\tau}_{2} \bar{\tau}_{21}=\frac{\lambda_{2} \lambda_{21} \bar{\tau}_{1}}{\lambda_{1}}+\frac{\lambda_{1} \lambda_{12} \bar{\tau}_{2}}{\lambda_{2}}-\lambda_{12} \lambda_{21} \\
\bar{\sigma}_{1} \bar{\sigma}_{12}=\bar{\sigma}_{2} \bar{\sigma}_{21}=\frac{\lambda_{2} \lambda_{21} \bar{\sigma}_{1}}{\lambda_{1}}+\frac{\lambda_{1} \lambda_{12} \bar{\sigma}_{2}}{\lambda_{2}}-\lambda_{12} \lambda_{21}
\end{array}\right.
$$

and the tangential coordinates $X_{12}$ of $N_{12}$ are of the form

$$
\lambda_{1} \lambda_{12} X_{12}=\lambda_{9} \lambda_{21} X_{1}+\lambda_{1} \lambda_{12} X_{2}-\lambda_{12} \lambda_{21} X .
$$

If equations similar to (20) are to be satisfied, we must have

$$
\left\{\begin{array}{l}
\lambda_{12}=\sum X_{1} x_{12}=\sum X_{1} x_{2}-\theta_{21} \\
\lambda_{21}=\sum X_{2} x_{12}=\sum X_{2} x_{1}-\theta_{12}
\end{array}\right.
$$

When these equations are differentiated, we find that the resulting equations are satisfied in virtue of the preceding formulas. Also we find that $x_{12}$, given by (III; 76), and $X_{12}$ given by (22) satisfy the condition $\sum X_{12} x_{12}=0$. Hence we may take $\lambda_{12}$ and $\lambda_{21}$ as just given.

Equations similar to (III, 65) and (15) are

$$
\begin{aligned}
y_{i j} & =x_{i j}-\frac{\boldsymbol{\tau}_{i j}}{\theta_{i j}} x_{i}, & z_{i j} & =x_{i j}-\frac{\sigma_{i j}}{\theta_{i j}} x_{i}, \\
\bar{X}_{1, i j} & =X_{i j}-\frac{\overline{\boldsymbol{\tau}}_{i j}}{\lambda_{i j}} X_{i}, & \bar{X}_{2, i j} & =X_{i j}-\frac{\bar{\sigma}_{i j}}{\lambda_{i j}} X_{i}
\end{aligned}
$$

From these equations, (20) and (23) we obtain

$$
\sum \bar{X}_{1, i j} y_{i j}-\tau_{i j}-\overline{\boldsymbol{\tau}}_{i j}=0, \quad \sum \bar{X}_{2, i j} z_{i j}-\sigma_{i j}-\bar{\sigma}_{i j}=0 .
$$


Consequently when $\lambda_{12}$ and $\lambda_{21}$ have the values (23), the expressions (22) are the tangential coordinates of $N_{12}$, whose point coordinates are given by (III, 76). From the form of (22) we are led at once to the theorem of $\S 23$, namely:

When $N, N_{1}, N_{2}$ aud $N_{12}$ form a quatern under transformations $F$ four corresponding tangent planes meet in a point.

When the point coordinates are cartesian, we make use of the preceding results by taking $w=-1$, and assuming that $X$ $Y, Z$ are direction-cosines. Then we have

$$
X x+Y y+Z z=W
$$

so that $W$ is the distance of the tangent plane from the origin.

From (III, 60) and (II, 20) we have in this case $u_{1}=\theta^{\prime} / \theta$ and $x_{1}$ in the formulas of Chapter 3 must be replaced by $-x_{1} \theta^{\prime} / \theta$, so as to obtain formulas of Chapter 2. From these results, (II, 2) and (20) we have $\lambda=\sum X x^{\prime 38}$ ), so that $\lambda$ is the distance from the origin to the tangent plane to the net $N^{\prime}$ determining the conjugate congruence of the transformation. If we call it $w$ and denote by $w^{-1}$ the corresponding tangential coordinate of $N_{1}^{\prime}$, parallel to $N_{1}$, and determining the same congruence, we have

$$
\lambda=\sum X x^{\prime}=w, \quad \sum X_{1} x_{1}^{\prime}=w^{-1},
$$

where $X_{1}, Y_{1}, Z_{1}$ are the direction-cosines of the normal to $N_{1}$. If $W_{1}$ denotes the other tangential coordinate of $N_{1}$, we can write (11) in the form

$$
\begin{cases}\frac{\partial}{\partial u}\left(\frac{X_{1}}{w^{-1}}\right)=\bar{\tau} \frac{\partial}{\partial u}\left(\frac{X}{w}\right), & \frac{\partial}{\partial v}\left(\frac{X_{1}}{w^{-1}}\right)=\bar{\sigma} \frac{\partial}{\partial v}\left(\frac{X}{w}\right), \\ \frac{\partial}{\partial u}\left(\frac{W_{1}}{w^{-1}}\right)=\bar{\tau} \frac{\partial}{\partial u}\left(\frac{W}{w}\right), & \frac{\partial}{\partial v}\left(\frac{W_{1}}{w^{-1}}\right)=\bar{\sigma} \frac{\partial}{\partial v}\left(\frac{W}{w}\right) .\end{cases}
$$

In this case equations (15) become

$$
\bar{X}_{1}=\frac{X_{1}}{w^{-1}}-\bar{\tau} \frac{X}{w}, \quad \bar{X}_{2}=\frac{X_{1}}{w^{-1}}-\bar{\sigma} \frac{X}{w} .
$$

38) When we are dealing with cartesian coordinates, the symbol $\Sigma$ denotes the sum of the terms in $x, y$ and $z$. 
From these expressions it follows that if $\varepsilon$ denotes the angle between the tangent planes of $N$ and $N_{1}$, a necessary and sufficient condition that the harmonic congruence of the transformation be normal is that

$$
\left(w^{-1}\right)^{2} \bar{\tau} \bar{\sigma}-w w^{-1} \cos \varepsilon(\bar{\tau}+\bar{\sigma})+w^{2}=0 .
$$

53. Transformations $\Omega$ of nets with equal tangential invariants. From (15) it follows that a necessary and sufficient condition that the focal planes of the harmonic congruence of the transformation be harmonic with respect to the tangent planes to $N$ and $N_{1}$ is that (cf. V, Ex. 8)

$$
\overline{\boldsymbol{\tau}}+\overline{\boldsymbol{\sigma}}=0 .
$$

In this case we have from (10)

$$
\frac{\partial}{\partial u} \log \frac{\lambda}{\mu}=2 \frac{\partial}{\partial u} \log \beta, \quad \frac{\partial}{\partial v} \log \frac{\lambda}{\mu}=2 \frac{\partial}{\partial v} \log \alpha .
$$

Recalling that $\alpha$ and $\beta$ are determined only to within factors which are respectively functions of $u$ alone and $v$ alone, we see that in all generality the tangential equation (1) can in this case be written

$$
\frac{\partial^{2} \lambda}{\partial u \partial v}+\frac{\partial \log \sqrt{\varrho}}{\partial v} \frac{\partial \lambda}{\partial u}+\frac{\partial \log \sqrt{\varrho}}{\partial u} \frac{\partial \lambda}{\partial v}+\gamma \lambda=0 .
$$

If we put $\mu=-2 \varrho w, w$ being the solution of (29) determining the transformation, equations (10) can be integrated in the form

$$
\overline{\boldsymbol{\tau}}=-\bar{\sigma}=-\varrho w^{2} .
$$

Now the tangential equation of $N_{1}$ is

$$
\frac{\partial^{2} \lambda_{1}}{\partial u \partial v}-\frac{\partial}{\partial v} \log \sqrt{\varrho} w \frac{\partial \lambda_{1}}{\partial u}-\frac{\partial}{\partial u} \log \sqrt{\varrho} w \frac{\partial \lambda_{1}}{\partial v}=0 .
$$

Thus the tangential equations of both $N$ and $N_{1}$ have equal invariants. We say that $N$ and $N_{1}$ are in the relation of a transformation $\Omega^{39}$ ).

39) Rendiconti di Palermo, vol. 39 (1915), p.161; cf. also Demoulin, Bull. Acad. de Belgique, 1919, p. 273. 
If we put

$$
\vartheta=\lambda \sqrt{\varrho}, \quad \bar{\vartheta}=\frac{\lambda_{1}}{\sqrt{\varrho} w}
$$

equations (29) and (31) are transformed into

$$
\frac{\partial^{2} \vartheta}{\partial u \partial v}=\left(\frac{1}{\sqrt{\varrho}} \frac{\partial^{2} \sqrt{\varrho}}{\partial u \partial v}-\gamma\right) \vartheta, \frac{\partial^{2} \bar{\vartheta}}{\partial u \partial v}=\left(\sqrt{\varrho} w \frac{\partial^{2}}{\partial u \partial v} \frac{1}{\sqrt{\varrho} w}\right) \bar{\vartheta}
$$

These equations are satisfied respectively by

$$
\begin{cases}\nu_{1}=\sqrt{\varrho} X, & \nu_{2}=\sqrt{\varrho} Y, \quad \nu_{3}=\sqrt{\varrho} Z, \\ \bar{\nu}_{1}=\frac{X_{1}}{\sqrt{\varrho} w}, & \bar{\nu}_{2}=\frac{Y_{1}}{\sqrt{\varrho} w}, \quad \bar{v}_{3}=\frac{Z_{1}}{\sqrt{\varrho} w} .\end{cases}
$$

In terms of these functions equations (11) are reducible to

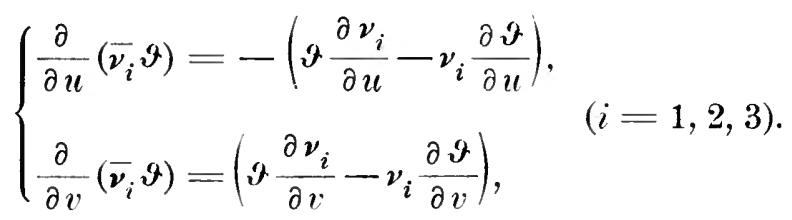

Since $N$ has equal tangential invariants, its spherical representation is the spherical representation also of the asymptotic lines on a surface $\mathbf{\Sigma}$ whose point coordinates, $\xi, \eta, \zeta$, are given by the Lelieuvre formulas $[\S 79]$, namely

$$
\frac{\partial \xi}{\partial u}=\nu_{2} \frac{\partial \nu_{3}}{\partial u}-\nu_{3} \frac{\partial \nu_{2}}{\partial u}, \quad \frac{\partial \xi}{\partial v}=-\nu_{2} \frac{\partial \nu_{3}}{\partial v}+\nu_{3} \frac{\partial \nu_{2}}{\partial v}
$$

Similar equations in the functions $\bar{\nu}_{i}$ give the point coordinates $\xi_{1}, \mu_{1}, \zeta_{1}$, of a surface $\Sigma_{1}$ with the same spherical representation of its asymptotic lines as the curves of the net $N_{1}$. Moreover, equations (33) express the condition that $\Sigma$ and $\Sigma_{1}$ are the focal surfaces of a $W$ congruence [ $[172]$. The surfaces $\Sigma$ and $\Sigma_{1}$ are associate to the respective nets $N$ and $N_{1}[\S 155]$. 
From the theory of $W$ congruences [\$ 172] it follows that if $X, Y, Z$ and $X_{1}, Y_{1}, Z_{1}$ are direction-cosines of the normals to $\Sigma$ and $\Sigma_{1}$, then

$$
\nu_{1}=\sqrt{\varrho} X, \quad \bar{\nu}_{1}=\sqrt{\varrho_{1}} X_{1},
$$

where $-1 / \varrho^{2}$ and $-1 / \varrho_{1}^{2}$ are the Gaussian total curvatures of $\Sigma$ and $\Sigma_{1}$ respectively. From these expressions, (26) and (32), we have

$$
w^{-1}=\frac{1}{\sqrt{\varrho \varrho_{1}} w} .
$$

Hence equations (26) become

$$
\left\{\begin{array}{l}
\frac{\partial}{\partial u}\left(\sqrt{\varrho \varrho_{1}} w X_{1}\right)=-\varrho w^{2} \frac{\partial}{\partial u}\left(\frac{X}{w}\right), \frac{\partial}{\partial v}\left(\sqrt{\varrho \varrho_{1}} w X_{1}\right)=\varrho w^{2} \frac{\partial}{\partial v}\left(\frac{X}{w}\right) \\
\frac{\partial}{\partial u}\left(\sqrt{\varrho \varrho_{1}} w W_{1}\right)=-\varrho u^{2} \frac{\partial}{\partial u}\left(\frac{W}{w}\right), \frac{\partial}{\partial v}\left(\sqrt{\varrho \varrho_{1} w} W_{1}\right)=\varrho w^{2} \frac{\partial}{\partial v}\left(\frac{W}{v}\right)
\end{array}\right.
$$

and we have the theorem:

Each solution $w$ of the tangential equation of a net with equal tangential invariants leads by quadratures (36) to the determination of a net $N_{1}$ which is an $\Omega$ transform of $N$.

From $[72, \$ 172]$ we have that the coordinates of the surface $\Sigma_{1}$ associate to $N_{1}$ are given by equations of the form

$$
\xi_{1}=\xi+\sqrt{\varrho \varrho_{1}}\left(Y Z_{1}-Z Y_{1}\right) \text {. }
$$

In [§ 172] it is shown that direction-parameters of the normals to the focal surfaces of any $W$ congruence satisfy equations of the form (33). Since these are of the form (11) with $\bar{\tau}+\bar{\sigma}=0$, we have

If two nets $N$ and $N_{1}$ with equal tangential invariants are in relation $F$, and if the surfaces $\mathbf{\Sigma}$ and $\Sigma_{1}$ with the same spherical representation of their asymptotic lines as the curves of $N$ and $N_{1}$ respectively can be so placed in space that they are the focal surfaces of a $W$ congruence, then $N$ and $N_{1}$ are in relation $\Omega$.

54. Theorems of permutability of transformations $\Omega$ and of $W$ congruences. Let $N$ be a net with equal tangential invariants, and $N_{1}$ and $N_{2}$ be $\Omega$ transforms of $N$ by means of solutions $w_{1}$ and $w_{2}$ of equation (1). 
The functions $w_{12}$ and $w_{81}$ defined by the quadratures

(38)

$$
\left\{\begin{array}{l}
\frac{\partial}{\partial u}\left(\sqrt{\varrho \varrho_{i}} w_{i} w_{i j}\right)=-\varrho w_{i}^{\frac{\partial}{\partial}} \frac{\partial}{\partial u}\left(\frac{w_{j}}{w_{i}}\right), \\
\frac{\partial}{\partial v}\left(\sqrt{\varrho \varrho_{i}} w_{i} w_{i j}\right)=\varrho w_{i}^{2} \frac{\partial}{\partial v}\left(\frac{w_{j}}{w_{i}}\right),
\end{array} \quad(i, j=1,2, i \neq j)\right.
$$

are evidently solutions of the tangential equations of $N_{1}$ and $N_{2}$ respectively. It is clear that $w_{i j}$ as thus defined is determined to within the additive function $\frac{c_{i}}{\sqrt{\varrho \varrho_{i} w_{i}}}$. The constants $c_{i}$ can be chosen so that there are $\infty^{1}$ pairs of functions $w_{18}$ and $v_{21}$ satisfying the condition

$$
\left.\sqrt{\varrho \varrho_{1}} w_{1} w_{12}+\sqrt{\varrho \varrho_{2}} w_{2} w_{21}=0^{40}\right) .
$$

By applying the results of $\S 52$ we shall show that each pair of functions determines a net $N_{12}$ which is an $\Omega$ transform of $N_{1}$ and $N_{2}$. In fact, the tangential coordinates $X_{12}, Y_{12}, Z_{12}, W_{12}$ of $N_{12}$ are given by the following equations analogous to (22):

$$
\sqrt{\varrho_{12}} X_{12}=\sqrt{\varrho}\left(X-\frac{w_{2}}{w_{19}} X_{1}-\frac{w_{1}}{w_{21}} X_{2}\right),
$$

where

(41) $\varrho_{12}=\varrho\left(1+\frac{w_{2}^{2}}{w_{12}^{2}}+\frac{w_{1}^{2}}{w_{21}^{2}}-2 \frac{w_{2}}{w_{12}} \cos \varepsilon_{1}-2 \frac{w_{1}}{w_{21}} \cos \varepsilon_{2}+2 \frac{w_{1} w_{2}}{w_{12} w_{21}} \sum X_{1} X_{2}\right)$.

It is readily shown that, in consequence of (36) and (39), the functions (40) satisfy the equations

$$
\left\{\begin{array}{l}
\frac{\partial}{\partial u}\left(\sqrt{\varrho_{i} \varrho_{i j}} w_{i j} X_{12}\right)=-\varrho_{i} w_{i j}^{2} \frac{\partial}{\partial u}\left(\frac{X_{i}}{w_{i j}}\right), \\
\frac{\partial}{\partial v}\left(\sqrt{\varrho_{i} \varrho_{i j}} w_{i j} X_{12}\right)=\varrho_{i} w_{i j}^{\mathbf{q}} \frac{\partial}{\partial v}\left(\frac{X_{i}}{w_{i j}}\right),
\end{array}(i, j=1,2, i \neq j) .\right.
$$

Hence we have established the theorem:

If $N$ has equal tangential invariants, and $N_{1}$ and $N_{2}$ are two $\Omega$ transforms of $N$, by a quadrature we can find $\infty^{1}$ nets $N_{12}$, which are $\Omega$ transforms of $N_{1}$ and $N_{2}$.

t0) The reason for this choice is to be found in (21). 
If $\Sigma, \Sigma_{1}$ and $\Sigma_{2}$ are the surfaces associate to $N, N_{1}$ and $N_{2}$ so that $\Sigma$ and $\Sigma_{1}$ are the focal surfaces of a $W$ congruence, and also $\Sigma$ and $\Sigma_{2}$, the associate $\Sigma_{12}$ of a net $N_{12}$ can be so placed in space that $\Sigma_{1}$ and $\Sigma_{12}$ are focal surfaces of a $W$ congruence, and likewise $\Sigma_{2}$ and $\Sigma_{12}$. From equations analogous to (34) and (37) we have for the coordinates, $\xi_{12}, \eta_{12}, \zeta_{12}$, of $\Sigma_{12}$

$$
\xi_{12}=\xi-\sqrt{\varrho \varrho_{1}} \frac{w_{1}}{w_{21}}\left(Y_{1} Z_{2}-Y_{2} Z_{1}\right)
$$

Hence we have the theorem of Bianchi $\left.{ }^{41}\right)$ :

If $\mathbf{\Sigma}$ and $\Sigma_{1}$ are focal surfaces of $a W$ congruence and $\mathbf{\Sigma}$ and $\Sigma_{2}$ of a second $W$ congruence, there can be found by quadratures an infinity of surfaces $\Sigma_{12}$ such that $\Sigma_{1}$ and $\Sigma_{12}$ are focal surfaces of "W congruence, and likewise $\Sigma_{1}$ and $\Sigma_{12}$.

55. Nets permanent in deformation. When the harmonic congruence of a transformation $\Omega$ is normal, the focal planes of the congruence are perpendicular, and consequently the angles between the tangent planes of the nets $N$ and $N_{1}$ are bisected by these focal planes. Now from (28), (30) and (35) we have $\varrho=\varrho_{1}$. Hence:

A necessary and sufficient condition that the harmonic congruence of a transformation $\Omega$ be normal is that the associate surfaces $\Sigma$ and $\Sigma_{1}$ of $N$ and $N_{1}$ have the same total curvature at corresponding points; moreover, the focal planes of the congruence bisect the angles between the tangent planes of $N$ and $N_{1}$.

If $\varrho=\varrho_{1}$, we have from (34)

$$
\sum \nu_{1} \bar{\nu}_{1}=\varrho \cos \varepsilon, \quad \sum \nu_{1}^{2}=\sum{\overline{\nu_{1}^{2}}}^{2}=\varrho .
$$

Multiplying the first of (33) by $\nu_{i}$ and summing; and also by $\overline{\nu_{i}}$ and summing, we get

$$
\begin{aligned}
& -\sum \nu_{i} \frac{\partial \bar{v}_{i}}{\partial u}=\frac{1}{2} \frac{\partial \varrho}{\partial u}+\varrho(\cos \varepsilon-1) \frac{\partial \log \vartheta}{\partial u}, \\
& -\sum \bar{v}_{i} \frac{\partial \nu_{i}}{\partial u}=\frac{1}{2} \frac{\partial \varrho}{\partial u}+\varrho(1-\cos \varepsilon) \frac{\partial \log \vartheta}{\partial u} .
\end{aligned}
$$

il) Lezioni, vol. 2, pp. 71-74. 
Adding, we have

$$
\frac{\partial}{\partial u} \sum \nu_{i} \bar{\nu}_{i}=-\frac{\partial \varrho}{\partial u}
$$

Differentiating the first of (44), we get

$$
\frac{\partial}{\partial u} \cos \varepsilon=-(1+\cos \varepsilon) \frac{\partial \log \varrho}{\partial u} .
$$

In like manner from the second of (33) we get

$$
\frac{\partial}{\partial v} \cos \varepsilon=(1-\cos \varepsilon) \frac{\partial \log \varrho}{\partial v}
$$

Expressing the condition of integrability of these two equations, we find that

$$
\varrho=U+V
$$

where $U$ and $V$ are functions of $u$ and $v$ alone respectively. Now the integral of equations (45) and (46) is

$$
\tan \frac{\varepsilon}{2}=\sqrt{\frac{U-c}{V+c}}
$$

where $c$ is an arbitrary constant.

From [ $\S 141]$ it follows that in this case both $N$ and $N_{1}$ are nets which admit an infinity of applicable nets, which we shall call nets permanent in deformation.

If $D$ and $D^{\prime \prime}$ are the second fundamental coefficients of $N$, the coefficients $D_{k}$ and $D_{k}^{\prime \prime}$ of the nets $N_{k}$ applicable to $N$ are given by [cf. $§ 141$ ].

$$
D_{k}=\tanh \varphi D, \quad D_{k}^{\prime \prime}=\operatorname{coth} \varphi D^{\prime \prime},
$$

where $\varphi$ is defined by

(50) $\frac{\partial \varphi}{\partial u}=-\frac{\partial \log \sqrt{\varrho}}{\partial u} \tanh \varphi, \quad \frac{\partial \varphi}{\partial v}=-\frac{\partial \log \sqrt{\varrho}}{\partial v} \operatorname{coth} \varphi$. 
Moreover,

$$
\frac{\partial \log \varrho}{\partial u}=-2\left\{\begin{array}{c}
12 \\
2
\end{array}\right\}^{\prime}, \quad \frac{\partial \log \varrho}{\partial v}=-2\left\{\begin{array}{c}
12 \\
1
\end{array}\right\}^{\prime},
$$

the Christoffel symbols being formed with respect to the linear element of the spherical representation of $N$, namely

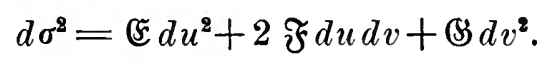

From the formulas of [§ 83], namely

$$
\mathfrak{F}=\frac{G D^{2}}{H^{2}}, \quad \mathfrak{F}=-\frac{F D D^{\prime \prime}}{H^{2}}, \quad \mathfrak{S}=\frac{E D^{\prime 2}}{H^{2}},
$$

it follows that the coefficients $\mathfrak{E}_{k}, \mathfrak{F}_{k}, \mathbb{G}_{k}$ of the linear element of the spherical representation of $N_{k}$ are given by

$$
\mathfrak{F}_{k}=\tanh ^{2} \varphi \mathfrak{E}, \quad \mathfrak{F}_{k}=\mathfrak{F}, \quad \mathbb{G}_{k}=\operatorname{coth}^{2} \varphi \mathbb{G} .
$$

The integral of equations (50) for $\varrho$ given by $(47)$ is

$$
\tanh \varphi=\sqrt{\frac{1+k V}{1-k U}}
$$

where $k$ is a constant, such that when $k=0$ we have the net $N$, that is $N_{0}=N$.

If we form the Christoffel symbols $\left\{\begin{array}{c}12 \\ 1\end{array}\right\}_{k}^{\prime}$ and $\left\{\begin{array}{l}12\}^{\prime} \\ 2\}_{k}\end{array}\right.$ with respect to (53), the equations analogous to (51) are

$$
\left\{\begin{array}{l}
\frac{\partial \log \varrho_{k}}{\partial u}=-2\left\{\begin{array}{c}
12 \\
2
\end{array}\right\}_{k}^{\prime}=\frac{U^{\prime}}{U+V} \frac{1+k V}{1-k U}, \\
\frac{\partial \log \varrho_{k}}{\partial v}=-2\left\{\begin{array}{c}
12 \\
1
\end{array}\right\}_{k}^{\prime}=\frac{V^{\prime}}{U+V} \frac{1-k U}{1+k V},
\end{array}\right.
$$

from which it follows that we may take

$$
\varrho_{k}^{\prime}=\frac{U+V}{(1-k U)(1+k V)}=\frac{1}{k}\left(\frac{1}{1-k U}-\frac{1}{1+k V}\right) .
$$


56. Transformations $\Omega$ of permanent nets for which $\mathbb{E} \neq \mathbf{0}$ and $\mathbb{S} \neq 0$. We write the linear element of the sphere in the form

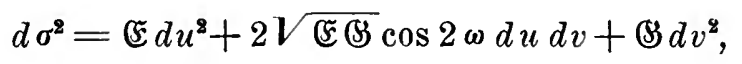

where $2 \omega$ is the angle between the parametric curves.

We denote by $X^{\prime}, Y^{\prime}, Z^{\prime} ; X^{\prime \prime}, Y^{\prime \prime}, Z^{\prime \prime}$ the direction-cosines of the bisectors of the angles between the parametric curves on the sphere, so that we have

$$
\left\{\begin{array}{l}
\frac{\partial X}{\partial u}=\sqrt{\mathfrak{E}}\left(\sin \omega X^{\prime}+\cos \omega X^{\prime \prime}\right), \\
\frac{\partial X}{\partial v}=\sqrt{\mathscr{S}}\left(-\sin \omega X^{\prime}+\cos \omega X^{\prime \prime}\right) .
\end{array}\right.
$$

If these equations be differentiated with respect to $v$ and $u$ respectively and we make use of $[(22) \S 66]$, we find

$(59)$

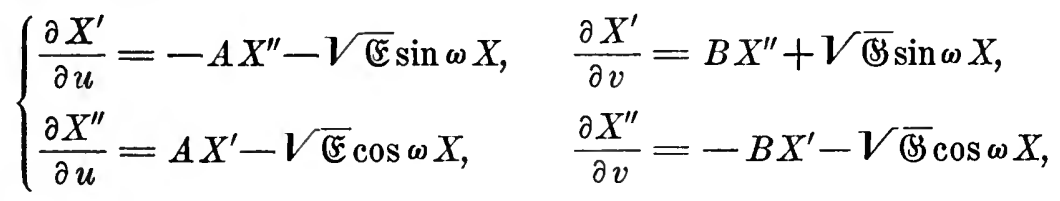

where

(60)

$$
\left\{\begin{array}{l}
A=\frac{\partial \omega}{\partial u}-\sqrt{\frac{\widetilde{F}}{\mathscr{S}}} \sin 2 \omega \frac{\partial \log \sqrt{\varrho}}{\partial v}, \\
B=\frac{\partial \omega}{\partial v}-\sqrt{\frac{\mathbb{G}}{\mathscr{E}}} \sin 2 \omega \frac{\partial \log \sqrt{\varrho}}{\partial u} .
\end{array}\right.
$$

The direction-cosines, $X_{1}, Y_{1}, Z_{1}$, of the normal to an $\Omega$ transform $N_{1}$ may be written in the form

$$
X_{1}=X \cos \varepsilon+\sin \varepsilon\left(\sin \alpha X^{\prime}-\cos \alpha X^{\prime \prime}\right) .
$$

Since $\varrho=\varrho_{1}$, equations (36) become

(62) $\frac{\partial}{\partial u}\left(\varrho w X_{1}\right)=-\varrho w^{2} \frac{\partial}{\partial u}\left(\frac{X}{w}\right), \quad \frac{\partial}{\partial v}\left(\varrho v X_{1}\right)=\varrho w^{2} \frac{\partial}{\partial v}\left(\frac{X}{w}\right)$.

When the expressions (61) are substituted in (62), we find the following equations: 


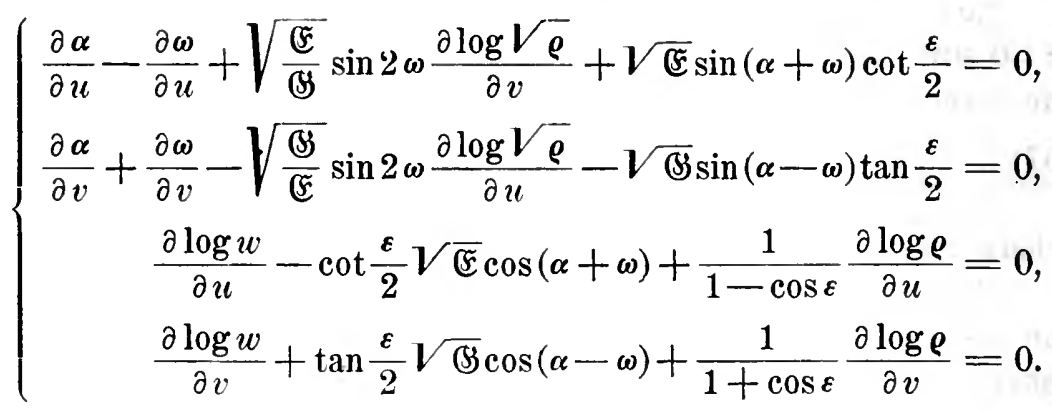

The condition of integrability of the first two equations is satisfied, and by means of them we show that the last two are consistent, and that $w$ satisfies the tangential equation of $N$, namely (29) with $\gamma=\sqrt{\sqrt{S}} \cos 2 \omega$. Hence:

If $N$ is a permanent net with the spherical representation (57), each pair of functions $\alpha$ and $w$ satisfying (63) leads by quadratures to an $\Omega$ transform $N_{1}$ which is a permanent net; and the associates surfaces $\Sigma$ and $\Sigma_{1}$ of $N$ and $N_{1}$ have the same total curvature at corresponding points and can be so placed in space that they are the focal surfaces of an $W$ congruence ${ }^{42}$ ).

From (37) it follows that the coordinates $\xi_{1}, \mu_{1}, \zeta_{1}$ of $\Sigma_{1}$ are of the form

$$
\xi_{1}=\xi+\sin \varepsilon\left(\cos \alpha X^{\prime}+\sin \alpha X^{\prime \prime}\right) .
$$

Since $w$ in (63) is not a general solution of (29), we say that the permanent nets whose tangential coordinates are $X, Y, Z$ and a function $w$ defined by (63) is a special permanent net.

Suppose that $N_{1}$ and $N_{2}$ are two $\Omega$ transforms of $N$ by means of functions $\alpha_{i}, w_{i}, \varepsilon_{i}(i=1,2)$. In order that one of the $\infty^{1}$ $\Omega$ transforms $N_{12}$ shall be a permanent net, it is necessary that $\varrho_{12}=\varrho$. From (39), (41) and (61) we have in this case

$w_{12}\left(\cos \varepsilon_{1}-\cos \varepsilon_{2}\right)=w_{2}\left[1-\cos \varepsilon_{1} \cos \varepsilon_{2}-\sin \varepsilon_{1} \sin \varepsilon_{2} \cos \left(\alpha_{1}-\alpha_{2}\right)\right]$.

It is readily shown that this function $w_{12}$ satisfies the corresponding equations (38), namely

42) 'The existence of such $W$ congruences was established by Bianchi Lezioni, vol. 2, pp. 74-80. 
(65) $\frac{\partial}{\partial u}\left(\varrho w_{1} w_{12}\right)=-\varrho w_{1}^{2} \frac{\partial}{\partial u}\left(\frac{w_{2}}{w_{1}}\right), \frac{\partial}{\partial v}\left(\varrho w_{1} w_{12}\right)=\varrho w_{1}^{2} \frac{\partial}{\partial v}\left(\frac{w_{2}}{w_{1}}\right)$.

Hence we have the following theorem of permutability:

If $N$ is a permanent net and $N_{1}$ and $N_{2}$ are permanent nets which are $\Omega$ transforms of $N$ by means of functions $\alpha_{1}, w_{1}, \varepsilon_{1}$ and $\alpha_{\mathbf{2}}, w_{2}, \varepsilon_{2}\left(\varepsilon_{2} \neq \varepsilon_{1}\right)$, of the $\infty^{1} \Omega$ transforms $N_{12}$ one is a permanent net and it can be found without quadratures.

Incidentally we have established the theorem of Bianchi ${ }^{43}$ ):

If $\Sigma$ and $\Sigma_{1}$ are focal surfaces of a $W$ congruence and $\Sigma$ and $\Sigma_{2}$ are focal surfaces of a second $W$ congruence such that the total eurvatures of $\Sigma, \Sigma_{1}$ and $\Sigma_{2}$ are the same, there can be found without quadratures a surface $\Sigma_{12}$ of the same total curvature such that $\Sigma_{1}$ and $\Sigma_{12}$ are focal surfaces of a W congruence, and likewise $\Sigma_{2}$ and $\Sigma_{12}$.

When in particular $\varrho=$ const., $N$ is the net of geodesics on a surface of Voss [ $\S 141]$. In this case $\varepsilon$ is a constant. Each set of solutions of the corresponding equations (63) determines an $Q$ transform $N_{1}$ which is a net of geodesics on a surface of Voss. Moreover, these transformations admit a theorem of permutability. The associated $W$ congruence is pseudospherical,-and the preceding theorem is the theorem of permutability of transformations of Bäcklund [ $\$ 121$ ].

57. Transformations $\Omega$ of a sequence of permanent nets. In $\S 56$ we saw that in order to obtain an $\Omega$ transformation of a permanent net $N$ info a permanent net $N_{1}$, it is necessary and sufficient to take for the net $N^{\prime}$ parallel to $N$ one of the special permanent nets determined by a pair of functions satisfying (63). Suppose now that we consider one of the deforms $N_{k}$ of $N(\S 55)$. The equations for the $\varrho$ transformations of $N_{k}$ analogous to (63) are

$$
\begin{aligned}
& \left(\frac{\partial \alpha_{k}}{\partial u}-\frac{\partial \omega}{\partial u}+\sqrt{\frac{\mathfrak{F}_{k}}{\mathfrak{G}_{k}}} \sin 2 \omega \frac{\partial}{\partial v} \log \sqrt{\varrho_{k}}+\sqrt{\mathfrak{F}_{k}} \sin \left(\alpha_{k}+\omega\right) \cot \frac{\varepsilon_{k}}{2}=0,\right. \\
& \frac{\partial \alpha_{k}}{\partial v}+\frac{\partial \omega}{\partial v}-\sqrt{\frac{\mathscr{S}_{k}}{\mathfrak{F}_{k}}} \sin 2 \omega \frac{\partial}{\partial u} \log \sqrt{\varrho_{k}}-\sqrt{\mathscr{G}_{k}} \sin \left(\alpha_{k}-\omega\right) \tan \frac{\varepsilon_{k}}{2}=0 \\
& \frac{\partial \log w_{k}}{\partial u}-\cot \frac{\varepsilon_{k}}{2} \sqrt{\mathfrak{E}_{k}} \cos \left(\alpha_{k}+\omega\right)+\frac{1}{1-\cos \varepsilon_{k}} \frac{\partial \log \varrho_{k}}{\partial u}=0, \\
& \frac{\partial \log w_{k}}{\partial v}+\tan \frac{\varepsilon_{k}}{2} \sqrt{\Theta_{k}} \cos \left(\alpha_{k}-\omega\right)+\frac{1}{1+\cos \varepsilon_{k}} \frac{\partial \log \varrho_{k}}{\partial v}=0,
\end{aligned}
$$

43) Lezioni, vol. 2 , pp. $80-82$. 
where $\mathfrak{E}_{k}, \mathbb{S}_{k}$ and $\varrho_{k}$ are given by ( 53$)$ and (56), and analogously to (48) we have

$$
\tan \frac{\varepsilon_{k}}{2}=\sqrt{\frac{\frac{1}{k U-1}+k c_{k}}{\frac{1}{k V+1}-k c_{k}}} .
$$

When the expressions for $\mathbb{E}_{k}, \mathbb{S}_{k}$ and $\varrho_{k}$ are substituted in the first two of (66), we find that $\alpha_{k}=\alpha$ is a solution, if

$$
\operatorname{coth} \varphi \cdot \tan \frac{\varepsilon_{k}}{2}=\tan \frac{\varepsilon}{2},
$$

which is consistent with (67), if we take

$$
k c_{k}(1-c k)=1 \text {. }
$$

On this hypothesis the last two of (66) are reducible to

$$
\left\{\begin{array}{l}
\frac{\partial \log w_{k}}{\partial u}=\frac{\partial \log w}{\partial u}-\frac{1}{2} \frac{U^{\prime} k}{1-k U} \\
\frac{\partial \log w_{k}}{\partial v}=\frac{\partial \log w}{\partial v}+\frac{1}{2} \frac{V^{\prime} k}{1+k V}
\end{array}\right.
$$

of which the integral is $w_{k}=\sigma w \sqrt{(1-k U)(1+k V)}$, where $\sigma$ is a constant. We shall show that when $\sigma=(1-k c)^{-1}$ the corresponding net $N_{k}^{\prime}$ parallel to $N_{k}$ is applicable to $N^{\prime}$. To this end we remark that the second fundamental coefficients $\left(D_{k}\right)^{\prime}$ and $\left(D_{k}^{\prime \prime}\right)^{\prime}$ of the net with the spherical representation (53) and determined by $w_{k}$ are given by [cf. $\S 67$ ]

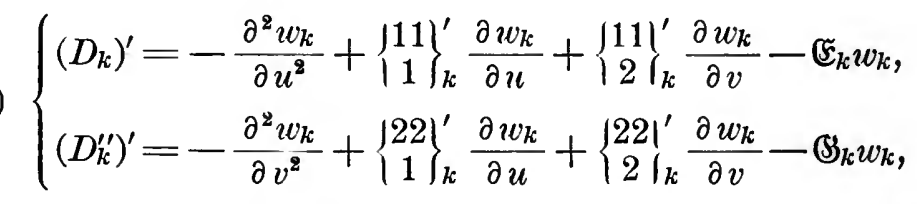

where the symbols $\left\{\begin{array}{c}r s s \\ t\end{array}\right\}_{k}^{\prime}$ are formed with respect to (53). When we put $k=0$ in these expressions, we obtain the expressions for the coefficients $(D)^{\prime}$ and $\left(D^{\prime \prime}\right)^{\prime}$ for $N^{\prime}$ by definition. 
When the epressions (66) are substituted in (69), we deduce the relations

$$
\frac{\left(D_{k}\right)^{\prime}}{w_{k}} \frac{1-k U}{1-k c}=\frac{(D)^{\prime}}{w}, \quad \frac{\left(D_{k}^{\prime \prime}\right)^{\prime}}{w_{k}} \frac{1+k V}{1-k c}=\frac{\left(D^{\prime \prime}\right)^{\prime}}{w}
$$

From (49) and (54) it follows that we must have

Hence

$$
\left(D_{k}\right)^{\prime}=\sqrt{\frac{1+k V}{1-k U}}(D)^{\prime}, \quad\left(D_{k}^{\prime \prime}\right)^{\prime}=\sqrt{\frac{1-k U}{1+k V}}\left(D^{\prime \prime}\right)^{\prime} .
$$

$$
w_{k}=\frac{\sqrt{(1-k U)(1+k V)}}{1-k c} w
$$

In $\S 27$ we saw that if $N(x)$ and $N_{k}\left(x_{k}\right)$ are applicable nets, and $N^{\prime}\left(x^{\prime}\right)$ and $N_{k}^{\prime}\left(x_{k}^{\prime}\right)$ are a pair of applicable nets parallel to $N$ and $N_{k}$ respectively, the $F$ transforms, $N_{1}$ and $N_{k, 1}$ of $N$ and $N_{k}$ determined by the nets $N^{\prime}$ and $N_{k}^{\prime}$ and the function $\theta^{\prime}=\sum x_{k}^{\prime 2}-\sum x^{\prime 2}$ are applicable to one another. In order to apply this result to the present case we calculate the expression for $\theta^{\prime}$. From [\$ 67] we have

$$
\begin{aligned}
& \sum x_{k}^{\prime 2}=w_{k}^{2}+\frac{1}{\mathfrak{S}^{2}}\left[\mathscr{S}_{k}\left(\frac{\partial w_{k}}{\partial u}\right)^{2}-2 \mathfrak{F}_{k} \frac{\partial w_{k}}{\partial u} \frac{\partial w_{k}}{\partial v}+\mathfrak{E}_{k}\left(\frac{\partial w_{k}}{\partial v}\right)^{2}\right] \\
& \sum x^{\prime 2}=w^{2}+\frac{1}{\mathfrak{S}^{2}}\left[\mathfrak{S}\left(\frac{\partial w}{\partial u}\right)^{2}-2 \mathfrak{F} \frac{\partial w}{\partial u} \frac{\partial w}{\partial v}+\mathfrak{E}\left(\frac{\partial w}{\partial v}\right)^{2}\right] .
\end{aligned}
$$

Substituting the values from the preceding equations, we find that

$$
\theta^{\prime}=\frac{k}{1-k c} \frac{w^{2} \sin 2 \omega}{\mathfrak{S}^{2}}[\mathfrak{E S}(U+V) \sin 2 \alpha
$$

$-\sqrt{\mathscr{S}}\left(S \cot \frac{\varepsilon}{2} \sin (\alpha-\omega) U^{\prime}+\mathscr{E} \sqrt{\mathscr{S}} \tan \frac{\varepsilon}{2} \sin (\alpha+\omega) V^{\prime}\right]$.

We note that $k$ appears in this expression only in the factor $k /(1-k c)$, and consequently it does not appear in the equation $x_{1}=x-\frac{\theta}{\theta^{\prime}} x^{\prime}$, since $\theta$ also involves this factor. Hence as $k$ 
varies and consequently $N_{k}$, we find that all the transforms $N_{k, 1}$ are applicable to $N_{1}{ }^{44}$ ).

58. Transformations $\Omega$ in point coordinates. Nets in relation $O$. In $\S 28$ we saw that if $N(x)$ and $\bar{N}(\bar{x})$ are two nets corresponding with orthogonality of linear elements, that is are in relation $O$, they have the same point equation, say

$$
\frac{\partial^{2} \theta}{\partial u \partial v}=\frac{\partial \log a}{\partial v} \frac{\partial \theta}{\partial u}+\frac{\partial \log b}{\partial u} \frac{\partial \theta}{\partial v} .
$$

Moreover, if $h$ and $l$ are any solutions of equations (I, 13), the corresponding parallel nets $N^{\prime}\left(x^{\prime}\right)$ and $\bar{N}^{\prime}(\bar{x})$ are in relation $O$, and $\theta^{\prime}$, given by

$$
\theta^{\prime}=\sum x^{\prime} \bar{x}^{\prime}
$$

is a solution of the common point equation of $N^{\prime}$ and $\overline{N^{\prime}}$ (cf. $\S 28$ ). Furthermore, if $\theta$ is the solution of (71) corresponding to $\theta^{\prime}$, then the nets $N_{1}\left(x_{1}\right)$ and $\bar{N}_{1}\left(\bar{x}_{1}\right)$ defined by

$$
x_{1}=x-\frac{\theta}{\theta^{\prime}} x^{\prime}, \quad \bar{x}_{1}=\bar{x}-\frac{\theta}{\theta^{\prime}} \bar{x}^{\prime}
$$

are in relation $O$ and are respective $F$ transforms of $N$ and $\bar{N}$.

Suppose now that $N$ and $\bar{N}$ are nets in 3-space. Then, as follows from [ $§ 157$ ] they have equal tangential invariants; similarly $N_{1}$ and $\overline{N_{1}}$. It is our purpose to show that $N$ and $N_{1}$ are in relation $\Omega$, and likewise $\bar{N}$ and $\overline{N_{1}}$, and thus obtain the equations of transformations $\Omega$ in terms of the point coordinates.

If $\bar{N}(\bar{x})$ is any net with equal tangential invariants, and $N(x)$ is in relation $O$ with $\bar{N}$, we have [cf. $\S 157$ ]

$$
\frac{\partial x}{\partial u}=\overline{z_{0}} \frac{\partial \bar{y}}{\partial u}-\bar{y}_{0} \frac{\partial \bar{z}}{\partial u}, \quad \frac{\partial x}{\partial v}=\overline{z_{0}} \frac{\partial \bar{y}}{\partial v}-\bar{y}_{0} \frac{\partial \bar{z}}{\partial v},
$$

and

$$
\frac{\partial \bar{x}}{\partial u}=z_{0} \frac{\partial y}{\partial u}-y_{0} \frac{\partial z}{\partial u}, \quad \frac{\partial \vec{x}}{\partial v}=z_{0} \frac{\partial y}{\partial v}-y_{0} \frac{\partial z}{\partial v}
$$

44) Cf. Transactions, vol. 19 (1918), p. 179. The existence of transformations $\Omega$ of permanent nets for which $\mathbb{E}=0, \mathbb{B} \neq 0$ is established also; the restriction in the theorem of page 183 is not necessary, since the second of equations (61) should include the term $-V^{\prime} /(1+\cos \sigma) V$. 
where $\bar{x}_{0}, \bar{y}_{0}, \overline{z_{0}}$ and $x_{0}, y_{0}, z_{0}$ are the point coordinates of the surfaces $\bar{\Sigma}$ and $\Sigma$ associate to $\bar{N}$ and $N$, and the asymptotic lines on $\bar{\Sigma}$ and $\boldsymbol{\Sigma}$ are parametric. From (74) and (75) we have also

$$
\begin{array}{ll}
\sum \bar{x}_{0} \frac{\partial x}{\partial u}=0, & \sum \bar{x}_{0} \frac{\partial x}{\partial v}=0, \\
\sum x_{0} \frac{\partial \bar{x}}{\partial u}=0, & \sum x_{0} \frac{\partial \bar{x}}{\partial v}=0 .
\end{array}
$$

Consequently $\bar{x}_{0}, \bar{y}_{0}, \overline{z_{0}}$ and $x_{0}, y_{0}, z_{0}$ are direction-parameters of the normals to $N$ and $\bar{N}$ respectively. Furthermore, if the expressions for the derivatives of $y$ and $z$ as given by (74) are substituted in (75), we have in consequence of (77)

$$
x_{0} \bar{x}_{0}+y_{0} \bar{y}_{0}+z_{0} \bar{z}_{0}+1=0 \text {. }
$$

Hence $\boldsymbol{\Sigma}$ and $\overline{\boldsymbol{\Sigma}}$ are polar reciprocal with respect to the imaginary sphere $^{45}$ )

$$
x^{2}+y^{2}+z^{2}+1=0 .
$$

If $\overline{N^{\prime}}$ and $N^{\prime}$ are nets parallel to $\bar{N}$ and $N$ by means of the same pair of solutions $h$ and $l$ of (I, 13), we have

$$
\frac{\partial x^{\prime}}{\partial u}=\bar{z}_{0} \frac{\partial \bar{y}^{\prime}}{\partial u}-\bar{y}_{0} \frac{\partial \bar{z}^{\prime}}{\partial u}, \quad \frac{\partial x^{\prime}}{\partial v}=\bar{z}_{0} \frac{\partial \bar{y}^{\prime}}{\partial v}-\bar{y}_{0} \frac{\partial \bar{z}^{\prime}}{\partial v}
$$

For the $F$ transforms $N_{1}$ and $\bar{N}_{1}$ defined by (73) we have

$$
\begin{array}{ll}
\frac{\partial x_{1}}{\partial u}=\bar{z}_{01} \frac{\partial \bar{y}_{1}}{\partial u}-\bar{y}_{01} \frac{\partial \bar{z}_{1}}{\partial u}, & \frac{\partial x_{1}}{\partial v}=\bar{z}_{01} \frac{\partial \bar{y}_{1}}{\partial v}-\bar{y}_{01} \frac{\partial \bar{z}_{1}}{\partial v} \\
\frac{\partial \bar{x}_{1}}{\partial u}=z_{01} \frac{\partial y_{1}}{\partial u}-y_{01} \frac{\partial z_{1}}{\partial u}, & \frac{\partial \bar{x}_{1}}{\partial v}=z_{01} \frac{\partial y_{1}}{\partial v}-y_{01} \frac{\partial z_{1}}{\partial v}
\end{array}
$$

where $\bar{x}_{01}, \bar{y}_{01}, \bar{z}_{01}$ and $x_{01}, y_{01}, z_{01}$ are the point coordinates of surfaces $\bar{\Sigma}_{1}$ and $\Sigma_{1}$ associate to $\bar{N}_{1}$ and $N_{1}$ with their asymptotic

45) Darboux, Leçons, vol. 4, p. 67; also [Ex. 15, p. 391]. 
lines parametric, and they are direction-parameters of the normals to $N_{1}$ and $\overline{N_{1}}$ respectively. Moreover, we have analogously to (78)

$$
x_{01} \bar{x}_{01}+y_{01} \bar{y}_{01}+z_{01} \bar{z}_{01}+1=0 .
$$

From (81) we have

$$
\frac{\partial x_{1}}{\partial u} \frac{\partial \bar{z}_{1}}{\partial v}-\frac{\partial x_{1}}{\partial v} \frac{\partial \bar{z}_{1}}{\partial u}=\bar{z}_{01}\left(\frac{\partial \bar{y}_{1}}{\partial u} \frac{\partial \bar{z}_{1}}{\partial v}-\frac{\partial \bar{y}_{1}}{\partial v} \frac{\partial \bar{z}_{1}}{\partial u}\right)
$$

On differentiating (73) we obtain

$$
\begin{aligned}
& \frac{\partial x_{1}}{\partial u}=\frac{1}{\theta^{\prime 2}}\left(\theta-\frac{\theta^{\prime}}{h}\right)\left(x^{\prime} \frac{\partial \theta^{\prime}}{\partial u}-\theta^{\prime} \frac{\partial x^{\prime}}{\partial u}\right), \frac{\partial x_{1}}{\partial v}=\frac{1}{\theta^{\prime 2}}\left(\theta-\frac{\theta^{\prime}}{l}\right)\left(x^{\prime} \frac{\partial \theta^{\prime}}{\partial v}-\theta^{\prime} \frac{\partial x^{\prime}}{\partial v}\right) \\
& \frac{\partial \bar{x}_{1}}{\partial u}=\frac{1}{\theta^{\prime 2}}\left(\theta-\frac{\theta^{\prime}}{h}\right)\left(\bar{x}^{\prime} \frac{\partial \theta^{\prime}}{\partial u}-\theta^{\prime} \frac{\partial \bar{x}^{\prime}}{\partial u}\right), \frac{\partial \bar{x}_{1}^{\prime}}{\partial v}=\frac{1}{\theta^{\prime 2}}\left(\theta-\frac{\theta^{\prime}}{l}\right)\left(\bar{x}^{\prime} \frac{\partial \theta^{\prime}}{\partial v}-\theta^{\prime} \frac{\partial \bar{x}^{\prime}}{\partial v}\right)
\end{aligned}
$$

When these expressions and analogous ones in $\bar{y}_{1}$ and $\bar{z}_{1}$ are substituted in (84) and $\theta^{\prime}$ is given the value (72), the resulting equation is reducible, by means of (80) and the fact that $x_{0}, y_{0}, z_{0}$ are directionparameters of the normal to $\bar{N}^{\prime}$, to the form

Similarly we have

$$
\bar{z}_{01}=\frac{x^{\prime} y_{0}-y^{\prime} x_{0}-\bar{z}^{\prime}}{\sum \bar{x}^{\prime} x_{0}} .
$$

$$
z_{01}=\frac{\bar{x}^{\prime} \bar{y}_{0}-\bar{y}^{\prime} \bar{x}_{0}-z^{\prime}}{\sum x^{\prime} \bar{x}_{0}}
$$

From (85) and (86) we have

$$
x_{0} \bar{x}_{01}+y_{0} \bar{y}_{01}+z_{0} \bar{z}_{01}+1=0, \quad \bar{x}_{0} x_{01}+\bar{y}_{0} y_{01}+\bar{z}_{0} z_{01}+1=0 .
$$

Consider now the surfaces $\Sigma$ and $\Sigma_{1}$. The quantities $\bar{x}_{0}, \bar{y}_{0}, \bar{z}_{0}$ and $\bar{x}_{01}, \bar{y}_{01}, \bar{z}_{01}$, are direction-parameters of the normals to these respective surfaces. Moreover, from (78), (83) and (87) it follows that

$$
\sum \bar{x}_{0}\left(x_{01}-x_{0}\right)=0, \quad \sum \bar{x}_{01}\left(x_{01}-x_{0}\right)=0
$$

Consequently $\Sigma$ and $\Sigma_{1}$ are the focal surfaces of the congruence of lines joining corresponding points on these surfaces, and it is 
$W$ congruence, since the asymptotic lines are parametric on $\Sigma$ and $\Sigma_{1}$. Hence from the last theorem of $\S 53$ it follows that $N$ and $N_{1}$ are in relation $\Omega$, and also $\bar{N}$ and $\bar{N}_{1}$.

When a net $\bar{N}$ with equal tangential invariants is known, the associate surface $\bar{\Sigma}$ can be found by quadratures [\$ 78]. Then by equations (74) we obtain a net $N$, with equal tangential invariants, which is in relation $O$ to $\bar{N}$. Each net $\bar{N}^{\prime}$ parallel to $\bar{N}$ leads by a quadrature to an $\Omega$ transformation of $\bar{N}$ and $N$. Hence:

Each net parallel to a net $N$ with equal tangential invariants determines an $\boldsymbol{\Omega}$ transformation of $N$, and the determination of the point coordinates of the transform requires only quadratures ${ }^{46}$ ).

59. Transformations $\Omega$ and $K$ of the focal nets of a $W$ congruence. We have seen that the surface $\Sigma$ of the preceding section is associate to the surface $S$ on which $N$ lies. Since this relation is reciprocal, there exists a surface $\tilde{S}$ in relation $O$ to $\Sigma$; its coordinates, $\tilde{x}, \tilde{y}, \tilde{z}$, are given by quadratures of the form (analogous to (75))

$$
\frac{\partial \bar{x}}{\partial u}=z \frac{\partial y_{0}}{\partial u}-y \frac{\partial z_{0}}{\partial u}, \quad \frac{\partial \tilde{x}}{\partial v}=z \frac{\partial y_{0}}{\partial v}-y \frac{\partial z_{0}}{\partial v} .
$$

From these equations and (75) we see that $\bar{S}$ can be so placed in space that

$$
\bar{x}=\bar{x}+z y_{0}-y z_{0} .
$$

From [\$ 157] it follows that the asymptotic lines correspond on $\bar{S}$ and $\bar{S}$ the surface on which $\bar{N}$ lies. Also since $x_{0}, y_{0}, z_{0}$ and $x, y, z$ are direction-parameters of the normals to $\bar{S}$ and $\tilde{S}$ respectively, it follows from (88) that $\bar{S}$ and $\bar{S}$ are the focal surfaces of a $W$ congruence.

Since $\bar{S}$ and $\mathbf{\Sigma}$ are in relation $O$, there can be found by means of equations analogous to (74), the coordinates $\tilde{x}_{0}, \tilde{y}_{0}, \tilde{z}_{0}$ of a surface $\tilde{\Sigma}$ associate to $\tilde{S}$; moreover, from [§ 156] it follows that the parametric curves on $\tilde{S}$ and $\bar{\Sigma}$ form nets with equal point invariants. Furthermore, since $\bar{\Sigma}$ and $S$ bear to $\bar{S}$ and $\Sigma$ relations analogous to the relations of $\Sigma$ and $\bar{\Sigma}$ to $S$ and $\bar{S}$, it follows that $\bar{\Sigma}$ and $S$ are polar reciprocal with respect to $(79)$.

46) Cf. the first theorem of $\S 53$. 
When the net $\bar{N}$ is subjected to a transformation $\Omega$, in accordance with the preceding section, we get surfaces $\overline{S_{1}}, S_{1}, \overline{\Sigma_{1}}, \tilde{S}_{1}$ and $\tilde{\Sigma}_{1}$ related to one another as in the preceding paragraph. Since $S$ and $\tilde{\Sigma}$, and $S_{1}$ and $\tilde{\Sigma_{1}}$, are polar reciprocal with respect to (79), it follows that $\tilde{\Sigma}$ and $\tilde{\Sigma}_{1}$ are in the relation of a transformation $K\left(\mathrm{~V}\right.$, Ex. 11). Moreover, $\tilde{S}$ and $\tilde{S_{1}}$ being associate to $\tilde{\Sigma}$ and $\tilde{\Sigma_{1}}$ respectively are also in relation $K$ (II, Ex. 18). Furthermore, $\bar{S}_{1}$ and $\tilde{S_{1}}$ are the focal surfaces of a $W$ congruence.

From [§ 172] it follows that if $\bar{S}$ and $\tilde{S}$ are the focal surfaces of any $W$ congruence, it is possible to find by quadratures two surfaces $S$ and $\Sigma$ associate to one another and in relation $O$ with $\bar{S}$ and $\bar{S}$ respectively. If $\Sigma$ is referred to its asymptotic lines, the parametric curves on $\bar{S}$ and $\tilde{S}$ form nets with equal tangential and point invariants respectively. Hence:

$A W$ congruence admits of transformations into $W$ congriences such that one pair of corresponding focal nets are in relation $\boldsymbol{\Omega}$ and the other pair in relation $K^{\mathbf{4 7}}$ ).

6o. Nets with equal point invariants and equal tangential invariants. In consequence of [(38) $\S 83]$ equations $[(36) \S 83]$ can be written in the form

Hence:

$$
\frac{\partial}{\partial v} \log D=\frac{\partial}{\partial v} \log a \alpha, \quad \frac{\partial}{\partial u} \log D^{\prime \prime}=\frac{\partial}{\partial u} \log b \beta .
$$

When a net has two of the following properties, it has the third also; equal point invariants, equal tangential invariants, isothermalconjugate.

We consider such a net, and put

$$
\left\{\begin{array}{c}
12 \\
1
\end{array}\right\}=\frac{\partial \log \sqrt{\varrho}}{\partial v}, \quad\left\{\begin{array}{c}
12 \\
2
\end{array}\right\}=\frac{\partial \log \sqrt{\varrho}}{\partial u}, \quad D=-D^{\prime \prime} .
$$

From $[(3) \S 63]$ and $\left[\left(13^{\prime}\right) \S 64\right]$ we have

$$
\begin{array}{ll}
\left\{\begin{array}{c}
11 \\
1
\end{array}\right\}=\frac{\partial}{\partial u} \log \frac{H}{\sqrt{\varrho}}, & \left\{\begin{array}{c}
11 \\
2
\end{array}\right\}=\frac{\partial}{\partial v} \log \frac{D}{\sqrt{\varrho}}, \\
\left\{\begin{array}{c}
22 \\
1
\end{array}\right\}=\frac{\partial}{\partial u} \log \frac{D}{\sqrt{\varrho}}, & \left\{\begin{array}{c}
22 \\
2
\end{array}\right\}=\frac{\partial}{\partial v} \log \frac{H}{\sqrt{\varrho}} .
\end{array}
$$

47) Cf. Demoulin, 1. c., p. 276. 
Consequently the point coordinates of the net satisfy the equations

where

$$
\left\{\begin{array}{l}
\frac{\partial^{2} \theta}{\partial u^{2}}+\frac{\partial^{2} \theta}{\partial v^{2}}=\frac{\partial \log r}{\partial u} \frac{\partial \theta}{\partial u}+\frac{\partial \log r}{\partial v} \frac{\partial \theta}{\partial v}, \\
\frac{\partial^{2} \theta}{\partial u \partial v}=\frac{\partial \log \sqrt{\varrho}}{\partial v} \frac{\partial \theta}{\partial u}+\frac{\partial \log \sqrt{\varrho}}{\partial u} \frac{\partial \theta}{\partial v}
\end{array}\right.
$$

From $\S 25$ we have:

$$
r=\frac{H D}{\varrho}
$$

If $N(x)$ is a net with equal point and equal tangential invariants, a parallel net of the same kind is given by the quadratures

$$
\frac{\partial \bar{x}}{\partial u}=-\frac{1}{\varrho} \frac{\partial x}{\partial u}, \quad \frac{\partial \bar{x}}{\partial v}=\frac{1}{\varrho} \frac{\partial x}{\partial v} .
$$

In order to establish transformations $F$ of a net $N$ of this type into nets of the same kind, we consider $N$ expressed in terms of homogeneous coordinates, the point and tangential equations being

$$
\left\{\begin{array}{l}
\frac{\partial^{2} \theta}{\partial u \partial v}+\frac{\partial \log \sqrt{\varrho}}{\partial v} \frac{\partial \theta}{\partial u}+\frac{\partial \log \sqrt{\varrho}}{\partial u} \frac{\partial \theta}{\partial v}+c \theta=0, \\
\frac{\partial^{2} \lambda}{\partial u \partial v}+\frac{\partial \log \sqrt{\bar{\varrho}}}{\partial v} \frac{\partial \lambda}{\partial u}+\frac{\partial \log \sqrt{\bar{\varrho}}}{\partial u} \frac{\partial \lambda}{\partial v}+\gamma \lambda=0 .
\end{array}\right.
$$

It is readily shown that the equations

$$
\left\{\begin{array}{l}
\frac{\partial x_{1}}{\partial u}=\varrho\left(\frac{\partial x}{\partial u} \sum X_{1} x-x \sum X_{1} \frac{\partial x}{\partial u}\right) \\
\frac{\partial x_{1}}{\partial v}=-\varrho\left(\frac{\partial x}{\partial v} \sum X_{1} x-x \sum X_{1} \frac{\partial x}{\partial v}\right) \\
\frac{\partial X_{1}}{\partial u}=\bar{\varrho}\left(\frac{\partial X}{\partial u} \sum X x_{1}-X \sum x_{1} \frac{\partial X}{\partial u}\right) \\
\frac{\partial X_{1}}{\partial v}=-\bar{\varrho}\left(\frac{\partial X}{\partial v} \sum X x_{1}-X \sum x_{1} \frac{\partial X}{\partial v}\right)
\end{array}\right.
$$

and similar equations in $y_{1}, z_{1}, w_{1}, Y_{1}, Z_{1}$ and $W_{1}$ form a completely integrable system. For each set of solutions the function $\sum x_{1} X_{1}$ is constant. Consequently each set of solutions, for which 


$$
\sum x_{1} X_{1}=0
$$

defines a net $N_{1}$ for which these functions are the point and tangential coordinates. Furthermore, for a set of solutions the functions

$$
\theta=\sum X_{1} x, \quad \lambda=\sum X x_{1}
$$

are solution of the respective equations (90), so that equations (91) are equivalent to (III, Ex. 25) and (36). Consequently $N_{1}$ is both a $K$ and $\Omega$ transform of $N$, and therefore has equal point and equal tangential invariants. Hence, since (92) is bilinear we have:

$A$ net with equal point and equal tangential invariants admits $\infty^{6}$ transformations $K$ and $\Omega$ into nets of the same kind.

If $N_{1}$ and $N_{2}$ aré two of these transforms of $N$, from (III, 73, 75) it follows that for a net $N_{12}$ to be a $K$ transform of $N_{1}$ and $N_{2}$, we must have $\theta_{12}+\theta_{21}=0$. In like manner for $N_{12}$ to be an $\Omega$ transform we have from the equations preceding (21) that $\lambda_{12}+\lambda_{21}=0$. Hence from (23) we have $\sum X_{1} x_{2}+\sum X_{2} x_{1}=0$. It is readily. shown that the left-hand member of this expression is constant. Only when it is zero is there a net $N_{12}$ which is both a $K$ and an $\Omega$ transform of $N_{1}$ and $N_{2}$, and then there are an infinity of such nets, since $\theta_{12}$ is determined only to within an additive constant, but $\theta_{21}, \lambda_{12}$ and $\lambda_{21}$ are then completely determined.

\section{Exercises.}

1. If $x^{(1)}, x^{(2)}, x^{(3)}$ are the cartesian coordinates of a net $N$ in 3-space and $\theta$ is any solution of the point equation of $N$, then $\frac{\partial x^{(1)}}{\partial u}, \frac{\partial x^{(2)}}{\partial u}, \frac{\partial x^{(3)}}{\partial u}$, $\frac{\partial \theta}{\partial u}$ are tangential coordinates of a net.

2. A necessary and sufficient condition that the curves $v=$ const. of a net be plane is that the minus first Laplace transform be a derelopable surface; the analytical condition is that the invariant $H$ of the tangential equation of the net be equal to zero.

3. A necessary and sufficient condition that the curves of a net be plane is that the tangential coordinates can be taken in the form

$$
X=U_{1}+V_{1}, \quad Y=U_{2}+V_{2}, \quad Z=U_{3}+V_{3}, \quad W=U_{4}+V_{4},
$$

where the $U^{\prime} s$ and $V^{\prime} s$ are functions of $u$ and $v$ respectively. 
4. When two nets $N$ and $N_{1}$ in relation $F$ are subjected to a polar reciprocal transformation, the resulting nets are in relation $F$; and the conjugate and harmonic congruences of the given relation $F$ are transformed into the harmonic and conjugate congruences of the resulting relation $F$.

5. In a transformation $F$ defined by (11) the functions $\frac{\partial}{\partial u}\left(\frac{X}{\lambda}\right), \ldots, \frac{\partial}{\partial u}\left(\frac{W}{\lambda}\right)$ and $\frac{\partial}{\partial v}\left(\frac{X}{\lambda}\right), \ldots, \frac{\partial}{\partial v}\left(\frac{W}{\lambda}\right)$ are tangential coordinates of the first and second focal surfaces of the conjugate congruence of the transformation.

6. A necessary and sufficient condition that the transformation defined by (11) be radial is that $\lambda=a X+b Y+c Z+d W$, where $a, b, c ; d$ are constants.

7. When in (26) we take $v=a X+b Y+c Z$, where $a, b$ and $c$ are constants, the conjugate congruence of the transformation consists of parallel lines.

8. Show that the functions $\bar{X}_{1}$ and $\bar{X}_{2}$ given by (15) are tangential coordinates of the focal surfaces of the harmonic congruence of the corresponding transformation $F$.

9. Each solution of the adjoint of the tangential equation of a net determines. a congruence harmonic to the net (cf. Ex. 8). What is the dual of this theorem?

10. If the direction equation of a congruence $G$ is the same as the tangential equation of a net $N$, the determination of nets harmonic to $G$ and congruences harmonic to $N$ are equivalent problems, namely the solution of the adjoint of the given equation.

11. When a polar reciprocal transformation is applied to two nets in relation $Q$, the resulting nets are in relation $K$, and conversely.

12. A necessary and sufficient condition that the axis curves of a net $N$ form a net is that $N$ have equal tangential invariants (cf. IV, Ex. 20, 22).

13. If four nets $N, N_{1}, N_{2}, N_{12}$ form a quatern under transformations $\Omega$ determined by $w_{1}, w_{2}, w_{12}$ and $w_{21}$, the four nets parallel to them determined by $w_{1}, 1 / \sqrt{\rho \rho_{1}} w_{1}, w_{21}$ and $1 / \sqrt{\rho_{2} \rho_{12}} w_{21}$ form a quatern; likewise the four nets determined by $w_{2}, 1 / \sqrt{\rho \rho_{2}} w_{2}, w_{12}$ and $1 / \sqrt{\rho_{1} \rho_{12}} w_{12}$.

14. Let $N(x)$ be a net with equal tangential invariants and $\bar{N}(\bar{x})$ the net of the same kind in relation $O$ to $N$, and $N^{\prime}\left(x^{\prime}\right), N^{\prime \prime}\left(x^{\prime \prime}\right)$ and $\bar{N}^{\prime}\left(\bar{x}^{\prime}\right), \bar{N}^{\prime \prime}\left(\bar{x}^{\prime \prime}\right)$ corresponding parallel nets to $N$ and $\bar{N}$. If we put $\theta_{1}^{\prime}=\Sigma x^{\prime} \bar{x}^{\prime}, \theta_{2}^{\prime \prime}=\Sigma x^{\prime \prime} \bar{x}^{\prime \prime}$, then the nets $N_{1}\left(x_{1}\right)$ and $N_{2}\left(x_{2}\right)$, where

$$
x_{1}=x-\frac{\theta_{1}}{\theta_{1}^{\prime}} x^{\prime}, \quad x_{2}=x-\frac{\theta_{2}}{\theta_{2}^{\prime \prime}} x^{\prime \prime}
$$

are $\Omega$ transforms of $N$. If we draw through points of $N_{1}$ and $N_{2}$ lines with direction-parameters of the respective forms (cf. $§ 21$ )

$$
x_{1}^{\prime \prime \prime}=x^{\prime \prime}-\frac{\theta_{1}^{\prime \prime}}{\theta_{1}^{\prime}} x^{\prime}, \quad x_{2}^{\prime \prime \prime \prime}=x^{\prime}-\frac{\theta_{2}^{\prime}}{\theta_{2}^{\prime \prime}} x^{\prime \prime},
$$

where $\theta_{1}^{\prime \prime}$ and $\theta_{2}^{\prime}$ are solutions of the point equations of $N^{\prime \prime}$ and $N^{\prime}$ corresponding to $\theta_{1}$ and $\theta_{2}$, the point $M_{12}$ of intersection of these corresponding lines generates a net $N_{12}$. Show that this net $N_{12}$ will be an $\Omega$ transform of $N_{1}$ and $N_{2}$ if

$$
\theta_{1}^{\prime \prime}+\theta_{2}^{\prime}=\Sigma x^{\prime \prime} \bar{x}^{\prime}+\Sigma x^{\prime} \bar{x}^{\prime \prime} \text {. }
$$


15. Show that all the conditions of the preceding example are satisfied, if we replace $\theta_{1}^{\prime \prime}$ and $\theta_{2}^{\prime}$ by $\theta_{1}^{\prime \prime}+c$ and $\theta_{2}^{\prime}-c$, where $c$ is an arbitrary constant, and consequently there are $\infty^{1}$ nets $N_{12}$ in relation $\Omega$ with $N_{1}$ and $N_{2}$; that corresponding points of these nets $N_{12}$ lie on a conic through the corresponding points of $N, N_{1}$ and $N_{2}$; also that the tangents planes to these nets $N_{12}$ at corresponding points form a pencil (cf. $\S 26$ and II, Ex. 19).

16. Let $N$ be a net with equal point invariants, $N_{1}$ and $N_{2}$ be $K$ transforms of $N$, and $N_{12}$ one of the $K$ transforms of $N_{1}$ and $N_{2}$ in accordance with the theorem of $\S 26$; show that the tangent planes to the $\infty^{1}$ nets $N_{12}$ at corresponding points envelop a quadric cone (cf. Ex. 15).

17. Prove directly that the surfaces $\tilde{S}$ and $\tilde{S}_{1}$ of $\S 59$ are in relation $K$.

18. Two nets $N$ and $\bar{N}$ with equal tangential invariants which are in relation $O$ determine twelve surfaces forming a closed system of Darboux [Ex. 19, p. 391]; similarly two nets $N_{1}$ and $\bar{N}_{1}$ which are $\Omega$ transforms of $N$ and $\bar{N}$ determine a second system; find the relations between the two systems of surfaces other than those treated in $\S 59$.

19. If $S$ and $\bar{S}$ two surfaces in relation $O$ are referred to their common nets, $N(x)$ and $\bar{N}(\bar{x})$, the equations of the form

$$
\hat{x}=x+e \bar{x},
$$

where $e$ is an infinitesimal, define a surface $\hat{S}$ which is an infinitesimal deform of $S$, and the parametric curves form a net $\hat{N}$ [cf. $\S 158$ ]. If $N^{\prime}\left(x^{\prime}\right)$ and $\bar{N}^{\prime}\left(\bar{x}^{\prime}\right)$ are corresponding parallel nets to $N$ and $\bar{N}$ respectively, the equations of the form.

$$
\hat{x}^{\prime}=x^{\prime}+e \bar{x}^{\prime}
$$

define a net $\hat{N}^{\prime}$ which is an infinitestimal deform of $N^{\prime}$. Then equations of the form

$$
\hat{x}_{1}=\hat{x}-\frac{\theta}{\theta^{\prime}} \hat{x}^{\prime},
$$

where $\theta^{\prime}=\Sigma_{x^{\prime} \bar{x}^{\prime}}$, define a net $\hat{N}_{1}$, which is an $F$ transform of $\hat{N}$ and an infinitestimal deform of $N_{\mathrm{l}}$ whose coordinates are given by (73).

20. When the spherical representation of a permanent net is such that $\mathbb{E} \neq 0, \mathbb{S} \neq 0$, the parameters can be chosen so that either (i) $\rho=a, a$ constant; (ii) $\rho=v$; (iii) $\rho=u+v$. In these respective cases the coefficients of the spherical representation can be given the respective forms

$$
\begin{gathered}
\mathfrak{F}=\mathbb{S}=1, \quad \mathfrak{F}=\cos 2 \omega ; \\
\mathfrak{S}=\frac{1}{v}, \quad \Re=-\frac{\partial \psi}{\partial u}, \quad \mathbb{S}=\frac{\psi}{v} ; \\
\mathfrak{F}=\frac{\partial \psi}{\partial u}(u+v)^{-1}, \quad \mathfrak{F}=-\frac{\partial^{2} \psi}{\partial u \partial v}, \quad \&=\frac{\partial \psi}{\partial v}(u+v)^{-1} .
\end{gathered}
$$

Determine the conditions which $\omega$ and $\psi$ must satisfy in each case. 
21. When the curves $v=$ const. of a permanent net are represented on the gaussian sphere by isotropic generators, the coefficients of (52) are reducible to the form

$$
\mathbb{E}=0, \quad \Re=\frac{2}{(1+u v)^{2}}, \quad \mathbb{B}=\frac{2 V^{\prime}}{\nabla v(1+u v)}+V_{1},
$$

where $V$ and $V_{1}$ are functions of $v$ alone; also the tangential coordinates of such a net are given by

$$
\begin{gathered}
X=\frac{a_{1}}{1+u v}+b_{1}, \quad Y=\frac{a_{2}}{1+u v}+b_{2}, \quad Z=\frac{a_{3}}{1+u v}+b_{3}, \\
W \sqrt{V}=2 \frac{v \varphi+u \psi^{\prime}}{1+u v}-\varphi^{\prime}-\psi^{\prime},
\end{gathered}
$$

where $\varphi$ and $\psi$ are arbitrary functions of $u$ and $v$ respectively, and the $a$ 's and $b$ 's are functions of $v$ alone subject to the conditions:

$$
\begin{aligned}
& {\underset{i}{i}}_{i} a_{i}^{2}=0, \quad \underset{i}{\mathbf{S}} a_{i}^{\prime 2}=\frac{4}{v^{2}}, \quad{\underset{i}{i}}_{i} a_{i}^{\prime \prime 2}=\frac{4}{v^{2}}\left(V_{1}-\frac{5}{4} \frac{V^{\prime 2}}{V^{2}}+\frac{2 V^{\prime}}{v V}+\frac{V^{\prime \prime}}{V}\right), \\
& b_{i}=\left(\frac{V^{\prime} v}{4 V}-\frac{1}{2}\right) a_{i}+\frac{v}{2} a_{i}^{\prime} \quad(i=1,2,3),
\end{aligned}
$$

the primes indicating differentiation.

Drach, Ann. de Toulouse, ser. 2, vol. 10 (1908), p. 135. 


\section{Chapter VI.}

\section{Orthogonal nets.}

61. Nets $O$ and $p, O$. Congruences $\mathrm{I}$ and $p, \mathrm{I}$. A net $N(x)$, whose cartesian coordinates $x$ satisfy an equation

$$
\frac{\partial^{2} \theta}{\partial u \partial v}=\frac{\partial \log a}{\partial v} \frac{\partial \theta}{\partial u}+\frac{\partial \log b}{\partial u} \frac{\partial \theta}{\partial v},
$$

is called an orthogonal net, or for the sake of brevity an $\left.O n e t^{48}\right)$, if

(2) $E=\Sigma\left(\frac{\partial x}{\partial u}\right)^{2} \neq 0, \quad F=\sum \frac{\partial x}{\partial u} \frac{\partial x}{\partial v}=0, \quad G=\Sigma\left(\frac{\partial x}{\partial v}\right)^{2} \neq 0$.

The second of these conditions is equivalent to the requirement that $\omega$, defined by

$$
2 \omega=\sum x^{2}
$$

is a solution of (1). We say that a net $N$ is $p, O$, if its point equation (1) admits $p-1$ solutions, $y_{1}, \ldots, y_{p-1}$ linearly independent of the $x$ 's, such that

$$
\left\{\begin{array}{c}
\sum\left(\frac{\partial x}{\partial u}\right)^{2}-\Sigma\left(\frac{\partial y}{\partial u}\right)^{2} \neq 0, \quad \sum\left(\frac{\partial x}{\partial v}\right)^{2}-\sum\left(\frac{\partial y}{\partial v}\right)^{2} \neq 0 \\
\sum \frac{\partial x}{\partial u} \frac{\partial x}{\partial v}-\sum \frac{\partial y}{\partial u} \frac{\partial y}{\partial v}=0
\end{array}\right.
$$

We call the $y$ 's the complementary functions ${ }^{49}$ ). This means that $\sum x^{2}-\sum y^{2}$ is a solution of (1). It is understood that none of

48) The results of $\$ \S 61-65$ are due to Guichard, Annales de L'École Norm. Sup., ser. 3, vol. 14 (1897), pp. 467-516; vol. 15 (1898), pp. 179-227.

49) Note that if each $y$ be replaced by $i y$, where $i^{2}=-1$, a net $p, O$ is the projection in $n$-space of an $O$ net in $(n+p-1)$-space. It is this point of view that Guichard takes in considering these systems. 
the $y$ 's are constant. Hence if we have a net so that the $x^{\prime}$ s and $p-1$ functions $y$ satisfy $\sum x^{2}-\sum y^{2}=$ const., we say that the net is $p, O$ and not $p+1, O$.

We say that the equations

$$
y_{i}=\alpha_{i 1} \bar{y}_{1}+\ldots+\alpha_{i p-1} \bar{y}_{p-1}, \quad(i=1 \ldots p-1),
$$

define an orthogonal substitution, when the constants $\alpha_{i j}$ satisfy the conditions

$$
\sum_{i=1}^{p-1} \alpha_{i j}^{2}=1, \quad \sum_{i=1}^{p-1} \alpha_{i j} \alpha_{i k}=0, \quad(j \neq k) .
$$

When such a substitution is effected upon the complementary functions $y$ of a net $p, O$, we have $\sum \bar{y}^{2}=\sum y^{2}$ and consequently the functions $\bar{y}$ serve equally well as complementary functions.

When there is a linear relation between the $y$ 's, such as

$$
a_{1} y_{1}+\ldots+a_{p-1} y_{p-1}=a
$$

where $\sum a_{i}^{2} \neq 0$, that is, when the relation is non-isotropic, we can assume that $\sum a_{i}^{2}=1$, so that in the new variables $\bar{y}$, we may take $\bar{y}_{p-1}=a$ where $a$ is a constant. Hence the net is $p-1,0$.

If, however, $\sum a_{i}^{2}=0$, that is, if the relation is isotropic, we can choose the orthogonal substitution so that (7) is reducible to

$$
\bar{a}_{p-2} \bar{y}_{p-2}+\bar{a}_{p-1} \bar{y}_{p-1}=a,
$$

where now $\bar{a}_{p-2}^{2}+\bar{a}_{p-1}^{2}=0$. Hence

$$
\bar{y}_{p-1}= \pm i \bar{y}_{p-2}+b
$$

where $b$ is a constant. Since any complementary function may be replaced by itself with an additive constant, we have

$$
y_{1}^{2}+\ldots+y_{p-1}^{2}=\bar{y}_{1}^{2}+\ldots+\bar{y}_{p-3}^{2} .
$$

Hence the net is $p-2, O$, and we have:

When for a net apparently $p, O$, there is an isotropic linear relation between the complementary functions, the net is in fact 
$p-2, O$; when there is a non-isotropic linear relation, the net is $p-1,0$.

We say that a congruence is $\mathrm{I}$, when its direction-parameters $X$ satisfy the conditions

$$
\left\{\begin{array}{c}
\sum X^{2}=0 \\
\sum\left(\frac{\partial X}{\partial u}\right)^{2} \neq 0, \quad \sum\left(\frac{\partial X}{\partial v}\right)^{2} \neq 0 .
\end{array}\right.
$$

Since the $X$ 's satisfy an equation of the form

$$
\frac{\partial^{2} \theta}{\partial u \partial v}=\frac{\partial \log A}{\partial v} \frac{\partial \theta}{\partial u}+\frac{\partial \log B}{\partial u} \frac{\partial \theta}{\partial v}+C \theta
$$

it follows from the first of (8) that

$$
\sum \frac{\partial X}{\partial u} \frac{\partial X}{\partial v}=0
$$

It is readily seen that these conditions are satisfied also when each $X$ is replaced by $\lambda X$, where $\lambda$ is any function of $u$ and $v$. A congruence is $p, I$ when there are $p-1$ functions $Y$ satisfying (9) such that

$$
\text { (11) }\left\{\begin{array}{c}
\sum X^{2}-\sum Y^{2}=0, \\
\sum\left(\frac{\partial X}{\partial u}\right)^{2}-\sum\left(\frac{\partial Y}{\partial u}\right)^{2} \neq 0, \quad \sum\left(\frac{\partial X}{\partial v}\right)^{2}-\sum\left(\frac{\partial Y}{\partial v}\right)^{2} \neq 0 .
\end{array}\right.
$$

The functions $Y$ are called the complementary functions of the congruence $p, \mathrm{I}$. We have also

$$
\sum \frac{\partial X}{\partial u} \frac{\partial X}{\partial v}-\sum \frac{\partial Y}{\partial u} \frac{\partial Y}{\partial v}=0
$$

When a congruence is $2, \mathrm{I}$, we may choose for the parameters the $X$ 's divided by the single complementary function. In this case we have

$$
\sum X^{2}=1, \quad \sum \frac{\partial X}{\partial u} \frac{\partial X}{\partial v}=0,
$$


so that a congruence $2, I$ is the generalization of the normal congruence in 3-space.

62. Nets conjugate to congruences $I$ and $p, I$. In $\$ 5$ we saw that if $\theta$ is a solution of (9), the functions $x^{\prime}$ defined by

$$
x^{\prime}=\frac{x}{\theta}
$$

are the cartesian coordinates of a net $N^{\prime}$ whose radii vectores from the origin are parallel to the lines of a congruence $G$ of directionparameters $X$.

If $G$ is a congruence I, we have from (8)

$$
\sum x^{\prime 2}=0
$$

and

$$
\left\{\begin{array}{c}
\sum\left(\frac{\partial x^{\prime}}{\partial u}\right)^{2}=\frac{1}{\theta^{2}} \sum\left(\frac{\partial X}{\partial u}\right)^{2} \neq 0, \quad \sum\left(\frac{\partial x^{\prime}}{\partial v}\right)^{2} \neq 0 \\
\sum \frac{\partial x^{\prime}}{\partial u} \frac{\partial x^{\prime}}{\partial v}=0
\end{array}\right.
$$

Hence $N^{\prime}$ is a net $O$. In consequence of the second theorem of $\S 5$ and the last of (16) we have:

All nets conjugate to a congruence $\mathrm{I}$ are $O$.

If $G$ is a congruence $p$, I, the $p-1$ functions $y^{\prime}$ defined by

$$
y^{\prime}=\frac{Y}{\theta}
$$

are solutions of the point equation of $N^{\prime}$. In consequence of (11) and (12) we have

$$
\left\{\begin{array}{c}
\sum\left(\frac{\partial x^{\prime}}{\partial u}\right)^{2}-\sum\left(\frac{\partial y^{\prime}}{\partial u}\right)^{2} \neq 0, \quad \sum\left(\frac{\partial x^{\prime}}{\partial v}\right)^{2}-\sum\left(\frac{\partial y^{\prime}}{\partial v}\right)^{2} \neq 0 \\
\sum \frac{\partial x^{\prime}}{\partial u} \frac{\partial x^{\prime}}{\partial v}-\sum \frac{\partial y^{\prime}}{\partial u} \frac{\partial y^{\prime}}{\partial v}=0
\end{array}\right.
$$

- Hence in general $N^{\prime}$ is a net $p, O$. When, however,

$$
\theta=a_{1} Y_{1}+\ldots+a_{p-1} Y_{p-1}, \quad\left(\sum a_{i}^{2} \neq 0\right)
$$


there is a non-isotropic linear relation between the functions $y^{\prime}$, and $N^{\prime}$ is $p-1, O$ (cf. $\S 61$ ). When in (19),

$$
\sum a_{i}^{q}=0,
$$

there is an isotropic linear relation between the functions $y^{\prime}$, and $N^{\prime}$ is $p-2, O$.

We have shown that the nets conjugate to the given congruence are parallel to nets of the type (14), and are given by equations of the form (I, 12). By means of the same equations we obtain $p-1$ functions $y$ from the functions $y^{\prime}$. It is readily seen that these functions satisfy equations of the same form as (18). Hence the nets conjugate to the congruence are of the same type as the parallel nets $N^{\prime}$. In accordance with the fifth theorem of $\S 5$ there are $\infty^{1}$ of these nets parallel to each net $N^{\prime}$. If we call them a parallel family, we have:

Of the nets conjugate to a congruence $p, I$, there are $\infty^{p-3}$ parallel families of nets $p-2, O$, $\infty^{p-2}$ parallel families of nets $p-1, O$, the others are $p, O$.

In particular, we have:

Of the nets conjugate to a congruence $2, I$ there is one parallel family of nets $O$, and all the others are 2, $O$.

The nets $O$ arise from the case when $\theta$ in (17) is equal to $Y$.

For a congruence $3, I$, when $\theta$ is equal to $Y_{1}+i Y_{2}$ or $Y_{1}-i Y_{2}$ condition (20) is satisfied, and only in this case. Hence:

Of the nets conjugate to a congruence $3, \mathrm{I}$ there are

two parallel families of nets $O$, $\infty^{1}$ parallel families of nets $2, O$, all the others are $3, O$.

63. Orthogonal determinants. Since $F=0$ for an $O$ net, it follows from (I, 3) that $a$ and $b$ in (1) may be chosen so that

$$
a=\sqrt{E}, \quad b=\sqrt{G},
$$

in which case the normal parameters $\xi^{i}$ and $\eta^{i}$ of the net, given by

$$
\frac{\partial x}{\partial u}=\sqrt{E} \xi, \quad \frac{\partial x}{\partial v}=\sqrt{G} \eta,
$$


are the direction-cosines and are in the relations

$$
\sum \xi^{2}=\sum \eta^{2}=1, \quad \sum \xi \eta=0 .
$$

In this case $(I, 6,7)$ become

$$
\frac{\partial \xi}{\partial v}=n \eta, \quad \frac{\partial \eta}{\partial u}=m \xi,
$$

and

$$
n=\frac{1}{\sqrt{E}} \frac{\partial \sqrt{G}}{\partial u}, \quad m=\frac{1}{\sqrt{G}} \frac{\partial \sqrt{E}}{\partial v}
$$

Consider now the orthogonal determinant

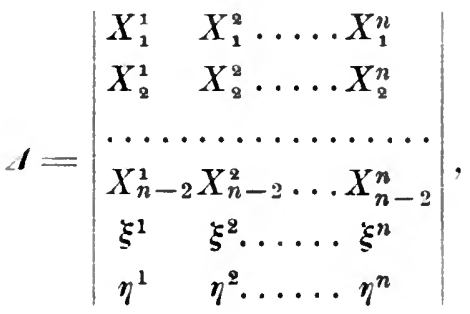

the $X$ 's being functions of $u$ and $v$ satisfying the conditions

(27) $\sum_{i=1}^{n} X_{k}^{i^{2}}=1, \quad \sum_{i=1}^{n} X_{k}^{i} X_{j}^{i}=0, \quad \sum_{i=1}^{n} \xi^{i} X_{k}^{i}=0, \quad \sum_{i=1}^{n} \eta^{i} X_{k}^{i}=0$.

Therefore we may look upon these functions as the directionparameters of $n$ mutually perpendicular lines. Since the first derivatives of the $X$ 's of any row are direction-parameters of some direction in the space, they are expressible as linear homogeneous functions of the other terms of the same column. It is our purpose to show that there is a set of $X$ 's such that the latter expressions take the simple form

(28) $\frac{\partial X_{k}}{\partial \imath}=a_{k} \xi ; \quad \frac{\partial X_{k}}{\partial v}=b_{k} \eta, \quad(k=1,2, \ldots n-2)$, and

$$
\frac{\partial \boldsymbol{\xi}}{\partial u}=-\sum_{k} a_{k} X_{k}-m \eta, \quad \frac{\partial \eta}{\partial v}=-\sum_{k} b_{k} X_{k}-n \xi,
$$


the $\xi, \eta$ and the $X$ 's in each equation having the same superscript, and $a_{k}, b_{k}, m$ and $n$ being the same for all values of the superscripts of $\xi, \eta$ and the $X$ 's.

From (24), (28), and (29) we get

$$
\begin{aligned}
\frac{\partial^{2} \xi}{\partial u \partial v} & =\frac{\partial n}{\partial u} \eta+m n \xi \\
& =-\sum_{k}\left(\frac{\partial a_{k}}{\partial v} X_{k}+a_{k} b_{k} \eta-m b_{k} X_{k}\right)-\eta \frac{\partial m}{\partial v}+m n \xi \\
\frac{\partial^{2} \eta}{\partial u \partial v} & =-\sum_{k}\left(\frac{\partial b_{k}}{\partial u} X_{k}+a_{k} b_{k} \xi-n a_{k} X_{k}\right)-\xi \frac{\partial n}{\partial u}+m n \eta \\
& =\frac{\partial m}{\partial v} \xi+m n \eta, \\
\frac{\partial^{2} X_{k}}{\partial u \partial v} & =\frac{\partial a_{k}}{\partial v} \xi+a_{k} n \eta=\frac{\partial b_{k}}{\partial u} \eta+b_{k} m \xi
\end{aligned}
$$

Since there are $n$ equations of each of these types, obtained by letting the superscript $i$ take values $1,2, \ldots n$, the following $2 n+3$ relations must be satisfied:

$$
\left\{\begin{aligned}
& \frac{\partial a_{k}}{\partial v}=m b_{k}, \frac{\partial b_{k}}{\partial u}=n a_{k} \\
& \frac{\partial m}{\partial v}+\frac{\partial n}{\partial u}+\sum_{k} a_{k} b_{k}=0 .
\end{aligned}\right.
$$

In consequence of (25) the last of these is equivalent to

(31) $\frac{\partial}{\partial v}\left(\frac{1}{\sqrt{G}} \frac{\partial \sqrt{E}}{\partial v}\right)+\frac{\partial}{\partial u}\left(\frac{1}{\sqrt{E}} \frac{\partial \sqrt{G}}{\partial u}\right)+\sum_{k} a_{k} b_{k}=0$.

Whenever we have a set of functions satisfying these equations, the system (24), (28) and (29) is completely integrable. Moreover, from the form of the equations of the system it follows that each set of solutions satisfies the conditions

$$
\left\{\begin{array}{l}
\sum_{k} X_{k}^{i^{2}}+\xi^{i^{2}}+\eta^{i^{2}}=\text { const. } \\
\sum_{k} X_{k}^{i} X_{k}^{j}+\xi^{i} \xi^{j}+\eta^{i} \eta^{j}=\text { coust. }
\end{array}\right.
$$


Hence by a suitable choice of the constants of integration the functions will satisfy (27). It can be shown, as in the case of three dimensions [cf. $\$ 65$ ], that the most general solution can be obtained from a particular solution by effecting an orthogonal substitution with constant coefficients on the elements of the determinant $\boldsymbol{\alpha}$; thus

$$
X_{k}^{i^{\prime}}=\sum_{j=1}^{n} \alpha_{i}^{j} X_{k}^{j}, \quad \xi \xi^{\prime}=\sum_{j=1}^{n} \alpha_{i}^{j} \xi^{j}, \quad \eta^{i^{\prime}}=\sum_{j=1}^{n} \alpha_{i}^{j} \eta^{j} .
$$

A determinant of the type (26) satisfying the conditions (24), (28), (29) and (30) is called an orthogonal determinant in space of $n$ dimensions.

Suppose we have $2 n$ functions $\xi$ and $\eta$ satisfying conditions of the form (23) and (24). We inquire whether there exists a corresponding orthogonal determinant $d$ for which these functions are the elements of the last two rows.

By purely algebraic processes we can find $n(n-2)$ functions $Y_{j}^{i},(i=1, \ldots \ldots n j=1, \ldots n-2)$ such that

$$
\left|\begin{array}{l}
Y_{1}^{1} \ldots \ldots . Y_{1}^{n} \\
-\ldots-\frac{-}{Y_{n}^{1}-2} \ldots . Y_{n-2}^{n} \\
\xi^{1} \ldots \ldots . \xi^{n} \\
\eta^{1} \ldots \ldots . \eta^{n}
\end{array}\right|
$$

is the determinant of an orthogonal substitution. Since

$$
\sum_{i=1}^{n} Y_{j}^{i} \xi^{i}=0, \quad \sum_{i=1}^{n} Y_{j}^{i} \eta^{i}=0
$$

we have equations of the form

$$
\left\{\begin{array}{c}
\frac{\partial Y_{j}^{i}}{\partial u}=\sum_{k} P_{j k} Y_{k}^{i}+A_{j} \xi^{i}, \\
\frac{\partial Y_{j}^{i}}{\partial v}=\sum_{k} Q_{j k} Y_{k}^{i}+B_{j} \eta^{i}, \\
\frac{\partial \xi^{i}}{\partial u}=-\sum A_{k} Y_{k}^{i}-m \eta^{i}, \quad \frac{\partial \xi^{i}}{\partial v}=n \eta^{i}, \\
\frac{\partial \eta^{i}}{\partial u}=m \xi^{i}, \quad \frac{\partial \eta^{i}}{\partial v}=-\sum B_{k} Y_{k}^{i}-n \xi^{i},
\end{array}\right.
$$


where $i=1, \ldots n ; j, k=1, \ldots n-2(j \neq k)$. Expressing the condition of integrability of the first two equations, we find that the functions $P, Q, A, B$ must satisfy the following equations:

$$
\left\{\begin{aligned}
\frac{\partial}{\partial v} P_{j l}-\frac{\partial}{\partial u} Q_{j l} & =\sum_{k} Q_{j l} \cdot P_{k l}-\sum_{k} P_{j k} \cdot Q_{k l}, \\
\frac{\partial B_{j}}{\partial u} & =\sum_{k} P_{j k} B_{k}+A_{j} n \\
\frac{\partial A_{j}}{\partial v} & =\sum_{k} Q_{j k} A_{k}+B_{j} m .
\end{aligned}\right.
$$

We are interested, however, in seeing whether there is a set of $Y$ 's for which the first two of (34) shall assume the form (28). Suppose we put

$$
X_{l c}^{i}=y_{1}^{k} Y_{1}^{i}+y_{2}^{k} Y_{2}^{i}+\ldots+y_{n-2}^{k} Y_{n-2}^{i}\left(\begin{array}{l}
i=1,2 \ldots n \\
k=1,2 \ldots n-2
\end{array}\right),
$$

where the $y$ 's are the elenents of an orthogonal substitution of order $n-2$. These functions $X_{k}^{i}$ serve with the $\xi$ 's and $\eta$ 's to make $\Delta(26)$ the determinant of an orthogonal substitution of order $n$. We have accordingly in the general case equations of the form (34). If, however, we wish the first two to be of the form (28), the functions $y$ must satisfy the equations

$$
\frac{\partial y_{l}^{k}}{\partial u}+\sum_{j=1}^{n-2} y_{j}^{k} P_{j l}=0, \quad \frac{\partial y_{l}^{k}}{\partial v}+\sum_{j=1}^{n-2} y_{j}^{k} Q_{j l}=0 .
$$

The conditions of integrability of these equations reduce to the first equation (35). But all of the latter are satisfied, and hence there exist solutions of (36). Hence we have the theorem:

When $2 n$ functions $\xi$ and $\eta$ are known satisfying (23) and (24), there exist functions $X_{j}^{i}$ which with $\xi$ and $\eta$ form an orthogonal determinant $\boldsymbol{A}$.

From the form of (36) it follows that the functions $y$ are determined to within an orthogonal substitution with constants coefficients. The effect of such a substitution on the $y$ 's is equivalent to an orthogonal substitution of the form 


$$
X_{k}^{\prime i}=\sum_{j=1}^{n-2} \alpha_{k}^{j} X_{j}^{i}
$$

on the elements of the columns of the matrix of the first $n-2$ rows of $A$. Hence the last two rows of an orthogonal determinant $A$ determine $\boldsymbol{A}$ to within such an orthogonal substitution with constant coefficients effected upon the matrix of the first $n-2$ rows of $A$.

When an $O$ net is known, the functions $\xi$ and $\eta$ can be found at once, and the further problem of putting its determinant in the canonical form requires the solution of $2(n-2)$ completely integrable equations of the form (36). Guichard ${ }^{50}$ ) calls this a problem of the order $n-2$. In particular for a net in 5 -space this is equivalent to finding the direction-cosines of an $O$ net in 3-space when the rotations of its trihedral are known. In [\$65] we saw that this requires the solution of a Riccati equation.

64. Determination of $O$ nets. We consider now the determination of the $O$ nets corresponding to a given orthogonal determinant. Evidently the coordinates $x$ of any net $N$ are expressible in the form

$$
x=\sum_{k=1}^{n-2} p_{k} X_{k}+q \xi \dot{\xi}+r \eta
$$

the superscripts of $x, X, \xi$, and $\eta$ being the same, and $p_{k}, q$ and $r$ being functions of $u$ and $v$ independent of the superscript of $x$. We have now to find the conditions to be satisfied by $p_{k}, q$ and $r$ in order that (37) be the equation of the net.

By differentiation of (37) we find

$$
\begin{aligned}
& \frac{\partial x}{\partial u}=\sum_{k=1}^{n-2}\left(\frac{\partial p_{k}}{\partial u}-a_{k} q\right) X_{k}+\xi\left(\sum_{k} a_{k} p_{k}+\frac{\partial q}{\partial u}+m r\right)+\eta\left(\frac{\partial r}{\partial u}-q m\right), \\
& \frac{\partial x}{\partial v}=\sum_{k=1}^{n-2}\left(\frac{\partial p_{k}}{\partial v}-b_{k} r\right) X_{k}+\xi\left(\frac{\partial q}{\partial v}-r n\right)+\eta\left(\sum_{k} b_{k} p_{k}+\frac{\partial r}{\partial v}+n q\right)
\end{aligned}
$$

Equating these expressions to those of (22), we see that we must have

so) L. c., p. 500. 


$$
\left\{\begin{array}{c}
\frac{\partial p_{k}}{\partial u}=a_{k} q, \quad \frac{\partial p_{k}}{\partial v}=b_{k} r \\
\frac{\partial q}{\partial u}=-\sum_{k} a_{k} p_{k}-m r+\sqrt{E}, \quad \frac{\partial q}{\partial v}=n r \\
\frac{\partial r}{\partial u}=m q, \quad \frac{\partial v}{\partial v}=-\sum_{k} b_{k} p_{k}-n q+\sqrt{G}
\end{array}\right.
$$

where $m$ and $n$ are given by (25).

By differentiating these equations, we find that they form a completely integrable system, and consequently their solution involves $n$ arbitrary constants.

We find also that a function $p_{k}$ and the $n$ functions $X_{k}^{i}$ are solutions of the equation

$$
\frac{\partial^{2} \theta_{k}}{\partial u \partial v}=\frac{\partial \log a_{k}}{\partial v} \frac{\partial \theta_{k}}{\partial u}+\frac{\partial \log b_{k}}{\partial u} \frac{\partial \theta_{k}}{\partial v} .
$$

Suppose now that we have a solution of any one of these $n-2$ equations, say $p_{l}$. From the equations

$$
\frac{\partial p_{l}}{\partial u}=a_{l} q, \quad \frac{\partial p_{l}}{\partial v}=b_{l} r
$$

we find two functions $q$ and $r$. Then from the other $n-3$ equations of this type we find by quadratures the functions $p_{k}$. When these values are substituted in (37), we have an $O$ net corresponding to the given orthogonal determinant. Hence:

The determination of the $O$ nets corresponding to a given orthogonal determinant is equivalent to the integration of any one of the $n-2$ equations of Laplace (39).

We shall speak of $p_{k}$ and $X_{k}^{i}$ as the tangential coordinates of the $O$ net defined by (37).

From (3) and (37) we have

$$
2 \omega=\sum p_{k}^{2}+q^{y}+r^{2} .
$$

Making use of (38), we find

$$
\frac{\partial \omega}{\partial u}=V \bar{E} q, \quad \frac{\partial \omega}{\partial v}=V \bar{G} r .
$$


As an immediate consequence of these equations we have the theorem:

$A$ necessary and sufficient condition that an O net corresponding to a given orthogonal determinant be such that $\sum x^{2}$ is constant is that its coordinates be given by

$$
x=\sum_{k=1}^{n-2} e_{k} X_{k}
$$

in which the e's are constant.

When we have a solution $\theta$ of the point equation (1) of an $O$ net $N$, the functions $q$ and $r$ given by

$$
\frac{\partial \theta}{\partial u}=V \bar{E} q, \quad \frac{\partial \theta}{\partial v}=\sqrt{G} r
$$

satisfy the fourth and fifth of equations (38). These functions and the functions $p_{k}\left(k_{i}=1, \ldots, n-2\right)$ obtained by the quadratures

$$
\frac{\partial p_{k}}{\partial u}=a_{k} q, \quad \frac{\partial p_{k}}{\partial v}=b_{k} r
$$

determine an $O$ net $N^{\prime}$, whose coordinates are of the form

$$
x^{\prime}=\sum p_{k} X_{k}+q \xi+r \eta
$$

and which corresponds to the same orthogonal determinant as $N$. Since the $p$ 's are determined only to within additive constants, there are $\infty^{n-2}$ nets $N^{\prime}$ corresponding to a solution $\theta$ of (1).

From (41) and (43) it follows that when $\theta=\omega, N^{\prime}$ coincides with $N$. When $\theta$ is a constant, we have the case of the preceding theorem. Hence we have:

Each solution of the point equation of an $O$ net $N$ other than () given by (3) leads by quadratures alone to $\infty^{n-2}$ nets $N^{\prime}$ corresponding to the same orthogonal determinant as $N$.

By means of (40) and (41) we establish the converse theorem:

Each net parallel to an $O$ net $N$ gives by quadratures a solution of the point equation of $N$.

65. Congruences conjugate to $O$ nets. From $\$ 5$ it follows that the direction-parameters of any congruence conjugate to an $O$ net $N$ can be chosen so that they are the cartesian coordinates 
of a net $N^{\prime}$ parallel to $N$, and therefore corresponding to the same orthogonal determinant. These nets $N^{\prime}$ are of three kinds.

$1^{\circ}$. When the coordinates of $N^{\prime}$ are of the form (42) with the $e^{\prime} \mathrm{s}$ in the isotropic relation

$$
\sum e_{k}^{2}=0
$$

the congruence is I. Evidently there are $\infty^{n-4}$ such congruences. $2^{\circ}$. When in (42) the constants satisfy

$$
\sum e_{k}^{2}=1,
$$

the congruence is $2, \mathrm{I}$; there are $\infty^{n-3}$ such congruences.

$3^{\circ}$. For the others, if we put

$$
X_{i}=x^{\prime}, \quad Y_{1}=\frac{1}{2}\left(\sum x^{\prime 2}+1\right), \quad Y_{2}=\frac{i}{2}\left(\sum x^{\prime 2}-1\right),
$$

we have

$$
\begin{gathered}
\sum X^{2}=Y_{1}^{2}+Y_{\mathrm{a}}^{2}, \\
\sum\left(\frac{\partial X}{\partial u}\right)^{2}-\left(\frac{\partial Y_{1}}{\partial u}\right)^{2}-\left(\frac{\partial Y_{2}}{\partial u}\right)^{2}=\sum\left(\frac{\partial x^{\prime}}{\partial u}\right)^{2} \neq 0, \\
\sum\left(\frac{\partial X}{\partial v}\right)^{2}-\left(\frac{\partial Y_{1}}{\partial v}\right)^{2}-\left(\frac{\partial Y_{2}}{\partial v}\right)^{2}=\sum\left(\frac{\partial x^{\prime}}{\partial v}\right)^{2} \neq 0 .
\end{gathered}
$$

Hence all the other congruences are 3,1 , and we have:

Of the congruences conjugate to an $O$ net in $n$-space, $\infty^{n-4}$ are $\mathrm{I}$, $\infty^{n-3}$ are $2, \mathrm{I}$, and all the rest are $3, \mathrm{I}$.

In view of the second theorem of $\S 64$ and the above results we remark:

$1^{\circ}$. In 3-space of the congruences conjugate to an $O$ net, one is $2, \mathrm{I}$ and the others are $3, \mathrm{I}$.

$2^{\circ}$. In 4-space of the congruences conjugate to an $O$ net, two are I, their parameters being $X_{1}+i X_{2}$ and $X_{1}-i X_{2} ; \infty^{1}$ are $2, \mathrm{I}$ and the others are $3, \mathrm{I}$.

66. Transformations $F$ of an $O$ net for which the conjugate congruence is normal to the net. A congruence of direction-parameters $X$ is said to be normal to a net $N(x)$, if

$$
\sum X \frac{\partial x}{\partial u}=0, \quad \sum X \frac{\partial x}{\partial v}=0
$$


When we take for the direction-parameters the cartesian coordinates $x^{\prime}$ of a suitably chosen net $N^{\prime}$ parallel to $N$, these equations of condition may be replaced by

$$
\sum x^{\prime} \frac{\partial x^{\prime}}{\partial u}=0, \quad \sum x^{\prime} \frac{\partial x^{\prime}}{\partial v}=0,
$$

from which follow

$$
\sum x^{\prime 2} \doteq l, \quad \sum \frac{\partial x^{\prime}}{\partial u} \frac{\partial x^{\prime}}{\partial v}=0, \quad \sum \frac{\partial x}{\partial u} \frac{\partial x}{\partial v}=0,
$$

where $k$ is a constant. Hence the congruence is I or 2, I, according as $k$ is zero or not, provided that

$$
\sum\left(\frac{\partial x}{\partial u}\right)^{2} \neq 0, \quad \sum\left(\frac{\partial x}{\partial v}\right)^{2} \neq 0,
$$

which we assume to be the case. Furthermole from (49) we see that $N$ and $N^{\prime}$ are $O$ nets. Hence:

A congruence conjugate to a net for which (50) holds can be normal to $N$ only in case $N$ is an $O$ net; then the congruence is $\mathrm{I}$ or $2, \mathrm{I}$.

Conversely :

$A$ congruence $\mathrm{I}$ or $2, \mathrm{I}$ is normal to the $O$ nets conjugate to it.

If a congruence $G$ conjugate to an $O$ net $N$ is normal to $N$, it follows from the second theorem of $\S 64$ that the coordinates $\hat{x}$ of the parallel net $\hat{N}$ determining the congruence are of the form

$$
\hat{x}=\sum_{k=1}^{n-2} e_{k} X_{k}
$$

where the $e$ 's are constants. By differentiation we have in consequence of (22) and (28)

$$
\frac{\partial \hat{x}}{\partial u}=\hat{h} \frac{\partial x}{\partial u}, \quad \frac{\partial \hat{x}}{\partial v}=\hat{\imath} \frac{\partial x}{\partial v},
$$

where

$$
\hat{h}=\sum_{k=1}^{n-2} \frac{e_{k} a_{k}}{\sqrt{E}}, \quad \hat{l}=\sum_{k=1}^{n-2} \frac{e_{k} b_{k}}{\sqrt{G}}
$$


An $F$ transform $N_{0}\left(x_{0}\right)$ of $N$ for which $G$ is the conjugate congruence is given by $(\S 15)$

$$
x_{0}=x-\frac{\theta}{\hat{\theta}} \hat{x},
$$

where $\theta$ is a solution of the point equation of $N$, and

$$
\frac{\partial \hat{\theta}}{\partial u}=\hat{h} \frac{\partial \theta}{\partial u}, \quad \frac{\partial \hat{\theta}}{\partial v}=\hat{l} \frac{\partial \theta}{\partial v} .
$$

If $p_{k}$ are the solutions of (43) and (44) for this function $\theta$, we find that these equations are satisfied by

$$
\hat{\theta}=\sum_{k=1}^{n-2} e_{k} p_{k} .
$$

We assume that it is this value of $\hat{\theta}$ which is used in (54) to determine $N_{0}$ (cf. $\S 17$ ).

From (II, 9) we have

(57) $\frac{\partial x_{0}}{\partial u}=\frac{\tau}{\hat{\theta}^{2}}\left(\hat{x} \frac{\partial \theta}{\partial u}-\hat{\theta} \frac{\partial x}{\partial u}\right), \quad \frac{\partial x_{0}}{\partial v}=\frac{\sigma}{\hat{\theta}^{2}}\left(\hat{x} \frac{\partial \theta}{\partial v}-\hat{\theta} \frac{\partial x}{\partial v}\right)$,

and consequently

$$
\sum \frac{\partial x_{0}}{\partial u} \frac{\partial x_{0}}{\partial v}=\frac{\boldsymbol{\tau} \sigma}{\hat{\theta}^{4}}\left(\sum \hat{x}^{2} \frac{\partial \theta}{\partial u} \frac{\partial \theta}{\partial v}-\sum \hat{x} \frac{\partial x}{\partial u} \hat{\boldsymbol{\theta}} \frac{\partial \boldsymbol{\theta}}{\partial v}-\sum \hat{x} \frac{\partial x}{\partial v} \hat{\boldsymbol{\theta}} \frac{\partial \boldsymbol{\theta}}{\partial v}\right) .
$$

When the congruence is $I$, that is when (46) holds, then $\sum \frac{\partial x_{0}}{\partial u} \frac{\partial x_{0}}{\partial v}=0$, whatever be $\theta$. Hence all the $F$ transforms are $O$ nets (cf. $\S 62$ ).

When $G$ is $2, \mathrm{I}$, that is when (47) holds, we have from (58)

$$
\sum \frac{\partial x_{0}}{\partial u} \frac{\partial x_{0}}{\partial v}-\frac{\partial \theta_{0}}{\partial u} \frac{\partial \theta_{0}}{\partial v}=0,
$$

where $\theta_{0}$, given by

$$
\theta_{0}=-\frac{\theta}{\hat{\theta}}
$$


is the solution of the point equation of $N_{0}$ which determines $N$ as an $F$ transform of $N_{0}(\S 16)$. Since (59) is the condition that $\sum x_{0}^{2}-\theta_{0}^{2}$ be a solution of the point equation of $N_{0}$, we have that $N_{0}$ is a net $2, O, \theta_{0}$ being the complementary function. Furthermore from (54) and (60) we have

$$
\sum\left(x-x_{0}\right)^{2}=\theta_{0}^{2} .
$$

From (44), (56), (59) and (60) it follows that, when $\theta$ in (43) is constant, $N_{0}$ is an $O$ net and is parallel to $N(\$ 16)$. Hence:

The $F$ transforms of an $O$ net for which the congruences of the transformations are normal to $N$ are $O$ nets and 2, $O$ nets; when the transform is 2, $O$, the complementary function is equal to the distance between corresponding points on $N$ and the transform.

When in particular $\theta=\omega$, we have from (52) $\sum x \hat{x}=\hat{\omega}$, where $\hat{\omega}$ is the corresponding solution of (55). Hence $\sum x_{0}^{2}=\theta_{0}^{2}$, that is, the hyperspheres with centers on $N_{0}$ and radii given by $\theta_{0}$ pass through the origin. Each of the $\infty^{n-3}$ congruences normal to an $O$ net is conjugate to a net of this kind ${ }^{51}$ ).

67. Transformations $F$ of $O$ nets into $O$ nets. In $\S 65$ we saw that in $n$-space the congruences conjugate to an $O$ net are $\mathrm{I}, 2, \mathrm{I}$ or $3, \mathrm{I}$. In the preceding section we discussed the transformations $F$ of $O$ nets for which the congruence of the transformation is I or 2, I. We consider now the case where the congruence is $3, \mathrm{I}$. We recall $(\S 62)$ that in addition to $N$ and its parallels there is one other family of parallel $O$ nets conjugate to the congruence. We shall make a study of the transformation $F$ of $N(x)$ into one of the nets of the second family, say $N_{1}\left(x_{1}\right)$.

We recall from $\S 15$ that the coordinates of $N_{1}$ are given by

$$
x_{1}=x-\frac{\theta}{\theta^{\prime}} x^{\prime},
$$

where

$$
\frac{\partial x^{\prime}}{\partial u}=h \frac{\partial x}{\partial u}, \quad \frac{\partial x^{\prime}}{\partial v}=l \frac{\partial x}{\partial v},
$$

51) Similar results follow when $\theta=\frac{1}{2} \Sigma(x-a)^{2}$, where the $a$ s are constant. In this case the spheres pass through the point whose coordinates are the $a$ 's. We shall refer to this as the case where $\theta=\omega$. 
and

Also we have

$$
\frac{\partial \boldsymbol{\theta}^{\prime}}{\partial u}=h \frac{\partial \theta}{\partial u}, \quad \frac{\partial \boldsymbol{\theta}^{\prime}}{\partial v}=l \frac{\partial \theta}{\partial v}
$$

(65) $\frac{\partial x_{1}}{\partial u}=\frac{\tau}{\theta^{\prime 2} h}\left(x^{\prime} \frac{\partial \theta^{\prime}}{\partial u}-\theta^{\prime} \frac{\partial x^{\prime}}{\partial u}\right), \quad \frac{\partial x^{\prime}}{\partial v}=\frac{\sigma}{\theta^{\prime 2} l}\left(x^{\prime} \frac{\partial \theta^{\prime}}{\partial v}-\theta^{\prime} \frac{\partial x^{\prime}}{\partial v}\right)$.

$\tau$ and $\sigma$ having the values

$$
\tau=h \theta-\theta^{\prime}, \quad \sigma=l \theta-\theta^{\prime} .
$$

From (65) it follows that a necessary and sufficient condition that $N_{1}$ be an $O$ net is that

$$
\frac{\partial \theta^{\prime}}{\partial u} \frac{\partial \theta^{\prime}}{\partial v} \sum x^{\prime 2}-\theta^{\prime} \frac{\partial \theta^{\prime}}{\partial u} \sum x^{\prime} \frac{\partial x^{\prime}}{\partial v}-\theta^{\prime} \frac{\partial \theta^{\prime}}{\partial v} \sum x^{\prime} \frac{\partial x^{\prime}}{\partial u}=0
$$

This equation is satisfied by

$$
\theta^{\prime}=\frac{1}{2} \sum x^{\prime 2}
$$

The coordinates $x^{\prime}$ of the net $N^{\prime}$ parallel to $N$ which are the direction-parameters of the congruence of the transformation are of the form

$$
x^{\prime}=\sum p_{k} X_{k}+q \xi+r \eta
$$

where $p_{k}, q$ and $r$ satisfy equations (38) with $E$ and $G$ replaced by $E^{\prime}$ and $G^{\prime}$, the first fundamental coefficients of $N^{\prime}$. Hence equation (68) may be written

$$
\theta^{\prime}=\frac{1}{2} \sum x^{\prime 2}=\frac{1}{2}\left(\sum p_{k}^{2}+q^{2}+r^{2}\right)
$$

The corresponding function $\theta$ of the transformation is given by the quadratures (64), which are reducible by means of (38) to

$$
\frac{\partial \theta}{\partial u}=\sqrt{E} q, \quad \frac{\partial \theta}{\partial v}=V \bar{G} r
$$


From these equations and the results of $\S 64$ it follows that $\theta$ is the solution of the point equation of $N$ which determines $N^{\prime}$. Hence $\boldsymbol{\theta}$ is given by a quadrature and the additive arbitrary constant leads to a family of parallel nets $N_{1}(\S 16)$. Accordingly:

Each net $N^{\prime}$ parallel to a given $O$ net determines $\infty^{1}$ parallel $O$ nets each of which is an $F$ transform of $N$; they are obtained by a quadrature.

Conversely, if $\theta$ is any solution of the point equation of $N$ other than $\omega$, and $N^{\prime}$ is any one of the $\infty^{n-2}$ nets parallel to $N$ determined by $\theta$ ( $(64)$, the function $\theta^{\prime}$ defined by (70) satisfies the corresponding equation (64). Hence:

Each solution of the point equation of $N$ other than $\omega$ leads to $\infty^{n-2} O$ nets $N_{1}\left(x_{1}\right)$ which are $F$ transforms of $N$; the coordinates $x_{1}$ are given by

where

$$
x_{1}=x-2 \frac{\theta x^{\prime}}{\sum x^{\prime 2}}
$$

$$
x^{\prime}=\sum_{k=1}^{n-2} p_{k} X_{k}+\frac{1}{\sqrt{E}} \frac{\partial \theta}{\partial u} \xi+\frac{1}{\sqrt{G}} \frac{\partial \theta}{\partial v} \eta,
$$

the functions $p_{k}$ being obtained by the quadratures

$$
\frac{\partial p_{k}}{\partial u}=\frac{a_{k}}{\sqrt{E}} \frac{\partial \theta}{\partial u}, \quad \frac{\partial p_{k}}{\partial v}=\frac{b_{k}}{\sqrt{G}} \frac{\partial \theta}{\partial v}
$$

Moreover, all of these nets $N_{1}\left(x_{1}\right)$ are harmonic to the congruence harmonic to $N$ determined by $\theta$.

68. Transformations $R$. The nets $N_{0}$ and $N_{1}$ defined by (54) and (62) respectively form with $N$ a triad $(\S 20)$. From (51), (69) and (56) we have

$$
\sum x^{\prime} \hat{x}=\sum e_{k} p_{k}=\hat{\theta}
$$

Consequently we obtain from (65) and (60)

$$
\begin{gathered}
\sum\left(x_{1}-x_{0}\right) \frac{\partial x_{1}}{\partial u}=0, \quad \sum\left(x_{1}-x_{0}\right) \frac{\partial x_{1}}{\partial v}=0, \\
\sum\left(x_{1}-x_{0}\right)^{2}=\left(\frac{\theta}{\hat{\theta}}\right)^{2}=\theta_{0}^{2} .
\end{gathered}
$$


From these equations and (61) we remark that the hyperspheres with centers at points of $N_{0}$ and radii determined by $\theta_{0}$ are tangent to $N$ and $N_{1}$. Since there are $\infty^{n-3}$ congruences $2, \mathrm{I}$ conjugate and normal to $N$, there are $\infty^{n-3}$ nets $N_{0}$ forming such a triad with $N$ and $N_{1}$. Hence :

If $N$ and $N_{1}$ are two $O$ nets in the relation of a transformation $F$, for which the congruence is $3, \mathrm{I}$, there are $\infty^{n-3}$ two-parameter families of hyperspheres tangent to $N$ and $N_{1}$; the locus of the centers of the hyperspheres of any family is a net $N_{0}$ which is $2, O$, the complementary function being the radius of the hypersphere; the nets $N, N_{1}$ and $N_{0}$ form a triad under transformations $F$.

In view of this result we call the transformation from $N$ into $N_{1}$ a generalized transformation of Ribaucour ${ }^{52}$ ), or simply a transformation $R$. We call the corresponding net $N_{0}$ a central net of the transformation.

From (63), (64) and (68) we have

$$
\sum x^{\prime} \frac{\partial x}{\partial u}=\frac{\partial \theta}{\partial u}, \quad \sum x^{\prime} \frac{\partial x}{\partial v}=\frac{\partial \theta}{\partial v} .
$$

By means of these equations, (57) and (75) we find that

Hence :

$$
\sum x^{\prime} \frac{\partial x_{0}}{\partial \imath}=0, \quad \sum x^{\prime} \frac{\partial x_{0}}{\partial v}=0 .
$$

The tangent planes of $N_{0}$ are normal bisectors of the joins of corresponding points of $N$ and $N_{1}$.

Also it follows from $\S 17$ that:

Corresponding tangent planes to $N, N_{1}$ and $N_{0}$ meet in a line generating a congruence harmonic to each of these nets.

By means of $N^{\prime}$ we can obtain a transformation $R$ of any net parallel to $N(\S 67)$. From the above results we obtain the theorem:

When a transformation $R$ of a net $N$ is known, a transformation $R$ of any net $\bar{N}$ parallel to $N$ can be found 'by a quadrature; the transforms of $N$ and $\bar{N}$ are parallel, as are also corresponding central nets of the transformations.

52) In his study of cyclic systems Ribaucour considered two-parameter families of spheres in 3-space upon the sheets of whose envelope the lines of curvature correspond. The relation between the two sheets has been called a transformation of Ribaucour. 
When in particular $\bar{N}$ is $N^{\prime}$, the transform of $\bar{N}$ is the origin, as follows from (62). This is the case treated at the end of $\S 66$. From (II, 1) and (68) we have

Hence:

$$
x_{1}^{\prime}=\frac{2 x^{\prime}}{\sum x^{\prime}} .
$$

When $N$ and $N_{1}$ are $O$ nets in the relation of a transformation $R$, the nets $N^{\prime}$ and $N_{1}^{\prime}$ whose coordinates are direction-parameters of the congruence of the transformation may be obtained from one other by inversion.

In $\S 75$ we shall show that only for certain types of $O$ nets do there exist transformations $F$ into non-parallel $O$ nets which are not transformations $R$. Hence:

In general an inversion is the only radial transformation of an $O$ net into an $O$ net.

The function $\omega_{1}$, where

$$
2 \omega_{1}=\sum x_{1}^{2},
$$

is a solution of the point equation of $N_{1}$. We wish to show, furthermore, that $\omega_{1}$ is the transform of $\omega$ by an equation analogous to (62), namely

$$
\omega_{1}=\omega \div \frac{\theta}{\theta^{\prime}} \omega^{\prime}
$$

where $\omega^{\prime}$ is defined by

$$
\frac{\partial \omega^{\prime}}{\partial u}=h \frac{\partial \omega}{\partial u}, \quad \frac{\partial \omega^{\prime}}{\partial v}=l \frac{\partial \omega}{\partial v} .
$$

When the expression (62) for $x_{1}$ is substituted in (78), the latter reduces to

$$
\theta+\omega^{\prime}-\sum x x^{\prime}=0 .
$$

By differentiating the left-hand member of this equation we find that it is constant. Hence the additive constant of $\omega^{\prime}$ can be chosen so that (80), and consequently (78), is satisfied.

69. Transformations $R$ in another form. From (65) and (68) we have

$$
\text { (81) } E_{1}=\sum\left(\frac{\partial x_{1}}{\partial u}\right)^{2}=\frac{\tau^{2} E}{\theta^{\prime 2}}, \quad G_{1}=\sum\left(\frac{\partial x_{1}}{\partial v}\right)^{2}=\frac{\sigma^{2} G}{\theta^{\prime 2}} .
$$


If we choose the algebraic signs of $\sqrt{E_{1}}$ and $\sqrt{G_{1}}$, so that

we have from (66)

$$
\tau=\theta^{\prime} \sqrt{\frac{E_{1}}{E}}, \quad \sigma=\theta^{\prime} \sqrt{\frac{G_{1}}{G}},
$$

$$
h=\frac{\theta^{\prime}}{\theta}\left(1+\sqrt{\frac{E_{1}}{E}}\right), \quad l=\frac{\theta^{\prime}}{\theta}\left(1+\sqrt{\frac{G_{1}}{G}}\right) .
$$

If we define a function $w$ by

$$
\theta^{\prime}=\theta w,
$$

equation (70) becomes

$$
\sum_{k=1}^{n-2} p_{k}^{2}+q^{2}+\dot{r}^{2}-2 \theta u=0
$$

The equations (62) of a transformation $R$ may be written

$$
x_{1}=x-\frac{1}{w}\left(\sum_{k=1}^{n-2} p_{k} X_{k}+q \xi+r \eta\right)
$$

where the functions involved satisfy (85) and

$$
\left\{\begin{array}{cc}
\frac{\partial \theta}{\partial u}=\sqrt{E} q, & \frac{\partial \theta}{\partial v}=\sqrt{G} r \\
\frac{\partial p_{k}}{\partial u}=a_{k} q, & \frac{\partial p_{k}}{\partial r}=b_{k} r \\
\frac{\partial q}{\partial u}=-\sum_{k=1}^{n-2} a_{k} p_{k}-\frac{1}{\sqrt{G}} \frac{\partial \sqrt{E}}{\partial v} r+w\left(\sqrt{E}+\sqrt{E_{1}}\right) \\
\frac{\partial q}{\partial v}=\frac{1}{\sqrt{E}} \frac{\partial \sqrt{G}}{\partial u} r, \quad \frac{\partial r}{\partial u}=\frac{1}{\sqrt{G}} \frac{\partial \sqrt{E}}{\partial v} q \\
\frac{\partial r}{\partial v}=-\sum_{k=1}^{n-2} b_{k} p_{k}-\frac{1}{\sqrt{E}} \frac{\partial \sqrt{G}}{\partial u} q+w\left(\sqrt{G}+\sqrt{G_{1}}\right) \\
\left.\frac{\partial \log w}{\partial u}=\sqrt{E_{1}} \frac{q}{\theta}, \quad \frac{\partial \log w}{\partial v}=\sqrt{G_{1}} \frac{r}{\theta}{ }^{33}\right)
\end{array}\right.
$$

33) The expressions for $\frac{\partial \log w}{\partial u}$ and $\frac{\partial \log w}{\partial v}$ follow from (64), (71), (83) and (84), and those for $\frac{\partial q}{\partial u}$ and $\frac{\partial r}{\partial v}$ are obtained by differentiating (85). 
The integrability conditions of this system of equations are satisfied provided that $\theta$ is a solution of the point equation of $N$ and that

$$
\left\{\begin{array}{l}
\frac{\partial \sqrt{E_{1}}}{\partial v}=\sqrt{G_{1}}\left(\frac{1}{\sqrt{G}} \frac{\partial \sqrt{E}}{\partial v}-\left(\sqrt{E}+\sqrt{E_{1}}\right) \frac{r}{\theta}\right), \\
\frac{\partial \sqrt{G_{1}}}{\partial v}=\sqrt{E_{1}}\left(\frac{1}{\sqrt{E}} \frac{\partial \sqrt{G}}{\partial u}-\left(\sqrt{G}+\sqrt{G_{1}}\right) \frac{q}{\theta}\right) .
\end{array}\right.
$$

as can be verified readily with the aid of (21), (30) and (31). Moreover, any set of functions satisfying (87) make the left-hand member of (85) constant, as can be shown by differentiation.

If we write

$$
\frac{\partial x_{1}}{\partial u}=\sqrt{E_{1}} \xi_{1}, \quad \frac{\partial x_{1}}{\partial v}=\sqrt{G_{1}} \eta_{1},
$$

it follows from (65) and (82) that

$$
\xi_{1}=\frac{q}{\theta^{\prime}} x^{\prime}-\xi, \quad \eta_{1}=\frac{r}{\theta^{\prime}} x^{\prime}-\eta .
$$

If we define functions $X_{1, k}$ by

$$
X_{1, k}=\frac{p_{k}}{\theta^{\prime}} x^{\prime}-X_{k} \quad(k=1, \ldots n-2),
$$

the functions $\xi_{1}, \eta_{1}$ and $X_{1, k}$ satisfy equations of the form (27). By differentiation we have

$$
\left\{\begin{aligned}
\frac{\partial \xi_{1}}{\partial v} & =n_{1} \eta_{1}, & \frac{\partial \eta_{1}}{\partial u} & =m_{1} \xi_{1}, \\
\frac{\partial X_{1, k}}{\partial u} & =a_{1, k} \xi_{1}, & \frac{\partial X_{1, k}}{\partial v} & =b_{1, k} \eta_{1},
\end{aligned}\right.
$$

where

$$
\left\{\begin{aligned}
n_{1} & =n-\frac{q}{\theta}\left(\sqrt{G}+\sqrt{G_{1}}\right), & m_{1} & =m-\frac{r}{\theta}\left(\sqrt{E}+\sqrt{E_{1}}\right), \\
a_{1, k} & =a_{k}-\frac{p_{k}}{\theta}\left(\sqrt{E}+\sqrt{E_{1}}\right), & b_{1, k} & =b_{k}-\frac{p_{k}}{\theta}\left(\sqrt{G}+V \overline{G_{1}}\right) .
\end{aligned}\right.
$$

Hence ( $\S 63$ ) the functions $\xi_{1}, \eta_{1}, X_{1, k}$ are the elements of the orthogonal determinant corresponding to $N_{1}$. 
70. Inverse of a transformation $R$. Let $p_{k}^{-1}, q^{-1}, r^{-1}$; $w^{-1}$ denote the functions determining the inverse transformation by means of which $N$ is obtained from $N_{1}$, so that

$$
x=x_{1}-\frac{1}{w^{-1}}\left(\sum p_{k}^{-1} X_{1, k}+q^{-1} \xi_{1}+r^{-1} \eta_{1}\right)
$$

When this expression for $\left(x-x_{1}\right)$ is equated to that given by (86), and $X_{1, k}, \xi_{1}$ and $\eta_{1}$ are replaced by their above values it is readily found that

$$
p_{k}^{-1}=\varrho p_{k}, \quad q^{-1}=\varrho q, \quad r^{-1}=\varrho \vartheta, \quad w^{-1}=-\varrho w
$$

where $\varrho$ is to be determined. When we require that these functions and $\theta^{-1}$ satisfy an equation analogous to (85), we find that $\theta^{-1}=-\varrho \theta$. Comparing this with (II, 19), we have $\varrho=1 / \theta^{\prime}=$ $1 / \theta w$. Hence

$$
\left\{\begin{array}{r}
p_{k}^{-1}=\frac{p_{k}}{\theta w}, \quad q^{-1}=\frac{q}{\theta w}, \\
v^{-1}=\frac{r}{\theta w}, \quad w^{-1}=-\frac{1}{\theta}, \quad \theta^{-1}=-\frac{1}{w} .
\end{array}\right.
$$

By means of (93) we show that these functions satisfy a system of equations of the form (87) for $N_{1}$.

From (51), (54), (56), (60) and (91) we find

$$
x_{1}-x_{0}=\theta_{0} \sum e_{k} X_{1, k}
$$

which shows that the parameters of the congruences conjugate to $N_{1}$ and $N_{0}$ and to $N$ and $N_{0}$ are the same linear functions of the quantities $X_{1, k}$ and $X_{k}$ respectively.

71. Transformations $R$ in tangential coordinates. Let $P_{k}, Q$ and $R$ denote the tangential coordinates of an $O$-net $N(x)$. They satisfy (cf. $§ 64$ )

$$
\left\{\begin{aligned}
\frac{\partial P_{k}}{\partial u} & =a_{k} Q, & & \frac{\partial P_{k}}{\partial v}=b_{k} R, \\
\frac{\partial \omega}{\partial u} & =V \bar{E} Q, & \frac{\partial \omega}{\partial v} & =\sqrt{\bar{G}} R,
\end{aligned}\right.
$$


where $\omega$ is given by

$$
2 \omega=\sum P_{k}^{2}+Q^{2}+R^{2}
$$

Also we have

$$
x=\sum P_{k} X_{k}+Q \xi+R \eta
$$

Equation (80) may be written

$$
\omega^{\prime}=\sum P_{k} p_{k}+Q q+R r-\theta
$$

where $p_{k}, q, r$ and $\theta$ determine a transformation $R$ of $N$ into an $O$-net $N_{1}\left(x_{1}\right)$, whose coordinates are of the form

$$
x_{1}=\sum P_{1, k} X_{1, k}+Q_{1} \xi_{1}+R_{1} \eta_{1}
$$

the functions $P_{1, k}, Q_{1}$ and $R_{1}$ being the tangential coordinates of $N_{1}$. In consequence of (86), (90) and (91) we have

$$
P_{1, k}=\frac{\omega^{\prime}}{\theta^{\prime}} p_{k}-P_{k}, \quad Q_{1}=\frac{\omega^{\prime}}{\theta^{\prime}} q-Q, \quad R_{1}=\frac{\omega^{\prime}}{\theta^{\prime}} r-R .
$$

These formulas and (91) define the given transformation $R$ in terms of the tangential coordinates of $N$ and $N_{1}$.

72. Theorem of permutability of transformations $R$. We apply the theorem of permutability of transformations $F$ to the case of transformations $R$, and assume that $N_{1}$ and $N_{2}$ are two $R$ transforms of $N$ determined by functions $\theta_{1}$ and $\theta_{2}$ respectively, and nets $N^{\prime}\left(x^{\prime}\right)$ and $N^{\prime \prime}\left(x^{\prime \prime}\right)$.

Now the analogues of (68) are

$$
\sum x^{\prime 2}=2 \theta_{1}^{\prime}, \quad \sum x^{\prime \prime 2}=2 \theta_{2}^{\prime \prime} .
$$

Making use of (II, 31), we obtain

$$
\begin{cases}\frac{\partial \theta_{1}}{\partial u}=\sum x^{\prime} \frac{\partial x}{\partial u}, & \frac{\partial \theta_{1}}{\partial v}=\sum x^{\prime} \frac{\partial x}{\partial v}, \\ \frac{\partial \theta_{2}}{\partial u}=\sum x^{\prime \prime} \frac{\partial x}{\partial u}, & \frac{\partial \theta_{\mathbf{2}}}{\partial v}=\sum x^{\prime \prime} \frac{\partial x}{\partial v} .\end{cases}
$$


By means of these results we establish by differentiation that $\sum x^{\prime} x^{\prime \prime}-\theta_{1}^{\prime \prime}-\theta_{2}^{\prime}$ is constant.

From the above and (II, 33) we have

$$
\sum\left(x_{1}^{\prime \prime \prime}\right)^{2}=2 \theta_{2}^{\prime \prime}-2 \frac{\theta_{1}^{\prime \prime}}{\theta_{1}^{\prime}}\left(\sum x^{\prime} x^{\prime \prime}-\theta_{1}^{\prime \prime}\right) .
$$

The functions $\theta_{1}^{\prime \prime}$ and $\theta_{2}^{\prime}$ are determined to within additive constants. Hence there are $\infty^{1}$ cases for which

$$
\sum x^{\prime} x^{\prime \prime}-\theta_{1}^{\prime \prime}-\theta_{2}^{\prime}=0 .
$$

Then, as follows from (II, 38),

$$
\theta_{12}^{\prime \prime \prime}=\frac{1}{2} \sum\left(x_{1}^{\prime \prime \prime}\right)^{2}
$$

Hence, by $\S 67, N_{12}$ is an $R$ transform of $N_{1}$.

As (105) is symmetrical in functions of the two transformations, $N_{12}$ is an $R$ transform of $N_{2}$ also. Consequently:

If $N_{1}$ and $N_{2}$ are $R$ transforms of an $O$ net $N$, there are $\infty^{1}$ $O$ nets $N_{12}$ which are $R$ transforms of $N_{1}$ and $N_{2}$, and they can be found by quadratures ${ }^{54}$ ).

The transformations from $N$ into $N_{1}$ and $N_{2}$ are given by equations of the form

$$
x_{i}=x-\frac{1}{w_{i}}\left(\sum p_{i, k} X_{k}+q_{i} \xi+r_{i} \eta\right) \quad(i=1,2),
$$

where the functions $p, q$ and $r$ are solutions of (87).

If we write the coordinates of $N_{1}^{\prime \prime \prime}\left(x_{1}^{\prime \prime \prime}\right)$, which are the directionparameters of the transformation of $N_{1}$ into a net $N_{12}$, in the form

$$
x_{1}^{\prime \prime \prime}=\sum_{k} p_{12, k} X_{1, k}+q_{12} \xi_{1}+r_{12} \eta_{1}
$$

54) This theorem is the generalization for $n$-space of the similar theorem for 3-space established by Bianchi by applying the Lie line-sphere transformation to the theorem of permutability of $W$ congruences; cf. Rendiconti dei Lincei, ser. 5, vol. $13^{1}$ (1904), p. 361. 
it follows from (II, 33), (90) and (91) that

(109) $p_{12, k}=\frac{\theta_{2}^{\prime}}{\theta_{1}^{\prime}} p_{1, k}-p_{2, k}, \quad q_{12}=\frac{\theta_{2}^{\prime}}{\theta_{1}^{\prime}} q_{1}-q_{2}, \quad r_{12}=\frac{\theta_{2}^{\prime}}{\theta_{1}^{\prime}} r_{1}-r_{9}$.

These quantities satisfy the relation

$$
\sum_{k} p_{12, k}^{2}+q_{12}^{2}+r_{12}^{2}=2 \theta_{12}^{\prime \prime \prime},
$$

and also because of (II, 37) the equations

$$
\begin{aligned}
\frac{\partial \theta_{12}}{\partial u} & =V_{E_{1}} q_{12}, & \frac{\partial \theta_{12}}{\partial v} & =\sqrt{G_{1}} r_{12}, \\
\frac{\partial p_{12, k}}{\partial u} & =a_{1, k} q_{12}, & \frac{\partial p_{12, k}}{\partial v} & =b_{1, k} r_{12} .
\end{aligned}
$$

The tangential coordinates of $N_{12}$ are given by the following formulas which are analogous to (91) and (101):

$$
\left\{\begin{aligned}
X_{12, k} & =\frac{p_{12, k}}{\theta_{12}^{\prime \prime \prime}} x_{1}^{\prime \prime \prime}-X_{1, k}, & P_{12, k} & =\frac{\omega_{1}^{\prime \prime \prime}}{\theta_{12}^{\prime \prime \prime}} p_{12, k}-P_{1, k}, \\
Q_{12} & =\frac{\omega_{1}^{\prime \prime \prime}}{\theta_{12}^{\prime \prime \prime}} q_{12}-Q_{1}, & R_{12} & =\frac{\omega_{1}^{\prime \prime \prime}}{\theta_{12}^{\prime \prime \prime}} r_{12}-R_{1},
\end{aligned}\right.
$$

where $\theta_{12}^{\prime \prime \prime}$ is given by (II, 38) and

$$
\left.\omega_{1}^{\prime \prime \prime}=\omega^{\prime \prime}-\frac{\theta_{1}^{\prime \prime}}{\theta_{1}^{\prime}} \omega\right)^{\prime} .
$$

When these expressions and those from (101) and (109) are substituted in

$$
\left\{\begin{array}{c}
X_{12, k}=X_{k}+\frac{\left(\theta_{2}^{\prime} x^{\prime \prime}-\theta_{2}^{\prime \prime} x^{\prime}\right) p_{1, k}+\left(\theta_{1}^{\prime \prime} x^{\prime}-\theta_{1}^{\prime} x^{\prime \prime}\right) p_{2, k}}{\theta_{1}^{\prime} \theta_{12}^{\prime \prime}} \\
P_{12, k}=P_{k}+\frac{\Omega_{2} p_{1, k}+\Omega_{1} p_{2, k}}{\theta_{1}^{\prime} \theta_{12}^{\prime \prime \prime}}, \quad Q_{12}=Q+\frac{\Omega_{2} q_{1}+\Omega_{1} q_{2}}{\theta_{1}^{\prime} \theta_{1:}^{\prime \prime \prime}} \\
R_{12}=R+\frac{\Omega_{2} r_{1}+\Omega_{1} r_{2}}{\theta_{1}^{\prime} \theta_{12}^{\prime \prime \prime}}
\end{array}\right.
$$

where

$$
\Omega_{1}=\theta_{1}^{\prime \prime} \omega^{\prime}-\theta_{1}^{\prime} \omega^{\prime \prime}, \quad \Omega_{2}=\theta_{2}^{\prime} \omega^{\prime \prime}-\theta_{2}^{\prime \prime} \omega^{\prime},
$$

we find that they are satisfied. 
Since the first of (112) involves the direction-cosines of $N$, the coordinates of $N^{\prime}$ and $N^{\prime \prime}$, and solutions of the point equations of $N^{\prime}$ and $N^{\prime \prime}$, we have the theorem:

If $N, N_{1}, N_{2}, N_{12}$ form a quatern under transformations $R, \bar{N}$ is any net parallel to $N$, and $\bar{N}_{1}$ and $\bar{N}_{2}$ are $R$ transforms of $\bar{N}$ parallel to $N_{1}$ and $N_{2}$ respectively, lines drawn through points of $\bar{N}_{1}$ and $\bar{N}_{2}$ parallel to the joins of $N_{1}$ and $N_{2}$ respectively with $N_{12}$ meet in points of a net $\bar{N}_{12}$ parallel to $N_{12}$.

73. Cyclic congruences. Let $N(x)$ be any net and $N_{1}\left(x_{1}\right)$ an $F$ transform defined by (62). The tangent planes to $N$ and $N_{1}$ meet in the lines of the congruence harmonic to $N$ determined by $\theta$; the coordinates $y$ and $z$ of the focal points $F_{1}$ and $F_{2}$ of the congruence are of the forms (II, 22)

$$
y=x-\frac{\theta}{\frac{\partial \theta}{\partial v}} \frac{\partial x}{\partial v}, \quad z=x-\frac{\theta}{\frac{\partial \theta}{\partial u}} \frac{\partial x}{\partial u} .
$$

If $M$ and $M_{1}$ denote corresponding points of $N$ and $N_{1}$, a necessary and sufficient condition that $F_{1} M=F_{1} M_{1}$ is that

$$
\sum x^{\prime 2} \frac{\partial \theta^{\prime}}{\partial v}-2 \sum x^{\prime} \frac{\partial x^{\prime}}{\partial v} \cdot \theta^{\prime}=0
$$

Hence in order that $F_{1} M=F_{1} M_{1}$ and $F_{2} M=F_{2} M_{1}$, it is necessary and sufficient that

$$
\boldsymbol{\theta}^{\prime}=c \sum x^{\prime 2}, \quad \text { or } \quad \sum x^{\prime 2}=0,
$$

where $c$ is a constant different from zero. In this case $N^{\prime}$ is an $O$ net, and $N$ and $N_{1}$ are $O$ nets. Consequently $(\S \S 66,67)$ :

When $N$ and $N_{1}$ are in relation $F$ a necessary and sufficient condition that each of the focal points of the harmonic congruence of the transformation be equidistant from the corresponding points of $N$ and $N_{1}$ is that $N$ and $N_{1}$ be $O$ nets in relation $R$, or conjugate to a congruence $\mathrm{I}$.

We consider the case for transformations $R$. In consequence of (22) and (87) the expressions (114) are reducible to the forms

$$
y=x-R_{2} \eta, \quad z=x-R_{1} \xi,
$$


where

$$
R_{1}=\frac{\theta}{q}, \quad R_{2}=\frac{\theta}{r} .
$$

From these equations and the preceding theorem it follows that the hyperspheres, $S_{1}$ and $S_{2}$, with centers at the focal points and of radii $R_{1}$ and $R_{2}$ pass through corresponding points of $N$ and of each of the $\infty^{n-2} R$ transforms of $N$ by meaus of $\theta(\$ 67)$. Moreover, since

$$
\sum(y-z)^{2}=R_{1}^{2}+R_{2}^{2}
$$

$S_{1}$ and $S_{2}$ meet orthogonally. From the fifth theorem of $\$ 10$ and the second of $\$ 67$ it follows that the hyperspheres with centers at the focal points of any congruence harmonic to an $O$ net $N$ and passing through points of $N$ meet orthogonally and pass through the $\infty^{n-2} R$ transforms of $N$ determined by the solution $\theta$ of the point equation of $N$ which determines the harmonic congruence. We call them focal hyperspheres of the congruence. In 3-space $S_{1}$ and $S_{2}$ are spheres mecting in circles orthogonal to $\infty^{1} R$ transforms of $N$, that is in circles of a cyclic system. Conversely, in [ $\S 177]$ it was seen that the $O$ nets orthogonal to the circles of a cyclic system are harmonic to the congruence of axes of the circles. Hence we say that for space of any order any congruence harmonic to an $O$ net is a cyclic congruence.

From the last theorem of $\$ 13$ we have the theorem:

If the direction-parameters $X$ of a cyclic congruence are solutions of the equation

$$
\frac{\partial^{2} \theta}{\partial u \partial v}=\frac{\partial \log A}{\partial v} \frac{\partial \theta}{\partial u}+\frac{\partial \log B}{\partial u} \frac{\partial \theta}{\partial v}+C \theta
$$

then

$$
\sum X^{2}=U^{2} A^{2}+V^{2} B^{2}
$$

where $U$ and $V$ are functions of $u$ and $v$ alone respectively.

In $\S 16$ we show that the condition (119) is characteristic of cyclic congruences.

If we put $X=y-z$, equation (117) is a special form of (119). 
In fact, from $(I, 54,55)$ we have

$$
\frac{\partial y}{\partial u}=\frac{\partial \log R_{2}}{\partial u}(y-z), \quad \frac{\partial z}{\partial v}=\frac{\partial \log R_{1}}{\partial v}(z-y) .
$$

If we express the condition that $X$ satisfy (118), we have

$$
R_{1}=A U, \quad R_{2}=B V,
$$

where $U$ and $V$ are functions of $u$ and $v$ alone respectively.

74. Multiply cyclic congruences. Let $N$ and $N_{1}$ be two $O$ nets in relation $F$ and let $G$ denote the harmonic congruence of the transformation $F$. If $N$ and $N_{1}$ are $R$ transforms of one another, the focal hyperspheres with centers on the focal nets of $G$ and passing through points of $N$ also pass through the corresponding points of $N_{1}$. If, however, $N_{1}$ is not an $R$ transform of $N$, then there are at least two pairs of focal hyperspheres associated with $G$. If we denote by $\bar{R}_{1}$ and $\bar{R}_{2}$ the radii of the focal hyperspheres passing through points of $N_{1}$, it follows from (121) that $\bar{R}_{1}=U_{1} R_{1}$ and $\bar{R}_{2}=V_{1} R_{2}$, where $U_{1}$ and $V_{1}$ are functions of $u$ and $v$ alone. From (111) it follows that $\bar{R}_{1}^{2}+\bar{R}_{2}^{\mathrm{a}}=R_{1}^{2}+R_{\mathrm{a}}^{2}$. From these two conditions we find that the functions $R$ must be of the forms

$$
R_{1}=U \lambda, \quad R_{2}=V \lambda ; \quad \bar{R}_{1}=\lambda \sqrt{U^{2}-c}, \quad \bar{R}_{2}= \pm \lambda V \overline{V^{2}+c}
$$

where $c$ is a constant and $\lambda$ is to be determined.

From (114), (115) and (122) we have

$$
\frac{\partial \theta}{\partial u}=\frac{V \bar{E}}{U} \frac{\theta}{\lambda}, \quad \frac{\partial \theta}{\partial v}=\frac{\sqrt{G}}{V} \frac{\theta}{\lambda} .
$$

Since $\theta$ must satisfy the point equation of $N$, namely

$$
\frac{\partial^{2} \theta}{\partial u \partial v}=\frac{\partial \log V \bar{E}}{\partial v} \frac{\partial \theta}{\partial u}+\frac{\partial \log V \bar{G}}{\partial u} \frac{\partial \theta}{\partial v},
$$

we must have

$$
\text { (125) } \frac{\partial}{\partial u} \log \frac{\theta}{\lambda}=\frac{V}{U} \frac{1}{\sqrt{G}} \frac{\partial \sqrt{E}}{\partial v}, \frac{\partial}{\partial v} \log \frac{\theta}{\lambda}=\frac{U}{V} \frac{1}{\sqrt{E}} \frac{\partial V \bar{G}}{\partial u} \text {. }
$$


The condition of integrability of these equations is

$$
\frac{\partial}{\partial v}\left(\frac{V}{\bar{U}} \frac{1}{\sqrt{G}} \frac{\partial \boldsymbol{V} \bar{E}}{\partial v}\right)=\frac{\partial}{\partial u}\left(\frac{U}{V} \frac{1}{\sqrt{E}} \frac{\partial \sqrt{G}}{\partial u}\right) .
$$

From this equation and (31) we have

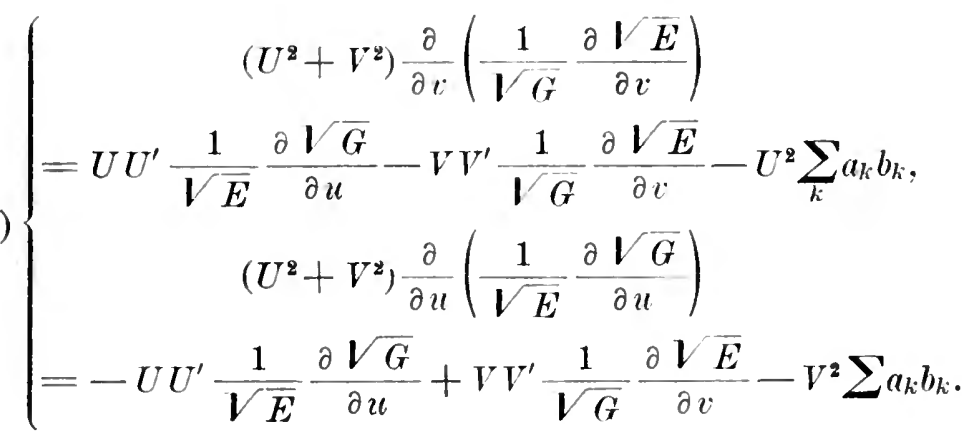

When a net $N$ satisfies conditions (127), the function $\theta / \lambda$ is found from (125) by a quadrature and then $\theta$ from (123) by another quadrature. Since these conditions do not involve the constant $c$ in (122), it follows that:

When a cyclic congruence admits more than one pair of focal hyperspheres cutting orthogonally it admits an infinity of pairs.

In this case we say that congruence is multiply cyclic.

The constant factor of integration of (125) can be taken equal to unity in all generality, but the additive constant in (123) gives a family of parallel multiply cyclic congruences. Hence:

When an $O$ net $N$ satisfies the conditions (127) there is a unique family of parallel multiply cyclic congruences harmonic to it, which can be found by quadratures.

75. Transformations $F$ of $O$ nets into $O$ nets which are not transformations $R$. We return to the consideration of the $O$ nets $N(x)$ and $N_{1}\left(x_{1}\right)$ harmonic to a multiply cyclic congruence, and such that the focal hyperspheres of radii $\bar{R}_{1}$ and $\bar{R}_{2}$ given by (122) pass through points of $N_{1}$. If we put

$$
E_{1}=\sum\left(\frac{\partial x_{1}}{\partial u}\right)^{2}, \quad G_{1}=\sum\left(\frac{\partial x_{1}}{\partial v}\right)^{2}
$$


then from (115) and analogous equations for $N_{1}$ we have (129) $x-\frac{R_{2}}{\sqrt{G}} \frac{\partial x}{\partial v}=x_{1}-\frac{\bar{R}_{2}}{\sqrt{G_{1}}} \frac{\partial x_{1}}{\partial v}, \quad x-\frac{R_{1}}{\sqrt{E}} \frac{\partial x}{\partial u}=x_{1}-\frac{\bar{K}_{1}}{\sqrt{E_{1}}} \frac{\partial x_{1}}{\partial u}$. Substituting in these equations from (62), (122) and (65), we get (130) $\sqrt{E_{1}}=-\frac{\tau}{\theta^{\prime}} \frac{\sqrt{E}}{U} \sqrt{U^{2}-c}, \quad \sqrt{G_{1}}=\mp \frac{\sigma}{\theta^{\prime}} \frac{V \bar{G}}{V} \sqrt{V^{2}+c}$

When these expressions and those given by (65) are substituted in (128), we get, in consequence of (63), (64) and (123),

(131) $\frac{\partial}{\partial u}\left(\frac{1}{\theta^{\prime}} \sum x^{\prime 2}\right)=\operatorname{ch} \frac{\sqrt{E}}{U^{\top}} \frac{\lambda}{\theta}, \frac{\partial}{\partial \imath}\left(\frac{1}{\theta^{\prime}} \sum x^{\prime 2}\right)=-c l \frac{\sqrt{G}}{\nabla} \frac{\lambda}{\theta}$.

If we put

$$
\frac{\partial \varrho}{\partial u}=\frac{\sqrt{E}}{U} \frac{\lambda}{\theta}, \quad \frac{\partial \varrho}{\partial v}=-\frac{\sqrt{G}}{V} \frac{\lambda}{\theta},
$$

we find that these equations are consistent, and that $\varrho$ so defined is a solution of (124). Consequently $\varrho^{\prime}$, defined by

$$
\frac{\partial \varrho^{\prime}}{\partial u}=h \frac{\partial \varrho}{\partial u}, \quad \frac{\partial \varrho^{\prime}}{\partial v}=l \frac{\partial \varrho}{\partial v},
$$

is a solution of the point equation of the net $N^{\prime \prime}$. Hence the integral of (131) is

$$
\sum x^{\prime 2}=c \theta^{\prime} \underline{o}^{\prime}
$$

where $\varrho^{\prime}$ is determined only to within an additive constant.

From (129) and (62) we have

$$
\frac{R_{2}}{\sqrt{G}} \frac{\partial x}{\partial v}-\frac{\theta}{\theta^{\prime}} x^{\prime}=\frac{\overline{R_{2}}}{\sqrt{G_{1}}} \frac{\partial x_{1}}{\partial v^{\prime}}, \quad \frac{R_{1}}{\sqrt{E}} \frac{\partial x}{\partial u}-\frac{\theta}{\theta^{\prime}} x^{\prime}=\frac{\overline{R_{1}}}{\sqrt{\bar{E}_{1}}} \frac{\partial x_{1}}{\partial u} .
$$

Squaring these equations and summing for the $x$ 's, we have, in consequence of (122) and (134),

(135) $2 \sum x^{\prime} \eta=\frac{c}{V}\left(\frac{\theta \varrho^{\prime}}{\lambda}-\frac{\theta^{\prime}}{\theta} \lambda\right), \quad 2 \sum x^{\prime} \xi=\frac{c}{U}\left(\frac{\theta \varrho^{\prime}}{\lambda}+\frac{\theta^{\prime}}{\theta} \lambda\right)$. 
The coordinates $x^{\prime}$ of $N^{\prime}$ are expressible in the form (69), where the functions $p_{k}, q, r$ are subject to the conditions (cf. 35):

(136) $\left\{\begin{array}{c}\frac{\partial p_{k}}{\partial u}=a_{k} q, \quad \frac{\partial p_{k}}{\partial v}=b_{k} r, \\ \frac{\partial q}{\partial u}=-\sum_{k} a_{k} p_{k}-\frac{1}{\sqrt{G}} \frac{\partial \sqrt{E}}{\partial v} r+h \sqrt{E}, \quad \frac{\partial q}{\partial v}=\frac{1}{\sqrt{E}} \frac{\partial \sqrt{G}}{\partial u} r, \\ \frac{\partial r}{\partial u}=\frac{1}{\sqrt{G}} \frac{\partial \sqrt{E}}{\partial v} q, \quad \frac{\partial r}{\partial v}=-\sum_{k} b_{k} p_{k}-\frac{1}{\sqrt{E}} \frac{\partial \sqrt{G}}{\partial u} q+l \sqrt{G} .\end{array}\right.$

From (135) and (69) we have

$$
2 q=\frac{c}{U}\left(\frac{\theta \varrho^{\prime}}{\lambda}+\frac{\theta^{\prime}}{\theta} \lambda\right), \quad 2 r=\frac{c}{V}\left(\frac{\theta \varrho^{\prime}}{\lambda}-\frac{\theta^{\prime}}{\theta} \lambda\right) .
$$

Substituting these expressions in the last four of equations (136), we obtain

$(138)$

$$
\left\{\begin{array}{l}
h \sqrt{E}\left(U^{2}-c\right)=U^{2} \sum_{k} a_{k} p_{k}-U U^{\prime} q+\left(U^{2}+V^{2}\right) \frac{1}{\sqrt{G}} \frac{\partial V \bar{E}}{\partial v} r, \\
\imath \sqrt{G}\left(V^{2}+c\right)=V^{2} \sum_{k} b_{k} p_{k}-V V^{\prime} r+\left(U^{2}+V^{2}\right) \frac{1}{\sqrt{E}} \frac{\partial \sqrt{G}}{\partial u} q .
\end{array}\right.
$$

In consequence of (30), (127) and (136) these expressions for $t$ and $l$ satisfy the equations

(139) $\frac{\partial h}{\partial v}=(l-h) \frac{\partial \log \sqrt{E}}{\partial v}, \quad \frac{\partial l}{\partial u}=(h-l) \frac{\partial \log \sqrt{G}}{\partial u}$.

Hence equations (123), (125), and the following in which $q, r, h$ and $l$ have the expressions (137) and (138) form a completely integrable system:

(140)

$$
\begin{cases}\frac{\partial p_{k}}{\partial u}=a_{k} q, & \frac{\partial p_{k}}{\partial v}=b_{k} r \\ \frac{\partial \varrho^{\prime}}{\partial u}=h \frac{\sqrt{E}}{U} \frac{\lambda}{\theta}, & \frac{\partial \varrho^{\prime}}{\partial v}=-l \frac{\sqrt{G}}{V} \frac{\lambda}{\theta} \\ \frac{\partial \theta^{\prime}}{\partial u}=h \frac{\sqrt{E}}{U} \frac{\theta}{\lambda}, & \frac{\partial \theta^{\prime}}{\partial v}=l \frac{\sqrt{G}}{V} \frac{\lambda}{\theta}\end{cases}
$$


In addition to $c$ the complete integral of the system involves $n+1$ constants of integration, since for each set of solutions the left-hand member of

$$
\sum p_{k}^{2}+q^{2}+r^{2}-c \theta^{\prime} \varrho^{\prime}=0
$$

is constant, and in order that (134) be satisfied, we must choose solutions satisfying (141). Therefore we have the theorem:

If an $O$ net $N$ satisfies the condition (127), it admits $\infty^{n+2}$ transformations $F$ into $O$ nets, which are not $R$ transforms of $N$; the harmonic congruences of these transformations are multiply cyclic.

76. Nets 2, $O$. Let $N(x)$ be a net $2, O$ in $n$-space, the complementary function being $y$. Then $x^{(1)}, \ldots, x^{(n)}$, iy are the coordinates of an $O$ net $N$ in $n+1$ space. From $\S 65$ it follows that there are $\infty^{n-3}$ nets $N^{\prime}$ parallel to $N$ for which

$$
\sum x^{\prime 2}=y^{\prime 2}
$$

We say that such a net $N^{\prime}$ is a special net 2, $O$. Hence there are $\infty^{n-3}$ special nets $2, O$ parallel to $N$. If $N^{\prime}$ is a special net, we effect the transformation $F$ of $N$ by means of the solution $y$ of its equation and the conjugate congruence of direction-parameters $x^{\prime}$, so that the coordinates $x_{1}$ of the transform $N_{1}$ are of the form

$$
x_{1}=x-\frac{y}{y^{\prime}} x^{\prime}
$$

In consequence of (142) we have

$$
\sum\left(x_{1}-x\right)^{2}=y^{2}
$$

Also in consequence of (142) and the equations

$$
\frac{\partial x^{\prime}}{\partial u}=h \frac{\partial x}{\partial u}, \frac{\partial x^{\prime}}{\partial v}=l \frac{\partial x}{\partial v}, \frac{\partial y^{\prime}}{\partial u}=h \frac{\partial y}{\partial u}, \frac{\partial y^{\prime}}{\partial v}=l \frac{\partial y}{\partial v}
$$

we have from (143) by differentiation $\sum \frac{\partial x_{1}}{\partial u} \frac{\partial x_{1}}{\partial v}=0$, that is $N_{1}$ is an $O$ net. 
Consider two of these transforms $N_{1}$ and $N_{2}$ by means of two parallel special nets $N^{\prime}\left(x^{\prime}\right)$ and $N^{\prime \prime}\left(x^{\prime \prime}\right)$ satisfying (142) and $\sum x^{\prime \prime 2}=y^{\prime \prime 2}$. In consequence of (145) and analogous equations we find that

$$
\sum x^{\prime} x^{\prime \prime}-y^{\prime} y^{\prime \prime}=c
$$

where $c$ is a constant. Since $N_{1}$ and $N_{2}$ are transforms of $N$ by means of $y$, the nets $N, N_{1}$ and $N_{2}$ form a triad $(\S 20)$, and $N_{2}$ is an $F$ transform of $N$ by means of $-y / y^{\prime}$, and the corresponding solution $-y^{\prime \prime} / y^{\prime}$ of the point equation of the net $N_{1}^{\prime \prime \prime}$, parallel to $N_{1}$, whose coordinates are given by (cf. II, 33)

$$
x_{1}^{\prime \prime \prime}=x^{\prime \prime}-\frac{y^{\prime \prime}}{y^{\prime}} x^{\prime} .
$$

In consequence of (146) and (142), we have

$$
\sum\left(x_{1}^{\prime \prime \prime}\right)^{2}=-2 c \frac{y^{\prime \prime}}{y^{\prime}} .
$$

Hence if $c=0$, the conjugate congruence of the transformation from $N_{1}$ into $N_{2}$ is I. If $c \neq 0, N_{1}$ and $N_{2}$ are in relation $R(\S 67)$. Hence in consequence of (144):

If $N$ is a net 2, $O$ in $n$-space, the complementary function being $y$, the net $N$ admits $\infty^{n-3} F$ transforms $N_{i}$ which are $O$ nets and the corresponding points of these nets lie on the hypersphere of radius $y$ and center at the corresponding point of $N$; moreover, any two nets $N_{1}$ and $N_{2}$ are $R$ transforms, unless the congruence of lines joining corresponding points of $N_{1}$ and $N_{2}$ is I.

When, in particular, $N$ is a net 2, $O$ in 3-space, it follows from the last remark of $\S 65$ that there are two special nets $N^{\prime}$ parallel to $N$. Moreover, there are no congruences I conjugate to an $O$ net in 3-space ( $(65)$. Hence:

If $N$ is a non-special 2, $O$ net in 3-space, the complementary function being $y$, on the envelope of the spheres of radius $y$ and centers on $N$ the parametric curves form $O$ nets in relation $R$ with one another and in relation $F$ with $N$.

We return to the consideration of a net $N$ in $n$-space which is $2, O$, the complementary function being $y$. Let $N^{\prime}$ be a parallel 
net; then equations (145) hold. If $\theta$ and $\theta^{\prime}$ are corresponding solutions of the point equations of $N$ and $N^{\prime}$, the equations of the form (62) define an $F$ transform $N_{1}$ of $N$, and the point equation of $N_{1}$ admits the solution

$$
y_{1}=y-\frac{\theta}{\theta^{\prime}} y^{\prime}
$$

From these equations we have

$$
\sum x_{1}^{2}-y_{1}^{2}=\sum x^{2}-y^{2}-2 \frac{\theta}{\theta^{\prime}}\left(\sum x x^{\prime}-y y^{\prime}\right)+\frac{\theta^{2}}{\theta^{\prime 2}}\left(\sum x^{\prime 2}-y^{\prime 2}\right) .
$$

Since $\sum x^{2}-y^{2}$ is a solution of the point equation of $N$, the function $\sigma^{\prime}$ defined by

(149) $\frac{\partial \sigma^{\prime}}{\partial u}=h \frac{\partial}{\partial u}\left(\sum x^{2}-y^{2}\right), \quad \frac{\partial \sigma^{\prime}}{\partial v}=l \frac{\partial}{\partial v}\left(\sum x^{2}-y^{2}\right)$

is a solution of the point equation of $N^{\prime}$. If $N^{\prime}$ is a special net, a solution of (149) is $\sigma^{\prime}=2\left(\sum x x^{\prime}-y y^{\prime}\right)$, and equation (148) becomes

$$
\sum x_{1}^{2}-y_{1}^{2}=\sum x^{2}-y^{2}-\frac{\theta}{\theta^{\prime}} \sigma^{\prime}
$$

Consequently $\sum x_{1}^{2}-y_{1}^{2}$ is the solution of the point equation of $N_{1}$ corresponding to the solution $\sum x^{2}-y^{2}$ of the point equation of $N$ whatever be $\theta$.

If $N^{\prime}$ is not a special net, a solution of its point equation is

$$
\theta^{\prime}=\frac{1}{2}\left(\sum x^{\prime 2}-y^{\prime 2}\right)
$$

and the corresponding solution of the point equation of $\mathrm{N}$ is given by

$$
\frac{\partial \theta}{\partial u}=\sum x^{\prime} \frac{\partial x}{\partial u}-y^{\prime} \frac{\partial y}{\partial u}, \quad \frac{\partial \theta}{\partial v}=\sum x^{\prime} \frac{\partial x}{\partial v}-y^{\prime} \frac{\partial y}{\partial v} .
$$

In this case a solution of (149) is

$$
\sigma^{\prime}=2\left(\sum x x^{\prime}-y y^{\prime}-\theta\right) .
$$

By means of this function equation (148) is reducible to (150). Hence: 
If $N$ is a net $2, O$ and $N^{\prime}$ is a parallel net which is not special, an $F$ transform $N_{1}$ of $N$ which is $2, O$ can be found by a quadrature; if $N^{\prime}$ is special, each solution of the point equation of $N$ determines an $F$ transform which is $2, O$.

\section{Exercises.}

1. A net $N^{\prime}$ parallel to a net $N$ which is $p, O$ is $p, O$, the complementary fonctions of $N^{\prime}$ being solutions of its point equation corresponding to the complementary functions of $N$.

2. Of the $O$ nets corresponding to a given orthogonal determinant, those defined by

$$
x=\sum_{k=1}^{n-2} e_{k} X_{k}, \quad \sum_{k} e_{k}^{2}=0,
$$

where the $e$ 's are constants, lie on the hypercone $\Sigma x^{2}=0$, and any such $O$ net is so determined; for such a net

$$
\sqrt{E}=\sum_{k} e_{k} a_{k}, \quad \sqrt{G}=\sum_{k} e_{k} b_{k} .
$$

3. If $N$ is an $O$ net ou the hypercone $\Sigma x^{2}=0$, and $G$ is any congruence conjugate to $N$, the developables of $G$ meet the hypercone in a net which is an $R$ transform of $N$; for this transformation

$$
\theta=\Sigma e_{k} p_{k}, \quad \omega^{\prime}=0 .
$$

4. If $N_{1}$ and $N_{2}$ are $R$ transforms of a net $N$ and all of these nets lie on the hypercone $\Sigma x^{2}=0$, so also do the nets $N_{12}$ which are $R$ transforms of $N_{1}$ and $N_{2}$, in accordance with $\$ 72$.

5. A congruence parallel to a cyclic congruence is cyclic, and for $\infty^{1}$ of the parallel cyclic congruences the circles of the cyclic system pass through a point (cf. § 13).

6. The equation of Laplace satisfied by the direction-parameters of a multiply cyclic congruence has equal invariants.

7. If $N$ is an $O$ net harmonic to a multiply cyclic congruence $G$, any net $N^{\prime}$ parallel to $N$ possesses the same property, and the multiply cyclic congruence harmonic to $N^{\prime}$ is determined by the solution of the point equation of $N^{\prime}$ corresponding to the solution determining $G$.

8. Any congruence parallel to a multiply cyclic congruence is multiply cyclic.

9. If $N$ is a net 2,O and $N_{1}$ is any $O$ net conjugate to a congruence 2, I conjugate to $N$, the distance between corresponding points of $N$ and $N_{1}$ is the complementary function of $N$. 
10. In order that the first and minus first Laplace transforms of a net $N$ with point equation (1) be $O$ nets it is necessary that $a$ and $b$ can be chosen so that

$$
\sum\left(\frac{\partial x}{\partial u}\right)^{2}=\left(\frac{\partial b}{\partial u}\right)^{2}, \quad \sum\left(\frac{\partial x}{\partial v}\right)^{2}=\left(\frac{\partial a}{\partial v}\right)^{2} .
$$

From $(I, 3)$ it follows that we must have

$$
\text { (ii) }\left\{\begin{aligned}
\left(\frac{\partial b}{\partial u}\right)^{2}\left(\frac{\partial a}{\partial v}\right)^{2}-\left(\sum \frac{\partial x}{\partial u} \frac{\partial x}{\partial v}\right)^{2} & =a\left(\frac{\partial b}{\partial u} \frac{\partial a}{\partial v} \frac{\partial^{2} b}{\partial u \partial v}-\frac{\partial^{2} a}{\partial u \partial v} \sum \frac{\partial x}{\partial u} \frac{\partial x}{\partial v}\right) \\
& =b\left(\frac{\partial b}{\partial u} \frac{\partial a}{\partial v} \frac{\partial^{2} a}{\partial u \partial v}-\frac{\partial^{2} b}{\partial u \partial v} \sum \frac{\partial x}{\partial u} \frac{\partial x}{\partial v}\right) .
\end{aligned}\right.
$$

The consistency of the equations (ii) necessitates the condition

$$
\frac{\partial b}{\partial u} \frac{\partial a}{\partial v}\left(\frac{a}{b}-\frac{b}{a}\right)=a \frac{\partial^{2} a}{\partial u \partial v}-b \frac{\partial^{2} b}{\partial u \partial v} .
$$

When this condition is satisfied, the equations

$$
\frac{\partial \log \sigma}{\partial u}=\frac{1}{a} \frac{\partial b}{\partial u}, \quad \frac{\partial \log \sigma}{\partial v}=\frac{1}{b} \frac{\partial a}{\partial v}
$$

are consistent (Cf. II, Ex. 12) and (ii) may be replaced by

$$
\sum \frac{\partial x}{\partial u} \frac{\partial x}{\partial v}=a b \frac{\partial^{2}}{\partial u \partial v} \log \sigma .
$$

When (i), (iii) and (v) are satisfied, the net $N$ possesses the desired property. We call it a $G$ net.

11. The point equation of a $G$ net $N$ aduits the solutions

$$
\theta_{1}=\Sigma x^{2}-a^{2}, \quad \theta_{2}=\mathbf{\Sigma} x^{2}-b^{2} .
$$

The first Laplace transform of $\theta_{1}$ is $\Sigma x_{1}^{2}$ and the minus first Laplace transform of $\theta_{2}$ is $\Sigma_{x_{-1}^{2}}$, where $x_{1}$ and $x_{-1}$ are the coordinates of the first and minus first Laplace transforms of $N$.

12. Any net parallel to a $G$ net is a $G$ net. From (II, Ex. 12) and (iii) of Ex. 10 it follows that two of these parallel nets have point equations with equal invariants, and are associates of one another. If the point equation of one of them is written

$$
\frac{\partial^{2} \theta}{\partial u \partial v}=\frac{\partial \log \sigma}{\partial v} \frac{\partial \theta}{\partial u}+\frac{\partial \log \sigma}{\partial u} \frac{\partial \theta}{\partial v}
$$

in place of (i) and (v) of Ex. 10, we have 
(ii) $\quad \sum\left(\frac{\partial x}{\partial u}\right)^{2}=\left(\frac{\partial \sigma}{\partial u}\right)^{2}, \sum\left(\frac{\partial x}{\partial v}\right)^{2}=\left(\frac{\partial \sigma}{\partial v}\right)^{2}, \sum \frac{\partial x}{\partial u} \frac{\partial x}{\partial v}=\sigma^{2} \frac{\partial^{2}}{\partial u \partial v} \log \sigma$.

A net $G$ of this type we call a net $G_{0}$.

13. The point equation of a net $G_{0}$ admits the solution $\theta_{0}=\mathbf{\Sigma} x^{2}-\sigma^{2}$. The first and minus first Laplace transforms of $\theta_{0}$ are $\Sigma_{x_{1}^{2}}$ and $\Sigma_{x_{-1}^{2}}$.

14. Show that the radial transform of a net $G_{0}$ by means of the function $\theta_{0}=\Sigma x^{2}-\sigma^{2}$ is a net $G_{0}$.

15. If a $G$ net $N$ is subjected to a transformation $F$ for which the directionparameters of the conjugate congruence of the transformation are the coordinates $x^{\prime}$ of one of the nets $G_{0}$ parallel to $N$ and the function $\theta^{\prime}$ of the transformation is S $x^{\prime 2}-\sigma^{2}$, the transform is a $G$ net, and its Laplace transforns are $R$ transforms of the corresponding Laplace transforms of $N$. 
Chapter VII.

\section{Transformations of Ribaucour.}

77. Orthogonal determinants and $O$ nets in 3-space. In the case of 3-space an orthogonal determinant is of the form

$$
A=\left|\begin{array}{c}
X^{1} \ldots \ldots X^{3} \\
\xi^{1} \ldots \ldots \xi^{3} \\
\eta^{1} \ldots \ldots \eta^{3}
\end{array}\right|
$$

Now

$$
\left\{\begin{array}{c}
\frac{\partial X}{\partial u}=a \xi, \quad \frac{\partial X}{\partial v}=b \eta \\
\frac{\partial \xi}{\partial u}=-a X-m \eta, \quad \frac{\partial \xi}{\partial v}=n \eta \\
\frac{\partial \eta}{\partial u}=m \xi, \quad \frac{\partial \eta}{\partial v}=-b X-n \xi
\end{array}\right.
$$

whereas it follows from $[\S 65]$ that

$$
\text { (3) }\left\{\begin{aligned}
a & =-\frac{D}{\sqrt{E}}=-\frac{\sqrt{E}}{\varrho_{1}}, & b & =-\frac{D^{\prime \prime}}{\sqrt{G}}=-\frac{\sqrt{G}}{\varrho_{2}}, \\
m & =\frac{1}{\sqrt{G}} \frac{\partial \sqrt{E}}{\partial v}, & n & =\frac{1}{\sqrt{E}} \frac{\partial \sqrt{G}}{\partial u}, \\
\frac{\partial a}{\partial v} & =m b, & \frac{\partial b}{\partial u} & =n a,
\end{aligned}\right.
$$

where $\varrho_{1}$ and $\varrho_{2}$ are the radii of principal curvature of an $O$ net corresponding to $A$.

When an orthogonal determinant is known, the coordinates of a corresponding net are of the form (cf. VI, 37) 


$$
x=P X+Q \xi+R \eta,
$$

where, as follows from (VI, 38), $P, Q$ and $R$ satisfy

(5) $\left\{\begin{array}{c}\frac{\partial P}{\partial u}=-\frac{D}{\sqrt{E}} Q, \quad \frac{\partial P}{\partial v}=-\frac{D^{\prime \prime}}{\sqrt{G}} R, \\ \frac{\partial Q}{\partial \imath}=\frac{D}{\sqrt{E}} P-\frac{1}{\sqrt{G}} \frac{\partial \sqrt{E}}{\partial v} R+\sqrt{E}, \quad \frac{\partial Q}{\partial v}=\frac{1}{\sqrt{E}} \frac{\partial \sqrt{G}}{\partial u} R, \\ \frac{\partial R}{\partial \imath}=\frac{1}{\sqrt{G}} \frac{\partial \sqrt{E}}{\partial v} Q, \quad \frac{\partial R}{\partial v}=\frac{D^{\prime \prime}}{\sqrt{G}} P-\frac{1}{\sqrt{E}} \frac{\partial \sqrt{G}}{\partial u} Q+\sqrt{G} .\end{array}\right.$

78. Transformations $R$ in 3-space. In 3-space the equations of a transformation $R$ of an $O$ net $N(x)$ are of the form

$$
x_{1}=x-\frac{1}{w}(p X+q \xi+r \eta),
$$

where, as follows from (VI, 87, 88), the functions satisfy the equations

$$
\begin{aligned}
& \frac{\partial \theta}{\partial u}=\sqrt{E} q, \quad \frac{\partial \theta}{\partial v}=\sqrt{G} r, \\
& \frac{\partial p}{\partial u}=-\frac{\sqrt{E}}{\varrho_{1}} q, \quad \frac{\partial p}{\partial v}=-\frac{\sqrt{G}}{\varrho_{2}} r, \\
& \frac{\partial q}{\partial u}=\frac{\sqrt{E}}{\varrho_{1}} p-\frac{1}{\sqrt{G}} \frac{\partial \sqrt{E}}{\partial v} r+w\left(\sqrt{E}+\sqrt{E_{1}}\right), \frac{\partial q}{\partial v}=\frac{1}{\sqrt{E}} \frac{\partial \sqrt{G}}{\partial u} r, \\
& \text { (7) }\left\{\frac{\partial r}{\partial u}=\frac{1}{\sqrt{G}} \frac{\partial \sqrt{E}}{\partial v} q, \quad \frac{\partial r}{\partial v}=\frac{\sqrt{G}}{\varrho_{2}} p-\frac{1}{\sqrt{E}} \frac{\partial \sqrt{G}}{\partial u} q+w\left(\sqrt{G}+V \overline{G_{1}}\right)\right. \text {, } \\
& \frac{\partial \log w}{\partial u}=\sqrt{E_{1}} \frac{q}{\theta}, \quad \frac{\partial \log w}{\partial v}=\sqrt{G_{1}} \frac{r}{\theta}, \\
& \frac{\partial \sqrt{E_{1}}}{\partial v}=\sqrt{G_{1}}\left[\frac{1}{\sqrt{G}} \frac{\partial \sqrt{E}}{\partial v}-\left(\sqrt{E}+V \overline{E_{1}}\right) \frac{r}{\theta}\right], \\
& \frac{\partial \sqrt{G_{1}}}{\partial u}=\sqrt{E_{1}}\left[\frac{1}{\sqrt{E}} \frac{\partial \sqrt{G}}{\partial u}-\left(\sqrt{G}+\sqrt{G_{1}}\right) \frac{q}{\theta}\right] \text {, }
\end{aligned}
$$


and also from (VI, 85)

$$
p^{2}+q^{2}+r^{2}-2 w \theta=0 .
$$

From (VI, 93) we have that the principal radii of curvature of the net $N_{1}$, denoted by $\varrho_{11}$ and $\varrho_{12}$, are given by

(9)

$$
\frac{\sqrt{E_{1}}}{\varrho_{11}}=\frac{\sqrt{E}}{\varrho_{1}}+\frac{p}{\theta}\left(\sqrt{E_{1}}+\sqrt{E}\right), \quad \frac{\sqrt{G_{1}}}{\varrho_{12}}=\frac{\sqrt{G}}{\varrho_{2}}+\frac{p}{\theta}\left(\sqrt{G_{1}}+\sqrt{G}\right)
$$

From (VI, 54, 56) we have that the coordinates $x_{0}, y_{0}, z_{0}$, of the central net and the radius $R$ of the spheres, are given by

$$
x_{0}=x-\frac{\theta}{p} X, \quad R=\frac{\theta}{p}
$$

We recall from $\S 68$ the following theorem:

When a transformation $R$ of a net $N$ is known, a transformation $R$ of any parallel net $\bar{N}$ can be found by a quadrature; the transforms of $N$ and $\bar{N}$ are parallel, as are also the central nets of the transformations.

The equations of the transformation of $\bar{N}$ are

$$
\bar{x}_{1}=\bar{x}-\frac{1}{\bar{w}}(p X+q \ddot{\xi}+\imath \cdot \eta)
$$

where $\bar{w}$ satisfies equations of the form (7). Since (8) must be satisfied, we have

$$
\bar{\theta} \bar{w}=\theta w
$$

Also we have the theorem:

When $N$ and $N_{1}$ are in relation $R$, the nets $N^{\prime}$ and $N_{1}^{\prime}$, whose coordinates are direction-parameters of the congruence of the transformation, may be obtained from one another by an inversion.

Conversely:

If two $O$ nets $N^{\prime}$ and $N_{1}^{\prime}$ are related by an inversion each net $N$ parallel to $N^{\prime}$ admits an $R$ transform $N_{1}$ parallel to $N_{1}^{\prime}$ which can be found by quadratures. 
From (VI, 91) we have that the direction-cosines of the normals to a net $N$ and to an $R$ transform $N_{1}$ are in the relation

$$
X_{1}=-X+\frac{p}{\theta^{\prime}} x^{\prime} ; \quad \theta^{\prime}=w \theta .
$$

From (VI, 28, 74) we have

$$
\begin{array}{ll}
\frac{\partial x^{\prime}}{\partial u}=\frac{V \overline{E^{\prime}}}{a} \frac{\partial X}{\partial u}, & \frac{\partial x^{\prime}}{\partial v}=\frac{V \overline{G^{\prime}}}{b} \frac{\partial X}{\partial v} ; \\
\frac{\partial \theta^{\prime}}{\partial u}=\frac{V \overline{E^{\prime}}}{a} \frac{\partial p}{\partial u}, & \frac{\partial \theta^{\prime}}{\partial v}=\frac{V \overline{G^{\prime}}}{b} \frac{\partial p}{\partial v},
\end{array}
$$

where $E^{\prime}$ and $G^{\prime}$ are the first fundamental coefficients of $N^{\prime}$. Hence (11) is of the form (II, 2), and we have:

When $N$ and $N_{1}$ are in relation $R$, their spherical representations are in relation $F$.

Conversely, we have the theorem:

If $N(x)$ is an orthogonal net on the unit sphere and $N^{\prime}\left(x^{\prime}\right)$ is an $O$ net with this spherical representation, the equations

$$
X_{1}=-X+2 \frac{\sum x^{\prime} X}{\sum x^{\prime 2}} x^{\prime}
$$

define an orthogonal net $N_{1}\left(x_{1}\right)$ on the sphere such that $N$ and $N_{1}$ are in relation $F$.

From (11) and (VI, 99, 101) it follows that the distance from a point of any $O$ net $N$ to the corresponding tangent plane to an $R$ transform $N_{1}$ is given by

$$
\sum X_{1}\left(x_{1}-x\right)=P_{1}+P-\frac{p}{\theta^{\prime}} \sum x x^{\prime}=-\frac{p \theta}{\theta^{\prime}} .
$$

79. The cyclic system associated with a transformation $R$. If $N$ and $N_{1}$ are two $O$ nets in relation $R$, the circles orthogonal to $N$ and $N_{1}$ at corresponding points have for axes the lines of the harmonic congruence $G$ of the transformation, that is the harmonic congruence determined by the solution $\theta$ of the point equation of $N(\S 73)$. In $\S 64$ we saw that there are 
$\infty^{1}$ nets $N^{\prime}$ parallel to $N$ determined by $\theta$. By the last theorem $\S 67$ each of these nets $N^{\prime}$ determines an $R$ transform $N_{1}$ of $N$ and all of these transforms are harmonic to $G$. Consequently the above circles are orthogonal to all of these nets $N_{1}$ and therefore form a cyclic system [§ 174]. Incidentally we have established the theorem:

If $N$ is an $O$ net and $G$ any congruence harmonic to it, the circles with lines of $G$ for axes and passing through corresponding points of $N$ form a cyclic system.

We call the planes of the circles orthogonal to two nets $N$ and $N_{1}$ in relation $R$ the circle-planes of the transformation. From the second theorem of $\S 11$ it follows that the circle-planes envelop a net $\bar{N}$. Since the circle-plane at a point $M$ of $N$ is determined by the normal to $N$ at $M$ and the line joining corresponding points of $N$ and $N_{1}$ it follows that any point on the circle-plane has coordinates of the form

$$
\bar{x}=x+j x^{\prime}+k X,
$$

and the direction-cosines of the plane are proportional to $r \xi-q \eta$. In order that the point $\bar{M}(\bar{x})$ be the point of contact of the plane with its envelope, the functions $j$ and $k$ must be such that

$$
\sum(r \xi-q \eta) \frac{\partial \bar{x}}{\partial u}=0, \quad \sum(r \xi-q \eta) \frac{\partial \bar{x}}{\partial v}=0 .
$$

By differentiation we find

$$
\left\{\begin{array}{l}
\frac{\partial \bar{x}}{\partial u}=\sqrt{E}\left(1+j h-\frac{k}{\varrho_{1}}\right) \xi+x^{\prime} \frac{\partial j}{\partial u}+X \frac{\partial k}{\partial u}, \\
\frac{\partial \bar{x}}{\partial v}=\sqrt{G}\left(1+j l-\frac{k}{\varrho_{\mathbf{2}}}\right) \eta+x^{\prime} \frac{\partial j}{\partial v}+X \frac{\partial k}{\partial v} .
\end{array}\right.
$$

Hence $j$ and $k$ are determined by

and we have

$$
1+j h-\frac{k}{\varrho_{1}}=0, \quad 1+j l-\frac{k}{\varrho_{2}}=0,
$$

$$
\frac{\partial \bar{x}}{\partial u}=x^{\prime} \frac{\partial j}{\partial u}+X \frac{\partial k}{\partial u}, \quad \frac{\partial \bar{x}}{\partial v}=x^{\prime} \frac{\partial j}{\partial v}+X \frac{\partial k}{\partial v} .
$$


If equations (16) are differentiated with respect to $v$ and $u$ respectively, we get

$$
h \frac{\partial j}{\partial v}-\frac{1}{\varrho_{1}} \frac{\partial k}{\partial v}=0, \quad l \frac{\partial j}{\partial u}-\frac{1}{\varrho_{2}} \frac{\partial l}{\partial u}=0 .
$$

Making use of these relations, we find from (17) by differentiation

$$
\frac{\partial^{2} \bar{x}}{\partial u \partial v}=x^{\prime} \frac{\partial^{2} j}{\partial u \partial v}+X \frac{\partial^{2} k}{\partial u \partial v}
$$

Hence $j$ and $l_{i}$ are solutions of the point equation of $\bar{N}$. We shall show that $\bar{\omega}$, defined by

$$
\overline{\mathbf{c}}=\boldsymbol{\theta}^{\prime} j+p k+\theta,
$$

also is a solution. In fact, we find that

$$
\left\{\begin{array}{c}
\frac{\partial \bar{\omega}}{\partial u}=\theta^{\prime} \frac{\partial j}{\partial u}+p \frac{\partial k}{\partial u}, \quad \frac{\partial \bar{\omega}}{\partial v}=\theta^{\prime} \frac{\partial j}{\partial v}+p \frac{\partial k}{\partial v}, \\
\frac{\partial^{2} \bar{\omega}}{\partial u \partial v}=\theta^{\prime} \frac{\partial^{2} j}{\partial u \partial v}+p \frac{\partial^{2} k}{\partial u \partial v} .
\end{array}\right.
$$

Moreover, the linear element of $\bar{N}$ may be written

$$
d \bar{s}^{2}=d k^{2}+2 d j d \bar{\omega}
$$

If we put

$$
j=m+i n, \quad 2 \bar{\omega}=m-i n,
$$

equation (22) becomes

$$
d s^{2}=d k^{2}+d m^{2}+d n^{2} .
$$

Since $k, m$ and $n$ are solutions of the point equation of $\bar{N}$, they are the coordinates of a net applicable to $\bar{N}$.

This result is in accord with [ $\S 141,176]$ where it was shown that the planes of the circles of a cyclic system envelope a net corresponding to the developables of the congruence of axes. Moreover, it was shown that the applicable net is known intrinsically when the cyclic system is given, and conversely when the appli- 
cable net is known a cyclic system can be found directly. For the sake of brevity we say with Guichard that a net is $C$ when it admits an applicable net.

We have just seen that $\bar{N}$ is a net $C$. It is harmonic both to the congruence normal to $N$ and to the congruence $G$ of the transformation from $N$ into $N_{1}(\S 11)$. The coordinates of the focal points of first rank of these two congruences are of the respective forms $x+\varrho_{1} X$ and $x-x^{\prime} / h$ (I, 37). By means of the preceding formulas we prove the theorem:

The normal congruence and the congruence $G$ are the harmonic congruences of $\bar{N}$ determined by the solutions $m+i n$ and $k$ of the point equation of $\bar{N}$.

Moreover from $\S 11$ it follows that the derived net of $\bar{N}$ by means of $m+i n$ and $k+$ const. is parallel to $N$.

Conversely, any net harmonic to a normal conguence $G_{0}$ is enveloped by the planes determined by $G_{0}$ and by any congruence conjugate to a net $N$ normal to $G_{0}$. But in $\S 65$ we saw that any other congruence conjugate to $N$ is 3 , I, and consequently leads to a transformation $R$ of $N$. Hence the resulting net harmonic to $G_{0}$ is a net $C$. Since the determination of congruences conjugate to $N$ is equivalent to finding nets parallel to $N$, we have:

The nets harmonic to a normal congruence are nets $C$; their. determination is equivalent to finding nets parallel to a net orthogonal to the congruence: when such a parallel net is known, the corvesponding harmonic net follows divectly and the coordinates of the applicable net can be found by quadratures ${ }^{55}$ ).

8o. When the circle-planes of a transformation $R$ pass through a point. Consider in particular the case for which the circle-planes pass through a point. From (17) it follows that $j$ and $k$ are constant. If the point is taken as the origin, we have from (14) that in all generality we may take

$$
p=P-l, \quad q=Q, \quad r=R,
$$

$d$ being a constant.

${ }^{55}$ ) In $\$ 117$ it will be shown that every net $C$ is harmonic to $\infty^{1}$ families of parallel normal congruences. 
From (VI, 96) and (7) it follows that $\theta=\omega-c$, where $c$ is a constant.

From these results and (10) we have

$$
\sum x_{0}^{\frac{2}{0}}=\frac{\theta^{2}}{p^{2}}-2 d \frac{\theta}{p}+2 c .
$$

Hence the spheres cut the fixed sphere with center at the origin and radius $r_{0}$ under the constant angle $\varepsilon$, where

$$
r_{0}^{2}=2 c, \quad r_{0} \cos \varepsilon=d .
$$

Conversely, it can be shown that when the spheres of a transformation $R$ meet under constant angle a fixed sphere with center at the origin, the functions of the transformation are of the form (25).

It is evident that any $O$ net admits such transformations $k$. In consequence of (25) the coordinates of any point on the line of the congruence $G$ of the transformation $R$ are of the form $(1+\lambda) x^{\prime}+d \cdot X$. When $\lambda=-1$, the point lies on the sphere of radius $d$ with center at the origin and describes the spherical representation of $N$. These lines meet the same sphere again in the point of coordinates $d \cdot\left(X-\frac{p}{\theta^{\prime}} x^{\prime}\right)$, which describes the spherical representation of $N_{1}$ (cf. 11).

81. The circles $K$ and congruence $K$. Let $N_{1}\left(x_{1}\right)$ and $N_{2}\left(x_{2}\right)$ be two $R$ transforms of an $O$ net $N(x)$, and write the equations of the transformations in the general forms

$$
\begin{cases}x_{1}=x-\frac{\theta_{1}}{\theta_{1}^{\prime}} x^{\prime}, & \theta_{1}^{\prime}=\frac{1}{2} \sum x^{\prime 2}=\theta_{1} u_{1}, \\ x_{2}=x-\frac{\theta_{2}}{\theta_{2}^{\prime \prime}} x^{\prime \prime}, & \theta_{2}^{\prime \prime}=\frac{1}{2} \sum x^{\prime \prime 2}=\theta_{2} w_{2},\end{cases}
$$

where $N^{\prime}\left(x^{\prime}\right)$ and $N^{\prime \prime}\left(x^{\prime \prime}\right)$ are the nets parallel to $N$ determining the conjugate congruences of the trausformations $\left.{ }^{56}\right)$. If $\theta_{1}^{\prime \prime}$ and $\theta_{2}^{\prime}$ are solutions of the point equations of $N^{\prime \prime}$ and $N^{\prime}$ respectively corre-

s6) Cf. Jonas, Sitz. Berl. Math. Gesell., vol. 14 (1915), p. 109. 
sponding to the solution $\theta_{1}$ and $\theta_{2}$ of the point equation of $N$, and the additive constants are chosen so that

$$
\theta_{1}^{\prime \prime}+\theta_{2}^{\prime}=\sum x^{\prime} x^{\prime \prime}
$$

then as shown in $\S 72$ the $\infty^{1}$ nets $N_{12}$, defined by (II, 46), namely

$$
x_{12}=x+\frac{\left(\theta_{1}^{\prime \prime} \theta_{2}-\theta_{2}^{\prime \prime} \theta_{1}\right) x^{\prime}+\left(\theta_{2}^{\prime} \theta_{1}-\theta_{1}^{\prime} \theta_{2}\right) x^{\prime \prime}}{\theta_{1}^{\prime} \theta_{2}^{\prime \prime}-\theta_{1}^{\prime \prime} \theta_{2}^{\prime}}
$$

are $O$ nets in relations $R$ with $N_{1}$ and $N_{2}$.

Consider the circle $K$ through three corresponding points, $M, M_{1}$ and $M_{2}$ of $N, N_{1}$ and $N_{2}$. The coordinates of its center are of the form

$$
x+\lambda x^{\prime}+\mu x^{\prime \prime}
$$

where $\lambda$ and $\mu$ are determined by the condition that the lines joining the center to the mid-points of the segments $M M_{1}$ and $M M_{2}$ are perpendicular to these segments. These conditions are reducible by means of (26) to

$$
2 \lambda \theta_{1}^{\prime}+\mu \sum x^{\prime} x^{\prime \prime}+\theta_{1}=0, \quad \lambda \sum x^{\prime} x^{\prime \prime}+2 \mu \theta_{2}^{\prime \prime}+\theta_{2}=0 .
$$

In like manner the condition that the line joining the center to the mid-point of the line joining $M_{1}$ to the corresponding point of one of the nets $N_{12}$ is perpendicular to this line is

$$
\Sigma\left(x+\lambda x^{\prime}+\mu x^{\prime \prime}-x_{1}+\frac{\theta_{12}}{\theta_{12}^{\prime \prime \prime}} \frac{x_{1}^{\prime \prime \prime}}{2}\right) x_{1}^{\prime \prime \prime}=0 .
$$

By means of (II, 33, 36), (VI, 106), and (27) we find that this condition is satisfied when (30) hold, and consequently the corresponding points of each of the nets $N_{12}$ lie on $K$. Hence:

If $N, N_{1}, N_{2}, N_{12}$ are four $O$ nets in the rolation of a quatern under transformations $R$, four corresponding points $M, M_{1}, M_{2}, M_{12}$ lie on a circle $K$; the four corresponding points on any four of the possible $\infty^{1}$ nets $N_{12}$, forming a quatern with $N, N_{1}, N_{2}$ are in constant cross-ratio ${ }^{57}$ ).

$\left.{ }^{57}\right)$ The last part of this theorem is due to Demoulin, Comptes Rendus, vol. $150(1910)$, p.156, and is left as an exercise. 
If we put $(\$ 21)$

$$
\theta_{c}=\theta_{1}+(c-1) \theta_{\Xi}, \quad x^{(c)}=x^{\prime}+(c-1) x^{\prime \prime},
$$

then .

$$
\theta_{c}^{(c)}=\frac{1}{2} \sum x^{(c)}, \quad \sum x^{(c)} x^{\prime \prime}=\theta_{c}^{\prime \prime}+\theta_{2}^{(c)}
$$

Hence the transformation $F$ determined by $\theta_{c}$ and the congruence of direction-parameters $x^{(c)}$ is a transformation $R$. Moreover, if $N_{c}$ is the transform, each of the $\infty^{1} O$ nets $N_{12}$ are $R$ transforms of $N_{c}$, and consequently corresponding points of $N, N_{1}, N_{2}$, each of the $\infty^{1}$ nets $N_{c}$ and each of the $\infty^{1}$ nets $N_{12}$ lie on a circle $K$. Hence:

If $\theta_{1}$ and $\theta_{2}$ are solutions of the point equation of an $O$ net $N$ determining two $R$ transforms, corresponding points of $N$ and of the $\infty^{1} R$ transforms $N_{c}$ of $N$ by means of $\theta_{1}+(c-1) \theta_{2}$ lie on a circle $K$, upon which lie also the corresponding points of the $\infty^{1}$ $O$ nets $N_{12}$ which are $R$ transforms of all the nets $N_{c}$.

Let $N_{c_{1}}$ and $N_{c_{0}}$ be any two of these transforms of $N$, and let $N_{01}$ and $N_{02}$ be the central nets of the corresponding $R$ transforms, both being conjugate to the congruence normal to $N$. From the third theorem of $\$ 68$ it follows that the tangent planes to $N_{01}$ and $N_{02}$ meet in the axis of the circle $K$, and consequently these axes form a congruence $K$ harmonic to the nets $N_{01}$ and $N_{02}$. Since the congruence $K$ is determined by the circles $K$, we have that it is harmonic to the central net of the transformation $R$ of $N$ into any net $N_{c}$. Since $N$ and any net $N_{12}$ may be looked upon as $R$ transforms of a net $N_{c}$, it follows that the central net of the transformation from $N_{c}$ into any net $N_{12}$ is harmonic to $K$.

From $\S 23$ it follows that corresponding tangent planes to $N$, to all the nets $N_{c}$ and to all the nets $N_{12}$ meet in a point generating a net $\bar{N}$, the derived net of $N$ determined by $\theta_{1}$ and $\theta_{2}$. Since the tangent plane of a central net passes through the intersection of the tangent planes to $N$ and a transform $N_{c}$, a point of $\bar{N}$ is on the corresponding tangent plane of each central net of all the transformations $R$. Consequently the congruence $K$ is conjugate to $\bar{N}$. Hence: 
Corresponding tangent planes of $N$, the $\infty^{1}$ nets $N_{c}$ and the $\infty^{1}$ nets $N_{12}$ meet in a point generating a net $\bar{N}$ conjugate to the congruence $K$ of the axes of the circles $K$ through corresponding points of these nets ${ }^{58}$ ).

Since the congruence $K$ is harmonic to the nets $N_{01}$, and $N_{02}$, and the tangents to the curves of these nets at corresponding points lie in the principal planes of $N$ at the corresponding point, the focal points of $K$ lie in these principal planes. In order to obtain the coordinates of these focal points, we remark $(\S 78)$ that the coordinates of $N_{01}$ and $N_{02}$ are of the forms

$$
x_{01}=x-\frac{\theta_{1}}{p_{1}} X, \quad x_{02}=x-\frac{\theta_{2}}{p_{2}} X .
$$

'The point equation of $N_{01}$ admits the solution $\theta_{01}=\theta_{2}-\theta_{1} p_{2} / p_{1}$, and from $\S 19$ it follows that $N_{02}$ is the $F$ transform of $N_{01}$, by means of $\theta_{01}$. Hence from (II, 22) and (7) we have for the coordinates of the focal nets $F_{1}\left(y_{1}\right), F_{2}\left(y_{2}\right)$ of the congiuence $K$, that is the intersections of the corresponding tangent planes to $N_{01}$ and $N_{02}$, expressions of the forms

$$
\left\{\begin{array}{l}
y_{1}=x_{01}-\frac{\theta_{01}}{\frac{\partial \theta_{01}}{\partial u}} \frac{\partial x_{01}}{\partial u}=x+\frac{\left(\theta_{1} p_{2}-\theta_{2} p_{1}\right) \xi+\left(\theta_{2} q_{1}-\theta_{1} q_{2}\right) X}{p_{1} q_{2}-p_{2} q_{1}} \\
y_{2}=x_{01}-\frac{\theta_{01}}{\frac{\partial \theta_{01}}{\partial v}} \frac{\partial x_{01}}{\partial v}=x+\frac{\left(\theta_{1} p_{2}-\theta_{2} p_{1}\right) \eta+\left(\theta_{2} r_{1}-\theta_{1} r_{2}\right) X}{p_{1} r_{2}-p_{2} r_{1}}
\end{array}\right.
$$

From the preceding theorem it follows that the normals to all the nets $N_{c}$ and to all the nets $N_{12}$ at points of a circle $K$ are tangent to the sphere $S$ with center at the corresponding point on $\bar{N}$ and passing through $K$. Moreover, the normal to any net $N_{c}$ meets the normal to any net $N_{12}$, in the center of the sphere tangent to $N_{c}$ and $N_{12}$. Hence these normals lie on a hyperboloid of revolution to which $S$ is tangent along $K$, unless all the normals lie in the plane of $K$. We consider the latter possibility.

58) Cf. Demoulin, 1. c., p. 310. 
If $\alpha, \beta, \gamma$ denote the direction-cosines of the congruence $K$, we have in consequence of (26) and (27)

$$
\alpha, \beta, \gamma=\frac{y^{\prime} z^{\prime \prime}-y^{\prime \prime} z^{\prime}, z^{\prime} x^{\prime \prime}-z^{\prime \prime} x^{\prime}, x^{\prime} y^{\prime \prime}-x^{\prime \prime} y^{\prime}}{\left[4 \theta_{1}^{\prime} \theta_{2}^{\prime \prime}-\left(\theta_{1}^{\prime \prime}+\theta_{2}^{\prime}\right)^{2}\right]^{\frac{1}{2}}}
$$

The direction-cosines of the normals to $N_{c}$ and $N_{12}$ are of the forms, by (11), (31) and (VI, 112),

$$
\begin{gathered}
X_{c}=\frac{p_{1}+(c-1) p_{2}}{\theta_{c}^{(c)}}\left(x^{\prime}+(c-1) x^{\prime \prime}\right)-X, \\
X_{12}=X+\frac{\left(\theta_{2}^{\prime} x^{\prime \prime}-\theta_{2}^{\prime \prime} x^{\prime}\right) p_{1}+\left(\theta_{1}^{\prime \prime} x^{\prime}-\theta_{1}^{\prime} x^{\prime \prime}\right) p_{2}}{\theta_{1}^{\prime} \theta_{2}^{\prime \prime}-\theta_{1}^{\prime \prime} \theta_{2}^{\prime}} .
\end{gathered}
$$

In consequence of (VI, 69) we have

$$
\sum X_{c} \alpha=-\sum X_{12} \alpha=\frac{q_{1} r_{2}-q_{2} r_{1}}{\left[4 \theta_{1}^{\prime} \theta_{2}^{\prime \prime}-\left(\theta_{1}^{\prime \prime}+\theta_{2}^{\prime}\right)^{2}\right]^{\frac{1}{2}}} .
$$

Hence either all the normals lie in the plane of the circle $K$, or none do. In order that they do, we must have

$$
q_{1} r_{2}-q_{2} r_{1}=0 .
$$

From $\S 79$ it follows that this is a necessary and sufficient condition that the circle-planes of the transformations of $N$ into $N_{1}$ and $N_{2}$ coincide. If we replace (35) by $q_{2}=\lambda q_{1}, r_{2}=\lambda r_{1}$, from the sixth and seventh of (7) it follows that $\lambda$ is a constant, which may be taken equal to unity. Then we have

(36) $\quad q_{2}=q_{1}, \quad r_{2}=r_{1}, \quad p_{2}=p_{1}+d, \quad \theta_{2}=\theta_{1}+e$,

where $d$ and $e$ are constants. When these expressions are substituted in (33), the latter become

$$
x-\frac{e}{d} X+\left(p_{1} \frac{e}{d}-\theta_{1}\right) \frac{\xi}{q_{1}}, \quad x-\frac{e}{d} X+\left(p_{1} \frac{e}{d}-\theta_{1}\right) \frac{\eta}{r_{1}} .
$$


Hence the congruence $K$ is harmonic to the $O$ net of coordinates $x-e X / d$, that is $K$ is a cyclic congruence $(\S 79)$, and the net is orthogonal to the circles of the corresponding cyclic system. Accordingly we have the theorem of Demoulin $^{59}$ ):

If $N$ is any $O$ net and $N_{1}$ and $N_{2}$ are tuo $R$ transforms of $N$, the circles $K$ determined by corresponding points of $N, N_{1}$ and $N_{2}$ are of two kinds; $1^{\circ}$, if the circle-planes of the two $R$ transforms are coincident, the normals to the $\infty^{1}$ nets $N_{c}$ and $\infty^{1}$ nets $N_{12}$ lie in the planes of the circles $K$, and the congruence $K$ is cyclic, the circles of the cyclic system being concentric with the circles $K$; $2^{\circ}$, if the circle-planes of the two transformations $R$ are distinct, the normals to the nets $N_{c}$ at points of a circle $K$ form one set of generators of a hyperboloid of revolution, and the normals to the nets $N_{12}$ the other family of generators ${ }^{60}$ ).

82. Transformations $D_{m}$ of isothermic surfaces. From $(\mathrm{VI}, 81)$ it follows that a necessary and sufficient condition that the correspondence between the two surfaces of a transformation $R$ be conformal is that $\tau^{2}=\sigma^{2}$. From (II, 8) and $\S 4$ it is seen that $\tau$ and $\sigma$ cannot be equal. Also from $\S 25$ it follows that when $\tau+\sigma=0$ the transformation is $K$ and the $O$ nets $N$ and $N_{1}$ have equal point invariants, that is they are isothermic. Hence we have the theorem of Cosserat ${ }^{61}$ ):

In order that the correspondence between two surfaces in relation $R$ be conformal it is necessary and sufficient that both surfaces be isothermic.

We proceed to the consideration of these transformations and put

$$
\sqrt{E}=\sqrt{G}=e^{\psi}
$$

where $\psi$ is a function thus defined. Now the point equation of $N$ is

$$
\frac{\partial^{2} \theta}{\partial u \partial v}=\frac{\partial \psi}{\partial v} \frac{\partial \theta}{\partial u}+\frac{\partial \psi}{\partial u} \frac{\partial \theta}{\partial v}
$$

59) I. C.

60) Cf. $\$ 108$.

(11) Annales de la Faculté des Sciences de Toulouse, vol. 8 (1894), p. E. 13. 
Comparing this equation with (II, 79), we have

$$
\frac{1}{\varrho}=m e^{-2 v},
$$

where $m$ is a constant.

From [\$ 65] it follows that the Codazzi equations of $N$ are

$$
\frac{\partial}{\partial v}\left(\frac{1}{\varrho_{1}}\right)=\left(\frac{1}{\varrho_{2}}-\frac{1}{\varrho_{1}}\right) \frac{\partial \psi}{\partial v}, \quad \frac{\partial}{\partial u}\left(\frac{1}{\varrho_{2}}\right)=\left(\frac{1}{\varrho_{1}}-\frac{1}{\varrho_{2}}\right) \frac{\partial \psi}{\partial u},
$$

from which it follows that

$$
\frac{\partial K_{0}}{\partial u}=-e^{-2 \psi} \frac{\partial L}{\partial u}, \quad \frac{\partial K_{0}}{\partial v}=e^{-2 \psi} \frac{\partial L}{\partial v},
$$

where we have put

$$
K_{0}=\frac{1}{\varrho_{1}}+\frac{1}{\varrho_{2}}, \quad L=e^{2 \phi}\left(\frac{1}{\varrho_{2}}-\frac{1}{\varrho_{1}}\right) .
$$

Expressing the condition of integrability of (40), we find that $L$ is a solution of (38).

The net conjugate to the congruence normal to $N$ which is in relation $K$ to $N$, that is the harmonic of $N$ with respect to the centers of curvature of $N$, is given by

$$
x+\frac{2}{K_{0}} X .
$$

From this result, (40) and (II, 82) it follows that the functions $\theta$ and $\hat{\theta}$ of this transformation $K$ are

$$
\theta=\frac{L}{2 m}, \quad \frac{\hat{\theta}}{\theta}=-\frac{K_{0}}{2} .
$$

We return to the consideration of transformations $R$ of $N$ and introduce the function $\nu$ by means of the equation

$$
w=\frac{\theta^{\prime}}{\theta}=m \nu
$$


We have from $(I, 82)$

$$
\frac{\partial \nu}{\partial u}=e^{-2 \psi} \frac{\partial \theta}{\partial u}=e^{-\psi} q, \quad \frac{\partial \nu}{\partial v}=-e^{-\psi} r .
$$

Comparing these equations and (7), we get

$$
\sqrt{E_{1}}=-\sqrt{G_{1}}=\frac{\theta}{\nu} e^{-\phi}
$$

In terms of these functions equations (7) become

$$
(47)\left\{\begin{array}{c}
\frac{\partial \theta}{\partial u}=e^{\psi} q, \quad \frac{\partial \theta}{\partial v}=e^{\psi} r \\
\frac{\partial p}{\partial u}=-\frac{e^{\psi}}{\varrho_{1}} q, \quad \frac{\partial p}{\partial v}=-\frac{e^{\psi}}{\varrho_{2}} r \\
\frac{\partial q}{\partial u}=\frac{e^{\psi}}{\varrho_{1}} p-\frac{\partial \psi}{\partial v} r+m\left(e^{\psi} v+e^{-\psi} \theta\right), \quad \frac{\partial q}{\partial v}=\frac{\partial \psi}{\partial u} r \\
\frac{\partial r}{\partial u}=\frac{\partial \psi}{\partial v} q, \quad \frac{\partial r}{\partial v}=\frac{e^{\psi}}{\varrho_{\mathbf{g}}} p-\frac{\partial \psi}{\partial u} q+m\left(e^{\psi} \nu-e^{-\phi} \theta\right) \\
\frac{\partial \nu}{\partial u}=e^{-\psi} q, \quad \frac{\partial \nu}{\partial v}=-e^{-\psi} r
\end{array}\right.
$$

and (6) assumes the form

$$
x_{1}=x-\frac{1}{m \nu}(p X+q \xi+r) .
$$

It is readily found that equations (47) form a completely integrable system, and that for any set of solutions the left-hand member of the following equation is constant:

$$
p^{2}+q^{2}+r^{2}-2 m \theta r=0 \text {. }
$$

The complete integration of (47) involves five constants in addition to $m$. When these constants are chosen so that (49) is satisfied, three of these constants are essentially arbitrary. Hence we have the theorem of Darboux ${ }^{62}$ ):

62) Annales de L'École Norm. Sup., ser. 3, vol. 16 (1899), p. 503. 
An isothermic net admits $\infty^{4}$ transformations $R$ into isothermic nets.

These transformations have been called $D_{m}$ by Bianchi ${ }^{63}$ ).

83. Theorem of permutability of transformations $D_{m}$. We consider two isothermic nets, $N_{1}$ and $N_{2}$, transforms of an isothermic net $N$ by means of two sets of solutions, $\theta_{i}, \nu_{i}, p_{i}, q_{i}, v_{i}, m_{i}$ $(i=1,2)$ of equations (47) and (49), and apply the results of $\$ \S 21,72$ to obtain a theorem of permutability of transformations $D_{n}$.

From (VI, 105) it follows that the additive constants of integration of $\theta_{2}^{\prime}$ and $\theta_{1}^{\prime \prime}$ must be chosen so that

$$
p_{1} p_{2}+q_{1} q_{2}+r_{1} r_{2}=\theta_{2}^{\prime}+\theta_{1}^{\prime \prime} .
$$

Since in the two transformations of $N$ we have taken $\varrho=e^{2 \psi} / m_{i}$, equations (II, 92) become

$$
\begin{aligned}
& \frac{\partial}{\partial u}\left(\theta_{12} \frac{\theta_{1}^{\prime}}{\theta_{1}}\right)=-m_{1} e^{-2 v} \theta_{1}^{2} \frac{\partial}{\partial u}\left(\frac{\theta_{2}}{\theta_{1}}\right), \\
& \frac{\partial}{\partial v}\left(\theta_{12} \frac{\theta_{1}^{\prime}}{\theta_{1}}\right)=m_{1} e^{-2 \vartheta} \theta_{1}^{2} \frac{\partial}{\partial v}\left(\frac{\theta_{2}}{\theta_{1}}\right) .
\end{aligned}
$$

From these and analogous equations in $\theta_{2}$, we find that the lefthand member of the following equation is constant; we consider in particular the case when

$$
\theta_{21} \nu_{2}+\theta_{12} \nu_{1}=0
$$

From this equation and (II, 36, 48) we have

$$
m_{1} \theta_{12}^{\prime \prime \prime}=m_{2} \theta_{12}\left(m_{1} \nu_{2}-\frac{\theta_{2}^{\prime}}{\theta_{1}}\right)=\frac{m_{2} \nu_{2}}{\nu_{1}} \theta_{21}\left(\frac{\theta_{2}^{\prime}}{\theta_{1}}-m_{1} \nu_{2}\right)
$$

from which follows, when $\theta_{21}$ and $\theta_{12}^{\prime \prime \prime}$ are replaced by their values (II, 38, 43),

$$
m_{1} m_{2}\left(\nu_{2} \theta_{1}+\nu_{1} \theta_{2}\right)=m_{2} \theta_{2}^{\prime}+m_{1} \theta_{1}^{\prime \prime}
$$

$\left.{ }^{63}\right)$ Annali, ser. 3, vol. 11 (1905), pp. 93-158. 
From (VI, 83) it follows that for any transformation $R$

$$
\left\{\begin{array}{l}
\frac{\partial \theta_{2}^{\prime}}{\partial u}=m_{1} v_{1}\left(\sqrt{E}+\sqrt{E_{1}}\right) q_{2}, \frac{\partial \theta_{2}^{\prime}}{\partial v}=m_{1} v_{1}\left(\sqrt{G}+\sqrt{G_{1}}\right) r_{2}, \\
\frac{\partial \theta_{1}^{\prime \prime}}{\partial u}=m_{2} v_{2}\left(\sqrt{E}+\sqrt{E_{2}}\right) q_{1}, \frac{\partial \theta_{1}^{\prime \prime}}{\partial v}=m_{2} v_{2}\left(\sqrt{G}+\sqrt{G_{2}}\right) r_{1} .
\end{array}\right.
$$

When equations (50) and (53) are solved for $\theta_{2}^{\prime}$ and $\theta_{1}^{\prime \prime}$, and the results are substituted in these equations, the latter are satisfied. Since $\theta_{1}^{\prime \prime}$ and $\theta_{2}^{\prime}$ are completely determined there is only one transform $N_{12}$, which is isothermic.

Making use of (54), we find

(55) $\frac{\partial}{\partial u}\left(\frac{\theta_{12}^{\prime \prime \prime}}{\theta_{12}}\right)=m_{2} \frac{\nu_{1}}{\theta_{1}} e^{\phi}\left(\frac{\theta_{2}^{\prime}}{\theta_{1}^{\prime}} q_{1}-q_{2}\right), \frac{\partial}{\partial v}\left(\frac{\theta_{12}^{\prime \prime \prime}}{\theta_{12}}\right)=-m_{2} \frac{\nu_{1}}{\theta_{1}} e^{\phi}\left(\frac{\theta_{2}^{\prime}}{\theta_{1}^{\prime}} r_{1}-r_{2}\right)$.

In consequence of (VI, 109) the expressions for $\frac{\partial \nu_{12}}{\partial u}$ and $\frac{\partial \nu_{12}}{\partial v}$ analogous to the last two of (47) differ from the right-hand members of (55) only by the factor $m_{2}$. Consequently

$$
\theta_{12}^{\prime \prime \prime}=m_{2} \theta_{12} v_{12}
$$

and the transformation from $N_{1}$ into $N_{12}$ is $D_{m_{2}}$. In like manner the transformation from $N_{2}$ into $N_{12}$ is $D_{m_{1}}$.

By means of (51) and (52) the expressions (II, 46) for the coordinates of $N_{12}$ are reducible to

$$
x_{12}=x+\frac{m_{2} \theta_{2} x^{\prime}-m_{1} \theta_{1} x^{\prime \prime}}{m_{1} \theta_{1}\left(\theta_{1}^{\prime \prime}-m_{2} \theta_{2} \nu_{1}\right)} .
$$

Making use of (50) and (53), we reduce this equation to

$$
x_{12}=x+\frac{m_{2}-m_{1}}{m_{1} m_{2}} \frac{m_{2} \theta_{2} x^{\prime}-m_{1} \theta_{1} x^{\prime \prime}}{p_{1} p_{2}+q_{1} q_{2}+r_{1} r_{2}-m_{1} \theta_{1} \nu_{2}-m_{2} \theta_{2} \nu_{1}} .
$$

From this it is seen that we must have $m_{y} \neq m_{1}$. Hence we have the following theorem of Bianchi ${ }^{64}$ ):

64) L. c., p. 120. 
If $N$ is an isothermic $O$ net, and $N_{1}$ and $N_{2}$ are two isothermic $O$ nets obtained from $N$ by transformations $D_{m_{1}}$ and $D_{m_{v}}$ there can be found without quadrature an isothermic $O$ net $N_{12}$ such that $N_{12}$ is a $D_{m_{2}}$ transform of $N_{1}$ and $D_{m_{1}}$ transform of $N_{2}$.

84. Special isothermic nets in 3-space. In $\S 81$ it is shown that in order that two transformations $R$ have the same circle-planes it is necessary that

$$
\theta_{2}=\theta_{1}+j, \quad p_{2}=p_{1}+k, \quad g_{2}=q_{1}, \quad r_{2}=r_{1},
$$

where $j$ and $k$ are constants. In $\S 119$ we shall show that in the tangent planes of a deform of a quadric, meeting the circle at infinity in four distinct points, there are eight points which describe isothermic surfaces, any one of which is in the relation of a transformation $R$ with three others, the corresponding cyclic systems admitting the tangent planes to the surface for circle-planes. We apply the preceding results to the determination of all isothermic surfaces admitting two transformations $D_{m}$ for which the circleplanes are the same.

From the last of (47) it follows that $\nu_{2}=\nu_{1}+n$, where $n$ is a constant. Moreover, since (49) must be satisfied by both transformations, we must have also

$$
p_{1} k+\frac{k^{2}}{2}=m_{2}\left(\theta_{1}+j\right)\left(v_{1}+n\right)-\theta_{1} v_{1} m_{1} .
$$

When this equation is differentiated with respect to $u$ and $v$, the two resulting equations are equivalent, by means of (47), to

$$
2\left(m_{2}-m_{1}\right) \nu_{1}+2 m_{2} n=-k K_{0}, \quad 2\left(m_{2}-m_{1}\right) \theta_{1}+2 m_{2} j=k L,
$$

where $K_{0}$ and $L$ are defined by (41). These equations may be replaced by

$$
\theta_{1}=L+e, \quad \nu_{1}=-K_{0}+f
$$

where $e$ and $f$ are constants. From the last two of (47) we find that

$$
q_{1}=-e^{\phi} \frac{\partial K_{0}}{\partial u}, \quad r_{1}=e^{\phi} \frac{\partial K_{0}}{\partial v} .
$$


In consequence of (40) these functions satisfy (47), if we take

$$
p_{1}=-\frac{1}{2} L K_{0}+g,
$$

where $g$ is a constant. In order that (49) hold we must have

(64) $e^{2 \psi}\left[\left(\frac{\partial K_{0}}{\partial u}\right)^{2}+\left(\frac{\partial K_{0}}{\partial v}\right)^{2}\right]+\frac{1}{4} K_{0}^{2} L^{2}+4 K_{0} L+2 B K_{0}+2 C L+D=0$, where
$A=2 m_{1}-g$
$B=m_{1} e$
$C=-m_{1} f$
$D=g^{2}-2 m_{1} e f$

Eliminating $e, f$ and $g$ from these equations, we have that $m_{1}$ must satisfy

$$
(A-2 m)^{2} m-D m+2 B C=0 .
$$

If then a net $N$ satisfies the condition (64) where $A, B, C, D$ are constants, there are in general three solutions $m$ of (66). When these are substituted in (65), we have three sets of values of $e$, $f$ and $g$ and the corresponding functions (61), (62) and (63) determine transformations of $N$ into three isothermic nets such that the circle-planes of the three transformations are the same. The foregoing results are due to Darboux ${ }^{65}$ ). Bianchi ${ }^{66}$ ) also has considered these surfaces, and in order to put in evidence the essential constants appearing in (64), he refers to such a surface as a special isothermic surface of class $(A, B, C, D)$.

85. Complementary transformations $D_{m}$ of special isothermic nets. The three nets obtained from $N$ by the transformations determined by the values (61), (62), (63) have been called the complementary nets by Bianchi. We shall show that they are special nets of class $(A,-B,-C, D)$. In fact, in consequence of (9) and (46) we find that the functions $K_{0,1}$ and $L_{1}$ for one of these nets $N_{1}$, defined by equations analogous to (41), have the values

$$
K_{0,1}=\frac{-\nu_{1} L+2 p_{1}}{\theta_{1}}, \quad L_{1}=-\frac{\theta_{1} K_{0}+2 p_{1}}{\nu_{1}} .
$$

${ }^{65)}$ L. c.: p. 507.

${ }^{66)}$ L. c., p. 130. 
From these and the foregoing formulas we find

$$
\begin{gathered}
L_{1}-e=-\frac{e f+2 g}{\nu_{1}}, \quad K_{0,1}+f=\frac{e f+2 g}{\theta_{1}}, \\
-\frac{1}{2} K_{0,1} L_{1}+g=(e f+2 g) \frac{p_{1}}{\nu_{1} \theta_{1}} .
\end{gathered}
$$

From these expressions and (VI, 94) it follows that $N$ may be obtained from $N_{1}$ by a transformation $D_{m_{1}}$ determined by the functions

$\left.\theta_{1}^{-1}=L_{1}-e, \quad \nu_{1}^{-1}=-\left(K_{0,1}+f\right), \quad p_{1}^{-1}=-\frac{1}{2} K_{0,1} L_{1}+g^{67}\right)$.

Since the effect of changing the signs of $e$ and $f$ is to change the signs of $B$ and $C$, but not to affect (66) we have that $N_{1}$ is a special net $(A,-B,-C, D)$.

Now we show, as Darboux has done ${ }^{68}$ ), that the circle-planes of a complementary transformation envelope a surface applicable to a general quadric. To this end we make use of the results of $\S 79$. From (VI, 83) and the preceding formulas, we find that for the present case

$$
\begin{gathered}
h=m\left(\nu+\theta e^{-2 \psi}\right), \quad l=m\left(\nu-\theta e^{-2 \psi}\right), \\
j=\frac{e-\theta}{m(\theta f-e \nu)}, \quad k=\frac{2 \theta}{\theta f-e \nu}, \quad \bar{\omega}=\theta \frac{2 g+e f-\nu e}{\theta f-e \nu} .
\end{gathered}
$$

From (23) it follows that if we put

$$
x=k, \quad y+i z=j, \quad y-i z=2 \bar{\omega},
$$

the point $(x, y, z)$ describes a surface $\Sigma$ applicable to the envelope of the circle-planes. When the above values of $j, k$ and $\bar{\omega}$ are substituted, and $\theta$ and $\nu$ are eliminated, we find that $\Sigma$ is the quadric

$$
(y-i z)[x+2 m(y+i z)]=2 g x^{2}+2 e x+2 m(2 g+e f) x(y+i z) .
$$

67) If $2 g+e f=0, N_{1}$ has constant mean curvature and the corresponding equation (64) is $\frac{1}{4} K_{0,1}^{2} L_{1}^{2}+A K_{0,1} L_{1}-2 B K_{0,1}-2 C L_{1}+D=0$ which is satisfied because of (65).

68) L. c., p. 508. 
86. Transformations $D_{m}$ of special isothermic nets. Let $N$ be a special isothermic net of class $(A, B, C, D)$ and $N_{1}$ a complementary transform by means of the functions given by (61), (62) and (63). We shall show that it is possible to find solutions $\boldsymbol{\theta}_{2}, \nu_{2}, p_{2}, q_{2}$ and $r_{2}$ of equations (47) such that the net $N_{2}$ is a special isothermic net. This is done by finding under what conditions a net $N_{12}$, arising from $N_{1}$ and $N_{2}$ in accordance with the theorem of permutability of $\S 83$ is a complementary transform of $N_{\mathbf{2}}$.

From (II, 43), (VI, 109) and (52) we have

$$
\begin{gathered}
\theta_{21}=\theta_{1}-\frac{\theta_{1}^{\prime \prime}}{\theta_{2}^{\prime \prime}} \theta_{2}, \quad p_{21}=\frac{\theta_{1}^{\prime \prime}}{\theta_{2}^{\prime \prime}} p_{2}-p_{1}, \\
\nu_{21}=\frac{1}{m_{1}} \frac{\theta_{21}^{\prime \prime \prime}}{\theta_{21}^{\prime \prime}}=\frac{1}{m_{2}}\left(m_{2} v_{1}-\frac{\theta_{1}^{\prime \prime}}{\theta_{2}}\right) .
\end{gathered}
$$

Also from (50) and (53) we obtain

$$
\left(m_{2}-m_{1}\right) \theta_{1}^{\prime \prime}=m_{2}\left(p_{1} p_{2}+q_{1} q_{2}+r_{1} r_{2}-m_{1} \nu_{2} \theta_{1}-m_{1} \nu_{1} \theta_{2}\right) .
$$

The analogues of (67) are

$$
K_{0,2}=-\frac{\nu_{2} L+2 p_{2}}{\theta_{2}}, \quad L_{2}=-\frac{\theta_{2} K_{0}+2 p_{2}}{\nu_{2}} .
$$

The equations

$$
\text { (68) } \theta_{21}=-L_{2}+e, \quad \nu_{81}=K_{0,2}+f, \quad p_{21}=\frac{1}{2} K_{0,2} L_{2}-q \text {, }
$$

are consistent with the above results, provided that

$p_{1} p_{2}+q_{1} q_{2}+r_{1} r_{2}-m_{2}\left(\nu_{2} \theta_{1}+\nu_{1} \theta_{2}\right)+\left(m_{2}-m_{1}\right)\left(\nu_{2} e+\theta_{2} f+2 p_{2}\right)=0$.

By differentiation we find that the left-hand member of this equation is constant for each set of solutions $\theta_{2}, \nu_{2}, p_{2}, q_{2}$ and $r_{2}$ of (47). Hence if the constants entering in the latter are chosen so that (69) is satisfied, the net $N_{12}$ is a complementary transform of $N_{2}$. The latter is a special isothermic net of class $(A$, $-B,-C, D)$. Hence we have the theorem of Bianchi ${ }^{69}$ ):

Of the $\infty^{4}$ isothermic nets obtained from a special isothermic net of class $(A, B, C, D)$ by transformations $D_{m}, \infty^{s}$ are nets of class $(A,-B,-C, D)$.

${ }^{69}$ L. c., p. 141. We note that Bianchi showed that $N_{2}$ is of the same class as $N$. This is due to his choice of directions in the triledron of $N_{3}$. 
87. Transformations $D_{m}$ of minimal surfaces. A minimal surface is isothermic, and the spherical representation of its lines of curvature is such that we may take $[\$ 109]$

$$
\frac{\sqrt{E}}{\varrho_{1}}=-\frac{\sqrt{G}}{\varrho_{2}}=-e^{-v},
$$

where $\sqrt{E}=\sqrt{G}=e^{\psi}$. From (9) and (46) it follows that the transform is minimal, if $\nu=p$, and only in this case. The equations of such a transformation are:

(71)

$$
\left\{\begin{array}{c}
\frac{\partial \theta}{\partial u}=e^{\psi} q . \quad \frac{\partial \theta}{\partial v}=e^{\psi} r, \\
\frac{\partial p}{\partial u}=e^{-\psi} q, \quad \frac{\partial p}{\partial v}=-e^{-\psi_{r} r} \\
\frac{\partial q}{\partial u}=-e^{-\psi} p-\frac{\partial \psi}{\partial v} r+m\left(c^{\psi^{\prime}} p+e^{\left.-\psi^{\prime} \theta\right)}, \quad \frac{\partial q}{\partial v}=\frac{\partial \psi}{\partial u} r,\right. \\
\frac{\partial r}{\partial u}=\frac{\partial \psi}{\partial v} q, \quad \frac{\partial r}{\partial v}=e^{-\psi} p-\frac{\partial \psi}{\partial u} q+m\left(e^{\psi} p-e^{-\psi} \theta\right) .
\end{array}\right.
$$

These equations form a completely integrable system. Since $p^{2}+q^{2}+r^{2}-2 m p \theta=0$ must be satisfied, we have:

$A$ minimal surface admits $\infty^{3}$ transformations $D_{n}$ into minimal surfaces ${ }^{70}$ ).

From (56), (52) and (VI, 109) we have $\nu_{12}=-p_{12}$, and therefore:

If a minimal suface is transformed into minimal surfaces $S_{1}$ and $S_{2}$ by transformations $D_{m_{1}}$ and $D_{m_{2}}$ there can be found without quadruture a minimal surface which is a $D_{m_{2}}$ transform of $S_{1}$ and a $D_{m_{1}}$ transform of $S_{2}{ }^{71}$ ).

88. Transformations $E_{m}$ of $O$ nets with isothermal spherical representation. Since the spherical representation of the $O$ net on a minimal surface is isothermal, each transformation $D_{m}$ of a minimal surface into a minimal surface leads by a quadrature to a transformation $R$ of an $O$ net with isothermal spherical representation into an $O$ net of the same type, in accor-

i0) Bianchi, Rendiconti dei Lincei, ser. 5, rol. $8^{1}$ (1899), p. 151.

ii) Cf. Bianchi, l. c. 
dance with the first theorem of $\S 78$. These transformations were studied by the author ${ }^{72}$ ) and later by Bianchi ${ }^{73}$ ), who called them transformations $E_{m}$. In this section we show that these transformations are the most general transformations $R$ of $O$ nets with isothermal spherical representation into nets of the same kind.

The tangential coordinates of $N$ satisfy $(\mathrm{V}, 29)$ with $\varrho=e^{2 \phi}$ and $\gamma=0$. From (VI, 94) we have that the functions $w$ and $w^{-1}$ of $\S 52$ are $p$ and $p / \theta^{\prime}$ respectively. Hence if a transformation $R$ of $N^{\top}$ is to be a transformation $\Omega$, that is if $N_{1}$ is to have isothermal spherical representation, its tangential equation must be of the form

$$
\frac{\partial^{2} \lambda_{1}}{\partial u \partial v}+\frac{\partial \psi_{1}}{\partial v} \frac{\partial \lambda_{1}}{\partial u}+\frac{\partial \psi_{1}}{\partial u} \frac{\partial \lambda_{1}}{\partial v}=0 .
$$

From $(V, 35)$ it follows that $\psi_{1}$ can be chosen so that

if we take

$$
e^{-\psi_{1}}=\frac{m p^{2}}{\boldsymbol{\theta}^{\prime}} e^{\psi}=\frac{p^{2}}{v^{\prime} \boldsymbol{\theta}} e^{\downarrow}
$$

If in accordance with (9) we put

$$
\sqrt{E_{1}}+\sqrt{E}=\frac{p}{\nu} e^{\psi}+\frac{\theta}{p} e^{-\psi}, \quad \sqrt{G_{1}}+1 \bar{G}=\frac{p}{\nu} e^{\psi}-\frac{\theta}{p} e^{-\psi},
$$

we find that the last two of equations (7) are satisfied, and consequently the following system of equations is completely integrable:

$$
\begin{aligned}
& \frac{\partial \theta}{\partial \imath}=\sqrt{E} q, \quad \frac{\partial \theta}{\partial v}=\sqrt{G} r, \\
& \frac{\partial p}{\partial u}=e^{-\downarrow} q, \quad \frac{\partial \underline{p}}{\partial \imath}=-e^{-\vartheta} r, \\
& \frac{\partial q}{\partial u}=-e^{-\psi} p-\frac{\partial \psi}{\partial v} r+m\left(p e^{\psi}+\frac{\theta \nu}{p} e^{-\psi}\right), \quad \frac{\partial q}{\partial v}=\frac{\partial \psi}{\partial u} r, \\
& \frac{\partial r}{\partial u}=\frac{\partial \psi}{\partial v} q, \quad \frac{\partial r}{\partial v}=e^{-\downarrow} p-\frac{\partial \psi}{\partial u} q+m\left(p e^{\psi}-\frac{\theta v}{p} e^{-\vartheta}\right), \\
& \frac{\partial \nu}{\partial \boldsymbol{u}}=\frac{q}{\boldsymbol{\theta}}\left(p e^{\psi}+\frac{\theta \nu}{p} e^{-\psi}-\sqrt{E} \nu\right), \quad \frac{\partial \nu}{\partial v}=\frac{r}{\theta}\left(p e^{\psi}-\frac{\theta \nu}{p} e^{-\psi}-\sqrt{G} \nu\right) .
\end{aligned}
$$

72) Transactions Amer. Math. Soc., vol. 9 (1908), pp. 149-177.

$\left.{ }^{73}\right)$ Rendiconti dei Lincei, ser. 5, rol. 24 (1915), p. 371. 
From these equations we have

$$
\frac{\partial}{\partial u}\left(\frac{\theta \nu}{p}\right)=e^{\psi} q, \quad \frac{\partial}{\partial v}\left(\frac{\theta v}{p}\right)=e^{\psi} v,
$$

and consequently $\theta y / p$ is a solution $\bar{\theta}$ of the point equation of the minimal surface $\bar{N}$ with the same spherical representation as $N$. In terms of this function the above expressions for the derivatives of $q$ and $r$ assume the same form as (71). Consequently the transformation of $\bar{N}$ by means of $p, q, r, \bar{\theta}$ is a $D_{m}$ into a minimal surface. Hence we have the theorem:

When an $O$ net $N$ with isothermal spherical representation admits a transformation $R$ into a net $N_{1}$ of the same lind, the minimal surface parallel to $N$ is transformed into the minimal surface parallel to $N_{1}$ by a transformation $D_{m}$.

Conversely, if $\bar{N}$ and $\bar{N}_{1}$ are $O$ nets on minimal surfaces in relation $D_{m}$, and $N$ is a net parallel to $\bar{N}$, the set of functions $p, q, r$ of this transformation and $\theta$ and $\nu$, where $\theta \nu=\bar{\theta} p$ determine transformations $E_{m}$ of $N$ into $\infty^{1}$ nets $N_{1}$ parallel to $\overline{N_{1}}$. For $\theta$ is determined to within an additive arbitrary constant by the first two of (75) and then $\nu$ is fixed. Hence:

An $O$ net with isothermal spherical representation admits $\infty^{2}$ transformations $E_{m}$, for each value of $m$, into $\infty^{1}$ parallel $O$ nets of the same kind.

From these results and the fifth theorem of $\S 78$ we have:

An isothermic net on a sphere admits $\infty^{3}$ transformations $F$ into isothermic nets on the sphere.

Consider a quatern of minimal surfaces in accordance with the last theorem of $\S 87$, and four nets $N, N_{1}, N_{2}, N_{12}$ parallel to the $O$ nets $\bar{N}, \bar{N}_{1}, \bar{N}_{2}, \bar{N}_{12}$ of the minimal surfaces in accordance with the last theorem of $\S 72$.

From (56) it follows that

$$
\bar{\theta}_{12}^{\prime \prime \prime}=-m_{2} \bar{\theta}_{12} p_{12}, \quad \bar{\theta}_{21}^{\prime \prime \prime \prime}=-m_{1} \bar{\theta}_{21} p_{21},
$$

where $\bar{\theta}_{12}$ and $\bar{\theta}_{21}$ determine the transformations from $\overline{N_{1}}$ and $\overline{N_{2}}$ into $\bar{N}_{12}$. From equations analogous to (76) and (49) we have

$$
\theta_{12} \nu_{12}=-p_{12} \bar{\theta}_{12}, \quad \theta_{21} \nu_{21}=-p_{21} \bar{\theta}_{21} \text {. }
$$


Hence, since $\theta_{12}^{\prime \prime \prime}=\bar{\theta}_{12}^{\prime \prime \prime}$ and $\theta_{21}^{\prime \prime \prime \prime}=\bar{\theta}_{21}^{\prime \prime \prime}$,

$$
\theta_{12}^{\prime \prime \prime}=m_{2} \theta_{12} \nu_{12}, \quad \theta_{21}^{\prime \prime \prime \prime}=m_{1} \theta_{21} \nu_{21},
$$

and consequently we have the theorem:

If $N_{1}$ and $N_{2}$ are obtained from an $O$ net $N$ with isothermal spherical representation by transformations $E_{m_{1}}$ and $E_{m_{2}}$, there can be found without quadratures a net $N_{12}$ of the same kind, such that $N_{12}$ is an $E_{m_{2}}$ transform of $N_{1}$ and an $E_{m_{1}}$ transform of $N_{\mathbf{2}}$.

89. Nets $\Omega$. By definition an $O$ net $N$ is a net $\Omega$ if there exists a net $N_{0}$ with equal point invariants conjugate to the congruence $G$ normal to $N$. Let $N$ be a net $\Omega$ and let $\theta_{0}$ be the solution of the point equation of $N$, namely

$$
\frac{\partial^{2} \theta}{\partial u \partial v}=\frac{\partial \log \sqrt{E}}{\partial v} \frac{\partial \theta}{\partial u}+\frac{\partial \log \sqrt{G}}{\partial u} \frac{\partial \theta}{\partial v},
$$

by means of which $N_{0}\left(x_{0}\right)$ is obtained from $N$ by a transformation $F$. The equations of the transformation are of the form

where $\hat{\theta_{0}}$ is defined by

$$
x_{0}=x-\frac{\theta_{0}}{\hat{\theta}_{0}} X,
$$

$$
\frac{\partial \hat{\theta}_{0}}{\partial u}=\hat{h} \frac{\partial \theta_{0}}{\partial u}, \quad \frac{\partial \hat{\theta}_{0}}{\partial v}=\hat{l} \frac{\partial \theta_{0}}{\partial v},
$$

and

$$
\hat{h}=-\frac{1}{\varrho_{1}}, \quad \hat{l}=-\frac{1}{\varrho_{2}},
$$

since [§51]

$$
\frac{\partial X}{\partial u}=-\frac{1}{\varrho_{1}} \frac{\partial x}{\partial u}, \quad \frac{\partial X}{\partial v}=-\frac{1}{\varrho_{2}} \frac{\partial x}{\partial v} .
$$

If the point equation of $N_{0}$ is written in the form

$$
\frac{\partial^{2} \theta}{\partial u \partial v}=\frac{\partial}{\partial v} \log V \varrho_{0} \frac{\partial \theta}{\partial u}+\frac{\partial}{\partial u} \log V \varrho_{0} \frac{\partial \theta}{\partial v},
$$


we have from (77) and $(\Pi, 12)$

$$
\sqrt{\varrho_{0}}=\frac{\tau_{0} \sqrt{E}}{\hat{\theta}_{0}} U=\frac{\sigma_{0} \sqrt{G}}{\hat{\theta}_{0}} V
$$

where $U$ and $V$ are functions of $u$ and $v$ respectively, and from (II, 8)

$$
\tau_{0}=-\left(\frac{\theta_{0}}{\varrho_{1}}+\hat{\theta_{0}}\right), \quad \sigma_{0}=-\left(\frac{\theta_{0}}{\varrho_{2}}+\hat{\theta_{0}}\right)
$$

When these expressions are substituted in (83), we obtain

(85)

$$
\hat{\theta}_{0}=\theta_{0} \frac{\varrho_{2} U \sqrt{E}-\varrho_{1} V \sqrt{G}}{\varrho_{1} \varrho_{2}(V \sqrt{G}-U \sqrt{E})}, \quad \sqrt{\varrho_{0}}=\frac{U V \sqrt{E G}\left(\varrho_{1}-\varrho_{2}\right)}{U \sqrt{E} \varrho_{2}-V V G \varrho_{1}}
$$

The Codazzi equations for $N$ are [§ 65]

(86)

$$
\frac{\partial}{\partial v}\left(\frac{\sqrt{E}}{\varrho_{1}}\right)=\frac{1}{\varrho_{2}} \frac{\partial \sqrt{E}}{\partial v}, \quad \frac{\partial}{\partial u}\left(\frac{\sqrt{G}}{\varrho_{2}}\right)=\frac{1}{\varrho_{1}} \frac{\partial \sqrt{G}}{\partial u}
$$

When we require that the function (85) satisfy (79), we find, in consequence of (86),

$$
\left\{\begin{array}{l}
\frac{\partial \log \theta_{0}}{\partial u}=\frac{U V \bar{E}}{V \sqrt{G}} \frac{\varrho_{1} \varrho_{2}}{\varrho_{1}-\varrho_{2}} \frac{\partial}{\partial u}\left(\frac{1}{\varrho_{1}}\right)+\frac{\partial}{\partial u} \log (U V \bar{E}-V \sqrt{G}) \\
\frac{\partial \log \theta_{0}}{\partial v}=\frac{V \sqrt{G}}{U \sqrt{E}} \frac{\varrho_{1} \varrho_{2}}{\varrho_{2}-\varrho_{1}} \frac{\partial}{\partial v}\left(\frac{1}{\varrho_{2}}\right)+\frac{\partial}{\partial v} \log (U \sqrt{E}-V \sqrt{G}) .
\end{array}\right.
$$

The condition of integrability of (87) is

(88) $\frac{\partial}{\partial v}\left(\frac{U V \bar{E}}{V \sqrt{G}} \frac{\varrho_{1} \varrho_{2}}{\varrho_{1}-\varrho_{2}} \frac{\partial}{\partial u}\left(\frac{1}{\varrho_{1}}\right)\right)=\frac{\partial}{\partial u}\left(\frac{V \sqrt{G}}{U \sqrt{E}} \frac{\varrho_{1} \varrho_{2}}{\varrho_{2}-\varrho_{1}} \frac{\partial}{\partial v}\left(\frac{1}{\varrho_{2}}\right)\right)$. 
When this condition is satisfied, we find that $\theta_{0}$ given by (87) satisfies (77), and consequently.$N$ is a net $\Omega$. Hence we have the theorem of Demoulin $\left.{ }^{74}\right)$ :

A necessary and sufficient condition that an $O$ net be a net $\Omega$ is that (88) be satisfied.

In consequence of the second theorem of $\S 25$ we have that when $N$ is a net $\Omega$, there is a second net $N_{\omega}$ with equal point invariants conjugate to the congruence $G$ normal to $N$, and that the points of $N_{0}$ and $N_{\omega}$ on a line of $G$ are harmonic to the focal points of $G$ on this line. Since the coordinates of these focal points are of the forms, $x+e_{1} X$ and $x+\varrho_{2} X$, we have from (78) and the analogous equations for $N_{\mathrm{\omega}}$, namely

the equation

$$
x_{\omega}=x-\frac{\theta_{\omega}}{\hat{\theta}_{\omega}} X,
$$

$$
\hat{\theta}_{\omega}=-\theta_{\omega} \frac{\varrho_{2} U V \bar{E}+\varrho_{1} V V \sqrt{G}}{\varrho_{1} \varrho_{2}(U \sqrt{E}+V \sqrt{G})} \text {. }
$$

The equations analogous to (87) are obtained from them by replacing $V$ by $-V$. From these equations we have

$$
\text { (90) } \theta_{\omega} \theta_{0}=U^{2} E-V^{2} G, \quad \sqrt{\varrho_{\omega}}=\frac{U V \sqrt{E G}\left(\varrho_{2}-\varrho_{1}\right)}{U V \bar{E} \varrho_{2}+V \sqrt{G} \varrho_{1}} \text {. }
$$

Since $N_{0}$ and $N_{\omega}$ are conjugate to the normal congruence to $N$, they are nets $2, O$, the respective complementary functions being $\theta_{0} / \hat{\theta}_{0}$ and $\theta_{\omega} / \hat{\theta}_{\mathrm{\omega}}(\S 66)$. Conversely, if $N_{0}$ is a net $2, O$ with equal invariants, the two sheets of the envelope of spheres with centers on $N_{0}$ and radii given by the complementary function of $N_{0}$ are surfaces $\Omega$ in relation $R$.

The foregoing results may be stated as follows:

When a net $N$ is a net $\Omega$, the nets with equal invariants conjugate to the congruence normal to $N$ can be found without quadratures; these nets are 2, $O$. Conversely, when a $2, O$ net $N_{0}$ with equal invariants is known, there can be found without quadratures two nets $\Omega$, in relation $R$, whose normal congruences are conjugate to $N_{0}$.

74) Comptes Rendus, vol. 153 (1911), pp. 590, 705, 927. 
From $(I I, 87)$ it follows that the net $N_{0}^{\prime}\left(x_{0}^{\prime}\right)$, where

$$
\frac{\partial x_{0}^{\prime}}{\partial u}=-\frac{1}{\varrho_{0}} \frac{\partial x_{0}}{\partial u}, \quad \frac{\partial x_{0}^{\prime}}{\partial v}=\frac{1}{\varrho_{0}} \frac{\partial x_{0}}{\partial r}
$$

has equal point invariants. From $\S 76$ we have that $N_{0}^{\prime}$ is $2, O$, the complementary function $t^{\prime}$ being given by a quadrature, and that the sheets of the envelope of the spheres with centers on $N_{0}^{\prime}$ and radius $t^{\prime}$ are parallel to the corresponding sheets of the envelope of the above congruence $R$ of spheres with centers on $N_{0}$. Hence:

When a net $\Omega$ is known, a parallel net $\Omega$ can be found by quadratures.

90. Transformations $R$ of nets $\Omega$. Let $N$ be a net $\Omega$ and $N_{1}$ an $R$ transform of $N$ by means of a set of solutions $\theta, p, q, r$ and $u$ of equations (7) and (8). As in the preceding. section, we denote by $N_{0}$ one of the nets with equal point invariants conjugate to the congruence normal to $N$; it is an $F$ transform of $N$ by means of $\theta_{0}$ and $\hat{\theta}_{0}$. In accordance with the theorem of permutability of transformations $F(\S 21)$ there exist $\infty^{2}$ nets $N_{01}$, which are $F$ transforms of $N_{0}$ and $N_{1}$.

From (11) and $\$ 21$ it follows that if we take $\hat{\theta}=p$ we obtain $\infty^{1}$ nets $N_{01}$ conjugate to the congruence normal to $N_{1}$, as the additive constant of $\theta_{0}^{\prime}$ varies. We seek the conditions that one of these nets be a $K$ transform of $N_{1}$ and thus has equal point invariants, in which case $N_{1}$ is a net $\Omega$.

From (II, 43,44) we have that the functions $\theta_{01}$ and $\theta_{01}^{\prime \prime \prime \prime}$ of the transformation from $N_{0}$ into a net $N_{01}$ are given by

$$
\theta_{01}=\theta-\frac{\theta_{0}}{\hat{\theta}_{0}} p, \quad \theta_{01}^{\prime \prime \prime}=\theta^{\prime}-\frac{\theta_{0}^{\prime}}{\hat{\theta}_{0}} p
$$

and from $(11,53)$ and $(80)$ we have

$$
h_{01}=-\frac{\frac{1}{\rho_{1}} \theta_{0}^{\prime}+h \hat{\theta_{0}}}{\tau_{0}}, \quad l_{01}=-\frac{\frac{1}{\rho_{2}} \theta_{0}^{\prime}+l \hat{\theta_{0}}}{\sigma_{0}}
$$


with

$$
\frac{\partial \theta_{01}^{\prime \prime \prime}}{\partial u}=h_{01} \frac{\partial \theta_{01}}{\partial u}, \quad \frac{\partial \theta_{01}^{\prime \prime \prime}}{\partial v}=l_{01} \frac{\partial \theta_{01}}{\partial v}
$$

A necessary and sufficient condition that $N_{01}$ be a $K$ transform of $N_{0}$ is that (II, 81)

$$
h_{01}=\frac{m \theta_{01}}{\varrho_{0}}+\frac{\theta_{01}^{\prime \prime \prime}}{\theta_{01}}, \quad l_{01}=-\frac{m \theta_{01}}{\varrho_{0}}+\frac{\theta_{01}^{\prime \prime \prime}}{\theta_{01}}
$$

where $m$ is a constant.

From (II, 36, 38) we have that the functions $\theta_{10}$ and $\theta_{10}^{\prime \prime \prime}$ of the transformation from $N_{1}$ into $N_{01}$ have the form

$$
\theta_{10}=\theta_{0}-\frac{\theta_{0}^{\prime}}{m \nu}, \quad \theta_{10}^{\prime \prime \prime}=\hat{\theta}_{0}-\frac{p \theta_{0}^{\prime}}{m \nu \theta}
$$

where $\theta^{\prime}=m \nu \theta$.

When we equate the expressions (92) and (94) for $h_{01}$ and $l_{01}$, the resulting equations are reducible by means of (VI, 83), (91) and (95)

$$
\left\{\begin{array}{l}
U \sqrt{E_{1}}=-U \sqrt{E} \frac{\theta_{10}}{\theta_{0}}-\frac{\theta_{01}}{\nu \sqrt{\varrho_{0}}}-\sqrt{\varrho_{0}} \frac{\theta}{\theta_{0}} \frac{\theta_{10}}{\theta_{01}} \\
V \sqrt{G_{1}}=-V \sqrt{G} \frac{\theta_{10}}{\theta_{0}}+\frac{\theta_{01}}{\nu \sqrt{\varrho_{0}}}-\sqrt{\varrho_{0}} \frac{\theta}{\theta_{0}} \frac{\theta_{10}}{\theta_{01}}
\end{array}\right.
$$

From (91) we have by differentiation

$$
\begin{aligned}
& \frac{\partial \theta_{01}}{\partial u}=-\frac{\sqrt{\varrho_{0}}}{U}\left(q-\frac{p}{\hat{\theta}_{0}} \frac{1}{\sqrt{E}} \frac{\partial \theta_{0}}{\partial u}\right) \\
& \frac{\partial \theta_{01}}{\partial v}=-\frac{\sqrt{\varrho_{0}}}{V}\left(r-\frac{p}{\hat{\theta}_{0}} \frac{1}{\sqrt{G}} \frac{\partial \theta_{0}}{\partial v}\right) .
\end{aligned}
$$

By means of these equations and the last two of (97), which are obtained by differentiating (95), we find that the expressions (96) satisfy the last two of (7). Hence the following system of equations in which $E_{1}$ and $G_{1}$ have the form (96) is completely integrable: 
91. Theorem of permutability of the transformations of nets $Q$

$$
\left\{\begin{array}{c}
\frac{\partial \theta}{\partial u}=V \bar{E} q, \quad \frac{\partial \theta}{\partial v}=\sqrt{G} r, \\
\frac{\partial p}{\partial u}=-\frac{\sqrt{E}}{\varrho_{1}} q, \quad \frac{\partial p}{\partial v}=-\frac{\sqrt{G}}{\varrho_{2}} r, \\
\frac{\partial q}{\partial u}=\frac{\sqrt{E}}{\varrho_{1}} p-\frac{1}{\sqrt{G}} \frac{\partial \sqrt{E}}{\partial v} r+m \nu\left(\sqrt{E}+\sqrt{E_{1}}\right), \frac{\partial q}{\partial v}=\frac{1}{\sqrt{E}} \frac{\partial \sqrt{G}}{\partial u} r \\
\frac{\partial r}{\partial u}=\frac{1}{\sqrt{G}} \frac{\partial \sqrt{E}}{\partial v} q, \frac{\partial r}{\partial v}=\frac{V \bar{G}}{\varrho_{2}} p-\frac{1}{\sqrt{E}} \frac{\partial \sqrt{G}}{\partial u} q+m \nu\left(\sqrt{G}+\sqrt{G_{1}}\right), \\
\frac{\partial \nu}{\partial u}=\sqrt{E_{1}} \frac{\nu q}{\theta}, \quad \frac{\partial \nu}{\partial v}=\sqrt{G_{1}} \frac{\nu r}{\theta} \\
\frac{\partial \theta_{10}}{\partial u}=-\sqrt{E_{1}}\left[\frac{q}{\theta}\left(\theta_{10}-\theta_{0}\right)+\frac{1}{\sqrt{E}} \frac{\partial \theta_{0}}{\partial u}\right] \\
\frac{\partial \theta_{10}}{\partial v}=-\sqrt{G_{1}}\left[\frac{r}{\theta}\left(\theta_{10}-\theta_{0}\right)+\frac{1}{\sqrt{G}} \frac{\partial \theta_{0}}{\partial v}\right] .
\end{array}\right.
$$

When we have a set of solutions $\theta, \nu, p, q, r, \theta_{10}$ satisfying the quadratic relation

$$
p^{2}+q^{2}+r^{2}-2 m \nu \theta=0,
$$

the $R$ transform $N_{1}$ is a net $\Omega$, and the coordinates of $N_{1}$ are given by equations of the form (48). The same functions multiplied by the same constant determine the same transformation. Hence in addition to $m$ there are four significant constants of integration, and consequently:

$A$ net $\Omega$ admits $\infty^{5}$ transformations $R$ into nets $\left.\Omega^{75}\right)$.

From (II, 86) (91) and (95) we find that the function $\varrho_{10}$ for $N_{10}$ has the expression

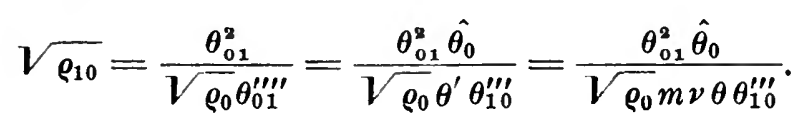

91. Theorem of permutability of the transformations of nets $\Omega$. Let $N$ be a net $\Omega$, and $N_{1}$ and $N_{2}$ two $R$ transforms of $N$ by means of functions $\theta_{i}, p_{i}, q_{i}, r_{i}, \nu_{i}, \theta_{i o}, m_{i}(i=1,2)$, solutions

${ }^{75)}$ Cf., Transactions of the Amer. Math. Soc., vol. 16 (1915), pp. 275-310. 
of (97) and (98). We wish to show that of the $\infty^{1} O$ nets $N_{12}$, which are $R$ transforms of $N_{1}$ and $N_{2}$ in accordance with $\S 72$, one is a net $\Omega$.

Consider the nets $N_{10}$ and $N_{20}$ which are $K$ transforms of $N_{0}$ by means of the pairs of functions $\theta_{01}, w_{01}$ and $\theta_{02}, w_{02}$. From $\S 24$ we have that there is a net $N_{120}$ which is an $F$ transform of each of the nets $N_{12}, N_{10}$ and $N_{20}$, and the functions $\theta_{10}^{\prime}$ and $\theta_{20}^{\prime}$ of the respective transformations from $N_{10}$ and $N_{20}$ are given by

$$
\begin{aligned}
& \theta_{0} \theta_{01} w_{01} \theta_{01}^{\prime}=w_{1}\left(\theta_{0} \theta_{01} \theta_{12}+\theta_{1} \theta_{10} \theta_{02}-\theta_{2} \theta_{10} \theta_{01}\right) \\
& \theta_{0} \theta_{02} w_{02} \theta_{02}^{\prime}=w_{2}\left(\theta_{0} \theta_{02} \theta_{21}+\theta_{2} \theta_{20} \theta_{01}-\theta_{1} \theta_{20} \theta_{02}\right)
\end{aligned}
$$

Moreover, from (II, 91) it follows that $N_{120}$ will be a $K$ transform of $N_{10}$ and $N_{20}$, and consequently have equal point invariants, if

$$
m_{1} \theta_{02}^{\prime} w_{02}+m_{2} \theta_{01}^{\prime} w_{01}=0 .
$$

Substituting the above values in this equation, we reduce the resulting equation to

$$
\left\{\begin{array}{c}
m_{2} \theta_{2}^{\prime}+m_{1} \theta_{1}^{\prime \prime}=\frac{m_{1} m_{\Xi}}{\theta_{0}}\left\{\nu_{1}\left[\frac{\theta_{1} \theta_{10} \theta_{02}}{\theta_{01}}-\theta_{2}\left(\theta_{10}-\theta_{0}\right)\right]\right. \\
+\nu_{2}\left[\frac{\theta_{2} \theta_{20} \theta_{01}}{\theta_{02}}-\theta_{1}\left(\theta_{20}-\theta_{0}\right)\right] .
\end{array}\right.
$$

If this equation is differentiated with respect to $u$ and $v$, we find that the resulting equations are satisfied identically.

Since $\theta_{2}^{\prime}$ and $\theta_{1}^{\prime \prime}$ are completely determined by (50) and (100), only one of the nets $N_{12}$ is an $\Omega$ net. Hence we have the theorem:

If $N_{1}$ and $N_{2}$ are $\Omega$ nets which are $R$ transforms of an $\Omega$ net $N$, there exists a unique $\Omega$ net $N_{12}$ which is an $R$ transform of $N_{1}$ and $N_{2}$; moreover, $N_{12}$ can be found directly, as soon as the coordinates of $N_{1}$ and $N_{2}$ are known.

92. Surfaces of Guichard of the first kind. In order that the $O$ net $N$ on a surface $S$ be defined by

$$
\begin{cases}\sqrt{E}=e^{\xi} \sinh \alpha, & \sqrt{G}=e^{\xi} \cosh \alpha, \\ \frac{1}{\varrho_{1}}=e^{-\xi}(\operatorname{coth} \alpha+h), & \frac{1}{\varrho_{2}}=e^{-\xi}(\tanh \alpha+h),\end{cases}
$$


it is necessary and sufficient that $h, \xi$ and $\alpha$ satisfy the equations

$$
\left\{\begin{array}{c}
\frac{\partial h}{\partial u}=(\operatorname{coth} \alpha+h) \frac{\partial \xi}{\partial u}, \quad \frac{\partial h}{\partial v}=(\tanh \alpha+h) \frac{\partial \xi}{\partial v} \\
\frac{\partial^{2} \xi}{\partial u \partial v}=\frac{\partial \xi}{\partial u} \frac{\partial \xi}{\partial v}+\operatorname{coth} \alpha \frac{\partial \xi}{\partial u} \frac{\partial \alpha}{\partial v}+\tanh \alpha \frac{\partial \xi}{\partial v} \frac{\partial \alpha}{\partial u} \\
\frac{\partial^{2} \alpha}{\partial u^{2}}+\frac{\partial^{2} \alpha}{\partial v^{2}}+\operatorname{coth} \alpha \frac{\partial^{2} \xi}{\partial u^{2}}+\tanh \alpha \frac{\partial^{2} \xi}{\partial v^{2}}-\operatorname{csch}^{2} \alpha \frac{\partial \alpha}{\partial u} \frac{\partial \xi}{\partial u} \\
+\operatorname{sech}^{2} \alpha \frac{\partial \alpha}{\partial v} \frac{\partial \xi}{\partial v}+(\cosh \alpha+h \sinh \alpha)(\sinh \alpha+h \cosh \alpha)=0
\end{array}\right.
$$

as follows from the Codazzi equations (86) and the Gauss equation [\$ 64]. These equations are satisfied also by $\bar{\xi}, \bar{\alpha}, h$, where

$$
e^{\bar{\xi}}=e^{-\xi}\left(1-h^{2}\right), \quad e^{\bar{\alpha}}=e^{-\alpha} \frac{1-h}{1+h} .
$$

An $O$ net $\vec{N}$ parallel to $N$ is defined by

$$
\begin{cases}V \overline{\bar{E}}=e^{\bar{\xi}} \sinh \bar{\alpha}, & V \overline{\bar{G}}=-e^{\bar{\xi}} \cosh \bar{\alpha}, \\ \frac{1}{\overline{\varrho_{1}}}=e^{-\bar{\xi}}(\operatorname{coth} \bar{\alpha}+h), & \frac{1}{\overline{\varrho_{2}}}=e^{-\bar{\xi}}(\tanh \bar{\alpha}+h) .\end{cases}
$$

By means of (101) and (104) we have

$$
\varrho_{1} \overline{\varrho_{2}}+\varrho_{2} \overline{\varrho_{1}}=-2 \text {. }
$$

Following Calapso ${ }^{76}$ ) we call $S$ a surface of Guichard of the first kind, and the parallel surface $\bar{S}$ its associate (cf. VII, Ex. 28).

When the expressions for $\sqrt{E}, \sqrt{G}, \varrho_{1}$ and $\varrho_{2}$ from (101) are substituted in (88), this condition is satisfied, if we take $U=V=1$. Hence we have the theorem of Demoulin ${ }^{77}$ ):

The surfaces of Guichard of the first kind are surfaces $\Omega$. From (87) and (85) we have

$$
\theta_{0}=e^{\xi}, \quad \hat{\theta}_{0}=1-h, \quad \sqrt{\varrho_{0}}=\frac{e^{\xi+\alpha}}{h-1} .
$$

$\left.{ }^{76}\right)$ Annali, ser. 3, vol. 11 (1905), p. 211.

77) L. c. 
We apply the results of $\S 90$ to establish transformations $R$ of surfaces of Guichard of the first kind. Analogously to (105) we have

$$
\theta_{10}=e^{\xi_{1}}, . \quad \theta_{10}^{\prime \prime \prime}=1-h_{1} .
$$

From (95) it follows that

$$
h_{1}+\frac{p}{\theta} e^{\xi_{1}}=h+\frac{p}{\theta} e^{\xi}=t
$$

where $t$ is thus defined. Now $\theta_{01}=\theta-e^{\xi} p /(1-h)$, and consequently (96) are reducible to

$$
\left\{\begin{array}{l}
\sqrt{E_{1}}=\frac{e^{\xi_{1}} \varphi}{1-t}+\frac{\theta}{\nu}(1-t) e^{-(\xi+a)}, \\
\sqrt{G_{1}}=\frac{e^{\xi_{1}} \psi}{1-t}-\frac{\theta}{\nu}(1-t) e^{-(\xi+a)},
\end{array}\right.
$$

where

$$
\varphi=\cosh \alpha+t \sinh \alpha, \quad \psi=\sinh \alpha+t \cosh \alpha .
$$

Since $\varphi^{2}-\psi^{2}=1-t^{2}$, when we require that $G_{1}-E_{1}=e^{2 \xi_{1}}$, we get

$$
e^{\xi_{1}}=\frac{\theta}{\nu} e^{-\xi}\left(t^{2}-1\right)
$$

If we put $\sqrt{\varrho_{10}}=e^{\xi_{1}+a_{1} / m}\left(h_{1}-1\right)$ in (99), we obtain

Hence we find

$$
e^{a_{1}}=e^{-a} \frac{t-1}{t+1}
$$

$$
V \overline{E_{1}}=e^{\xi_{1}} \sinh \alpha_{1}, \quad V \overline{G_{1}}=-e^{\xi_{1}} \cosh \alpha_{1} .
$$

Furthermore, equations (9) are satisfied by

$\frac{\sqrt{E_{1}}}{\varrho_{11}}=-\left(\cosh \alpha_{1}+h_{1} \sinh \alpha_{1}\right), \quad \frac{\sqrt{G_{1}}}{\varrho_{12}}=\sinh \alpha_{1}+h_{1} \cosh \alpha_{1}$. 
Hence the surface $S_{1}$ is a surface of Guichard of the first kind. By means of (108) the expressions (107) are reducible to

$$
V \overline{E_{1}}=-\frac{\theta}{\nu} e^{-\xi}(\varphi t+\psi), \quad \sqrt{G_{1}}=-\frac{\theta}{\nu} e^{-\xi}(\psi t+\varphi) .
$$

Consequently the functions determining a transformation $R$ of a surface of Guichard of the first kind into surface of this type are solutions of the completely integrable system:

(110)

$$
\left\{\begin{array}{c}
\frac{\partial \theta}{\partial u}=e^{\xi} \sinh \alpha q, \quad \frac{\partial \theta}{\partial v}=e^{\xi} \cosh \alpha r \\
\frac{\partial p}{\partial u}=-(\cosh \alpha+h \sinh \alpha) q, \quad \frac{\partial p}{\partial v}=-(\sinh \alpha+h \cosh \alpha) r \\
\frac{\partial q}{\partial u}=(\cosh \alpha+h \sinh \alpha) p-\left(\tanh \alpha \frac{\partial \xi}{\partial v}+\frac{\partial \alpha}{\partial v}\right) \cdot r \\
+m\left[-\theta e^{-\xi}(\varphi t+\psi)+\nu e^{\xi} \sinh \alpha\right] \\
\frac{\partial q}{\partial v}=\left(\operatorname{coth} \alpha \frac{\partial \xi}{\partial u}+\frac{\partial \alpha}{\partial u}\right) r, \quad \frac{\partial r}{\partial u}=\left(\tanh \alpha \frac{\partial \xi}{\partial v}+\frac{\partial \alpha}{\partial v}\right) q \\
\frac{\partial r}{\partial v}=(\sinh \alpha+h \cosh \alpha) p-\left(\operatorname{coth} \alpha \frac{\partial \xi}{\partial u}+\frac{\partial \alpha}{\partial u}\right) \cdot q \\
+m\left[-\theta e^{-\xi}(\psi t+\varphi)+\nu e^{\xi} \cosh \alpha\right] \\
\frac{\partial \nu}{\partial u}=-e^{-\xi}(\varphi t+\psi) q, \quad \frac{\partial \nu}{\partial v}=-e^{-\xi}(\psi t+\varphi) r .
\end{array}\right.
$$

The equations of the transformation are of the form (48) in these functions. Accordingly we have the theorem ${ }^{78}$ ):

$A$ surface of Guichard of the first kind admits $\infty^{3}$ transformations $R_{m}$, for each value of $m \neq 0$, into surfaces of the same kind.

When $S$ is transformed into $S_{1}$, the associate surface $\bar{S}$ is transformed into a surface $\overline{S_{1}}$, parallel to $S_{1}$, by means of the functions $p, q, r, \bar{\theta}$ and $\bar{\nu}$, where in consequence of (49) $\bar{\theta} \bar{\nu}=\theta \nu$. The equations in $\bar{\theta}$ are

$$
\frac{\partial \bar{\theta}}{\partial \imath}=e^{\bar{\xi}} \sinh \bar{\alpha} q, \quad \frac{\partial \bar{\theta}}{\partial v}=-e^{\bar{\xi}} \cosh \bar{\alpha} r
$$

78) Annali, ser. 3, vol. 22 (1914), p. 205; also Transactions of Amer. Math. Soc. vol. 17 (1916), p. 68 . 
It is readily found that

$$
\bar{\theta}=\nu-\frac{p^{2}}{\theta}, \quad \bar{\nu}=\frac{\nu \theta^{2}}{\nu \theta-p^{2}} .
$$

The associate of $S_{1}$ is determined by equations analogous to (103), namely

$$
\begin{gathered}
e^{\bar{\xi}_{1}}=e^{-\xi_{1}}\left(1-h_{1}^{2}\right)=-\frac{\nu}{\theta} e^{\xi}+\frac{2 p t}{\theta}+\frac{p^{2}}{\theta \nu} e^{-\xi}\left(1-t^{2}\right), \\
e^{\bar{\alpha}_{1}=e^{-\alpha_{1}} \frac{1-h_{1}}{1+h_{1}}}=e^{\alpha} \frac{t+1}{t-1} \frac{1-t+\frac{p}{\theta} e^{\xi_{1}}}{1+t-\frac{p}{\theta} e^{\xi_{1}}}, \\
\bar{h}_{1}=h_{1} .
\end{gathered}
$$

If this associate is to be the $R$ transform of $\bar{S}$ by means of $\bar{\theta}, \bar{\nu}, p, q, r$, then analogously to (106), (108) and (109) we must have

$$
\begin{gathered}
h_{1}+\frac{p}{\bar{\theta}} e^{\overline{\xi_{1}}}=h+\frac{p}{\bar{\theta}} e^{\bar{\xi}}=\bar{t} \\
e^{\overline{\xi_{1}}}=\frac{\bar{\theta}}{\bar{\nu}} e^{-\bar{\xi}}\left(\overline{t^{2}}-1\right), . \quad e^{\bar{\alpha}_{1}}=e^{-\bar{\alpha}} \frac{\bar{t}-1}{\bar{t}+1} .
\end{gathered}
$$

It is readily shown that these expressions are equivalent to the preceding. Hence:

When a surface of Guichard of the first kind is transformed into a surface of the same kind, the associate surfaces are in relation $R^{79}$ ).

\section{Exercises.}

1. If $N$ is a net $2, O$ the complementary function being $y$, the lines of curvature on the sheets of the envelope of the spheres of radius $y$ and centers on $N$ are represented on the unit sphere by the central projections on this sphere of the two special nets $2, O$ parallel to $N$; the coordinates of the spherical representations are $x^{\prime} / y^{\prime}$ and $x^{\prime \prime} / y^{\prime \prime}$.

2. Corresponding tangent planes to two pairs of $O$ nets in relation $R$ whose central nets are parallel are parallel.

3. A necessary and sufficient condition that the spheres, tangent to a surface $S$ and with centers at the mid-points of the segments of the normals to $S$

79) Annali, 1. c. p. 209. 
included between the centers of principal curvature of $S$, determine an $R$ trausform of $S$ is that the lines of curvature of $S$ have isothermic spherical representation (cf. $§ 6$ and I, Ex. 8).

4. A necessary and sufficient condition that the spheres tangent to a surface $S$ with centers at points harmonic to the points on $S$ with respect to the centers of principal curvature determine an $R$ transform of $S$ is that $S$ be an isothermic surface (cf. $\S 25$ ).

Darboux, Annales Norm., ser. 3, vol. 16 (1899) p. 504.

5. Show that the first three theorems of $\S 81$ are equally true for the transformations $R$ of nets $O$ in $n$-space.

6. For a system of circles $K$ of the second kind (\$ 81) the focal points of the congruence $K$ are harmonic to the foci of the meridian sections of the hyperboloid of revolution whose generators are the normals to the uets $N_{c}$ and $N_{12}$.

Demoulin, Comptes Rendus, vol. 150 (1910), p. 159.

7. If $N$ is an isothermic $O$ net, the point equation of $N$ admits the solution $\theta_{0}=e^{2 \psi}\left(\frac{1}{\rho_{1}}-\frac{1}{\rho_{2}}\right)$, and the corresponding parallel $O$ nets $N_{0}\left(x_{0}\right)$ are defined by equations of the form

$$
x_{0}=\left(\frac{1}{2} \theta_{0} K_{0}+c\right) X+e^{\phi}\left(\frac{\partial K_{0}}{\partial u} \xi-\frac{\partial K_{0}}{\partial v} \eta\right),
$$

where $c$ is an arbitrary constant and $K_{0}=\frac{1}{\rho_{1}}+\frac{1}{\rho_{2}}$.

8. If $N$ is an isothermic $O$ net defined by (37), the Christoffel transform $\bar{N}$ [§ 159] is defined by

$$
\sqrt{\bar{E}}=-V \overline{\bar{G}}=e^{-\psi}, \quad \bar{\rho}_{1}=\rho_{1} e^{-2 \phi}, \quad \bar{\rho}_{2}=-\rho_{2} e^{-2 \vartheta} .
$$

Show that if $N_{1}$ is a $D_{m}$ transform of $N$, a $D_{m}$ transform $\bar{N}_{1}$ of $\bar{N}$ is given by

$$
\bar{p}=p, \quad \bar{q}=q, \quad \bar{r}=r, \quad \bar{\theta}=\nu, \quad \bar{\nu}=\theta, \quad \bar{m}=m,
$$

and that $\bar{N}_{1}$ is the Christoffel transforn of $N_{1}$. Also if $M, M_{1}$ and $\bar{M}, \bar{M}_{1}$ denote corresponding poiuts on these nets, the product of the segments $M M_{1}$ and $\bar{M} \overline{M_{1}}$ is equal to $2 / m$.

Biancli, l. c., p. 105.

9. If $M, M_{1}, M_{2}, M_{12}$ are corresponding points of four nets satisfying the theorem of permutability of transformations $D_{m}(\S 83)$, the cross-ratio $\left(M M_{12}\right.$, $M_{1} M_{2}$ ) is equal to $m_{2} / m_{1}$.

Demoulin, l. c., p. 157.

10. Show that the $O$ net of a minimal surface may be considered of class $(0,0,0,0)(\S 84)$ and obtain the results of $\S 87$ from those of $\S 86$.

Bianchi, l. c., p. 149.

11. From [§ 125] we have for a surface of mean curvature $K_{0}=1$,

$$
\frac{1}{\rho_{1}}=e^{-\psi} \sinh \psi, \quad \frac{1}{\rho_{2}}=e^{-\psi} \cosh \psi .
$$

In this case from (47) it follows that $\theta-\nu+2 p=a$, where $a$ is a constant. Show that when $a=0$, the mean curvature of $N_{1}$ is -1 . 
12. Show that the $O$ net of a surface of mean curvature $K_{0}=1$ may be considered a special isothermic net of class $\left(-\frac{1}{2}, 0,0, \frac{1}{4}\right)$, and apply the results of $\S 86$ to obtain those of Ex. 11 .

Bianchi, l. c., p. 151.

13. The Christoffel transform of a special isothermic surface of class $(A, B$, $C, D)$ is a special surface of class $(A,-C,-B, D)$. Bianchi, l. c., p. 131 .

14. If from a special isothermic net $N$ of class $(A, B, C, D)$, we obtain two nets of $N_{1}, N_{2}$, of class $(A,-B,-C, D)$ by transformations $D_{m_{1}}$ and $D_{m_{k}}$, the surface $N_{12}$ is of class $(A, B, C, D)$.

Bianchi, l. c., p. 146.

15. Determine the special isothermic surfaces for which the circle-planes of the complementary transformations pass through a point.

16. Show that the nets $N^{\prime}$ of transformations $D_{m}$ of isothermic nets and of transformations $E_{m}$ of $O$ nets with isothermal spherical representation have the property

$$
E^{\prime}-G^{\prime}=2 m\left(x^{\prime 2}+y^{\prime 2}+z^{\prime 2}\right) \text {. }
$$

Calapso, Annali, ser. 3, vol. 26 (1917), p. 168.

17. If $N_{\mathrm{l}}$ is a $D_{m}$ transform of an isothermic $O$ net $N$, an isothermic $O$ net $\bar{N}$ is defined by

$$
\sqrt{\bar{E}}=\sqrt{\bar{G}}=\frac{e^{\phi}}{\theta}, \quad \frac{1}{\bar{\rho}_{1}}=p+\frac{\theta}{\rho_{1}}, \quad \frac{1}{\overline{\rho_{2}}}=p+\frac{\theta}{\rho_{2}} .
$$

Bianchi, Annali, ser. 3, vol. 12 (1906) p. 22.

18. If $N$ and $N_{1}$ are two $O$ nets in relation $E_{m}$, the minimal surfaces $\boldsymbol{\Sigma}$ and $\boldsymbol{\Sigma}_{1}$, whose asymptotic lines have the same spherical representation as the curves of $N$ and $N_{1}$ respectively, can be so placed in space that they are the focal surfaces of a $W$ congruence $(\$ 53)$.

19. When two $O$ nets $N$ and $N_{1}$ are in relation $E_{m}$, the spherical representation of these nets are isothermal orthogonal nets on the unit sphere in relation $F$, and these two nets are related conformally. The equations of the transformation are of the form

$$
X_{1}=-X+\frac{p}{m \theta \nu}(p X+q \xi+r \eta)
$$

where $\theta, \nu, p, q$ and $r$ are solutions of (75).

20. A necessary and sufficient condition that the spherical representations of two $O$ nets in relation $R$ be conformal is that the transformation be $E_{m}$.

Annals, Ser. 2, vol. 17 (1915) p. 69:

21. If four nets $N, N_{1}, N_{2}, N_{12}$, form a quatern under transformations $E_{m}$, and $N_{3}$ is an $E_{m_{3}}$ transform, then the nets $N_{3}, N_{13}, N_{23}, N^{\prime}$ of $\S 24$ form a similar quatern.

Transactions, vol. 9 (1908), p. 170.

22. If $N$ is an $O$ net with isothermal spherical representation, the point equation of $N$ admits the solution $\theta_{0}=E-G$, and the corresponding parallel $O$ nets $N_{0}$ are defined by equations of the form

$x_{0}=\left[2 e^{-\psi}(\sqrt{E}+\sqrt{G})+c\right] X+\frac{1}{\sqrt{E}}\left(\frac{\partial E}{\partial u}-\frac{\partial G}{\partial u}\right) \xi+\frac{1}{\sqrt{G}}\left(\frac{\partial E}{\partial v}-\frac{\partial G}{\partial v}\right) \eta$,

where $c$ is a constant. 
23. If in (88) we put $\sqrt{E}=\sqrt{G,} U=-V=1$, this condition is satisfied, that is isothermic surfaces are surfaces $\Omega$. In this case

$$
\theta_{0}=e^{2 \psi}\left(\frac{1}{\rho_{2}}-\frac{1}{\rho_{1}}\right), \quad \hat{\theta}_{0}=-\theta_{0} \frac{\rho_{1}+\rho_{2}}{2 \rho_{1} \rho_{2}} .
$$

In order that the transformations deternined by $(97)$ be $D_{m}$, we must have

$$
e^{\psi} \theta_{10} \nu \sqrt{\rho_{0}}+\theta_{0} \theta_{01}=0 .
$$

24. Determine the character of the surfaces $\Omega$ which are $R$ transforms of an isothermic surface other than $D_{m}$ transforms.

25. If in (88) we put

$$
\sqrt{E}=-\rho_{1} e^{-t}, \quad V \bar{G}=\rho_{2} e^{-\psi}, \quad U=V=1,
$$

the equation is satisfied in consequence of (86); that is a surfase with isothermal representation of its lines of curvature is a surface $\Omega$. In this case $2 \theta_{0}=-\hat{\theta}_{0}\left(\rho_{1}+\rho_{2}\right)$; consequently the mid-point of the segment between centers of principal curvature of the surface describes a net $N_{0}$ with equal point invariants; also $N_{\omega}$ is at infinity. When we express the condition $\sqrt{E_{1}} / \rho_{11}=\sqrt{G_{1}} / \rho_{12}$ we get

$$
2 e^{-\psi} \frac{\theta}{p}+(\sqrt{G}-\sqrt{E})\left(1-\frac{\theta_{10}}{\theta_{0}}\right)+2 \frac{\theta_{01}}{\nu \sqrt{\rho_{0}}}=0 .
$$

By means of this relation the expressions (74) are obtainable from (96), and these transformations $R_{m}$ are $E_{m}$.

26. Determine the character of the surfaces $\Omega$ which are $R$ transforms of a surface with isothermal spherical representation of its lines of curvature other than the $\boldsymbol{E}_{m}$ transforns.

27. If $S_{1}$ and $S_{2}$ are surfaces of Guichard of the first kind obtained from such a surface $S$ by transformation $R_{m_{1}}$ and $R_{m_{2}}$, there can be found without quadratures a surface $S_{12}$ of the same kind which is in relations $R_{m_{2}}$ and $R_{m_{1}}$ with $S_{1}$ and $S_{2}$.

Annali, ser. 3, vol. 22 (1914), p. 212.

28. In order that the $O$ net $N$ on a surface $S$ be defined by

$$
\sqrt{E}=e^{\xi} \sin \alpha, \sqrt{G}=e^{\xi} \cos \alpha, \frac{1}{\rho_{1}}=e^{-\xi}(\cot \alpha+h), \frac{1}{\rho_{2}}=e^{-\xi}(-\tan \alpha+h),
$$

it is necessary and sufficient that $h, \xi$ and $\alpha$ satisfy

$$
\begin{gathered}
\frac{\partial h}{\partial u}=(h+\cot \alpha) \frac{\partial \xi}{\partial u}, \quad \frac{\partial h}{\partial v}=(h-\tan \alpha) \frac{\partial \xi}{\partial v}, \\
\frac{\partial^{2} \xi}{\partial u \partial v}=\frac{\partial \xi}{\partial u} \frac{\partial \xi}{\partial v}+\cot \alpha \frac{\partial \alpha}{\partial v} \frac{\partial \xi}{\partial u}-\tan \alpha \frac{\partial \alpha}{\partial u} \frac{\partial \xi}{\partial v}, \\
\frac{\partial^{2} \alpha}{\partial u^{2}}-\frac{\partial^{2} \alpha}{\partial v^{2}}+\cot a \frac{\partial^{2} \xi}{\partial u^{2}}+\tan \alpha \frac{\partial^{2} \xi}{\partial v^{2}}-\csc ^{2} \alpha \frac{\partial \alpha}{\partial u} \frac{\partial \xi}{\partial u}+\sec ^{2} \alpha \frac{\partial a}{\partial v} \frac{\partial \xi}{\partial v} \\
-(\cos \alpha+h \sin \alpha)(\sin \alpha-h \cos \alpha)=0 .
\end{gathered}
$$


These equations are satisfied by $\bar{\xi}, \bar{a}$ and $h$ defined by

$$
\begin{aligned}
e^{\bar{\xi}} & =e^{-\xi}\left(1+h^{2}\right), \\
\sin \bar{a} & =-\frac{1}{1+h^{2}}\left[\sin a\left(1-h^{2}\right)-2 h \cos a\right], \\
\cos \bar{a} & =\frac{1}{1+h^{2}}\left[\cos a\left(1-h^{2}\right)+2 h \sin a\right] .
\end{aligned}
$$

An $O$ net $\bar{N}$ parallel to $N$ is defined by

$$
\begin{array}{rlrl}
\sqrt{\bar{E}} & =e^{\bar{\xi}} \sin \bar{a}, & \sqrt{\bar{G}}=-e^{\bar{\xi}} \cos \bar{\alpha}, \\
\frac{1}{\bar{\rho}_{1}}=e^{-\bar{\xi}}(\cot \bar{a}+h), & \frac{1}{\bar{\rho}_{2}}=e^{-\bar{\xi}}(-\tan \bar{a}+h)
\end{array}
$$

and $\rho_{1} \overline{\rho_{2}}+\rho_{2} \overline{\rho_{1}}=-2$. The surface $S$ is called a surface of Guichard of the second kind, and $\bar{S}$ its associate. Calapso, Annali, ser. 3, vol. 11 (1905), p. 216.

29. Show that a surface of Guichard of the second kind is a surface $\boldsymbol{Q}$ and determine the transformations $R$ of such a surface into surfaces of the same kind. Annali, ser. 3, vol. 22 (1914), p. 205.

30. A necessary and sufficient condition that a surface of Guichard of the first kind be a spherical surface $[\$ 115]$ is that $e^{\xi}=a, h=0$, where $a$ is a constant; then $\rho_{1}=a \tanh a, \rho_{2}=a \operatorname{coth} a$. In this case the associate surface is homothetic to the given one.

31. If $S$ is a spherical surface (cf. Ex. 30), equations (106), (108) and (110) are consistent when $\nu=\left(a^{2} p^{2}-\theta^{2}\right) / \theta a^{2}$. Thus a spherical surface admits $\infty^{2}$ transformations $R_{m}$, for each value of $m \neq 0$, into spherical surfaces.

Annali, l. c. p. 230.

32. For the circle-plane of a trausformation $R_{m}$ of a spherical surface (Ex. 31), the functions $j, k$ and $\bar{\omega}$ of $\S 79$ have the values $j=a^{2} / 2 m \theta, \quad k=-p a^{2} / \theta$, $\bar{\omega}=\left(\theta^{2}-p^{2} a^{2}\right) / 2 \theta$. Consequently the planes of these circles envelope a surface applicable to the quadric of revolution $x^{2}+2 m\left(y^{2}+z^{2}\right)=a^{2}$.

Annali, 1. c. p. 235.

33. If $S$ is a surface of Guichard of the first kind and $S_{1}$ is an $R_{m}$ transform of the same kind, a surface of Guichard of the first kind is defined by

$$
\begin{aligned}
& \sqrt{\bar{E}}=\frac{e^{\xi}}{\theta} \sinh a, \quad \sqrt{\bar{G}}=\frac{e^{\xi}}{\theta} \cosh \alpha, \\
& \frac{1}{\overline{\rho_{1}}}=\theta e^{-\xi}(1-2 m)\left(\operatorname{coth} \alpha+h+e^{\xi} \frac{p}{\theta}\right) \\
& \frac{1}{\overline{\rho_{2}}}=\theta e^{-\xi}(1-2 m)\left(-\tanh \alpha+h+e^{\xi} \frac{p}{\theta}\right) . \\
& \text { Calapso, Annali, ser. 3, vol. } 29(1920), \text { p. } 84 .
\end{aligned}
$$


Chapter VIII.

\section{Circles and spheres.}

93. Coordinates of a sphere. The equation of a sphere in 3-space can be written in the form

$$
\begin{gathered}
2 \alpha_{1} y_{1}+2 \alpha_{2} y_{2}+2 \alpha_{3} y_{3}+i \alpha_{1}\left(y_{3}^{2}+y_{2}^{2}+y_{3}^{2}+1\right) \\
+\alpha_{5}\left(y_{1}^{2}+y_{2}^{2}+y_{3}^{2}-1\right)=0
\end{gathered}
$$

where $\alpha_{1}, \ldots, \alpha_{5}$ are constants, and $y_{1}, y_{2}, y_{3}$ are current coordinates. We call the five constants $\alpha$ the coordinates of the sphere. If $x_{1}, x_{2}, x_{3}$ are the coordinates of the center of the sphere and $R$ its radius, we have

(2) $\left\{\begin{array}{c}\alpha_{1}=h x_{1}, \quad \alpha_{2}=h x_{2}, \quad \alpha_{3}=h x_{3}, \\ \alpha_{4}=\frac{i h}{2}\left(x_{1}^{2}+x_{2}^{2}+x_{3}^{2}-R^{2}+1\right), \quad \alpha_{5}=\frac{h}{2}\left(x_{1}^{2}+x_{2}^{2}+x_{3}^{2}-R^{2}-1\right),\end{array}\right.$

where $h$ is a factor of proportionality. These equations can be written also thus

(3) $\left\{\begin{array}{c}x_{1}=-\frac{\alpha_{1}}{\alpha_{5}+i \alpha_{4}}, \quad x_{2}=-\frac{\alpha_{2}}{\alpha_{5}+i \omega_{4}}, \quad x_{3}=-\frac{\alpha_{3}}{\alpha_{5}+i \alpha_{4}}, \\ x_{1}^{2}+x_{2}^{2}+x_{3}^{2}-R^{2}=-\frac{\alpha_{5}-i \alpha_{4}}{\alpha_{5}+i \alpha_{4}}, \quad h=-\left(\alpha_{5}+i \alpha_{4}\right) .\end{array}\right.$

An exception arises when

$$
\alpha_{5}+i \alpha_{4}=0,
$$

which is a necessary and sufficient condition that (1) defines a plane. From (3) we have

$$
R=\frac{\sqrt{\sum \alpha^{2}}}{\alpha_{5}+i \alpha_{4}},
$$


where we assume the sign of the radical chosen so that $R$ is positive for a real sphere. It follows that a necessary and sufficient condition that a sphere defined by (1) be a point-, or null-sphere, is

$$
\alpha_{1}^{2}+\alpha_{3}^{2}+\alpha_{3}^{2}+\alpha_{4}^{2}+\alpha_{5}^{2}=0 .
$$

By definition the power of a point with respect to a sphere is the product of the distances to any two points of the sphere collinear with it, that is the square of the tangent to the sphere when the point is outside. Hence the left-hand member of (1) divided by $i \alpha_{4}+\alpha_{5}$ is the power of the point $\left(y_{1}, y_{2}, y_{3}\right)$ with respect to the sphere (1).

Consider the sphere (1) and another of center $\left(x_{1}^{1}, x_{9}^{1}, x_{8}^{1}\right)$ and radius $R^{\prime}$ defined by

$$
\left\{\begin{array}{c}
2 \alpha_{1}^{1} y_{1}+2 \alpha_{2}^{1} y_{2}+2 \alpha_{3}^{1} y_{3}+i \alpha_{4}^{1}\left(y_{1}^{2}+y_{8}^{2}+y_{3}^{2}+1\right) \\
+\alpha_{5}^{1}\left(y_{1}^{2}+y_{2}^{2}+y_{3}^{2}-1\right)=0
\end{array}\right.
$$

The angle under which they cut is given by

$$
\cos \theta=\frac{R^{2}+R^{\prime 2}-\sum_{i=1}^{3}\left(x_{i}-x_{i}^{\prime}\right)^{2}}{2 R R^{\prime}} .
$$

With the aid of (3) and (5) one shows that this is expressible in terms of the coordinates $\alpha$ and $\alpha^{\prime}$ of the two spheres in the form

$$
\cos \theta=\frac{\sum_{i=1}^{5} \alpha_{i} \alpha_{i}^{\prime}}{\left(\sum \alpha_{i}^{2} \cdot \sum \alpha_{i}^{2 \prime}\right)^{\frac{1}{2}}}
$$

In certain discussions it is advantageous to introduce a sixth coordinate $\alpha_{6}$ defined by

$$
i \alpha_{6}=R\left(\alpha_{5}+i \alpha_{4}\right)
$$

- Comparing this equation with (5), we note that

$$
\alpha_{1}^{2}+\ldots+\alpha_{6}^{2}=0
$$


Now equation (9) becomes

$$
\cos \theta=-\frac{\sum_{i=1}^{5} \alpha_{i} \alpha_{i}^{\prime}}{\alpha_{6} \alpha_{6}^{\prime}}
$$

94. Pentaspherical coordinates of a point. When the sphere (1) is not a null-sphere, its coordinates $\alpha$ may be chosen so that $\sum \alpha^{2}=1$. These coordinates $\alpha$ are direction-cosines of a line in 5-space and from (9) it follows that the angle between two spheres in 3-space is equal to the angle between two lines in 5 -space whose direction-cosines are the corresponding $\alpha$ 's and $\alpha^{\prime \prime}$ s. Accordingly we can choose a set of five mutually orthogonal spheres in 3 -space whose coordinates $\alpha_{i j}$ satisfy the conditions

$$
\sum_{i=1}^{5} \alpha_{i j}^{2}=1, \quad \sum_{i=1}^{5} \alpha_{i j} \alpha_{i k}=0\left(\begin{array}{c}
j, k=1 \ldots, 5 \\
j \neq k
\end{array}\right) .
$$

With this choice we consider the functions

(14) $2 \lambda z_{j}=2 \sum_{i=1}^{8} \alpha_{i j} y_{i}+i \alpha_{4 j}\left(y_{1}^{2}+y_{2}^{2}+y_{3}^{2}+1\right)+\alpha_{5 j}\left(y_{1}^{2}+y_{2}^{2}+y_{3}^{2}-1\right)$.

The right-hand member is the power of the point $\left(y_{1}, y_{2}, y_{3}\right)$ with respect to the sphere $S_{j}$, divided by its radius $R_{j}=1 /\left(\alpha_{5 j}+i \alpha_{4 j}\right)$. When

$$
i \alpha_{4 j}+\alpha_{5 j}=0,
$$

the sphere $S_{j}$ is a plane and $\lambda z_{j}$ is the distance from the point $\left(y_{1}, y_{2}, y_{3}\right)$ to the plane.

The quantities $z_{j}(j=1, \ldots 5)$ are called the pentaspherical coordinates of the point, $\lambda$ being a parameter. In consequence of (13) we have the fundamental relation

$$
\sum_{j=1}^{5} z_{j}^{2}=0 .
$$

From equations (14) we have

$$
\left\{\begin{array}{c}
y_{1}=\lambda \sum_{j=1}^{5} \alpha_{1 j} z_{j}, \quad y_{2}=\lambda \sum_{j=1}^{5} \alpha_{2 j} z_{j}, \quad y_{3}=\lambda \sum_{j=1}^{5} \alpha_{3 j} z_{j} \\
1+\lambda \sum_{j=1}^{5}\left(\alpha_{5 j}+i \alpha_{4 j}\right) z_{j}=0, \quad y_{2}^{2}+y_{2}^{2}+y_{3}^{2}=\lambda \sum_{j=1}^{5}\left(\alpha_{5 j}-i \alpha_{4 j}\right) z_{j} .
\end{array}\right.
$$


Hence when five quantities satisfying (16) are given, they are the pentaspherical coordinates of a point, whose cartesian coordinates are given by $(17)^{80}$ ).

As an immediate consequence of these results, we have:

The equation of a general sphere is of the form

$$
\alpha_{1} z_{1}+\alpha_{2} z_{2}+\ldots+\alpha_{5} z_{5}=0
$$

where $z_{i}$ are the pentaspherical coordinates of a point on the sphere, and the $\alpha$ 's are constants.

If $z_{j}$ and $z_{j}^{\prime}$ are the pentaspherical coordinates of two points $M$ and $M^{\prime}$, whose respective cartesian coordinates are $y_{i}$ and $y_{i}^{\prime}$ the distance between them is given by

$$
\overline{M M^{\prime}}=\sum_{i=1}^{3}\left(y_{i}-y_{i}^{\prime}\right)^{2}=-2 \lambda \lambda^{\prime} \sum_{j=1}^{5} z_{j} z_{j}^{\prime}=\lambda \lambda^{\prime} \sum_{j=1}^{5}\left(z_{j}-z_{j}^{\prime}\right)^{2},
$$

in consequence of (17), where, because of (5) and (14)

$$
1+\lambda \sum_{j=1}^{5} \frac{z_{j}}{R_{j}}=0, \quad 1+\lambda^{\prime} \sum_{j=1}^{5} \frac{z_{j}^{\prime}}{R_{j}}=0
$$

$R_{j}$ being the radius of the sphere of coordinates $\alpha_{j}$.

If $M^{\prime}$ approaches $M$ along a curve, the linear element is given by

$$
d s^{2}=\frac{\sum_{j=1}^{5}\left(d z_{j}\right)^{2}}{\left(\sum_{j=1}^{5} \frac{z_{j}}{R_{j}}\right)^{2}}=\frac{\sum_{j=1}^{5}\left(d z_{j}\right)^{2}}{\left[\sum_{j=1}^{5} z_{j}\left(\alpha_{5 j}+i \alpha_{4 j}\right)\right]^{2}}
$$

When in (17) we take $a_{i i}=1(i=1, \ldots 5), a_{i j}=0(i \neq j)$, these equations reduce to

$$
\left\{\begin{array}{c}
y_{1}=\lambda z_{1}, \quad y_{2}=\lambda z_{2}, \quad y_{3}=\lambda z_{3} \\
1+\lambda\left(z_{5}+i z_{4}\right)=0, \quad y_{1}^{2}+y_{2}^{2}+y_{8}^{2}=\lambda\left(z_{5}-i z_{4}\right)
\end{array}\right.
$$

80) An exception is afforded by $z_{j}=a_{5 j}+i a_{4 j}$ in which case the point is at infinity (cf. VIII, Ex. 1). 
From (4) it follows that the fundamental spheres of coordinates $\alpha_{i 1}, \alpha_{i 2}, \alpha_{i 3}$ are planes. In this case we say that the $z$ 's are special pentaspherical coordinates. For this case equation (20) reduces to

$$
d s^{2}=\frac{\sum\left(d z_{j}\right)^{2}}{\left(z_{5}+i z_{4}\right)^{2}} .
$$

95. Pentaspherical coordinates of an $O$ net. If $y_{1}, y_{2}, y_{3}$ are the cartesian coordinates of a point on a surface referred to its lines of curvature, the functions $\lambda z_{j}$ satisfy the point equation of the surface, as follows from (14); and consequently, if $\lambda$ involves $u$ and $v$, the $z$ 's satisfy an equation of the form

$$
\frac{\partial^{2} \theta}{\partial u \partial v}=\frac{\partial \log a}{\partial v} \frac{\partial \theta}{\partial u}+\frac{\partial \log b}{\partial u} \frac{\partial \theta}{\partial v}+c \theta .
$$

Conversely, if we have five solutions of this equation satisfying (16), say $z_{j}$, and an independent solution $1 / \lambda$, then the functions $z_{j}^{\prime}$, where $z_{j} \lambda=z_{j}^{\prime}$, satisfy an equation of this form with $c=0$, and consequently $y_{1}, y_{2}, y_{3}, y_{1}^{2}+y_{2}^{2}+y_{3}^{\mathrm{a}}, 1$, given by (17) or (21), are solutions of the latter equation. Hence:

If five particular solutions of an equation of the form (23) satisfy the relation

$$
z_{1}^{2}+z_{2}^{2}+\ldots+z_{5}^{2}=0,
$$

they are the pentaspherical coordinates of a surface referred to its lines of curvature.

If the invariants of (23) are equal, the $O$ net is isothermic. In this case the equation is reducible to the Moutard form

$$
\frac{\partial^{2} \theta}{\partial u \partial v}=M \theta
$$

Hence:

If an equation of the Moutard form admits five particular. solutions $z_{j}$ satisfying the relations $\sum z_{j}^{2}=0$, the quantities are the pentaspherical coordinates of an isothermic net.

96. Congruences of spheres. When the quantities $\alpha$ in (1) are functions of two parameters $u_{1}$ and $v_{1}$, we say that (1) defines 
a congruence of spheres. When the $\alpha$ 's are independent, we can find by differentiation alone an equation of the form

$$
D \frac{\partial^{2} \theta}{\partial u_{1}^{2}}+2 E \frac{\partial^{2} \theta}{\partial u_{1} \partial v_{1}}+F \frac{\partial^{2} \theta}{\partial v_{1}^{2}}+G \frac{\partial \theta}{\partial u_{1}}+H \frac{\partial \theta}{\partial v_{1}}+K \theta=0,
$$

of which the five functions $\alpha$ are solutions. If we take as independent variables $u$ and $v$, defined by

$$
u=\varphi\left(u_{1}, v_{1}\right), \quad v=\psi\left(u_{1}, v_{1}\right)
$$

such that $\varphi$ and $\psi$ satisfy the differential equation

$$
D\left(\frac{\partial \varphi}{\partial u_{1}}\right)^{2}+2 E \frac{\partial \varphi}{\partial u_{1}} \frac{\partial \varphi}{\partial v_{1}}+F\left(\frac{\partial \varphi}{\partial v_{1}}\right)^{2}=0,
$$

the above equation is transformed into the Laplace form

$$
\frac{\partial^{2} \theta}{\partial u \partial v}=\frac{\partial \log A}{\partial v} \frac{\partial \theta}{\partial u}+\frac{\partial \log B}{\partial u} \frac{\partial \theta}{\partial v}+C \theta .
$$

The determination of $u$ and $v$ requires the integration of the differential equation

$$
F d u_{1}^{2}-2 E d u_{1} d v_{1}+D d v_{1}^{2}=0 .
$$

The curves on the envelope of the spheres uniquely defined by this equation are called the principal curves.

We assume hereafter that the parameters of a congruence of spheres are such that the spherical coordinates $\alpha$ satisfy an equation of the Laplace form (26). Then the principal curves on the envelope are parametric. The equation of the congruence is of the form

$$
\sum_{i=1}^{5} \alpha_{i} z_{i}=0
$$

We consider the congruence of spheres defined by (28). As $u$ varies a sphere touches a tubular envelope along the circle $C_{-1}$, given by

$$
\sum \alpha_{i} z_{i}=0, \quad \sum \frac{\partial \alpha_{i}}{\partial u} z_{i}=0,
$$


and as $v$ varies, along the circle $C_{1}$ given by

$$
\sum \alpha_{i} z_{i}=0, \quad \sum \frac{\partial \alpha_{i}}{\partial v} z_{i}=0 .
$$

The intersections $A_{1}$ and $A_{2}$ of these circles are the points of tangency of the sphere (28) with the envelope of the congruence of spheres, that is, the points defined by

$$
\sum \alpha_{i} z_{i}=0, \quad \sum \frac{\partial \alpha_{i}}{\partial u} z_{i}=0, \quad \sum \frac{\partial \alpha_{i}}{\partial v} z_{i}=0 .
$$

The circles (29) and (30) are called the focal circles of the first and second rank respectively of the congruence of spheres.

Differentiating the first two of (31) with respect to $v$ and making use of the third, we get

$$
\sum \epsilon_{i} \frac{\partial z_{i}}{\partial v}=0, \quad \sum \frac{\partial \alpha_{i}}{\partial u} \frac{\partial z_{i}}{\partial v}=0 .
$$

Hence as $v$ varies the points $A_{1}$ and $A_{2}$ begin to move along the circle $C_{-1}$. Similarly as $u$ varies, these points begin to move along $C_{1}$. But as $u$ and $v$ vary, the points $A_{1}$ and $A_{2}$ trace out the principal curves on the envelope of the sphere of coordinates $\alpha$. Hence we have the theorem of Darboux ${ }^{81}$ ):

On the envelope of a congruence of spheres, when a displacement is made along one of the principal curves, the four points of contact of two infinitely near spheres with the envelope lie on a circle which is a focal circle of the congruence.

We have also from the above that the line $A_{1} A_{2}$ and the line $A_{1}^{\prime} A_{2}^{\prime}$, joining nearby points, meet in the plane of $C_{-1}$ or $C_{1}$ as $v$ or $u$ varies. Hence these planes are the focal planes of the congruence of lines $A_{1} A_{2}$. Accordingly in view of the preceding theorem we have the theorem of Ribaucour:

The chords of contact of the spheres of a congruence with its envelope generate a congruence whose focal planes are the planes of the circles of contact $u=$ const., $v=$ const., and the developables of the congruence are parametric.

${ }^{81)}$ Leçons, $2^{\text {nd }}$ ed., vol. 2, p. 335. 
97. Derived congruences of spheres. Since the five functions $\alpha$ satisfy equation (26), they are the direction-parameters

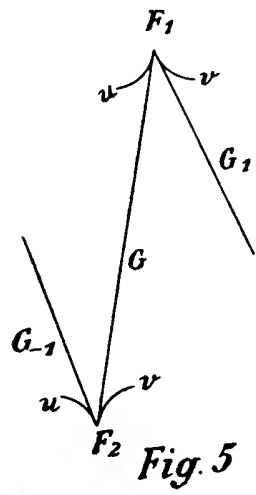
of a congruence $G$ of lines in 5-space. Conversely, the direction-parameters of such a congruence determine a congruence of spheres in 3 -space. From $(I, 60)$ it follows that the directionparameters of the congruence $G_{1}$ and $G_{-1}$ of tangents to the curves of parameter $v$ on the first focal surface of $G$ and of parameter $u$ on the second focal surface may be given the respective forms

$$
\alpha_{i} \frac{\partial}{\partial v} \log \frac{\alpha_{i}}{A}, \quad \alpha_{i} \frac{\partial}{\partial \imath} \log \frac{\alpha_{i}}{B}
$$

These congruences $G_{1}$ and $G_{-1}$ in 5-space correspond to the congruences of spheres in 3-space with the respective equations

$$
\sum \alpha_{i} \frac{\partial}{\partial v} \log \frac{\alpha_{i}}{A} \cdot z_{i}=0, \quad \sum \alpha_{i} \frac{\partial}{\partial u} \log \frac{\alpha_{i}}{B} \cdot z_{i}=0
$$

Hence we speak of these congruences of spheres as the first and minus first derived congruences of the congruence defined by (28).

Evidently the circles (29) are the intersections of corresponding spheres of the given congruence and its minus first derived congruence, and similarly the circles (30) of spheres of the given congruence and its first derived congruence.

Consider now the congruence of spheres defined by the first of (33). As $u$ varies, a sphere describes a tubular surface, to which the sphere is tangent along the circle defined by (33) and

$$
\sum \frac{\partial}{\partial u}\left(\alpha_{i} \frac{\partial}{\partial v} \log \frac{\alpha_{i}}{A}\right) \cdot z_{i}=0
$$

which is readily seen to be the circle (30). In like manner for the congruence of spheres defined by the second of (33) as $v$ varies a sphere describes a tubular surface to which the sphere is tangent along the circle (29). 


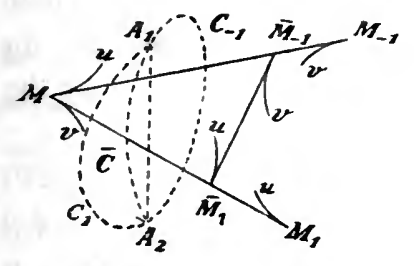

Fig. 6

From (3) it follows that $\alpha_{1}, \alpha_{2}, \alpha_{3}$, $-\left(\alpha_{5}+i \alpha_{4}\right)$ are homogeneous coordinates of the center $M$ of the sphere. Since they satisfy equation (26), $M$ describes a net $N$. When we compare (32) with (III, 2) we remark that the centers $M_{1}$ and $M_{-1}$ of these spheres describe nets which are the first and minus first Laplace transforms of $N$. Since the lines $M M_{-1}$ and $M M_{1}$ are necessarily the axes of the circles (29) and (30) respectively, we have the theorem:

If $G$ is a congruence of spheres, and $G_{-1}$ and $G_{1}$ its minus first and first derived congricnces, as $u$ or $v$ varies a sphere describes a tubular surface tangent to the sphere along a circle which is the intersection of the sphere and the corresponding sphere of the minus first or first derived congruence respectively. The centers $M, M_{-1}$ and $M_{1}$ of corresponding spheres describe nets in 3-space forming a Laplace sequence, and the joins $M M_{-1}$ and $M M_{1}$ are the axes of the circles of intersection.

Consider further the circle (29). We saw in $\S 96$ that its plane is the focal plane of the congruence $A_{1} A_{2}$ tangent to the second focal surface of this congruence. Hence on the envelope of the plane the parametric curves form a net $\bar{N}$. This circle lies also on the corresponding sphere given by the second of (33). If $B_{1}$ and $B_{2}$ are the points where this sphere touches its envelope, this plane is a focal plane of the congruence of lines $B_{1} B_{2}$ and $\bar{N}$ is the first focal net of the congruence. Hence:

The planes of the focal circles of either rank of a congruence of spheres envelope a net whose tangents are the chords of contact of the spheres and the corresponding derived congruence of spheres with their envelopes.

98. Congruences of circles. By definition the circles of intersection of corresponding spheres of two congruences of spheres determined by the congruences of tangents to a net in 5-space form a congmience of circles, for which the spheres are the focal spheres. The centers of the spheres are the focal points of the congruence of axes of the circles $(\S 97)$. Moreover, as we have just seen, the planes of the circles envelop a net, whose tangents 
meet the circles in the points where the focal spheres touch their envelopes. Equations (29) define a congruence of circles, as do also equations (30). Moreover, any congruence of circles may be so defined.

When for the congruences of circles $C_{-1}$ as defined by (29) the parameter $v$ varies, the circles $C_{-1}(u, v)$ and $C_{-1}(u, v+d v)$ meet in the points, $A_{1}$ and $A_{2}$, where the sphere of parameter $\alpha$ tonches its envelope; they are defined by (31). When the parameter $u$ varies, the circles $C_{-1}(u, v)$ and $C_{-1}(u+d u, v)$ meet in the points, $A_{1}^{\prime}, A_{2}^{\prime}$, of intersection of the spheres of parameters

$$
\alpha_{i}, \frac{\partial \alpha_{i}}{\partial u}, \frac{\partial^{2} \alpha_{i}}{\partial u^{2}}
$$

Hence as $u$ and $v$ varies the circle $C_{-1}$ meets the infinitely near circle in two points.

Consider, conversely, a two parameter family of circles such that as either parameter, $u$ or $v$, varies, two infinitely near circles meet in two points. Hence as $u$ or $v$ varies the axes of the circles generate developables. Let $F_{1}$ and $F_{2}$ be the focal points of this congruence. The spheres $S_{1}$ with centers $F_{1}$ and containing the circles of the system envelope, as $u$ varies, a tubular surface tangent along the circles. If the equation of the spheres $S_{1}$ is $\sum \beta_{i} z_{i}=0$, the circles are defined by this equation and $\sum \frac{\partial \beta_{i}}{\partial u} z_{i}=0$.

In like manner, the spheres $S_{2}$ with centers $F_{2}$ and passing through the circles envelop, as $v$ varies, a tubular surface to which they are tangent along the circles. Thus the circles are defined also by

$$
\sum \boldsymbol{\alpha}_{i} z_{i}=0, \quad \sum \frac{\partial \alpha_{i}}{\partial v} z_{i}=0
$$

where the first equation defines the spheres $S_{2}$.

Since the circles are the same in both cases, we must have

$$
\frac{\partial \boldsymbol{\beta}_{i}}{\partial u}=l \boldsymbol{\alpha}_{i}+m \boldsymbol{\beta}_{i}, \quad \frac{\partial \boldsymbol{\alpha}_{i}}{\partial v}=n \boldsymbol{\alpha}_{i}+r \boldsymbol{\beta}_{i} .
$$

But from $\S 2$ it follows that the $\alpha$ 's and $\beta$ 's are direction-parameters of a net in 5-space. Combining this result with the second theorem of the preceding section, we have: 
A congruence of circles is characterized by the property that as $u$ or $v$ varies a circle meets the infinitely near circle in two points, which are the points of intersection of the circle and the tangents to the net enveloped by the plane of the circle.

We call these points the focal points of the congruence of circles.

99. Congruences of spheres and circles in cartesian coordinates. From (3) it follows that the cartesian coordinates $x_{1}, x_{2}, x_{3}$ of the center of the sphere, and also the function

$$
2 \theta=x_{1}^{2}+x_{2}^{2}+x_{3}^{2}-R^{2}
$$

are solutions of the equation

$$
\frac{\partial^{2} \theta}{\partial u \partial v}=\frac{\partial \log a}{\partial v} \frac{\partial \theta}{\partial u}+\frac{\partial \log b}{\partial u} \frac{\partial \theta}{\partial v},
$$

where as follows from (3) and (26)

$$
a=\frac{A}{h}, \quad b=\frac{B}{h} .
$$

Conversely, if $x_{1}, x_{2}, x_{3}$ and $\theta$ are four solutions of an equation (36), it follows that the quantities $\alpha$ defined by (2) are solutions of an equation of the form (26). Hence:

If $\theta$ is a solution of the point equation of a net $N$, the spheres with centers on $N$ and radii given by

$$
R^{2}=x_{1}^{2}+x_{2}^{2}+x_{3}^{2}-2 \theta
$$

form a congruence upon whose envelope the parametric curves are the principal curves.

As a corollary we have:

The points of an $O$ net in 3-space form a congruence of null spheres.

When the equation of a congruence of spheres is written in the form

$$
\sum(y-x)^{2}-R^{2}=0,
$$


the $y$ 's being current coordinates, the equations of the focal circles $C_{-1}$ and $C_{1}$ of the congruence are given by (39) and the respective equations

$$
\sum(y-x) \frac{\partial x}{\partial u}+R \frac{\partial R}{\partial u}=0, \quad \sum(y-x) \frac{\partial x}{\partial v}+R \frac{\partial R}{\partial v}=0,
$$

which shows that the axes of the circles are tangent to the curves of the net of centers. Since equations (39) and (40) are equivalent to (31), we have:

Equations (40) define the focal planes of the congmence of lines joining corresponding points on the envelope of the spheres (39), the developables being parametric.

By means of (38) equations (40) may be written

$$
\sum y \frac{\partial x}{\partial u}-\frac{\partial \theta}{\partial u}=0, \quad \sum y \frac{\partial x}{\partial v}-\frac{\partial \theta}{\partial v}=0 .
$$

If we write

$$
\theta_{-1}=\theta-\frac{b}{\frac{\partial b}{\partial u}} \frac{\partial \theta}{\partial u}, \quad \theta_{1}=\theta-\frac{a}{\frac{\partial a}{\partial v}} \frac{\partial \theta}{\partial v},
$$

in consequence of $(I, 48)$, the preceding equations are equivalent to

$$
\sum y \frac{\partial x_{-1}}{\partial v}-\frac{\partial \theta_{-1}}{\partial v}=0, \quad \sum y \frac{\partial x_{1}}{\partial u}-\frac{\partial \theta_{1}}{\partial u}=0 .
$$

Hence:

If $\theta$ is a solution of the point equation of a net $N$, the spheres with centers on the minus first and first Laplace transforms of $N$ and with radii given by the respective equations

$$
R_{-1}^{2}=\sum x_{-1}^{2}-2 \theta_{-1}, \quad R_{1}^{2}=\sum x_{1}^{2}-2 \theta_{1}
$$

meet the spheres with centers on $N$ and radius given by (38) in congruences of circles.

When the central net $N(x)$ of a congruence of spheres is an $O$ net, the congruence of lines joining the corresponding points of contact on the envelopes of the spheres is normal, since the focal 
planes are perpendicular. A surface $\bar{N}$ normal to the congruence is given by equations of the form [§67]

$$
\bar{x}=W X+\frac{\partial W}{\partial u} \frac{1}{\mathfrak{E}} \frac{\partial X}{\partial u}+\frac{\partial W}{\partial v} \frac{1}{\mathbb{B}} \frac{\partial X}{\partial v},
$$

where the $X$ 's are the direction-cosines of the congruence and also of the normal to the given net $N$. The function $W$ is to be determined by the condition that the point of $\bar{N}$ lies on the line whose equations are (40). Making use of (41), we find that this condition reduces to

$$
-\varrho_{1} \frac{\partial W}{\partial u}=\frac{\partial \theta}{\partial u}, \quad-\varrho_{2} \frac{\partial W}{\partial v}=\frac{\partial \theta}{\partial v},
$$

where $\varrho_{1}$ and $\varrho_{2}$ are the principal radii of curvature of $N$. Hence $W$ is found by a quadrature.

Conversely, if a net $\bar{N}$ parallel to $N$ is known, we find by the quadrature (42) the radius of the corresponding sphere. Hence we have the following theorem of Ribaucour ${ }^{82}$ ):

If $S$ and $\bar{S}$ are two surfaces with the same spherical representation of their lines of curvature, the normals of either are the chords of contact with their envelope of a family of spheres having their centers on the other surface; the radii of the spheres are found by a quadrature.

Ioo. Congruences $R$ of spheres. We are in a position now to establish the theorem:

If the principal curves form a net on each of the sheets of the envelope of a congruence of spheres, they are $O$ nets in relation $R$, provided that the radii of the spheres are not constant.

If the quantities $y$ in (39) are the coordinates of the point of contact of the spheres with one sheet of the envelope, we have

$$
\sum(y-x) \frac{\partial y}{\partial u}=0, \quad \sum(y-x) \frac{\partial y}{\partial v}=0 .
$$

If equation (41) be differentiated with respect to $v$ and $u$, respectively we obtain

$$
\sum \frac{\partial y}{\partial v} \frac{\partial x}{\partial u}=0, \quad \sum \frac{\partial y}{\partial u} \frac{\partial x}{\partial v}=0 .
$$

82) Comptes Rendus, vol. 67 (1868), p. 1334. 
Differentiating the first of (43) with respect to $v$ or the second with respect to $u$, we get

$$
\sum(y-x) \frac{\partial^{2} y}{\partial u \partial v}+\sum \frac{\partial y}{\partial u} \frac{\partial y}{\partial v}=0 .
$$

Hence if the $y$ 's are to satisfy an equation of the form (36), we must have $\sum \frac{\partial y}{\partial u} \frac{\partial y}{\partial v}=0$, which is the condition that the net $\bar{N}(y)$ be an $O$ net. In consequence of this result the central net is $2, O$, and by the last theorem of $\S 66$ and the second theorem of $\S 76$, the theorem is established.

By definition a congruence of spheres is a congruence $R$ if the central net $N_{0}$ is 2,O and the complementary function of $N_{0}$ is the radius of a representative sphere.

From $\S 68$ we have:

The spheres of a transformation $R$ form a congruence $R$.

When two congruences $R$ of spheres have parallel central nets we say that the congruences of spheres are parallel. Hence from the last theorem of $\S \mathbf{7 6}$ we have:

If $G$ and $G^{\prime}$ are parallel congruences $R$ of spheres and the spheres $G^{\prime}$ do not pass through a point, there can be found by a quadrature a congruence $R$ of spheres $G_{1}$ such that the central nets of $G$ and $G_{1}$ are in relation $F$.

In this sense the congruences $G$ and $G_{1}$ are in relation $F$.

The spheres with centers on any surface $S$ and passing through a point form a congruence $R$, the central net $2, O$ on $S$ being determined by the equation of the lines of curvature of the envelope of the spheres; it is the net on $S$ determined by the function $\sqrt{x^{2}+y^{2}+z^{2}}(\S 3)$. In like manner the spheres with centers on a surface $S$ and tangent to a plane $\pi$, or a fixed sphere, form a congruence $R$, the central $2, O$ net on $S$ corresponding to the lines of curvature on the other sheet of the envelope.

The spheres tangent to two fixed spheres form a congruence $R$ with centers on a central quadric of revolution, whose foci are the centers of the fixed spheres. When one of the fixed spheres is replaced by a plane, the surface of centers is a paraboloid of revolution. 
If the centers of the fixed spheres are the points $(a, 0,0)$ and $(-a, 0,0)$ and the radii are $r_{1}$ and $r_{2}$, we have

$(x-a)^{2}+y^{2}+z^{2}=\left(R+\varepsilon_{1} r_{1}\right)^{2}, \quad(x+a)^{2}+y^{2}+z^{2}=\left(R+\varepsilon_{2} r_{2}\right)^{2}$,

where $\varepsilon_{1}= \pm 1$ and $\varepsilon_{2}= \pm 1$. From these equations it follows that

$$
x^{2}+y^{2}+z^{2}-R^{2}=b x+c, \quad R=d x+e,
$$

where $b, c, d$ and $e$ are constants. Hence these two functions are solutions of the point equation of any net on the quadric. Similar results hold for a paraboloid of revolution. Consequently:

On a quadric of revolution any net is $2, O$.

Conversely, if every net on a surface $S$ is $2, O$, the functions $x^{2}+y^{2}+z^{2}-R^{2}$ and $R$ must be linear functions of the coordinates. Consequently :

If every net on a surface is 2,0 , the surface is a quadric of revolution.

ror. Plücker line coordinates. If $x_{1}, x_{2}, x_{3}, x_{4}$ and $y_{1}, y_{2}$, $y_{3}, y_{4}$ are the homogeneous point coordinates of two points $P$ and $Q$, the numbers $p_{12}, p_{13}, p_{14}, p_{23}, p_{34}, p_{42}$ defined by

$$
p_{i j}=\left|\begin{array}{cc}
x_{i} & x_{j} \\
y_{i} & y_{j}
\end{array}\right|(i, j=1,2,3,4, i \neq j)
$$

are called the Plicker coordinates of the line $P Q$. Evidently the ratios only of these coordinates are so determined. Since these ratios are unaltered, if $x_{1}, x_{2}, x_{3}, x_{4}$, are replaced by $\lambda x_{1}+\mu y_{1}$, $\ldots ., \lambda x_{4}+\mu y_{1}$, any two points of the line determine the same set of line coordinates. The six coordinates satisfy the equation

$$
p_{12} p_{34}+p_{13} p_{42}+p_{14} p_{93}=0,
$$

as may be seen by expanding in terms of two-rowed minors the identity

$$
\left|\begin{array}{llll}
x_{1} & x_{2} & x_{3} & x_{4} \\
y_{1} & y_{2} & y_{3} & y_{4} \\
x_{1} & x_{2} & x_{3} & x_{4} \\
y_{1} & y_{2} & y_{3} & y_{4}
\end{array}\right|=0 .
$$


Conversely, if we have any six numbers $p_{i j}$ satisfying (45), we can find two points, $\left(x_{1}, x_{2}, x_{3}, 0\right)$ and $\left(y_{1}, 0, y_{3}, y_{4}\right)$ satisfying (44), and consequently there is a line with these coordinates.

Consider two lines $p$ and $p^{\prime}$ meeting in the point $\left(x_{i}\right)$ and determined by $Q\left(y_{i}\right)$ and $Q^{\prime}\left(y_{i}^{\prime}\right)$. Since

we have

$$
\left|\begin{array}{llll}
x_{1} & x_{2} & x_{3} & x_{4} \\
y_{1} & y_{2} & y_{3} & y_{4} \\
x_{1} & x_{2} & x_{3} & x_{4} \\
y_{1}^{\prime} & y_{2}^{\prime} & y_{3}^{\prime} & y_{4}^{\prime}
\end{array}\right|=0
$$

\section{$p_{12} p_{34}^{\prime}+p_{13} p_{42}^{\prime}+p_{14} p_{23}^{\prime}+p_{34} p_{12}^{\prime}+p_{42} p_{13}^{\prime}+p_{23} p_{14}^{\prime}=0$,}

where $y_{i j}^{\prime}=\left(x_{i} y_{j}^{\prime}-x_{j} y_{i}^{\prime}\right)$. Conversely, if two sets of line coordinates satisfy this condition, any two points on one line are coplanar with two points on the other. The line coordinates of the line joining $P$ and the point $\left(\lambda y_{i}+\mu y_{i}^{\prime}\right)$ are of the form $\lambda p_{i j}+\mu p_{i j}^{i}$. Hence:

A flat pencil of lines consists of the lines whose coordinates are $\lambda p_{i j}+\mu p_{i j}^{\prime}$, if $p$ and $p^{\prime}$ are lines of the pencil.

Consider the lines of space whose coordinates satisfy a linear relation

$$
a_{34} p_{12}+a_{42} p_{13}+a_{23} p_{14}+a_{12} p_{34}+a_{13} p_{42}+a_{14} p_{28}=0
$$

where the $a$ 's are constants. If $\left(b_{1}, b_{2}, b_{3}, b_{4}\right)$ is any point of space, the coordinates $\left(x_{1}, x_{2}, x_{3}, x_{4}\right)$ of any point of the lines through the given point for which (47) holds, satisfy a linear equation whose coefficients involve the $a$ 's and the $b$ 's. Hence all the lines through the given point lie in a plane. Consequently the lines satisfying (47) form a linear complex. The plane in which all the lines of the complex through a point lie is called the null-plane of the point.

When the $a$ 's in (47) satisfy (45), they are the coordinates of a line which all the lines of the complex meet. In this case the complex is called special. 
102. The Lie line-sphere transformation. Consider the relation between two 3 -spaces $r$ and $R$ whose cartesian coordinates $x_{i}$ and $X_{i}$ are in the relations

$$
\left\{\begin{array}{c}
\left(X_{1}+i X_{2}\right)+X_{3} x_{1}+x_{3}=0 \\
\left.\left(X_{1}-i X_{2}\right) x_{1}-x_{2}-X_{3}=0^{83}\right)
\end{array}\right.
$$

To each point of either space corresponds a line of the other. If $\left(x_{i}\right)$ and $\left(x_{i}^{\prime}\right)$ are two points of a line of $r$ corresponding to a point $\left(X_{i}\right)$ of $R$, we find that equations (48) and similar equations in $x_{i}^{\prime}$ are consistent, provided that

$$
p_{12}-p_{34}=0
$$

here $p_{i j}=x_{i} x_{j}^{\prime}-x_{j} x_{i}^{\prime}$ and $x_{4}=x_{i}^{\prime}=1$. Hence to the points of $R$ correspond lines of the linear complex (49), which is called the complex of the transformation. If we have any line of the complex and substitute the coordinates of any two of its points in (48), we find the corresponding point in $R$.

In like manner the coordinates $X_{i}$ and $X_{i}^{\prime}$ of two points in $R$ whose line corresponds to a point in $r$, satisfy the condition

$$
\sum\left(X_{i}-X_{i}^{\prime}\right)^{2}=0,
$$

that is to a point in $r$ corresponds a minimal line in $R$.

To the points of a line $a$ of the complex (49) correspond minimal lines in $R$ passing through the point $A$ which corresponds to $a$. Also to the points of a minimal line $M$ in $R$ correspond the lines of the complex through the point of $r$ corresponding to $M$. Moreover, since the transformation is continuous, to the tangents of a minimal curve in $R$ correspond the points in $r$ of a curve whose tangents are lines of the complex (49).

A general line in $r$ is defined by

$$
\left\{\begin{array}{l}
x_{1} p_{34}+x_{3} p_{41}+p_{13}=0 \\
x_{2} p_{34}+x_{3} p_{42}+p_{23}=0
\end{array}\right.
$$

83) Lie, Geometrie der Berührungstransformationen, p. 453 et seq. 
Eliminating the $x$ 's from these equations and (48), we get

where

$$
\begin{gathered}
2 \alpha_{1} X_{1}+2 \alpha_{2} X_{2}+2 \alpha_{3} X_{3}+i \alpha_{4}\left(X_{1}^{2}+X_{2}^{2}+X_{3}^{2}+1\right) \\
+\alpha_{5}\left(X_{1}^{3}+X_{2}^{2}+X_{3}^{2}-1\right)=0
\end{gathered}
$$

$$
\left\{\begin{array}{lll}
p_{12}=\alpha_{3}+i \alpha_{6}, & p_{13}=\alpha_{1}+i \alpha_{2}, & p_{14}=\alpha_{5}+i \alpha_{4} \\
p_{34}=\alpha_{3}-i \alpha_{6}, & p_{42}=\alpha_{1}-i \alpha_{2}, & p_{23}=\alpha_{5}-i \alpha_{4}
\end{array}\right.
$$

Since the $p$ 's satisfy (45), the $\alpha$ 's satisfy (11), and consequently:

To a general line in $r$ correspond a sphere in $R$.

From these results and (10) it follows that to a line of complex (49) of the transformation corresponds a null-sphere in $R$.

From (46) and (52) it follows that to two intersecting lines of coordinates $p_{i j}$ and $p_{i j}^{\prime}$ correspond two spheres $S$ and $S^{\prime}$ whose coordinates $\alpha$ and $\alpha^{\prime}$ satisfy the condition

$$
\alpha_{1} \alpha_{1}^{\prime}+\alpha_{2} \alpha_{2}^{\prime}+\ldots+\alpha_{6} \alpha_{6}^{\prime}=0,
$$

that is, as follows from (12), the spheres are tangent.

From (52) it follows that the line of coordinates $p_{i j}^{\prime}$, given by

$$
\left\{\begin{array}{lll}
p_{12}^{\prime}=\alpha_{3}-i \alpha_{6}, & p_{13}^{\prime}=p_{13}, & p_{14}^{\prime}=p_{14}, \\
p_{34}^{\prime}=\alpha_{3}+i \alpha_{6}, & p_{42}^{\prime}=p_{42}, & p_{23}^{\prime}=p_{23},
\end{array}\right.
$$

corresponds to the sphere (51). The equations of this line are

$$
\left\{\begin{array}{l}
x_{1} p_{12}+x_{3} p_{41}+p_{13}=0 \\
x_{2} p_{12}+x_{3} p_{42}+p_{23}=0
\end{array}\right.
$$

The lines of the complex (49) meeting the line (50) form a congruence. The line coordinates $\bar{p}_{i j}$ of a line of this congruence satisfy (49) and

$$
p_{34} \bar{p}_{12}+p_{42} \bar{p}_{13}+p_{23} \bar{p}_{14}+p_{12} \bar{p}_{34}+p_{13} \bar{p}_{42}+p_{14} \bar{p}_{28}=0 .
$$

It is readily seen that all of these lines meet the line (55). Hence the lines (50) and (55) are the directrices of the congruence. We refer to two such lines as conjugate with respect to the complex (49). Hence: 
To a sphere in $R$ corresponds two lines in $r$ conjugate with respect to the complex of the transformation.

From the foregoing discussion it follows that to the points on the conjugate lines correspond the minimal lines of the sphere and to the lines of the complex meeting the conjugate lines correspond the points of the sphere.

Consider two intersecting lines in $r$ of coordinates $p_{i j}$ and $p_{i j}^{\prime}$, not of the complex (49), and the two tangent spheres corresponding to them. The homogeneous point coordinates of the centers, $C$ and $C^{\prime}$, of the spheres are $\left(\alpha_{1}, \alpha_{2}, \alpha_{3},-\alpha_{5}-i \alpha_{4}\right)$ and $\left(\alpha_{1}^{\prime}, \alpha_{2}^{\prime}, \alpha_{3}^{\prime}\right.$, $\left.-\alpha_{5}^{\prime}-i \alpha_{4}^{\prime}\right)$, and the point of tangency of the spheres is the intersection of the line $C C^{\prime}$ with either sphere. To a line of coordinates $i p_{i j}+\mu p_{i j}^{\prime}$ corresponds a sphere of coordinates $\lambda \alpha_{i}+\mu \alpha_{i}^{\prime}$. Hence:

To a flat pencil of lines in $r$ corresponds a pencil of spheres tangent to one another at the same point; moreover, the centers of the spheres form a linear pencil of points projective with the given pencil of lines.

From (4) it follows that the sphere of the pencil which is the tangent plane to all the spheres of the pencil is determined by $\lambda / \mu=-\left(\alpha_{5}^{\prime}+i \alpha_{4}^{\prime}\right) /\left(\alpha_{5}+i \alpha_{4}\right)$.

Suppose that this is the sphere of coordinates $\alpha$. From (52) and (54) it follows that to a plane in $R$ corresponds in $r$ two lines of the special complex $p_{14}=0$ conjugate with respect to the complex (49). All the lines of this special complex meet the line $l$ at infinity in the plane $x_{1}=0$, that is the line $x_{1}=0, x_{4}=0$; this line is in the complex (49). Hence:

To a plane in $R$ corresponds in $r$ two lines of the special complex $p_{14}=0$ conjugate with respect to the complex of the transformation, and to the points of these lines the two pencils of minimal lines in the given plane.

Let $g$ and $g^{\prime}$ be the conjugate lines corresponding to a sphere $S$. A line $a$ of the complex (49) meeting $g$ and $g^{\prime}$ corresponds to a point $A$ of $S$, and to the minimal generators of $S$ through $A$ correspond the points $m$ and $m^{\prime}$ where $a$ meets $g$ and $g^{\prime}$. Since $g$ and $g^{\prime}$ are conjugate with respect to the complex, all the lines of the complex through $m^{\prime}$ meet $g$ : that is, $m^{\prime}$ and $g$ determine the null-plane of $m^{\prime}$. In like manner $m$ and $g^{\prime}$ determine the nullplane of $m$. 
If $S_{1}$ is a sphere tangent to $S$ at $A$, the conjugate lines $g_{1}$ and $g_{1}^{\prime}$ corresponding to $S_{1}$ pass through $m$ and $m^{\prime}$ respectively. Hence:

To all the spheres tangent to $S$ at $A$ correspond a pencil of lines through $m$ lying in the null-plane of $m^{\prime}$ and a pencil of lines through $m^{\prime}$ lying in the null-plane of $m$.

ro3. The Lie transformations of surfaces. A point and a plane through it constitute a surface element of which the point is the center. We consider the surface elements consisting of the points of a surface $\mathbf{S}$ in $R$ and the corresponding tangent planes. By the Lie transformation the minimal curves of $\Sigma$ correspond to. two one parameter families of curves $c$ and $c^{\prime}$ in $r$ admitting lines of the complex (49) for tangents; these curves lie on two surfaces $\sigma$ and $\sigma^{\prime}$. To a point $A$ of $\Sigma$ corresponds a line $a$ of the complex tangent to a curve $c$ and to a curve $c^{\prime}$ at points $m$ and $m^{\prime}$ corresponding to the respective minimal tangents to $\Sigma$ at $A$. Hence $\sigma$ and $\sigma^{\prime}$ are focal surfaces of a congruence of lines of the complex. Two nearby tangents to $c$ determine a plane tangent to $\sigma^{\prime}$, that is the null-plane of $m$ in the tangent plane to $\sigma^{\prime}$ at $m^{\prime}$. In like manner the null-plane at $m^{\prime}$ is the tangent plane to $\sigma$ at $m$. 'The spheres tangent to $\Sigma$ at $A$ correspond to two pencils of lines with centers at $m$ and $m^{\prime}$, as shown in the preceding section. Hence to the surface elements of $\Sigma$ correspond two sets of surface elements whose centers are the points of $\sigma$ and $\sigma^{\prime}$, and whose planes are the tangent planes to $\sigma$ and $\sigma^{\prime}$ at corresponding points. Therefore:

When a Lie transformation is applied to a surface $\mathbf{\Sigma}$ in $R$, the two corresponding surfaces $\sigma$ and $\sigma^{\prime}$ in $v$ are the focal surfaces of a congruence of lines of the complex of the transformation; corresponding directions in the tangent planes to $\sigma$ and $\sigma$ correspond to spheres tangent to $\Sigma$; and the surface elements of $\Sigma$ correspond to the surface elements of $\sigma$ and $\boldsymbol{\sigma}^{\prime}$.

Thus the correspondence is a contact transformation.

As a point $m$ moves along a curve $\gamma$ on $\sigma$, the tangent planes intersect in directions conjugate to $\gamma$. Hence only in case $\gamma$ is an asymtotic line do nearby surface elements have a corresponding direction in common. Two nearby surface elements of $\boldsymbol{\Sigma}$ are tangent to the same sphere only in case the center of the element moves along a line of curvature of $\Sigma$. Consequently we have the following important property of the Lie transformation: 
The spheres with centers at the centers of principal curvature of $\Sigma$ correspond to the tangents to the asymptotic lines on $\sigma$ and $\sigma^{\prime}$; the lines of curvature of $\mathbf{\Sigma}$ correspond to the asymptotic lines on $\sigma$ and $\sigma^{\prime}$, which are the focal surfaces of a $W$ congruence consisting of lines of the complex of the transformation.

Since the tangents to $\sigma$ at a point $m$ are projective with the centers of the spheres tangent to $\Sigma(102)$, we have:

The centers of spheres corresponding to conjugate directions at $m$ are harmonic with respect to the centers of principal curvature of $\mathbf{S}$.

104. Congruences $R$ of spheres. $W$ congruences of lines. From $\S 100$ it follows that a necessary and sufficient condition that a congruence of spheres be $R$ is that the radius $R$ be a solution of (36), which from (10) is equivalent to the condition that $\alpha_{6}$-be a solution of (26). Hence:

A necessary and sufficient condition that a congruence of spheres be $R$ is that the six coordinates of the congruence satisfying (11) be solutions of the same equation of Laplace.

If $\alpha_{i}$ and $\alpha_{i}^{\prime}(i=1, \ldots 6)$ are the coordinates of two congruences $R$ of spheres tangent to the same surface, we have

$$
\sum \alpha^{2}=0, \quad \sum \alpha^{\prime 2}=0, \quad \sum \alpha \alpha^{\prime}=0 .
$$

Hence the quantities $\alpha_{i}$ and $\alpha_{i}^{\prime}$ are homogeneous coordinates of two nets, $N(\alpha)$ and $N^{\prime}\left(\alpha^{\prime}\right)$, in 5-space lying on the hyperquadric $\sum x^{2}=0$, and in relation $F$ for which the lines of the congruence of the transformation are generators of the hyperquadric (cf. III, Ex. 23).

As an immediate consequence of the results of the preceding section we have the theorem:

If two surfaces $\Sigma$ and $\Sigma_{1}$ are in relation $R$, and $\sigma, \sigma^{\prime}$ and $\sigma_{1}, \sigma_{1}^{\prime}$ are the transforms of $\Sigma$ and $\Sigma$ respectively by a Lie line-sphere transformation, the surfaces $\sigma$ and $\sigma_{1}$ are the focal surfaces of $a W$ congruence and likewise $\sigma^{\prime}$ and $\sigma_{1}^{\prime}$ moreover, $\sigma$ and $\sigma^{\prime}$ are focal surfaces of a $W$ congruence consisting of lines of the complex of the transformation and likewise $\sigma_{1}$ and $\sigma_{1}^{\prime}$.

Conversely if $\sigma$ and $\sigma_{1}$ are the focal surfaces of a $W$ congruence, and $\Sigma$ and $\Sigma_{1}$ are transforms of $\sigma$ and $\sigma_{1}$ by a Lie transformation 
then $\Sigma$ and $\Sigma_{1}$ are in relation $R$. Hence we have the following theorem of Darboux ${ }^{84}$ ) as a consequence of the preceding theorems:

$A$ necessary and sufficient condition that a congruence be a $W$ congruence is that the Pliicker coordinates of the lines be solutions of an equation of Laplace.

Since a $W$ congruence is transformed into a W-congruence by a projective transformation, we have:

$A$ net $N(x)$ in 5-space lying on a quadratic variety of four dimensions whose equation is

$$
\sum a_{i j} x^{(i)} x^{(j)}=0
$$

represents a $W$ congruence.

From the results of $\S 38$ we have:

Each transform $F$ of a net representing a $W$ congruence leads directly to a net representing another $W$ congruence.

Consider in particular a surface $\sigma$ upon which there lies an $R$ net $N(\S 45)$, that is a net for which the tangents to the curves of the net in each system form $W$ congruences. When a Lie transformation is applied to $\sigma$, to the tangents to either family of curves of $N$ correspond spheres tangent to $\Sigma$ with centers describing nets $2, O$ conjugate to the congruence of normals to $\boldsymbol{S}$. Moreover, corresponding points of these central nets, $C$ and $C^{\prime}$, are harmonic to the centers of principal curvature of $\Sigma$ and the nets have equal invariants $(\S 25)$, that is $\Sigma$ is a surface $\Omega(\S 89)$. Conversely, if a Lie transformation is applied to a surface $\Omega$, there result two nets $R$. Consequently we have the theorem of Demoulin ${ }^{85}$ ):

$A$ net $R$ is transformed into an $O$ net $\Omega$ by a Lie transformation and conversely.

Since the nets $C$ and $C^{\prime}$ are in the relation of a transformation $K$ (§ 25), the coordinates $\alpha_{i}$ and $\alpha_{i}^{\prime}(i=1, \ldots 6)$, of the spheres with centers on $C$ and $C^{\prime}$ tangent to $\Sigma$ satisfy equations of Laplace with equal invariants. Conversely, if the coordinates $\alpha_{i}$ of a sphere are solutions of such an equation, the central net $C$ has equal invariants, and consequently ( $\$ 89)$ the sheets of the envelope are surfaces $\Omega$. Hence:

84) Leçons, $2^{\text {nd }}$ ed., vol. 2, p. 358.

$\left.{ }^{85}\right)$ Comptes Rendus, vol. 153 (1911), p. 590. 
When the Laplace equation of a congruence $R$ of spheres has equal invariants, the sheets of the envelope are surfaces $\boldsymbol{\Omega}$, and conversely.

As a corollary we have:

$A$ necessary and sufficient condition the a net be $R$ is that the Pliicker line coordinates of the tangents to either family of curves of the net are solutions of a Laplace equation with equal invariants.

105. Harmonic congruences of spheres and circles. Suppose we have a congruence of circles $C_{1}$, whose axes are tangent to the curves $u=$ const. of a net $N$, the circles being determined by a solution $\theta$ of the point equation of $N$, as discussed in $\S 99$.

Any net $\bar{N}$ conjugate to this congruence of axes is defined by equations of the form

$$
\bar{x}=x-\frac{\theta_{1}}{\frac{\partial \theta_{1}}{\partial v}} \frac{\partial x}{\partial v},
$$

where $\boldsymbol{\theta}_{1}$ is another solution of the point equation (36) of $N(\S 8)$. Evidently $\bar{\theta}$ given by $\bar{\theta}=\theta-\theta_{1} \frac{\partial \theta}{\partial v} / \frac{\partial \theta_{1}}{\partial v}$ is a solution of the point equation of $\bar{N}$. Hence the spheres $\bar{S}$ with centers on points of $\bar{N}$ and radii determined by

$$
\sum \bar{x}^{2}-\overline{R^{2}}=2 \bar{\theta}
$$

form a congruence. It is readily found that the circles $C_{1}$ lie on the corresponding spheres of this congruence.

We say that a congruence of spheres and a congruence of circles are harmonic, when the centers of the spheres describe a net conjugate to the axes of the circles, and the latter lie on the corresponding spheres ${ }^{86}$ ):

We may state the preceding results as follows:

If a congruence of circles is determined by a solution $\theta$ of the point equation of the net $N$ whose curves $u=$ const. are tangent to the axes of the circles, and $\theta_{1}$ is the solution determining a net $\bar{N}$ conjugate to the axes, the congruence of spheres harmonic to the circles and with centers on $\bar{N}$ is determined by

$$
\bar{\theta}=\theta-\frac{\theta_{1}}{\frac{\partial \theta_{1}}{\partial v}} \frac{\partial \theta}{\partial v} .
$$

$\left.{ }^{86}\right)$ Cf. Guichard, Annales L'École Norm. Sup., ser. 3, vol. 20 (1903), p. 197. 
Consider the converse problem when the spheres are given with centers on a net $\bar{N}$ and radii determined by a solution $\bar{\theta}$ of its point equation. A congruence $G$ conjugate to $\bar{N}$ is obtained by drawing through points of $\bar{N}$ lines parallel to the lines joining the origin to points of a parallel net $\bar{N}^{\prime}$ whose coordinates $\bar{x}^{\prime}$ are given by

$$
\frac{\partial \bar{x}^{\prime}}{\partial u}=h \frac{\partial \bar{x}}{\partial u}, \quad \frac{\partial \bar{x}^{\prime}}{\partial v}=l \frac{\partial \bar{x}}{\partial v} .
$$

A function $\overline{\theta^{\prime}}$ is given by the quadratures

$$
\frac{\partial \overline{\boldsymbol{\theta}^{\prime}}}{\partial u}=h \frac{\partial \overline{\boldsymbol{\theta}}}{\partial u}, \quad \frac{\partial \overline{\boldsymbol{\theta}^{\prime}}}{\partial v}=l \frac{\partial \overline{\boldsymbol{\theta}}}{\partial v}
$$

From (I, 37) we have that the focal point generating a net $N$ whose curves $u=$ const. are tangent to the congruence $G$ is given by equations of the form

$$
x=\bar{x}-\frac{1}{l} \bar{x}^{\prime}
$$

Moreover, $1 / l$ is the solution of the point equation of $N$ determining $\bar{N}$ as a Levy transform of $N(\S 8)$, that is the function $\theta_{1}$ referred to in the above theorem. Hence it is readily seen that the function

$$
\theta=\bar{\theta}-\frac{1}{l} \bar{\theta}^{\prime}
$$

enables us to express $\bar{\theta}$ in the form (58). Consequently the spheres with centers on the net $N$ and radii determined by $\theta$ meet the corresponding spheres of the given congruence in circles harmonic to the latter. Accordingly we have:

The determination of congruences of circles harmonic to a congruence of spheres is the problem of finding congruences conjugate to the net of centers of the spheres and a quadrature.

Since $\overline{\theta^{\prime}}$ is determined by (60) to within an additive constant, there are $\infty^{1}$ congruences of circles harmonic to the given congruence of spheres such that corresponding circles have the same axis.

This result may be obtained in another manner which brings to light the role of transformations $F$ in this theory. Let $\bar{N}_{1}\left(\bar{x}_{1}\right)$ 
be an $F$ transform of $\bar{N}$ by means of a solution $\hat{\theta}$ of the point equation of $\bar{N}$, the congruence $G$ of the transformation having parameters $\bar{x}^{\prime}$. Thus

$$
\bar{x}_{1}=\bar{x}-\frac{\hat{\theta}}{\hat{\theta}^{\prime}} \bar{x}^{\prime}
$$

From (II, 7) we have

where

$$
\frac{\partial \bar{x}_{1}^{\prime}}{\partial u}=h_{1} \frac{\partial \bar{x}_{1}}{\partial u}, \quad \frac{\partial \bar{x}_{1}^{\prime}}{\partial v}=l_{1} \frac{\partial \bar{x}_{1}}{\partial v},
$$

$$
h_{1}=\frac{h}{\hat{\theta}^{\prime}-h \dot{\theta}}, \quad l_{1}=\frac{l}{\hat{\theta}^{\prime}-l \hat{\theta}} .
$$

Consequently from (61) we have

$$
x=\bar{x}_{1}-\frac{1}{l_{1}} \bar{x}_{1}^{\prime}
$$

We call the functions

$$
\overline{\theta_{1}}=\bar{\theta}-\frac{\hat{\theta}}{\hat{\theta}^{\prime}} \overline{\theta^{\prime}}, \quad \frac{\overline{\theta^{\prime}}}{\hat{\theta}^{\prime}},
$$

the solutions of the point equations of $\bar{N}_{1}$ and $\overline{N_{1}^{\prime}}$ corresponding to the solution $\bar{\theta}$ of the point equation of $\bar{N}$. We find from (62) that

$$
\theta=\overline{\theta_{1}}-\frac{1}{l_{1}}-\frac{\overline{\theta^{\prime}}}{\hat{\theta}^{\prime}}
$$

Consequently we have:

If $\bar{N}$ and $\overline{N_{1}}$ are $F$ transforms of one another, and $\bar{\theta}$ and $\overline{\theta_{1}}$ are corresponding solutions of the point equations of $\bar{N}$ and $\bar{N}_{1}$ respectively, the congruences of spheres $S$ and $S_{1}$ with centers on $\bar{N}$ and $\bar{N}_{1}$ and radii determined by $\bar{\theta}$ and $\bar{\theta}_{1}$ respectively, intersect in a congruence of circles having the lines of congruence of the transformation $F$ for axes.

When, in particular, $\hat{\theta}=\bar{\theta}$, the function $\bar{\theta}_{1}=0$, and the spheres $S_{1}$ pass through the origin. Hence:

If the central net $\bar{N}$ of a congruence of spheres $S$ whose radii are determined by a solution $\bar{\theta}$ of the point equation of $\bar{N}$ is subjected to a transformation $F$ determined by $\bar{\theta}$, the spheres $S_{1}$ with centers on the transform $\bar{N}_{1}$ and passing through the origin meet 
the spheres $S$ in a congruence of circles harmonic to $S$; moreover, the $\infty^{1}$ congruences of circles harmonic to the spheres $S$ and with the same axes are obtained in this way by varying the additive constant of integration in $\bar{\theta}^{\prime}$.

From this result and (38) it follows that the circles are the intersections of the spheres

where

$$
\sum x_{0}^{2}-2 \sum x_{0} \bar{x}+2 \bar{\theta}=0, \quad \sum x_{0}^{2}-2 \sum x_{0} \bar{x}_{1}=0
$$

$$
\overline{x_{1}}=\bar{x}-\frac{\bar{\theta}}{\bar{\theta}^{\prime}} \bar{x}^{\prime}
$$

and the $x_{0}$ 's are current coordinates. Moreover, in consequence of (63) the equation of the planes of the circles is

$$
\sum x_{0} \bar{x}^{\prime}-\bar{\theta}^{\prime}=0 \text {. }
$$

Consequently the coordinates $x_{0}$ of the net $N_{0}$ enveloped by these planes are found by solving (64) and

$$
\sum x_{0} \frac{\partial \bar{x}}{\partial u}-\frac{\partial \overline{\boldsymbol{\theta}}}{\partial u}=0, \quad \sum x_{0} \frac{\partial \bar{x}}{\partial v}-\frac{\partial \overline{\boldsymbol{\theta}}}{\boldsymbol{\theta} v}=0,
$$

in consequence of (59) and (60). From (41) it follows that (65) are the equations of the chord of contact of the spheres $S$ with their envelope. Consequently $N_{0}$ is conjugate to the congruence of these chords of contact and we have the theorem of Guichard ${ }^{87}$ ):

The congruence of lines joining the points of contact of a congruence of spheres with their envelope is conjugate to the net enveloped by the planes of any congruence of circles harmonic to the spheres.

The quantities $\bar{x}^{\prime}$ and $\bar{\theta}^{\prime}$ are tangential coordinates of $N_{0}$. The tangential coordinates of the net $N_{10}$ enveloped by the planes of any other congruence of circles harmonic to the spheres are given by

$$
\begin{cases}\frac{\partial \bar{x}^{\prime \prime}}{\partial u}=\frac{h^{\prime}}{h} \frac{\partial \bar{x}^{\prime}}{\partial u}, & \frac{\partial \bar{x}^{\prime \prime}}{\partial v}=\frac{l^{\prime}}{l} \frac{\partial \bar{x}^{\prime}}{\partial v}, \\ \frac{\partial \overline{\boldsymbol{\theta}}{ }^{\prime \prime}}{\partial u}=\frac{h^{\prime}}{h} \frac{\partial \overline{\boldsymbol{\theta}^{\prime}}}{\partial u}, & \frac{\partial \overline{\boldsymbol{\theta}}^{\prime \prime}}{\partial v}=\frac{l^{\prime}}{l} \frac{\partial \overline{\boldsymbol{\theta}^{\prime}}}{\partial v},\end{cases}
$$

${ }^{87}$ ) L. c., p. 197. 
in consequence of (59) and similar equations defining the other congruence of circles. But from these equations it follows that $N_{0}$ and $N_{10}$ are in relation $F$ or are parallel. Hence:

When two congruences of circles are harmonic to the same congruence of spheres, the nets enveloped by the planes of the circles are in relation $F$ or are parallel.

Conversely since the tangential coordinates of any two nets in relation $F$ can be chosen so that they satisfy equations of the form (66), it follows that the tangential coordinates of any other net conjugate to the lines joining corresponding points of $N_{0}$ and $N_{10}$ satisfy equations of the form (66). Hence:

If $S$ is a sphere describing a congruence and $G$ is the congruence of lines joining the points of contact of $S$ with its envelope, the tangent planes of any net conjugate to $G$ meet the spheres $S$ in a congruence of circles harmonic to the spheres ${ }^{88}$ ).

106. Representation in 5-space. When we interpret the results of $\S 105$ in terms of nets and congruences in 5-space, we find that a net representing the congruence of circles is harmonic to a congruence representing the congruence of spheres. We shall establish this result.

From (2), (32), (37) and (38) it follows that the parameters of the spheres giving rise to the circles $C_{1}$ are of the form

$$
\begin{aligned}
& \alpha_{i}^{\prime}=h x_{i}(i=1,2,3), \quad \alpha_{4}^{\prime}=i h\left(\theta+\frac{1}{2}\right), \quad \alpha_{5}^{\prime}=h\left(\theta-\frac{1}{2}\right) \\
& \boldsymbol{\beta}_{i}^{\prime}=h\left(\frac{\partial x_{i}}{\partial v}-\frac{\partial \log a}{\partial v} x_{i}\right)(i=1,2,3), \\
& \boldsymbol{\beta}_{4}^{\prime}=i h\left(\frac{\partial \theta}{\partial v}-\left(\theta+\frac{1}{2}\right) \frac{\partial \log a}{\partial v}\right), \quad \beta_{5}^{\prime}=h\left(\frac{\partial \theta}{\partial v}-\left(\theta-\frac{1}{2}\right) \frac{\partial \log a}{\partial v}\right) .
\end{aligned}
$$

Since the functions $\boldsymbol{\alpha}^{\prime}$ and $\boldsymbol{\beta}^{\prime}$ are the direction-parameters of the tangents to a net, they are solutions of a system of equations of the form $(I, 4)$. Any other solution $\theta_{1}$ of $(36)$ gives two other solutions of this system, namely

$$
p^{\prime}=h \theta_{1}, \quad q^{\prime}=h\left(\frac{\partial \theta_{1}}{\partial v}-\frac{\partial \log a}{\partial v} \theta_{1}\right) .
$$

s9) Cf. Gnichard, l. c. p. 197. 
We make use of this fact to obtain the parameters of the congruence in 5-space representing the congruence of spheres $S$ harmonic to the circles $C_{1}$ in $\S 105$. In fact, from (57) and (58) it follows. that the parameters $\bar{\alpha}$ of the congruence are given by

$$
\begin{aligned}
& \bar{\alpha}_{i}=\bar{h} \bar{x}_{i}=\frac{\bar{h}}{h^{2} \frac{\partial \bar{\theta}_{1}}{\partial v}}\left(\alpha_{i}^{\prime} q^{\prime}-\beta_{i}^{\prime} p^{\prime}\right) \quad(i=1,2,3) \\
& \bar{\alpha}_{4}=i \bar{h}\left(\bar{\theta}+\frac{1}{2}\right)=\frac{\bar{h}}{h^{2} \frac{\partial \theta_{1}}{\partial v}}\left(\alpha_{4}^{\prime} q^{\prime}-\beta_{4}^{\prime} p^{\prime}\right) \\
& \bar{\alpha}_{\overline{5}}=\bar{h}\left(\bar{\theta}-\frac{1}{2}\right)=\frac{\bar{h}}{h^{2} \frac{\partial \theta_{1}}{\partial v}}\left(\alpha_{5}^{\prime} q^{\prime}-\beta_{5}^{\prime} p^{\prime}\right)
\end{aligned}
$$

Hence $(\S 13)$ the congruence and net in 5-space corresponding to the congruences of spheres and circles are harmonic.

In order to prove conversely that congruences of circles and spheres represented by a net and a congruence harmonic to it in 5 -space are harmonic to one another, we take the direction-parameters, $\alpha$ and $\beta$, of the net in the normal form, so that

$$
\frac{\partial \boldsymbol{\alpha}}{\partial v}=n \boldsymbol{\beta}, \quad \frac{\partial \boldsymbol{\beta}}{\partial u}=m \boldsymbol{\alpha} .
$$

The parameters of any harmonic net are of the form

$$
\bar{\alpha}=\alpha q-\beta p,
$$

where $p$ and $q$ are a pair of solutions of (67). From these expressions it follows that the spheres $S$ of coordinates $\bar{\alpha}$ pass through the circles of intersection of the corresponding spheres of coordinates $\alpha$ and $\beta$. Hence their net of centers is conjugate to the congruence of the axes of these circles, since the curves of the net and the developables of the congruence are parametric.

From (68) we have by differentiation

$$
\frac{\partial \bar{\alpha}}{\partial u}=q \frac{\partial \alpha}{\partial u}-\frac{\partial p}{\partial u} \boldsymbol{\beta}, \quad \frac{\partial \bar{\alpha}}{\partial v}=\frac{\partial q}{\partial v} \alpha-p \frac{\partial \boldsymbol{\beta}}{\partial v} .
$$


When $u$ varies, the characteristics of the spheres $S$ are circles, $\Gamma_{1}$, the intersection of the spheres of coordinates $\bar{\alpha}$ and $\frac{\partial \bar{\alpha}}{\partial u}$, which in consequence of (67), (68) and (69) pass through the corresponding points, $A_{1}$ and $A_{2}$, where the spheres of coordinates $\alpha$ touch their envelope. Similarly, when $v$ varies the characteristics of $S$ are circles $\Gamma_{2}$ passing through the corresponding points $B_{1}$ and $B_{2}$, where the spheres of coordinates $\beta$ touch their envelope. We have seen that the lines $A_{1} A_{2}$ and $B_{1} B_{2}$ are the tangents to the parametric net $\bar{N}$ on the envelope of the circle $C_{1}(\S 97)$. Moreover, we know that the lines of intersection of the planes of the circles $\Gamma_{1}$ and $\Gamma_{2}$, that is the joins of points of contact of the spheres $S$ with their envelope, form a congruence with the developables parametric. Hence we have the fifth theorem of $\S 105$.

107. Conjugate congruences of spheres and circles. We return to the consideration of the congruence of spheres $S$ whose centers describe a net $N$ and whose radius is determined by a solution $\theta$ of (36), the point equation of $N$. By means of a solution $\theta_{1}$ of (36) we determine Levy transforms $\bar{N}_{-1}$ and $\bar{N}_{1}$ of $N$, such that $M \bar{M}_{-1}$ and $M \bar{M}_{1}$ are tangent to the curves of parameter $u$ and $v$ respectively of $N$. By means of the functions

$$
\bar{\theta}_{-1}=\theta-\frac{\theta_{1}}{\frac{\partial \theta_{1}}{\partial u}} \frac{\partial \theta}{\partial u}, \quad \bar{\theta}_{1}=\theta-\frac{\theta_{1}}{\frac{\partial \theta_{1}}{\partial v}} \frac{\partial \theta}{\partial v}
$$

we determine spheres $\bar{S}_{-1}$ and $\bar{S}_{1}$ with centers on $\bar{N}_{-1}$ and $\bar{N}_{1}$ respectively. The spheres $S$ meet the spheres $\bar{S}_{-1}$ and $\bar{S}_{1}$ in congruences of circles $C_{-1}$ and $C_{1}$ respectively, harmonic to $\bar{S}_{-1}$ and $\bar{S}_{1}$. Moreover, corresponding circles $C_{-1}$ and $C_{1}$ meet in the points $A_{1}$ and $A_{2}$ where $S$ touches its envelope (cf. fig. 6).

Since the nets $\bar{N}_{-1}$ and $\bar{N}_{1}$ are Laplace transforms of one another, the same is true of the functions $\bar{\theta}_{-1}$ and $\overline{\theta_{1}}$ (I, Ex. 11). Consequently by the fourth theorem of $\S 99$ the circles $\bar{C}$ of intersection of the spheres $\bar{S}_{-1}$ and $\bar{S}_{1}$ form a congruence. Since the spheres $\bar{S}_{-1}$ and $\bar{S}_{1}$ pass through $A_{1}$ and $A_{2}$, so also does their intersection $\bar{C}$.

From $\S 97$ it follows that the points of contact, $B_{-1}, B_{-1}^{\prime}$ and $B_{1}, B_{1}^{\prime}$ respectively, of the spheres $\bar{S}_{-1}$ and $\bar{S}_{1}$ with their 


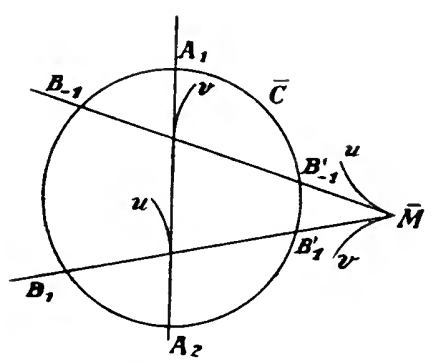

Fig. 7

envelopes lie on $\bar{C}$, and that the lines $B_{-1} B_{-1}^{\prime}$ and $B_{1} B_{1}^{\prime}$ are the tangents to the net $\bar{N}$ enveloped by the planes of $\bar{C}$. Since $\bar{S}_{-1}$ is harmonic to $C_{-1}$, it follows from $\S 105$ that the line $B_{-1} B_{-1}^{\prime}$ passes through the net enveloped by the plane of $C_{-1}$, that is the second focal net of $A_{1} A_{2}$. Similarly the line $B_{1} B_{1}^{\prime}$ passes through the first focal net of $A_{1} A_{2}$ (cf. fig. 7). Consequently the congruence $A_{1} A_{2}$ is harmonic to $\bar{N}$. Hence ${ }^{89}$ ):

If $A_{1}$ and $A_{2}$ are the points of contact with its envelope of a congruences of spheres $S$ with centers at points of a net $N$ and $G$ is any congruence harmonic to $N$, the circles with the lines of $G$ for axes and passing through the corresponding points $A_{1}$ and $A_{2}$ form a congruence. Moreover, the congruence $A_{1} A_{2}$ is harmonic to the net enveloped by the planes of the circles.

Consider now the representation in 5-space. To the spheres $S$ corresponds a congruence $G$, to $C_{1}$ and $C_{-1}$ the focal nets $F_{1}$ and $F_{2}$ of $G$, to $\bar{S}_{1}$ and $\bar{S}_{-1}$ congruences harmonic to $F_{1}$ and $F_{2}$, which are tangents to a net $P$ representing the congruence of circles $\bar{C}$.

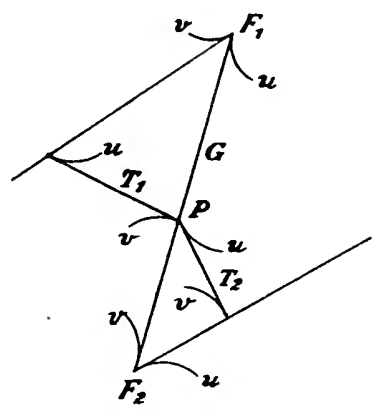

Fig. 8

Hence the net $P$ is conjugate to $G$.

Conversely, if we have a congruence $G$ and a conjugate net $P$ in 5-space, to $G$ there corresponds in 3-space a congruence of spheres $S$ with centers at points of a net $N$. It touches its envelope at the points, $A_{1}$ and $A_{2}$, of intersection of $C_{1}$ and $C_{-1}$, the characteristics of $S$ when $v$ and $u$ vary respectively. Since the tangents $T_{1}$ and $T_{2}$ to the curves of parameter $v$ and $u$ of $P$ are harmonic to the focal nets $F_{1}$ and $F_{2}$ of $G$, they generate congruences represented in 3 -space by congruences of spheres harmonic to the circles $C_{1}$ and $C_{-1}$ respectively, the centers of the spheres being on nets $\bar{N}_{1}$ and $\bar{N}_{-1}$ conjugate to the tangents to

$\left.{ }^{89}\right)$ Cf. Guichard, 1. c., p. 198. 
the curves of parameter $v$ and $u$ respectively of $N$. However, since $T_{1}$ and $T_{2}$ are tangents to the net $P$, the two congruences of spheres with centers on $\bar{N}_{1}$ and $\bar{N}_{-1}$ meet in a congruence of circles, and corresponding points of $\bar{N}_{1}$ and $\bar{N}_{-1}$ are the foci of the axes of the circles $(\$ 97)$. Hence $(\$ 10)$ the congruence of axes is harmonic to $N$. In view of these results we say, with Guichard, that congruences of spheres and circles related as in the preceding theorem are conjugate to one another ${ }^{90}$ ).

In order to obtain a congruence of circles conjugate to a congruence of spheres, we note that the coordinates of $\bar{N}_{1}$ are of the form (57) where $\theta_{1}$ is a solution of the point equation of $N$, the net of centers of the spheres. The function $\bar{\theta}_{1}$ determining the radius of $\overline{S_{1}}$ is given by $(70)$. Hence the circles are defined by the equations

$$
\sum\left(y^{2}-2 y \bar{x}_{1}\right)+2 \bar{\theta}_{1}=0, \quad \sum y \frac{\partial \bar{x}_{1}}{\partial u}-\frac{\partial \bar{\theta}_{1}}{\partial u}=0,
$$

where the $y$ 's are current coordinates.

Conversely, if we have a congruence of circles, any net harmonic to the axes of the circles is given by equations of the form (61) and the radius of the spheres of the conjugate congruence is determined by (62). The converse may be stated in geometrical form, if we observe that it follows from the representation in 5-space that there is a reciprocal correspondence between the congruence harmonic to $\bar{N}$ and the nets harmonic to the congruence of axes of the circles. In view of this fact and the preceding results we have:

If $G$ is a congruence harmonic to the net $\vec{N}$ enveloped by the planes of the circles of a congruence, and $A_{1}$ and $A_{2}$ are the points of meeting of a line of $G$ and the corresponding circle of the congruence, the plane determined by the mid-point of the segment $A_{1} A_{2}$ and the axis of the circle envelopes a net $N$; the spheres with centers on $N$ and passing through $A_{1}$ and $A_{2}$ form a congruence conjugate to the given congruence of circles $^{91}$ ).

ro8. Congruences of circles $K$ determined by transformations $R$. In $\S 81$ we found that if $N_{1}$ and $N_{2}$ are $R$ trans-

${ }^{90}$ ) L. c., p. 197.

${ }^{91)}$ Cf. Guichard, l. c., p. 198. 
forms of an $O$ net $N$, there can be found by quadratures alone a set of $\infty^{1}$ nets $N_{c}$, of which $N_{1}$ is a member, which are $R$ transforms of a family of $\infty^{1}$ nets $N_{12}$, of which $N$ is a member; corresponding points of these $\infty^{2}$ nets lie on a circle $K$; the tangent planes to the nets at points of $K$ pass through a point $M_{d}$ which describes a net $N_{d}$ conjugate to the congruence $K$ of the axes of the circles $K$, this congruence being harmonic to the central nets of the transformation of $N$ into $N_{c}$; the normals to the nets $N_{12}$ and $N_{c}$ at points of $K$ are tangent to the sphere $S$ with center at $M_{d}$ and passing through $K$. We wish to show that the circles $K$ form a congruence of circles and the spheres $S$ a congruence of spheres harmonic to the former.

Since $\omega=\frac{1}{2} \sum x^{2}$ is a solution of the point equation of $N$, the function (cf. $\S 81$ and VI, 96)

$$
\omega_{01}=\omega-\frac{\theta_{1}}{p_{1}} P
$$

is a solution of the point equation of $N_{01}$, and consequently

$$
\begin{aligned}
& \omega_{y_{1}}=\omega_{01}-\frac{\theta_{01}}{\frac{\partial \theta_{01}}{\partial u}} \frac{\partial \omega_{01}}{\partial u}=\omega+\frac{\left(\theta_{1} p_{2}-\theta_{2} p_{1}\right) Q+\left(\theta_{2} q_{1}-\theta_{1} q_{2}\right) P}{p_{1} q_{2}-p_{2} q_{1}}, \\
& \omega_{y_{2}}=\omega_{01}-\frac{\theta_{01}}{\frac{\partial \theta_{01}}{\partial v}} \frac{\partial \omega_{01}}{\partial v}=\omega+\frac{\left(\theta_{1} p_{2}-\theta_{2} p_{1}\right) R+\left(\theta_{2} r_{1}-\theta_{1} r_{2}\right) P}{p_{1} r_{2}-p_{2} r_{1}}
\end{aligned}
$$

are solutions of the point equations of the focal nets $F_{1}$ and $F_{2}$ of the congruence $K$, and either is a Laplace transform of the other. From (VII, 33) it follows that the radii of the spheres with centers on $F_{1}$ and $F_{2}$ and passing through the corresponding circles $K$ are given by

$$
\begin{aligned}
& R_{y_{1}}^{2}=\frac{\left(\theta_{1} p_{2}-\theta_{2} p_{1}\right)^{2}+\left(\theta_{2} q_{1}-\theta_{1} q_{2}\right)^{2}}{\left(p_{1} q_{2}-p_{2} q_{1}\right)^{2}}, \\
& R_{y_{2}}^{2}=\frac{\left(\theta_{1} p_{2}-\theta_{2} p_{1}\right)^{2}+\left(\theta_{2} r_{1}-\theta_{1} r_{2}\right)^{2}}{\left(p_{1} r_{2}-p_{2} r_{1}\right)^{2}} .
\end{aligned}
$$


From these equations and (VII, 33) we find that

$$
\sum y_{1}^{2}-R_{y_{1}}^{2}=2 \omega_{y_{1}}, \quad \sum y_{2}^{2}-R_{y_{2}}^{2}=2 \omega_{y_{2}} .
$$

Hence (§ 99) the circles $K$ form a congruence, and the spheres $S$ form a congruence harmonic to these circles $(\S 105)$.

From the second theorem of $\S 11$ it follows that the lines joining the focal points of the same rank of corresponding lines of the congruences of the transformations $R$ of $N$ into two nets of the class $N_{c}$ meet in a point $\bar{M}$ which describes the net $\bar{N}$ enveloped by the planes of the circles $K$. If $A_{1}$ and $A_{2}$ are the points of contact of the sphere $S$ with its envelope, the lines $A_{1} A_{2}$ form a congruence $\bar{G}$ which is conjugate to the net $\bar{N}(\S 105)$.

Since normals to a pair of nets $N$ and $N_{1}$ are tangent to the corresponding sphere $S$, the circles orthogonal to $N$ and $N_{1}$ form a cyclic system ( $(79)$ and lie on the spheres $S$. In $\S 113$ it is shown that a cyclic system is a congruence, and consequently this cyclic system is harmonic to the sphere. From the results of $\S 105$ it follows that the axes of these circles form a congruence. conjugate to $N_{d}$.

rog. Orthogonal congruences of spheres and circles. Consider the congruence of spheres $\bar{S}$ with centers on $\bar{N}(\bar{x})$ and radii determined by a solution $\bar{\theta}$ of the point equation of $\bar{N}$, and also the harmonic circles whose planes are defined by (64). The spheres $S$ with centers on the net $N$ enveloped by these planes and with radii determined by a solution $\varphi$ of the point equation of $N$ are orthogonal to the corresponding spheres $\overline{S,}$ when, and only when,

$$
y=\sum \bar{x} x-\bar{\theta} .
$$

In consequence of (65) we have

$$
\frac{\partial \varphi}{\partial u}=\sum \bar{x} \frac{\partial x}{\partial u}, \quad \frac{\partial \varphi}{\partial v}=\sum \bar{x} \frac{\partial x}{\partial v} .
$$

Because of these equations it follows that the spleres $\bar{S}$ are orthogonal also to the first and minus first derived spheres of $S$ (§ 99). Moreover, equations (73), being of the form (41), show 
that the line joining the points where $S$ touches its envelope passes through the corresponding point of $\bar{N}$ and hence is the axis of the circle.

Conversely, let $\varphi$ be any solution of the point equation of $N$, and let $S$ denote the spheres with centers on $N$, and radii determined by $\varphi$. Let $\bar{N}(\bar{x})$ be any net conjugate to the line joining the points of contact of $S$ with its envelope, then equations (73) hold. By differentiating them with respect to $v$ and $u$ respectively, we get

$$
\sum \frac{\partial \bar{x}}{\partial v} \frac{\partial x}{\partial u}=0, \quad \dot{\sum} \frac{\partial \bar{x}}{\partial u} \frac{\partial x}{\partial v}=0
$$

In consequence of these equations $\bar{\theta}$ given by (72) is a solution of the point equation of $\bar{N}$, and therefore the spheres $\bar{S}$ with centers on $\bar{N}$ and radii determined by $\bar{\theta}$ meet $S$ and the two derived congruences of spheres orthogonally. We say that the congruence of spheres $S$ are orthogonal to the congruence of circles in which the spheres $\bar{S}$ are cut by the tangent planes of $N$.

By definition the poles of a circle are the two points on its axis, equidistant from the plane of the circle, which are the centers of the null spheres passing through the circle; any sphere with center in the plane of a circle and orthogonal to a sphere through the circle passes through the poles of the circle ${ }^{92}$ ). For the present case the points of contact of $S$ with its envelope are the poles of the circles, since the spheres $\bar{S}$ are orthogonal to $S$ and its derived spheres. Hence:

The circles in the tangent planes of the surface of centers of a congruence of spheres which have for poles the points of contact of the spheres with their envelope form a congruence of circles whose focal parameters are the same as for the congruence of spheres.

\section{Also:}

If $C$ describes a congruence of circles, the spheres with centers in the net enveloped by the plane of $C$ and passing through the poles of $C$ form a congruence orthogonal to the congruence of circles $^{93}$ ).

We have seen that the spheres $S$ orthogonal to a congruence of circles $C$ are orthogonal to any congruence of spheres $\bar{S}$ har-

${ }^{92}$ ) Note that when the circles are real the poles are imaginary and vice-rersa.

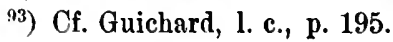


monic to $C$, as are also the first and minus first derived spheres $S_{1}$ and $S_{-1}$ of $S$. Hence if the equation of $S$ is $\sum \gamma z=0$, and the equations of $C$ are $\sum \alpha z=0, \sum \beta z=0$, where the $\alpha$ 's and $\beta$ 's satisfy (67), it follows from (68) that

$$
\begin{array}{clc}
\sum \gamma \alpha=0, & \sum \gamma \beta=0, & \sum \frac{\partial \gamma}{\partial u} \alpha=0, \\
\sum \frac{\partial \gamma}{\partial u} \beta=0, & \sum \frac{\partial \gamma}{\partial v} \alpha=0, & \sum \frac{\partial \gamma}{\partial v} \beta=0 .
\end{array}
$$

Consequently the spheres $S, S_{1}$ and $S_{-1}$ are represented in 5-space by a congruence and its first and minus first derived congruences whose lines are perpendicular to the tangents to the net representing $C$. Conversely, if we have such a configuration in 5-space, the corresponding spheres $S, S_{1}, S_{-1}$, in 3-space are orthogonal to the congruences of spheres harmonic to the circles $C$ represented by the net. Consequently the points of contact of $S$ with its envelope are the poles of $C$. Since the centers of the spheres lie in the planes of $C$, and these planes are the tangent planes of the central net of $S$, the spheres are orthogonal to the circles. Hence:

A necessary and sufficient condition that a congruence of spheres and a congruence of circles be orthogonal is that the congruence of spheres and its first and minus first derived congruences be represented in 5-space by congruences of lines perpendicular to the tangents to the curves of the net representing the congruence of circles.

I 10. Transformations $F$ of congruences of circles. Consider the congruence $\bar{S}$ of spheres and the circles $C$ harmonic to them, as defined in the preceding section, and in addition a second congruence of circles $C_{1}$ harmonic to $\bar{S}$. Let $N_{1}\left(x_{1}\right)$ be the net enveloped by the planes of these circles and $\varphi_{1}$ be the solution of the point equation of $N_{1}$ determining the congruence of spheres with centers on $N_{1}$ and orthogonal to $\bar{S}$. Then

$$
\rho_{1}=\sum \bar{x} x_{1}-\bar{\theta},
$$

and

$$
\frac{\partial \boldsymbol{\varphi}_{1}}{\partial u}=\sum \bar{x} \frac{\partial x_{1}}{\partial u}, \quad \frac{\partial \boldsymbol{\varphi}_{1}}{\partial v}=\sum \bar{x} \frac{\partial x_{1}}{\partial v} .
$$


From the fifth theorem of $\S 105$ it follows that $N_{1}$ is an $F$ transform of $N$, or is parallel to $N$, the nets $N$ and $N_{1}$ being conjugate to the congruence $G$ of lines joining the points where $\bar{S}$ touches its envelope. Consider first the case where $N$ and $N_{1}$ are not parallel, then

$$
x_{1}=x-\frac{\theta}{\theta^{\prime}}{ }^{\prime} x^{\prime}
$$

where $\theta$ is a solution of the point equation of $N$, the direction-parameters $x^{\prime}$ of $G$ and $\theta^{\prime}$ being in the relations
$\frac{\partial x^{\prime}}{\partial u}=h \frac{\partial x}{\partial u}$
$\frac{\partial x^{\prime}}{\partial v}=l \frac{\partial x}{\partial v}$
$\frac{\partial \theta^{\prime}}{\partial u}=h \frac{\partial \theta}{\partial u}$
$\frac{\partial \boldsymbol{\theta}^{\prime}}{\partial v}=l \frac{\partial \theta}{\partial v}$

Since $G$ is normal to the tangent plane to $\bar{N}$, we have

$$
\sum x^{\prime} \frac{\partial \bar{x}}{\partial u}=0, \quad \sum x^{\prime} \frac{\partial \bar{x}}{\partial v}=0
$$

and consequently $\varphi^{\prime}$ defined by

satisfies the equations

$$
\varphi^{\prime}=\sum x^{\prime} \bar{x}
$$

$$
\frac{\partial \varphi^{\prime}}{\partial u}=h \frac{\partial \varphi}{\partial u}, \quad \frac{\partial \varphi^{\prime}}{\partial v}=l \frac{\partial \varphi}{\partial v} .
$$

From (72), (74), (76) and (79) we have

$$
\varphi_{1}=\varphi-\frac{\theta}{\theta^{\prime}} \varphi^{\prime}
$$

that is $\varphi_{1}$ is the transform of $\varphi$.

Confversely, let $N$ and $N_{1}$ be two nets in relation $F$, and $\varphi$ and $\varphi_{1}$ be corresponding solutions of their point equations in the relation (81). Let $C$ and $C_{1}$ be congruences of circles in the planes of $N$ and $N_{1}$ determined by the functions $\varphi$ and $\varphi_{1}$ respectively. The equations of the axes of these circles are 


$$
\sum y \frac{\partial x}{\partial u}-\frac{\partial \varphi}{\partial u}=0, \quad \sum y \frac{\partial x}{\partial v}-\frac{\partial \varphi}{\partial v}=0
$$

and

$$
\sum y \frac{\partial x_{1}}{\partial u}-\frac{\partial \varphi_{1}}{\partial u}=0, \quad \sum y \frac{\partial x_{1}}{\partial v}-\frac{\partial \varphi_{1}}{\partial v}=0
$$

These two lines are found to intersect in consequence of (76) and (81). If we denote by $\bar{x}$ the coordinates of the point of intersection $\bar{M}$, we find that they satisfy (82) and (79).

From (79) and (80) equations (78) follow. In view of this result and the fact that $x^{\prime}$ and $\varphi^{\prime}$ are solutions of the point equation of $N^{\prime}$, equation (79) is the tangential equation of a net, and therefore $\bar{M}$ describes a net conjugate to both axes.

From the preceding section it follows that the spheres $\bar{S}$ with centers $\bar{M}$ and radius determined by $\bar{\theta}$ given by (72) are harmonic to the circles $C$. But by means of (76), (79) and (81) we find that (74) is a consequence of (72). Hence the-spheres $\bar{S}$ are likewise harmonic to $C_{1}$.

We say that the circles $C$ and $C_{1}$ whose planes envelope nets in relation $F$ and which are determined by solutions of the point equations of these nets in - the relation (81) are $F$ transforms of one another, or simply in relation $F$. Hence:

- Two congruences of circles which are F transforms of one another are harmonic to a congruence of spheres, and two congruences of circles harmonic to a congruence of spheres are $F$ transforms of one another unless the corresponding circles lie in parallel planes.

\section{Incidentally we have:}

When two congruences of circles are in relation $F$, corresponding circles meet in the two points where each circle cuts the corresponding line of the harmonic congruence of the transformation $F$ of the nets enveloped by the planes of the circles.

If $N, N_{1}, N_{2}$ and $N_{12}$ are four nets of a quatern in relations $F$ $(\$ 21)$ and the planes of congruences of circles $C, C_{1}$ and $C_{2}$ envelope the respective nets $N, N_{1}$ and $N_{2}$ and are determined by functions $\varphi, \varphi_{1}$ and $\varphi_{2}$ respectively, where

$$
\varsigma_{1}=\varsigma-\frac{\theta_{1}}{\theta_{1}^{\prime}} \varphi^{\prime}, \quad \varphi_{2}=\varphi-\frac{\theta_{2}}{\theta_{2}^{\prime \prime}} \varphi^{\prime \prime},
$$


the function $\varphi_{12}$, defined by

$$
\theta_{1} \theta_{12}^{\prime \prime \prime} \varsigma_{12}=\frac{\theta_{2}^{\prime \prime}}{\theta_{2}}\left(\theta_{2} \theta_{21} \varphi_{1}+\theta_{1} \theta_{12} \varphi_{2}-\theta_{12} \theta_{21} \varphi\right)
$$

is the corresponding solution of the point equation of $N_{12}$, whose coordinates are given by (II, 49). Hence the circles $C_{12}$ whose planes envelope $N_{12}$ and which are determined by $\varphi_{12}$ are $F$ transforms of $C_{1}$ and $C_{2}$.

We shall give another geometric interpretation of transformations $F$ of congruences of circles. Take two congruences of circles conjugate to the same congruence of spheres. Since their axes are harmonic to the net of centers of the spheres, the intersections of corresponding axes form a net, and consequently the spheres with centers at points of this net and containing the circles form a congruence of spheres harmonic to the two congruences of circles. Hence:

Two congruences of circles conjugate to a congruence of spheres are in the relation of a transformation $F$.

As a corollary we have:

The nets enveloped by the planes of the circles of two congruences of circles conjugate to a congruence of spheres are in the relation of a transformation $F$.

In consequence of the last theorem of $\S 107$ and the second of $\S 110$ we have:

Two congruences of circles in the relation of a transformation $F$ are conjugate to a unique congrience of spheres.

From the foregoing theorems follows the theorem:

Any two congruences of circles harmonic to a congruence of spheres are conjugate to a congruence of spheres, and conversely.

Since two congruences of circles in relation $F$ are conjugate to a congruence of spheres, it follows from that the nets in 5-space representing the congruences of circles are conjugate to the congruence of lines representing the spheres. Hence:

The nets in 5-space representing congruences of circles in relation $F$ are $F$ transforms of one another; and any two nets in 5-space in relation $F$ correspond to congruences of circles in relation $F$.

The theorem preceding this one is a consequence of the latter and the fact that two nets in relation $F$ are harmonic to a congruence. 
I I I. Parallel transformations of congruences of circles. We consider the exceptional case where the corresponding planes of two congruences of circles harmonic to a congruence of spheres are parallel. Now

$$
\frac{\partial x_{1}}{\partial u}=h \frac{\partial x}{\partial u}, \quad \frac{\partial x_{1}}{\partial v}=l \frac{\partial \varphi}{\partial v},
$$

and consequently (75) is satisfied by

$$
\frac{\partial \varphi_{1}}{\partial u}=h \frac{\partial \varphi}{\partial u}, \quad \frac{\partial \varphi_{1}}{\partial v}=l \frac{\partial \varphi}{\partial v} .
$$

Suppose, conversely, that we have two congruences of circles, $C$ and $C_{1}$, whose planes envelope parallel nets $N(x)$ and $N_{1}\left(x_{1}\right)$, and the circles are determined by corresponding functions $\varphi$ and $\varphi_{1}$, that is in the relation (86), we say they are parallel transforms of one another.

We consider the point $\bar{M}$ of coordinates $\bar{x}$ defined by (73) and

$$
\sum \bar{x}\left(x_{1}-x\right)=\varphi_{1}-\varphi .
$$

By differentiating this equation and making use of (73), (85) and (86), we find

$$
\sum \frac{\partial \bar{x}}{\partial u}\left(x_{1}-x\right)=0, \quad \sum \frac{\partial \bar{x}}{\partial v}\left(x_{1}-x\right)=0 .
$$

Since $x_{1}-x$ and $\varphi_{1}-\varphi$ satisfy an equation of the Laplace type, they are the tangential coordinates of a net, the locus of $\bar{M}$. From $\S 109$ and (87) it follows that the spheres of center $\bar{M}$ and radius determined by $\bar{\theta}$ given by (72) are harmonic to the circles $C$ and $C_{1}$. Hence:

Two congruences of circles which are parallel transforms of one another are harmonic to a sphere; and congruences of circles harmonic to a sphere such that corresponding planes are parallel, are parallel transforms of one another.

I12. Congruences of spheres with applicable central nets. Let $N(x)$ and $\bar{N}(\bar{x})$ be two applicable nets in 3-space. Since they have the same point equation, the function 


$$
2 \theta_{0}=\sum x^{2}-\sum x^{2}
$$

is a solution of this equation. If $\theta$ is a solution of this equation, the spheres $\bar{S}$ with centers on $\bar{N}$ and radii given by

$$
R^{2}=\sum \bar{x}^{2}-2 \theta
$$

form a congruence. Since this may be written

$$
R^{2}=\sum x^{2}-2\left(\theta+\theta_{0}\right)
$$

the spheres $S$ of radius $R$ and centers on $N$ also form a congruence. Moreover, corresponding spheres of these two congruences touch their envelopes at the same points when $N$ and $\bar{N}$ are applied to one another. This result follows from the following theorem of Beltrami which is a consequence of the last theorem of [\$ 164]:

When the surface of centers of a two parameter family of spheres is deformed, the points of contact of the spheres with their envelope (which itself changes in the deformations) are fixed points with respect to the spheres.

When $\theta=0$ in (89), the spheres $\bar{S}$ pass through the origin. The envelope of these spheres is the origin $O$ and the locus of the point $O^{\prime}$ symmetric to $O$ with respect to the tangent planes to $\bar{N}$. In accordance with the above theorem, when $\bar{N}$ is applied to $N$, the loci of $O$ and $O^{\prime}$ are the sheets of the envelope of the spheres. Since $O$ and $O^{\prime}$ are the points of contact, we have by $\S 109$ that the null spheres with centers at $O$ and $O^{\prime}$ meet the tangent planes to $N$ in a congruence of circles orthogonal to the spheres $S$. Moreover, as $\vec{N}$ may be displaced in space the null sphere at the origin may be replaced by any fixed null sphere. Hence:

If $N$ and $\bar{N}$ are applicable nets, the circles, in which the tangent planes of $\bar{N}$ are met by a fixed null sphere, become a congrience of circles when $\bar{N}$ is applied to $N$.

I 3. Generation of cyclic systems. It is our purpose now to show that the congruences of circles referred to in the last theorem are cyclic systems $(\S 79)$ and that all cyclic systems can be obtained in this manner. 
Suppose we have a surface $S$ referred to a general moving trihedral. From $[\S 69,(51)]$ it follows that if $\left(x_{0}, y_{0}, z_{0}\right)$ is a point fixed in space, it is necessary and sufficient that

$$
\begin{array}{ll}
\frac{\partial x_{0}}{\partial u}+\xi+q z_{0}-r y_{0}=0, & \frac{\partial x_{0}}{\partial v}+\xi_{1}+q_{1} z_{0}-r_{1} y_{0}=0, \\
\frac{\partial y_{0}}{\partial u}+\eta+r x_{0}-p z_{0}=0, & \frac{\partial y_{0}}{\partial v}+\eta_{1}+r_{1} x_{0}-p_{1} z_{0}=0, \\
\frac{\partial z_{0}}{\partial u}+p y_{0}-q x_{0}=0, & \frac{\partial z_{0}}{\partial v}+p_{1} y_{0}-q_{1} x_{0}=0 .
\end{array}
$$

If we put

$$
\begin{array}{ll}
A=\frac{\partial x_{0}}{\partial u}+\xi-r y_{0}, & A_{1}=\frac{\partial x_{0}}{\partial v}+\xi_{1}-r_{1} y_{0}, \\
B=\frac{\partial y_{0}}{\partial u}+\eta+r x_{0}, & B_{1}=\frac{\partial y_{0}}{\partial v}+\eta_{1}+r_{1} x_{0},
\end{array}
$$

the first four of the above equations may be written

(91) $q z_{0}=-A, \quad q_{1} z_{0}=-A_{1} . \quad p z_{0}=B, \quad p_{1} z_{0}=B_{1}$,

and the last two

$$
z_{0} \frac{\partial z_{0}}{\partial u}=-\left(x_{0} A+y_{0} B\right) . \quad z_{0} \frac{\partial z_{0}}{\partial v}=-\left(x_{0} A_{1}+y_{0} B_{1}\right) .
$$

It follows from (91) that

$$
\left(p q_{1}-p_{1} q\right) z_{0}^{2}-\left(A B_{1}-A_{1} B\right)=0 .
$$

Comparing these results with equations [§ 174, (12), (13)], we have that the circles of radius $i z_{0}$ and center $\left(x_{0}, y_{0}\right)$ lying in the plane $z=0$ of the moving trihedral form a cyclic system. Evidently these are the circles in which these planes are met by the fixed null sphere

$$
\sum\left(x-x_{0}\right)^{2}=0 .
$$

Conversely suppose we have any cyclic system consisting of circles, of center $\left(x_{0}, y_{0}\right)$ and radius $R$, lying in the plane $z=0$; then $x_{0}, y_{0}, R$ must satisfy $[\S 174,(12),(13)]$. Since these equations 
involve only the first fundamental coefficients of $S$, an infinity of cyclic systems is obtained by the deformation of the envelope of the planes of the circles. As the values of $p, q, p_{1}, q_{1}$ given by (91) satisfy $[\S 69,(48)]$ one of the deforms of $S$ is determined by these values. The point whose coordinates with respect to its moving trihedral are $x_{0}, y_{0},-i R$ is fixed in space and consequently we have the theorem of Darboux ${ }^{94}$ ):

$A$ general cyclic system of circles situated in the tangent planes to a surface $S$ is obtained by taking a deform of $S$, say $\bar{S}$, constructing the circles $\bar{C}$ of intersection of the tangent planes of $\bar{S}$ by a fixed null sphere and then taking the positions of $\bar{C}$ when $\bar{S}$ is deformed into $S$.

If the center of the fixed null sphere is $\left(a_{1}, a_{2}, a_{3}\right), 2 \theta$ in (89) is $2 \sum a \bar{x}-\sum a^{2}$, and consequently we have:

If $N(x)$ and $\overline{N(} \bar{x})$ are applicable nets, the circles in the tangent planes of $N(x)$ determined by the solution $\sum x^{2}-\sum(\bar{x}-a)^{2}$ of its point equation form a cyclic system.

In other words we have:

If $N(x)$ and $\overline{N(} \bar{x})$ are applicable nets, the circles in the tangent planes of $N$ which have for poles the points of contact with their envelope of spheres with centers on $N$ and radii given by

$$
R^{2}=\sum(\bar{x}-a)^{2}
$$

form a cyclic system.

I 14. Transformations $F$ of cyclic systems. In $\$ 110$ we established transformations $F$ of congruences of circles. Now we wish to show that certain of these transformations transform a cyclic system into cyclic systems ${ }^{95}$ ).

Let $N(x)$ be the net enveloped by the planes of the cyclic system $C, \bar{N}(\bar{x})$ the applicable net and $\varphi$ given by

$$
2 \varphi=\sum x^{2}-\sum \bar{x}^{2}
$$

the function determining the cyclic system, the point equation of $N$ and $\bar{N}$ being (36).

94) Leçons, vol. 3, p. 354 .

$\left.{ }^{95}\right)$ Proc. Nat. Acad. Sci., vol. 5 (1919), p. 555. 
In $\S 27$ we showed that if $h$ and $l$ is any pair of solutions of the system

$$
\frac{\partial h}{\partial v}=(l-h) \frac{\partial \log a}{\partial v}, \quad \frac{\partial l}{\partial u}=(h-l) \frac{\partial \log b}{\partial u},
$$

the nets $N^{\prime}\left(x^{\prime}\right)$ and $\bar{N}^{\prime}\left(\bar{x}^{\prime}\right)$, whose coordinates are given by

(94) $\frac{\partial x^{\prime}}{\partial u}=l \frac{\partial x}{\partial u}, \quad \frac{\partial x^{\prime}}{\partial v}=l \frac{\partial x}{\partial v} ; \quad \frac{\partial \bar{x}^{\prime}}{\partial u}=h \frac{\partial \bar{x}}{\partial u}, \quad \frac{\partial \bar{x}^{\prime}}{\partial v}=l \frac{\partial \bar{x}}{\partial v}$,

are applicable, and parallel to $N$ and $\bar{N}$ respectively. Also, if $\theta^{\prime}$ is the solution of the point equations of $N^{\prime}$ and $\overline{N^{\prime}}$ defined by

$$
\theta^{\prime}=\sum x^{\prime 2}-\sum \bar{x}^{\prime 2}
$$

and $\theta$ is given by

$$
\frac{\partial \theta^{\prime}}{\partial u}=h \frac{\partial \theta}{\partial u}, \quad \frac{\partial \theta^{\prime}}{\partial v}=l \frac{\partial \theta}{\partial v},
$$

the nets $N_{1}\left(x_{1}\right)$ and $\bar{N}_{1}\left(\bar{x}_{1}\right)$, whose coordinates are defined by

$$
x_{1}=x-\frac{\theta}{\theta^{\prime}} x^{\prime}, \quad \bar{x}_{1}=\bar{x}-\frac{\theta}{\theta^{\prime}} \bar{x}^{\prime},
$$

are applicable, and are $F$ transforms of $N$ and $\bar{N}$ respectively.

The circles $C_{1}$ lying in the tangent planes of $N_{1}$ and determined by the function $\varphi_{1}$, given by (81), form a congruence which is an $F$ transform of $C$ (§ 110). These circles form a cyclic system, if

$$
2 \varphi_{1}=\sum x_{1}^{2}-\sum \bar{x}_{1}^{2}
$$

Substituting the values from (81) and (97), we find

$$
\varphi^{\prime}=\sum x x^{\prime}-\sum \bar{x} \bar{x}^{\prime}-\frac{\theta}{2} .
$$

As this function satisfies (80), we have:

If $N(x)$ and $\bar{N}(\bar{x})$ are applicable nets and $N^{\prime}\left(x^{\prime}\right)$ and $\bar{N}^{\prime}\left(\bar{x}^{\prime}\right)$ are applicable nets parallel to them, and $N_{1}$ is the F transform of 
$N$ by means of $N^{\prime}$ and the corresponding functions $\theta$ and $\theta^{\prime}=\sum x^{\prime}$ $-\sum \bar{x}^{\prime 2}$, the circles in the tangent planes of $N_{1}$ determined by $\varphi_{1}$, where

$$
2 \varphi_{1}=\sum x^{2}-\sum \bar{x}^{2}-2 \frac{\theta}{\theta^{\prime}}\left(\sum x x^{\prime}-\sum \bar{x} \bar{x}^{\prime}-\frac{\theta}{2}\right),
$$

form a cyclic system which is an $F$ transform of the cyclic system of circles in the planes of $N$ determined by $2 \varphi=\sum x^{\mathbf{2}}-\sum \bar{x}^{2}$.

We remark that similar results hold for the cyclic systems of circles in the tangent planes of $\bar{N}$ and $\bar{N}_{1}$ determined by $\bar{\varphi}=-\varphi$ and $\bar{\varphi}_{1}=-r_{1}$ respectively.

We shall show that these transformations admit a theorem of permutability. To this end we take two nets $N^{\prime}\left(x^{\prime}\right)$ and $N^{\prime \prime}\left(x^{\prime \prime}\right)$ parallel to $N$ and the nets $\overline{N^{\prime}}$ and $\bar{N}^{\prime \prime}$ parallel to $\bar{N}$ and applicable to $N^{\prime}$ and $N^{\prime \prime}$ respectively. By means of these we determine two $F$ transforms, $N_{1}$ and $N_{2}$, of $N$, and $\bar{N}_{1}$ and $\bar{N}_{2}$ of $\bar{N}$ such that $N_{1}$ and $\bar{N}_{1}$ are applicable, and also $N_{2}$ and $\bar{N}_{2}$. If we choose the additive constants in the functions $\theta_{1}^{\prime \prime}$ and $\theta_{2}^{\prime}$ so that (cf. II, 97)

$$
\theta_{1}^{\prime \prime}+\theta_{2}^{\prime}-2\left(\sum x^{\prime} x^{\prime \prime}-\sum \bar{x}^{\prime} \bar{x}^{\prime \prime}\right)=0
$$

there are $\infty^{1}$ pairs of applicable nets $N_{12}$ and $\bar{N}_{12}$ such that $N$, $N_{1}, N_{2}, N_{12}$ and $\bar{N}, \overline{N_{1}}, \overline{N_{2}}, \bar{N}_{12}$ are quaterns under transformations $F$.

In accordance with the preceding theorem, we set up a cyclic system in the planes of $N_{1}$ with circles determined by $\rho_{1}$ given by (98), and a cyclic system in the planes of $N_{2}$ with circles determined by

$$
\varphi_{2}=\varphi-\frac{\theta_{2}}{\theta_{2}^{\prime \prime}}\left(\sum x x^{\prime \prime}-\sum \bar{x} \bar{x}^{\prime \prime}-\frac{\theta_{2}}{2}\right)
$$

Since $\varphi_{1}$ and $\varphi_{2}$ are transforms of $\varphi$ by (81) and an analogous equation, it follows that a solution $\varphi_{12}$ of the point equation of $N_{12}$ is given by (84).

In order that $\varphi_{12}$ shall define a cyclic system for $N_{12}$, we must have, in accordance with (98),

$$
\varphi_{12}=\varphi_{1}-\frac{\theta_{12}}{\theta_{12}^{\prime \prime \prime}}\left(\sum x_{1} x_{1}^{\prime \prime \prime}-\sum \bar{x}_{1} \bar{x}_{1}^{\prime \prime \prime}-\frac{\theta_{12}}{2}\right) .
$$

It is readily found that this condition is satisfied identically. Hence: 
If $C_{1}$ and $C_{2}$ are two cyclic systems which are $F$ transforms of a cyclic system $C$, there can be found by quadratures $\infty^{1}$ cyclic system $C_{12}$ which are $F$ transforms of $C_{1}$ and $C_{2}$.

115. Cyclic systems in 3-space and nets $O$ in 5-space. From the theorem of $[\S 177]$ it follows that the spheres focal to a cyclic system are orthogonal. Consequently a cyclic system is represented in 5-space by an $O$ net $(\$ 98)$. We consider the converse problem, taking an $O$ net $N$ in 5 -space corresponding to the determinant

We recall that

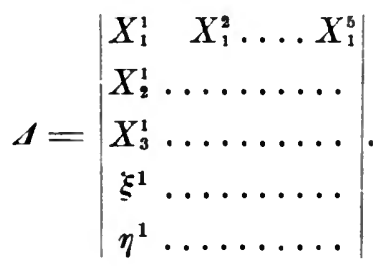

$$
\left\{\begin{array}{rlrl}
\frac{\partial \xi}{\partial v} & =n \eta, & & \frac{\partial \eta}{\partial u}=m \xi, \\
\frac{\partial X_{k}}{\partial u} & =a_{k} \xi, & & \frac{\partial X_{k}}{\partial v}=b_{k} \eta \\
\frac{\partial a_{k}}{\partial v} & =m b_{k}, & & \frac{\partial b_{k}}{\partial u}=n a_{k} .
\end{array} \quad(k=1,2,3),\right.
$$

Let $\Sigma_{1}$ and $\Sigma_{2}$ denote the spheres of coordinates $\xi$ and $\eta$ respectively. If $\alpha$ are the coordinates of the congruence of spheres $S$ orthogonal to the circles $C$ represented by $N$, we have

$$
\sum \alpha \xi=0, \quad \sum \alpha \eta=0 .
$$

Since the two derived spheres of $S$ must also be orthogonal to $\Sigma_{1}$ and $\Sigma_{2}$, we must have also (§97)

(102) $\sum \frac{\partial \alpha}{\partial u} \xi=0, \quad \sum \frac{\partial \alpha}{\partial v} \xi=0, \quad \sum \frac{\partial \alpha}{\partial u} \eta=0, \quad \sum \frac{\partial \alpha}{\partial v} \eta \doteq 0$.

In order that (101) be satisfied, we must have

$$
\alpha=\sum_{k=1}^{3} \bar{x}_{k} X_{k}
$$


where $\bar{x}_{1}, \bar{x}_{2}, \bar{x}_{3}$ are determined by (102). Making use of (100), we find that they must satisfy

$$
\sum \bar{x}_{k} a_{k}=0, \quad \sum \bar{x}_{k} b_{k}=0,
$$

and consequently they are determined to within a factor, say $t$. Now

$$
\begin{array}{cc}
\frac{\partial \alpha}{\partial u}=\sum \frac{\partial \bar{x}_{k}}{\partial u} X_{k} & \frac{\partial \alpha}{\partial v}=\sum \frac{\partial \bar{x}_{k}}{\partial v} X_{k}, \\
\sum \frac{\partial \bar{x}_{k}}{\partial v} a_{k}=0, & \sum \frac{\partial \bar{x}_{k}}{\partial u} b_{k}=0 .
\end{array}
$$

From these equations it is readily shown that the $\bar{x}$ 's satisfy the same Laplace equation as the $\alpha$ 's. If we choose the factor $t$ so that $\alpha_{1}, \alpha_{2}, \alpha_{3}$ are the coordinates $x_{1}, x_{2}, x_{3}$ of the net $N$ of centers of $S$, this equation assumes the form (36), and consequently $\bar{x}_{1}, \bar{x}_{2}, \bar{x}_{3}$ are the coordinates of a net $\bar{N}$. Moreover, from (5), (104) and (105) we have

$$
R^{2}=\sum \alpha^{2}=\sum \bar{x}_{k}^{2}, \quad \sum d \alpha^{2}=\sum d x^{2}=\sum d \bar{x}^{2} .
$$

Hence $N$ and $\bar{N}$ are applicable, and the conditions of the second theorem of $\S 113$ are satisfied, so that we have:

$A$ necessary and sufficient condition that a congruence of circles be a cyclic system is that it be represented in 5-space by an $O$ net.

From this result and the last theorem of $\S 110$ we have: The problem of transformations $F$ of cyclic systems is the same as of transformations $F$ of $O$ nets into $O$ nets in 5-space.

The centers of the spheres $\Sigma_{1}$ and $\Sigma_{2}$ are the focal points of the congruence $G$ of axes of the cyclic system. Their homogeneous coordinates are

$$
\xi^{1}, \xi^{2}, \xi^{3},-\left(\xi^{5}+i \xi^{4}\right) ; \quad \eta^{1}, \eta^{2}, \eta^{3},-\left(\eta^{5}+i \eta^{4}\right) .
$$

Since the homogeneous coordinates of the net $\hat{N}_{k}$ of the centers of the sphere $S_{k}$ of coordinates $X_{k}$ are

$$
X_{k}^{1}, X_{k}^{2}, X_{k}^{3},-\left(X_{k}^{5}+i X_{k}^{4}\right),
$$

it follows from (100) and $\S 33$ that the nets $\hat{N}_{k}$ are harmonic to $G$. 
Moreover, the cartesian coordinates $\hat{x}$ of $\hat{N}_{k}$ satisfy an equation of the form (36), where

$$
a=\frac{a_{k}}{X_{k}^{b}+i X_{k}^{4}}, \quad b=\frac{b_{k}}{X_{k}^{3}+i X_{k}^{4}} .
$$

As $R_{k}=1 /\left(X_{k}^{5}+i X_{k}^{4}\right)$ and $\left(X_{k}^{5}-i X_{k}^{4}\right) /\left(X_{k}^{5}+i X_{k}^{4}\right)$ are solutions, so also is $\sum \hat{x}_{k}^{2}-R_{k}^{2}$. Hence the nets $\dot{N}_{k}$ are $2, O$.

The equation of the sphere $S_{1}$ is

$$
\sum X_{1} z=0
$$

The pentaspherical coordinates $z$ and $z_{1}$ of the points where it touches its envelope are common solutions of this equation, and

$$
\sum \xi z=0, \quad \sum \eta z=0
$$

Since they must satisfy $\sum z^{2}=0$ also, we have that they may be taken as

$$
z=X_{2}+i X_{3}, \quad z_{1}=X_{2}-i X_{3}
$$

These points lie on the circle of intersection of the spheres $\Sigma_{1}$ and $\Sigma_{2}$, namely (106), and since their pentaspherical coordinates satisfy an equation of the Laplace type (26), the points describe $O$ nets $N$ and $N_{1}(\S 95)$. As in the case of $\hat{N}_{k}$, these nets are harmonic to the congruence $G$, and consequently the net is orthogonal to the circles. Similar results follow for the spheres $S_{2}$ and $S_{3}$, which proves again that the circles of intersection of the spheres $\Sigma_{1}$ and $\Sigma_{2}$ form a cyclic system.

In the same manner it is shown that if $a_{1}, a_{2}, a_{3}$ are any constants satisfying the isotropic relation $\sum a^{2}=0$, the quantities $\sum a_{k} X_{k}$ are pentaspherical coordinates of an $O$ net orthogonal to the circles. Hence:

The last two rows of an orthogonal determinant of the fifth order determine a cyclic system; any isotropic linear functions of the terms of the first three rows are pentaspherical coordinates of an $O$ net orthogonal to the cyclic system. 
I 16. Cyclic congruences. In $\S 73$ we found that a necessary condition that a congruence be cyclic is that the direction-parameters satisfy the relation

$$
\sum X^{2}=A^{2} U^{2}+B^{2} V^{2}
$$

where $U$ and $V$ are functions of $u$ and $v$ alone respectively, and $A$ and $B$ are the functions which appear in the direction-equation of the congruence (26). Guichard ${ }^{96}$ ) has shown that this condition is also sufficient for space of any order. We adapt his proof to the case of 3-space.

In $\S 30$ we saw that the homogeneous coordinates $z^{i}$ and $y^{i}$, of the foci of a congruence can be chosen so that

$$
\frac{\partial z}{\partial u}=m y, \quad \frac{\partial y}{\partial v}=n z
$$

in which case the direction-parameters

$$
X^{i}=\frac{y^{i}}{y^{4}}-\frac{z^{i}}{z^{4}}(i=1,2,3)
$$

satisfy an equation (26) with

$$
A=\frac{1}{y^{4}}, \quad B=\frac{1}{z^{4}} .
$$

Since $A$ and $B$ are determined to within respective factors $U$ and $V$, functions of $u$ and $v$ alone, the condition (108) may be written

$$
\sum X^{2}=\frac{1}{y^{2_{4}}}+\frac{1}{z^{2_{4}}}
$$

If we define functions $\xi$ and $\eta$ by the equations

$$
\begin{gathered}
\xi^{i}=y^{i}, \quad \eta^{i}=z^{i}(i=1,2,3), \\
-\left(\xi^{5}+i \xi^{4}\right)=y^{4}, \quad-\left(\eta^{5}+i \eta^{4}\right)=z^{4}, \\
\sum_{i=1}^{5} \xi^{2}=1, \quad \sum_{i=1}^{5} \eta^{2}=1,
\end{gathered}
$$

96) Annales L'École Norm. Sup., ser. 3, vol. 15 (1898), p. 203. 
the condition (110) reduces to

$$
\sum_{i=1}^{5} \xi \eta=0 .
$$

Moreover, from (109) it follows that the functions $\xi$ and $\eta$ satisfy the equations (100). Hence the $\xi$ 's and $\eta$ 's are the elements of the last two rows of a determinant $\boldsymbol{A}$ of the fifth order. In consequence of the preceding section, we have that the congruence is harmonic to $O$ nets. Hence:

A necessary and sufficient condition that the direction-parameters of a congruence in 3-space be cyclic, that is harmonic to a net $O$, is that its parameters satisfy a condition of the form (108).

\section{Exercises.}

1. Show that $z_{i}=\frac{1}{R_{i}}$ are the pentaspherical coordinates of any point of the plane at infinity not on the circle at infinity; and that a point on the circle of inflnity has an infinite set of coordinates of the form $z_{i}=z_{i}^{\prime}+h / R_{i}$, where $h$ is any constant and $\Sigma z_{i}^{\prime 2}=0, \Sigma z_{i}^{\prime} / R_{i}=0$.

Darboux, Leçons, $2^{\text {nd }}$ ed., vol. 1, p. 270.

2. When two spheres are orthogonal, the center of either is the pole of their radical plane with respect to the other; consequently any one of the five spheres of $\S 94$ is conjugate with respect to the tetrahedron whose vertices are the centers of the other four spheres. Darboux, Principes de Géom. Anal. p. 384.

3. If a sphere $S$ of center $(a, b, c)$ and radius $R$ is subjected to an inversion with respect to the sphere $S_{0}$ of center $(0,0,0)$ and radius $k$, the center $\left(a^{\prime}, b^{\prime}, c^{\prime}\right)$ and radius $R^{\prime}$ of the transform $S^{\prime}$ are given by

$$
\frac{a^{\prime}}{a}=\frac{b^{\prime}}{b}=\frac{c^{\prime}}{c}=\frac{R^{\prime}}{R}=\frac{k^{2}}{a^{2}+b^{2}+c^{2}-R^{2}} .
$$

When $S$ is orthogonal to $S_{0}, S^{\prime}$ coincides with $S$.

Darboux, l. c., p. 386.

4. When in Ex. 3 we replace $S$ by $S_{0}$, we have $R^{\prime}=-R$. Consequently the pentaspherical coordinates $z_{k}$ of a point $P$ and its inverse $P^{\prime}$ with respect to the fundamental sphere $S_{k}$ differ in sign, but the other four coordinates of $P$ and $P^{\prime}$ are respectively equal. Hence five successive inversions with respect to the five fundamental spheres transform a point into itself. Darboux, l. c., p. 387.

5. From (19) it follows that the equation in pentaspherical coordinates $z$ of a sphere of radius $\rho$ and center of pentaspherical coordinates $z_{i}^{\prime}$ is

$$
\underset{i}{2 \Sigma_{i} z_{i}^{\prime}+\rho^{2}} \sum_{i} \frac{z_{i}}{R_{i}} \sum_{i} \frac{z_{i}^{\prime}}{R_{i}}=0
$$


Hence the pentaspherical coordinates of the center and the radius of the splere $\Sigma a_{i} z_{i}=0$ are given by

$$
z_{i}^{\prime}=a_{i}-\frac{1}{2 R_{i}} \frac{\sum a_{i}^{2}}{\sum \frac{a_{i}}{R_{i}}}, \quad \rho=\frac{\sqrt{\Sigma a_{i}^{2}}}{\sum \frac{a_{i}}{R_{i}}} .
$$

Darboux, 1. c., p. 390.

6. If in (20) we replace $d z_{j}$ by $d z_{j}+k \delta z_{j}$ and equate the coefficients of $k$ on both sides of the equation, we get

$$
d s \delta s \cos (d s, \delta s)=\frac{\Sigma d z_{j} \cdot \delta z_{j}}{\left(\sum \frac{z_{j}}{R_{j}}\right)^{2}}
$$

for the angle between two displacements.

Darboux, 1. c., p. 389.

7. A homogeneous equation $\varphi\left(z_{1}, \ldots, z_{3}\right)=0$ defines a surface. For a displacement on the surface $\sum \frac{\partial \varphi}{\partial z_{i}} d z_{i}=0$. If we put $\delta z_{i}=\lambda \frac{\partial \varphi}{\partial z_{i}}$, we have $\Sigma z_{i} \grave{\delta} z_{i}=\lambda \Sigma_{i} \frac{\partial \varphi}{\partial z_{i}}=0$, since $\varphi$ is homogeneous. From Ex. 6 it follows that the displacement $\delta z_{i}$ is normal to the surface.

Darboux, 1. c., p. 403.

8. If $\varphi\left(z_{1}, \ldots z_{5}\right)=0$ and $\downarrow\left(z_{1}, \ldots z_{5}\right)=0$ define two surfaces, the angle $\theta$ between their tangent planes at any point is given by (cf. Exs. 6, 7)

$$
\cos \theta=\frac{\sum_{i} \frac{\partial \varphi}{\partial z_{i}} \frac{\partial \psi}{\partial z_{i}}}{\sqrt{\sum\left(\frac{\partial \varphi}{\partial z_{i}}\right)^{2} \cdot \sum\left(\frac{\partial \psi}{\partial z_{i}}\right)^{2}}} .
$$

9. The equation

Darboux, l. c., p. 404.

$$
2(u-v) \frac{\partial^{2} \theta}{\partial u \partial v}+\frac{\partial \theta}{\partial u}+\frac{\partial \theta}{\partial v}=0
$$

admits the solutions $\theta=A \sqrt{(u-a)(v-a)}$, where $A$ and $a$ are arbitrary-constants. If we put $f(w)=\sum_{i}\left(w-a_{i}\right)$ where $a_{i}(i=1, \ldots 5)$ are constants, the five functions

$$
z_{i}=\sqrt{\frac{\left(a_{i}-u\right)\left(a_{i}-v\right)\left(a_{i}-h\right)}{f^{\prime}\left(a_{i}\right)}}(i=1, \ldots 5)
$$

satisfy (24) and for each value of the constant $h$ are the peutaspherical coordinates of a surface referred to its lines of curvature. The equation of this one parameter family of surfaces is

$$
\sum \frac{z_{i}^{2}}{a_{i}-h}=0
$$

which, in consequence of (21), is expressible in cartesian coordinates as follows:

(ii) $\frac{y_{1}^{2}}{a_{1}-h}+\frac{y_{8}^{2}}{a_{2}-h}+\frac{y_{3}^{2}}{a_{3}-h}-\frac{\left(y_{1}^{2}+y_{2}^{2}+y_{3}^{2}+1\right)^{2}}{4\left(a_{4}-h\right)}+\frac{\left(y_{1}^{2}+y_{2}^{2}+y_{2}^{8}-1\right)^{2}}{4\left(a_{5}-h\right)}=0$. 
These surfaces of the fourth degree admitting the circle at infinity as a double line are called cyclides.

Darboux, Leçons, $2^{\text {nd }}$ ed., vol. 1, p. 258.

10. Show that

$$
\left(h-h^{\prime}\right) \sum \frac{z_{i}^{2}}{\left(a_{i}-h\right)\left(a_{i}-h^{\prime}\right)}=\sum \frac{z_{i}^{2}}{a_{i}-h}-\sum \frac{z_{i}^{2}}{a_{i}-h^{\prime}},
$$

and consequently two cyclides meet orthogonally along their line of intersection. Show that three cyclides of the family (ii) Ex. 9 pass through a point, and that this equation defines a triply orthogonal system of surfaces.

Darboux, Principes de Géom. Anal., p. 478.

11. A cyclide defined by (i) Ex. 9 is transformed into itself by an inversion with respect to any of the five coordinate spheres (Ex. 4). Darboux, l. c., p. 421.

12. Show that transformations $R$ of an $O$ net defined in pentaspherical coordinates is the same problem as finding congruences conjugate to a net in 5-space on the hypercone (24) (cf. VI, Ex. 3).

13. A necessary and sufficient condition that the spheres of a congruence be orthogonal to a fixed sphere or pass through a point is that $\theta$ in (38) be a linear function of the $x$ 's. Show that in the former case either sheet of the envelope is obtainable from the other by an inversion with respect to the fixed sphere.

14. If the spheres $S$ of a congruence cut a fixed sphere $\Sigma$ under constant angle, different from 0 and $\pi$, by the addition of a constant to the radius of each sphere $S$ the new spheres $S_{1}$ are orthogonal to a fixed sphere $\Sigma_{1}$ concentric with $\Sigma$. Hence the sheets of the envelope of the spheres $S$ being parallel to the sheets of the enrelope of the spheres $S_{1}$ are in relation $R$.

Darboux, Leçons, $2^{\text {nd }}$ ed., vol. 1, p. 310.

15. When the equations (50) of a line in $r$ are written in the form $x=r z+\rho$, $y=s z+\sigma$, the equation of the corresponding sphere in $R$ is

$$
\left(X-\frac{\rho+s}{2 r}\right)^{2}+\left(Y-i \frac{s-\rho}{2 r}\right)^{2}+\left(Z-\frac{\eta-1}{2 r}\right)^{2}=\left(\frac{\eta+1}{2 r}\right)^{2}
$$

where $\eta=s \rho-r \sigma$.

Lie, l. c.

16. To the points in $R$ correspond in $r$ the lines defined by the Pfaffian equation

$$
x d y-y \ddot{d} d x+d z=0 .
$$

Lie, l. c.

17. The surface elements of a surface $\sigma(\S 103)$ are defined by $x, y, z, p, q$. From Ex.16 it follows that the null-plane of the point $(x, y, z)$ is $y x^{\prime}-x y^{\prime}+z-z^{\prime}=0$, where $x^{\prime}, y^{\prime}, z^{\prime}$ are current coordinates. Since this is the tangent plane to $\sigma^{\prime}$, the corresponding surface element of the latter is defined by

$$
x^{\prime}=-q, \quad y^{\prime}=p, \quad z^{\prime}=z-x p-y q, \quad p^{\prime}=y, \quad q^{\prime}=-x .
$$

Lie, l. c., p. 467.

18. The lines through $m(x, y, z)$ of $\sigma$ lying in the tangent plane are defined by

$$
\left(x^{\prime}-x\right)=r\left(z^{\prime}-z\right), \quad y^{\prime}-y=s\left(z^{\prime}-z\right),
$$


where $x^{\prime}, y^{\prime}, z^{\prime}$ are current coordinates, and $r$ and $s$ are such that $p r+q s=1$. To these lines correspond the spheres

(i) $r\left(X^{2}+Y^{2}+Z^{2}\right)-(s+x-r z) X-i(s-x+r z) Y+(1-s x+r y) Z+y-s z=0$. Iie, l. c. that is

19. The tangent plane to $\Sigma$ is defined by (i) Ex. 18 when $r=0, q s=1$,

$$
(x q+1) X-i(x q-1) Y+(x-q) Z+z-q y=0 .
$$

This plane is tangent to each of the spheres (i) at the point $(X, Y, Z)$, where

$$
X+i Y=-z+x \frac{p x+q y}{q+x}, \quad X-i Y=\frac{y-p}{q+x}, \quad Z=-\frac{p x+q y}{q+x},
$$

and the other functions, $P, Q$, of the surface elements of $\Sigma$ are

$$
P=\frac{x q+1}{q-x}, \quad Q=-i \frac{x q-1}{q-x} .
$$

These five equations determine the surface element of $\Sigma$ corresponding to a surface element of $\sigma$.

Lie, l. c.

20. Show that when the equations of Ex. 19 are applied to the surface $\sigma^{\prime}$ we get the same result as for $\sigma$.

Lie, 1. c.

21. Let a line $d$ generate a congruence $G$, its focal points being $A$ and $B$; and let $d_{1}$ and $d_{-1}$ be the corresponding lines of the first and minus first derived congruences of $G$. When this configuration is subjected to a Lie transformation, spheres $S, S_{1}$ and $S_{-1}$ correspond to $d, d_{1}$ and $d_{-1}$; the spheres $S$ and $S_{1}$ are tangent at a point $M_{1}$ of one sheet $\Sigma_{1}$ of the envelope of the spheres $S$, and $S$ and $S_{-1}$ at a point $M_{-1}$ of the other sheet $\Sigma_{-1}$; moreover, the centers $C$ and $C_{1}$ of $S$ and $S_{1}$ are harmonic to the centers of principal curvature of $\Sigma_{1}$ at $M_{1}$ and the centers $C$ and $C_{-1}$ are harmonic to the centers of principal curvature of $\Sigma_{-1}$ at $M_{-1}$. Demoulin, Comptes Rendus, vol. 153 (1911), p. 590.

22. If in the preceding exercise the lines $d$ belong to the linear complex of the transformation, the spheres $S$ are points $M$, the two surfaces $\Sigma_{1}$ and $\Sigma_{-1}$ coincide and are the locus $\Sigma$ of these points; the spheres $S_{1}$ and $S_{-1}$ coincide, are tangent to $\Sigma$ and their centers are harmonic to points of $\Sigma$ with respect to the centers of principal curvature of $\Sigma$; and the lines $d_{1}$ and $d_{-1}$ are conjugate with respect to the complex. Demoulin, 1. c.

23. To a surface with isothermal spherical representation of its linez of curvature corresponds by a Lie transformation a surface upon which there is an $R$ net for which the tangents to all the curves is one family meet a fixed line (cf. VII, Ex. 25).

Demoulin, l. c.

24. To an isothermic surface corresponds by a Lie transformation a surface upon which there is an $R$ net for which the tangents to all the curves in one family are lines of the complex of the transformation.

Demoulin, l. e.

25. When a congruence in 5 -space is $I$, the corresponding congruence of spheres in 3-space is formed by point-spheres, whose centers describe a surface upon which the parametric net $N$ consists of its lines of curvature. The orthogonal 
congruence of circles consists of circle points with centers on $N$ and in the tangent plane of $N$.

Guichard, l. c., p. 199.

26. The spheres of a transformation $R$ in 3-space are represented in 5-space by a congruence 2 , I, such that if the parameters of the congruence are given by (2) with $h=1$, the complementary function is $R$. Conversely any congruence 2, I of spheres gives a transformation of Ribaucour of the sheets of its envelope.

27. A necessary and sufficient condition that on an envelope of spheres the lines of curvature in both systems be spherical is that the six coordinates of the sphere be of the form $a_{i}=U_{i}+V_{i}$, where $U_{i}$ and $V_{i}$ are functions of $u$ and $v$ respectively subject to the condition $\Sigma\left(U_{i}+V_{i}\right)^{2}=0$.

Darboux, Leçons, $2^{\text {nd }}$ ed., vol. 2, p. 357.

28. A necessary and sufficient condition that on an envelope of spheres the lines of curvature in one system be spherical is that the six coordinates $a_{i}$ of the spheres satisfy an equation of Laplace for which one of the invariants is equal to zero.

Darboux, l. c.

29. When the envelope $S$ of the planes of a congruence of circles is referred to a moving trihedral, and the coordinates of the center are $(a, b)$ and the radius is $R$, the coordinates of the poles of the circles are $(a, b, i R)$ and $(a, b,-i R)$. The radii of the spheres with centers on $S$ orthogonal to the circles are given by $\sqrt{a^{2}+b^{2}-R^{2}}$. In this case equations (40) assume the form $[\S 174,(14)]$.

30. By means of the preceding exercise and $[\S 175,20]$ show that a necessary and sufficient condition that the circles form a cyclic system is that the focal points of the congruence of lines joining corresponding points on the envelope of spheres are harmonic with respect to the points of contact.

Ribaucour, Journ. de Math., ser. 4, vol. 7 (1891), p. 260.

31. From the preceding exercise and $\S 82$ it follows that a necessary and sufficient condition that the circles orthogonal to the spheres of a transformation $R$ form a cyclic system is that it be a transformation $D_{m}$ of isothermal $O$ nets.

32. If $N$ and $N_{1}$ are two $O$ nets in relation $R$, the circles orthogonal to $N$ and $N_{1}$ form a cyclic system $(\S 79)$; the poles $P_{0}$ and $P_{0}^{\prime}$ of the circles have coordinates of the form

$$
x_{0}=x-\theta \frac{\xi+i \eta}{q+i r}, \quad x_{0}^{\prime}=x-\theta \frac{\xi-i \eta}{q-i r} ;
$$

the points $P_{0}$ and $P_{0}^{\prime}$ describe the sheets of the envelope of the spheres through $P_{0}$ and $P_{0}^{\prime}$ with centers on the net $\bar{N}(\bar{x})$ enveloped by the plane of the circles (cf. § 79); the radius of the spheres is given by

also

$$
R^{2}=2 j\left(j \theta^{\prime}+k p+\theta\right)+k^{2}=2 j \bar{\omega}+k^{2} ;
$$

$$
\Sigma \bar{x}^{2}-R^{2}=\Sigma x^{2}+2 j \omega^{\prime}+2 P k,
$$

where $\omega^{\prime}$ is given by $(\mathrm{VI}, 80)$. Show that the right-hand member of this equation is a solution of the point equation of $\bar{N}$. Show also that $P_{0}$ and $P_{0}^{\prime}$ are harmonic with respect to the focal points of the congruence of axes of the circles. 
33. A necessary and sufficient condition that the poles $P_{0}$ and $P_{0}^{\prime}$ of Ex. 32 describe $O$ nets in relation $\mathrm{R}$ is that the point equation of $\bar{N}$ admit as solution $R=\sqrt{2 j \bar{\omega}+k^{2}}$. This condition is reducible to

$$
\theta^{2}+p \theta\left(\rho_{1}+\rho_{2}\right)+\rho_{1} \rho_{2}\left[\theta(h+l)-q^{2}-r^{2}\right]=0 .
$$

Show that the two sheets of the envelope are conformally represented upon one another and the transformation $R$ is $D_{m}$.

34. If $S$ and $S_{1}$ are two surfaces of Guichard of the first kind in the relation of a transformation $R_{m}$ (§92), the condition (i) of Ex. 33 is satisfied when $m=\frac{1}{2}$. The same is true for the associate surfaces $\bar{S}$ and $\bar{S}_{1}$. The points $P_{0}$ and $\overline{P_{0}}$ describe isothermic nets $N_{0}$ and $\overline{N_{0}}$ which are Christoffel transforms of one another; likewise $P_{0}^{\prime}$ and $\bar{P}_{0}^{\prime}$; and the product of the segments $P_{0} P_{0}^{\prime}$ and $\bar{P}_{0} \bar{P}_{0}^{\prime}$ is equal to 4 . The nets $N_{0}$ and $N_{0}^{\prime}$ are in relation $D_{\frac{1}{2}}$ and likewise $\bar{N}_{0}$ and $\bar{N}_{0}^{\prime}$.

Calapso, Annali, ser. 3, vol. 24 (1915), p. 25.

35. If two applicable nets $N$ and $\bar{N}$ are $O$, the cyclic congruences determined by the method of $\S 112$ are normal, and parallel to congruences of normals to a spherical surface [cf. § 178].

36. The problem of finding cyclic systems whose planes envelope a sphere is equivalent to the determination of surfaces $S$ applicable to the sphere. If $x_{1}, x_{2}, x_{8}$ are the coordinates of a surface applicable to a sphere of radius $a$, the circles in the tangent planes of the sphere determined by $2 \varphi=a^{2}-\Sigma x_{i}^{2}$ form such a cyclic system.

37. Let $S$ be a surface applicable to a sphere $S_{0}$, and $x_{i}(i=1,2,3)$ the coordinates of $S$; then $x_{1}, x_{2}, x_{8}, \Sigma x_{i}^{2}$ are the tangential coordinates of a surface whose lines of curvature correspond to the lines of curvature of $S$.

Ribaucour, l. c., p. 55.

38. Consider a $G_{0}$ net $N$ in 3-space as defined by (VI, Ex. 12), and take the congruence of spheres of radius $\sigma$ with centers on $N$. Show that corresponding points of the Laplace transforms of $N$ lie on the corresponding sphere; that the developables of the congruence $G$ of lines joining corresponding points of contact of the sphere with its envelope are parametric and the focal planes of $G$ are tangent to the sphere at corresponding points of the Laplace transforms, $N_{1}$ and $N_{-1}$ of $N$; that the focal point of the first rank of $G$ is the center of the osculating sphere of the curve $v=$ const. of $N_{1}$ and the center of normal curvature of the curve $u=$ const. of $N_{-1}$, and similarly for the focal point of second rank; and that the curves $u=$ const. of $\mathrm{N}_{-1}$ and $v=$ const. of $N_{1}$ have common osculating circles at corresponding points.

Stetson, Annals, vol. 19 (1917), p. 123.

39. If the curves of two nets $N_{1}$ and $N_{2}$ correspond and the osculating planes of the curves $v=$ const. of $N_{1}$ osculate the curves $u=$ const. of $N_{2}$ at corresponding points, then $N_{1}$ and $N_{2}$ are $O$ nets, and $N_{1}$ is the first Laplace transform and $N_{2}$ the minus first transform of a net $N$, which is a net $G_{0}$.

Stetson, Annals, vol 19 (1917), p. 123. 
Chapter IX.

\section{Rolling surfaces.}

117. Congruences harmonic to nets $C$. Let $N(x)$ be a net $C$ in 3-space and $\bar{N}(\bar{x})$ the applicable net. If $\theta$ is a solution of their common point equation, the congruences $G$ and $\bar{G}$ harmonic to these respective nets and determined by $\theta$ have directionparameters of the form

$$
X_{i}=\frac{\partial x_{i}}{\partial u} \frac{\partial \theta}{\partial v}-\frac{\partial x_{i}}{\partial v} \frac{\partial \theta}{\partial u}, \quad \bar{X}_{i}=\frac{\partial \bar{x}_{i}}{\partial u} \frac{\partial \theta}{\partial v}-\frac{\partial \bar{x}_{i}}{\partial v} \frac{\partial \theta}{\partial u} .
$$

These functions satisfy the same direction-equation and are in the relation

$$
\sum X_{i}^{2}=\sum \overline{X_{i}^{2}}
$$

since $N$ and $\bar{N}$ are applicable.

If $\theta=\bar{x}_{3}$, then $\bar{X}_{3}=0$; if $\theta=\bar{x}_{1}+i \bar{x}_{2}$, then $\bar{X}_{1}^{2}+\bar{X}_{2}^{2}=0$. In the latter case $G$ is $2, \mathrm{I}$, that is a normal congruence. The intersections of corresponding lines of $G$ and of the harmonic congruence $G^{\prime}$ determined by $\theta=\bar{x}_{3}$ generate a net $N_{d}$ conjugate to $G$ and $G^{\prime}$, being a derived net of $N(\S 11)$. From (I, Ex. 15) it follows that $N_{d}$ is parallel to the net of coordinates

$$
\frac{X_{i}}{\frac{\partial \bar{x}_{8}}{\partial u} \frac{\partial \theta}{\partial v}-\frac{\partial \bar{x}_{3}}{\partial v} \frac{\partial \theta}{\partial u}}=\frac{X_{i}}{\bar{X}_{3}}
$$

and the complementary function $y^{\prime}$, defined by $(\mathrm{VI}, 17)$ is equal to 1 . Hence this net and consequently $N_{d}$, is an $O$ net. Therefore the $\infty^{1} O$ nets normal to $G$ are determined by the congruences harmonic to $N$ for which $\theta=\bar{x}_{3}+$ const. Then by applying an orthogonal substitution to the $x^{\prime}$, , or what is the same thing displacing $\bar{N}$ in space, we have: 
If $N(x)$ is a net $C$ and $\bar{N}(\bar{x})$ the applicable net, of the congruences harmonic to $N$ :

$\infty^{1}$ families of parallel congruences are 2, I;

$\infty^{2}$ families are $3, \mathrm{I}$ and the others are 4, I;

in the first and second cases $\theta=\sum a \bar{x}+d$ with $\sum a^{2}=0$ and $\sum a^{2} \neq 0$ respectively. The $O$ nets normal to a congruence $2, \mathrm{I}$ are generated by its points of intersection with the parallel harmonic congruences determined by $\theta=\sum b \bar{x}+e$, where $\sum a b=0, \sum a_{0} b=0$, $a_{0}$ being the conjugate imaginary of $a$.

If now we consider the congruence $\bar{G}$ harmonic to $\bar{N}$ determined by $\theta=\bar{x}_{1}+i \bar{x}_{2}$, we find that the lines of this congruence lie in the plane $x_{1}+i x_{2}=0$. Moreover the points of these lines corresponding to the points on the congruence $\bar{G}$ which generate the $O$ nets normal to $G$ are the intersections of these lines in the plane $x_{1}+i x_{2}=0$ and the planes $x_{3}=$ const. In the general case when $\theta=\sum a \bar{x}+d$, with $\sum a^{2}=0$, the lines of the congruence $\bar{G}$ lie in the isotropic plane $\sum a x+d=0$, and the points corresponding to the points on $G$ describing the $O$ nets are the intersections of these lines and the $\infty^{1}$ isotropic lines lying in this plane.

I r8. Rolling surfaces. Let $S$ and $\bar{S}$ be two applicable surfaces, and $M$ and $\bar{M}$ corresponding points. Imagine $S$ held fixed and $\bar{S}$ moved in space so that $\bar{M}$ comes into coincidence with $M$, and the tangent planes at $\bar{M}$ and $M$ come into coincidence, and likewise corresponding linear elements arising from $\bar{M}$ and $\boldsymbol{M}$. For each pair of points $M$ and $\bar{M}$ we have a different position of $\bar{S}$. Consequently $\bar{S}$ assumes $\infty^{2}$ different positions, unless $S$ and $\bar{S}$ are ruled surfaces with generators in correspondence [cf. 142], in which case there are $\infty^{1}$ different positions. In this particular case the two surfaces do not have a net in common; it will be assumed that this case is excluded in what follows, unless mention is made of it. As $\bar{S}$ undergoes this rigid motion of two parameters it is said to roll on $S$. The common tangent plane at the point of coincidence of $S$ and $\bar{S}$ is called the plane of contact.

The results of the last paragraph of the preceding section may be stated as follows ${ }^{97}$ ):

97) Darboux, Annales L'École Norm. Sup., ser. 3, vol. 16 (1899), p. 467. 
When a surface $\bar{S}$ rolls over an applicable surface $S$, a plane $\pi$ invariably fixed to $\bar{S}$, cuts the plane of contact of $S$ and $\bar{S}$ in a line d which generates a congruence, which is harmonic to the net common to $S$ and $\bar{S}$. When $\pi$ is isotropic, the congruence of lines $d$ is normal to a family of surfaces whose lines of curvature correspond to the net common to $S$ and $\bar{S}$; moreover, every isotropic line in $\pi$ meets $d$ in the point which describes one of these normal surfaces.

Any line fixed to $\bar{S}$ may be looked upon as the intersection of two planes fixed to $\bar{S}$. Since furthermore, there is a unique isotropic plane through an isotropic line, we have:

When a surface $\bar{S}$ rolls over an applicable surface $S$, a line $l$ invariably fuxed to $\bar{S}$ meets the plane of contact in a point which generates a derived net of the net common to $S$ and $\bar{S}$; if $l$ is an isotropic line this derived net is an $O$ net, the normals to which are the positions of the intersections of the tangent planes to $\bar{S}$ and the unique isotropic plane through $l$; all derived $O$ nets are so determined.

As a corollary we have:

If two parallel isotropic lines are invariably bound to a surface $\bar{S}$ as it rolls over $S$, the two $O$ nets generated by the points of meeting of these lines and the plane of contact have the same spherical representation.

We may now restate the first theorem of $\S 113$ as follows:

If a surface $\bar{S}$ rolls over an applicable surface $S$ and $Q$ is a point invariably fixed to $\bar{S}$, the isotropic lines through $Q$ (null. sphere) meet the plane of contact in points $P$, on a circle $C$, which generate the $O$ nets orthogonal to the cyclic system of the circles $C$.

Also:

If $S$ and $\bar{S}$ are applicable surfaces and lines $l$ are drawn through a fixed point normal to the tangent planes to $\bar{S}$, as $\bar{S}$ rolls over $S$ the lines $l$ generate a cyclic congruence whose developables correspond to the net common to $S$ and $\bar{S}$.

If $N(x)$ and $N_{1}\left(x_{1}\right)$ are nets in relation $F$ by means of a net $N^{\prime}\left(x^{\prime}\right)$ and corresponding solutions $\theta$ and $\theta^{\prime}$ of the point equations of $N$ and $N^{\prime}$ respectively, and $\varphi, \varphi^{\prime}, \varphi_{1}$ and $\psi, \psi^{\prime}, \psi_{1}$ are sets of corresponding solutions of the point equations of $N^{\prime}, N^{\prime}$ and $N_{1}$ so that

$$
x_{1}=x-\frac{\theta}{\theta^{\prime}} x^{\prime}, \quad \varphi_{1}=\varphi-\frac{\theta}{\theta^{\prime}} \varphi^{\prime}, \quad \psi_{1}=\psi-\frac{\theta}{\theta^{\prime}} \psi^{\prime} .
$$


the derived nets of $N$ and $N_{1}$ by means of the respective pairs of functions $\varphi, \psi$ and $\varphi_{1}, \psi_{1}$ are in relation $F$ (I, Ex. 6). Suppose now that $N, N^{\prime}$ and $N_{1}$ are nets $C$, and that the applicable nets are $\bar{N}, \overline{N^{\prime}}$ and $\overline{N_{1}}$ so that

$$
\bar{x}_{1}=\bar{x}-\frac{\theta}{\theta^{\prime}} \bar{x}^{\prime}
$$

the functions $\theta$ and $\theta^{\prime}$ being chosen in accordance with $\S 27$. If we take

$$
\begin{array}{ll}
\varphi=a_{1} \bar{x}+b_{1} \bar{y}+c_{1} \bar{z}+d_{1}, & \varphi^{\prime}=a_{1} \bar{x}^{\prime}+b_{1} \bar{y}^{\prime}+c_{1} \bar{z}^{\prime} \\
\psi=a_{2} \bar{x}+b_{2} \bar{y}+c_{2} \bar{z}+d_{2}, & \psi^{\prime}=a_{2} \bar{x}^{\prime}+b_{2} \bar{y}^{\prime}+c_{2} \bar{z}^{\prime}
\end{array}
$$

where $a_{1} b_{1}, \ldots d_{2}$ are arbitrary constants, then

$$
\varphi_{1}=a_{1} \bar{x}_{1}+b_{1} \bar{y}_{1}+c_{1} \bar{z}_{1}+d_{1}, \quad \psi_{1}=a_{2} \bar{x}_{1}+b_{2} \bar{y}_{1}+c_{2} \bar{z}_{1}+d_{2} .
$$

In this case the derived nets of $N$ and $N_{1}$ are the loci of the points of meeting of the line

$$
a_{1} x+b_{1} y+c_{1} z+d_{1}=0, \quad a_{2} x+b_{2} y+c_{2} z+d_{2}=0
$$

with the respective planes of contact as $\bar{N}$ rolls over $N$ and $\overline{N_{1}}$ over $N_{1}$. Hence:

If $N$ and $\bar{N}$ are applicable nets, and $N_{1}$ and $\overline{N_{1}}$ are $F$ transforms of $N$ and $\bar{N}$ respectively, by means of $\theta^{\prime}=\sum x^{\prime 2}-\sum \bar{x}^{\prime 2}$, such that they are applicable, and $l$ is a line invariably bound to $\bar{N}$ and $\vec{N}_{1}$, as $\vec{N}_{1}$ rolls on $N_{1}$ the point where $l$ meets the plane of contact of $N_{1}$ and $\bar{N}_{1}$ generates a net which is an $F$ transform of the net generated by the point where $l$ meets the plane of contact when $\bar{N}$ rolls on $N$.

If the line $l$ is isotropic, the two nets thus found are $O$ nets in the relation $R$. Hence:

If $N$ and $\bar{N}$ are applicable nets and $N_{1}$ and $\overline{N_{1}}$ are $F$ transforms by means of $\theta^{\prime}=\sum x^{\prime 2}-\sum \bar{x}^{\prime 2}$, the cyclic systems in which a point sphere invariably bound to $\vec{N}$ and $\bar{N}_{1}$ meets the common tangent planes as $\bar{N}$ rolls on $N$ and as $\bar{N}_{1}$ rolls on $N_{1}$ are in relation $F$; moreover, the two surfaces orthogonal to these cyclic systems determined by each generator of the null sphere are in relation $R$. 
I19. Special isothermic surfaces. We apply these results to the case where $\bar{S}$ is a quadric $Q$ which meets the circle at infinity in four distinct points $P_{i}$. Through each of these points pass two isotropic generators, $d_{i}$, $d_{i}^{\prime}$. The twelve points of intersection of lines $d_{i}$ and $d_{k}^{\prime}$, where $i \neq k$, are the umbilical points of $Q$. If $m_{i}$ and $m_{i}^{\prime}$ are the points where $d_{i}$ and $d_{i}^{\prime}$ meet the plane of contact of $Q$ and $S$, and $\Sigma_{i}$ and $\Sigma_{i}^{\prime}$ denote the loci of $m_{i}$ and $m_{i}^{\prime}$ as $Q$ rolls over $S$, on the eight surfaces $\Sigma_{i}$ and $\Sigma_{i}^{\prime}$ the lines of curvature correspond to the nets $\bar{N}$ and $N$ common to $Q$ and $S$. Two surfaces $\Sigma_{i}$ and $\Sigma_{k}(k \neq i)$ are normal to the circles of intersection of the planes of contact with the point sphere having its center at the umbilical point of intersection $P_{i k}$ of $d_{i}$ and $d_{k}^{\prime}$. Two surfaces $\Sigma_{i}$ and $\Sigma_{i}$ have the same spherical representation of their lines of curvature, by the third theorem of $\S 118$. The $O$ nets on $\Sigma_{i}$ and $\Sigma_{i}^{\prime}$ are conjugate to the congruence of the lines of intersection of the plane of contact and the plane of the lines $d_{i}$ and $d_{i}^{\prime}$. Likewise, the $O$ nets on $\Sigma_{i}$ and $\Sigma_{i}^{\prime}$ are conjugate to the congruence of lines of intersection of the plane of contact and the plane of the lines $d_{i}$ and $d_{k}^{\prime}$, that is the tangent plane to $Q$ at $P_{i k}$. The congruence of lines $m_{i} m_{i}^{\prime}$ is harmonic to the net $N$ on $S$, and its focal points divide harmonically the segment $m_{i} m_{i}^{\prime}$, since the points $m_{i}$ and $m_{i}$ lie on the generators of $Q$. Similar results hold for the congruence of lines $m_{i} m_{k}^{\prime}$. Hence $\Sigma_{i}$ and $\Sigma_{i}^{\prime}$ are isothermic surfaces in the relation of a Christoffel transformation [§ 159]. Moreover $\boldsymbol{\Sigma}_{i}$ and $\boldsymbol{\Sigma}_{k}^{\prime}$ are in the relation of a transformation $K(\S 25)$ which is a transformation $D_{m}$ since the nets are $O$ nets. Hence we have the theorem of Darboux ${ }^{98}$ ):

When a quadric $Q$ which meets the circle at infinity in four. distinct points rolls on an applicable surface $S$, the eight surfaces generated by the points of intersection of the isotropic generators and the plane of contact are isothermic, their lines of curvature corresponding to the net common to $Q$ and $S$; two of these surfaces determined by generators through a point at infinity are Christoffel transforms of one another: two determined by generators through an umbilical point are in the relation of a transformation $D_{m}$.

In $\S 84$ we considered these isothermic surfaces from another

98) L. c., p. 492. 
point of view and found that they are the type called special by Bianchi.

120. Rolling of a surface applicable to a surface of revolution. Let $\bar{S}$ be a surface of revolution defined by

$$
\bar{x}=r \cos v, \quad \bar{y}=r \sin v, \quad \bar{z}=\varphi(r) .
$$

Its linear element is

$$
d s^{2}=\left(1+\varphi^{\prime 2}\right) d r^{2}+r^{2} d v^{2} .
$$

Let $S$ be a surface applicable to $\bar{S}$. If we put

$$
d u=\sqrt{1+\varphi^{\prime 2}} d r, \quad U=r,
$$

the linear element assumes the form

$$
d s^{2}=d u^{2}+U^{2} d v^{2} .
$$

The tangents to the deforms of the meridians of $\bar{S}$, that is the curves $v=$ const. form a normal congruence $G$ for which the other focal surface, $S_{1}$, called the complementary surface to $S$, is given by equations of the form [cf. $\S 76$ ]

$$
x_{1}=x-\frac{U}{U^{\prime}} \frac{\partial x}{\partial u} .
$$

We recall from [\$124] that this surface $S_{1}$ is applicable to a surface of revolution, whose linear element is

$$
d s_{1}^{2}=U^{2}\left(\frac{1}{U^{\prime}}\right)^{\prime 2} d u^{2}+\frac{1}{U^{\prime 2}} d w^{2},
$$

that the normal congruence $G$ is a $W$ congruence and that any normal $W$ congruence may be obtained in this manner.

When we apply equations (3) to $\bar{S}$, we obtain

$$
\bar{x}_{1}=\bar{y}_{1}=0, \quad \bar{z}_{1}=\varphi-r \varphi^{\prime},
$$

which is the point of intersection of the tangent to the meridian at a given point and the axis of revolution of $\bar{S}$. Hence: 
When a surface of revolution $\bar{S}$ rolls on an applicable surface $S$, the point in which the axis of $\bar{S}$ meets the plane of contact generates the complementary surface $S_{1}$ of $S$, and the line joining this point to the corresponding point of $S$ generates a normal $W$ congruence of which $S$ and $S_{1}$ are the focal surfaces; moreover, any normal $W$ congruence may be generated in this manner.

If we put

$$
r_{1}=k \sqrt{1+\varphi^{\prime 2}}, \quad w=k v_{1}
$$

where $k$ is any constant, by means of (2) equation (4) is reducible to

$$
d s_{1}^{2}=\frac{r^{2}}{k^{2}} d r_{1}^{2}+r_{1}^{2} d v_{1}^{2}
$$

If $\bar{S}$ is the quadric of revolution

$$
\frac{z^{2}}{a}+\frac{r^{2}}{b}=1
$$

we have

$$
1+\varphi^{\prime 2}=\frac{(a-b) r^{2}+b^{2}}{b\left(b-r^{2}\right)} \text {. }
$$

Substituting this expression in the first of (5), solving for $r^{2}$ and putting $k^{2}=b^{2} /(b-a)$, we have

$$
\frac{r^{2}}{k^{2}}=\frac{(a-b) r_{1}^{2}+b^{2}}{b\left(b-r_{2}^{2}\right)} \text {. }
$$

Hence (6) assumes the same form as (1) for the expression (8), that is $S_{1}$ is applicable to $\bar{S}$.

Similarly, if $\bar{S}$ is the paraboloid of revolution

$$
2 z=m r^{2}
$$

we have $1+\varphi^{\prime 2}=1+m^{2} r^{2}$ and by taking $k^{2}=-1 / m^{2}$, we find that $S_{1}$ is applicable to $\bar{S}$. Hence:

When a non-developable quadric of revolution $\bar{S}$ rolls on an applicable surface $S$, the point in which the axis of $\bar{S}$ meets the 
common tangent plane generates the complementary surface $S_{1}$ of $S$, and $S_{1}$ is applicable to $\bar{S}$.

As a consequence of the results of $\S 100$ we have the theorem:

When a non-developable quadric of revolution $\bar{S}$ rolls on an applicable surface $S$, the spheres with centers on $\bar{S}$ passing through either focus are tangent to two surfaces in relation $R$.

121. The fundamental theorems of Guichard. Let $S$ be a surface applicable to the quadric of revolution $\bar{S}$ which is defined by (7). Denote by $A$ and $A^{\prime}$ the extremities of the axis of revolution of $\bar{S}$, and let $S$ be in contact with $\bar{S}$ at a point $M$. Denote by $d, d_{1}$ and $d^{\prime}, d_{1}^{\prime}$ the isotropic generators of $\bar{S}$ through $A$ and $A^{\prime}$ respectively. These lines meet the common tangent plane $\pi$ in the respective points $a, a_{1}, a^{\prime}, a_{1}^{\prime}$. The lines $a a_{1}^{\prime}$ and $a^{\prime} a_{1}$ are the generators of $\bar{S}$ through $M$. The lines $a a^{\prime}$ and $a_{1} a_{1}^{\prime}$ are the intersections with the plane $\pi$ of the isotropic planes through $A A^{\prime}$ and the pairs of parallel generators $d, d^{\prime}$ and $d_{1}, d_{1}^{\prime}$.

From the theorems of $\S 118$ it follows that as $\bar{S}$ rolls on $S$, the lines $a a^{\prime}$ and $a_{1} a_{1}^{\prime}$ generate normal congruences, and the points $a, a^{\prime}$ and $a_{1}, a_{1}^{\prime}$ generate $O$ nets normal to these congruences. Moreover, these congruences are harmonic to the net $N$ common to $S$ and $\bar{S}$. Since the lines $a a_{1}^{\prime}$ and $a^{\prime} a_{1}$ are the generators of $\bar{S}$ and therefore form a harmonic pencil with the tangents to any net at $M$ it follows that the focal points of the lines $a a^{\prime}$ and $a_{1} a_{1}^{\prime}$ divide harmonically the segments $a a^{\prime}$ and $a_{1} a_{1}^{\prime}$ both of which are of the constant length $A A^{\prime}$. It can be shown by the methods of [§ 73], that the surfaces generated by $a, a^{\prime}, a_{1}, a_{1}^{\prime}$, have constant mean curvature $\pm \frac{2}{A A^{\prime}}$.

The lines $a a^{\prime}$ and $a_{1} a_{1}^{\prime}$ meet in the point $M^{\prime}$ in which the axis of $\bar{S}$ meets the common tangent plane. Hence $M^{\prime}$ describes a derived net $N^{\prime}$ of $N$, and as shown in the preceding section $N^{\prime}$ lies on a surface applicable to $\bar{S}$.

From the fourth theorem of $\S 118$ it follows that $a$ and $a_{1}$ describe two of the $O$ nets orthogonal to the cyclic system of circles in which the null sphere at $A$ meets the common tangent plane. Hence these $O$ nets are in relation $R$, and the net $N^{\prime}$ is the central net. Similarly $a^{\prime}$ and $a_{1}^{\prime}$ describe $O$ nets in relation $R$ with the same central net. 
Hence we have the theorem ${ }^{99}$ :

When a central quadric of revolution $\bar{S}$ rolls on an applicable surface $S$, the points $a, a_{1}$ and $a^{\prime}, a_{1}^{\prime}$ in which the isotropic generators through the extremities, $A, A^{\prime}$, of the axis of $\bar{S}$ meet the comnon tangent plane, generate surfaces of constant mean curvature, such that $(a)$ and $\left(a_{1}\right)$, and $\left(a^{\prime}\right)$ and $\left(a_{1}^{\prime}\right)$, are pairs in relation $R$; and $(a)$ and $\left(a^{\prime}\right)$, and $\left(a_{1}\right)$ and $\left(a_{1}^{\prime}\right)$, are pairs of parallel surfaces.

From the theorem of Bonnet [§ 73] it follows that the mean points of the segments $a a^{\prime}$ and $a_{1} a_{1}^{\prime}$ generate surfaces of constant total curvature in relation $R$.

Since the tangent planes to $S$ and its complementary surface $S_{1}$ are normal to one another, when $\bar{S}$ rolls on $S$, the tangent planes $\pi_{1}$ to $S_{1}$ pass through the axis of $\bar{S}$, an infinity of them corresponding to one plane. When $S_{1}$ is applied to $\bar{S}$ the planes $\pi_{1}$ become tangent to $\bar{S}$ and the lines which coincided with the axis of $\bar{S}$ when $S$ was applied to $\bar{S}$ become a family of generators of $\bar{S}$, since an infinity of the planes $\pi_{1}$ pass through each line. The isotropic planes through the axis become the isotropic tangent planes to $\bar{S}$ which have the property of passing through the foci of $\bar{S}$. Consequently when $S_{1}$ is applied to $\bar{S}$ either the lines $a a_{1}^{\prime}$ (or $a^{\prime} a_{1}$ ) coincide with the axis of $\bar{S}$ and $a$ and $a_{1}^{\prime}$ (or $a^{\prime}$ and $a_{1}$ ) become the foci of $\bar{S}$. Accordingly as $\bar{S}$ rolls on $S_{1}$ the surfaces generated by $a, a_{1}$ and by $a^{\prime} a_{1}^{\prime}$ are the sheets of the envelope of spheres with centers on $\bar{S}$ and passing through one and the other focus of $\bar{S}$ respectively. Since $a$ and $a_{1}$ are symmetric with respect to the common tangent plane, we have the above results in the following form as given by Guichard ${ }^{100}$ ):

If $\bar{S}$ is a central quadric of revolution whose axis is of length $2 a, F_{1}$ and $F_{2}$ its foci, and $\varphi_{1}$ and $\varphi_{2}$ the symmetric points of $F_{1}$ and $F_{2}$ with respect to the tangent planes of $\bar{S}$, then as $\bar{S}$ rolls over an applicable surface the points $F_{1}, F_{2}, \varphi_{1}, \varphi_{2}$ describe surfaces of constant mean curvature $\pm 1 / a$.

When $\bar{S}$ is the paraboloid (9), two of the points $a^{\prime}$ and $a_{1}^{\prime}$ are at infinity, and consequently the points $a$ and $a_{1}$ bisect the focal segments of the normal congruences generated by the lines in which

39) Darboux, 1. c., p. 477.

${ }^{100}$ ) Comptes Rendus, vol. 128 (1899), p. 232; cf. also Darboux, 1. c., p. 477. 
the isotropic planes through the axis of $\bar{S}$ and the isotropic generators through the vertex of $\bar{S}$ meet the common tangent planes of $S$ and $\bar{S}$. Hence:

When a paraboloid of revolution $\bar{S}$ rolls on an applicable surface $S$, the points in which the two isotropic generators through the vertex meet the common tangent plane generate two minimal surfaces in relation $R$, and the central net of the transformation $R$ is described by the point in which the axis of $\bar{S}$ meets the common tangent planes.

In $\$ 120$ we showed that this central net lies on a surface also applicable to $\bar{S}$. By reasoning similar to that for a central quadric of revolution, we obtain the theorem of Guichard ${ }^{101}$ ):

When a paraboloid of revolution rolls on a surface applicable to the paraboloid, the focus and the point symmetric to it with respect to the common tangent plane generate minimal surfaces.

In $\S 127$ we give analytical proofs of these theorems of Guichard.

122. Deformable transformations $R$ of the first type. Let $S_{0}$ be the surface of centers of a congruence of spheres of radius R. Corresponding points on the sheets, $S$ and $S_{1}$, of the envelope are symmetric with respect to the tangent plane to $S_{0}$ at the corresponding point $M$. Let $S_{0}$ be referred to a moving trihedral whose plane $y=0$ is the plan $M M_{0} M_{1}$, and let the parametric lines on $S_{0}$ be chosen so that the $x$ and $y$ axes are tangent to the curves $v=$ const. and $u=$ const. respectively. If $\sigma$ denotes the angle which the line $M M_{0}$ makes with the plane $z=0$, the coordinates of $M$ are $R \cos \sigma, 0, R \sin \sigma$.

The displacements of a point $P$ of coordinates $t \cos \sigma, 0, t \sin \sigma$ are given by $[\S 69]$

(10) $\left\{\begin{array}{l}\delta x=d(t \cos \sigma)+\xi d u+\left(q d u+q_{1} d v\right) t \sin \sigma, \\ \delta y=\eta_{1} d v+\left(r d u+r_{1} d v\right) t \cos \sigma--\left(p d u+p_{1} d v\right) t \sin \sigma, \\ \delta z=d(t \sin \sigma)-\left(q d u+q_{1} d v\right) t \cos \sigma .\end{array}\right.$

When we replace $t$ by $R$ and require that

$$
\cos \sigma \delta x+\sin \sigma \delta z=0
$$

101) I. C. 
for all displacements of $M_{0}$, we find

$$
\frac{\partial R}{\partial u}+\xi \cos \sigma=0, \quad \frac{\partial R}{\partial v}=0
$$

In order that $M$ describe a line of curvature on $S$, it is necessary that there be a point $P$ such that

$$
\frac{\delta x}{\cos \sigma}=\frac{\delta y}{0}=\frac{\delta z}{\sin \sigma}
$$

These conditions are equivalent to

(12) $\left\{\begin{aligned} \eta_{1} d v+\left[\left(r d u+r_{1} d v\right) \cos \sigma-\left(p d u+p_{1} d v\right) \sin \sigma\right] t & =0, \\ \xi \sin \sigma d u+\left[q d u+q_{1} d v-d \sigma\right] t & =0 .\end{aligned}\right.$

Eliminating $t$ from these equations, we obtain as the equation of the lines of curvature on $S$

$$
\left\{\begin{aligned}
\xi \sin \sigma & (p \sin \sigma-r \cos \sigma) d u^{2}+\eta_{1}\left(q_{1}-\frac{\partial \sigma}{\partial v}\right) d v^{2} \\
+ & {\left[\eta_{1}\left(q-\frac{\partial \sigma}{\partial u}\right)+\xi \sin \sigma\left(p_{1} \sin \sigma-r_{1} \cos \sigma\right)\right] d u d v=0 }
\end{aligned}\right.
$$

The equation of the lines of curvature on $S_{1}$ is obtained from (13) by replacing $\sigma$ by $-\sigma$. In order that the lines of curvature on $S$ and $S_{1}$ correspond, that is that the congruence of spheres be $R$, it is necessary and sufficient that

$$
\frac{r \cos \sigma}{p \sin \sigma}=\frac{\frac{\partial \sigma}{\partial v}}{q_{1}}=\frac{\eta_{1} \frac{\partial \sigma}{\partial u}+\xi \sin \sigma \cos \sigma r_{1}}{\eta_{1} q+\xi p_{1} \sin ^{2} \sigma}
$$

We recall that, in accordance with the theorem of Beltrami ( $\$ 112)$, if $S_{0}$ is deformed, the spheres touch their envelope at the same points of the spheres as for $S_{0}$. We investigate under what conditions a congruence $R$ of spheres remains a congruence $R$ in all deformations of the central surface $S_{0}$. Evidently $\sigma$ remains unaltered, as do also the functions $\xi, \eta_{1}, r, r_{1}$, since they depend 
only on the linear element of $S_{0}$ [§ 72]. However, the functions $p, q$, $p_{1}, q_{1}$ vary in the deformation. Consequently if equations (14) are to hold for all deformations, we must have [cf. $\$ 72,(75)$ ]

$$
r=-\frac{1}{\eta_{1}} \frac{\partial \dot{\xi}}{\partial v}=0, \quad \frac{\partial \sigma}{\partial v}=0, \quad \eta_{1} \frac{\partial \sigma}{\partial u}+\sin \sigma \cos \sigma \frac{\partial \eta_{1}}{\partial u}=0
$$

Hence $\sigma$ must be a function of $u$ alone. Then from the last of (15) it follows that the parameter $v$ can be chosen so that $\eta_{1}$ is a function of $u$ alone. Moreover, from the first of (15) we find that $\xi$ is a function of $u$ alone. Hence $S_{0}$ is applicable to a surface of revolution. If we take the linear element of $S_{0}$ in the form

$$
d s_{0}^{2}=U^{2} d u^{2}+u^{2} d v^{2}
$$

from the last of (15) we obtain

$$
\tan \sigma=\frac{c}{u}
$$

where $c$ is a constant. Then from (11) we have

$$
R=-\int \frac{U u d u}{\sqrt{u^{2}+c^{2}}}+\text { const }
$$

Hence we have the theorem of Bianchi $\left.{ }^{102}\right)$ :

In order that a congruence $R$ of spheres remain a congruence $R$ in all deformations of the central surface $S_{0}$, it is necessary and sufficient that $S_{0}$ be applicable to a surface of revolution and that the radius of the spheres be given by (18).

123. Deformable transformations $R$ of the second type. In this section we determine every congruence $R$ of spheres whose central net $N_{0}$ admits an applicable net $\overline{N_{0}}$ which is $2, O$ and consequently is the central net of another congruence $R$ of spheres ${ }^{103}$ ). To this end we make use of the equations of a transformation $R$ as given in $\S 78$.

102) Lezioni, vol. 2, p. 117.

${ }^{103}$ ) Cf. Trans. Amer. Math. Soc. vol. 17 (1916), pp. 437--458; also, Calapso, Annali, vol. 26 (1917), pp. 151-190. 
From (VII, 7) we have

$$
\frac{\partial}{\partial u}\left(\frac{\theta}{p}\right)=\frac{q}{p} L_{1}, \quad \frac{\partial}{\partial v}\left(\frac{\theta}{p}\right)=\frac{r}{p} L_{2},
$$

where

(20)

$$
L_{1}=V \bar{E}\left(1+\frac{1}{\varrho_{1}} \frac{\theta}{p}\right), \quad L_{2}=\sqrt{G}\left(1+\frac{1}{\varrho_{\underline{a}}} \frac{\theta}{p}\right) .
$$

Since the radius of the spheres of the transformation is $\theta / p$, and this radius must be the same for the spheres of centers on $\bar{N}_{0}$ applicable to $\mathrm{N}_{0}$, by the theorem of Beltrami ( $\S 112$ ), we must have for the functions $\bar{\theta}$ and $\bar{p}$ of the corresponding transformation

$$
\bar{\theta}=\varrho \theta, \quad \bar{p}=\varrho p,
$$

where $\varrho$ is a factor of proportionality to be determined.

From (VII, 10) we find that the first fundamental coeficients $E_{0}$, $F_{0}, G_{0}$ of $N_{0}$ are

(22) $\quad E_{0}=L_{1}^{2}\left[1+\left(\frac{q}{p}\right)^{2}\right], \quad F_{0}=L_{1} L_{2} \frac{q v}{p^{2}}, \quad G_{0}=L_{2}^{3}\left[1+\left(\frac{r}{p}\right)^{2}\right]$.

If we denote by $\bar{S}$ and $\overline{S_{1}}$ the sheets of the envelope of spheres of radius $\bar{\theta} / \bar{p}$ with centers on $\bar{N}_{0}$ and by $\bar{q}, \bar{r}, \bar{w}$ the functions of the $R$ transformation from $\bar{S}$ into $\overline{S_{1}}$, the latter functions must satisfy equations of the form (VII, 7). From (19), (20) and the analogous equations for the transformation of $\bar{S}$, we get

(23)

$$
\left\{\begin{array}{l}
\sqrt{E} q \varrho\left(1+\frac{1}{\varrho_{1}} \frac{\theta}{p}\right)=\sqrt{\bar{E}} \bar{q}\left(1+\frac{1}{\bar{\varrho}_{1}} \frac{\theta}{p}\right), \\
\sqrt{G} \cdot \varrho\left(1+\frac{1}{\varrho_{2}} \frac{\theta}{p}\right)=\sqrt{\bar{G}} \bar{r}\left(1+\frac{1}{\varrho_{2}} \frac{\theta}{p}\right) .
\end{array}\right.
$$

From these equations and the ones obtained by expressing the equality of the first fundamental coefficients of $S_{0}$ and $\bar{S}_{0}$ as givell by equations of the form (22), we have

$$
\bar{q}=\varrho q, \quad \bar{r}=\varrho v, \quad \bar{w}=\varrho w,
$$


by making a suitable choice of signs of $\sqrt{\bar{E}}$ and $\sqrt{\bar{G}}$, and requiring that the quadratic relation (VII, 8 ) be satisfied also by the functions $\bar{p}, \bar{q}, \bar{r}$ and $\bar{w}$.

When the above expressions for $\bar{q}$ and $\bar{r}$ are substituted in the equations analogous to (VII, 7), we obtain

$$
\frac{\partial \log \varrho}{\partial u}=\frac{q}{\theta}(\sqrt{\bar{E}}-V \bar{E}), \quad \frac{\partial \log \varrho}{\partial v}=\frac{r}{\theta}(\sqrt{\bar{G}}-\sqrt{G})
$$

$$
\left\{\begin{array}{l}
\frac{1}{\sqrt{\bar{G}}} \frac{\partial \sqrt{\bar{E}}}{\partial v}-\frac{1}{\sqrt{G}} \frac{\partial \sqrt{E}}{\partial v}=\frac{r}{\theta}(\sqrt{\bar{E}}-\sqrt{E}), \\
\frac{1}{\sqrt{\bar{E}}} \frac{\partial \sqrt{\bar{G}}}{\partial u}-\frac{1}{\sqrt{E}} \frac{\partial \sqrt{G}}{\partial u}=\frac{q}{\theta}(\sqrt{\bar{G}}-\sqrt{\bar{G}}),
\end{array}\right.
$$

$$
V \overline{\bar{E}_{1}}-V \bar{E}_{1}=\sqrt{\overline{\bar{E}}}-V \overline{\bar{E}}, \quad \sqrt{\overline{G_{1}}}-V \bar{G}_{1}=V \overline{\bar{G}}-V \overline{\bar{G}}
$$

$$
\left\{\begin{array}{c}
2^{\sqrt{\bar{E} \bar{G}}+}+\sqrt{\bar{E}}\left(\sqrt{G_{1}}-\sqrt{\bar{G}}\right)+\sqrt{\bar{G}}\left(\sqrt{E_{1}}-\sqrt{E}\right) \\
-\sqrt{E_{1}} \sqrt{G}-\sqrt{G_{1}} \sqrt{E}=0
\end{array}\right.
$$

The Gauss and Codazzi equations for $S$ are $[\S 65]$

$$
\left\{\begin{array}{l}
\frac{\partial}{\partial v}\left(\frac{1}{\sqrt{G}} \frac{\partial \sqrt{E}}{\partial v}\right)+\frac{\partial}{\partial u}\left(\frac{1}{\sqrt{E}} \frac{\partial \sqrt{G}}{\partial u}\right)+\frac{\sqrt{E G}}{\varrho_{1} \varrho_{2}}=0 \\
\frac{\partial}{\partial v}\left(\frac{\sqrt{E}}{\varrho_{1}}\right)=\frac{1}{\varrho_{2}} \frac{\partial \sqrt{E}}{\partial v}, \quad \frac{\partial}{\partial u}\left(\frac{\sqrt{G}}{\varrho_{2}}\right)=\frac{1}{\varrho_{1}} \frac{\partial \sqrt{G}}{\partial u}
\end{array}\right.
$$

Similar equations for $\bar{S}$ are satisfied by the functions $\bar{E}, \bar{G}, \bar{\varrho}_{1}$ and and $\bar{\varrho}_{2}$ given by (23) and (26), provided (28) is satisfied. Hence if (23), (26) and (28) hold, the conditions of the problem are satisfied.

The central nets $N_{0}$ and $\bar{N}_{0}$ of the two transformations $R$ are $2, O$, the complementary function being the radius $R$ of the spheres for each net. In $\S 27$ it was shown that any net $\bar{N}_{0}^{\prime}$ parallel to $\overline{N_{0}}$ is applicable to a net $N_{0}^{\prime}$ determined by a quadrature, which is parallel to $N_{0}$. From (VI, Ex. 1) it follows that $N_{0}^{\prime}$ and $\bar{N}_{0}^{\prime}$ are nets $2, O$, and that they have the same complementary function $R^{\prime}$ which is 
obtained by a quadrature. Moreover, from $\S 76$ it follows that two of the nets $\bar{N}_{0}^{\prime}$ parallel to $\bar{N}_{0}$ are special, that is $\sum \bar{x}_{0}^{\prime 2}=R^{\prime 2}$. Hence if the central nets $N_{0}$ and $\bar{N}_{0}$ of two transformations $R$ are applicable, there exist two transformations $R$ whose central nets $N_{0}^{\prime}$ and $\bar{N}_{0}^{\prime}$ are parallel to $N_{0}$ and $\bar{N}_{0}$, such that the spheres of the transformation with centers on $\bar{N}_{0}^{\prime}$ pass through the origin. We apply the preceding formulas to this case.

If in (27) we put $V \overline{\bar{E}_{1}^{\prime}}=\sqrt{\overline{\bar{G}}_{1}^{\prime}}=0$, we get

$$
\sqrt{\overline{E^{\prime}}}=\sqrt{E^{\prime}}-\sqrt{E_{1}^{\prime}}, \quad \sqrt{\overline{G^{\prime}}}=\sqrt{G^{\prime}}-\sqrt{G_{1}^{\prime}} .
$$

Now equation (28) reduces to

$$
\sqrt{E_{1}^{\prime}} \sqrt{G^{\prime}}+\sqrt{G_{1}^{\prime}} \sqrt{E^{\prime}}=0,
$$

that is $N^{\prime}$ and $N_{1}^{\prime}$ are conformal. But as shown in $\S 82$ this is possible only in case $N^{\prime}$ and $N_{1}^{\prime}$ are isothermic nets in the relation of a transformation $D_{m}$. If we make use of the results of $\S 82$ and put

$$
\sqrt{E^{\prime}}=\sqrt{G^{\prime}}=e^{\psi}, \quad \sqrt{E_{1}^{\prime}}=-\sqrt{G_{1}^{\prime}}=\frac{\theta^{\prime}}{\nu^{\prime}} e^{-\psi},
$$

in (30) we find that (26) is satisfied. Hence we have the theorem of Bianchi ${ }^{104}$ ):

The central net $N_{0}^{\prime}$ of a transformation $D_{m}$ of an isothermic net $N^{\prime}$ into an isothermic net $N_{1}^{\prime}$ admits an applicable net $\overline{N_{0}^{\prime}}$ such that as $N_{0}^{\prime}$ rolls on $\bar{N}_{0}^{\prime}$ the spheres of the transformation pass through a fixed point $O$.

This result may be looked as follows: When $\overline{N_{0}^{\prime}}$ rolls on $N_{0}^{\prime}$, the point $O$ invariably fixed to $\bar{N}_{0}^{\prime}$ generates the surface $S_{1}^{\prime}$. In the terminology of Bianchi ${ }^{105}$ ) $S_{1}^{\prime}$ is a surface of rolling, being generated by a point fixed to one surface as the latter rolls over an applicable surface. From the foregoing results we have also the converse theorem $\left.{ }^{106}\right)$ :

101) Rendiconti dei Lincei, ser. 5, vol. 24 (1915), p. 303.

19:) Rendiconti dei Lincei, ser. 5, vol. $23^{1}$ (1914), p. 4.

${ }^{106)}$ Rendiconti dei Lincei, ser. 5, vol. 24 (1915), p. 349. 
Transformations $D_{m}$ are the only transformations $R$ for which one of the two sheets of the envelope of the spheres is a surface of rolling as the central net rolls on its applicable net.

In $\S 78$ we saw if $N_{0}$ is any net parallel to $N_{0}^{\prime}$ and $R$ is the complementary function, the sheets of the envelope of the spheres of centers on $N_{0}$ and radius $R$ are parallel to the isothermic nets $N^{\prime}$ and $N_{1}^{\prime}$. Hence we have the theorem:

$A$ necessary and sufficient condition that an $O$ net $N$ admits an $R$ transform $N_{1}$ such that the central net of the transformation is applicable to a net is that $N$ have the same spherical representation as an isothermic net.

From $(V \Pi, 3)$ it follows that the functions $\frac{1}{\sqrt{G}} \frac{\partial \sqrt{E}}{\partial v}$ and $\frac{1}{\sqrt{E}} \frac{\partial \sqrt{G}}{\partial u}$ have the same values for all parallel $O$ nets. Consequently we must have

$$
\frac{1}{\sqrt{G}} \frac{\partial \sqrt{E}}{\partial v}=\frac{\partial \psi}{\partial v}, \quad \frac{1}{\sqrt{E}} \frac{\partial \sqrt{G}}{\partial u}=\frac{\partial \psi}{\partial u} .
$$

From (31) it follows that

$$
\left\{\begin{array}{l}
\frac{1}{\sqrt{G_{1}}} \frac{\partial \sqrt{E_{1}}}{\partial v}=-\frac{\partial}{\partial v} \log \left(\frac{\theta^{\prime}}{\nu^{\prime}} e^{-\psi}\right), \\
\frac{1}{\sqrt{E_{1}}} \frac{\partial \sqrt{G_{1}}}{\partial u}=-\frac{\partial}{\partial u} \log \left(\frac{\theta^{\prime}}{\nu^{\prime}} e^{-\psi}\right) .
\end{array}\right.
$$

We remark that $p, q, r$ of the $R$ transformation of $N$ into $N_{1}$ and $N^{\prime}$ into $N_{1}^{\prime}$ are the same $(\S 78)$. Hence if in (VII, 8) we put $w=m v$, where $m$ is a constant, it follows from this equation and (VII, 49) written $p^{2}+q^{2}+r^{2}=2 m \theta^{\prime} \nu^{\prime}$ that

$$
\theta \nu=\theta^{\prime} \nu^{\prime}
$$

Consequently from (33), (VII, 47) and the last two of equations (VII, 7) we get

$$
\sqrt{E_{1}}+V E=e^{\psi} \frac{\theta}{\theta^{\prime}}+e^{-\downarrow} \frac{\theta^{\prime}}{\nu}, \quad \sqrt{G_{1}}+\sqrt{G}=e^{\psi} \frac{\theta}{\theta^{\prime}}-e^{-\psi} \frac{\theta^{\prime}}{\nu} .
$$


The equations of the $R$ transformation from $N$ into $N_{1}$ are

$$
x_{1}=x-\frac{1}{m \nu}(p X+q \xi+r \eta)
$$

where the functions satisfy the system

$$
\begin{aligned}
& \frac{\partial \theta}{\partial u}=\sqrt{E} q, \quad \frac{\partial \theta}{\partial v}=\sqrt{G} r, \\
& \frac{\partial \boldsymbol{\theta}^{\prime}}{\partial u}=e^{\natural} q, \quad \frac{\partial \boldsymbol{\theta}^{\prime}}{\partial v}=e^{\natural} r, \\
& \frac{\partial p}{\partial u}=-\frac{\sqrt{E}}{\varrho_{1}} q, \quad \frac{\partial p}{\partial v}=-\frac{\sqrt{G}}{\underline{o}_{2}} r, \\
& \frac{\partial \boldsymbol{q}}{\partial u}=\frac{\sqrt{\boldsymbol{E}}}{\varrho_{1}} p-\frac{\partial \psi}{\partial v} r+m\left(e^{\psi} \frac{\boldsymbol{\theta}}{\boldsymbol{\theta}^{\prime}} \nu+e^{-\psi} \boldsymbol{\theta}^{\prime}\right), \quad \frac{\partial q}{\partial v}=\frac{\partial \psi}{\partial u} r, \\
& \frac{\partial r}{\partial u}=\frac{\partial \psi}{\partial v} q, \quad \frac{\partial r}{\partial v}=\frac{\sqrt{G}}{\varrho_{2}}-\frac{\partial \psi}{\partial u} q+m\left(e^{\psi} \frac{\theta}{\theta^{\prime}} \nu-e^{-\psi} \theta^{\prime}\right), \\
& \frac{\partial \nu}{\partial u}=\frac{q}{\theta}\left[\nu\left(e^{\psi} \frac{\theta}{\theta^{\prime}}-V \bar{E}\right)+e^{-\psi} \theta^{\prime}\right], \\
& \frac{\partial \nu}{\partial v}=\frac{r}{\theta}\left[\nu\left(e^{\psi} \frac{\theta}{\theta^{\prime}}-\sqrt{G}\right)-e^{-\varphi} \theta^{\prime}\right] \text {. }
\end{aligned}
$$

Hence:

Any $O$ net having the same spherical reprensentation as an isothermic net admits $\infty^{5} R$ transformations for which the central net $N_{0}$ is applicable to a net $\bar{N}_{0}$ which is 2,0 .

For the case of $N^{\prime}$ and $\overline{N^{\prime}}$ equations (25) can be integrated with the result $\varrho=1 / \nu^{\prime}$. Since $p, q, r$ are the same for the transformation from $N^{\prime}$ into $N_{1}^{\prime}$ and $N$ into $N_{1}$, it follows that $\varrho$ has this value in general. Hence the transformation functions from $\overline{N^{\prime}}$ into $\overline{N_{1}^{\prime}}$ are

(37) $\bar{p}=\frac{p}{\nu^{\prime}}, \quad \bar{q}=\frac{q}{\nu^{\prime}}, \quad \bar{r}=\frac{r}{\nu^{\prime}}, \quad \overline{\theta^{\prime}}=\frac{\theta^{\prime}}{\nu^{\prime}}, \quad \bar{\nu}^{\prime}=1$, and for $\bar{N}$ into $\overline{N_{1}}$

$$
\bar{p}, \bar{q}, \bar{r}, \bar{\theta}=\frac{\theta}{\nu^{\prime}}, \quad \bar{\nu}=\frac{\nu}{\nu^{\prime}} .
$$


From (30), (31) and (23) we find

$$
\left\{\begin{aligned}
\sqrt{\overline{E^{\prime}}}=e^{\psi}-\frac{\theta^{\prime}}{\nu^{\prime}} e^{-\psi}, & \sqrt{\overline{\overline{G^{\prime}}}}=e^{\psi}+\frac{\theta^{\prime}}{\nu^{\prime}} e^{-\psi}, \\
\frac{\sqrt{\overline{E^{\prime}}}}{\overline{\varrho_{1}^{\prime}}}=\frac{e^{\psi}}{\varrho_{1}^{\prime}}+\frac{p}{\nu^{\prime}} e^{-\psi}, & \frac{\sqrt{\overline{G^{\prime}}}}{\overline{\varrho_{2}^{\prime}}}=\frac{e^{\psi}}{\varrho_{2}^{\prime}}-\frac{p}{\nu^{\prime}} e^{-\psi},
\end{aligned}\right.
$$

where $\varrho_{1}^{\prime}, \varrho_{2}^{\prime}$ and $\bar{\varrho}_{1}^{\prime}, \bar{\varrho}_{2}^{\prime}$ are the principal radii of the isothermic net $N^{\prime}$ and the net $\overline{N^{\prime}}$ respectively.

Since the left-hand members of (26) are the same for $N^{\prime}, \overline{N^{\prime}}$ and $N, \bar{N}$, we have from this equation, (39) and (34)
(40) $\left\{\begin{array}{l}\sqrt{\bar{E}}=\sqrt{\bar{E}}-\frac{\theta^{\prime}}{\nu} e^{-\vartheta,}, \\ \frac{\sqrt{\bar{E}}}{\bar{\varrho}_{1}}=\frac{\sqrt{E}}{\varrho_{1}}+\frac{p}{\theta} \frac{\theta^{\prime}}{\nu} e^{-\downarrow,},\end{array}\right.$

$$
\sqrt{\bar{G}}=\sqrt{G}+\frac{\theta^{\prime}}{\nu} e^{-\psi}
$$$$
\frac{\sqrt{\bar{G}}}{\bar{\varrho}_{2}}=\frac{\sqrt{\bar{G}}}{\varrho_{2}}-\frac{p}{\theta} \frac{\theta^{\prime}}{\nu} e^{-\psi} .
$$

Also from (27), (35) and (VII, 9)

$$
\begin{cases}\sqrt{\overline{E_{1}}}=\frac{\theta}{\theta^{\prime}} e^{\psi}-V \bar{E}, & \sqrt{\overline{G_{1}}}=\frac{\theta}{\theta^{\prime}} e^{\psi}-V \bar{G}, \\ \frac{\sqrt{\bar{E}_{1}}}{\varrho_{11}}=\frac{V \bar{E}}{\varrho_{1}}+e^{\psi} \frac{p}{\theta^{\prime}}, & \frac{V \overline{\overline{G_{1}}}}{\overline{\varrho_{12}}}=\frac{V \bar{G}}{\varrho_{2}}+e^{\psi} \frac{p}{\theta^{\prime}} .\end{cases}
$$

Since the nets $\bar{N}^{\prime}$ and $\bar{N}$ are defined intrinsically, the determination of the cartesian coordinates of these nets requires the solution of a Riccati equation.

124. Deformable transformations of the second type of minimal surfaces. We apply the results of the preceding section to the case when the transformation $D_{m}$ is the one considered in $\S 87$ which transforms the $O$ net $N^{\prime}$ of a minimal surface into the $O$ net $N_{1}^{\prime}$ of a minimal surface. Then $\nu^{\prime}=p$ and from (39) and (VII, 70) we have

$$
\frac{\sqrt{\overline{E^{\prime}}}}{\overline{\varrho_{1}^{\prime}}}=\frac{\sqrt{\overline{G^{\prime}}}}{\overline{\varrho_{2}^{\prime}}}=0
$$


that is $\bar{N}^{\prime}$ is a plane net. We have seen in the preceding section that $\overline{N_{1}^{\prime}}$ is a point net. Hence the spheres of the transformation pass through a point and are tangent to a plane; consequently the central net lies on a paraboloid of revolution. In order to obtain the equation of the paraboloid, we find the distance from the point $\bar{N}_{1}^{\prime}$ to the plane $\overline{N^{\prime}}$. From (VII, 48) and the condition $\nu^{\prime}=p$ we find that this distance is $1 / \mathrm{m}$. Hence the equation of the paraboloid is $m r^{2}=2 z$. Therefore we have the theorem:

The surface of centers of a transformation $D_{m}$ of a minimal surface into a minimal surface is applicable to the paraboloid of revolution $\left.m\left(x^{2}+y^{2}\right)=2 z^{107}\right)$.

125. Deformable transformations $E_{m}$. From $\S 88$ and (32) it is seen that a transformation $E_{m}$ of a surface with isothermal representation of its lines of curvature into a surface of the same kind is a deformable transformation $R$ of the second type. We apply the results of $\S 88$ to this case.

Comparing equations (VII, 76) and (36), we find that the functiou $\theta^{\prime}$ in (36) is given by

$$
\theta^{\prime}=\frac{\theta \nu}{p}
$$

From (40) it follows that $\sqrt{\bar{E}} / \bar{\varrho}_{1}=\sqrt{\bar{G}} / \varrho_{2}=0$, that is $\bar{N}$ is a planar net. This result may be stated as follows: As $\bar{S}_{0}$ rolls over $S_{0}$, a plane invariably fixed to $\bar{S}_{0}$ has $S$ for its envelope. Bianchi ${ }^{108}$ ) calls $S$ an envelope of rolling in this case. Hence we have the theorem of Bianchi ${ }^{109}$ ):

$A$ surface $S$ with isothermal representation of its lines of curvature is an envelope of rolling, when the central net $N_{0}$ of any $E_{m}$ transformation of $S$ rolls on its applicable net.

We shall prove the converse theorem ${ }^{110}$ ):

Transformations $E_{m}$ are the only transformations $R$ for which the given surface is an envelope of rolling as the central net $N_{0}$ rolls on its applicable net.

107) Cf. Bianchi, Memoire dei Lincei, ser. 5, rol. 12 (1918), p. 532.

$\left.{ }^{108}\right)$ Rendiconti dei Lincei, ser. 5, vol. $23^{1}$ (1914), p. 3.

$\left.{ }^{109}\right)$ Rendiconti dei Lincei, ser. 5, vol. 24 (1915), p. 367.

11n) Annals of Mathematics, ser. 2, vol. 17 (1915), p. 64. 
If $\bar{S}$ is to be a plane, we must have from (40)

$$
\frac{\sqrt{E}}{\varrho_{1}}+\frac{\sqrt{G}}{\varrho_{2}}=0, \quad \frac{\sqrt{E}}{\varrho_{1}}=-\frac{p}{\theta} \cdot \frac{\theta^{\prime}}{\nu} e^{-\psi} .
$$

From the first it follows that the spherical representation of $S$ is isothermal. Replacing the first by (VII, 70), we have from the second that (42) must hold, and consequently the transformation is $E_{m}{ }^{111}$ ).

126. Transformations $F$ of deformable transformations $R$ of the second type. Consider a deformable congruence $R$ of spheres of the second type $G$, and denote by $G^{\prime}$ the parallel deformable congruence $R$ which gives a transformation $D_{m}(\S 123)$. Let $N_{0}$ denote the central net of $G$ and $\bar{N}_{0}$ of the deform $\bar{G}$; likewise $N_{0}^{\prime}$ and $\bar{N}_{0}^{\prime}$. Then, as we have seen

$$
\sum{\overline{x_{0}^{\prime}}}^{2}=R^{\prime 2}
$$

From $\S 76$ it follows that if we take the $F$ transform of $N_{0}$ by means of $\theta^{\prime}=\frac{1}{2}\left(\sum x_{0}^{\prime 2}-R^{\prime 2}\right)$, the coordinates $x_{0}^{\prime}$ being the direction-parameters of the conjugate congruence of the transformation, we get a net $N_{10}$ which is $2, O$, the complementary function being

$$
R_{1}=R-\frac{\theta}{\theta^{\prime}} R^{\prime}
$$

Moreover, in consequence of (43) we have

$$
\theta^{\prime}=\frac{1}{2}\left(\sum x_{0}^{\prime 2}-\sum \bar{x}_{0}^{\prime 2}\right)
$$

Hence from $\S 27$ it follows that the $F$ transform of $\bar{N}_{0}$ by means of $\theta^{\prime}$, the coordinates $\bar{x}_{0}^{\prime}$ being the direction-parameters of the conjugate congruence of the transformation, is applicable to $N_{10}$. Then $\sum x_{10}^{2}-\sum \bar{x}_{10}^{2}$ is a solution of the common point equation

111) Similar results follow, if we require that $\bar{S}_{1}$ be a plane. 
of $N_{10}$ and $\overline{N_{10}}$. Since $\sum x_{10}^{2}-R_{1}^{2}$ is a solution of this equation (§ 76), so also is $\sum \bar{x}_{10}^{2}-R_{1}^{2}$, and consequently $\bar{N}_{10}$ is $2, O$. Hence:

When a deformable congruence $R$ of spheres of the second type is known, another congruence of the same type can be found by quadratures ${ }^{112}$ ).

127. Converses of the theorems of Guichard. In this section we determine under what conditions the two sheets of the envelope of a congruence of spheres have the same constant mean curvature for all deformations of the central surface $S_{0}$. We consider first the case when the sheets are minimal surfaces.

If $\varrho_{1}$ and $\varrho_{2}$ denote the principal radii of curvature of $S$, then

$$
\varrho_{1}=R-t_{1}, \quad \varrho_{2}=R-t_{2},
$$

where $t_{1}$ and $t_{2}$.are the roots of the equation obtained by eliminating $d u$ and $d v$ from (12). In consequence of (15) this equation is reducible to

$$
\left\{\begin{array}{c}
t^{2}\left[\left(p q_{1}-p_{1} q\right) \sin \sigma+r_{1} \cos \sigma\left(q-\frac{\partial \sigma}{\partial u}\right)+p_{1} \sin \sigma \frac{\partial \sigma}{\partial u}\right] \\
+t\left[\eta_{1}\left(q-2 \frac{\partial \sigma}{\partial u}\right)-\xi \sin ^{y} \sigma p_{1}\right]+\xi \eta_{1} \sin \sigma=0 .
\end{array}\right.
$$

If $S$ is to be a minimal surface, we must have $2 R=t_{1}+t_{2}$, that is

$$
2 \mathrm{R}=\frac{\xi \sin ^{2} \sigma p_{1}-\eta_{1}\left(q-2 \frac{\partial \sigma}{\partial u}\right)}{\left(p q_{1}-\dot{p}_{1} q\right) \sin \sigma+r_{1} \cos \sigma\left(q-\frac{\partial \sigma}{\partial u}\right)+p_{1} \sin \sigma \frac{\partial \sigma}{\partial u}} .
$$

If $S_{\mathbf{1}}$ also is to be minimal, this equation must be satisfied also when $\sigma$ is replaced by $-\sigma$. This gives the two equations

$$
\left\{\begin{array}{c}
{\left[\left(p q_{1}-p_{1} q\right) \sin \sigma-r_{1} \cos \sigma \frac{\partial \sigma}{\partial u}\right] R=\eta_{1} \frac{\partial \sigma}{\partial u}} \\
\left(2 R r_{1} \cos \sigma+\eta_{1}\right) q+\left(2 R \sin \sigma \frac{\partial \sigma}{\partial u}-\xi \sin ^{2} \sigma\right) p_{1}=0 .
\end{array}\right.
$$

112) Rendiconti dei Lincei, ser. 5 , vol. $30^{2}$ (1921). 
The first of these equations involves only functions which do not vary as $S_{0}$ is deformed. In order that the second hold for all deformations of $S_{0}$, we must have

$$
2 R r_{1} \cos \sigma+\eta_{1}=0, \quad 2 R \frac{\partial \sigma}{\partial u}-\xi \sin \sigma=0 .
$$

Eliminating $R$, we get the third of (15).

From (16) we have $\eta_{1}=u, r_{1}=1 / U$. In consequence of (17) the first of (46) becomes $2 R=-U \sqrt{c^{2}+u^{2}}$. When we substitute this expression in (11), we obtain $U=c_{1} \sqrt{c^{2}+u^{2}}$, where $c_{1}$ is an arbitrary constant. Hence by a suitable choice of the parameters the linear element of $S_{0}$ is reducible to the form

$$
d s_{0}^{2}=\left(1+m^{2} u u^{2}\right) d u^{2}+u^{2} d v^{2} .
$$

This is the linear element of the paraboloid of revolution $Q$, defined by (9). Also we find

$$
R=\frac{1}{2 m}\left(1+m^{2} u^{2}\right)
$$

These expressions satisfy the first of (45) and thus all the conditions of the problem are satisfied. Moreover, it can be shown that $\boldsymbol{R}$ equals the distance from a point of $Q$ to its focus on the axis of revolution. Hence we have the theorem ${ }^{113}$ ):

The spheres with centers on a paraboloid of revolution and passing through the focus constitute the only congruences $R$ of spheres the sheets of whose envelope are minimal surfaces in all deformations of the central surface.

We consider next the case when the two sheets have the same constant mean curvature $1 / a$ for all deformations of $S_{0}$. In this case

$$
R^{2}-2 a R+(a-R)\left(t_{1}+t_{2}\right)+t_{1} t_{2}=0 .
$$

Substituting the expressions for $t_{1}+t_{2}$ and $t_{1} t_{2}$ from (44) and requiring that this condition hold for both $S$ and $S_{1}$, we get

$\left.{ }^{113}\right)$ Cf. Bianchi, Memoire dei Lincei, ser. 5, rol. 12 (1918), p. 479. 


$$
\text { (49) }\left\{\begin{array}{r}
\left(R^{2}-2 a R\right)\left[\left(p q_{1}-p_{1} q\right) \sin \sigma-r_{1} \cos \sigma \frac{\partial \sigma}{\partial u}\right] \\
+2(a-R) \eta_{1} \frac{\partial \sigma}{\partial u}+\xi \eta_{1} \sin \sigma=0, \\
{\left[\left(R^{2}-2 a R\right) r_{1} \cos \sigma-(a-R) \eta_{1}\right] q} \\
+\left[\left(R^{2}-2 a R\right) \frac{\partial \sigma}{\partial u}+(a-R) \xi \sin \sigma\right] p_{1} \sin \sigma=0 .
\end{array}\right.
$$

Since these equations must be true for all deformations of $S_{0}$, the coefficients of $q$ and $p_{1}$ in the second must be equal to zero. In consequence of the third of (15) this is equivalent to the single condition

$(50)$

$$
\left(R^{2}-2 a R\right) \cos \sigma+U u(R-a)=0 .
$$

On differentiating this equation with respect to $u$ and making use of (11) and (15), we obtain

$$
(R-a)\left(U \cos ^{2} \sigma-u U^{\prime}\right)+u U^{2} \cos \sigma=0 .
$$

Eliminating $R$ from (50) and (51) and substituting for $\cos \sigma$ its expression from (17), we find

$$
U^{\prime 2}-u U^{\prime}\left(\frac{2 U}{u^{2}+c^{2}}+\frac{U^{3}}{a^{2}}\right)+\frac{U^{2} u^{2}}{\left(u^{2}+c^{2}\right)^{2}}=0 .
$$

When (50) and (52) are satisfied, so also is the first of (49).

The general integral of (52) is

$$
U=a \sqrt{\frac{u^{2}+c^{2}}{\left(l-c^{2}\right)\left(u^{2}+l\right)}},
$$

where $l$ is an arbitrary constant. Hence the linear element of $S_{0}$ is

$$
d s_{0}^{2}=\frac{a^{2}\left(u^{2}+c^{2}\right)}{\left(l-c^{2}\right)\left(u^{2}+l\right)} d u^{2}+u^{2} d v^{2} .
$$

This is the linear element also of the quadric $Q$ of revolution of the conic

$$
\frac{x^{2}}{a^{2}}-\frac{y^{2}}{l}=1,
$$


about the $x$-axis, provided that

$$
c^{2}=\frac{l^{2}}{a^{2}+l}
$$

Hence to each choice of $l$ there corresponds a value of $c$.

Equation (51) reduces to

$$
R-a+\sqrt{\frac{a^{2}+l}{l}} \sqrt{u^{2}+l}=0 .
$$

Furthermore since $a^{2}$ enters only in (52), we have that $a$ in (50) and (51) may be replaced by - a. Accordingly we have also

$$
R+a+\sqrt{\frac{a^{2}+l}{l}} \sqrt{u^{2}+l}=0 .
$$

Hence the spheres with centers on the quadric $Q$ may pass through either of the foci on the $x$-axis, and we have the theorem ${ }^{114}$ ):

The spheres with centers on a central quadric of revolution and passing through either focus on the axis of revolution constitute the only congruences $R$ of spheres the sheets of whose envelope have the same constant mean curvature for all deformations of the central surface.

128. Theorems of Ribaucour and Bianchi. In accordance with the theorem of Beltrami $(\S 112)$ the tangent planes to either sheet of the envelope of a congruence of spheres envelop a surface for all deformations of the surface of centers, if these planes are understood to be carried along in the deformation. We seek the general solution of the problem: To determine all cases for which the surface elements of a surface $S_{1}$ associated with a surface $S$ continue to be the surface elements of a surface in any deformation of $\left.S^{115}\right)$.

Let $S$ be referred to any system of parametric lines. Between the coordinates, $x, y, z$, of $S$ and $x_{1}, y_{1}, z_{1}$, of $S_{1}$ we have relations of the form

114) Cf. Bianchi, 1. c., p. 481.

$\left.{ }^{115}\right)$ Cf. Bianchi, Rendiconti dei Lincei, ser. 5, vol. 24 (1915), p. 3. 


$$
x_{1}=x+l \frac{\partial x}{\partial u}+m \frac{\partial x}{\partial v}+n X
$$

where $l, m$ and $n$ are the same for $y_{1}$ and $z_{1}$. Differentiating this equation and making use of $[\S 64,(7),(8)]$, we obtain

$$
\left\{\begin{array}{c}
\frac{\partial x_{1}}{\partial u}=\left(L+n \frac{F D^{\prime}-G D}{H^{2}}\right) \frac{\partial x}{\partial u} \\
+\left(M+n \frac{F D-E D^{\prime}}{H^{2}}\right) \frac{\partial x}{\partial v}+\left(\frac{\partial n}{\partial u}+D l+D^{\prime} m\right) X \\
\frac{\partial x_{1}}{\partial v}=\left(P+n \frac{F D^{\prime \prime}-G D^{\prime}}{H^{2}}\right) \frac{\partial x}{\partial u} \\
+\left(Q+n \frac{F D^{\prime}-E D^{\prime \prime}}{H^{2}}\right) \frac{\partial x}{\partial v}+\left(\frac{\partial n}{\partial v}+D^{\prime} l+D^{\prime \prime} m\right) X
\end{array}\right.
$$

where

(58)
$\left\{L=\frac{\partial l}{\partial \imath}+\left\{\begin{array}{c}11 \\ 1\end{array}\right\} l+\left\{\begin{array}{c}12 \\ 1\end{array}\right\} m+1\right.$
$M=\frac{\partial m}{\partial \imath}+\left\{\begin{array}{c}11 \\ 2\end{array}\right\} l+\left\{\begin{array}{c}12 \\ 2\end{array}\right\} m$,

the Christoffel symbols being formed with respect to the linear element of $S$.

If $X_{1}, Y_{1}$ and $Z_{1}$ denote the direction-cosines of the normal to $S_{1}$, we have

$$
X_{1}=\lambda \frac{\partial x}{\partial u}+\mu \frac{\partial x}{\partial v}+\nu X
$$

and similar equations for $Y_{1}$ and $Z_{1}$, where $\lambda, \mu, \nu$ must be such that

$$
\sum \frac{\partial x_{1}}{\partial u} X_{1}=0, \quad \sum \frac{\partial x_{1}}{\partial v} X_{1}=0
$$

Substituting from the above equations, we get

j0)

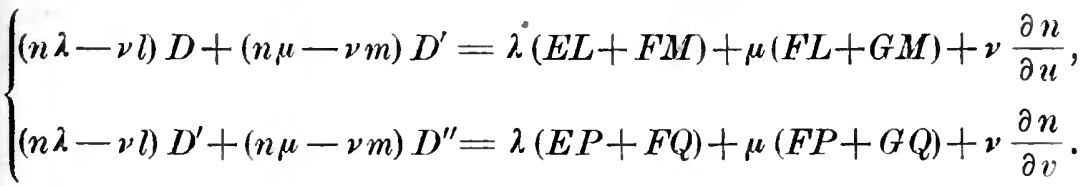


If $S$ is deformed in any manner carrying the surface-elements of $S_{1}$ invaribly bound to it, for a deform $\bar{S}$ of $S$ we have

$$
\left\{\begin{array}{l}
\bar{x}_{1}=\bar{x}+l \frac{\partial \bar{x}}{\partial u}+m \frac{\partial \bar{x}}{\partial v}+n \bar{X}, \\
\bar{X}_{1}=\lambda \frac{\partial \bar{x}}{\partial u}+\mu \frac{\partial \bar{x}}{\partial v}+\nu \bar{X},
\end{array}\right.
$$

where $l, m, n, \lambda, \mu, \nu$ are unaltered by the deformation. This fact is of prime importance.

The right-hand members of (60) involve only quantities unaltered by the deformation. Consequently we must have

$$
n \lambda=\nu l, \quad n \mu=\nu m .
$$

There are two cases to be considered,

$I^{\circ}$. When $\nu \neq 0$, then

$$
x_{1}=x+\frac{n}{\nu} X_{1}
$$

which expresses the fact that $S_{1}$ is a sheet of the envelope of spheres with centers on $S$, that is the theorem of Beltrami.

$2^{\circ}$. When $v=0$, then $n=0$. In this case the points of $S_{1}$ lie in the corresponding tangent planes to $S$, and since $\sum X X_{1}=0$, corresponding tangent planes to $S$ and $S_{1}$ are perpendicular. The existence of this case was established by Ribaucour $\left.{ }^{116}\right)$. Hence we have the theorem of Bianchi ${ }^{117}$ ):

A necessary and sufficient condition that $\infty^{2}$ surface-elements invaribly associated with a surface $S$ continue to be the surface-elements of a surface in all deformations of $S$ is that they are the elements of a sheet of the envelope of a congruence of spheres with centers on $S$, or of a surface $S_{1}$ such that corresponding tangent planes to $S$ and $S_{1}$ are perpendicular and points of $S_{1}$ lie in the corresponding tangent planes to $S$.

We remark that if equations.(62) hold for one deformation of $S$, they hold for every deformation.

$\left.{ }^{116}\right)$ Journ. de Math., ser. 4, vol. 7 (1891), p. 92.

117) L. c., p. 4. 
129. The surface generated by a point in the tangent plane to a surface $\bar{S}$ as $\bar{S}$ rolls on an applicable surface $S$. The formulas of the preceding section can be used to find the surface generated by a point $P$ associated with $\bar{S}$ as $\bar{S}$ rolls on an applicable surface $S$. When in particular $P$ lies in the tangent plane to $\bar{S}$, we have $n=0$. We consider this case.

If we put

$\left\{\begin{array}{c}\Omega_{1}=L^{2} E+2 L M F+M^{2} G, \quad \Omega_{2}=L P E+(L Q+M P) F+M Q G \\ \Omega_{3}=P^{2} E+2 P Q F+Q^{2} G\end{array}\right.$

the first fundamental coefficients of $S_{1}$ are found from (57) to be

$$
\left\{\begin{array}{c}
E_{1}=\Omega_{1}+\left(l D+m D^{\prime}\right)^{2}, \quad F_{1}=\Omega_{2}+\left(l D+m D^{\prime}\right)\left(l D^{\prime}+m D^{\prime \prime}\right) \\
G_{1}=\Omega_{9}+\left(l D^{\prime}+m D^{\prime \prime}\right)^{2}
\end{array}\right.
$$

Also the coefficients of $\overline{S_{1}}$ are

$$
\left\{\begin{array}{c}
\overline{E_{1}}=\boldsymbol{\Omega}_{1}+\left(l \bar{D}+m \overline{D^{\prime}}\right)^{2}, \quad \overline{F_{1}}=\boldsymbol{\Omega}_{2}+\left(l \bar{D}+m \overline{D^{\prime}}\right)\left(l \bar{D}^{\prime}+m \overline{D^{\prime \prime}}\right) \\
\overline{G_{1}}=\boldsymbol{\Omega}_{\mathbf{3}}+\left(l \bar{D}^{\prime}+m \overline{D^{\prime \prime}}\right)^{2}
\end{array}\right.
$$

where $\bar{D}, \overline{D^{\prime}}, \overline{D^{\prime \prime}}$ are the second fundamental coefficients of $\bar{S}$.

Since the functions $\Omega_{1}, \Omega_{2}, \Omega_{3}$ are the same for both surfaces, we have from (64) and (65)

$$
\left\{\begin{array}{c}
d s_{1}^{2}-d \bar{s}_{1}^{2^{-}}=\left[\left(l D+m D^{\prime}\right) d u+\left(l D^{\prime}+m D^{\prime \prime}\right) d v\right]^{2} \\
-\left[\left(l \bar{D}+m \overline{D^{\prime}}\right) d u+\left(l \overline{D^{\prime}}+m \bar{D}^{\prime \prime}\right) d v\right]^{2}
\end{array}\right.
$$

As an application of this result we consider the case when $\bar{S}$ is a ruled surface and $S$ is not ruled, and we take for the curves $v=$ const. the generators of $\bar{S}$, that is $\bar{D}=0$. We take the point $P$ determined by the condition that it lies on a generator of $\bar{S}$ and is such that as $\bar{S}$ rolls on $S$ it describes a line of length zero on $\bar{S}$. Then we have $m=0, d \bar{s}_{1}=0$. Since $D D^{\prime \prime}-D^{\prime 2}$ $=-\overline{D^{\prime 2}}$, we have from $(66)$

$$
d s_{1}^{2}=l^{2} D\left(D d u^{2}+2 D^{\prime} d u d v+D^{\prime \prime} d v^{2}\right) .
$$

Hence we have the theorem of Darboux $\left.{ }^{118}\right)$ :

118) Annales de l'École Norm. Sup., ser. 3, vol. 16 (1899), p. 497. 
When a ruled surface $\bar{S}$ rolls on a non-ruled applicable surface $S$, the points where the different lines of length zero of $\bar{S}$ meet the generators of $\bar{S}$ describe surfaces which are conformably represented on one another; and their lines of length zero correspond to the asymptotic lines on $S$.

The surfaces described by the points $a, a_{1}, a^{\prime}, a_{1}^{\prime}$ in $\S 121$ are examples of this theorem.

130. Kinematically conjugate directions on rolling surfaces. If on a surface $\bar{S}$ we take a curve $C$ of the family determined by an equation of the form $d v=M d u$, and at each point of $C$ draw the tangents to the curves of another family determined by an equation $\delta v=N \delta u$, the coordinates of this ruled surface $\bar{R}$ are given by (61), when we put $l=t \delta u, m=t \delta v$, $n=0, t$ being the parameter of a point on the line. As $\bar{S}$ rolls on an applicable surface $S$, along $C$, these lines generate a ruled surface $R$ tangent to $S$. From (66) it follows that a necessary and sufficient condition that $R$ and $\bar{R}$ be applicable is that

(68) $(D+\bar{D}) d u \delta u+\left(D^{\prime}+\bar{D}^{\prime}\right)(d u \boldsymbol{\delta} v+d v \delta u)+\left(D^{\prime \prime}+\overline{D^{\prime \prime}}\right) d v \boldsymbol{\delta} v=0$, 01

(69) $(D-\bar{D}) d u \boldsymbol{\delta} u+\left(D^{\prime}-\bar{D}^{\prime}\right)(d u \boldsymbol{\delta} v+d v \boldsymbol{\delta} u)+\left(D^{\prime \prime}-\bar{D}^{\prime \prime}\right) d v \boldsymbol{\delta} v=0$.

If we desire the condition to be satisfied for all deformations of $\bar{S}$ and in particular when $S$ and $\bar{S}$ are congruent, we must take (69). In fact (68) is obtained from (69), if $\bar{S}$ is replaced by its symmetric with respect to the origin.

Equation (69) coordinates with every family of curves on $\bar{S}$ a second family, and the relation is involutoric. Beltrami ${ }^{119}$ ) has called two such families kinematically conjugate. The preceding results may be stated as follows:

If $S$ and $\bar{S}$ are applicable surfaces, and $C$ and $\bar{C}$ are any two corresponding curves, the ruled surfaces, $R$ and $\bar{R}$, consisting of the tangents to $S$ and $\bar{S}$ in directions kinematically conjugate to $C$ and $\bar{C}$ are applicable.

As $\bar{S}$ rolls on $S$ and $\bar{C}$ rolls on $C$, coincident lines of $R$ and $\bar{R}$ form the instantaneous axis of rotation of the rolling. Hence:

$\left.{ }^{119}\right)$ Giornale di Battagline, vol. 10 (1872), p. 103. 
When a surface $\bar{S}$ rolls on an applicable surface $S$, the instantaneous axis of rotation lies in the plane of contact and its direction is kinematically conjugate to the direction of motion of the instantaneous center.

Since the common conjugate system of curves on $S$ and $\bar{S}$ satisfies the equations

$$
\begin{aligned}
& D d u \delta u+D^{\prime}(d u \delta v+d v \delta u)+D^{\prime \prime} d v \delta v=0, \\
& \bar{D} d u \delta u+\overline{D^{\prime}}(d u \delta v+d v \delta u)+\overline{D^{\prime \prime}} d v \delta v=0,
\end{aligned}
$$

we have the theorem:

The common conjugate system on two applicable surfaces is kinematically conjugate.

When $\bar{S}$ rolls along a curve of either family of the common conjugate system, the surfaces $R$ and $\bar{R}$ are developables whose edges of regression correspond, since the points of these edges are Laplace transforms of points of $C$ and $\bar{C}$.

The equation

$$
(D-\bar{D}) d u^{2}+2\left(D^{\prime}-\overline{D^{\prime}}\right) d u d v+\left(D^{\prime \prime}-\overline{D^{\prime \prime}}\right) d v^{2}=0
$$

defines two families of curves on $S$ and $\bar{S}$ which are kinematically auto-conjugate, that is as $\bar{S}$ rolls on $S$ along one of these curves the instantaneous axis is tangent to the curve itself. In this case -also the surfaces $R$ and $\bar{R}$ are developable. Since $S$ and $\bar{S}$ are applicable, the curves $C$ and $\bar{C}$ have the same geodesic curvature, at corresponding points. Also as follows from (70) and [§ 49] their normal curvatures are equal, and consequently their first curvatures.

As an example of the foregoing we consider two applicable surfaces $S$ and $\bar{S}$, and assume that the curves $u=$ const. are the asymptotic lines on $\bar{S}$. Then

$$
\bar{D}^{\prime 2}=D^{\prime 2}-D D^{\prime \prime} \text {. }
$$

In consequence of this relation we have that the equation of the asymptotic lines on $S$ may be written

$$
\left[\left(D^{\prime}-\overline{D^{\prime}}\right) d u+D^{\prime \prime} d v\right]\left[\left(D^{\prime}+\overline{D^{\prime}}\right) d u+D^{\prime \prime} d v\right]=0 .
$$

From this equation it follows that the curves kinematically conjugate to $u=$ const. on $\bar{S}$ correspond to one family of asymptotic lines 
on $S$. Bianchi has called them virtual asymptotic lines on $\bar{S}$. Moreover, the other family of virtual asymptotic lines on $\bar{S}$ satisfy (68). Hence we have the theorem ${ }^{120}$ ):

If $S$ and $\bar{S}$ are applicable surfaces, the ruled surface $\bar{R}$ consisting of the tangents to the asymptotic lines in one family where they meet a virtual asymptotic line of $\bar{S}$ is applicable to the ruled surface $R$ of tangents to the corresponding virtual asymptotic lines of $S$ where they are met by its corresponding asymptotic line.

If $\bar{S}$ is a ruled surface, we may take it for $\bar{R}$, and then $R$ consists of the tangents to the corresponding virtual asymptotic lines on $S$, which are geodesics. Hence as a corollary of the above we have the theorem of Chieffi ${ }^{121}$ ):

If $S$ is applicable to a ruled surface $\bar{R}$, the ruled surface consisting of tangents to the geodesic virtual asymptotic lines of $S$ at points of meeting of any asymptotic line a of $S$ is applicable to $S$ with a rigid in the applicability.

I3I. Congruences of rolling. When a non-ruled surface $\bar{S}$ rolls on an applicable surface $S$, a line $l$ invaribly fixed to $\bar{S}$ generates a congruence $G$ called by Bianchi ${ }^{122}$ ) a congruence of rolling. In order to find the focal points of $G$, we note that there are two instantaneous axes of rotation in the plane of contact $\pi$ at a point $M$ for which $l$ and a nearby position $l^{\prime}$ meet, namely the direction $l_{1}$ joining $M$ and the point $P_{1}$ in which $l$ meets $\pi$, and the direction $l_{2}$ normal to the plane through $l$ perpendicular to $\pi$. When $l_{1}$ is the axis of rotation, the point $P_{1}$ generates one of the focal surfaces. When $l_{2}$ is the axis, the lines $l$ and $l^{\prime \prime}$ meet in the foot $P_{2}$ of the common perpendicular of $l$ and $l_{2}$, that is the foot of the perpendicular from $M$ to $l$; and thus $P_{2}$ generates the second focal surface of $G$. The plane of the lines $l$ and $l^{\prime \prime}$ is the tangent plane to the locus of $P_{1}$ and is perpendicular to $\pi$. The plane of $l$ and $l^{\prime}$ is the tangent plane to the locus of $P_{2}$ and is normal to the line $M P_{2}$. Hence we have the theorem of Bianchi $\left.{ }^{123}\right)$ :

For any congruence of rolling one focal surface is generated.

${ }^{120}$ ) Cf. Bianchi, Memoire dei Lincei, ser. 5, vol. 12 (1918), p. 445.

121) Giomale di Battagline, vol. 43 (1905), p. 9.

${ }^{122}$ ) Rendiconti dei Lincei, ser. 5, vol. 24 (1915), p. 15.

${ }^{123}$ ) L. c. These results have been established by him by analytical processes in the Rendiconti di Palermo, vol. 39 (1915), p. 187. 
by the point $P_{1}$ in which $l$ meets the plane of contact $\pi$, and the other by the foot $P_{2}$ of the perpendicular from the point of contact $M$ to $l$; the tangent planes to these respective surfaces are the plane through $l$ nornal to $\pi$ and the plane through $P_{2}$ perpendicular. to $M P_{2}$.

From the above treatment it is seen that the focal surfaces of $G$ are generated by the same surface-elements attached to $\bar{S}$ whatever be the surface $S$ on which $\bar{S}$ rolls.

From the results of $\S 130$ and the above considerations we have that the developables of a congruence of rolling correspond to the curves kinematically conjugate to the curves on $S$ whose tangents are the lines $l_{1}$ and $l_{2}$ for a point of contact. The latter are the curves corresponding to the curves in which $\bar{S}$ is met by the pencil of planes through $l$ and by the parallel pencil of planes normal to $l$. Bianchi has called them the meridian profiles and the curves of level respectively. Hence:

For a congruence of rolling the developables correspond to the curves on $S$ kinematically conjugate to the deforms of the meridian profiles and the curves of level on $\bar{S}$.

Since a right conoid is the only surface posessing a family of meridian profiles which are also curves of level, we have the theorem of Bianchi:

The congruences of rolling with coincident developables are those, and only those, generated by the axis of a right conoid as it rolls on an applicable surface.

From the preceding considerations it is evident that the developables of a congruence of rolling are real. We remark also that the focal surfaces of a congruence of rolling afford examples of the theorems of Beltrami and Ribaucour $(\S 128)$.

\section{Exercises.}

1. Show that the theorem of Bonnet [\$ 73] may be interpreted as follows: When a sphere rolls on an applicable surface, its center describes a surface of constant mean curvature.

2. When a quadric $\bar{S}$ tangent to the circle at infinity at a single point $P$ rolls on an applicable surface $S$, the two isotropic generators of $\bar{S}$ through $P$ meet the common tangent plane in two points, $a$ and $a^{\prime}$, which describe two parallel surfaces of constant mean curvature $\pm 1 / a a^{\prime}$.

Darboux, Annales L'École Norm. Sup., ser. 3, vol. 16 (1899), p. 468. 
3. If $S$ and $\bar{S}$ are applicable surfaces and the spheres with centers on $\bar{S}$ and passing through the origin become tangent to the plane $z=0$ as $\bar{S}$ rolls on $S$, it is necessary that

If we put [cf. $\S 47$ ]

$$
\bar{x}^{2}+\bar{y}^{2}+\bar{z}^{2}=z^{2}
$$

$$
\begin{aligned}
\bar{x}=z \frac{\alpha+\beta}{\alpha \beta+1}, \quad \bar{y} & =i z \frac{\beta-\alpha}{\alpha \beta+1}, \quad \bar{z}=z \frac{\alpha \beta-1}{\alpha \beta+1}, \\
x+i y & =\omega \quad x-i y=\omega_{0},
\end{aligned}
$$

then since $S$ and $\bar{S}$ are applicable

$$
\frac{4 z^{2} d \alpha d \beta}{(\alpha \beta+1)^{2}}=d \omega d \omega_{0}
$$

Hence the projection of $\bar{S}$ on the unit sphere and of $S$ on the plane $z=0$ are conformal. The general solution is given by

$$
\omega=f(\alpha), \quad \omega_{0}=f_{0}(\beta), \quad z=\frac{1}{2}(1+a \beta) \sqrt{f^{\prime}(\alpha) f_{0}^{\prime}(\beta)},
$$

where $f$ is arbitrary and $f_{0}$ is the conjugate function.

Calo, Annali, ser. 3, vol. 4 (1900), pp. 123-130.

4. If $S$ and $\bar{S}$ are applicable surfaces and the spheres with centers on $\bar{S}$ and passing through the origin become tangents to a sphere of radius $a$ with center at the origin as $\bar{S}$ rolls on $S$, is is necessary that $\bar{x}^{2}+\bar{y}^{2}+\bar{z}^{2}=R^{2}$, $x^{2}+y^{2}+z^{2}=(R \pm a)^{2}$.

If we put

$$
\begin{aligned}
\bar{x}=R \frac{\bar{a}+\bar{\beta}}{\bar{a} \bar{\beta}+1}, & \bar{y}=i R \frac{\bar{\beta}-\bar{\alpha}}{\bar{a} \bar{\beta}+1}, & \bar{z}=R \frac{\bar{\alpha} \bar{\beta}-1}{\bar{a} \bar{\beta}+1}, \\
x=(R \pm a) \frac{\alpha+\beta}{\alpha \beta+1}, & y=i(R \pm a) \frac{\beta-a}{\alpha \beta+1}, & z=(R \pm a) \frac{a \beta-1}{\alpha \beta+1},
\end{aligned}
$$

since $S$ and $\bar{S}$ are applicable, we have

$$
\frac{R^{2} d \bar{\alpha} d \bar{\beta}}{(1+\bar{\alpha} \bar{\beta})^{2}}=(R \pm a)^{2} \frac{d \alpha d \beta}{(1+a \beta)^{2}} .
$$

Hence the projections of $S$ and $\bar{S}$ on the unit sphere are conformal. The general solution is given by $\alpha=f(\bar{\alpha}), \beta=f_{0}(\bar{\beta})$, where $f$ is an arbitrary function and $f_{0}$ is the conjugate function. Then $R$ can be found directly from $(i)$.

Calo, l. c.

5. When the Christoffel transform (cf. VII, Ex. 8) of $N^{\prime}$ in $\S 123$ is taken as $N$, the corresponding net $\bar{N}$ is a point, and consequently the central net of this transformation is the other special net $2, O$ parallel to $\bar{N}_{0}^{\prime}$.

6. Show by means of (VII, 3) that a necessary and sufficient condition that an $O$ net have the same spherical representation as an isothermic $O$ net is that

$$
\frac{\partial}{\partial u}\left(\frac{1}{\sqrt{G}} \frac{\partial \sqrt{E}}{\partial v}\right)=\frac{\partial}{\partial v}\left(\frac{1}{\sqrt{E}} \frac{\partial \sqrt{G}}{\partial u}\right) .
$$

Trans. Amer. Math. Soc., vol. 17 (1916), p. 447. 
7. A necessary and sufficient condition that an $O$ net on the unit sphere be the spherical representation of an isothermic net is

$$
\frac{\partial}{\partial u}\left(\frac{1}{\sqrt{\mathscr{B}}} \frac{\partial \sqrt{\mathscr{E}}}{\partial v}\right)=\frac{\partial}{\partial v}\left(\frac{1}{\sqrt{\mathscr{E}}} \frac{\partial \sqrt{\mathscr{B}}}{\partial u}\right) \text {. }
$$

8. Show that the net $\overline{N^{\prime}}$ of $\S 123$ is parallel to two isothermic nets $(\bar{N})^{\prime}$ and $(\bar{N})^{\prime \prime}$ for which

$$
\sqrt{(\bar{E})^{\prime}}=-\sqrt{(\bar{G})^{\prime}}=\frac{e^{-\psi}}{\nu}, \quad \sqrt{(\bar{E})^{\prime \prime}}=\sqrt{(\bar{G})^{\prime \prime}}=e^{\psi} \nu ;
$$

that a transformation $D$ of $(\bar{N})^{\prime}$ is determined by the functions $\bar{p}, \bar{q}, \bar{r}$ of (37) and $(\bar{\theta})^{\prime}=-1 / \nu^{\prime},(\bar{\nu})^{\prime}=\theta^{\prime},(\bar{m})^{\prime}=-m$; and that the resulting transform is the net obtained from the given transformation of $N^{\prime}$ referred to in (VII, Ex. 17).

9. Show that there exists an $O$ net, with the same spherical representation as an isothermic net, for which the tangential coordinate $P$ is equal to $\sqrt{5}-\mathbb{B}$.

Calapso, Annali, ser. 3, vol. 26 (1917), p. 171.

10. If we have an $O$ net $N$ of the kind in Ex. 9 and put

$$
\sqrt{E}=\frac{\sqrt{E}}{\rho_{1}}=\sqrt{P} \cosh \varphi, \quad \sqrt{B}=\frac{\sqrt{G}}{\rho_{2}}=\sqrt{P} \sinh \varphi,
$$

from (VII, 3,5) we have

$$
\frac{\partial \varphi}{\partial u}-\frac{\partial \psi}{\partial u}=\frac{1}{2} \frac{Q}{\sqrt{P}} \sinh \varphi, \quad \frac{\partial \varphi}{\partial v}-\frac{\partial \psi}{\partial v}-=\frac{1}{2} \frac{R}{\sqrt{P}} \cosh \varphi .
$$

From (VII, 9) and (35) we have for an $R$ transform of this $O$ net

$$
\frac{E_{1}}{\rho_{11}^{2}}-\frac{G_{1}}{\rho_{12}^{2}}=P+2 \sqrt{P} \frac{p}{\theta}\left(e^{\psi-\varphi} \frac{\theta}{\theta^{\prime}}+e^{-\psi+\varphi} \frac{\theta^{\prime}}{\nu}\right)+4 \frac{p^{2}}{\nu \theta} \text {. }
$$

In order that this expression be equal to $P_{1}$, namely (VI, 101)

$$
P_{1}=P-\frac{p}{m \nu \theta}(P p+Q q+R r-\theta),
$$

we must have

$$
P p+Q q+R r-\theta+2 \sqrt{P} m \nu\left(e^{\psi-\varphi} \frac{\theta}{\theta^{\prime}}+e^{-\psi+\varphi_{\frac{\theta}{}}} \frac{\theta^{\prime}}{\nu}\right)+4 m p=0 .
$$

The left-hand member is found by differentiation to be a constant. Hence there are $\infty^{4}$ transformations $R$ of $N$ into nets of the same kind.

Calapso, 1. c.; p. 152.

11. If $N^{\prime}$ and $N^{\prime \prime}$ are isothermic $O$ nets in the relation of Christoffel (cf. VII, Ex. 8), the mid-point of the join of corresponding points on $N^{\prime}$ and $N^{\prime \prime}$ describes an $O$ net $N$ parallel to $N^{\prime}$ and $N^{\prime \prime}$ for which $\sqrt{E}=\cosh \psi, \sqrt{G}=\sinh \psi$. When these expressions are substituted in (36), the function $2 \theta-\theta^{\prime}-\nu \theta / \theta^{\prime}$ is constant. Show that there are $\infty^{4}$ transformations $R$ for which this constant is equal to 
zero, and that for each of these transformations $E_{1}-G_{1}=1$; also that $N_{1}$ is the locus of the mid-point of the joins of corresponding points of $N_{1}^{\prime}$ and $N_{1}^{\prime}$.

Calapso, Annali, ser. 3, vol. 26 (1917), p.152.

12. A necessary and sufficient condition that the parametric curves of two applicable surfaces $S$ and $\overline{S^{\prime}}$ be kinematically conjugate is that $D^{\prime}=\overline{D^{\prime}}$.

13. A necessary and sufficient condition that the kinematically auto-conjugate curves for two applicable surfaces, $S, \bar{S}$, reduce to a single family is that $S$ aud $\bar{S}$ be ruled surfaces applicable with generators corresponding.

Bianchi, Memoirie dei Lincei, ser. 5, vol. 12 (1918), p. 441.

14. When two applicable surfaces are referred to their kinematically autoconjugate-system, $D=\bar{D}, D^{\prime}=-\overline{D^{\prime}}, D^{\prime \prime}=\overline{D^{\prime \prime}}$, and $\frac{\partial}{\partial u}\left\{\begin{array}{c}12 \\ 1\end{array}\right\}=\frac{\partial}{\partial v}\left\{\begin{array}{c}12 \\ 2\end{array}\right\}$, the Christoffel symbols being formed with respect to their linear element.

15. Given two quadratic differential forms

Bianchi, l. c., p. 445.

$$
a_{11} d u^{2}+2 a_{12} d u d v+a_{22} d v^{2}, \quad b_{11} d u^{2}+2 b_{12} d u d v+b_{92} d v^{2} .
$$

When the Jacobian of these forms is equated to zero, the resulting equation, namely

$$
\left|\begin{array}{ll}
a_{11} d u+a_{12} d v, & a_{12} d u+a_{92} d v \\
b_{11} d u+b_{12} d v, & b_{12} d u+b_{22} d v
\end{array}\right|=0
$$

defines two fuuctions $\bar{u}=\varphi(u, v), \quad \vec{v}=\psi(u, v)$ in terms of which the forms (1) become

$$
\bar{a}_{11} d \bar{u}^{2}+\bar{a}_{92} d \bar{v}^{2}, \quad \bar{b}_{11} d \bar{u}^{2}+\bar{b}_{22} d \bar{v}^{2} .
$$

Bianchi, Lezioni, vol. 1, p. 82

16. If $S$ and $\bar{S}$ are applicable surfaces, the curves defined by equating to zero the Jacobian of the forms

$$
\begin{gathered}
E d u^{2}+2 F d u d v+G d v^{2}, \\
(D-\bar{D}) d u^{2}+2\left(D^{\prime}-\bar{D}^{\prime}\right) d u d v+\left(D^{\prime \prime}-\bar{D}^{\prime \prime}\right) d v^{2}
\end{gathered}
$$

form an orthogonal kinematically conjugate system. Bianchi, Lezioni, vol. 2, p.38.

17. On two applicable surfaces of constant mean curvature $\pm 1 / a$ whose lines of curvature correspond and for which $\rho_{1}$ and $\rho_{2}$ are the radii of principal curvature of one surface and $-\rho_{2},-\rho_{1}$ of the other [cf. $\S 125$ ], every orthogonal system is kinematically conjugate; moreover, this is the only case where every orthogoulal systen is kinematically conjugate.

Bianchi, l. c., p. 39.

18. The lines of curvature on a surface of rolling described by a point $O$ fixed with respect to a rolling surface $\bar{S}$ correspond to that kinematically conjugate system on $\bar{S}$ which projects into an orthogonal system on a sphere with center at $O$, the projection being from $O$ as center.

Bianchi, Memorie dei Lincei, ser. 5, vol. 12 (1918), p. 448.

19. The lines of curvature on an envelope of rolling described by a plane $\pi$ fixed with respect to a rolling surface $\bar{S}$ correspond to that kinematically conjugate system on $\bar{S}$ which projects orthogonally into an orthogonal system on $\pi$. 
20. When a surface $\bar{S}$ rolls orer an applicable surface $S$, any two parallel lines fixed with respect to $S$ generate congruences for which one family of developables correspond, namely, those corresponding to the curres kinematically conjugate to the transforms of the curves of level of $S$.

Bianchi, Rendiconti dei Lincei, ser. 5, vol. 24 (1915), p. 16.

21. When a surface of revolution $\bar{S}$ rolls ou an applicable surface $S$, the axis of $\bar{S}$ generates a normal congruence, since the focal planes are perpendicular. One of the focal surfaces is the complementary surface $S_{1}$ of $S$ corresponding to the deforms of the meridians of $S$.

Bianchi, Rendiconti di Palermo, vol. 39 (1915), p. 205.

22. For the surface $\bar{S}$ defined by

$$
\bar{x}=r \cos \theta, \quad \bar{y}=r \sin \theta, \quad \bar{z}=c \log r+f(\theta),
$$

where $f$ is an arbitrary function, the distance cut off on the $z$-axis by the perpendicular from any point $P$ of the surface to the axis and the tangent plane at $P$ is equal to $c$. Hence when $\bar{S}$ rolls on an applicable surface, the axis generates a congruence of rolling for which the focal distance is constant.

23. When the surface $\bar{S}$ defined by

Bianchi, l.' c., p. 207.

$$
\bar{x}=r \cos \theta, \quad \bar{y}=r \sin \theta, \quad \bar{z}=c \log r+m \theta
$$

rolls on an applicable surface $S$, the $z$-axis generates a congruence $G$ of rolling for which the distance between the focal points is $c$ and the angle between the focal planes is $\sin ^{-1} c / \sqrt{c^{2}+m^{2}}$. Hence $G$ is a pseudospherical congruence whose focal surfaces have the same gaussian curvature $-1 /\left(c^{2}+m^{2}\right)$ [cf. $\left.§ 171\right]$. Bianchi, l. c., p. 208. 
Chapter X.

\section{Surfaces applicable to a quadric.}

132. Transformations $F$ of nets on a quadric. Consider a net $N$ on the general quadric $Q$, whose equation is

$e x^{2}+f y^{2}+g z^{2}+2 a y z+2 b z x+2 c x y+2 r x+2 s y+2 t z+w=0$.

Since the coordinates are solutions of an equation of the form (II, 6), we have on differentiating (1) with respect to $u$ and $v$

$$
\left\{\begin{array}{c}
e \frac{\partial x}{\partial u} \frac{\partial x}{\partial v}+f \frac{\partial y}{\partial u} \frac{\partial y}{\partial v}+g \frac{\partial z}{\partial u} \frac{\partial z}{\partial v}+a\left(\frac{\partial y}{\partial u} \frac{\partial z}{\partial v}+\frac{\partial y}{\partial v} \frac{\partial z}{\partial u}\right) \\
+b\left(\frac{\partial z}{\partial u} \frac{\partial x}{\partial v}+\frac{\partial z}{\partial v} \frac{\partial x}{\partial u}\right)+c\left(\frac{\partial x}{\partial u} \frac{\partial y}{\partial v}+\frac{\partial x}{\partial v} \frac{\partial y}{\partial u}\right)=0
\end{array}\right.
$$

Any net $N^{\prime}\left(x^{\prime}\right)$ parallel to $N$ is given by equations of the form (II, 3). Consequently we have an equation of the form (2) in which $x, y, z$, are replaced by $x^{\prime}, y^{\prime}, z^{\prime}$. From this it follows that the function

$$
\theta^{\prime}=e x^{\prime 2}+f y^{\prime 2}+g z^{\prime 2}+2\left(a y^{\prime} z^{\prime}+b z^{\prime} x^{\prime}+c x^{\prime} y^{\prime}\right)
$$

is a solution of the point of equation of $N^{\prime 124}$ ). It is readily found that $\theta^{\prime}$ and $\theta$, given by

$$
\left\{\begin{array}{c}
\theta=2\left[e x x^{\prime}+f y y^{\prime}+g z z^{\prime}+a\left(y z^{\prime}+y^{\prime} z\right)+b\left(z x^{\prime}+z^{\prime} x\right)\right. \\
\left.+c\left(x y^{\prime}+x^{\prime} y\right)+r x^{\prime}+s y^{\prime}+t z^{\prime}\right]
\end{array}\right.
$$

satisfy the equations

$$
\frac{\partial \boldsymbol{\theta}^{\prime}}{\partial u}=h \frac{\partial \boldsymbol{\theta}}{\partial u}, \quad \frac{\partial \boldsymbol{\theta}^{\prime}}{\partial v}=l \frac{\partial \theta}{\partial v} .
$$

124) The function $\theta^{\prime} \neq 0$, since $N^{\prime}$ cannot lie on a cone. 
When these values are substituted in (II, 2), it is found that the $F$ transform $N_{1}\left(x_{1}\right)$ lies on $Q$. It has been shown in $\S 5$ that any congruence conjugate to a net $N$ can be obtained by drawing through points of $N$ lines whose direction-parameters are the coordinates of some net parallel to $N$. Hence we have the theorem of Ribaucour:

Any congruence conjugate to a net on a quadric meets the quadric again in a net to which it is conjugate.

We apply the results of $\S 21$ to the particular case when $N$ is on the quadric $Q$, and also $N_{1}$ and $N_{2}$, that is when $\theta_{1}$ and $\theta_{2}$ are of the form (4). In order that $\theta_{12}$ and $\theta_{12}^{\prime \prime \prime}$ be of the form (3) and (4) with $x^{\prime} y^{\prime} z^{\prime} ; x y z$ replaced by $x_{1}^{\prime \prime \prime}, y_{1}^{\prime \prime \prime}, z_{1}^{\prime \prime} ; x_{1}, y_{1}, z_{1}$ respectively, we must have

$$
\left\{\begin{array}{c}
\theta_{1}^{\prime \prime}+\theta_{2}^{\prime}-2\left[e x^{\prime} x^{\prime \prime}+f y^{\prime} y^{\prime \prime}+g z^{\prime} z^{\prime \prime}+a\left(y^{\prime} z^{\prime \prime}+y^{\prime \prime} z^{\prime}\right)\right. \\
\left.+b\left(z^{\prime} x^{\prime \prime}+z^{\prime \prime} x^{\prime}\right)+c\left(x^{\prime} y^{\prime \prime}+x^{\prime \prime} y^{\prime}\right)\right]=0
\end{array}\right.
$$

By differentiation it is found that the left-hand member of this equation is constant, and consequently the additive constants in $\theta_{1}^{\prime \prime}$ and $\theta_{2}^{\prime}$ can be chosen in $\infty^{1}$ ways so that (6) shall hold. Hence:

If $N_{1}$ and $N_{\varepsilon}$ are $F$ transforms of $N$ and all three nets lie on $Q(1)$, there are $\infty^{1}$ other nets $N_{12}$ on $Q$ which are $F$ transforms of $N_{1}$ and $N_{2}$; they can be found by a quadrature.

133. Permanent nets on a quadric. Let $Q$ be a quadric referred to its asymptotic lines, and write its linear element in the form

$$
d s_{0}^{2}=E_{0} d \alpha^{2}+2 F_{0} d \alpha d \beta+G_{0} d \beta^{2},
$$

and its second quadratic form

$$
\Phi=2 D_{0}^{\prime} d \alpha d \beta .
$$

If $\bar{S}$ is an applicable surface, its linear element is (7) and its second quadratic form is

$$
\bar{\Phi}=\overline{D_{0}} d \alpha^{2}+2 \overline{D_{0}^{\prime}} d \alpha d \beta+\overline{D_{0}^{\prime \prime}} d \beta^{2} .
$$

Since the asymptotic lines on $Q$ are straight lines, we must have [§ 85] 


$$
\left\{\begin{array}{c}
11 \\
2
\end{array}\right\}_{0}=\left\{\begin{array}{c}
22 \\
1
\end{array}\right\}_{0}=0
$$

the Christoffel symbols $\left\{\begin{array}{c}r s\}^{\prime} \\ t\}_{0}\end{array}\right.$ being formed with respect to (7). If we put

$$
H_{0}=\sqrt{E_{0} G_{0}-F_{0}^{2}}, \quad \sigma^{2}=\frac{D_{0}^{\prime}}{H_{0}}=\sqrt{-K},
$$

from $[\S \S 63,77]$ we have

$$
\begin{cases}\left\{\begin{array}{c}
12\} \\
1\}_{0}=-\frac{\partial \log \sigma}{\partial \beta},
\end{array}\right. & \left\{\begin{array}{c}
12 \\
2\}_{0}
\end{array}\right\}_{0}-\frac{\partial \log \sigma}{\partial \alpha}, \\
\left\{\begin{array}{c}
11 \\
1\}_{0}
\end{array}\right\}_{0} \frac{\partial}{\partial \alpha} \log H_{0} \sigma, & \left\{\begin{array}{c}
22\}_{1} \\
2\}_{0}=\frac{\partial}{\partial \beta} \log H_{0} \sigma .
\end{array}\right.\end{cases}
$$

Hence the Codazzi equations $[\S 64]$ for $\bar{S}$ are reducible to

$$
\left\{\begin{array}{l}
\frac{\partial \bar{D}_{0}}{\partial \beta}-\frac{\partial \bar{D}_{0}^{\prime}}{\partial \alpha}+\frac{\partial \log \sigma}{\partial \beta} \overline{D_{0}}+\frac{\partial \log D_{0}^{\prime}}{\partial \alpha} \overline{D_{0}^{\prime}}=0 \\
\frac{\partial \overline{D_{0}^{\prime \prime}}}{\partial \alpha}-\frac{\partial \overline{D_{0}^{\prime}}}{\partial \beta}+\frac{\partial \log \sigma}{\partial \alpha} \overline{D_{0}^{\prime \prime}}+\frac{\partial \log D_{0}^{\prime}}{\partial \beta} \overline{D_{0}^{\prime}}=0
\end{array}\right.
$$

Since $Q$ and $\bar{S}$ are applicable,

If we put

$$
\bar{D}_{0} \overline{D_{0}^{\prime \prime}}-\overline{D_{0}^{\prime 2}}=-D_{0}^{\prime 2}=-H_{0}^{2} \sigma^{4} \text {. }
$$

we have

$$
\Delta=\sigma \overline{D_{0}}, \quad A^{\prime \prime}=\sigma \overline{D_{0}^{\prime \prime},} \quad A^{\prime}=\frac{\overline{D_{0}^{\prime}}}{\bar{D}_{0}^{\prime}}
$$

$$
\sqrt{A A^{\prime \prime}}=\sigma D_{0}^{\prime} \sqrt{{A^{\prime 2}-1}^{\prime}}
$$

Then (13) may be replaced by

$$
\frac{\partial A}{\partial \beta}=\sigma D_{0}^{\prime} \frac{\partial A^{\prime}}{\partial \alpha}, \quad \frac{\partial A^{\prime \prime}}{\partial \alpha}=\sigma D_{0}^{\prime} \frac{\partial A^{\prime}}{\partial \beta} .
$$

The equation of the common conjugate system of $Q$ and $\bar{S}$ is $[\S 56]$

$$
\Delta d \alpha^{2}-A^{\prime \prime} d \beta^{2}=0 \text {. }
$$


By means of the above formulas we find that the Gaussian curvature of the left-hand member of this equation is zero, and consequently it can be given the form $d u d v$. Hence we have the theorem of Servant ${ }^{125}$ ):

If $\bar{S}$ is a surface applicable to a quadric $Q$ and the latter is referred to its generators, the common conjugate system can be found by quadratures.

If we put

17) $e^{-\infty}\left(\sqrt{\boldsymbol{A}} d \boldsymbol{\alpha}-\boldsymbol{V} \overline{\boldsymbol{A}^{\prime \prime}} d \boldsymbol{\beta}\right)=d u$, $e^{\omega}\left(\boldsymbol{V} \overline{\boldsymbol{A}} d \boldsymbol{\alpha}+\boldsymbol{V} \overline{\boldsymbol{A}^{\prime \prime}} d \boldsymbol{\beta}\right)=d v$ we find, in consequence of (16),

$$
e^{2 \omega}=A^{\prime}+\sqrt{A^{\prime 2}-1}, \quad e^{-2 \omega}=A^{\prime}-\sqrt{A^{\prime 2}-1} .
$$

From equations (17) we have

$$
\left\{\begin{array}{c}
2 \sqrt{\boldsymbol{\Delta}} d \boldsymbol{\alpha}=e^{\infty} d u+e^{-\infty} d v \\
2 \sqrt{\boldsymbol{\Delta}^{\prime \prime}} d \beta=-e^{\infty} d u+e^{-\infty} d v .
\end{array}\right.
$$

In terms of $u$ and $v$ the second quadratic forms of $Q$ and $\bar{S}$ are necessarily of the form

$$
\Phi=D d u^{2}+D^{\prime \prime} d v^{2}, \quad \bar{\Phi}=\bar{D} d u^{2}+\bar{D}^{\prime \prime} d v^{2} .
$$

In consequence of (8), (9) and (19) we have

(20) $D=-\frac{1}{2 \sigma}\left(\frac{A^{\prime}}{\sqrt{A^{\prime 2}-1}}+1\right), D^{\prime \prime}=\frac{1}{2 \sigma}\left(\frac{A^{\prime}}{\sqrt{A^{\prime 2}-1}}-1\right)$,

$$
\bar{D}=-\bar{D}^{\prime \prime}=\frac{-1}{2 \sigma \sqrt{{A^{\prime 2}}^{2}-1}} .
$$

From these follow the theorems $\left.{ }^{126}\right)$ :

If a net upon a quadric admits an applicable net, then

$$
D+D^{\prime \prime}=-\frac{1}{\sigma} \text {. }
$$

125) Bull. Soc. Math. de France, vol. 30 (1902), p. 19.

126) Cf. Servant, l. c., p. 20. 
The permanent net on a deform of a quadric is isothermalconjugate.

From (19) we have

(23) $\frac{\partial \alpha}{\partial u}=\frac{e^{\omega}}{2 \sqrt{\boldsymbol{A}}}, \frac{\partial \alpha}{\partial v}=\frac{e^{-\omega}}{2 \sqrt{\boldsymbol{A}}} ; \frac{\partial \beta}{\partial u}=-\frac{e^{\omega}}{2 \sqrt{\boldsymbol{A}^{\prime \prime}}}, \frac{\partial \beta}{\partial v}=\frac{e^{-\infty}}{2 \sqrt{\boldsymbol{A}^{\prime \prime}}}$.

In consequence of (15) and (18) we have from these equations

$$
\left\{\begin{array}{l}
\frac{\partial \alpha}{\partial u} \frac{\partial \boldsymbol{\beta}}{\partial v}+\frac{\partial \alpha}{\partial v} \frac{\partial \boldsymbol{\beta}}{\partial u}=0 \\
\frac{\partial \alpha}{\partial u} \frac{\partial \boldsymbol{\beta}}{\partial u}+\frac{\partial \alpha}{\partial v} \frac{\partial \boldsymbol{\beta}}{\partial v}=-\frac{1}{2 \sigma D_{0}^{\prime}} .
\end{array}\right.
$$

Conversely, if we have a solution of this system, and define functions $\omega, A$ and $A^{\prime \prime}$ by (23) and $A^{\prime}$ by (15), we find that $\omega$ and $A^{\prime}$ satisfy (18). Since the corresponding equations (17) are consistent, we obtain (16). Then a surface $\bar{S}$ applicable to $Q$ is defined intrinsically by (14), and we have:

The determination of permanent nets on a quadric is equivalent to the solution of equations (24).

134. The permanent net on a deform of a quadric. When a quadric $Q$ and an applicable surface $\bar{S}$ are referred to the permanent nets $N$ and $\bar{N}$, we have, in consequence of (21),

$$
\sigma^{4}=-\frac{D D^{\prime \prime}}{H^{2}}=\frac{\overline{D^{2}}}{H^{2}}, \quad H^{2}=E G-F^{2},
$$

where $\sigma^{4}=-K, K$ being the total curvature of $Q$ and $\bar{S}$, and the linear element of $Q$ and $\bar{S}$ is

$$
d s^{2}=E d u^{2}+2 F d u d v+G d v^{2} .
$$

If we define two functions $a$ and $b$ by means of the equations

we have from (22)

$$
D=-\sigma a^{2}, \quad D^{\prime \prime}=\sigma b^{2}
$$

$$
a^{2}-b^{2}=\frac{1}{\sigma^{2}}
$$


In consequence of (21) and (25), we may take

(29)

$$
-\bar{D}=\overline{D^{\prime \prime}}=\sigma a b, \quad H \sigma=a b .
$$

The Codazzi equations for $N$ and $\bar{N}$ are [cf. $\S 64$ ]

(30) $\begin{cases}\frac{\partial D}{\partial v}=D\left\{\begin{array}{c}12 \\ 1\end{array}\right\}-D^{\prime \prime}\left\{\begin{array}{c}11 \\ 2\end{array}\right\}, & \frac{\partial D^{\prime \prime}}{\partial u}=-\left\{\begin{array}{c}22 \\ 1\end{array}\right\} D+\left\{\begin{array}{c}12 \\ 2\end{array}\right\} D^{\prime \prime}, \\ \frac{\partial \bar{D}}{\partial v}=\bar{D}\left(\begin{array}{c}12\} \\ 11\}\end{array}\right\}+\left\{\begin{array}{c}11 \\ 2\end{array}\right\}, & \frac{\partial \bar{D}}{\partial u}=\bar{D}\left(\left\{\begin{array}{c}22 \\ 1\end{array}\right\}+\left\{\begin{array}{c}12 \\ 2\end{array}\right\}\right),\end{cases}$

the Christoffel symbols $\left\{\begin{array}{c}r s \\ t\end{array}\right\}$ being formed with respect to (26).

From these equations, in which $D, D^{\prime \prime}$ and $\bar{D}$ are replaced by their expressions from (27) and (29), and the identities [cf. $§ 63$ ]

$$
\frac{\partial \log H}{\partial u}=\left\{\begin{array}{c}
11 \\
1
\end{array}\right\}+\left\{\begin{array}{c}
12 \\
2
\end{array}\right\}, \quad \frac{\partial \log H}{\partial v}=\left\{\begin{array}{c}
22 \\
2
\end{array}\right\}+\left\{\begin{array}{c}
12 \\
1
\end{array}\right\}
$$

we obtain, in consequence of (28),
$\left\{\begin{array}{c}11\} \\ 1\end{array}\right\}=\frac{\partial}{\partial u} \log \frac{a}{\sigma}$
$\left\{\begin{array}{c}12 \\ 1\end{array}\right\}=\frac{\partial}{\partial v} \log a$,
$\left\{\begin{array}{c}22 \\ 1\end{array}\right\}=\frac{b^{2}}{a^{2}} \frac{\partial}{\partial u} \log b \sigma$,
$\left\{\begin{array}{c}11 \\ 2\end{array}\right\}=\frac{a^{2}}{b^{2}} \frac{\partial}{\partial v} \log a \sigma$,
$\left\{\begin{array}{c}12 \\ 2\end{array}\right\}=\frac{\partial}{\partial u} \log b$,
$\left\{\begin{array}{c}22 \\ 2\end{array}\right\}=\frac{\partial}{\partial v} \log \frac{b}{\sigma}$.

If $\bar{x}, \bar{y}, \bar{z}$ denote the cartesian coordinates of $\bar{S}$, from the Gauss equations $[\S 64,(7)]$ for $\bar{S}$ we have that $\bar{x}, \bar{y}, \bar{z}$ are solutions of the equations:

(33)

$$
\left\{\begin{aligned}
\frac{\partial^{2} \theta}{\partial u^{2}}+\frac{\partial^{2} \theta}{\partial v^{2}} & =2 \frac{\partial \log a}{\partial u} \frac{\partial \theta}{\partial u}+2 \frac{\partial \log b}{\partial v} \frac{\partial \theta}{\partial v} \\
\frac{\partial^{2} \theta}{\partial u \partial v} & =\frac{\partial \log a}{\partial v} \frac{\partial \theta}{\partial u}+\frac{\partial \log b}{\partial u} \frac{\partial \theta}{\partial v}
\end{aligned}\right.
$$

Hence from $\S 45$ we have:

The permanent net on a deform of a quadric is an $R$ net $\left.{ }^{\mathbf{1 2 \pi}}\right)$.

127) Cf. Tzitzeica, Comptes Rendus, vol. 152 (1911), p. 1077; also Bianchi, Rendiconti dei Lincei, ser. 5, vol. 22 (1913), p. 3. 
We desire to prove the converse theorem:

When the functions $a$ and $b$ in the equations (33) of an $R$ net satisfy the condition (28), the net is applicable to a net on a quadric.

From (31) and (33) we have for any $R$ net $\bar{N}$

$$
\begin{array}{lll}
\left\{\begin{array}{c}
11 \\
1
\end{array}\right\}=\frac{\partial}{\partial u} \log \frac{H}{b}, & \left\{\begin{array}{c}
12 \\
1
\end{array}\right\}=\frac{\partial \log a}{\partial v}, & \left\{\begin{array}{c}
22 \\
1
\end{array}\right\}=\frac{\partial}{\partial u} \log \frac{a^{2} b}{H}, \\
\left\{\begin{array}{c}
11 \\
2
\end{array}\right\}=\frac{\partial}{\partial v} \log \frac{a b^{2}}{H}, & \left\{\begin{array}{c}
12 \\
2
\end{array}\right\}=\frac{\partial \log b}{\partial u}, & \left\{\begin{array}{c}
22 \\
2
\end{array}\right\}=\frac{\partial}{\partial v} \log \frac{H}{a} .
\end{array}
$$

When these values are substituted in the second set of (30), we find that the second fundamental coefficients of an $R$ net satisfying (33) are of the form $-\bar{D}=\bar{D}^{\prime \prime}=a^{2} b^{2} / H$. Then since $\bar{D} \overline{D^{\prime \prime}}=-\sigma^{4} H^{2}$, we may take $H \sigma=a b$, in which case we have (29) and the above expressions for the Christoffel symbols are reducible to (32), in consequence of (28). As a result we have that (27) furnishes a solution of the first set of (30) in which the symbols have the values (32); that is $\bar{N}$ admits an applicable net $N$, for which the second fundamental coefficients are given by (27).

If $\alpha$ and $\beta$ are the parameters of the asymptotic lines of the surface $S$ on which $N$ lies, we have

$$
\lambda d \alpha=a d u-b d v, \quad \mu d \beta=a d u+b d v
$$

where the integrating factors $\lambda, \mu$ are subject to the conditions

$$
\frac{\partial}{\partial v}\left(\frac{a}{\lambda}\right)+\frac{\partial}{\partial u}\left(\frac{b}{\lambda}\right)=0, \quad \frac{\partial}{\partial v}\left(\frac{a}{\mu}\right)-\frac{\partial}{\partial u}\left(\frac{b}{\mu}\right)=0 .
$$

In consequence of (27), (29) and $[\S 64,(7)]$ the coordinates of $S$ satisfy the equations

$$
\begin{aligned}
b^{2} \frac{\partial^{2} \theta}{\partial u^{2}}+a^{2} \frac{\partial^{2} \theta}{\partial v^{2}} & =b^{2} \frac{\partial}{\partial u} \log a b \frac{\partial \theta}{\partial u}+a^{2} \frac{\partial}{\partial v} \log a b \frac{\partial \theta}{\partial v} \\
\frac{\partial^{2} \theta}{\partial u \partial v} & =\frac{\partial \log a}{\partial v} \frac{\partial \theta}{\partial u}+\frac{\partial \log b}{\partial u} \frac{\partial \theta}{\partial v} .
\end{aligned}
$$


If we express these equations in terms of $\alpha$ and $\beta$, we obtain

$$
\begin{gathered}
a b\left(\frac{1}{\lambda^{2}} \frac{\partial^{2} \theta}{\partial \alpha^{2}}+\frac{1}{\mu^{2}} \frac{\partial^{2} \theta}{\partial \beta^{2}}\right)+\frac{1}{2} \frac{\partial \theta}{\partial \alpha}\left[b \frac{\partial}{\partial u}\left(\frac{1}{\lambda}\right)-a \frac{\partial}{\partial v}\left(\frac{1}{\lambda}\right)-\frac{1}{\lambda} \frac{\partial b}{\partial u}+\frac{1}{\lambda} \frac{\partial a}{\partial v}\right] \\
+\frac{1}{2} \frac{\partial \theta}{\partial \beta}\left[b \frac{\partial}{\partial u}\left(\frac{1}{\mu}\right)+a \frac{\partial}{\partial v}\left(\frac{1}{\mu}\right)-\frac{1}{\mu} \frac{\partial b}{\partial u}-\frac{1}{\mu} \frac{\partial a}{\partial v}\right]=0, \\
a b\left(-\frac{1}{\lambda^{2}} \frac{\partial^{2} \theta}{\partial \alpha^{2}}+\frac{1}{\mu^{2}} \frac{\partial^{2} \theta}{\partial \beta^{2}}\right)+\frac{\partial \theta}{\partial \alpha}\left[a \frac{\partial}{\partial v}\left(\frac{1}{\lambda}\right)+\frac{1}{\lambda} \frac{\partial b}{\partial u}\right] \\
+\frac{\partial \theta}{\partial \beta}\left[a \frac{\partial}{\partial v}\left(\frac{1}{\mu}\right)-\frac{1}{\mu} \frac{\partial b}{\partial u}\right]=0 .
\end{gathered}
$$

Adding and substracting these equations, the resulting equations are reducible in consequence of the above conditions on $\lambda$ and $\mu$ to the form

$$
\frac{\partial^{2} \theta}{\partial \alpha^{2}}=A \frac{\partial \theta}{\partial \alpha}, \quad \frac{\partial^{2} \theta}{\partial \beta^{2}}=B \frac{\partial \theta}{\partial \beta},
$$

and consequently $S$ is a quadric.

135. Transformations $F_{k}$ of permanent nets on a central quadric. For the central quadric $Q$, whose equation is

$$
e x^{2}+f y^{2}+g z^{2}=1
$$

we have

$$
X, Y, Z=\frac{e x, f y, g z}{\sqrt{\sum e^{2} x^{2}}}
$$

and the Gaussian curvature is given by ${ }^{128}$ )

$$
K=-\frac{1}{c^{4}\left(\sum e^{2} x^{8}\right)^{2}}, \quad \frac{1}{\sigma}=c \sqrt{\sum e^{2} x^{2}}
$$

where $c^{4}=-1 / e f g$.

If $Q$ is referred to a net $N$ whose point equation is the second of (33), we have

(37) $\frac{\partial}{\partial v} \log \left[\sum e\left(\frac{\partial x}{\partial u}\right)^{2}\right]=\frac{\partial}{\partial v} \log a^{2}, \quad \frac{\partial}{\partial u} \log \left[\sum e\left(\frac{\partial x}{\partial v}\right)^{2}\right]=\frac{\partial}{\partial u} \log b^{2}$,

$\left.{ }^{128}\right)$ Cf. C. Smith, Solid Geometry, $9^{\text {th }}$ edition, p. 223. 
and also

$$
\sum e \frac{\partial x}{\partial u} \frac{\partial x}{\partial v}=0
$$

Hence

$$
\sum e\left(\frac{\partial x}{\partial u}\right)^{2}=a^{2} U, \quad \sum e\left(\frac{\partial x}{\partial v}\right)^{2}=b^{2} V
$$

where $U$ and $V$ are functions of $u$ and $v$ alone.

In consequence of (27), (32), and the Gauss equations for $Q$ we find

$$
\left\{\begin{array}{l}
\frac{\partial}{\partial u}\left[\sum e\left(\frac{\partial x}{\partial u}\right)^{2}\right]=\frac{\partial}{\partial u} \log \frac{a^{2}}{\sigma^{2}} \sum e\left(\frac{\partial x}{\partial u}\right)^{2}-2 \sigma a^{2} \sum e X \frac{\partial x}{\partial u} \\
\frac{\partial}{\partial v}\left[\sum e\left(\frac{\partial x}{\partial v}\right)^{2}\right]=\frac{\partial}{\partial v} \log \frac{b^{2}}{\sigma^{2}} \sum e\left(\frac{\partial x}{\partial v}\right)^{2}+2 \sigma b^{2} \sum e X \frac{\partial x}{\partial v}
\end{array}\right.
$$

From (35) and (36) we have

$$
\sum e X \frac{\partial x}{\partial u}=\frac{1}{c} \frac{\partial}{\partial u}\left(\frac{1}{\sigma}\right), \quad \sum e X \frac{\partial x}{\partial v}=\frac{1}{c} \frac{\partial}{\partial v}\left(\frac{1}{\sigma}\right) .
$$

When the expressions (39) are substituted in (40), the result is reducible by means of (41) to

$$
\frac{1}{2} \frac{\partial U}{\partial u}=-\left(U-\frac{1}{c}\right) \frac{\partial \log \sigma}{\partial \imath}, \quad \frac{1}{2} \frac{\partial V}{\partial v}=-\left(V+\frac{1}{c}\right) \frac{\partial \log \sigma}{\partial v} .
$$

Also on differentiating equation (38) with respect to $u$ and $v$ we get

$$
\begin{aligned}
& (U+V) \frac{\partial \log a}{\partial v}+\left(V+\frac{1}{c}\right) \frac{\partial \log \sigma}{\partial v}=0, \\
& (U+V) \frac{\partial \log b}{\partial u}+\left(U-\frac{1}{c}\right) \frac{\partial \log \sigma}{\partial u}=0 .
\end{aligned}
$$

From these two sets of equations it follows that $U=-V=1 / c$, and consequently

$$
\sum e\left(\frac{\partial x}{\partial u}\right)^{2}=\frac{a^{2}}{c}, \quad \sum e\left(\frac{\partial x}{\partial v}\right)^{2}=-\frac{b^{2}}{c} .
$$


Hence from (28) and (36) we have the theorem:

For any permanent net on a central quadric (34) the coordinates satisfy the condition

$$
\sum e\left(\frac{\partial x}{\partial u}\right)^{2}+\sum e\left(\frac{\partial x}{\partial v}\right)^{2}=c \sum e^{2} x^{2}
$$

where $\left.c^{4}=-1 / e f g^{129}\right)$.

This equation may be written

$$
a^{2}-b^{2}=c^{2} \sum e^{2} x^{2}
$$

Suppose, conversely, we have a net $N$ on the quadric (34) satisfying the condition (43). Since equations (39) hold for any net on the quadric, the functions $a$ and $b$ in the point equation of $N$ can be chosen so that we have (42). If these equations are differentiated with respect to $u$ and $v$ respectively, and the Gauss equations $[\S 64,(7)]$ are used, the resulting equations are reducible by means of $(41)$ to

$$
\left\{\begin{array}{l}
a^{2}\left\{\begin{array}{c}
11 \\
1
\end{array}\right\}+\frac{\partial}{\partial u}\left(\frac{1}{\sigma}\right) D-a \frac{\partial a}{\partial u}=0, \\
b^{2}\left\{\begin{array}{c}
22 \\
2
\end{array}\right\}-\frac{\partial}{\partial v}\left(\frac{1}{\sigma}\right) D^{\prime \prime}-b \frac{\partial b}{\partial v}=0 .
\end{array}\right.
$$

From (38) and (42) we have

$-\frac{a^{2} b^{2}}{c^{2}}=\sum e f\left(\frac{\partial x}{\partial u} \frac{\partial y}{\partial v}-\frac{\partial x}{\partial v} \frac{\partial y}{\partial u}\right)^{2}=H^{2} \sum e f Z^{2}=-\frac{H^{2} \sigma^{2}}{c^{2}}$.

Hence, if we take $H \sigma=a b$, from (31) we obtain

$$
\left\{\begin{array}{c}
11 \\
1
\end{array}\right\}=\frac{\partial}{\partial u} \log \frac{a}{\sigma}, \quad\left\{\begin{array}{c}
22 \\
2
\end{array}\right\}=\frac{\partial}{\partial v} \log \frac{b}{\sigma} .
$$

In consequence of these expressions equations (45) give $D=-\sigma a^{2}, D^{\prime \prime}=\sigma b^{2}$. Substituting these expressions in the first

${ }^{129}$ ) Cf. Calapso, Annali, ser. 3, vol. 19 (1912), p. 62. 
set of equations (30) and making use of (28), which is a consequence of (42) and (43), we get the expressions (32). When these values are substituted in the second set of (30), a solution is $-\bar{D}=\bar{D}^{\prime \prime}=\sigma a b$. Hence there exists a net $\bar{N}$ applicable to $N$. Therefore:

When a net on a central quadric (34) satisfies the condition (43), it is a permanent net.

Let $N$ be a permanent net on the quadric $Q(34)$. From (3) and (4) it follows that if in the equations of the form

$$
x_{1}=x-\frac{\theta}{\theta^{\prime}} x^{\prime}
$$

we put

(48) $\quad \boldsymbol{\theta}=2\left(e x x^{\prime}+f y y^{\prime}+g z z^{\prime}\right) \equiv 2 \sum e x x^{\prime}, \quad \theta^{\prime}=\sum e x^{\prime 2}$,

the $F$ transform $N_{1}$ of $N$ lies on $Q$. In order that $N_{1}$ be a permanent net it is sufficient that

$$
\sum e\left(\frac{\partial x_{1}}{\partial u}\right)^{2}+\sum e\left(\frac{\partial x_{1}}{\partial v}\right)^{2}=c \sum e^{2} x_{1}^{2}
$$

From (47) we have by differentiation

(50) $\frac{\partial x_{1}}{\partial u}=\frac{\boldsymbol{\tau}}{\boldsymbol{\theta}^{\prime 2}}\left(x^{\prime} \frac{\partial \theta}{\partial u}-\boldsymbol{\theta}^{\prime} \frac{\partial x}{\partial u}\right), \quad \frac{\partial x_{1}}{\partial v}=\frac{\sigma}{\theta^{\prime 2}}\left(x^{\prime} \frac{\partial \theta}{\partial v}-\theta^{\prime} \frac{\partial x}{\partial v}\right)$, where

$$
\tau=h \theta-\theta^{\prime}, \quad \sigma=l \theta-\theta^{\prime} .
$$

Substituting these expressions in (49), we can reduce the resulting equation by means of (42) to

$$
\tau^{2} a^{2}-\sigma^{2} b^{2}=c^{2} \dot{\theta}^{\prime 2} \sum e^{2} x_{1}^{2} .
$$

In consequence of (II, 16) we have from (51)

$$
\left\{\begin{array}{l}
\frac{\partial}{\partial v}\left(\frac{\boldsymbol{\tau}}{\boldsymbol{\theta}^{\prime}}\right)=\frac{\sigma-\boldsymbol{\tau}}{\boldsymbol{\theta}^{\prime}} \frac{\partial}{\partial v} \log a-\frac{h \sigma}{\boldsymbol{\theta}^{\prime \mathbf{2}}} \frac{\partial \boldsymbol{\theta}}{\partial v}, \\
\frac{\partial}{\partial u}\left(\frac{\sigma}{\boldsymbol{\theta}^{\prime}}\right)=\frac{\boldsymbol{\tau}-\boldsymbol{\sigma}}{\boldsymbol{\theta}^{\prime}} \frac{\partial}{\partial u} \log b-\frac{l \boldsymbol{\tau}}{\boldsymbol{\theta}^{\prime \mathbf{2}}} \frac{\partial \boldsymbol{\theta}}{\partial u} .
\end{array}\right.
$$


Differentiating (52) and making use of (53), the resulting equations are reducible to

$$
\begin{aligned}
\frac{\partial \varphi}{\partial u}+\frac{1}{\theta} \frac{\partial \theta}{\partial u}\left[-\frac{b^{2}}{\theta^{\prime 2}} l \theta(\tau-\sigma)+c^{2} \sum e^{2} x_{1}\left(x_{1}-x\right)\right. & \\
& \left.+c^{2} \frac{\tau}{\theta^{\prime}} \sum e^{2} x\left(x_{1}-x\right)\right]=0,
\end{aligned}
$$$$
\frac{\partial \varphi}{\partial v}+\frac{1}{\theta} \frac{\partial \theta}{\partial v}\left[\frac{a^{2}}{\theta^{\prime 2}} h \theta(\sigma-\tau)+c^{2} \sum e^{2} x_{1}\left(x_{1}-x\right)\right.
$$$$
\left.+c^{2} \frac{\sigma}{\theta^{\prime}} \sum e^{2} x\left(x_{1}-x\right)\right]=0
$$

where

$$
\varphi=a^{2} \frac{\tau}{\theta^{\prime}}-b^{2} \frac{\sigma}{\theta^{\prime}}+c^{2} \sum e^{2} x x_{1}
$$

By means of (44), (51), (52) and (54) these equations are reducible to

$$
\text { (55) } \frac{\partial \varphi}{\partial u}+\frac{\varphi}{\theta} \frac{\partial \theta}{\partial u}\left(\frac{h \theta}{\theta^{\prime}}-2\right)=0, \quad \frac{\partial \varphi}{\partial v}+\frac{\varphi}{\theta} \frac{\partial \theta}{\partial v}\left(\frac{l \theta}{\theta^{\prime}}-2\right)=\dot{0} \text {, }
$$

which can be integrated in the form

$$
\varphi \theta^{\prime}=-\frac{k c^{2}}{2} \theta^{2}
$$

where $k$ is an arbitrary constant. When this value for $\varphi$ is substituted in (54), this equation and (52) are equivalent, in consequence of (47) and (51), to

(57) $h^{2} a^{2}-l^{2} b^{2}=c^{2} \sum\left(e^{2}-k e\right) x^{\prime 2}, \quad h a^{2}-l b^{2}=c^{2} \sum\left(e^{2}-k e\right) x x^{\prime}$.

Differentiating the second of these equations, we obtain

$$
\text { (58) }\left\{\begin{array}{l}
\frac{\partial h}{\partial u}+\frac{\partial \log a}{\partial u} h-\frac{b^{2}}{a^{2}} \frac{\partial \log b}{\partial u} l-\frac{c^{2}}{a^{2}} \sum\left(e^{2}-k e\right) x^{\prime} \frac{\partial x}{\partial u}=0 \\
\frac{\partial l}{\partial v}+\frac{\partial \log b}{\partial \imath} l-\frac{a^{2}}{b^{2}} \frac{\partial \log a}{\partial v} h+\frac{c^{2}}{b^{2}} \sum\left(e^{2}-k e\right) x^{\prime} \frac{\partial x}{\partial v}=0
\end{array}\right.
$$


(59)

$$
\left\{\begin{array}{rlrl}
\frac{\partial h}{\partial v}=(l-h) \frac{\partial \log a}{\partial v}, & \frac{\partial l}{\partial u}=(h-l) \frac{\partial \log b}{\partial u} \\
\frac{\partial x^{\prime}}{\partial u}=h \frac{\partial x}{\partial u}, & \frac{\partial x^{\prime}}{\partial v}=l \frac{\partial x}{\partial v} ; & \frac{\partial y^{\prime}}{\partial u}=h \frac{\partial y}{\partial u}, \frac{\partial y^{\prime}}{\partial v}=l \frac{\partial y}{\partial v} \\
\frac{\partial z^{\prime}}{\partial u}=h \frac{\partial z}{\partial u}, & \frac{\partial z^{\prime}}{\partial v}=l \frac{\partial z}{\partial v}
\end{array}\right.
$$

It is readily found that equations (58) and (59) form a completely integrable system, in consequence of (44). Moreover, for every set of solutions of this system equations (57) are satisfied to within additive constants, as is found by differentiation. Hence each set of solutions satisfying (57) determines an $F$ transform which is a permanent net. From (47), (48) and (59) it is seen that if $x^{\prime}, y^{\prime}, z^{\prime}, h$ and $l$ are multiplied by the same constant, the transform $N_{1}$ is unaltered. Hence when $k$ is any constant different from $e, f$ and $g$, there are $\infty^{2}$ sets of solutions satisfying (57) and giving distinct transforms.

When $k=e$, there are $\infty^{1}$ sets of solution $s, y^{\prime}, z^{\prime}, h$ and $l$ of (58) and (59) satisfying (57). Then $x^{\prime}$ is given by a quadrature (59) and involves an additive constant, say $m$. In this case each set of solution $s, y^{\prime}, z^{\prime}, h$ and $l$ determines $\infty^{1}$ transformations, such that the corresponding points of the $\infty^{1}$ transforms lie on a conic, the section of quadric by a plane parallel to the lines from the origin to the points $\left(x^{\prime}+m, y^{\prime}, z^{\prime}\right)$ as $m$ varies. Similar results hold when $k=f$ or $k=g$. Hence:

A permanent net on a central quadric ex $x^{2}+f y^{2}+g z^{2}=1$ admits $\infty^{2}$ transformations $F_{k}$ into permanent nets on the quadric for each value of the constant $k$; when $k$ is equal to $e, f$ or $g$, the transforms $N_{1}$ may be grouped into $\infty^{1}$ families of $\infty^{1}$ transforms each such that corresponding points of the nets of a family lie on a conic $^{\mathbf{1 3 0}}$ ).

136. Transformations $F_{k}$ of surfaces applicable to a central quadric. It is our purpose to show that each transformation $F$ of a permanent net $N$ on a quadric $Q$ into a permanent net $N_{1}$ on $Q$ leads directly to a transformation $F$ of the net $\bar{N}$ applicable to $N$ into the net $\bar{N}_{1}$ applicable to $N_{1}$. In fact, we

${ }^{130}$ ) Cf. Journ. de Math., ser. 8, vol. 4 (1921), pp. 37-66. 
shall show that it is possible to find without quadratures a net $\bar{N}^{\prime}$ parallel to $\bar{N}$ such that $\theta^{\prime}$ given by (48) can be put in the form (cf. II, 95)

$$
\theta^{\prime}=k\left(\sum x^{\prime 2}-\sum \bar{x}^{\prime 2}\right),
$$

and then the desired transform is defined by

$$
\bar{x}_{1}=\bar{x}-\frac{\theta}{\theta^{\prime}} \bar{x}^{\prime} .
$$

Equating these expressions for $\theta^{\prime}$, we have

$$
(e-k) x^{\prime 2}+(f-k) y^{\prime 2}+(g-k) z^{\prime 2}+k \sum \bar{x}^{\prime 2}=0 .
$$

Differentiating this expression and assuming that equations of the form

$$
\frac{\partial \bar{x}^{\prime}}{\partial u}=h \frac{\partial \bar{x}}{\partial u}, \quad \frac{\partial \bar{x}^{\prime}}{\partial v}=l \frac{\partial \bar{x}}{\partial v},
$$

hold, we obtain

$$
\left\{\begin{array}{l}
(e-k) x^{\prime} \frac{\partial x}{\partial u}+(f-k) y^{\prime} \frac{\partial y}{\partial u}+(g-k) z^{\prime} \frac{\partial z}{\partial u}+k \sum \bar{x}^{\prime} \frac{\partial \bar{x}}{\partial u}=0 \\
(e-k) x^{\prime} \frac{\partial x}{\partial v}+(f-k) y^{\prime} \frac{\partial y}{\partial v}+(g-k) z^{\prime} \frac{\partial z}{\partial v}+k \sum \bar{x}^{\prime} \frac{\partial \bar{x}}{\partial v}=0 .
\end{array}\right.
$$

If these equations are differentiated with respect to $u$ and $v$ and in the reduction use is made of equations $[\S 64,(7)]$ for $N$ and $\bar{N}$, two of the resulting equations are satisfied identically in consequence of (64) and the other two are reducible to

(65)

$$
\left\{\begin{array}{l}
h \sum e\left(\frac{\partial x}{\partial u}\right)^{2}+D \sum(e-k) x^{\prime} X+k \bar{D} \sum \bar{x}^{\prime} \bar{X}=0, \\
l \sum e\left(\frac{\partial x}{\partial v}\right)^{2}+D^{\prime \prime} \sum(e-k) x^{\prime} X+k \bar{D}^{\prime \prime} \sum \bar{x}^{\prime} \bar{X}=0 .
\end{array}\right.
$$

In consequence of (27), (28), (29), (35), (42) and the second of (57) these two equations are equivalent to

$$
(h-l) \sigma a b+c l \sum \bar{x}^{\prime} \bar{X}=0 .
$$


Solving equations (64) and (66) for $\bar{x}^{\prime}, \bar{y}^{\prime}, \bar{z}^{\prime}$, we have expressions of the form

$$
\left\{\begin{array}{c}
k \bar{x}^{\prime}=\frac{\sigma a b}{c}(l-h) \bar{X}+\frac{1}{H^{2}}\left[\frac{\partial \bar{x}}{\partial u} \sum(e-k) x^{\prime}\left(F \frac{\partial x}{\partial v}-G \frac{\partial x}{\partial u}\right)\right. \\
\left.+\frac{\partial \bar{x}}{\partial v} \sum(e-k) x^{\prime}\left(F \frac{\partial x}{\partial u}-E \frac{\partial x}{\partial v}\right)\right]
\end{array}\right.
$$

If we differentiate these expressions, we find that $\bar{x}^{\prime}, \bar{y}^{\prime}, \bar{z}^{\prime}$ satisfy equations of the form (63), by making use of equations of $\S \S 134$, 135 and of $[\S 63]$.

Also from (67) we have, with the aid of $[\S 48,(11)]$,

(68) $k^{2} \sum \bar{x}^{\prime 2}=\frac{\sigma^{2} a^{2} b^{2}}{c^{2}}(l-h)^{2}+\sum(e-k)^{2} x^{\prime 2}-\left(\sum(e-k) x^{\prime} X\right)^{2}$.

Substituting this expression in (62), we find that it is satisfied in virtue of (57).

From these results and the last theorem of $\S 135$ we have, when $k$ is not equal to $0, e, f$ or $g$, the theorem:

If $\bar{N}$ is a net applicable to a net $N$ on a central quadric $e x^{2}+f y^{2}+g z^{2}=1$, for each value of $k$ different from $e, f, g$ and zero, there exist $\infty^{2}$ transformations $F_{k}$ of $\bar{N}$ into nets $\bar{N}_{1}$ applicable to the quadric; these transforms are conjugate to $\infty^{2}$ congruences $G$; their determination requires the finding of solutions of a completely integrable system of equations subject to a quadratic and a linear homogeneous relations ${ }^{\mathbf{1 3 1}}$ ).

When $k=e$, the function $x^{\prime}$ is determined to within an additive constant $m$, as seen in $\S 135$. There are only $\infty^{1}$ sets of solutions $y^{\prime}, z^{\prime}, h$ and $l$, and in consequence from (67) it follows that there are only $\infty^{1}$ congruences $G$ of the $\infty^{2}$ transformations. As $m$ varies we obtain $\infty^{1}$ transforms $N_{1}$ conjugate to the same congruence. They are defined by (61) with

$$
\theta=2 \sum e x x^{\prime}+2 m e x, \quad \theta^{\prime}=e\left(x^{\prime}+m\right)^{2}+f y^{\prime 2}+g z^{\prime 2} .
$$

From this expression for $\theta$ and the results of $\$ 23$ it follows that the tangent planes at corresponding points of these nets $N_{1}$ enve-

131) Journ. de Math. l. c. p. 53. 
lope a cone. If $\xi_{0}, \eta_{0}, \zeta_{0}$ are the coordinates of the vertex, the equation of the tangent plane is

$$
\left(\xi-\xi_{0}\right) X_{1}+\left(\eta-\eta_{0}\right) Y_{1}+\left(\zeta-\zeta_{0}\right) Z_{1}=0,
$$

where $\xi, \eta, \zeta$ are current coordinates, and $X_{1}, Y_{1}$ and $Z_{1}$ are direction-parameters of the normal to $N_{1}$. When their expressions are calculated, it is found that they involve $m$ to the second degree, and consequently the cone is a quadric.

When $k=e, x^{\prime}$ does not appear in (62) and (64). Solving the latter for $y^{\prime}$ and $z^{\prime}$, and substituting in (62), we obtain a homogeneous quadric equation in $\bar{x}^{\prime}, \bar{y}^{\prime}, \bar{z}^{\prime}$. Hence the lines of the congruences $G$ through a point of $\bar{N}$ form a quadric cone. Since similar results hold when $k$ is equal to $f$ or $g$, we have in consequence of the last theorem of $\$ 135$ the theorem:

If $\bar{N}$ is a net applicable to a net $N$ on the central quadric $Q$ (34), there are $\infty^{2}$ sets of solutions of equations (57), (58), (59) with $k$ equal to e, $f^{\prime} m^{\prime} g$ : there are thus three families of $\infty^{2}$ transforms $\vec{N}_{1}$ of $\overrightarrow{N^{\prime}}$ which are applicable to $Q$ : the transforms of each family are conjuyate to $\infty^{1}$ congruences $G$, there being $\infty^{1}$ transforms conjugate to each $G$ : the lines of the congruences $G$ through a point of $\bar{N}$. form a quallvic cone; the tangent planes at points of a line of $G$ of the nets $\bar{N}_{1}$ comjugate to it envelop a quadric cone, and the points on $Q$ corresponding to these points of the nets $\bar{N}_{1}$ on a line of $G$ lie on a conic ${ }^{132}$ ).

When $Q$ is the quadric of revolution $e\left(x^{2}+y^{2}\right)+y z^{2}=1$, the transformations of the type described in the first of the above theorems exist. There is, however, only one family of transformations of the type described in the second theorem; they are $F_{g}$. When $l=e$, equation (62) may be written

$$
\sum \bar{x}^{\prime 2}-\left(1-\frac{g}{e}\right) z^{\prime 2}=0
$$

132) The two types of transformation set forth in this section were discovered by Guichard in a different manner in his Mémoire sur la deformation. des quadrics, Mémoires à L'Académie des Sciences de France, ser. 2, vol. 24 (1909). His method did not reveal the relation between the nets $N$ and $N_{1}$ on the quadric in either case, nor did he show that the nets $\bar{N}$ and $\bar{N}_{1}$ are in relation $F$ when $k=e, f$ or $g$. These results were announced by the auther in 1919, Trans. Amer. Math. Soc., vol. 20 (1919). pp. 323-338. 
From the results of $\$ 100$ it follows that $N$ is a net $2, O$ and consequently $\bar{N}$ is 2,O. From (69) it follows that $\bar{N}^{\prime}$ is one of the two special nets $2, O$ parallal to $\bar{N}$, the complementary function being

$$
\overline{t^{\prime}}=\sqrt{1-\frac{g}{e}} z^{\prime}
$$

Suppose conversely that we take for $\bar{N}^{\prime}$ one of these two special nets 2, $O$ and define $z^{\prime}$ by (70). Equations (64) and (65) hold for $k=e=f$. Adding equations (65), we have in consequence of (27), (28), (29), (35) and (42), the second of (57). From these follow (66), (67), (68), and then the first of (57), as in the general case. Thus $h$ and $l$ determined by $\bar{N}^{\prime}$ satisfy the equations of $\$ 135, z^{\prime}$ is given directly by (70) and $x^{\prime}$ and $y^{\prime}$ by the quadratures (59), and thus involve additive arbitrary constants.

Each of the two parallel special 2, $O$ nets $\bar{N}^{\prime}$ determines a normal congruence conjugate to $\bar{N}(\$ 61)$. In accordance with the theorem of Beltrami $(\$ 112)$ and the results of $\S 100$, when the net $\bar{N}$ is applied to its applicable net $N$ on $Q$, the lines of these two normal congruences coincide with the lines joining points of $N$ to the foci on the axis of revolution of $Q$. Hence we have the theorem:

Let $\bar{N}$ be a net applicable to a net $N$ on a central quadric of revolution $Q$; the lines joining points of $N$ to the foci of $Q$ on the axis of revolution become lines of two normal congruences $G_{1}$ and $G_{2}$, conjugate to $\bar{N}$ when $N$ is applied to $\bar{N}$; there can be found by two quadratures $\infty^{2}$ nets $\bar{N}_{1}$ conjugate to $G_{1}$ and $\infty^{2}$ nets $\bar{N}_{2}$ conjugate $G_{2}$ which are applicable to $\infty^{2}$ nets $N_{1}$ and $\infty^{2}$ nets $N_{2}$ on $Q$; the nets $\bar{N}_{1}$, or $\bar{N}_{2}$, can be grouped into $\infty^{1}$ families of $\infty^{1}$ nets such that their tangent planes at points on the same line of the congruence form a quadric cone and the corresponding points of the applicable nets on $Q$ lie on a conic $^{\mathbf{1 3 3}}$ ).

When $Q$ is a sphere, real or imaginary, with the equation $e\left(x^{2}+y^{2}+z^{2}\right)=1$, an applicable net $\bar{N}$ consists of the lines of curvature on a surface of constant gaussian curvature, since every

${ }^{133}$ ) In order to obtain one of these families we hold fixed the additive constant in $x^{\prime}$ and let the additive constant of $y^{\prime}$ vary, and vice-versa; cf. Trans. Amer. Math. Soc., l. c., p. 337. 
net on $Q$ is orthogonal. If we take $k=e$, it follows from (62) that $\bar{N}^{\prime}$ is a cone; hence there are no transformations of the type of the second theorem. When $k \neq e$, we have from (60) and (62) $\theta^{\prime}=\frac{e k}{k-e} \sum \bar{x}^{\prime 2}$, that is the transformation is $R(\S 67)$. Hence:

A surface of constant gaussian curvature admits $\infty^{3}$ transformations $R$ into surfaces of the same curvature.

137. Theorem of permutability of transformations $F_{k}$ of surfaces applicable to a central quadric. Let $\bar{N}_{1}\left(\bar{x}_{1}\right)$ and $\bar{N}_{2}\left(\bar{x}_{2}\right)$ be obtained from a net $\bar{N}(\bar{x})$ applicable to a net $N(x)$ on $Q$ by means of transformations $F_{k_{1}}$ and $F_{k_{2}}$, and let $N_{1}\left(x_{1}\right)$ and $N_{2}\left(x_{2}\right)$ be the nets on $Q$ to which $\bar{N}_{1}$ and $\bar{N}_{2}$ are applicable. Let $\theta_{1}$ and $\theta_{2}$ be the functions of these transformations, where

$\bar{x}^{\prime}, \bar{x}^{\prime \prime} ; x^{\prime}, x^{\prime \prime}$ being obtained from $\bar{x}$ and $x$ by the quadratures

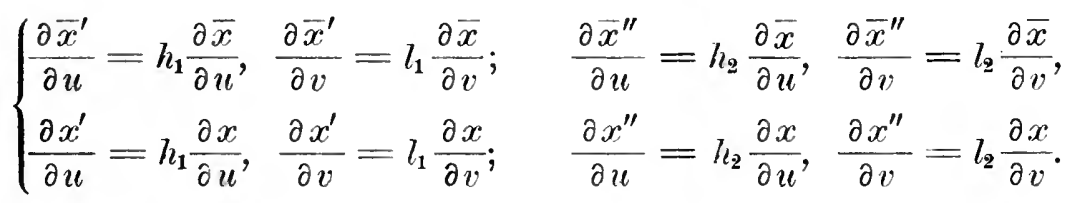

We seek under what conditions the net $\overline{N_{12}}$ whose coordinates are of the form

where (cf. $\S \S 20,21$ )

$$
\left\{\begin{array}{l}
\bar{x}_{1}^{\prime \prime \prime}=\bar{x}^{\prime \prime}-\frac{\theta_{1}^{\prime \prime}}{\theta_{1}^{\prime}} \bar{x}^{\prime}, \quad \theta_{12}=\theta_{2}-\frac{\theta_{2}^{\prime}}{\theta_{1}^{\prime}} \theta_{1}, \quad \theta_{12}^{\prime \prime \prime}=\theta_{2}^{\prime \prime}-\frac{\theta_{1}^{\prime \prime}}{\theta_{1}^{\prime}} \theta_{2}^{\prime}, \\
\frac{\partial \theta_{1}^{\prime \prime}}{\partial u}=h_{2} \frac{\partial \theta_{1}}{\partial u}, \frac{\partial \theta_{1}^{\prime \prime}}{\partial v}=l_{2} \frac{\partial \theta_{1}}{\partial v}, \quad \frac{\partial \theta_{2}^{\prime}}{\partial u}=h_{1} \frac{\partial \theta_{2}}{\partial u}, \frac{\partial \theta_{2}^{\prime}}{\partial v}=l_{1} \frac{\partial \theta_{2}}{\partial v}
\end{array}\right.
$$

is applicable to a net $N_{12}$ on $Q$, defined by

$$
\text { If we put }
$$

$$
x_{12}=x_{1}-\frac{\theta_{12}}{\theta_{12}^{\prime \prime}} x_{1}^{\prime \prime \prime}, \quad x_{1}^{\prime \prime \prime}=x^{\prime \prime}-\frac{\theta_{1}^{\prime \prime}}{\theta_{1}^{\prime}} x^{\prime} .
$$

$$
\theta_{12}^{\prime \prime \prime}=7_{i_{2}}\left(\sum x_{1}^{\prime \prime \prime 2}-\sum \bar{x}_{1}^{\prime \prime \prime 2}\right)
$$


we find that this expression is equivalent to that of (74) if

$$
k_{2} \theta_{1}^{\prime \prime}+k_{1} \theta_{2}^{\prime}-2 k_{1} k_{2}\left(\sum x^{\prime} \cdot x^{\prime \prime}-\sum \bar{x}^{\prime} \bar{x}^{\prime \prime}\right)=0 \text {. }
$$

When the left-hand member of this equation is differentiated, it is found to be constant in consequence of (71), (72) and (74). Hence the additive constants entering in $\theta_{1}^{\prime \prime}$ and $\theta_{2}^{\prime}$ can be chosen so that (77) holds.

From (6) it follows that $N_{12}$ defined by (75) is on $Q$, if

$$
\theta_{1}^{\prime \prime}+\theta_{2}^{\prime}-2\left(e y^{\prime} x^{\prime \prime}+f y^{\prime} y^{\prime \prime}+g z^{\prime} z^{\prime \prime}\right)=0 .
$$

Solving equations (77) and (78) for $\theta_{1}^{\prime \prime}$ and $\theta_{2}^{\prime}$, we find expressions which satisfy (74). Hence:

If $\bar{N}_{1}$ and $\bar{N}_{2}$ are transforms of a net $\bar{N}$ applicable to a net $N$ on a central quadric $Q$ by means of transformations $F_{k_{2}}$ and $\boldsymbol{F}_{\boldsymbol{k}_{2}}$ $\left(k_{2} \neq l_{1}\right)$, there can be found directly a net $\bar{N}_{12}$, applicable to a net $N_{12}$ on $Q$, which is in relations $F_{k_{3}}^{\prime}$ and $F_{k_{1}}^{\prime}$ with $\bar{N}_{1}$ and $\bar{N}_{2}$ respectively.

This theorem holds when $k_{1}$ or $k_{2}$, or both, is equal to $e, f$ or $g$, but $h_{1} \neq l_{2}$. It holds equally when $Q$ is a central quadric of revolution, or a sphere, real or imaginary ${ }^{134}$ ).

138. Transformations $B_{k}$ of surfaces applicable to a central quadric. Let $\bar{N}(\bar{x})$ be a net applicable to a net $N(x)$ on the central quadric (34). We consider a transformation $F_{k}$ of $\bar{N}$ and $N$ as treated in $\$ 136$. We have

$$
\theta=2 \sum e x x^{\prime}
$$

From (35) and (36) we have

$$
\sum e x^{\prime} \mathrm{X}=r \sigma \sum e^{2} x x^{\prime}
$$

By differentiation of (79) we have

$$
\frac{\partial \theta}{\partial u}=2 \sum e r^{\prime} \frac{\partial x}{\partial u}, \quad \frac{\partial \theta}{\partial r}=2 \sum e x^{\prime} \frac{\partial x}{\partial r},
$$

15t) Trans. Aner. Math. Soc.. 1. c., pp. 324. 335. 
and with the aid of (27). (28), (42) and (57) we find

$$
\left\{\begin{array}{l}
\frac{\partial^{2} \theta}{\partial u^{2}}=\left\{\begin{array}{c}
11 \\
1
\end{array}\right\} \frac{\partial \theta}{\partial u}+\left\{\begin{array}{c}
11\} \\
2
\end{array}\right\} \frac{\partial \theta}{\partial c}-c k \sigma^{2} a^{2} \theta+\frac{2 \sigma^{2} a^{2} b^{2}}{v}(l-l), \\
\frac{\partial^{2} \theta}{\partial v^{2}}=\left\{\begin{array}{c}
22 \\
1
\end{array}\right\} \frac{\partial \theta}{\partial u}+\left\{\begin{array}{c}
22 \\
2
\end{array}\right\} \frac{\partial \theta}{\partial v}+c k \sigma^{2} b^{2} \theta+\frac{2 \sigma^{2} a^{2} b^{2}}{c}(h-l) .
\end{array}\right.
$$

Because of (28) and (32) we have

$$
\frac{\partial^{2} \theta}{\partial u^{2}}+\frac{\partial^{2} \theta}{\partial r^{2}}=2 \frac{\partial \log u}{\partial u} \frac{\partial \theta}{\partial u}+2 \frac{\partial \log b}{\partial r} \frac{\partial \theta}{\partial v}-r k \theta .
$$

From the results $\$ 49$ it follows that if we take two transformations $F_{k}$ of $\bar{N}(k \neq e, f ; g)$ and write

$$
\theta_{1}=2 \sum e x x^{\prime}, \quad \theta_{2}=2 \sum e x x^{\prime \prime},
$$

the net $\hat{N}(\hat{x})$, defined by

$$
\hat{x}=x+p \frac{\partial \dot{x}}{\partial u}+q \frac{\partial \bar{x}}{\partial v},
$$

where

$$
\left\{\begin{array}{c}
p=\frac{1}{\lambda}\left(\theta_{1} \frac{\partial \theta_{2}}{\partial v}-\theta_{2} \frac{\partial \theta_{1}}{\partial v}\right), \quad q=\frac{1}{I}\left(\theta_{2} \frac{\partial \theta_{1}}{\partial u}-\theta_{1} \frac{\partial \theta_{2}}{\partial u}\right), \\
\quad I=\frac{\partial \theta_{2}}{\partial \imath} \frac{\partial \theta_{1}}{\partial v}-\frac{\partial \theta_{1}}{\partial u} \frac{\partial \theta_{2}}{\partial v},
\end{array}\right.
$$

is a $W$ transform of $\bar{N}$, that is these two nets are on the focal surfaces of a $W$ congruence.

On substituting the expressions for the derivatives of $\theta_{1}$ and $\theta_{2}$ analogous to $(81)$ and $(82)$ in $(I, 68)$, we reduce the resulting expressions to

$$
\begin{aligned}
& \left(\frac{\partial \hat{x}}{\partial u}=p\left\{\bar{D} \bar{X}+\frac{2 \sigma^{2} a^{2}}{c \bar{A}}\left[\left(\frac{\partial \bar{x}}{\partial u} \frac{\partial \theta_{2}}{\partial v}-\frac{\partial \bar{x}}{\partial v} \frac{\partial \theta_{2}}{\partial u}\right)\left(b^{2}\left(l_{1}-h_{1}\right)-c^{2} k_{i} \frac{\theta_{1}}{2}\right)\right.\right.\right. \\
& \left.\left.-\left(\frac{\partial \bar{x}}{\partial u} \frac{\partial \theta_{1}}{\partial v}-\frac{\partial \bar{x}}{\partial v} \frac{\partial \theta_{1}}{\partial u}\right)\left(b^{2}\left(l_{2}-h_{2}\right)-r \cdot 2 ; \frac{\theta_{2}}{2}\right)\right]\right\}, \\
& \frac{\partial \hat{x}}{\partial v}=q\left\{\bar{D}^{\prime \prime} \bar{X}-\frac{2 \sigma^{2} b^{2}}{c \Delta}\left[\left(\frac{\partial \bar{x}}{\partial u} \frac{\partial \theta_{2}}{\partial v}-\frac{\partial \bar{x}}{\partial v} \frac{\partial \theta_{2}}{\partial u}\right)\left(a^{2}\left(l_{1}-l_{1}\right)-c^{2} l_{i} \frac{\theta_{1}}{2}\right)\right.\right. \\
& \left.\left.-\left(\frac{\partial \bar{x}}{\partial u} \frac{\partial \theta_{1}}{\partial v}-\frac{\partial \bar{x}}{\partial v} \frac{\partial \theta_{1}}{\partial u}\right)\left(a^{2}\left(l_{2}-l_{2}\right)-c^{2} k \frac{\theta_{\mathbf{g}}}{2}\right)\right]\right\} \text {. }
\end{aligned}
$$


By means of the same functions $\theta_{1}$ and $\theta_{2}$ we obtain a derived net $\tilde{N}(\tilde{x})$ of $N$. Its equations are

$$
\tilde{x}=x+r \frac{\partial x}{\partial u}+q \frac{\partial x}{\partial r}
$$

where $\nu$ and $q$ are given by (86). On substituting the expressions for $\theta_{1}$ and $\theta_{2}$, as given by (84) in the expression (86) for $A$, we find, in consequence of (35) and (cf. (29))

$$
H \sigma=a b,
$$

that

$(90)\left\{\begin{array}{c}\boldsymbol{I}=4 \sum e f\left(x^{\prime \prime} y^{\prime}-x^{\prime} y^{\prime \prime}\right)\left(\frac{\partial x}{\partial u} \frac{\partial y}{\partial v}-\frac{\partial x}{\partial v} \frac{\partial y}{\partial u}\right) \\ =4 H \sum e f\left(x^{\prime \prime} y^{\prime}-x^{\prime} y^{\prime \prime}\right) Z=4 \text { cabefg } \sum x\left(y^{\prime \prime} z^{\prime}-y^{\prime} z^{\prime \prime}\right) .\end{array}\right.$

Hence the expression for $\tilde{x}$ is reducible to

From this we have

$$
\tilde{x}=\frac{y^{\prime \prime} z^{\prime}-y^{\prime} z^{\prime \prime}}{e \sum x\left(y^{\prime \prime} z^{\prime}-y^{\prime} z^{\prime \prime}\right)} \text {. }
$$

$$
\sum e \tilde{x} x=1 \text {. }
$$

The equations analogous to (57) are

$$
\begin{cases}h_{1}^{2} a^{2}-l_{1}^{2} b^{2}=c^{2} \sum\left(e^{2}-l_{i} e\right) x^{\prime 2}, & h_{2}^{2} a^{2}-l_{2}^{2} b^{2}=c^{2} \sum\left(e^{2}-k e\right) x^{\prime \prime 2}, \\ h_{1} a^{2}-l_{1} b^{2}=c^{2} \sum\left(e^{2}-k e\right) x x^{\prime}, & h_{2} a^{2}-l_{2} b^{2}=c^{2} \sum\left(e^{2}-k e\right) x x^{\prime \prime} .\end{cases}
$$

By differentiation it can be shown that the left-hand member of the following equation is constant for any two transformations $\boldsymbol{F}_{\boldsymbol{k}}$

$$
l_{1} h_{2} a^{2}-l_{1} l_{2} b^{2}-r^{2} \sum\left(e^{2}-k e\right) x^{\prime} x^{\prime \prime}=0 \text {. }
$$

We choose the nets $N^{\prime}$ and $N^{\prime \prime}$ so that (94) is satisfied. By means of these relations we show that

$$
\left\{\begin{array}{c}
\sum\left(f^{2}-k_{2} f\right)\left(g^{2}-k g\right)\left(y^{\prime} z^{\prime \prime}-y^{\prime \prime} z^{\prime}\right)^{2}=\sum\left(e^{2}-k e\right) x^{\prime 2} \cdot \sum\left(e^{2}-k e\right) x^{\prime \prime 2} \\
-\left(\sum\left(e^{2}-k e\right) x^{\prime} x^{\prime \prime}\right)^{2}=-\frac{a^{2} b^{2}}{c^{4}}\left(l_{1} l_{2}-l_{2} h_{1}\right)^{2}
\end{array}\right.
$$


138. Transformations $B_{k}$ of surfaces applicable to a central quadric 343

also that

$$
\left\{\begin{array}{c}
\left(e^{2}-k e\right)\left(f^{2}-k \cdot f\right)\left(g^{2}-k \cdot g\right)\left[\sum x\left(y^{\prime \prime} z^{\prime}-y^{\prime} z^{\prime \prime}\right)\right]^{2} \\
=\frac{k a^{2} b^{2}}{c^{4}}\left(l_{1} h_{2}-l_{2} h_{1}\right)^{2}
\end{array}\right.
$$

In consequence of these identities we have

$$
\sum \frac{\tilde{x}^{2}}{\frac{1}{e}-\frac{1}{k}}=1
$$

that is $\tilde{N}(\tilde{x})$ lies on a quadric confocal with $Q$.

The equations for $\tilde{N}$ analogous to (87) are obtained by removing the bars from the quantities $\bar{x}, \bar{X}, \bar{D}$ and $\bar{D}^{\prime \prime}$. Substituting from (81) the expressions for the derivatives of $\theta_{1}$ and $\theta_{2}$ and making use of $(35),(36),(84),(89)$ and $(90)$, we reduce the resulting equations to

(98)

$$
\left\{\begin{aligned}
& \frac{\partial \tilde{x}}{\partial u}= \frac{4 \sigma^{2} a^{3} b p^{\prime} f^{\prime} g}{A}\left\{c^{2}\left(e^{2}-k e\right) x \sum x\left(y^{\prime} z^{\prime \prime}-y^{\prime \prime} z^{\prime}\right)+c^{2} k\left(y^{\prime} z^{\prime \prime}-y^{\prime \prime} z^{\prime}\right)\right. \\
&\left.+b^{2}\left[\left(z y^{\prime \prime}-y z^{\prime \prime}\right)\left(l_{1}-h_{1}\right)-\left(z y^{\prime}-y z^{\prime}\right)\left(l_{2}-h_{2}\right)\right]\right\}, \\
& \frac{\partial \tilde{x}}{\partial v}=- \frac{4 \sigma^{2} a b^{3} q f q}{I}\left\{c^{2}\left(e^{2}-k e\right) x \sum x\left(y^{\prime} z^{\prime \prime}-y^{\prime \prime} z^{\prime}\right)+c^{2} k\left(y^{\prime} z^{\prime \prime}-y^{\prime \prime} z^{\prime}\right)\right. \\
&\left.+a^{2}\left[\left(z y^{\prime \prime}-y z^{\prime \prime}\right)\left(l_{1}-h_{1}\right)-\left(z y^{\prime}-y z^{\prime}\right)\left(l_{2}-h_{2}\right)\right]\right\} .
\end{aligned}\right.
$$

From (93) we have

(99) $\left\{\begin{array}{c}\left(z y^{\prime \prime}-y z^{\prime \prime}\right)\left(h_{1} a^{2}-l_{1} b^{2}\right)-\left(z y^{\prime}-y z^{\prime}\right)\left(l_{2} a^{2}-l_{2} b^{2}\right) \\ =c^{2}\left\{\left(e^{2}-k e\right) x \sum x\left(y^{\prime} z^{\prime \prime}-y^{\prime \prime} z^{\prime}\right)+\left(\frac{1}{c^{2} \sigma^{2}}-k\right)\left(z^{\prime} y^{\prime \prime}-z^{\prime \prime} y^{\prime}\right)\right\} .\end{array}\right.$

Adding the left-hand member of this expression to the expressions in parentheses in (98) and subtracting the right-hand member, we get in consequence of (28)

(100)

$$
\left\{\begin{array}{l}
\frac{\partial \tilde{x}}{\partial u}=\frac{4 a^{3} b p f g}{\Delta}\left[h_{1}\left(z y^{\prime \prime}-y z^{\prime \prime}\right)-h_{2}\left(z y^{\prime}-y z^{\prime}\right)+\left(z^{\prime \prime} y^{\prime}-z^{\prime} y^{\prime \prime}\right)\right] \\
\frac{\partial \tilde{x}}{\partial \imath}=-\frac{4 a b^{3} q f y}{d}\left[l_{1}\left(z y^{\prime \prime}-y z^{\prime \prime}\right)-l_{2}\left(z y^{\prime}-y z^{\prime}\right)+\left(z^{\prime \prime} y^{\prime}-z^{\prime} y^{\prime \prime}\right)\right] .
\end{array}\right.
$$


For $\tilde{N}$ the expressions amalogons to $\sum e\left(\frac{\partial x}{\partial u}\right)^{2}$ and $\sum e\left(\frac{\partial x}{\partial v}\right)^{2}$ for $N$ are $\sum \frac{k e}{k-e}\left(\frac{\partial \tilde{x}}{\partial u}\right)^{2}$ and $\sum \frac{k e}{k-e}\left(\frac{\partial \tilde{x}}{\partial v}\right)^{2}$. Making use of wellknown theorems on determinants, we find ultimately that

$$
\left\{\begin{array}{c}
\sum \frac{k e}{k-e}\left(\frac{\partial \tilde{x}}{\partial u}\right)^{2}=-l_{i} a^{2} p^{2} . \quad \sum \frac{k e}{k-e}\left(\frac{\partial \tilde{x}}{\partial v}\right)^{2}=k b^{2} q^{2}, \\
\sum \frac{k e}{k-e} \frac{\partial \tilde{x}}{\partial n} \frac{\partial \tilde{x}}{\partial r}=0 .
\end{array}\right.
$$

From (87) and the analogous equations for $\tilde{N}$ we have, in consequence of (27) and (29),

(102)

$$
\left\{\begin{array}{c}
\sum\left(\frac{\partial \hat{x}}{\partial u}\right)^{2}-\sum\left(\frac{\partial \tilde{x}}{\partial u}\right)^{2}=p^{2}\left(\bar{D}^{2}-D^{2}\right)=-a^{2} p^{2}, \\
\sum \frac{\partial \hat{x}}{\partial u} \frac{\partial \hat{x}}{\partial r}-\sum \frac{\partial \tilde{x}}{\partial u} \frac{\partial \tilde{x}}{\partial r}=0 \\
\sum\left(\frac{\partial \hat{x}}{\partial v}\right)^{2}-\sum\left(\frac{\partial \tilde{x}}{\partial v}\right)^{2}=q^{2}\left(\bar{D}^{\prime \prime 2}-D^{\prime \prime 2}\right)=b^{2} q^{2} .
\end{array}\right.
$$

From (101) and (102) we have

$$
\left\{\begin{array}{c}
\sum\left(\frac{\partial \hat{x}}{\partial u}\right)^{2}=\sum \frac{k}{k-e}\left(\frac{\partial \tilde{x}}{\partial u}\right)^{2}, \quad \sum \frac{\partial \hat{x}}{\partial u} \frac{\partial \hat{x}}{\partial r}=\sum \frac{k}{k-e} \frac{\partial \tilde{x}}{\partial u} \frac{\partial \tilde{x}}{\partial v}, \\
\sum\left(\frac{\partial \hat{x}}{\partial v}\right)^{2}=\sum \frac{k}{k-e}\left(\frac{\partial \tilde{x}}{\partial v}\right)^{2}
\end{array}\right.
$$

Hence if we put

$$
\tilde{x}_{0}=\sqrt{\frac{k}{k-e}} \tilde{x}, \quad \tilde{y}_{0}=\sqrt{\frac{k}{k-f}} \tilde{y}, \quad \tilde{z}_{0}=\sqrt{\frac{k}{k-g}} \tilde{z},
$$

the net $\hat{N}(\hat{x})$ is applicable to the net $\tilde{N}_{0}\left(\tilde{x}_{0}\right)$, which in consequence of (97) lies on $Q$. The equations (104) define the relation of Ivory between a quadric and a confocal quadric; the point of coordinates $\tilde{x}_{0}, \tilde{y}_{0}, \tilde{z}_{0}$ is the intersection with $Q$ of the orthogonal trajectory of the family of confocal quadrics which passes through the point of coordinates $\tilde{x}, \tilde{y}$, $\tilde{z}$ of $(97)^{135}$ ).

135) Bianchi, Lezioni, vol.3, p. 59. 
The functions of a transformation $W$ are $x^{\prime}, y^{\prime}, z^{\prime}, x^{\prime \prime}, y^{\prime \prime}, z^{\prime \prime}$, $h_{1}, l_{1}, h_{2}$ and $l_{2}$. They satisfy a completely integrable system of the form (58) and (59). Moreover, the five conditions (93) and (94) must be satisfied. However, these equations are satisfied also by the functions

(105) $\left\{\begin{array}{lllll}\alpha x^{\prime}+\beta x^{\prime \prime}, & \alpha y^{\prime}+\beta y^{\prime \prime}, & \alpha z^{\prime}+\beta z^{\prime \prime}, & \alpha l_{1}+\beta h_{2}, & \alpha l_{1}+\beta l_{2}, \\ \gamma x^{\prime}+\boldsymbol{d} x^{\prime \prime}, & \gamma y^{\prime}+\delta y^{\prime \prime}, & \gamma z^{\prime}+\delta z^{\prime \prime}, & \gamma h_{1}+\delta l_{2}, & \gamma l_{1}+\delta l_{2},\end{array}\right.$

where $\alpha, \beta, \gamma$ and $\delta$ are constants. In this case, as follows from (85), (86) and (88) we get the same nets $\hat{N}$ and $\hat{N}$. Consequently for each value of $k$ there are $\infty^{1}$ transformations of the kind sought.

If $N_{1}\left(x_{1}\right)$ and $N_{2}\left(x_{2}\right)$ denote the $F_{k}$ transforms of $\lambda$. we have

$$
x_{1}=x-\frac{\theta_{1}}{\theta_{1}^{\prime}} x^{\prime}, \quad x_{2}=x-\frac{\theta_{2}}{\theta_{2}^{\prime \prime}} x^{\prime \prime} .
$$

In consequence of $(91)$ and (92) we have

$$
\sum_{e} \tilde{x}_{1}=1, \quad \sum_{e} \tilde{x}_{2}=1 .
$$

Hence the point of coordinates, $\bar{x}, \bar{y}, \tilde{z}$ is the pole of the plane of the corresponding points on $N, N_{1}$ and $N_{2}$.

We may state the foregoing results as follows.

If $N$ is a permanent net on a rentral quadric $Q$, there are ${ }^{1}$ sets of transformations $F_{k}$ of $N$ into nets $N_{1}$ and $N_{2}$ so that the condition (94) is satisfied for each value of $k$ different firom zero; the locus of the pole $M_{k}$ of the plane $M M_{1} M_{2}$ with respect to $Q$ is a net $\tilde{N}$ on a quadric confocal to $Q ;$ as $N$ rolls on its applicable net $\bar{N}$, the point $M_{k}$ describes a net $\hat{N}$, such that $\bar{N}$ and $\hat{N}$ are the focal nets of a $W$ congruence, and $\hat{N}$ is copplicable to the nut an $Q$ which is the Ivory transform of the net $\tilde{N}$.

These are the transformations $B_{k}$ found by Bianchi by entirely different processes ${ }^{136}$ ).

Let $N$ be a permanent net on the quadric (34) and $N_{1}$ an $F_{k}$ transform of $N$ by means of the functions $x^{\prime}, y^{\prime}, z^{\prime}, h_{1}$ and $l_{1}$ satisfying (57), (58) and (59). From the form of equations (93) and (94) and the observations conceming (105) it follows that

${ }^{136}$ ) Lezioni, vol. 3, (haps. 1, 2, 3; ef. also Journ. de Matl., I. c.. p. (61. 
there exist only two sets of functions $x^{\prime \prime}, y^{\prime \prime}, z^{\prime \prime}, h_{2}, l_{2}$ and $x^{\prime \prime \prime}, y^{\prime \prime \prime}$, $z^{\prime \prime \prime}, h_{3}, l_{3}$ each satisfying (93) and (94) with $x^{\prime}, y^{\prime}, z^{\prime} h_{1}, l_{1}$ and determining different $B_{k}$ transforms of $N$, and also of the net $\bar{N}$ applicable to $N$.

The corresponding transforms $\hat{N}_{2}$ and $\hat{N}_{3}$ of $\bar{N}$ are determined by $\theta_{1}, \theta_{2}$ and $\theta_{1}, \theta_{3}$ where $\theta_{1}$ and $\theta_{2}$ are given by (84) and $\theta_{3}=\sum e x x^{\prime \prime \prime}$. Consequently these transforms are conjugate to the congruence harmonic to $N$ determined by $\theta_{1}$. The same is true of the transforms $\tilde{N}_{2}$ and $\tilde{N}_{3}$ of $N$ determined by $\theta_{1}, \theta_{2}$ and $\theta_{1}, \theta_{3}$. The congruence harmonic to $N$ determined by $\theta_{1}$ consists of the lines of intersection of the corresponding tangent planes to $N^{\prime}$ and its $F_{k}$ transform $N_{1}$ by means of $x^{\prime}, y^{\prime}, z^{\prime}, l_{1}$ and $l_{1}$. In view of the preceding results we have the theorem:

If $N$ is a permanent net on a central quadric $Q$ and $N_{1}$ is an $F_{k}$ transform of $N$, and $P_{1}$ and $P_{2}$ are the points of intersection with the confocal quadric $Q_{k}$ of the line of intersection of corresponding tangent planes of $N$ and $N_{1}$, then as $N$ rolls on its applicable net $\bar{N}$, the points $P_{1}$ and $P_{2}$ generate nets applicable to $Q$, each of which is a $B_{k}$ transform of $\bar{N}$.

139. Permutability to transformations $F_{k}$ and $B_{k}$. Let $\bar{N}(\bar{x})$ be the permanent net on a deform of the quadric $Q$ (34) and $\hat{N}(\hat{x})$ its $B_{k}$ transform by means of functions $x^{\prime}, y^{\prime}, z^{\prime} ; x^{\prime \prime}, y^{\prime \prime}, z^{\prime \prime}$; $h_{1}, l_{1}, h_{2}, l_{2}$. Let $x^{\prime \prime \prime}, y^{\prime \prime \prime}, z^{\prime \prime \prime}, h_{3}, l_{3}$, be a set of solutions of (50) and (59) with $l_{i}$ replaced by $k^{\prime}$ satisfying the conditions

(106) $h_{3}^{2} a^{2}-l_{3}^{2} b^{2}=c^{2} \sum\left(e^{2}-l_{i}^{\prime} e\right) x^{\prime \prime \prime 2}, \quad h_{3} a^{2}-l_{3} b^{2}=c^{2} \sum\left(e^{2}-l_{i}^{\prime} e\right) x x^{\prime \prime \prime}$,

so that the $F_{k^{\prime}}$ transform $N_{3}$ of $N$, defined by equations of the form

$$
x_{3}=x-\frac{\theta_{3}}{\theta_{3}^{\prime \prime \prime}} x^{\prime \prime \prime}
$$

where

$$
\theta_{3}=2 \sum e x x^{\prime \prime \prime}, \quad \theta_{3}^{\prime \prime \prime}=\sum e x^{\prime \prime \prime},
$$

is on $Q$.

From (II, 33) it follows that the equations of the forms

$$
x_{3}^{\prime}=x^{\prime}-\frac{\theta_{3}^{\prime}}{\theta_{3}^{\prime \prime \prime}} x^{\prime \prime \prime}, \quad x_{3}^{\prime \prime}=x^{\prime \prime}-\frac{\theta_{3}^{\prime \prime}}{\theta_{3}^{\prime \prime \prime}} x^{\prime \prime \prime}
$$


define nets parallel to $N_{3}$; the corresponding functions $h_{31}, l_{31}$ and $h_{32}, l_{32}$ are given by (cf. II, 50)

$$
\begin{array}{ll}
l_{31}=\frac{h_{3} \theta_{3}^{\prime}-h_{1} \theta_{3}^{\prime \prime \prime}}{l_{3} \theta_{3}-\theta_{3}^{\prime \prime \prime}}, & l_{31} \doteq \frac{l_{3} \theta_{3}^{\prime}-l_{1} \theta_{3}^{\prime \prime \prime}}{l_{3} \theta_{3}-\theta_{3}^{\prime \prime \prime}} \\
l_{32}=\frac{l_{3} \theta_{3}^{\prime \prime}-h_{2} \theta_{3}^{\prime \prime \prime}}{h_{3} \theta_{3}-\theta_{3}^{\prime \prime \prime}}, & l_{32}=\frac{l_{3} \theta_{3}^{\prime \prime}-l_{2} \theta_{3}^{\prime \prime \prime}}{l_{3} \theta_{3}-\theta_{3}^{\prime \prime \prime}} .
\end{array}
$$

The functions $a_{3}$ and $b_{3}$ appearing in the point equation of $N_{3}$ are of the form (cf. II, 12)

$$
a_{3}=a\left(h_{3} \frac{\theta_{3}}{\theta_{3}^{\prime \prime \prime}}-1\right), \quad b_{3}=b\left(l_{3} \frac{\theta_{3}}{\theta_{3}^{\prime \prime \prime}}-1\right) \text {. }
$$

In order that these functions may satisfy equations analogous to (93) and (94), namely

$$
\begin{aligned}
& a_{3}^{2} h_{31}^{2}-b_{3}^{2} l_{31}^{2}=c^{2} \sum\left(e^{2}-l e\right) x_{3}^{\prime 2}, \quad a_{3}^{2} h_{32}^{2}-l_{3}^{2} l_{32}^{2}=c^{2} \sum\left(e^{2}-k e\right) x_{3}^{\prime \prime 2}, \\
& a_{3}^{2} l_{31}-b_{3}^{2} l_{31}=c^{2} \sum\left(e^{2}-k e\right) x_{3} x_{3}^{\prime}, \quad a_{3}^{2} l_{32}-b_{3}^{2} l_{32}=c^{2} \sum\left(e^{2}-j e\right) x_{3} x_{3}^{\prime \prime} \text {, } \\
& a_{3}^{2} h_{31} h_{32}-b_{3}^{2} l_{31} l_{32}=c^{2} \sum\left(e^{2}-k e\right) x_{3}^{\prime} x_{3}^{\prime \prime},
\end{aligned}
$$

it is necessary and sufficient that

$$
\left\{\begin{array}{l}
a^{2} h_{1} h_{3}-b^{2} l_{1} l_{3}-c^{2} \sum\left(e^{2}-k e\right) x^{\prime} x^{\prime \prime \prime}-\frac{c^{2}}{2}\left(k-k^{\prime}\right) \theta_{3}^{\prime}=0 \\
a^{2} h_{2} h_{3}-b^{2} l_{2} l_{3}-c^{2} \sum\left(e^{2}-k e\right) x^{\prime \prime} x^{\prime \prime \prime}-\frac{c^{2}}{2}\left(k-k^{\prime}\right) \theta_{3}^{\prime \prime}=0 .
\end{array}\right.
$$

Differentiating these equations with respect to $u$ and $v$, and making use of (58), (59) and analogous equations, we find that the lefthand members are constant.

If $k^{\prime} \neq k$, the functions $\theta_{3}^{\prime}$ and $\theta_{3}^{\prime \prime}$ are uniquely determined by (110), and consequently $x_{3}^{\prime}$ and $x_{3}^{\prime \prime}$ are uniquely determined by (109). Then by means of the functions

$$
\theta_{31}=2 \sum e x_{3} x_{3}^{\prime}, \quad \theta_{32}=2 \sum e x_{3} x_{3}^{\prime \prime}
$$

we obtain a $B_{k}$ transform $\hat{N}_{3}$ of $\overline{N_{3}}$ which is applicable to $Q$. 
It is readily found by differentiation that the left-hand members of the equations

$$
\theta_{3}^{\prime}+\theta_{1}^{\prime \prime \prime}-2 \sum e x^{\prime} x^{\prime \prime \prime}=0 . \quad \theta_{3}^{\prime \prime}+\theta_{2}^{\prime \prime \prime}-2 \sum e x x^{\prime \prime} x^{\prime \prime \prime}=0
$$

are constants. If we take $\theta_{1}^{\prime \prime \prime}$ and $\theta_{2}^{\prime \prime \prime}$ as given by (112), we find that

$$
\theta_{31}=\theta_{1}-\frac{\theta_{3}}{\theta_{3}^{\prime \prime \prime}} \theta_{1}^{\prime \prime \prime} . \quad \theta_{32}=\theta_{2}-\frac{\theta_{3}}{\theta_{3}^{\prime \prime \prime}} \theta_{2}^{\prime \prime \prime} .
$$

These are the conditions that $\hat{N}^{\text {and }} \hat{N}_{3}$ are in relation $F$ (cf. $\$ 22$ ), We desire to show that this transformation is in fact a transformation $F_{k}$, as defined in $\$ 136$.

From $\$ 22$ we have that the functions $\hat{\theta}_{3}$ and $\hat{\theta}_{3}^{\prime \prime \prime}$ of the transformation $F$ from $\hat{N}$ into $\hat{N}_{3}$ are given by

(114) $\quad \hat{\theta_{3}}=\theta_{3}+p \frac{\partial \theta_{3}}{\partial \imath}+q \frac{\partial \theta_{3}}{\partial r}, \quad \hat{\theta}_{3}^{\prime \prime \prime}=\theta_{3}^{\prime \prime \prime}+p^{\prime \prime \prime} \frac{\partial \theta_{3}^{\prime \prime \prime}}{\partial \imath}+q^{\prime \prime \prime} \frac{\partial \theta_{3}^{\prime \prime \prime}}{\partial v}$,

where $p$ and $q$ are given by (86) and

$$
\begin{gathered}
p^{\prime \prime \prime}=\frac{1}{\boldsymbol{t}^{\prime \prime \prime}}\left(\theta_{1}^{\prime \prime \prime} \frac{\partial \theta_{2}^{\prime \prime \prime}}{\partial r}-\theta_{2}^{\prime \prime \prime} \frac{\partial \theta_{1}^{\prime \prime \prime}}{\partial v}\right), \quad q^{\prime \prime \prime}=\frac{1}{\boldsymbol{L}^{\prime \prime \prime}}\left(\theta_{2}^{\prime \prime \prime} \frac{\partial \theta_{1}^{\prime \prime \prime}}{\partial u}-\theta_{1}^{\prime \prime \prime} \frac{\partial \theta_{2}^{\prime \prime \prime}}{\partial u}\right) \\
\boldsymbol{l}^{\prime \prime \prime}=\frac{\partial \theta_{2}^{\prime \prime \prime}}{\partial u} \frac{\partial \theta_{1}^{\prime \prime \prime}}{\partial r}-\frac{\partial \theta_{2}^{\prime \prime \prime}}{\partial v} \frac{\partial \theta_{1}^{\prime \prime \prime}}{\partial u},
\end{gathered}
$$

and

$$
\frac{\partial \boldsymbol{\theta}_{i}^{\prime \prime \prime}}{\partial \boldsymbol{u}}=h_{3} \frac{\partial \boldsymbol{\theta}_{i}}{\partial \boldsymbol{u}}, \quad \frac{\partial \boldsymbol{\theta}_{i}^{\prime \prime \prime}}{\partial v}=l_{3} \frac{\partial \boldsymbol{\theta}_{i}}{\partial r}(i=1,2,3) .
$$

Moreover, the direction-parameters of the conjugate congruence of this transformation are the coordinates $\hat{x}^{\prime \prime \prime}, \hat{y}^{\prime \prime \prime}, \hat{z}^{\prime \prime \prime}$ of a net $\hat{N}_{3}^{\prime \prime \prime}$, parallel to $\hat{N}_{3}$, where

$$
\hat{r}^{\prime \prime \prime}=x^{\prime \prime \prime}+p^{\prime \prime \prime} \frac{\partial \bar{x}^{\prime \prime \prime}}{\partial u}+q^{\prime \prime \prime} \frac{\partial \bar{x}^{\prime \prime \prime}}{\partial v} .
$$

From $\$ 22$ corresponding to (85) and (116). we obtain

$$
\frac{\partial \hat{x}^{\prime \prime \prime}}{\partial u}=\hat{h} \frac{\partial \hat{x}}{\partial u}, \quad \frac{\partial \hat{x}^{\prime \prime \prime}}{\partial v}=\hat{\imath} \frac{\partial \hat{x}}{\partial r},
$$


where

(118) $\hat{h}=\frac{1}{p \Lambda}\left(\theta_{1}^{\prime \prime \prime} \frac{\partial \theta_{\mathbf{z}}}{\partial r}-\theta_{2}^{\prime \prime \prime} \frac{\partial \theta_{1}}{\partial r}\right) . \quad \hat{l}=\frac{1}{q^{\prime}}\left(\theta_{2}^{\prime \prime \prime} \frac{\partial \theta_{1}}{\partial u}-\theta_{1}^{\prime \prime \prime} \frac{\partial \theta_{\mathbf{2}}}{\partial u}\right)$.

Substituting the expressions (108) for $\theta_{3}$ and $\theta_{3}^{\prime \prime \prime}$ in (114), we get, in consequence of $(88)$ and (115)

$$
\hat{\theta}_{3}=2 \sum e \cdot x^{\prime \prime \prime} \tilde{x}
$$

and

$$
\left\{\begin{aligned}
\hat{\theta}_{3}^{\prime \prime \prime} & =\theta_{3}^{\prime \prime \prime}+\frac{1}{\boldsymbol{T}}\left[\theta_{1}^{\prime \prime \prime}\left(\frac{\partial \theta_{2}}{\partial r} \frac{\partial \theta_{3}}{\partial u}-\frac{\partial \theta_{2}}{\partial u} \frac{\partial \theta_{3}}{\partial v}\right)-\theta_{2}^{\prime \prime \prime}\left(\frac{\partial \theta_{1}}{\partial v} \frac{\partial \theta_{3}}{\partial u}-\frac{\partial \theta_{1}}{\partial u} \frac{\partial \theta_{3}}{\partial v}\right)\right] \\
& =\theta_{3}^{\prime \prime \prime}+\frac{4 e f g a b c}{t}\left[\theta_{1}^{\prime \prime \prime} \sum x\left(y^{\prime \prime \prime} z^{\prime \prime}-y^{\prime \prime} z^{\prime \prime \prime}\right)-\theta_{2}^{\prime \prime \prime} \sum x\left(y^{\prime \prime \prime} z^{\prime}-y^{\prime} z^{\prime \prime \prime}\right)\right]
\end{aligned}\right.
$$

this second expression for $\hat{\theta_{3}^{\prime \prime \prime}}$ is derived from the first with the aid of (29), (35) and (36).

From (110) and (112) we get

$$
\left\{\begin{array}{l}
a^{2} h_{1} h_{3}-b^{2} l_{1} l_{3}=c^{2} \sum\left(e^{2}-k^{\prime} e\right) x^{\prime} x^{\prime \prime \prime}+\frac{c^{2}}{2}\left(k^{\prime}-l_{i}\right) \theta_{1}^{\prime \prime \prime} \\
a^{2} h_{2} h_{3}-b^{2} l_{2} l_{3}=c^{2} \sum\left(e^{2}-k^{\prime} e\right) x^{\prime \prime} x^{\prime \prime \prime}+\frac{c^{2}}{2}\left(k^{\prime}-l^{\prime}\right) \theta_{2}^{\prime \prime \prime} .
\end{array}\right.
$$

From equations (121), (100), (106) and (90) we deduce

$$
\left\{\begin{array}{c}
\theta_{1}^{\prime \prime \prime}\left(y^{\prime \prime} z-y z^{\prime \prime}\right)-\theta_{2}^{\prime \prime \prime}\left(y^{\prime} z-y z^{\prime}\right)=\frac{1}{2 c^{2}\left(k^{\prime}-k\right) a b f g} \\
{\left[\frac{h_{3}}{p} \frac{\partial \tilde{x}}{\partial u}+\frac{l_{3}}{q} \frac{\partial \tilde{x}}{\partial r}+c x^{\prime \prime \prime}\left(e-k^{\prime}\right)\right]}
\end{array}\right.
$$

By means of (122) and analogous equations equation (120) is reducible to

$$
\left\{\begin{array}{c}
\hat{\boldsymbol{\theta}}_{3}^{\prime \prime \prime}=\frac{1}{k^{\prime}-\boldsymbol{l}_{i}}\left[\sum\left(2 e^{2}-k e-k^{\prime} e\right) x^{\prime \prime \prime 2}+\frac{2 h_{3}}{c p} \sum e x^{\prime \prime \prime} \frac{\partial \tilde{x}}{\partial u}\right. \\
\left.+\frac{2 l_{3}}{c q} \sum e x^{\prime \prime \prime} \frac{\partial \tilde{x}}{\partial r}\right]
\end{array}\right.
$$


Equations of the form

$$
\tilde{x}^{\prime \prime \prime}=x^{\prime \prime \prime}+x^{\prime \prime \prime} \frac{\partial x^{\prime \prime \prime}}{\partial u}+q^{\prime \prime \prime} \frac{\partial x^{\prime \prime \prime}}{\partial v}
$$

define a net $\tilde{N}^{\prime \prime \prime}$ parallel to $\tilde{N}$. This expression is reducible to $\left\{\begin{aligned} \tilde{x}^{\prime \prime \prime} & =x^{\prime \prime \prime}+\frac{1}{d}\left[\theta_{1}^{\prime \prime \prime}\left(\frac{\partial \theta_{2}}{\partial v} \frac{\partial x}{\partial u}-\frac{\partial \theta_{2}}{\partial u} \frac{\partial x}{\partial v}\right)-\theta_{2}^{\prime \prime \prime}\left(\frac{\partial \theta_{1}}{\partial v} \frac{\partial x}{\partial u}-\frac{\partial \theta_{1}}{\partial u} \frac{\partial x}{\partial v}\right)\right], \\ & =x^{\prime \prime \prime}+\frac{2 f g a b c}{d}\left[\theta_{1}^{\prime \prime \prime}\left(y^{\prime \prime} z-y z^{\prime \prime}\right)-\theta_{2}^{\prime \prime \prime}\left(y^{\prime} z-y z^{\prime}\right)\right]\end{aligned}\right.$

which in consequence of (122) becomes

$$
\tilde{x}^{\prime \prime \prime}=\frac{1}{k^{\prime}-l_{k}}\left[(e-k) x^{\prime \prime \prime}+\frac{l_{3}}{c p} \frac{\partial \tilde{x}}{\partial u}+\frac{l_{3}}{c q} \frac{\partial \tilde{x}}{\partial v}\right] \text {. }
$$

Since $\hat{N}$ is applicable to $\tilde{N_{0}}$, defined by (104), it follows from the results of $\$ 136$ that a transformation $F_{k^{\prime}}$ of $\hat{N}$ is given by the functions

$$
\left\{\begin{array}{c}
\tilde{\theta}_{3}=2 \sum e \tilde{x}_{0} \tilde{x}_{0}^{\prime \prime \prime}=2 k \sum \frac{e}{k-e} \tilde{x} \tilde{x}^{\prime \prime \prime}, \\
\tilde{\theta}_{3}^{\prime \prime \prime}=\sum e \tilde{x}_{0}^{\prime \prime \prime 2}=k \sum \frac{e}{k-e} \tilde{x}^{\prime \prime \prime \prime}
\end{array}\right.
$$

provided that analogonsly to (57) and because of (101)

$$
\text { (127) }\left\{\begin{array}{l}
\hat{h}^{2} a^{2} p^{2}-\hat{l}^{2} b^{2} q^{2}=-\frac{c}{k} \sum\left(e^{2}-k^{\prime} e\right) \tilde{x}_{0}^{\prime \prime \prime}=c \sum \frac{e^{2}-k^{\prime} e}{e-k} \tilde{x}^{\prime \prime \prime}, \\
\hat{h}_{a} a^{2} p^{2}-\hat{l} b^{2} q^{2}=-\frac{c}{k} \sum\left(e^{2}-k^{\prime} e\right) \tilde{x}_{0} \tilde{x}_{0}^{\prime \prime \prime}=c \sum \frac{e^{2}-k^{\prime} e}{e-k} \tilde{x} \tilde{x}^{\prime \prime \prime} .
\end{array}\right.
$$

When the expression (125) for $\tilde{x}^{\prime \prime \prime}$ is substituted in (126), the resulting equations are reducible by means of $(97),(101)$ and (106) to

$$
(128)\left\{\begin{aligned}
& \tilde{\theta}_{3}= \frac{2 k}{k-k^{\prime}} \sum e x^{\prime \prime \prime} \tilde{x} \\
& \tilde{\theta}_{3}^{\prime \prime \prime}=-\frac{k}{\left(k-k^{\prime}\right)^{2}}\left[\sum\left(2 e^{2}-k e-k^{\prime} e\right) x^{\prime \prime \prime}{ }^{2}\right. \\
&\left.\quad+\frac{2 l_{3}}{c p} \sum e x^{\prime \prime \prime} \frac{\partial \tilde{x}}{\partial u}+\frac{2 l_{3}}{c q} \sum e x^{\prime \prime \prime} \frac{\partial \tilde{x}}{\partial v}\right] .
\end{aligned}\right.
$$


In consequence of (118) we can write the first of (124) in the form

$$
\tilde{x}^{\prime \prime \prime}-x^{\prime \prime \prime}=\hat{h} p \frac{\partial x}{\partial \imath}+\hat{\imath} q \frac{\partial x}{\partial \imath}
$$

From this equation, (38) and (42) we obtain

$$
\sum e\left(\tilde{x}^{\prime \prime \prime}-x^{\prime \prime \prime}\right)^{2}=\frac{1}{c}\left(\hat{h}^{2} a^{2} p^{2}-\hat{l}^{2} b^{2} q^{2}\right) .
$$

In order that this equation be consistent with the first of (127), we must have

$$
\left(k-k^{\prime}\right) \sum \frac{e}{e-k} \tilde{x}^{\prime \prime \prime 2}+2 \sum e \tilde{x}^{\prime \prime \prime} x^{\prime \prime \prime}-\sum e x^{\prime \prime \prime 2}=0 .
$$

Because of (101) and (106) this condition is satisfied identically when the expression (125) for $\tilde{x}^{\prime \prime \prime}$ is substituted. Again from (129) and (88) we have

$$
\sum e\left(\tilde{x}^{\prime \prime \prime}-x^{\prime \prime \prime}\right)(\tilde{x}-x)=\frac{1}{c}\left(\hat{h} a^{2} p^{2}-i b^{2} q^{2}\right) .
$$

It is readily found that this equation is consistent with the second of (127). Moreover, when we compare the functions (128) giving an $F_{k^{\prime}}$ transform of $\hat{N}$ with the functions (119) and (123) determining a derived net of $N_{3}$, we find that they differ by the same constant factor. Hence:

Let $N$ be a permanent net on the central quadric (34) and $\bar{N}$ the applicable net; if $\hat{N}$ is a $B_{k}$ transform of $\bar{N}$ and $\overline{N_{3}}$ is an $F_{k^{\prime}}$ transform of $\bar{N}$, there can be found divectly a net $\hat{N}_{3}$ which is a $B_{k}$ transform of $\overline{N_{3}}$ and an $F_{k^{\prime}}$ transform of $\hat{N}$.

140. Theorem of permutability of transformations $B_{k}$. Let $\bar{N}$ be a net applicable to a net $N$ on the central quadric $Q$ (34), and $\hat{N}_{1}$ and $\hat{N}_{3}$ nets applicable to nets on $Q$ and obtained from $\bar{N}$ by transformations $B_{k}$ and $B_{k^{\prime}}$ respectively, determined by the respective pairs of functions $\theta_{1}, \theta_{2}$ and $\theta_{3}, \theta_{4}$, where

(130) $\theta_{1}=2 \sum e x x^{\prime}, \quad \theta_{2}=2 \sum e x x^{\prime \prime}, \quad \theta_{3}=2 \sum e x x^{\prime \prime \prime}, \quad \theta_{4}=2 \sum e_{x} x^{\prime \prime \prime \prime}$. 
From $\S 50$ it follows that the functions $\dot{\theta}_{3}$ and $\dot{\theta}_{4}$, defined by

$$
\left\{\begin{array}{c}
\hat{\theta}_{i}=\theta_{i}+\left[\frac{\partial \theta_{i}}{\partial u}\left(\theta_{j} \frac{\partial \theta_{k}}{\partial v}-\theta_{k} \frac{\partial \theta_{j}}{\partial v}\right)+\frac{\partial \theta_{i}}{\partial v}\left(\theta_{k} \frac{\partial \theta_{j}}{\partial u}-\theta_{j} \frac{\partial \theta_{k}}{\partial u}\right)\right] \\
\cdot\left(\frac{\partial \theta_{k}}{\partial u} \frac{\partial \theta_{j}}{\partial v}-\frac{\partial \theta_{k}}{\partial v} \frac{\partial \theta_{j}}{\partial u}\right)^{-1}
\end{array}\right.
$$

for $i=3,4 ; j=1, k=2$, determine a $W$ transform of $\hat{N}_{1}$ which is also the $W$ transform of $\hat{N}_{8}$ defined by the two functions given by (131) for $i=1,2 ; j=3, k=4$.

From (127) it follows that conditions analogous to (93) are satisfied. The analogue of (94) is

$$
\hat{h}_{3} \hat{h}_{4} a^{2} p^{2}-\hat{i}_{3} i_{4} b^{2} q^{2}=c \sum \frac{e^{2}-k^{\prime} e}{e-k} \tilde{x}^{\prime \prime \prime} \tilde{x}^{\prime \prime \prime \prime},
$$

which can be shown to be satisfied identically, by the use of methods similar to those used at the end of $\S 139$. Consequently the above $W$ transform of $\hat{N}_{1}$ is in fact a $B_{k^{\prime}}$ transform. Hence we have:

If $\hat{N}_{1}$ and $\hat{N}_{\underline{1}}$ are obtained from the permanent net $\bar{N}$ of " deform of a central quadric $Q$ by transformations $B_{k}$ and $B_{k^{\prime}}$ $\left(k^{\prime} \neq k\right)$, there can be found directly a net $\hat{N}_{12}$ applicable to a net on $Q$ such that $\hat{N}_{1}$ and $\hat{N}_{12}$, and $\hat{N}_{2}$ and $\hat{N}_{12}$ are in relations $B_{k^{\prime}}^{\prime}$ und $B_{l i}^{\prime}$ respertively ${ }^{137}$ ).

141. Transformations $F_{k}$ of permanents nets on a paraboloid and of surfaces applicable to a paraboloid. For the paraboloid $P$,

$$
e x^{2}+f y^{2}+2 z=0 .
$$

we have

$$
X, Y, Z=\frac{e x, f y, 1}{\sqrt{e^{2} x^{2}+f^{2} y^{2}+1}},
$$

and

$$
K=-\frac{1}{c^{4}\left(e^{2} x^{2}+f^{2} y^{2}+1\right)^{2}}, \quad \frac{1}{\sigma}=\sqrt{e^{2} x^{2}+f^{2} y^{2}+1},
$$

where $r^{4}=-1 / 4$ ef.

137) Cf., Bianchi, 1. c.. chap. 4. 
If $P$ is referred to a net $N$ whose point equation is the second of $(33)$, we have

$$
e \frac{\partial x}{\partial u} \frac{\partial x}{\partial v}+f \frac{\partial y}{\partial u} \frac{\partial y}{\partial v}=0
$$

and by processes analogous to those used in $\$ 135$ we find

(135) $e\left(\frac{\partial x}{\partial u}\right)^{2}+f\left(\frac{\partial y}{\partial u}\right)^{2}=\frac{a^{2}}{c^{2}}$

$$
e\left(\frac{\partial x}{\partial v}\right)^{2}+f^{\prime}\left(\frac{\partial y}{\partial v}\right)^{2}=-\frac{b^{2}}{c}
$$

and we prove the theorem:

$A$ necessary and sufficient condition that a net $N$ on a paraboloid (132) be permanent is that the parameters can be chosen so that

$$
e\left(\frac{\partial x}{\partial u}\right)^{2}+f\left(\frac{\partial y}{\partial u}\right)^{2}+e\left(\frac{\partial x}{\partial v}\right)^{2}+f\left(\frac{\partial y}{\partial v}\right)^{2}=e\left(e^{2} x^{2}+f^{2} y^{2}+1\right)
$$

In order to obtain $F_{k}$ transforms of $N$ which are permanent nets on $P$, we take in place of (48)

$$
\theta=2\left(e x x^{\prime}+f y y^{\prime}+z^{\prime}\right), \quad \theta^{\prime}=e x^{\prime 2}+f y^{\prime \prime} .
$$

Each set of functions satisfying the completely integrable system of equations (59) and (58) with $g=0$, and the conditions

$$
\left\{\begin{array}{l}
h^{2} a^{2}-l^{2} b^{2}=c^{2}\left[\left(e^{2}-k e\right) x^{2}+\left(f^{2}-k \cdot f\right) y^{\prime 2}\right] \\
h a^{2}-l b^{2}=c^{2}\left[\left(e^{2}-k e\right) x x^{\prime}+\left(f^{2}-k f\right) y y^{\prime}-k z^{\prime}\right]
\end{array}\right.
$$

determine a transformation $F_{k}$ of $N$ into a net $N_{1}$ on $P$. When $k=e$ or $f$ we have two special types of transformations, as described in the last theorem of $\$ 135$.

Consider now the net $\bar{N}$ applicable to $N$. From (60) and (13i) we have in place of $(62)$

$$
(e-k) x^{\prime 2}+(f-k) y^{\prime 2}-k z^{\prime 2}+k \sum \overline{x^{\prime 2}}=0 .
$$

Proceeding as in the case of (62), we find equations obtained from (64), (65), (67) and (68) by putting $g=0$. Hence there exists for transformations $F_{k}$ of $\bar{N}$ theorems similar to the first two theorems 
of $\$ 136$, but there are only two types of transformations as described in the second theorem; they are $F_{e}$ and $F_{f}$.

When $e=f, P$ is a paraboloid of revolution and the transformations $F_{k}(k \neq e)$ are described by the first theorem of $\S 136$. When $l_{i}=e$, equation (139) reduces to $\sum \bar{x}^{\prime 2}-z^{\prime 2}=0$. Hence $\overline{N^{\prime}}$ is a special net $2, O$, the complementary function being $z^{\prime}$. In this ease, as for the central quadric of revolution, the transforms of $\bar{N}$ are conjugate to the two normal congruences conjugate to $\vec{N}$. When $\vec{N}$ is applied to $P$, the lines of one of these congruences pass through the focal point of $P$ on the axis of revolution, and the lines of other congruence are normal to the tangent plane to $P$ at its vertex (cf. $\$ 100$ ). Hence:

Let $\vec{N}$ be a net applicable to a net $N$ on a paraboloid of revolution $P$; the lines joining points of $N$ to the focus of $P$ and the lines parallel to the axis of $P$ become two normal congruences, $G_{1}$ and $G_{2}$, conjugate to $\vec{N}$, when $N$ is applicd to $\bar{N}$; there can be found by two quadratures $\infty^{2}$ nets $\bar{N}_{1}$ conjugate to $G_{1}$ and $\infty^{2}$ nets $\bar{N}_{2}$ conjugate to $G_{2}$ which are applicable to $\infty^{2}$ nets $N_{1}$ and $\infty^{2}$ nets $N_{2}$ on $P$; the nets $\overline{N_{1}}$ or $\vec{N}_{2}$ can be grouped into $\infty^{1}$ families of $\infty^{1}$ nets such that their corresponding tangent planes envelope a quadric cone, and the corresponding points on $P$ lie on a conic ${ }^{138}$ ).

142. Transformations $B_{k}$ of surfaces applicable to a paraboloid. In this section we establish for surfaces applicable to $P(132)$ transformations analogous to those treated in $\$ 138$. Equations (82) and (83) hold in this case also. In place of (84) we have

(140) $\theta_{1}=2\left(e x x^{\prime}+f y y^{\prime}+z^{\prime}\right), \quad \theta_{2}=2\left(e x x^{\prime \prime}+f y y^{\prime \prime}+z^{\prime \prime}\right)$.

In place of (90) and (91) we have

and

$$
\boldsymbol{t}=4 \operatorname{efabc}\left(x^{\prime \prime} y^{\prime}-x^{\prime} y^{\prime \prime}\right)
$$

$$
\begin{aligned}
\tilde{x} & =\frac{1}{e} \frac{y^{\prime \prime} z^{\prime}-y^{\prime} z^{\prime \prime}}{x^{\prime \prime} y^{\prime}-x^{\prime} y^{\prime \prime}}, \quad \tilde{y}=\frac{1}{f} \frac{z^{\prime \prime} x^{\prime}-z^{\prime} x^{\prime \prime}}{x^{\prime \prime} y^{\prime}-x^{\prime} y^{\prime \prime}} \\
\tilde{z} & =-z+\frac{y\left(x^{\prime \prime} z^{\prime}-x^{\prime} z^{\prime \prime}\right)+x\left(y^{\prime} z^{\prime \prime}-y^{\prime \prime} z^{\prime}\right)}{x^{\prime \prime} y^{\prime}-x^{\prime} y^{\prime \prime}} .
\end{aligned}
$$

Now $e x \tilde{x}+f y \tilde{y}+z+\tilde{z}=0$.

${ }^{138}$ ) Trans. Amer. Math. Soc., l. c., p. 338. 
The first two of equations (93) with $g=0$ hold and in place of the second two we have

$$
\left\{\begin{array}{l}
h_{1} a^{2}-l_{1} b^{2}=c^{2}\left[\left(e^{2}-k e\right) x x^{\prime}+\left(f^{2}-k f\right) y y^{\prime}-k_{z^{\prime}}\right] \\
h_{2} a^{2}-l_{2} b^{2}=c^{2}\left[\left(e^{2}-k e\right) x x^{\prime \prime}+\left(f^{2}-k f\right) y y^{\prime \prime}-k_{z} z^{\prime \prime}\right]
\end{array}\right.
$$

and (94) with $g=0$ holds.

By making use of the expressions for $x\left(y^{\prime} z^{\prime \prime}-y^{\prime \prime} z^{\prime}\right)$ and $y\left(z^{\prime} x^{\prime \prime}-z^{\prime \prime} x^{\prime}\right)$ which are obtainable from (141), we find that $\dot{r}$, $\tilde{y}$ and $\tilde{z}$ satisfy the condition

$$
\frac{\tilde{x}^{2}}{\frac{1}{e}-\frac{1}{k}}+\frac{\tilde{y}^{2}}{\frac{1}{f}-\frac{1}{k}}+2 \tilde{z}+\frac{1}{k}=0,
$$

that is the net $\tilde{N}$ lies on a quadric confocal with $P$.

In place of (100) we have

$$
\frac{\partial \tilde{x}}{\partial \imath}=\frac{4 p a^{3} b f}{d}\left(y^{\prime \prime} l_{l_{1}}-y^{\prime} h_{2}\right), \quad \frac{\partial \bar{x}}{\partial v}=-\frac{4 q a b^{3} f}{I}\left(y^{\prime \prime} l_{1}-y^{\prime} l_{2}\right) .
$$

From these and analogous equations we obtain, by means of equations similar to (95),

$$
\begin{gathered}
\frac{e k}{k-e}\left(\frac{\partial \tilde{x}}{\partial u}\right)^{2}+\frac{f k}{k-f}\left(\frac{\partial \tilde{y}}{\partial u}\right)^{2}=-k a^{2} p^{2}, \\
\frac{e k}{k-e} \frac{\partial \tilde{x}}{\partial u} \frac{\partial \tilde{x}}{\partial v}+\frac{f k}{k-f} \frac{\partial \tilde{y}}{\partial u} \frac{\partial \tilde{y}}{\partial v}=0, \\
\frac{e k}{k-e}\left(\frac{\partial \tilde{x}}{\partial v}\right)^{2}+\frac{f k}{k-f}\left(\frac{\partial \tilde{y}}{\partial v}\right)^{2}=k b^{2} q^{2} .
\end{gathered}
$$

From these equations and (102) we have

$$
\begin{gathered}
\sum\left(\frac{\partial \hat{x}}{\partial u}\right)^{2}=\sum\left(\frac{\partial \tilde{x}_{0}}{\partial u}\right)^{2}, \quad \sum \frac{\partial \dot{x}}{\partial u} \frac{\partial \hat{x}}{\partial v}=\sum \frac{\partial \tilde{x}_{0}}{\partial u} \frac{\partial \tilde{x}_{0}}{\partial v} \\
\sum\left(\frac{\partial \dot{x}}{\partial v}\right)^{2}=\sum\left(\frac{\partial \tilde{x}_{0}}{\partial v}\right)^{2}
\end{gathered}
$$


Here the symbol $\Sigma$ refers to the three variables, where

$$
\tilde{x}_{0}=\sqrt{\frac{k}{k-e}} \tilde{x}, \quad \tilde{y}_{0}=\sqrt{\frac{k}{k-f}} \tilde{y}, \quad \tilde{z}_{0}=\tilde{z}+\frac{1}{2 k} .
$$

These are the equations of the transformation of Ivory for $P^{139}$ ).

The other observations for transformations of a central quadric hold also for the case of the paraboloid, and consequently we have the analogous theorem:

If $N$ is a permanent net on a paraboloid $P$, there are $\infty{ }^{1}$ pairs of transformations $F_{k}$ of $N$ into nets $N_{1}$ and $N_{2}$ so that the condition

$$
h_{1} h_{2} a^{2}-l_{1} l_{2} b^{2}=r^{2}\left[\left(e^{2}-l_{i}\right) x^{\prime} \cdot x^{\prime \prime}+\left(f^{2}-k f\right) y^{\prime} y^{\prime \prime}\right]
$$

holds; the locus of the pole $\tilde{M}$ of the plane $M M_{1} M_{2}$ with respect to $P$ is a net $\tilde{N}$ on a paraboloid confocal to $P$; as $N^{\top}$ rolls on its applicuble net $\bar{N}$ the point $\tilde{M}$ describes a net $\hat{N}$ such that $\bar{N}$ and $\hat{N}$ are the focal nets of a $W$ congruence, and $\hat{N}$ is applicable to the net on $P$ which is the Ivory transform of the net $\tilde{N}$.

These are the transformations $B_{k}$ of surfaces applicable to a paraboloid, as found by Bianchi in another manner ${ }^{140}$ ).

143. Determination of the asymptotic lines on a surface. When a surface $S$ is referred to a general system of parametric lines, the translations and rotations of a general moving trihedral satisfy the conditions [cf. $\$ 69]$

$$
(142) \begin{cases}\frac{\partial p}{\partial r}-\frac{\partial p_{1}}{\partial u}=q r_{1}-q_{1} r, & \frac{\partial \xi}{\partial v}-\frac{\partial \xi_{1}}{\partial u}=\eta r_{1}-\eta_{1} r, \\ \frac{\partial q}{\partial \imath}-\frac{\partial q_{1}}{\partial u}=r p_{1}-p r_{1}, & \frac{\partial \eta}{\partial v}-\frac{\partial \eta_{1}}{\partial u}=\xi_{1} r-\xi r_{1}, \\ \frac{\partial r}{\partial r}-\frac{\partial r_{1}}{\partial u}=p q_{1}-\mu_{1} q, & \eta_{1} p-\eta \nu_{1}+\xi q_{1}-\xi_{1} q=0 .\end{cases}
$$

The equation of asymptotic lines on $S$ is $[\S 71,(68)]$

$$
(p \eta-q \xi) d u^{2}+\left(p \eta_{1}-q \xi_{1}+p_{1} \eta-q_{1} \xi\right) d u d v+\left(p_{1} \eta_{1}-q_{1} \xi_{1}\right) d v^{2}=0 .
$$

$\left.{ }^{139}\right)$ Cf. Bianclii, 1. c., p. 30 .

140) Lezioni, vol. 3, chàp. 1. 
If we replace this equation by the two

$$
\left\{\begin{array}{l}
p d u+p_{1} d v=\lambda\left(\Xi d u+\xi_{1} d v\right) \\
q d u+q_{1} d v=\lambda\left(\eta d u+\eta_{1} d v\right)
\end{array}\right.
$$

the function $\lambda$ is determined by

$$
\lambda^{2}=-\frac{1}{\varrho_{1} \varrho_{2}}=l^{2}, \quad \lambda= \pm k,
$$

as is seen by the elimination of $d u$ and $d v$ from (143) and [s 70]. If $\alpha$ and $\beta$ are the parameters of the asymptotic lines on $S$, we must have

$$
\left\{\begin{array}{l}
p \frac{\partial u}{\partial \boldsymbol{c}}+p_{1} \frac{\partial v}{\partial \boldsymbol{\alpha}}=k\left(\xi \frac{\partial u}{\partial \boldsymbol{c}}+\xi_{1} \frac{\partial v}{\partial \boldsymbol{\alpha}}\right), \\
q \frac{\partial u}{\partial \boldsymbol{\alpha}}+q_{1} \frac{\partial v}{\partial \boldsymbol{\alpha}}=k\left(\eta \frac{\partial u}{\partial \boldsymbol{\alpha}}+\eta_{1} \frac{\partial v}{\partial \boldsymbol{c}}\right), \\
p \frac{\partial u}{\partial \boldsymbol{\beta}}+p_{1} \frac{\partial v}{\partial \boldsymbol{\beta}}=-l_{i}\left(\xi \frac{\partial u}{\partial \boldsymbol{\beta}}+\xi_{1} \frac{\partial v}{\partial \boldsymbol{\beta}}\right), \\
q \frac{\partial u}{\partial \boldsymbol{\beta}}+q_{1} \frac{\partial v}{\partial \boldsymbol{\beta}}=-k\left(\eta \frac{\partial u}{\partial \boldsymbol{\beta}}+\eta_{1} \frac{\partial v}{\partial \boldsymbol{\beta}}\right) .
\end{array}\right.
$$

If these equations are solved for $p, q, p_{1}, q_{1}$ and the results are substituted in (142), we obtain the equations which $u$ and $v$ must satisfy.

This calculation is simplified, if we note that the first of (142) is equivalent to

$$
\begin{gathered}
\frac{\partial}{\partial \boldsymbol{\beta}}\left(p \frac{\partial u}{\partial \iota}+p_{1} \frac{\partial v}{\partial \boldsymbol{\alpha}}\right)-\frac{\partial}{\partial \iota}\left(p \frac{\partial u}{\partial \boldsymbol{\beta}}+p_{1} \frac{\partial v}{\partial \boldsymbol{\beta}}\right) \\
=\left(q \frac{\partial u}{\partial \boldsymbol{\alpha}}+q_{1} \frac{\partial v}{\partial \boldsymbol{\alpha}}\right)\left(r \frac{\partial u}{\partial \boldsymbol{\beta}}+r_{1} \frac{\partial v}{\partial \boldsymbol{\beta}}\right)-\left(q \frac{\partial u}{\partial \boldsymbol{\beta}}+q_{1} \frac{\partial v}{\partial \boldsymbol{\beta}}\right)\left(r \frac{\partial u}{\partial \boldsymbol{c}}+r_{1} \frac{\partial v}{\partial \boldsymbol{\alpha}}\right) .
\end{gathered}
$$

On replacing the expressions in parentheses by their values from (144), we obtain

$$
\begin{gathered}
\xi \frac{\partial^{2} u}{\partial \alpha \partial \beta}+\xi_{1} \frac{\partial^{2} v}{\partial \alpha \partial \beta}+\frac{\partial u}{\partial \varkappa} \frac{\partial u}{\partial \beta}\left(\frac{1}{k} \frac{\partial k \xi}{\partial u}-\eta \cdot\right) \\
+\frac{\partial v}{\partial \alpha} \frac{\partial v}{\partial \beta}\left(\frac{1}{k} \frac{\partial k \xi_{1}}{\partial v}-\eta_{1} r_{1}\right) \\
+\frac{1}{2}\left(\frac{\partial u}{\partial \alpha} \frac{\partial v}{\partial \beta}+\frac{\partial u}{\partial \beta} \frac{\partial v}{\partial \alpha}\right)\left(\frac{1}{k} \frac{\partial k \xi}{\partial v}+\frac{1}{k} \frac{\partial k_{i} \xi_{1}}{\partial u}-\eta r_{1}-\eta_{1} v\right)=0 .
\end{gathered}
$$


This relation may be replaced by two equations because the translations $\xi, \xi_{1}, \eta, \eta_{1}$ are not completely determined. In fact, they are subject only to the conditions

$$
E=\xi^{2}+\eta^{2}, \quad F=\xi_{1}+\eta \eta_{1}, \quad G=\xi_{1}^{2}+\eta_{1}^{2} .
$$

It we equate $\xi$ and $\xi_{1}$ to zero successively, we obtain the following equations due to Darboux $\left.{ }^{141}\right)$ :

$$
\begin{aligned}
& \left(\frac{\partial^{2} u}{\partial \boldsymbol{\kappa} \partial \beta}+\frac{\partial u}{\partial \alpha} \frac{\partial u}{\partial \beta}\left(\frac{\partial \log k}{\partial u}+\left\{\begin{array}{c}
11 \\
1
\end{array}\right\}\right)\right. \\
& +\left(\frac{\partial u}{\partial \alpha} \frac{\partial v}{\partial \beta}+\frac{\partial u}{\partial \beta} \frac{\partial v}{\partial u}\right)\left(\frac{1}{2} \frac{\partial \log l}{\partial v}+\left\{\begin{array}{c}
12 \\
1
\end{array}\right\}\right)+\frac{\partial v}{\partial \alpha} \frac{\partial v}{\partial \beta}\left\{\begin{array}{c}
22\} \\
1
\end{array}\right\}=0, \\
& \frac{\partial^{2} v}{\partial \alpha \partial \beta}+\frac{\partial t}{\partial \alpha} \frac{\partial v}{\partial \beta}\left(\frac{\partial \log k}{\partial v}+\left\{\begin{array}{c}
22 \\
2
\end{array}\right\}\right) \\
& +\left(\frac{\partial u}{\partial \alpha} \frac{\partial v}{\partial \beta}+\frac{\partial u}{\partial \beta} \frac{\partial v}{\partial \alpha}\right)\left(\frac{1}{2} \frac{\partial \log k}{\partial u}+\left\{\begin{array}{c}
12 \\
2
\end{array}\right\}\right)+\frac{\partial u}{\partial \alpha} \frac{\partial u}{\partial \beta}\left\{\begin{array}{c}
11 \\
2\}
\end{array}\right\}=0,
\end{aligned}
$$

where the symbols $\left\{\begin{array}{c}r s \mid \\ t \mid\end{array}\right.$ are formed with respect to the linear element of $S$, $"$ and " being parametric.

Since the coefficients in (145) involve only $E, F, G$ and their derivatives, each pair of solutions enables us to transform the linear element, so that it becomes the linear element of a surface referred to its asymptotic lines; that is, the complete solution of (145) gives not only the asymptotic lines on $S$ but each family of curves which correspond to the asymptotic lines on a surface applicable to $S$. Bianchi calls these curves the virtual asymptotic lines on $S$.

From the results of [S 77] it follows that when a system of virtual asymptotic lines are known on $S$, the function $\overline{D^{\prime}}$ of the surface $\bar{S}$, applicable to $S$ and upon which the parametric lines are the asymptotic lines, ean be found by a quadrature. Hence we have:

The deformation of a surface and the determination of its virtual asymptotir lines are equivalent problems.

144. Deformations of paraboloids and central quadrics of revolution. We apply the results of the preceding section to the case when $S$ is a quadric referred to its gemerators. If $\boldsymbol{\alpha}_{0}$ and $\boldsymbol{\beta}_{0}$

i4) Leçons, vol. 3, p. 290. 
are the parameters of a family of virtual asymptotic lines on $S$, in consequence of (10), (11) and (12), equations (145) become, since $k=\sigma^{2}$.

(146) $\frac{\partial^{2} \alpha}{\partial \alpha_{0} \partial \beta_{0}}+\frac{\partial \alpha}{\partial \alpha_{0}} \frac{\partial \alpha}{\partial \beta_{0}} \frac{\partial}{\partial \varkappa} \log \sigma D_{0}=\frac{\partial^{2} \beta}{\partial \kappa_{0} \partial \beta_{0}}+\frac{\partial \beta}{\partial \alpha_{0}} \frac{\partial \beta}{\partial \beta_{0}} \frac{\partial}{\partial \beta} \log \sigma D_{0}^{\prime}=0$.

It is readily found that the first integral of these equations is

$$
\frac{\partial \iota}{\partial \boldsymbol{\iota}_{0}} \frac{\partial \boldsymbol{\beta}}{\partial \boldsymbol{\iota}_{0}}=\frac{\partial \boldsymbol{\omega}}{\partial \beta_{0}} \frac{\partial \beta}{\partial \beta_{0}}=\frac{1}{\sigma \bar{D}_{0}^{\prime}}{ }^{14 \boldsymbol{\varepsilon}} \text {. }
$$

In accordance with these equations we introduce a function $\omega$, thus

$$
\frac{\partial \boldsymbol{\alpha}}{\partial \boldsymbol{\epsilon}_{0}}=e^{\omega} \frac{\partial \beta}{\partial \beta_{0}}, \quad \frac{\partial \alpha}{\partial \beta_{0}}=e^{\omega} \frac{\partial \beta}{\partial \alpha_{0}} .
$$

Differentiating these equations with respect to $\beta_{0}$ and $\alpha_{0}$ respectively, and substituting in the first of (146), we get in consequence of (147)

(149) $\frac{\partial^{2} \beta}{\partial \beta_{0}^{2}}+\frac{\partial \omega}{\partial \beta_{0}} \frac{\partial \beta}{\partial \beta_{0}}-\frac{\partial}{\partial \varkappa} \frac{1}{\sigma D_{0}^{\prime}}=0 . \quad \frac{\partial^{2} \beta}{\partial \alpha_{0}^{2}}+\frac{\partial \omega}{\partial \alpha_{0}} \frac{\partial \beta}{\partial \alpha_{0}}-\frac{\partial}{\partial \alpha}-\frac{1}{\sigma D_{0}^{\prime}}=0$

Expressing the consistency of these equations and the second of (146), we obtain

$$
\frac{\partial^{2} \omega}{\partial \alpha_{0} \partial \beta_{0}}=e^{\omega} \frac{\partial^{2}}{\partial \alpha^{3}}\left(\frac{1}{\sigma D_{0}^{\prime}}\right)-e^{-\omega} \frac{\partial^{2}}{\partial \beta^{2}}\left(\frac{1}{\sigma D_{0}^{\prime}}\right) .
$$

For the paraboloid $e x^{2}-f y^{2}+2 z=0$, we have

$$
x=\frac{\alpha-\beta}{\sqrt{e}}, \quad y=\frac{\alpha+\beta}{\sqrt{t}}, \quad z=2 \alpha \beta .
$$

From these expressions we find

Also

$$
H_{0}^{2}=E_{0} G_{0}-F_{0}^{2}=\frac{4}{e f}\left[f(\alpha+\beta)^{2}+e(\alpha-\beta)^{2}+1\right]
$$

$$
\begin{aligned}
D_{0}^{\prime} & =\frac{4}{\sqrt{e f} H_{0}}, \quad \sigma^{2}=\frac{4}{\sqrt{e f}} \frac{1}{H_{c}^{*}} ; \\
\frac{1}{D_{0}^{\prime} \sigma} & =\frac{1}{2 \sqrt[4]{e f}}\left[f(\alpha+\beta)^{2}+e(\alpha-\beta)^{2}+1\right] .
\end{aligned}
$$

142) Servant, Bull. Soc. Math. France, vol. 29 (1901), p. 232. 
Hence $\frac{\partial^{2}}{\partial \alpha^{2}}\left(\frac{1}{\sigma D_{0}^{\prime}}\right)$ and $\frac{\partial^{2}}{\partial \beta^{2}}\left(\frac{1}{\sigma D_{0}^{\prime}}\right)$ are equal to the same constant and equation (150) is reducible to the equation of surfaces of constant curvature [§§ 118, 119]. For each solution of $(150)$ the above equations for the determination of $\alpha$ and $\beta$ as functions of $\boldsymbol{\alpha}_{0}$ and $\boldsymbol{\beta}_{0}$ are completely integrable. Hence:

The determination of surfaces applicable to a paraboloid and to a sphere, real or imaginary, are equivalent problems.

We remark that when the paraboloid is a surface of revolution $(-e=f)$, equation (150) can be integrated directly.

In like manner for the central quadric

we have

$$
e x^{2}+f y^{2}-g z^{2}=1
$$

$$
\begin{gathered}
x=\frac{1}{\sqrt{e}} \frac{1+\alpha \beta}{\alpha+\beta}, \quad y=\frac{1}{\sqrt{f}} \frac{\alpha-\beta}{\alpha+\beta}, \quad z=\frac{1}{\sqrt{g}} \frac{1-\alpha \beta}{\alpha+\beta}, \\
H_{1}^{2}=4 \frac{g(1-\alpha \beta)^{2}+e(1+\alpha \beta)^{2}+f(\alpha-\beta)^{2}}{e f g(\alpha+\beta)^{6}}, \\
D_{0}^{\prime}=-\frac{4}{(\alpha+\beta)^{4} \sqrt{e f g} H_{0}}, \quad \sigma^{2}=\frac{4}{\sqrt{e f g}(\alpha+\beta)^{4} H_{0}^{2}}, \\
\frac{1}{D_{0}^{\prime} \sigma}=\frac{-1}{2 \sqrt[4]{e f g}}\left[g(1-\alpha \beta)^{2}+e(1+\alpha \beta)^{2}+f(\alpha-\beta)^{2}\right] .
\end{gathered}
$$

When $g=-e$, the coefficients of $e^{\omega}$ and $e^{-\omega}$ in $(150)$ are equal to the same constant and we have:

The determination of surfaces applicable to a central quadric of revolution and to the sphere, real or imaginary, are equivalent problems.

I45. Surfaces conjugate in deformation. Following Bianchi ${ }^{143}$ ), we say that two non-developable surfaces, $S$ and $\overline{S \text {, are }}$ conjugate in deformation, when the asymptotic lines correspond on $S$ and $\bar{S}$ and to every system of virtual asymptotic lines on $S$

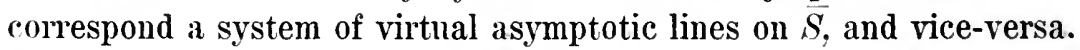
In order that the latter condition be satisfied, the coefficients of the equations (145) must be equal to the corresponding coefficients of the similar equations for $\overline{S .}$ These conditions may be written

1+3) Lezioni, vol. 3, p. 201. 
(151)

$$
\left\{\begin{array}{l}
\frac{\partial \log \lambda}{\partial u}=2\left[\left\{\begin{array}{c}
12 \\
2
\end{array}\right\}-\left\{\begin{array}{c}
|\overline{12}| \\
|2|
\end{array}\right]=\left\{\begin{array}{c}
11\}-|\overline{11}| \\
1\}
\end{array}\right\}\right. \\
\frac{\partial \log \lambda}{\partial v}=2\left[\left\{\begin{array}{c}
12 \\
1
\end{array}\right\}-\left\{\begin{array}{c}
\mid \overline{12}\} \\
\mid 1
\end{array}\right\}=\left\{\begin{array}{c}
22\} \\
2
\end{array}\right\}-\left\{\begin{array}{c}
22 \mid \\
2
\end{array}\right\}\right.
\end{array}\right.
$$

$$
\left\{\begin{array}{c}
11\} \\
2\}
\end{array}\right\}\left\{\begin{array}{c}
111 \\
2
\end{array}\right\}, \quad\left\{\overline{22} \mid=\left\{\begin{array}{c}
22 \mid \\
11\}
\end{array},\right.\right.
$$

where the symbols $\left\{\begin{array}{c}\overline{r s} \\ t\end{array}\right\}$ are formed with respect to the linear element of $\bar{S}$, and

$$
i=\frac{\bar{k}}{k} \text {. }
$$

From $[\S 85]$ we have that the equation of the geodesic lines on $S$ is

$$
\left\{\begin{array}{c}
\frac{d^{2} v}{d u^{2}}-\left\{\begin{array}{c}
22\} \\
1
\end{array}\right\}\left(\frac{d v}{d u}\right)^{3}+\left(\left\{\begin{array}{c}
22\} \\
2\}
\end{array}-2\{12\}\right)\left(\frac{d v}{d u}\right)^{2}\right. \\
+\left(2\left\{\begin{array}{c}
12\} \\
2
\end{array}\right\}-\left\{\begin{array}{c}
11 \\
1
\end{array}\right\}\right) \frac{d v}{d u}+\left\{\begin{array}{c}
11\} \\
2
\end{array}\right\}=0 .
\end{array}\right.
$$

Hence from (151) and (152) it follows that the geodesics on $S$ and $\bar{S}$ correspond.

Suppose, conversely, that the geodesics on two surfaces $S$ and $\bar{S}$ correspond and also the asymptotic lines. Then from (154) we have (152) and

(155) $\left\{\begin{array}{c}22 \\ 2\end{array}\right\}-2\left\{\begin{array}{c}\overline{12} \mid \\ 1\end{array}\right\}=\left\{\begin{array}{c}22 \\ \mid 2\end{array}\right\}-2\left\{\begin{array}{c}12\} \\ 1\}\end{array}, \quad\left\{\overline{11\}}-2\left\{\begin{array}{c}12 \\ 2\end{array}\right\}=\left\{\begin{array}{c}11\} \\ \mid 1\}\end{array}\right\}-2\left\{\begin{array}{c}12\} \\ \mid 2\end{array}\right\}\right.\right.$, and also

$$
\frac{\bar{D}}{\bar{H}}=\lambda \frac{D}{H}, \quad \frac{\bar{D}^{\prime}}{\bar{H}}=\lambda \cdot \frac{D^{\prime}}{H}, \quad \frac{\bar{D}^{\prime \prime}}{\bar{H}}=\lambda \frac{D^{\prime \prime}}{H},
$$

where $\lambda$ has the value (153). The Codazzi equations for $S$ are [\$ 64$]$

$$
\begin{aligned}
& \frac{\partial}{\partial v}\left(\frac{D}{H}\right)-\frac{\partial}{\partial u}\left(\frac{D^{\prime}}{H}\right)+\left\{\begin{array}{c}
22 \\
2
\end{array}\right\} \frac{D}{H}-2\left\{\begin{array}{c}
12 \\
2
\end{array}\right\} \frac{D^{\prime}}{H}+\left\{\begin{array}{c}
11\} \\
2
\end{array}\right\} \frac{D^{\prime \prime}}{H}=0, \\
& \frac{\partial}{\partial u}\left(\frac{D^{\prime \prime}}{H}\right)-\frac{\partial}{\partial v}\left(\frac{D^{\prime}}{H}\right)+\left\{\begin{array}{c}
22\} \\
1
\end{array}\right\} \frac{D}{H}-2\left\{\begin{array}{c}
12 \\
1
\end{array}\right\} \frac{D^{\prime}}{H}+\left\{\begin{array}{c}
11 \\
1
\end{array}\right\} \frac{D^{\prime \prime}}{H}=0 .
\end{aligned}
$$


and similar equations for $\bar{S}$. Substituting in the latter from (156), we find

$$
\frac{\partial \log \lambda}{\partial u}=\left\{\begin{array}{c}
11 \\
1
\end{array}\right\}-\left\{\overline{11} \mid, \quad \frac{\partial \log \lambda}{\partial v}=\left\{\begin{array}{c}
22 \\
2\}
\end{array}\right\}-\left\{\begin{array}{c}
22 \\
2
\end{array}\right\}\right.
$$

on condition that $S$ is not developable. Equations (151) are satisfied and we have the theorem of Servant ${ }^{144}$ ) and Bianchi ${ }^{145}$ ):

A necessary and sufficient condition that two non-elevelopable sufaces be conjugate in deformation is thut the geolesic lines and the asymptotic lines respectively correspond on the surfaces.

When two surfaces $S$ and $\bar{S}$ are conjugate in deformation, each set of solutions of equations (145) leads to a pair of surfaces $S^{\prime}$ and $\overline{S^{\prime}}$, applicable to $S$ and $\bar{S}$ respectively, upon which the asymptotic lines correspond, and since conditions (151) and (152) hold for these surfaces, they too are conjugate in deformation. Hence:

If two surfuces are conjugate in deformation. each set of solutions "f the corresponding equations (145) determine two other surfaces conjugate in deformation and applicable to the respective given surfaces.

Dini ${ }^{146}$ ) solved the problem of finding pairs of surfaces upon which the geodesics correspond. We reproduce his results.

We assume that the surfaces are referred to their common orthogonal system. Then equations (155) can be integrated, thus

$$
\frac{E}{G^{2}}=\frac{\bar{E}}{\overline{G^{2}}} \frac{1}{V^{3}}, \quad \frac{G}{E^{2}}=\frac{\bar{G}}{\overline{E^{2}}} \frac{1}{U^{3}},
$$

where $U$ and $V$ are arbitrary functions of $u$ and $r$ respectively. From these we have

$$
\bar{E}=\frac{E}{V U^{2}}, \quad \bar{G}=\frac{G}{U V^{2}} .
$$

By the substitution of the above values of $\bar{E}$ and $G$ in (152), we get

$$
\frac{\partial E}{\partial r}(U-V)=-V^{\prime} E, \quad \frac{\partial G}{\partial u}(U-V)=U^{\prime} G .
$$

34) Colnptes Rendus, vol. 136 (1903), p. 1239.

14j) I. c., p. 206.

146) Annali, ser. 3, vol. 3 (1869), p. 269. 
Neglecting the case where $U=V=$ const., that is when $S$ and $\bar{S}$ are homothetic, we have on integration

$$
E=U_{1}^{2}(U-V), \quad G=V_{1}^{2}(U-V)
$$

where $U_{1}$ and $V_{1}$ are arbitrary functions of $u$ and $v$ respectively.

If we choose the parameters so that

$$
U=u+h, \quad V=r+h .
$$

where $h$ is a constant, we find that the two linear elements are

$$
\left\{\begin{array}{l}
d s^{2}=(u-v)\left(U_{1}^{2} d u^{2}+V_{1}^{2} d v^{2}\right), \\
d s^{2}=\left(\frac{1}{v+h}-\frac{1}{u+h}\right)\left(\frac{U_{1}^{2} d u^{2}}{u+h}+\frac{V_{1}^{2} d v^{2}}{v+h}\right),
\end{array}\right.
$$

both of the Liouville form [cf. \$ 93]. Since $h$ is arbitrary, there are $\infty^{1}$ surfaces $\bar{S}$ corresponding to $S$ with geodesics in correspondence.

We inquire under what condition two such surfaces are conjugate in deformation ${ }^{147}$ ). We exclude the case where either $U$ or $V$ is constant, that is when $S$ is applicable to a surface of revolution (cf. X, Ex. 6). Substituting the valnes from (158) in (151), we find

$$
\frac{\overline{h^{2}}}{l^{2}}=\frac{1}{a}(u+h)^{2}(v+h)^{2},
$$

where $a$ is a constant. From $[\$ 64,(12)]$ we have

$$
k^{2}=\frac{1}{\sqrt{E G}}\left[\frac{\partial}{\partial u}\left(\frac{1}{\sqrt{E}} \frac{\partial \sqrt{G}}{\partial u}\right)+\frac{\partial}{\partial \imath}\left(\frac{1}{\sqrt{G}} \frac{\partial \sqrt{E}}{\partial v}\right)\right],
$$

and similarly for $\overline{k^{2}}$. Calculating the expressions for $k$ and $\bar{k}$, and substituting in the above equation, the resulting equation is reducible to, on dropping the subscripts of $U_{1}$ and $V_{1}$,

(159)

$$
\left\{\begin{array}{c}
\left(\frac{1}{u+h}-\frac{1}{a}\right) \frac{1}{U^{2}}\left(\frac{U^{\prime}}{U}+\frac{1}{u-v}\right)+\frac{1}{2(u+h)^{2}} \frac{1}{U^{2}} \\
-\left(\frac{1}{v+h}-\frac{1}{a}\right) \frac{1}{V^{2}}\left(\frac{V^{\prime}}{V}-\frac{1}{u-v}\right)-\frac{1}{2(v+h)^{2}} \frac{1}{V^{2}}=0 .
\end{array}\right.
$$

147) Cf. Serrant, l. c.; also Bianchi, Rend. dei Lincei, ser. 5, vol. 11 (1902), p. 265 . 
Differentiating with respect to $u$ and $v$, we find ultimately that $U$ and $V$ must be such that

$$
\left\{\begin{array}{l}
\left(\frac{1}{u+h}-\frac{1}{a}\right) \frac{1}{U^{2}}=a u^{2}+2 \beta u+\gamma \\
\left(\frac{1}{r+h}-\frac{1}{a}\right) \frac{1}{V^{2}}=-\left(\alpha v^{2}+2 \beta v+\gamma\right)
\end{array}\right.
$$

where $\alpha, \beta$ and $\gamma$ are constants which are arbitrary, since these expressions satisfy (159).

When $\alpha \neq 0$ in (160), the functions $U^{2}$ and $V^{2}$ are of the form

$$
\left\{\begin{array}{l}
U^{2}=\frac{A u}{(u+a)(u+a-b)(u+a-c)}, \\
V^{2}=\frac{-A v}{(v+a)(v+a-b)(v+a-c)},
\end{array}\right.
$$

where $A, b$ and $c$ are constants different from zero, and the linear element of $S$ is

$$
\left\{\begin{aligned}
d s^{2}= & A(u-v)\left[\frac{u d u^{2}}{(u+a)(u+a-b)(u+a-c)}\right. \\
& \left.-\frac{v d v^{2}}{(v+a)(v+a-b)(v+a-c)}\right]
\end{aligned}\right.
$$

When the expressions (161) are substituted in (160), we find that $h=a$. If we put

$$
u_{1}+\frac{1}{a}=\frac{1}{u+a}, \quad v_{1}+\frac{1}{a}=\frac{1}{v+a},
$$

the linear element of $\bar{S}$ is reducible to

$$
\begin{aligned}
{d \varepsilon^{2}}^{2} & =\frac{d a}{b c}\left(u_{1}-v_{1}\right)\left[\frac{u_{1} d u_{1}^{2}}{\left(u_{1}+\frac{1}{a}\right)\left(u_{1}+\frac{1}{a}-\frac{1}{b}\right)\left(u_{1}+\frac{1}{a}-\frac{1}{c}\right)}\right. \\
& \left.-\frac{v_{1} d v_{1}^{2}}{\left(v_{1}+\frac{1}{a}\right)\left(v_{1}+\frac{1}{a}-\frac{1}{b}\right)\left(v_{1}+\frac{1}{a}-\frac{1}{c}\right)}\right]
\end{aligned}
$$


Comparing (162) and (164) with $[\$ 96,(14)]$ and taking $A=-\frac{1}{4}$. we note that $S$ and $\bar{S}$ are applicable to the quadrics $Q$ and $\bar{Q}$ whose respective equations are

$$
\left\{\begin{array}{l}
\frac{x^{2}}{a}+\frac{y^{2}}{a-b}+\frac{z^{2}}{a-c}=1 \\
x^{2}+\frac{b \bar{y}^{2}}{b-a}+\frac{c z^{2}}{c-a}=\frac{b c}{a^{2}} .
\end{array}\right.
$$

From these equations it follows that when $Q$ is an ellipsoid, $\bar{Q}$ is an hyperboloid of two sheets, and vice-versa; when $Q$ is a hyperboloid of one sheet, so also is $\bar{Q}$. Moreover, if $Q$ is a surface of revolution $(b=c)$, so also is $\bar{Q}$.

We remark that $Q$ and $\bar{Q}$ are transformable into one another by the projectivity

$$
\bar{x}=\sqrt{\frac{b c}{a}} \frac{1}{x}, \quad \bar{y}=\sqrt{\frac{c}{a}} \frac{y}{x} . \quad \bar{z}=\sqrt{\frac{b}{a}} \frac{z}{x} .
$$

If in (161) we take $c=0$, and proceed as in the general case, we obtain (162) with $c=0$ and in place of (164) we have

$$
d \bar{s}^{2}=\left(v_{1}-u_{1}\right) \frac{A a}{b}\left[\frac{u_{1} d u_{1}^{2}}{\left(u_{1}+\frac{1}{a}\right)\left(u_{1}+\frac{1}{a}-\frac{1}{b}\right)}-\frac{v_{1} d v_{1}^{2}}{\left(u_{1}+\frac{1}{a}\right)\left(u_{1}+\frac{1}{a}-\frac{1}{b}\right)}\right] .
$$

Hence, as follows from $[\$ 97,(27)]$, the quadric $\bar{Q}$ is a paraboloid.

If in (160) we take $\alpha=0$, we note that $U^{2}$ is of the form $A u /(u+a)(u+a-b)$ that is $Q$ is a paraboloid. Proceeding as above, we find that $\bar{Q}$ is a central quadric of revolution. These results show, as in $\$ 144$, that the deformations of paraboloids and central quadrics of revolution are equivalent problems.

The foregoing results lead to the theorem of Servant:

Aside from surfaces applicable to a surface of revolution, the only surfaces admitting surfaces conjugate in deformation are surfuces applicable to a quadric, and any such surface has this property.

146. Transformations $H$ of surfaces applicable to a quadric. Let $Q$ and $\bar{Q}$ be two quadrics conjugate in deformation. If a surface $S$ applicable to $Q$ is known, we have a set of virtual 
asymptotic lines on $Q$, that is a solution of equations (145), and consequently a deform $\bar{S}$ of $\bar{Q}$ is thereby determined intrinsically. Bianchi ${ }^{148}$ ) calls the relation between $S$ and $\vec{S}$ a transformation $H$.

Suppose that $S$ is a ruled deform of $Q$. Since the transformation $H$ of $S$ into $\bar{S}$ preserves geodesics and asymptotic lines it follows that $\bar{S}$ also is ruled. Hence:

The transformation $H$ changes every ruled deform of $Q$ into a ruled deform of $\bar{Q}$.

Since asymptotic lines correspond on $S$ and $\overline{S,}$ to each conjugate system on $S$ corresponds a conjugate system on $\bar{S}$; the same is true for $Q$ and $\bar{Q}$. Hence to the conjugate system on $S$ permanent in its deformation from $Q$ corresponds the permanent conjugate system on $\bar{S}$. Therefore:

The transformation $H$ changes the permanent net on $S$ into the permanent net on $\bar{S}$.

Let $N$ and $\bar{N}$ denote these permanent nets on $S$ and $\bar{S}$, and $N_{0}$ and $\bar{N}_{0}$ the nets on $Q$ and $\bar{Q}$ to which they are applicable. Let $N_{01}$ denote an $F_{k}$ transform of $N_{0}$, and $N_{1}$ the corresponding $F_{k}$ transform of $N$ in accordance with the results of $\S \S 136,141$. Since $N_{01}$ admits the applicable net $N_{1}$, the net $\bar{N}_{01}$ on $\bar{Q}$ corresponding to $N_{01}$ admits an applicable net $\bar{N}_{1}$ conjugate in deformation to $N_{1}$. But $\bar{N}_{01}$ is obtained from $N_{01}$ by a projectivity, so that $\overline{N_{01}}$ is an $F_{k}$ transform of $\overline{N_{0}}$, since any transformation $F$ is transformed into a transformation $F$ by a projectivity. Consequently $\bar{N}_{1}$, applicable to $\bar{N}_{01}$, can be so placed in space that it is an $\mathrm{F}_{k}$ transform of $\bar{N}$. Hence:

If $N$ and $\bar{N}$ are nets in relation $H$, and $N_{1}$ is an $F_{k}$ transform of $N$, there exists a net $\bar{N}_{1}$ which is an $F_{k}$ transform of $\bar{N}$ and an $H$ transform of $N_{1}$.

Thus we have established the permutability of the transformations $F_{k}$ and $H$ (cf. X, Ex. 12).

147. Isothermal-conjugate nets on a quadric. From (12) it follows that the coordinates of a quadric $Q$ referred to its asymptotic lines satisfy the two equations

(167) $\frac{\partial^{2} \theta}{\partial \alpha^{2}}=2 \frac{\partial}{\partial \alpha} \log a \frac{\partial \theta}{\partial \alpha}, \quad \frac{\partial^{2} \theta}{\partial \beta^{2}}=2 \frac{\partial}{\partial \beta} \log a \frac{\partial \theta}{\partial \beta}$,

145) Lezioni, vol. 3 , p. 214. 
where $a^{2}=H_{0} \sigma$. If we put

$$
u=a+\beta, \quad v=\alpha-\beta,
$$

the parametric lines on $Q$ form an isothermal-conjugate system, and every system of this kind is obtained by replacing $\alpha$ and $\beta$ by arbitrary functions of $\alpha$ and $\beta$ respectively.

In terms of $u$ and $v$ equations (167) are equivalent to

$$
\left\{\begin{aligned}
\frac{\partial^{2} \theta}{\partial u^{2}}+\frac{\partial^{2} \theta}{\partial v^{2}} & =2 \frac{\partial}{\partial u} \log a \frac{\partial \theta}{\partial u}+2 \frac{\partial}{\partial v} \log a \frac{\partial \theta}{\partial v}, \\
\frac{\partial^{2} \theta}{\partial u \partial v} & =\frac{\partial \log a}{\partial v} \frac{\partial \theta}{\partial u}+\frac{\partial \log a}{\partial u} \frac{\partial \theta}{\partial v} .
\end{aligned}\right.
$$

These equations are of the form (IV, 73). Hence every isothermalconjugate net on a quadric is a net $R$ with equal point invariants.

When we apply to (169) the conditions (IV, 22) that (169) admit three independent solutions, we find that $a^{2}$ must satisfy the equation of Liouville

$$
\frac{\partial^{2} \log \varphi}{\partial u^{2}}-\frac{\partial^{2} \log \varphi}{\partial v^{2}}=k \varphi
$$

where $k$ is a constant. The general solution of this equation is known [§ 151]. When a solution is substituted in (169), the resulting equations are reducible to (167) by (168). Since equations (169) are the most general of the form (IV, 73) for which $a=b$, we have:

Every isothermal-conjugate net on a quadric is a net $R$ with equal point invariants, and these are the only nets $R$ with equal point invariants.

When a quadric is transformed into a sphere by a projective transformation, each net with equal point invariants of the quadric becomes an isothermal-orthogonal net on the sphere; this net is also isothermal-conjugate. Since an isothermal-conjugate system is transformed by a projectivity into an isothermal-conjugate system [\$§ 82, 84], we have the converse theorem:

Every net with equal point invariants on a quadric is isothermalconjugate. 
From the first theorem of $\$ 60$ and the preceding considerations we have the theorem:

Every isothermal-conjugate system on a quadric has equal tangential invariants; and every net on a quadric with equal tangential inrariants is isothermal-conjugate and has equal point invariants.

In $\$ 88$ we saw that an isothermal net on a sphere admits $\infty^{3}$ trausformations $F$ into nets of the same kind on the sphere. Hence the same is true of nets with equal point invariants on any quadric, in view of the above remarks (cf. $\S 148$ ).

148. Transformations $F$ and $W$ of isothermal-conjugate nets on a central quadric. Let $N$ be an isothermal-conjugate net on the central quadric $Q$ (34). An $F$ transform $N_{1}$ also on $Q$ is given by (47) and (48). From (II, 81) and (169) we have that $N_{1}$ is a $K$ transform, that is $N_{1}$ has equal point invariants and is isothermal-conjugate, if

$$
l=\frac{k \theta}{4 a^{2}}+\frac{\theta^{\prime}}{\theta}, \quad l=-\frac{k \theta}{4 a^{2}}+\frac{\theta^{\prime}}{\theta},
$$

where $k$ is a constant. In consequence of (48) these equations are equivalent to

$$
a^{2}(h-l)-k \sum e x x^{\prime}=0, \quad a^{2}\left(h^{2}-l^{2}\right)-k \sum e x^{\prime 2}=0 .
$$

Differentiating these equations and making use of (59), we have

$$
\left\{\begin{array}{l}
\frac{\partial h}{\partial u}=\frac{k}{a^{2}} \sum e x^{\prime} \frac{\partial x}{\partial \imath}+(l-h) \frac{\partial}{\partial u} \log a, \\
\frac{\partial l}{\partial \imath}=-\frac{k}{a^{2}} \sum e x^{\prime} \frac{\partial x}{\partial \imath}+(h-l) \frac{\partial}{\partial v} \log a .
\end{array}\right.
$$

These equations and (59) form a completely integrable system. For each set of solutions of these equations the left-hand members of (171) are constants. Since all of the equations are homogeneous, there are $\infty^{2}$ sets of solutions satisfying (171) for each value of $k$. Then the conditions (170) are satisfied and we have the theorem:

An isothermal-conjugate net on a central quadric admits $\infty^{3}$ transformations $K$ into isothermal-conjugate nets on the quadric ${ }^{\mathbf{1 4 9}}$ ).

$\left.{ }^{149}\right)$ Cf. 'Tzitzeica, Bull. Sciences Math., ser. 2, vol. 36 (1912), pp. 151-164. 
From equations (169) and (31) we have the following expressions for the Christoffel symbols formed with respect to the linear element (26) of the net $N$ :

$$
\left\{\begin{array}{lll}
\left\{\begin{array}{c}
11 \\
1
\end{array}\right\}=\frac{\partial}{\partial u} \log \frac{H}{a}, & \left\{\begin{array}{c}
12 \\
1
\end{array}\right\}=\frac{\partial \log a}{\partial v}, & \left\{\begin{array}{c}
22 \\
1
\end{array}\right\}=\frac{\partial}{\partial u} \log \frac{a^{3}}{H}, \\
\left\{\begin{array}{c}
11 \\
2
\end{array}\right\}=\frac{\partial}{\partial v} \log \frac{a^{3}}{H}, & \left\{\begin{array}{c}
12\} \\
2
\end{array}\right\}=\frac{\partial \log a}{\partial u}, & \left\{\begin{array}{c}
22 \\
2
\end{array}\right\}=\frac{\partial}{\partial v} \log \frac{H}{a} .
\end{array}\right.
$$

The Codazzi equations for $N$ call be written as the second set of (30); then we have by integration $D=-D^{\prime \prime}=-a^{4} / H$, since $a$ is determined by (169) only to within a constant factor. Hence if $\sigma^{4}=-K$, we have

$$
H \sigma=a^{2}, \quad D=-D^{\prime \prime}=-\sigma a^{2} .
$$

In consequence of (173) and (174) we show by methods similar to those used in $\S 135$ that

(175) $\sum e\left(\frac{\partial x}{\partial u}\right)^{2}=\frac{a^{2}}{c}, \quad \sum e \frac{\partial x}{\partial u} \frac{\partial x}{\partial v}=0, \quad \sum e\left(\frac{\partial x}{\partial v}\right)^{2}=-\frac{a^{2}}{c}$,

where $c^{4}=-1 / e f g$.

Suppose now that we have two sets of solutions of (59) and (172), namely $x^{\prime}, y^{\prime}, z^{\prime}, h_{1}, l_{1} ; x^{\prime \prime}, y^{\prime \prime}, z^{\prime \prime}, h_{2}, l_{2}$, satisfying the collditions

$$
\begin{cases}a^{2}\left(h_{1}-l_{1}\right)-k \sum e x x^{\prime}=0, & a^{2}\left(h_{1}^{2}-l_{1}^{2}\right)-k \sum e x^{\prime 2}=0 \\ a^{2}\left(h_{2}-l_{2}\right)-k \sum e x x^{\prime \prime}=0, & a^{2}\left(h_{2}^{2}-l_{2}^{2}\right)-k \sum e x^{\prime 2}=0\end{cases}
$$

and also

$$
a^{2}\left(h_{1} h_{2}-l_{1} l_{2}\right)-k \sum e x^{\prime} x^{\prime \prime}=0 .
$$

By means of (173), (174), (175) and (176) we show as in $\S 138$ that the functions

$$
\theta_{1}=2 \sum e x x^{\prime}, \quad \theta_{2}=2 \sum e x x^{\prime \prime}
$$

are solutions of the equation

$$
\frac{\partial^{2} \theta}{\partial u^{2}}+\frac{\partial^{2} \theta}{\partial v^{2}}=2 \frac{\partial \log a}{\partial u} \frac{\partial \theta}{\partial u}+2 \frac{\partial \log a}{\partial v} \frac{\partial \theta}{\partial v}+\frac{k}{c} \theta,
$$


and the second of (169). Consequently (§ 49) $\theta_{1}$ and $\theta_{2}$ determine a $W$ transform $\tilde{N}$ of $N$. Its coordinates $\tilde{x}, \tilde{y}, \tilde{z}$ are given by (88), which in turn are reducible to (91).

From (176) and (177) we have

$$
\begin{aligned}
\sum f^{\prime} g\left(y^{\prime \prime} z^{\prime}-y^{\prime} z^{\prime \prime}\right)^{2}=\sum e x^{\prime 2} \cdot \sum e x^{\prime \prime 2}-\left(\sum e x^{\prime} x^{\prime \prime}\right)^{2} & =-\frac{a^{4}}{k^{2}}\left(h_{1} l_{2}-h_{\mathbf{2}} l_{1}\right)^{2} \\
\operatorname{efg}\left[\sum x\left(y^{\prime \prime} z^{\prime}-y^{\prime} z^{\prime \prime}\right)\right]^{2} & =-\frac{a^{4}}{k^{2}}\left(h_{1} l_{2}-h_{2} l_{1}\right)^{2}
\end{aligned}
$$

In consequence of these identities we have that $\tilde{N}$ lies on the given quadric (34). Hence the congruence of lines joining corresponding points on $N$ and $\tilde{N}$ degenerates into one set of generators of the quadric.

$$
\begin{aligned}
& \text { From (34), (92), (88); (175) and } \sum e \tilde{x}^{2}=1 \text {, we have } \\
& \frac{1}{c}\left(p^{2} a^{2}-q^{2} b^{2}\right)=\sum e\left(p \frac{\partial x}{\partial u}+q \frac{\partial x}{\partial v}\right)^{2}=\sum e(\tilde{x}-x)^{2}=0 .
\end{aligned}
$$

Hence (cf.IV, 75) $\tilde{N}$ is a net with equal point invariants.

As at the close of $\S 138$, we remark that there are only two sets of solutions $x^{\prime \prime}, y^{\prime \prime}, z^{\prime \prime}, h_{2}, l_{2} ; x^{\prime \prime \prime}, y^{\prime \prime \prime}, z^{\prime \prime \prime}, h_{3}, l_{3}$ each of which gives with $x^{\prime}, y^{\prime}, z^{\prime}, h_{1}, l_{1}$, a net $\tilde{N}$, say nets $\tilde{N}_{2}$ and $\tilde{N}_{3}$. These nets are conjugate to the congruence of the lines of intersection of the tangents planes to $N$ and its $F$ transform $N_{1}$ by means of $x^{\prime}, y^{\prime}, z^{\prime}, h_{1}, l_{1}$; and their points are the intersections of the lines of this congruence with the generators of the quadric. Accordingly we have the theorem:

If $N$ and $N_{1}$ are two isothermal-conjugate nets on a central quadric in relation $F$, the points in which the quadric is met by the intersections of corresponding tangent planes of $N$ and $N_{1}$ generate. two isothermal-conjugate nets in relation $F^{150}$ ).

\section{Exercises}

1. A necessary and sufficient condition that $\bar{N}$ whose coordinate 5 satisfy (33) lie on a ruled deform of $Q$ is that $a / b$ be a function of $u+v$ or $u-v$.

2. A necessary and sufficient condition that the nets permanent in deformation on every surface applicable to a surface $S$ be isothermal-conjugate is that $S$ be a quadric.

Terracini, Annali, vol. 30 (1921), p. 145.

150) Tzitzeica, 1. c., has given a geometrical proof of this theorem. 
3. When $k^{\prime}=k$ in $\$ 139$, the solutions of (106) and (110) are

$$
\begin{gathered}
h_{3}=\alpha h_{1}+\beta h_{2}, \quad l_{3}=\alpha l_{1}+\beta l_{2}, \\
x^{\prime \prime \prime}=\alpha x^{\prime}+\beta x^{\prime \prime}, \quad y^{\prime \prime \prime}=\alpha y^{\prime}+\beta y^{\prime \prime}, \quad z^{\prime \prime \prime}=\alpha z^{\prime}+\beta z^{\prime \prime} .
\end{gathered}
$$

In this case the transforms $\hat{N}$ and $\hat{N}_{3}$ coincide.

4. Show that the transformations $F_{k}$ and $B_{k}$ of a deform of a paraboloid are permutable.

5. If $\hat{N}_{1}$ and $\hat{N}_{2}$ are obtained from the pernanent net of a deform of a paraboloid $P$ by transformations $B_{k}$ and $B_{k^{\prime}}\left(k^{\prime} \neq k\right)$, there can be found directly a net $\hat{N}_{12}$ applicable to a net on $P$, such that $\hat{N}_{1}$ and $\hat{N}_{12}$, and $\hat{N}_{2}$ and $\hat{N}_{12}$ are in relations $B_{k^{\prime}}^{\prime}$ and $B_{k}^{\prime}$ respectively. Bianchi, Lezioni, vol. 3, chap. 4.

6. The surfaces of revolution admitting a conjugate in deformation have the linear element

$$
d s^{2}=\frac{h\left[1-c^{2}\left(a r^{2}+1\right)\right]}{a r^{2}+1} d r^{2}+r^{2} d r^{2},
$$

where $a, c$ and $h$ are arbitrary constants. The linear element of the conjugate surface is

$$
d \bar{s}^{2}=\frac{h\left[1-c^{2}\left(a r^{2}+1\right)\right]}{\left(a r^{2}+1\right)^{3}} d r^{2}+\frac{r^{2}}{a r^{2}+1} d r^{2} .
$$

Bianchi, Rend. dei Lincei, ser. 5, vol. 11 (1902), p. $2 \pi 2$.

7. If two surfaces applicable to a surface of revolution are conjugate in deformation, so also are their complementary surfaces [\$76]. Bianchi, l. c., p. 273.

8. If two quadrics, $Q$ and $\bar{Q}$, correspond in a projectivity which transforms the quadries confocal with $Q$ into the quadrics confocal with $\bar{Q}$, then $Q$ and $\bar{Q}$ are conjugate in deformation.

Bianchi, Lezioni, vol. 3, p. 208.

9. If $S$ and $\bar{S}$ are conjugate in deformation and $S$ is deformed so that one of its asymptotic lines remains rigid, then $\bar{S}$ admits a deformation in which the corresponding asymptotic line is rigid. Bianchi, l. c., p. 215.

10. If at points of two corresponding asymptotic lines on two surfaces conjugate in deformation tangents are drawn to the geodesics which are the deforms of generators of the applicable quadrics, $Q$ and $\underline{Q}$, the two ruled surfaces formed by these tangents are applicable to $Q$ and $\bar{Q}$, and are conjugate in deformation.

Bianchi, l. c., p. 215.

11. When the first of equations (165) is written in the form (34) and the second $\bar{e} \bar{x}^{2}+\bar{f} \bar{y}^{2}+\bar{g} \bar{z}^{2}=1$, the equations of the projectivity (166) are

also

$$
\bar{x}=\frac{1}{\sqrt{e \bar{e}}} \frac{1}{x}, \quad \bar{y}=\sqrt{\frac{-f}{e \bar{f}}} \frac{y}{x}, \quad \bar{z}=\sqrt{\frac{-g}{e \bar{g}}} \frac{z}{x}:
$$

$$
\bar{c}^{4}=-1 / \bar{e} \bar{f} \bar{g}=\frac{e^{2}}{\bar{e}^{2}} c^{4}
$$

and the point equation of a net on $\bar{Q}$ is

$$
\frac{\partial^{2} \theta}{\partial u \partial v}=\frac{\partial}{\partial v} \log \frac{a}{x} \frac{\partial \theta}{\partial u}+\frac{\partial}{\partial u} \log \frac{b}{x} \frac{\partial \theta}{\partial v},
$$

if the equation of the corresponding net on $Q$ is the second of (33). 
12. If a permanent net on $Q$ admits a transformation $F_{k}$ by means of a set of functions $x^{\prime}, y^{\prime}, z^{\prime}, h, l$, the corresponding net on $\bar{Q}$ (cf. Fx. 11) admits a transformation $F_{\hat{k}}$ determined by

$$
\begin{gathered}
\bar{x}^{\prime}=-\frac{1}{\sqrt{e \bar{e}}} \frac{x^{\prime}}{x}, \quad y^{\prime}=\sqrt{\frac{\overline{-f}}{e f}}\left(y^{\prime}-\frac{x^{\prime} y}{x}\right), \quad \overline{z^{\prime}}=\sqrt{\frac{\overline{-g}}{e \bar{g}}}\left(z^{\prime}-\frac{x^{\prime} z}{x}\right), \\
\bar{h}=h x-x^{\prime}, \quad \bar{l}=l x-x^{\prime}, \quad \bar{k}=\frac{\bar{e}(e-k)}{e} .
\end{gathered}
$$

13. If $\hat{\Gamma}$ is a $B_{k}$ transform of a net $N$ applicable to $Q$ by means of functions $x^{\prime}, y^{\prime}, z^{\prime} ; x^{\prime \prime}, y^{\prime \prime}, z^{\prime \prime} ; h_{1}, l_{1} ; h_{2}, l_{2}$, the functions analogous to those of Ex. 12 determine a $B \bar{k}$ transform of $\bar{N}$ applicable to $\bar{Q}$, and this transform and $\hat{N}$ are in relation $H$.

Bianchi, l. c., p. 231.

14. Show by means of $[\S 12 \pi]$ that the pencils of planes whose axes are polar with respect to a quadric meet the latter in an isothermal-conjugate net.

Bianchi, l. c., p. 244.

15. When a quadric $Q$ is referred to an isothermal-conjugate net, so also is a quadric conjugate in deformation to $Q$, and $\bar{H}_{0} \sigma^{3}=H_{0} \sigma^{3} c$, where $c$ is a constant.

16. In order that a net $S$ on a quadric be permanent in more than one deformation of the quadric, it is necessary that $N$ be isothermal-conjugate.

Servant, Bull. Soc. de France, vol. 30 (1902), p. 21.

17. If ${ }^{\circ} N_{1}$ and $N_{2}$ are isothermal-conjugate nets on a central quadric $Q$ (34) obtained from an isothermal-conjugate net on $Q$ by transformations $K_{k}$ and $K_{k}$; $\left(k^{\prime} \neq k\right)$ of $\S 148$, there can be found directly a net $N_{12}$ on $Q$, which is a $K_{k^{\prime}}$ transform of $N_{1}$ and a $K_{k}$ transform of $N_{2}$; it is determined by the functions $\theta_{1}^{\prime \prime}$ and $\theta_{2}^{\prime}$ given by $(78)$ and

$$
k \theta_{1}^{\prime \prime}+k^{\prime} \theta_{2}^{\prime}=k \frac{\theta_{2}^{\prime \prime} \theta_{1}}{\theta_{2}}+k^{\prime} \frac{\theta_{1}^{\prime} \theta_{2}}{\theta_{1}} .
$$

18. Derive the equations of transformations $K_{k}$ of isothermal-conjugate nets on a paraboloid similar to those for a central quadric $\$ 148$.

19. If a ruled surface $S$ is subjected to an infinitesimal deformation which leaves the surface ruled and through each point of $S$ and in the corresponding tangent plane a line is drawn perpendicular to the direction of the deformation, these lines form a $W$ congruence for which the other focal surface is ruled; this construction gives the most general $W$ congruence with ruled focal surfaces and generators corresponding. Bianchi, Comptes Rendus, rol. 143 (1906), p. 635.

20. If the focal surfaces, $S_{1}$ and $S_{2}$, of a $W$ congruence are ruled, and if the curved asymptotic lines of $S_{2}$ correspond to the generators of $S_{1}$, then $S_{1}$ is a quadric.

Segre, Atti di Torino, Dec. 28, 1913;

Tortorici, Rend. di Napoli, vol. 28 (1922), Jan. 21. 


\section{Index}

The numbers refer to pages.

Adjoint equation, 9, 22, 88, 93.

Angle, of two lines 1 ; of two hyperplanes, 2; of two spheres, 234; of two surfaces in pentaspherical coordinates, 282.

Applicable nets, definition, 61 ; parallel to given pair, 62; transformations $F$ of, $61,275,290,325,334$; and congruences of circles, 272; and cyclic systems, 274 ; which are $O, 286$; which are $2, O, 298$. See Nets $C$ and Nets permanent in deformation.

Asymptotic lines, definition, 98; parametric, 99,123 ; in Lie line-sphere transformation, 252; determination of, 356 . Axis curres, definition, 125 ; property of, 126 ; form a net, $125,153$.

Axis congruence, definition, 125.

Beltrami, 272, 299, 310, 312, 314.

Bianchi, 112, 138, 142, 143, 180, 209, $210,212,214,215,216,229,230,292$, $298,301,305,308,310,312,316,317$, $320,327,344,345,352,356,360,362$, $363,366,371,372$.

Bonnet, 295, 317.

Calapso, 225, 230, 232, 286, 298, 319, $320,331$.

Calo, 318.

Chieffi, 316.

Christoffel transformations of isothermic surfaces, 229, 286 ; and transformations $D_{m}, 229$; nets determined by, 319 ; of special isothermic nets, $230,291$.

Circles $\boldsymbol{K}$, definition, 202 ; normals to nets at points of, 204; of the first kind, 206; of the second kind, 206, 229; form a congruence, 2933.
Circle-planes of a transformation $k$, definition, 198; envelope of, 199, 213, 285 , 286; pass through a point, 200 , 230 ; coincident for two transformations, $206,211$.

Congruence, definition, 11; developables of, 11; focal points of, 11; focal surfaces of, 11; conjugate to a net, 12, 15,34 ; direction equation of 13,153 ; parallel, 15; middle surface, $16,21,32$; larmonic to a net, $22,24,25,29,80$, 81, 153; harmonic to a point net, 28; conjugate, of a transformation $F, 34$; harmonic, of a transformation $F, 40$, 153; conjugate to $O$ nets, 167 ; normal to a net, 168.

Congruences I and $p$, I, definition, 15s; 2,I, normal, 159, 294; nets conjugate to, $159,168$.

Congruence $K$, definition, 203; cyclic, 206 ; focal points of, 229 .

Congruence $R$, definition, 113 ; focal surfaces. 115 .

Congruences of circles, definition, 241; characteristic property, 243; focal spheres of, 241 ; focal points of, 243 ; in cartesian coordinates, 243 ; transformations $F$ of, 269,270 ; parallel transformations of, 272 ; a cyclic system, $272,274,278,285$; envelope of planes of, $258,259,269,270,271,274$.

Congruence of rolling, 316; focal surfaces of, 317; developables of, 317; with coincident developables, 317; parallel, 321 ; normal, 321 ; with constant focal segment, 321; pseudospherical, 321. 
Congruences of spheres, definition, 238; principal curves of, 238 ; focal circles of, 239,241 ; chords of contact of, 239 ; lerived, 240, 243; in cartesian coordinates, 243 ; mull-splieres, 243 , 284; with applicable central nets, 271 ; cutting a fixed sphere under constant angle, 283.

Congruences $R$ of spheres, definition, 246 ; parallel, 246; transformations $F$ of, 246; direction-equation witl equal invariants, $25 \overline{5}$; representation in 5-space, 285; permanent in deformation, 298, 308,310 .

Congruences of spheres and circles, harmonic, 255-259, 264, 265, 267, $269-271$; conjugate, 261-263, 270; orthogonal, 266, 267, 285.

Conics, pencil of, 95 .

Coordinates, cartesian, 1; lomogeneous point, 69; tangential, 97, 166, 286; of a sphere, 233; pentasplerical, see Pentaspherical.

Cosserat, 206.

Curve, definition, 2, 71; parametric, 2.

Cyclic congruences, definition, 183; parallel, 191; characteristic property, 280, 285; generation of, 289; multiply, 185, 188, 191.

Cyclic systems, definition, 183; circles pass through a point, 191 ; of a trans. formation $R, 197,206,285$; generation of, $272-274,285,289$; transformations $F$ of, 275, 277, 278, 290; representation in 5-space, 277-279; envelope of planes of, 199, 213, 285, 286.

Cyclides, 283.

Darboux, 6, 92, 147, 154, 208, 212, 213, $239,254,274,281,282,283,285,288$, $291,295,313,317,358$.

Deform of a quadric, which meets the circle at infinity in 4 points, 291; of revolution, 293-296, 305, 360; tangent to the circle at infinity, 317 ; permanent nets on, $326-329,353 ; R$ nets on,
327, 328; transformations $F_{k}$ of, $334-340,346$, 353, 354; transformations $B_{k}$ of, $340-352,354-356$; transformations $H$ of, $366,371,372$.

Demoulin, 107, 108, 112, 113, 115, 119, $124,134,150,202,204,220,225,229$, $254,284$.

Derivant net, definition, 25 ; of two transformations $F, 54$; of two transformations $K, 61$; of two transformations $R, 265$. Derived congruences, definition, 21; of a $W$ congruence, 108; of spheres, $240,241$.

Derived net, definition, 25; Laplace transforms of a, 32, 33; parallel, 33, 53; transformations $F$ of, 54,66 ; of two transformations $F, 54$; reciprocally, 116 ; which is $O, 289$.

Developable surface, definition, 10 ; of a congruence, 11.

Dini, 362.

Direction-parameters, of a line, 1; of a congruence, 12, 14, 20, 30; of a cyclic congruence, 183, 281.

Drach, 1 อ5.

Envelope of rolling, definition, 305; lines of curvature, 320 .

Envelopes of spheres, with $O$ nets corresponding, 174, 189, 229; conformal mapping of the two sheets, 206, 286; principal curves on, 238, 239, 243; congruence of chords of contact, 239 , $241,242,244,245,258,259,262,285$; with applicable central nets, 272, 274, 298, 302, 307, 308, 310; cutting a fixed sphere under constant angle, 283; with spherical lines of curvature, 285.

Equation, point, of a net, 3, 72; direction, of a congruence, 13, 153; tangential, of a net, 128, 153.

Focal circles of a congruence of spheres, $239,241$.

Focal nets of a congruence, 11, 15; of parallel collgruences, 21 ; radial transforms, 33 ; in relation $F, 34$. 
Focal points, of first and second rank, 11 ; coordinates of, $10,14,29$; of a ray congruence, 124; of a congruence of circles, 245.

Focal spheres of a congruence of circles, 241; meeting orthogonally, 183.

Focal surfaces of a congruence, 11, 15. Green, 124, 125, 126.

Guichard, 5, 12, 26, 124, 156, 165, 200, $255,258,262,263,266,280,285,295$, $296,337$.

Hammond, 77, 85, 87, 93, 94.

Homogeneous point coordinates, 69 ; of a net in 2-space, 96 ; of a net in 3-space, 101, 103; of an $R$ net, 107.

Hyperplane, definition 2; at infinity, 69 . Hyperquadric, net on, 90,254 ; transformations $F$ of nets on, 90, 94 .

Hyperspheres, in transformations $R, 171$, 174, 189; focal, 183, 185.

Invariants of an equation of Laplace, definition, 16 ; equal to zero, 73,92 ; in a sequence of Laplace, 75, 92.

Inversion, in transformations $R, 175$; of a sphere, 281; in pentaspherical coordinates, 281; of a cyclide, 283.

Isothermal-conjugate nets, definition, 104; preserved by projective transformation, 104; $R$ nets are, 107; on a quadric, $112,367,372$; and nets with equal point invariants, 125,150 ; characteristic property, 126 ; on a deform of a quadric, 326 .

Isothermic $O$ nets, definition, 206; special, $212-214,291$; are nets $\Omega, 231$; in pentaspherical coordinates, 237; Lie transformation of, 284 ; nets with the same spherical representation as, 302-304, 318,319 ; spherical representation of, 319 . See Transformations $D_{m}$ and Curistoffel trausformations.

Isotropic, relation, 157; line, 289, 291, 296; plane, 289, 296.

Jonas, 42, 47, 110, 113, 119, 201.

Kinematically conjugate directions, definition, 314; axes of rotation, 315; auto-conjugate, 315, 320; for congruences of rolling; 317,321 ; parametric, 320 ; orthogonal, 320 .

Koenigs, 58, 123.

Laplace equations, 3,12 ; invariants of, 16; seqnence of, 74; Moutard form, 237. Laplace transforms, definition, 17 ; in cartesian coordinates, 17; of parallel nets, 18 ; of a function, 19, 32 ; of a deriver net, 32, 33; of a radial trausform, 33; trausformations $F$ of, 66,94 ; in homogeneous coordinates, 72 ; of a net $R$, 108; in tangential coordinates, 129; which are $O$ nets, $192,193,286$. See Sequences of Laplace and Levy sequences.

Lery sequences, of the first order, 81, 93 ; of higher order, $83-85$, 93 ; periodic, 85,86 .

Levy transforms, definition, 19 ; of parallel nets, 32; of radial transforms, 33; and transformations $F, 66$; in homogeneous coordinates, 81 ; in a hyperplane, 81 . Lie, 249, 283, 284.

Lie line-sphere transformation, equations of, 249,283 ; linear complex of, $249,284$. Lie transformations of surfaces, definition, 252 ; equations of, 283,284 ; W congruence of, 253.

Linear complex, definition, 248; null-plane of, $248,251,252$; special, 248; lines coljugate with respect to, 250, 251, 284 ; tangents to a curve lines of a, 284.

Martiu, 32.

Minimal surfaces, transformations $D_{m}$ of, 215,217 ; $O$ net of, 229; focal sheets of a $W$ congruence, 230 ; in relation $R$. 296, 208; deformable transformations $R$ of, 304, 305 .

Nets, definition, 3 ; characteristic property, 4 ; tallgent planes of, 4 ; point, 5, 28, 30; in 3-space, 6, 100-103, 105; parallel, $7-12$, 124; planar, $9,96,123$; colljugate to a congruence, 12, 14, 19, 32,159 ; harmonic to a congruence, 22 , $24,27,30,34,200$; of translation, 32, 
66 ; minimal. 32,66 ; in relation 0,63 , 146. 154; in homogeneous coordinates, 72 ; periodic, 76, 94 ; in tangential coordinates, 127, 128; of plane curves, 152. See Applicable nets, Derivant nets, Derived nets, Isothermal-conjugate nets.

Nets corresponding with orthogonality of linear elemeuts, 63, 146; parallel, 64; transformatious $F$ of, $64-66$.

Nets $C$, definition, 200; harmonic to a normal congruence, 200 ; on the envelope of planes of a cyclic system, 200 ; congruences harmonic to, 287, 288. See Applicable nets.

Nets $G$, definition, 192; parallel, 192; Laplace transforms of, 192,286 ; radial transforms of, 193; transformations $F$ of, 193.

Nets $O$, definition, 156, 194; conjugate to congruences I, 2, I and $3, \mathrm{I}, 160$; determination of, $165-167$; parallel, 167 ; on a hypersphere, 167 ; congruences conjugate to, 168 ; transformations $F$ of, $168-175,185-188$; radial transformations of, 175; inversion of, 175; transformations $R$ of, 174-182, 195-197; on a lypercone, 191, 283; with isothermal spherical representation, 215-218, 229; in pentaspherical coordinates, 237, 279; in 5-space and cyclic systems, 277 ; generated by a rolling surface, 289, 291, 294; derived, 289. See Isothermic $O$ nets.

Nets 2, $O$, definition, 156 ; as cential nets of transformations $R, 171,189$; special, 188, 228; transformations $F$ of, $189-191$; on quadrics of revolution, 247 ; associated with cyclic systems, 279 ; applicable, 298.

Nets $p, O$, definition, 156 ; conjugate to congruences, $p, \mathrm{I}, 160$; parallel, 191.

Nets permanent in deformation, definition, 139 ; fundamental coefficients, $139-140$; transformations $\Omega$ of, $141-146$; special,
142 ; spherical representation of, 154 , 155.

Nets $R$, definition, 107; equations, 107 ; Laplace transforms of, 108 ; on a quadric, 112, 367; reciprocally derived, 118; transformations $W$ of, 115-123, 125; transformations $F$ of, 124, 125; and nets $\Omega, 254$; in line coordinates, 255 ; with tangents in one family meeting a fixed line, 284; on deforms of a quadric, $327,328$.

Nets $\Omega$, definition, 218 ; equations of, 219 , 220 ; parallel, 221 ; transformations $R$ of, 221-224; isothermic, 231; with isothermal spherical representation, 231 ; on surfaces of Guichard, 225, 232; and nets $R, 254$.

Nets with equal point invariants, 16, 32, 67, 68, 95, 106, 125; characteristic property, 124 ; associate, 59,67 ; planar, 123; and equal tangential invariants, 150 ; which are $O$ nets, 206 ; on a quadric, 366-368. See Transformations $K$.

Nets with equal tangential invariants, definition, 128; which are permanent in deformation, 139; and equal point invariants, 150; characteristic property, 153 ; on a quadric, $366-368$. See Transformations $\boldsymbol{Q}$.

Nets with same spherical representation as isothermic $O$ nets, 302,303 ; transformations $R$ of, 303, 319, 320 .

Normal parameters of a net, 5 .

Osculating planes of a net, 11 ; conjugate to a congruence, 23 ; and Laplace transforms of a derived net, 32 ; in homogeneous coordinates, 71 ; and nets $G_{0}$, 286.

Orthogonal determinant, definition, 163; in 5-space, 165, 279; in 3-space, 194. Parallel, lines, 1; hyperplanes, 2; nets, 7-12, 32; congruences, 15, 28.

Pentaspherical coordinates, of a point, 235; fundamental spheres, 235, 231; equation of a sphere in, 236, 281; 
special, 237; of an $O$ net, 237, 279; of an isothermic net, 237; of a point of the plane at infinity, 281; angle between displacements in, 282 ; equation of a surface in, 283 ; transformations $R$ in, 283.

Perpendicular, lines, 1 ; hyperplanes, 2. Plane, in cartesian coordinates, 2; tangent, of a surface, 3 ; tangent, of a net, 4 ; in homogeneous coordinates. 71 .

Plücker line coordinates, 247.

Point equation of a net, in cartesian coordinates, 3 ; in homogeneous coordinates, 72.

Point nets, definition, 5; congruences harmonic to 28,29 ; harmonic to a congruence, 30 .

Polar transformation, 100, 125, 153.

Poles of a circle, definition, 266; points on an envelope of spleres, 266, 274: of a cyclic system, 285, 286.

Principal curves on an envelope of spheres, $238,239,243$; form a net, 245.

Problem of order $n-2,165$.

Projective transformations, of space, 70; of nets in relations $F, 93$; of nets in 2-space, 97 ; of asymptotic lines, 100 ; of nets in 3-space, 103, 128; of isothermal-conjugate nets, 104 .

Quadrics, isothermal-conjugate nets on, 112 , 366-370, 372; nets $R$ on, 112, 367 ; osculating, 124; of revolution, 247 , 293--296, 305, 337, 360; deformation of, $291,293,305,326,360$; rolling, 291-296, 317; tangent to the circle at infinity, 317; transformations $F$ of, $322,329-334,352,353,368$; permanent nets on, 323-326, 329-334, 353, 370,372 ; referred to generators, 323-325; conjugate in deformation, 365,371 ; nets with equal point invariants on, 367 , 368 ; nets with equal tangential invariants on, 367,368 ; trausformations $W$ of, $368-370,372$. Quatern of transformations $F^{\prime}$, definition, 48 .
Radial transformations, definition, 31 ; harmonic congruences of, 31 ; of Laplace transforms, 33; of Levy transforms, 33; and transformations $F, 40-42,67$, 153; of every net on a surface, 124; of nets $O, 175$; of nets $G_{0}, 193$.

Ray congruence, definition, 105; focal points, 124.

Ray curres, definition, 105; property of, 124.

Relation, $F, 34 ; K, 58 ; O, 63,146$; $R, 174$; isotropic, 157.

Ribaucour, 16, 89, 174, 239, 245, 285. 286, 312, 323.

Rolling surfaces, definition, 288; plane of contact, 288 ; and cyclic systems, 289 ; on a quadric, 291-296, 317; on a surface of revolution, 293-298, 321; kinematically conjugate directions on, 314,320 ; ruled, 314, 315; axes of rotation, 315; on a sphere, 317 ; spheres associated with, 318 .

Ruled surfaces, referred to asymptotic lines, 123; osculating quadrics of, 124; rolling, 314; applicable to a non-ruled surface, 314,316 ; applicable, 320 ; infinitesimal deformation of, $372 ; W$ congruences whose focal surfaces are, 372 . Segre, 372.

Sequences of Laplace, definition, 74; equatious of $75,76,92$; invariants of, 75, 76, 92; periodic, $76-80,93,94$; derived nets of, $83-85$, 93; transformations $F$ of, 94.

Servant, 325, 359, 362, 363, 365, 372.

Spheres, coordinates of, 233; point-, 234; null-, 234, 289, 294; power with respect to, 234; equations in pentapherical coordinates, 236; inversion of, 281 ; rolling, 317. See Congruences of spheres. Stetson, 286.

Surface, definition, 2 ; tangent planes, 3 ; determination of uets on, $5-7 ; R, 111$; of Voss, 143 ; determination of asymptotic lines on, 356-358; element, 252. 
Surfaces applicable to a quadric, sce Deforms of a quadric.

Surfaces applicable to a surface of revolution, 293-298, 321; complementary, 292, 294-296, 321; conjugate in leformation, 371.

Surfaces conjugate in deformation, definition, 360; cliaracteristic property, 362 ; letermination of, $362-365,371$; quadrics, $365,372$.

Surfaces of constant mean curvature, transformations $R$ of, $229,295,310$; special isothermic nets on, 230; generation of, 294,317 ; kinematically conjugate lines on, 320 .

Surfaces of constant total curvature, equations of, 232; transformations $R$ of, $232,295,339$; cyclic systems associated with, 286; determination of 360 .

Surfaces of Guichard, definition, 225, 232 ; associated surfaces, $225,228,232$; are surfaces $Q, 225,232$; transformations $R$ of, $227,231,232,286$; isothermic surfaces associated with, 286.

Surface of rolling, definition, 301; one sheet of envelope of congruence $R$ a, 302 ; equations of, 313 ; lines of curvature on, 320 .

Tangential coordinates, of a surface, defiuition, 98; referred to asymptotic lines, 100 ; of a net, 127; Laplace transforms in, 129; transformations $F$ in, $129-134,153$; of a net $O, 166$; transformations $R$ in, 178-182.

Terracini, 370.

Theorem of permutability, of transformations $F, 45-51,90,132$; for parallel nets, 50, 67; extended, 55-57, 68, 224,230 ; of $W$ congruences, 124,138 , 143. For references to the theorem of permutability of any type of transformation see this transformation.

'Tortorici, 372.

Transformations $B_{k}$ of surfaces applicable to a quadric, $340-346,354-356$; permutability witl transformations $F_{k}$, 346,371 ; theorem of permutability of, $352,371$.

Transformations $D_{m}, 208,209,231$; theorem of permutability of, $209-211$, 215,230 ; of special isothermic surfaces, $212-214,230,291$; of minimal surfaces, 215,304 ; isothermic nets determined by, 230 ; parallel nets of, 230 ; characteristic property, 285; induced, 286, 319 ; generation of, 291 ; and surfaces of rolling, 301, 302; deformable, 304. Transformations $E_{m}, 215-217,231$; theorem of permutability of, 218,230 ; characteristic property, 230 ; deformable, 305 ; and envelopes of rolling, 305 .

Transformations $\boldsymbol{F}$, equations in cartesian coordinates, $34-37$; conjugate congruence of, $34,42,89$; inverse of, 37 ; parallel, 38, 50, 67; harmonic congruence of, 40, 182; and radial transformations, 40-42, 67, 153 ; triads of, 44,90 ; theorem of permutability of, $45-51,55-57,67,90,132$; of derived nets, 54, 66; of applicable nets, 61-63; of nets in relation $O, 64,146,154$; of Laplace transforms, 66,94 ; of Levy transforms, 66; in homogeneous coordinates, 87-91; of nets on a hyperquadric, 90,94 ; projective transformations of, 93 ; of periodic Laplace sequences, 94 ; of nets $R, 124,125$; in tangential coordinates, 129-134, 153; and polar transformations, 153 ; of nets $O, 168-175,185-188$; of nets $2, O$, $189-191$; of nets $G, 193$; of nets on a sphere, 197, 217; of congruences $R$ of spheres, 246; of congruences of circles, 269-271; of cyclic systems, $274-278,290$; of deformable transformations $R, 306$; of nets on a quadric, 322,323 ; of isothermal-conjugate nets on a quadric, 368, 372. References to particular types of transformations $F$ are given under these transformations. 
Transformations $F_{k}$, of nets on a quadric, $329-334,353$; of surfaces applicable to a quadric, 334-339, 354; theorem of permutability of, $323,339,340$.

Transformations $H$ of surfaces applicable to a quadric, definition 366 ; of ruled deforms, 366 ; permutability with transformations $F_{k}, 366,372$; permutability with transformations $B_{k}, 372$.

Transformation, $\theta_{1}$, definition, 113 ; of surfaces of constant curvature, 124; of isothermal systems on a sphere, 124 .

Transformations $K$, definition, 58 ; in cartesian coordinates, $57-59,67$; theorem of permutability of, $60,61,67,68,154$, 372; of associate surfaces, 68,124 ; of Levy transforms, 68; in homogeneous coordinates, 94,95 ; characteristic property, 95 ; of nets with equal point and equal tangential invariants, $150-152$; polar transformations of, 153 ; with normal conjugate congruences, 206,221; of nets on a quadric, 368-370, 372 . See Transformations $D_{m}$.

Transformations $R$, in cartesian coordinates, 173-177, 195; central nets, 174, $189,196,228$; of parallel nets $O, 174$, 196, 228; inverse of, 178 ; in tangential coordinates, 178,181 ; theorem of permutability of, $180-182,202-206$; of Laplace transforms of a net $G, 193$; spherical representation of, 197; cyclic systems of, 197, 206, 285; circle-planes of, see Circle-planes; circles $K$ of, see Circles $K$; congruences $K$ of, see Congruences $K$; conformal, 206 ; of isothermic $O$ nets, see Transformations $D_{m}$; of minimal surfaces, 215, 296, 304, 305; of nets $\Omega, 221-224$; of surfaces of Giuchard, 227, 231, 232, 286; with conformal spherical representation, 215, 230,306 ; of surfaces of constant mean curvature, 229, 295, 310; of surfaces of constant total curvature, 232,295 , 339 ; in pentaspherical coordinates, 283 ; deformable of the first type, 296, 308, 310 ; deformable of the second type, 298-306; with applicable central nets, 297,302 ; of nets with the same spherical representation as isothermic $O$ nets, 303, 319 ; and surfaces of rolling, 302,318 ; and envelopes of rolling, 305 ; transformations $F$ of, 306 .

Transformations $W$, of nets $R, 115-123$, 125 ; of isothermal-conjugate nets on a quadric, 370 ; of ruled surfaces, 372 . See Transformations $B_{k}$.

Transformations $\Omega$, in tangential coordinates $134-136$; theorem of permutability of, 137, 138, 153, 154; of nets permanent in deformation, 141-146; in point coordinates 146-149; of a focal surface of a $W$ congruence, 149, 150; of nets with equal point and equal tangential invariants, $150-152$; polar transformation of, 153.

Tzitzeica, 33, 77, 93, 95, 107, 116, 124, $327,368,370$.

Umbilical points of a quadric, 291.

Virtual asymptotic lines, definition, 316 : determination of, 358 .

$W$ congruences, definition, 106 ; of tangents to a net $R, 107,113$; derived, 108 ; and reciprocally derived nets, 116; quatern of, 124, 138, 143; with focal surfaces of equal curvature, 138,142 , 143 ; transformations $\Omega$ and $K$ of, 150 ; with minimal focal surfaces, 230 ; whose lines belong to a linear complex, 252, 253 ; in Lie transformations of surfaces, 252,253 ; representation in 5 -space, 254 ; in Plücker line-coordinates, 254 ; normal, 292,293 ; with ruled focal surfaces, 372 . Wilczynski, 105, 123, 125, 126. 


\section{Erratum}

\$59. Change "focal nets" to "focal surfaces". 




\section{PLEASE DO NOT REMOVE CARDS OR SLIPS FROM THIS POCKET UNIVERSITY OF TORONTO LIBRARY}

PraA Sal 


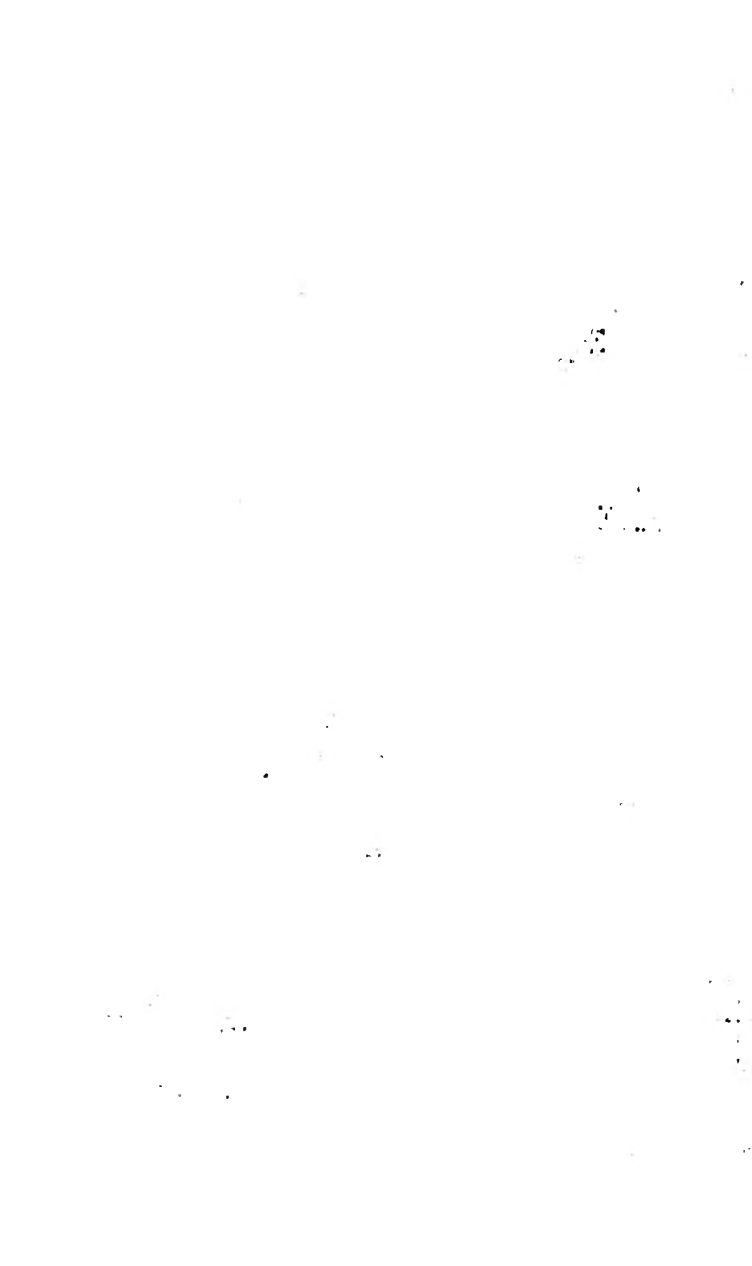

